\title{
Being Outside and Inside: Dialogic Identity and Intercultural Communication through Drama in Teaching English as an International Language
}

The copyright of this thesis rests with the author.

No quotation from it should be published without

his prior written consent and information derived

from it should be acknowledged.

Yoon-Jeong Choi

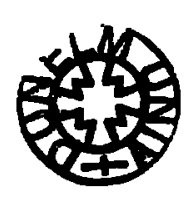

1

A Thesis Submitted for the Degree of Doctor of Philosophy

\author{
School of Education \\ The University of Durham
}

2003 


\section{Declaration}

I declare that this thesis, which I submit for the degree of Doctor of Philosophy at the University of Durham, is my own work and is not the same as any which has previously been submitted for a degree in this or another university.

\section{Copyright by Yoon-Jeong Choi}

The copyright of this thesis rests with the author. No quotation or data from it should be published without her prior written consent and information derived from it should be acknowledged. 
I have asked myself what the word "culture" actually means to me in the light of the different experiences I have lived through, and it gradually becomes clear that this amorphous term in fact covers three broad cultures: one which is basically the culture of the state; another which is basically that of the individual, and then there is a "third culture"....

For the "third culture" is the culture of links. It is the force that can counterbalance the fragmentation of our world. It has to do with the discovery of relationships where such relationships have become submerged and lost - between man and sociely, between one race and another, between the microcosm and the macrocosm, between humanity and machinery, between the visible and the invisible, between categories, languages, genres. What are these relationships? Only cultural acts can explore and reveal these vital truths. 


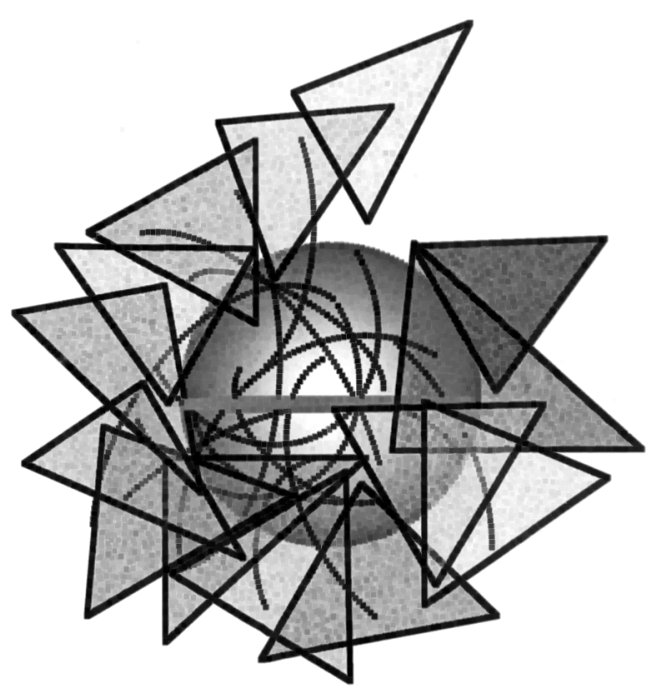

Life by its very nature is dialogic.

To live means to participate in dialogue: to ask questions, to heed, to respond, to agree, and so forth.

[Bakhtin (1984) Problems of Dostoevsky's Poetics: 293]. 
Being Outside and Inside: Dialogic Identity and Intercultural Communication through Drama in Teaching English as an International Language

By Yoon-Jeong Choi

Abstract

Intercultural communication (ICM) through drama is explored through six central concepts: ICM, otherness, outsidedness, duality, dialecticality, and dialogicality. These concepts are examined in the context of intercultural drama in teaching English as an international language (EIL). The characteristics of intercultural drama are presented in relation to those concepts; the dynamics of intercultural drama are examined in dialogical-cum-cyclical perspective; drama is identified as an arena for participants to interact with dialogic identity in ICM.

Chapters One and Two discuss the current problem in teaching English as a foreign language in Korea and the position of EIL in a global context.

Chapter Three discusses how drama can be applied to ICM in two ways: one is that drama can deliver rich contexts in teaching and learning EIL and ICM; the other is that drama can provide socio-cultural and intercultural practices more appropriately than any other forms. Here I highlight the dynamic of dialecticality, dialogicality, and in a cyclical process by exploring otherness and outsidedness. Also, I present an integrated approach through drama to ICM.

Chapter Four addresses the research method to be applied to this study, where I emphasise the multiplicity of drama activity and its relevant research methods in exploring the dynamics of intercultural drama. I suggest SPAR (students in participatory action research) and PAR (participatory action research), which are combined with grounded theory. Drama is also used with the above methods, and the ATLAS.ti programme is applied to data analysis in this research.

Chapters Five and Six present what I found through my research project with reference to ICM through drama: first, participants' drama experiences are artistic, therapeutic, and socio-cultural/intercultural; second, the dynamics through intercultural drama are dialectical, dialogical, and cyclical. Students gained an improved understanding of their own responses to ICM and deeper awareness of their own and other cultures.

Chapter Seven discusses the conclusions to be drawn and recommendations in summary: [1] intercultural education is vital for social inclusion/integration; [2] intercultural drama is a valuable means of changing the young person's sensitivity to the global world to improve their ICM; [3] drama educators or intercultural educators should improve their professional competences in teaching and develop more imaginative approaches to the curriculum. 


\section{Acknowledgements}

Like other $\mathrm{PhD}$ research students,

I had only 24 hours a day.

Like other $\mathrm{PhD}$ research students,

I took a demanding PhD course.

Although I was far away from my hometown,

I had to keep moving forward.

However, I learned how to put through this,

From everyone, every object, and every place around me,

I was neither a spiritualist nor an animist, though.

Whoever I was with,

Whatever I was by, and

Wherever I was,

They have guided me,

They have taught me, and

They have kept me peaceful.

Here, I dare to call names as representatives of all-

My mother,

My supervisors, Prof. Michael Byram and Dr. Michael Fleming, and

The Durham Cathedral.

At long last, it is time to return to them:

First, they gave me an assignment of a PhD;

Now, I will do my further assignment for them.

I should like to thank all of you. 


\section{CONTENTS}

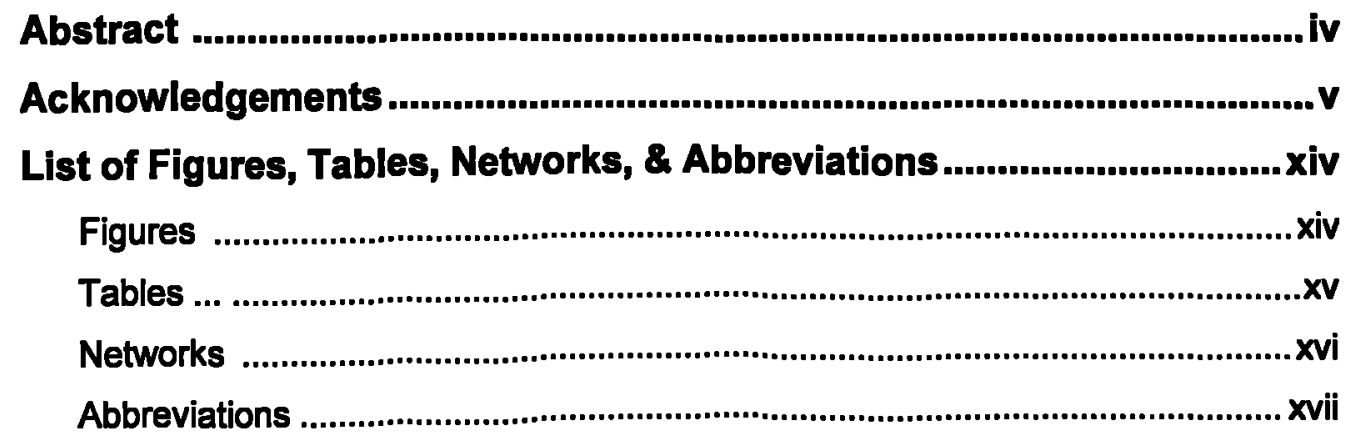

\section{PART I. INTRODUCTION}

Chapter 1. Introduction ............................................................2

1.1 Statement of Current Problems ......................................................2

1.2 Specific Practical Problems in Intercultural Education in Teaching English as an International Language (EIL)...................3

1.3 Introducing My Empirical Research.............................................4

1.4 Summary: EIL learners and Drama .............................................6

PART II. THE ENGLISH LANGUAGe AND INTERCULTURAL COMMUNICATION THROUGH DRAMA

Chapter 2. The English Language and ICM (Intercultural Communication) ...................................................8

2.1 Teaching English as an International Language (EIL) for ICM (Intercultural Communication) .....................................................10

2.1.1 The English Language in a Global Context ...................................... 10

2.1.2 Situational Teaching and Learning EIL.............................................. 15

2.1.3 Multimodal Communication in Situational Teaching and Learning

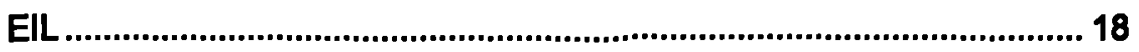

2.1.4 ElL Leamers as Critical Ethnographers .............................................20

2.2 ElL Learners to be Intercultural Actors ........................................22 
2.2.1.1 Relations between Culture, ICM, Individuals, and Society. 23

2.2.1.2 The Self-Others Relations to ICM: Othemess in Symbolic Interactionism.

2.2.1.3 The Self-Identity Relation to ICM: Otherness \& Outsidedness in Dialogism.

Language as Social Communicative Process.................................................. 30

The Dialogic or Interlocutive Self and All the Others........................................ 30

Open Otherness: Dialectical and Dialogical........................................................ 32

Outsidedness for Creative Understanding of the Self.......................................... 33

Thirdness between the Centre and the Not-Centre ......................................... 35

2.2.2 The Framework of ICM for EIL Learners ............................................. 39

2.2.2.1 Factors of ICM (Intercultural Communication) ............................................39

2.2.2.2 Structure and Process of ICM: Triadic, Third and Spiral............................42

2.2.2.3 Pathway to Intercultural Actors with Dialogic Identity................................... 47

2.3 Summary: ElL Learners to Intercultural Actors ............................51

\section{Chapter 3. Drama and its Approach to ICM (Intercultural Communication) ...................................................53}

3.1 Drama and its Double with ICM..................................................54

3.1.1. Art and Life: Figure and Ground of Drama ........................................ 54

3.1.2 Drama as Cultural Configurations ....................................................... 56

3.1.3 Situatedness: Situated Learning by Doing through Drama.................... 59

\subsection{Drama Activity and ICM (Intercultural Communication)...............61}

3.2.1 The Total Imaginative Activity through Drama in Intercultural Discourses.....................................................................................61 61

3.2.2 Juxtaposition in the Duality of Drama .................................................68

3.2.3 The Dialectic in the Duality of Drama.................................................... 71

3.2.4 Distancing and Defamiliarisation to Outsidedness and Reflexivity in Drama.

3.3 Drama Approaches to ICM (Intercultural Communication)

3.3.1 Approaches to Interculturalism in the Contemporary Theatre and Drama Education....................................................................................... 80

3.3.2 Integrated Approach to ICM through Drama in Schools ....................... 82

3.4 Summary: Dual, Dialectical, and Dialogical to Holistic .................85 


\section{Part III. Practicum: Methodology and Findings}

Chapter 4. Research Methods ..................................................88

4.1 Overview of the Research .....................................................................88

4.1.1 Parallelism in the Research Method .................................................. 88

4.1.2 Drama: Action Learning by Situatedness.......................................... 92

4.1.3 Intercultural Interactions through Drama.............................................. 93

4.1.4 Interest in the Effectiveness of Drama ................................................95

4.2 Research Design .................................................................................96

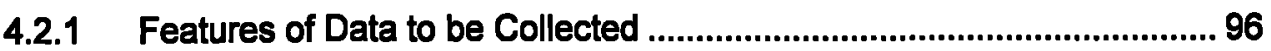

4.2.1.1 Ethnographic Data: Intercultural Interaction through Drama.......................96

4.2.1.2 ICM through Drama in Dramaturgical Perspective .................................... 98

4.2.2 Research Paradigm for Social Interaction/ICM ................................... 99

4.2.2.1 Socio-Cultural Literacy through Action Research ......................................99

4.2.2.2 The Aims of the Literacy in Action Research .........................................99

4.2.2.3 The Cycle of Action Research for the Literacy....................................... 102

4.2.3 The Less Illiterate, the More Literate through Drama .......................... 104

4.2.3.1 Our Illiteracy through Action Research in Socio-Cultural Context........... 104

4.2.3.2 Grounded Action Research is more Literate.......................................... 105

4.2.3.3 Drama as a Research Tool for ICM .................................................. 107

4.2.4 Students in Participatory Action Research with Drama ...................... 108

4.3 Contexts of the Research ................................................................ 109

4.3.1 Construction of the Project Group...................................................... 110

4.3.1.1 The Project Group: Participants as Co-Researchers................................ 110

Representativity of the Project Group .............................................................. 110

Backgrounds of the Project Group....................................................................111

The Perspective of the Project Group ....................................................................... 112

4.3.1.2 The Teacher as Researcher .................................................................. 113

4.3.1.3 Observers ................................................................................................ 114

4.3.2 Situational Contexts of the Researchers............................................ 114

4.3.2.1 The Environment inside the School: the School, the Teachers, and the Curriculum .............................................................................................. 114

4.3.2.2 the Environment outside the School: the Local Educational Council and the Community.................................................................................... 115

4.3.3 The Natural History of My Research Study......................................... 117

4.3.3.1 My Achilles' Heel in Teaching English as a Foreign Language: the Personal Context of the Research Topic .................................................. 117

4.3.3.2 Long Joumey into Drama Education: Trials and Errors ............................ 118

4.3.3.3 My Springboard for Education through Drama in Korea ......................... 119 
4.4.1 Students, Observers, and Me in Participatory Activity ........................ 120

4.4.2 Procedures and Arrangements ...................................................... 120

4.4.3 Action and Research in the Pilot Study.......................................... 121

4.4.4 Report on the Pilot Study ................................................................ 122

4.4.5 Implications for the Main Fieldwork.................................................. 124

4.5 Data Collection ........................................................................ 125

4.5.1 Instrumentation for Data Collection.................................................. 127

4.5.1.1 Field Diary ................................................................................................. 127

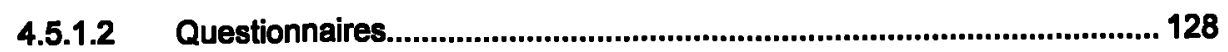

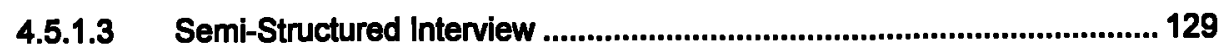

4.5.1.4 Self-Questioning Form............................................................................ 129

4.5.1.5 Observation Checklist......................................................................... 130

4.5.2 Drama Sessions ................................................................................ 131

4.5.3 Procedures of Data Collection ........................................................ 134

4.5.3.1 Stage 1: Preparation for the Main Fieldwork........................................... 134

4.5.3.2 Stage 2: Process-Centred Drama I.......................................................... 134

4.5.3.3 Stage 3: Process-Centred Drama II......................................................... 135

4.5.3.4 Stage 4: Product-Centred Drama ............................................................. 136

4.5.3.5 Stage 5: Review and Publication .......................................................... 136

4.5.3.6 Completion ............................................................................................. 136

4.5.4 Management of Data Collection: Triangulation ................................... 137

4.5.4.1 The Macro Level: Researcher in PAR (the BGCHS project)................... 139

Data Collected from the Reseancher Myself ................................................... 139

Data Collected from Student Participants by the Researcher............................. 140

Data Collected from Observers and Others by the Researcher........................ 141

4.5.4.2 The Micro Level: Students in PAR (the EDIE Project) ............................ 142

Data Collected from Students as Researchers................................................ 142

Data Collected from Students as Participants.................................................. 142

Data Collected from Non-Participants by Student Researchers ....................... 142

4.5.4.3 Triangular Data in the Study................................................................ 144

Tripodic Data Collection: Methodological Triangulation ...................................... 144

Triplex Data Collection: Combined Levels of Triangulation.................................. 145

Trilateral Data Collection: Investigator Triangulation ..........................................145

Triform Data Collection: Form Triangulation ...................................................... 146

4.6 Data Analysis .......................................................................... 147

4.6.1 Drama and its Analysis .................................................................... 148

4.6.1.1 Contemporary Trends in Data Analysis of Drama .................................... 148

4.6.1.2 Limitations of Previous Data Analyses of Drama ....................................... 149 
4.6.2 Approach to Drama Data Analysis in the Study.

4.6.2.1 GTA (Grounded Theory Analysis) and Drama ...................................... 151

Relationship between GTA and Drama .......................................................... 151

Objectives of GTA with Drama Data................................................................. 153

Principles of GTA and Drama ...................................................................... 154

4.6.2.2 Multiple Triadic Approach to Data Analysis of Drama............................ 157

Formulation between Triangulation, Limitations of Drama Research, and Integrated Approach to Drama ...................................................................... 158

Tripodic Data Analysis in Methodological Triangulation.................................... 158

Triplex Data Analysis at Combined Levels of Triangulation ............................... 158

Trilateral Data Analysis in Investigator Triangulation ......................................... 159

Triform Data Analysis in Form Triangulation................................................... 159

4.6.3 General Procedures to Analyse Data................................................. 159

4.6.3.1 Step 1: Preparative Activities for Data Analysis.................................... 160

4.6.3.2 Step 2: A Starting Point for Analysing Data ......................................... 160

4.6.3.3 Step 3: Arrangement of my Data .......................................................... 161

4.6.3.4 Step 4: Developing Coding Procedures................................................ 161

4.6.3.5 Step 5: Theoretical Sampling............................................................ 161

4.6.3.6 Step 6: Theoretical Saturation ......................................................... 162

4.6.3.7 Step 7: Evaluating Emergent Theories ................................................ 162

4.6.4 Management of Data Analysis .................................................. 162

4.6.4.1 Manual Data Analysis and its Limitations ............................................ 163

Using Computer Aided Qualitative Data Analysis Software
(CAQDAS) and ATLAS.ti Programme ................................................... 163

4.7 Trustworthiness: Confirmability and Dependability .................... 164

4.8 Summary: Metadrama, SPAR, and GTA .....................................1 166

\section{Chapter 5. Findings A: Drama Experiences in ICM} (Intercultural Communication) .........................168

5.1 Artistic Experience in ICM: Drama as an Art Form .................... 169

5.1.1 Expressing One's Own Emotions in Doing Drama: Knowing

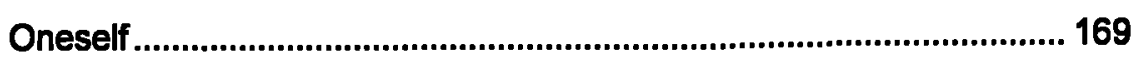

5.1.2 The Total Imaginative Activity in Doing Drama: Making Oneself......... 173

5.2 Therapeutic Experience in ICM: Drama as a Reflexive Tool ...... 177

5.2.1 Drama is Emancipatory............................................................... 177

5.2.2 Drama is Self-Reflexive................................................................. 179

\subsection{Socio-Cultural Experience in ICM: Drama as Cultural}

Morphology 
5.3.1 Drama to Individualise in a Whole Socio-Cultural Context 184

5.3.2 The Drama World and Everyday Life 190

5.4 Summary: Total Imaginative Activity through Drama-

Artistic, Therapeutic, and Socio-Cultural 202

Chapter 6. Findings B: Dynamics of Drama in ICM (Intercultural Communication)

6.1 Duality of Drama Actions in ICM 204

6.1.1 The Duality of Actuality and Theatricality in Bi-Dual ContextsEveryday Life, the EDIE Project, Drama Sessions, and Drama

Activities 205

6.1.1.1 Interaction between Contexts of Drama, Drama Sessions, and Everyday Life.

6.1.1.2 Interaction between Contexts of Drama, the EDIE Project, and Everyday Life

6.1.2 The Duality of Production and Perception in Bi-Dual Contexts-

Everyday Life, the EDIE project, Drama Sessions, and Drama

Activities 210

6.1.2.1 Production and Perception at the Extroversive Level of a Participant in Drama 210

6.1.2.2 Production and Perception at the Introversive Level of a Participant in Drama

6.2 Dialecticality of Drama Actions in ICM

6.2.1 Dialecticality between Everyday Life, the EDIE Project, and Drama

Sessions

6.2.1.1 Participants in Everyday Life-Co-Researchers in the EDIE Project [Play A] .

6.2.1.2 Participants in Everyday Lifo-Participants-in-Role in Drama Sessions [Play B's T].

6.2.2 Dialecticality in the Dual Drama Structure [Play B in Play A] in ICM ... 222

6.2.2.1 Sub-Text, Text, and Meta-Text in Participants' Drama Writing.................222

6.2.2.2 Co-Researchers [Play A's T], Performers [Play B's R], and Characters [Play B's T]

6.2.2.3 Reception and Production of Participants as Spectators in Play B's $R$ and $T$.

6.3 Dialogicality of ICM in Drama Activities .236

6.3.1 Dialogicality of ICM in Drama Activities 236

6.3.1.1 Dialogicality in the Extroversive Interaction of Participants with Intercultural Factors in Drama Activities 
6.3.2 Otherness and Outsidedness: Dialogicality of Participants in

Drama Activities

6.3.2.1 Otherness: Extroversive Dialogicality-Reflection of CoResearchers on the Event of 'English Zone'.

6.3.2.2 Otherness: Introversive Dialogicality-Self-Reflection of Performers through Characters in the Play, War Paint.

6.3.2.3 Outsidedness: Extroversive and Introversive Dialogicality-

Reflexivity of Participants in Drama activities of the Intemational

Business Meeting.

6.4 Cyclical Process with Dialectical and Dialogical Interactions

in ICM through Drama

6.4.1 Dialectical Cycles in Drama Activities 271

6.4.2 Dialogical Spirals in Drama Activities 275

6.5 Summary: Dual, Dialectical, Dialogical, and Cyclical 283

\section{PART IV. CONCLUSION}

Chapter 7. Conclusion and Discussion...............................286

7.1 Conclusion: Drama as an Arena of ICM.................................. 286

7.1.1 Drama and its Effectiveness .............................................................. 286

7.1.2 Reflections on the Study: Active, Reactive, \& Proactive...................... 291

7.2 Implications: Drama and its Holistic Effectiveness in the

National Curriculum

7.2.1 Cross-Curricular or Integrated Projects of Drama in Intercultural Education with Subjects

7.2.2 Drama in Intercultural Education with Ethics 294

7.3 Recommendations: Intercultural Education through Drama

for National and World Citizenship

7.3.1 Ethical Issues-ICM in Intercultural Discourses and the Hidden Curriculum surrounding Young People

7.3.2 Dialogic Identity through Drama—ICM into Integration/Inclusion in Balanced Intercultural Discourses. 
Bibliography ......................................................................300

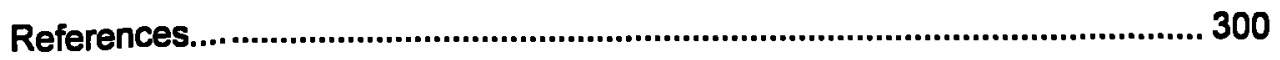

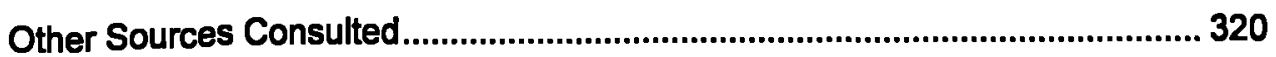

Appendices .............................................................................333

Appendix A: Q1 (ASQ) and Q2 (Post-Questionnaire)....................................... 333

Appendix B: Self-Questioning Form (an Example) ............................................ 350

Appendix C: Observation Checklist ................................................................ 351

Appendix D: Drama Sessions in the Study ..................................................... 352

Appendix E: Photographs of Drama Activities ................................................ 353

Appendix F: Previous Researches on the Effectiveness of Drama Education .... 356

Appendix G: How to Read Networks of ATLAS.ti ............................................. 357 


\section{List of Figures, Tables, and Networks}

\section{Figures}

Figure 2.1 Factors and Progresion of ICM in the Spiral Curriculum for ICP................... 46

Figure $3.1 \quad$ Rubin's Double Profiles .................................................................................55

Figure 3.2 The Infinity Loop between Social Drama and Aesthetic Drama...................72

Figure 4.1 The Spiral of Action Research Cycles ...................................................... 103

Figure 4.2 Key Concepts in GTA(grounded theory analysis) ..................................... 155

Figure 5.1 Still-Image of a Japanese Firm (4 April 2001)............................................ 192

Figure 5.2 Still-Image of an American Firm (4 April 2001) ....................................... 192

Figure 5.3 Marked Still-Image of a Japanese Firm (4 April 2001)................................ 193

Figure 5.4 Marked Still-Image of an American Firm (4 April 2001) ............................. 193

Figure 6.1 Duality of Participants' Drama Experiences: Actuality and Theatricality in Contexts, Bi-Dual and Intercultural ...........................................................209

Figure 6.2 Duality of Participants' Drama Experiences: Production-and-Perception Cycle in Bi-Dual Contexts ...............................213

Figure 6.3 Being Dialectical in the Dual Attitudes to English
(Students' Uneasiness vs. Co-Researchers' Willingness) ...........................216

Figure 6.4 Being Dialectical in the Cultural Clash of KimJA (Performers' Korean Emotion vs. Character's American Action on 4 April 2001) ........................218

Figure 6.5 Dialecticality between Sub-Text and Text in an Extract of the Intemational Business Meeting

Figure 6.6 Otherness from Dialogicality, Extroversive and Introversive, in Drama Activities

Figure 6.7 A Dialectical-cum-Dialogical Circle (DDC) and its Cone-Shaped Framework of Dialectical Dialogicality in Drama Dynamics throughout the EDIE project

Figure 6.8 The Cyclical Process for Dialectical Resolutions between Two Different Factors through Drama

Figure 6.9 The Simplified Dialectical-cum-Dialogical Circle (DDC) of the ConeShaped Framework in Dynamics of Drama ..................................... . . 282

Figure 6.10 The Spiral Cycles with Dialectical Dialogicality through Drama .... .. .. 282 


\section{Tables}

Table 2.1 Stages in the Development of Self-Awareness in Meadian

Perspective.

Table 2.2 Comparison of the Concept, Sociological Dialogue, between Mead's and Bakhtin's Perspectives.

Table 4.1 Analogy of the TV series Columbo to the Research Study. 91

Table 4.2 Research Questions and their Foci ....................................................... 126

Table 4.3 Procedures of Data Collection ..................................................................... 138

Table 4.4 Dual Triangulation of Data Collection ........................................................... 143

Table 5.1 Comparison of Individualising the Situation between $\mathrm{OHHJ}$ and KimHJ_Double Casting........................................................................... 186

Table 5.2 Comparison between Two Still-Images of Figures 5.3 and 5.4................. 194

Table 5.3 General Evaluation of the EDIE Project by Participants ............................. 196

Table 6.1 The Cyclical Process of Dialectical Resolutions in Characterising 'Mum' in the English Play, War Paint, at the Fifth Saturday Meeting ......... 274 


\section{Networks}

Network 5.1 Feeling through Consciousness into Total Imaginative Activity (Artistic Experience through Drama) by the Semantic Layout

Network 5.2 Therapeutic Experience through Drama to KimMS by the Semantic Layout.

Network 5.3 KimJA's Individualising the Dating Situation in Intercultural Context by the Semantic Layout.

Network 5.4 KimJA's Individualising the Dating Situation in Intercultural Context by the Topological Layout

Network 5.5 Resolution for ElL Education and the English Zone in the Projects by the Semantic Layout.

Network 5.6 Resolution for EIL Education and the English Zone in the Projects by the Topological Layout

Network 6.1 Dialecticality between a Participant in her Real World and her Drama World in ICM by the Semantic Layout

Network 6.2 Dialecticality between a Participant in her Real World and her Drama World in ICM by the Topological Layout.

Network 6.3 Dialecticality of MunJM between a Performer and its Character in ICM by the Semantic Layout.

Network 6.4 Extroversive Dialogical Interactions at the Stages of the Task for the English Zone by the Semantic Layout

Network 6.5 Extroversive Dialogical Interactions at the Stages of the Task for the English Zone by the Topological Layout.

Network 6.6 External Factors against the Room for the Club EDIE in the School by the Semantic Layout.

Network 6.7 External Factors against the Room for the Club EDIE in the School by the Topological Layout

Network 6.8 Rod [Cross-Character to RyuKW] by the Semantic Layout ........................ 258

Network 6.9 Greg [Cross-Character to KimMS] by the Sementic Layout 258

Network 6.10 Comparison Activity of Co-Researchers at Level 2 Sensibility by the Semantic Layout

Network 6.11 Dialogicality of MunJM as the Japanese President in the Drama Event of the Intemational Business Meeting by the Semantic Layout

Network 6.12 Dialogicality of MunJM as the Japanese President on a Korean President in the Drama Event of the Intemational Business Meeting by the Semantic Layout

Network 6.13 Dialetical-cum-Dialogical Circle: Reflexive Dialogicality through Outsidedness of Participants in the Event of the Intemational Business Meeting through Drama by the Semantic Layout.

Network 6.14 The Cyclical Process with Dialectical Dialogicality in the Drama Event of the International Business Meeting through Drama by the Semantic Layout.

Network 6.15 The Cyclical Process with Dialectical Dialogicality in the Drama Event of the Intemational Business Meeting through Drama by the Topological Layout 


\section{Abbreviations}

* In alphabetic order

\begin{tabular}{ll} 
AR & Action Research \\
ASQ & Analytic Surveying Questionnaire [Q1] \\
BGCHS & Busanjin Girls' Commercial High School \\
CAQDAS & Computer Aided Qualitative Data Analysis Software \\
DDC & Dialectical-cum-Dialogical Circle \\
EDIE & Educational Drama in English \\
EFL & English as a Foreign Language \\
EIL & English as an International Language \\
ESL & English as a Second Language \\
GTA & Grounded Theory Analysis \\
ICC & Intercultural Communicative Competence \\
ICM & Intercultural Communication \\
ICP & Intercultural Competence \\
PAR & Participatory Action Research \\
Play A's R & Realism in Play A \\
Play A's T & Theatricalism in Play A \\
Play B's R & Realism in Play B \\
Play B's T & Theatricalism in Play B \\
SI & Symbolic Interactionism \\
SPAR & Students in Participatory Action Research \\
SQF & Self-Questioning Form \\
\hline
\end{tabular}




\section{PART I.}

\section{INTRODUCTION}




\section{Chapter 1. \\ Introduction}

Internationalisation and globalisation have been affecting people's lives in every country on the globe, in their daily living as well as political and economic systems. This whole phenomenon links with 'the increasing interdependence of world society' (Giddens 1997: 65).

People want to understand other cultures by reducing culture shock, which implies that the experience of a new culture is an unpleasant surprise or shock, partly because it is unexpected and partly because it may lead to a negative evaluation of one's own culture' (Furnham and Bochner 1986: 47). It is said that 'globalisation has not produced a unified world, and many societies in the world co-exist with various cultural factors and conflicts that differ from their own (Giddens 1997: 65).

\subsection{Statement of Current Problems}

In order to reduce social divisions and conflicts and to have a better understanding of other cultures as well as our own culture, our society needs to take intercultural communication, or interaction, into consideration in the global world.

Therefore, educators and teachers, who take responsibility for teaching the young generation cultural specificity, or their own identity in each cultural area, should also consider encouraging them to study at school and live in society as preparation for the future, that is, for the more globalised world.

Moreover, it seems to be important for them to communicate with people in intercultural situations at the crossroads of cultures. Therefore, the objectives of intercultural education seem to be founded on understanding their own culture as well as others, but on the basis of cosmopolitanism rather than nationalism. With regard to this standpoint of intercultural education, I would like to mention two perspectives as a starting point in this study: one is that education is regarded as a cultural system and at the same time involves the formation of the cultural system; the other is that education should be concerned for the young generation, who live with the, not static but changing, cultural system, and should provide for them in the multicultural society and for the global world. 
However, at this stage, the first thing to be considered is that education is related to its milieu wherein there is a multitude of discourses, socio-cultural, economic, and political. First of all, I would like to explain the Korean educational situation. Secondly, I am going to raise an important issue relevant to teacher education concerning intercultural and drama education in the present educational milieu in South Korea. This issue could be considered in other educational situations and in other countries, as well.

\subsection{Specific Practical Problems in Intercultural Education in Teaching English as an International Language (EIL)}

There has been a tendency for educators as well as educational policy-makers to pay little attention to intercultural education in the National Curriculum in Korea.

Moreover, educational policy-makers and scholars disagreed in the 1990s whether it is appropriate or not that English is taught as a subject in the National Curriculum at the primary level. Now, pupils from the fourth grade in the primary schools learn English as a foreign language in a form of bilingual education.

However, there remain problems at both primary and secondary level, regarding teaching methods and intercultural education. I wonder whether Korean teachers of English pay attention to teaching something beyond language in their English classroom, in other words, teaching language in relation to its culture. Also, I wonder whether English should still be taught as a foreign language or rather as an international language, thirdly there are arguments about the ways there are to teach English in schools. As for me, I believe drama can provide language teachers with a good teaching method, whereby cultural dimensions can be added, because drama has been already applied as an art form, a learning medium, a socialising activity, or a therapeutic tool in various areas including the education field.

In spite of the various applications of drama, education in Korea has not paid attention to drama apart from being taught as an optional subject at the secondary level in the National Curriculum. However, there still remain problems in teaching drama to students at that level in Korea; for example, there are neither textbooks nor appropriate drama teachers. Although some teachers try to use drama in their classrooms, drama is taught as the subject of drama per $s e$, but it should be included in other subjects such as Language Arts, Literature, and EIL (English as an international language). 
In this regard, I propose to argue that native or non-native teachers of English, who have neglected drama activities or plays, should consider introducing drama to their students like other genres, and integrating it into other subjects or educational activities.

In short, I intend also to argue that we should consider making educational policies for teaching EIL and drama education with reference to intercultural education for the younger generation, who live with internationalisation and globalisation.

\subsection{Introducing My Empirical Research}

In this study, I am going to explore an effective method of intercultural education in order to make the young generation prepare for a society, that is multicultural, various, decentralised, and post-modern. I intend to investigate how teachers can be involved in intercultural education through drama in teaching EIL at the secondary level. My intention here is that, firstly, EIL should be taught in intercultural contexts, in other words, for intercultural communication, secondly, drama can be effective in introducing language and culture simultaneously to teaching of EIL, and thirdly, drama should be applied with intercultural imagination, which I adapt from 'sociological imagination,' in an integrated way (Mills 1970: 7).

From this viewpoint, I have the following research questions in this study.

\section{What Kinds of Role can Drama Take in Teaching and Learning ElL?}

Drama education has four different perspectives in introducing drama into the educational milieu: an art form, a learning medium, a socialising activity, and a therapeutic tool. These perspectives are complementary to each other, not separate from each other. $I$ intend to investigate how we can apply drama to teaching EIL in order to develop the intercultural communication of EIL learners.

\section{Can Drama be Effective for Intercultural Education?}

Drama provides students with different cultures in their authentic fullness and the Cultural Mazeway,' which leads to intercultural experiences (Seelye 1997: 97). They understand their own culture as well as other cultures through drama in the Cultural Mazeway. I intend to investigate how participants produce the total imaginative activity through intercultural imagination in drama. The intercultural imagination will be related to three levels of experience: sensation, sensibility, and sensitivity. 


\section{Why can Drama be Effective for Developing Intercultural Communication?}

Drama by its nature, and particularly, its duality, generates dynamics, such as dialecticality, spectatorship, self-spectatorship, distancing (otherness), and dialogicality, and consequently provides EIL learners with better intercultural communication (ICM). Therefore, I focus on ICM rather than on intercultural competence and intercultural communicative competence. This is because the first is within the process of intercultural experiences, whereas the last two are the product of intercultural experiences and the last is more emphasised on linguistic competence than socio-cultural competence. In this regard, I intend to investigate how participants experience intercultural situations and achieve ICM through drama.

\section{How do Participants do Drama Activity in the Intercultural Context?}

I intend to observe and analyse ICM (intercultural communication) through drama of participants, on the basis of a symbolic interactionism and dialogic perspective. But the main focus is on how participants communicate or act the total imaginative activity in their intercultural drama contexts.

\section{Do Participants take Roles as Intercultural Actors with an Intercultural Identity?}

I will identify changes of their identity level from mono-cultural attitudes into intercultural identity. At the end of this research project, I hope to confirm how participants change in terms of ICM and ICP (intercultural competence). However, I shall not produce the size of the quantitative effect in the level of their intercultural sensitivity because the key point in this study is how rather than how much drama is effective for developing ICM and ICP. In other words, I am going to identify the ways in which participants of this project are able to behave adequately and in a flexible manner in intercultural settings and how both adequacy and flexibility help them to develop intercultural awareness. The conclusive point is that through drama activity participants develop ICM sufficiently to become intercultural actors with ICP, which is capable of 'stabilising one's self-identity in the process of cross-cultural mediation, and of helping others to stabilise their self-identity' (Meyer 1991: 137).

\section{The Procedure}

In order to examine my research questions, I will explore three key concepts: 'ICM,' 'duality of drama' and 'drama experience.' I plan to investigate how participants cope with their drama activities in relation to ICM in learning EIL at the secondary level. Also, $I$ intend to draw conclusions about how drama can be effective in developing the ICM and ICP of EIL learners. 
The first chapter introduces the study. The second chapter surveys the status of English in a global context, the interrelation between an individual EIL learner and the global society in relation to ICM, and the role of education for members of a society in the intercultural dimension.

In the third chapter, I will explore what characteristics of drama are related to ICM, how participants are involved in ICM through drama and how teachers, including drama teachers, apply drama to their classroom activities.

In the fourth chapter, I address the research method to be applied to this study, wherein I identify the limitations of textualism or logocentrism in the information society and emphasise the multiplicity of drama activity and its relevant research methods in exploring the dynamics of intercultural drama. Regarding the aforementioned research method, I discuss the function of students as co-researchers in this study-SPAR (students in participatory action research), and the software for data analysisATLAS.ti, which can analyse qualitative data to visualise and integrate those textual, graphical and audio data.

In the fifth and sixth chapters, I present what I found throughout my research project with reference to ICM through drama: participants' drama experiences are artistic, therapeutic, and socio-cultural/ intercultural in the fifth; the dynamics through intercultural drama are dialectical, dialogical, and cyclic in the sixth.

In the seventh chapter, I present the conclusion. This leads me to extend the discussion into my view of directions needed in intercultural education, intercultural education through drama, an integrated approach to ICM through drama, and finally, the need to improve teaching methods and teaching programmes, by dint of reconstructing the curriculum, National as well as Hidden.

\subsection{Summary: ElL Learners and Drama}

There is a need to reconsider the status of EFL in educating the young generation in the fast-globalising world and its teaching methods should be reconsidered in terms of EIL.

In consideration of this, I will argue English should be related to plurilingual education and intercultural discourses and drama can be an effective method for intercultural education in the contexts. 


\section{Part II.}

\section{The English Language and Intercultural Communication through Drama}




\section{Chapter 2.}

\section{The English Language and ICM (Intercultural Communication)}

Since the 1960s, we have seen two enormous impacts: one is 'the Third Wave,' which is designated as the impact of computers and technologies on our daily life by Alvin Toffler (1980); the other, 'the Fourth Wave,' is the impact of multiculturalism (Phillion 1999: 129). Both affect people in daily life.

For instance, 'cyberspace,' seems a simple technological product, but it prevents the binary relationship between space and time from continuing as it has been. We do not need to consider where and when in cyberspace in the present as much as we did in the past (Toffler 1980). This means two contemporary concepts, spatiality and temporality, are different from those conceptualised in the past; globalising processes make those concepts more compacted. Space is not static and 'space-time compressions are many and varied' in the processes of trans-national and internationalised conditions' (Usher 2002: 50). That is to say, it enables for example English people to communicate with antipodal people, such as Koreans or Brazilians, and permits them to access and exchange data at the same time.

Under the influence of technologies and multiculturalism, there is a multitude of phenomena that we meet in the 'traditional' and the 'modern' in daily life. For instance, Korean teenagers are embarrassed with clashes between cultural values learnt from their parents and popular culture from Internet or mass media. Moreover, there are various global factors between the 'East' and the 'West,' by which the 'modern' can be sometimes replaced. These phenomena lead them metaphorically to the negotiating table in order to explore their differences or to relieve tension between them, to have a better understanding.

With regard to the contemporary social and global situations, how can educationists prepare their young people to participate effectively in this world? In other words, how can educationists educate their young people in order to live with others in those situations?

Regarding these questions, firstly, we should consider that there is a multitude of cultures, which have their own unique cultural values in the world. Therefore, it is 
desirable 'to avoid ethnocentrism, which is judging other cultures by comparison with one's own' in intercultural situations [author's bolds](Giddens 1997a: 24). Secondly, we should regard the globalising process as 'a form of institutionalization of the twofold process involving the universalization of particularism and the particularisation of universalism' (Robertson 1997: 77). Thirdly, this globalising world is 'characterized by human diversity, cross-cultural interaction, dynamic change, and global interdependence' (Merryfield 1995: 1). Judging from these points, intercultural education seems to be relevant to ethnorelativism and the mutual process between the local and the global culture.

It is clear that we should consider as a basis for intercultural education the fact that schools interact with, influence and are influenced by the broader society, i.e., the globalising world. Therefore, we can raise further specific questions from the standpoint of schooling:

-What can teachers select for intercultural education in a fastglobalising world?

- What kind of criteria can they use for the selection?

- How can they teach their students?

The first two questions have been already researched in terms of ICM, ICP, intercultural sensitivity, intercultural identity, and so on, in various perspectives. Nevertheless, the last question is not explored sufficiently enough, for instance, for many teachers who rely on their traditional teaching methods such as delivering pieces of information in their classrooms. Therefore, I shall focus on the how question, in relation to intercultural education, to introduce drama in ICM in this study. In the first place, I will review theoretical underpinnings and search for appropriate answers to this question in Chapters 2 and 3, and explore the answers in practice in Chapters 4, 5, and 6.

The present chapter will review the nature of communication through EIL (English as an international language), and ICM, and the discussion will lead to ICM through drama

This chapter will be presented in two sections. In the first, I shall discuss learning and teaching EIL. The discourse will show what role the English language can take in communication among people who have different mother tongues and what factors should be regarded for better communication. 
Second, I shall present a framework of ICM to help me deal with my research questions. My intention here is to point out what factors we could take into more consideration in ICM; for instance, the relationship of the self and all others. Also, I shall draw on approaches to intercultural education, which considers how to improve intercultural competence (hereafter interchangeable with ICP).

The following discussion emphasises psychological and sociological dimensions of ICM in the matters of otherness and outsidedness. The discussions are based on symbolic interactionism and Bakhtinian concepts.

\subsection{Teaching English as an International Language (EIL) for Intercultural Communication (ICM)}

\subsubsection{The English Language in a Global Context}

There is a multitude of cultures in the world and every culture has its own unique patterns of behaviour, which seem strange to those who have different cultural identities. Similarly, there is a multitude of languages and every language has its own unique patterns, which seem to be a foreign language for those who are non-native speakers.

With regard to this diversity of culture and language, first, I wondered how we can understand each other in a fast globalisng world, second, how we could communicate with those who speak in different languages, and third, how we could treat the English language, which is internationally dominant in the contemporary world. In the present section, I intend to discuss the last two questions, whereas the first question will be treated in the approach to drama in the next section.

In the matter of contemporary linguistic discourses in a global context and the globalising process, on one hand, the role of English is changing from EFL or ESL into an International Language (EIL) (Said 1983; Day 1985; Kachru 1992; Phillipson 1992; Pennycook 1994, 2001; Smith 1999). On the other hand, plurilingual education and World Englishes are becoming a focus of interest for policy-makers and educators in foreign language education (Jarvis 2000; Graddol 2001; Byram 2002; Brutt-Griffler 2002; Block and Cameron 2002; Council of Europe 2002; Bourne and Reid 2003).

Foreign language education or English language education is becoming a main issue in the globalising process and is under the influence of the globalising process, which is regarded as 'the universalisation of particularism and the particularisation of 
universalism' (Robertson 1997: 77). For instance, Pennycook points out that English is such a predominant linguistic paradigm in the world that the language can be inextricably bound up with the world. He describes its global predominant spread as 'English is in the world and the world is in English' (Pennycook 2001: 78).

Scholars discuss the spread of English in the globalising process from three different critical viewpoints: monolingual reductionism, arguments for linguistic diversity, and argument against diversity.

The first viewpoint is based on 'monolingual reductionism,' which rationalises the reduction of the number of languages, for instance, 'one state establishes one nation wherein the people speak one language.' Skutnabb-Kangas explicates the ideology of monolingual reductionism as follows:

\begin{abstract}
At an intra-state level, the ideology of monolingual reductionism is connected with and supports the mythical homogenous nation-state.... At a global level, it is connected with the purported desirability of an efficient global market place, of a global information society, and of international cooperation and peace... Monolingual reductionism is characterized by several myths which glorify monolingualism, stigmatise multilingualism and/or real linguistic diversity, and rationalise linguistic homogenisation.... These claim or imply that monolingualism at both the individual level and the societal level is normal, desirable, sufficient and inevitable (unavoidable) [author's bolds] (SkutnabbKangas 2000: 238-239).
\end{abstract}

In the above perspective, the English language among big languages was chosen as thelan official language in 45 countries in 1992 (Krauss 1992) and in over 70 countries in 1997 (Crystal 1997). That is, English as the official language is used in more than half of the countries in the world until 1997. Then I am wondering how many countries have joined this group since 1997. According to the British Council (1995), 94.4\% of respondents accept that English will retain its role as the dominant language in world media and communications. However, we need to consider, above all, the claim that monolingualism is unavoidable. According to Skutnabb-Kangas (2000), at an individual level, monolingualism by the English language results from a wrong educational policy, and an ideology of linguicism. At a societal level, monolingualism is a social construction, which can prevent political and economic global development, justice, equity, cooperation and democracy.

Moreover, from a social structuration viewpoint of monolingualism by the English language, monolingual reductionism, at the end, leads to the concept of linguistic imperialism whereby there is a strong interrelationship between the English language and the global world in that English is the most powerful language spoken throughout 
the world. Furthermore, English is the official language of the leading countries in the world. Day (1985) and Auerbach et al. (1996) argue that this leads to linguistic genocide by English of other languages in the world because of its prevalence.

In even stronger terms, Phillipson, in his book, Linguistic Imperialism, criticises the global spread of English and English Language Education. He regards linguicism as discrimination, analogous to sexism and racism, because of the language one speaks. From this standpoint, he defines English linguistic imperialism as follows' 'the dominance of English is asserted and maintained by the establishment and continuous reconstitution of structural and cultural inequalities between English and other languages' [author's italics] (Phillipson 1992: 47).

In relation to this concept of English linguistic imperialism, there is a fact that 'the dominance of English is therefore not only a result of politico-economic inequalities between the center and periphery; it is also a cause of these inequalities' [author's italics](Canagarajah 1999: 41). Therefore, there is little choice but to accommodate this linguistic imperialism. This structuralist determinism is true in practical terms.

In response to these negative aspects of linguicism, linguistic genocide and linguistic imperialism from monolingual reductionism (Barnet and Cavanagh 1994) and structuralist determinism (Day 1985 and Phillipson 1992), there is a counter-argument for linguistic diversity.

Let me take an example as Dascal's arguments for linguistic diversity. This perspective is based on the argument that one's language or the mother tongue is connected with identity development in that it functions as a central cultural core value to co-create the Self and others in us. Second, we should preserve the various linguistic and cultural heritages, which exist in the world from the viewpoint of human rights awareness.

Dascal (1996) illustrates his claims for multilingualism with arguments presented in terms of practical evidence, and ethical, ontological, epistemological and cognit ve points. For instance, he shows three pieces of practical evidence: one is that communication across languages is possible; another is that translation, albelt imperfect, is possible, and the other is that there is no reason to fear Babel an confusion.

However, we cannot ignore our reality and practicality of linguist c discourses in the world in the present and in the future. As for the former, we have to cope with lingu stic 
imperialism whereby ' $90 \%$ of today's languages might be extinct or "on the death row" in 100 years' time' (Skutnabb-Kangas 2000: 82). As for the latter, we should consider the cost and efficiency of languages as 'our most cost-effective communication tools' (Skutnabb-Kangas 262). In addition to the above two, we need to cons der a th rd standpoint between monolingual reductionism and linguistic diversity, which takes advantages from both. This is a resistant perspective to linguistic imperialism. That is to say, we acknowledge there is English linguistic imperialism, but need to res st the imperialism (Pennycook and Canagarajah).

Pennycook acknowledges English linguistic imperialism by arguing that 'English is in the world and plays an important role in the reproduction of global inequalities' (Pennycook 2001: 86). Equally, he asserts the importance of acknowledging culture and discourse as principal factors in understanding the global world. This is based on avoiding, above all, the aforementioned two perspectives, monoligual reductionism and structuralist determinism.

There is no doubt that English as an international language (EIL) is connected with international discourses. This is because English and international discourses facilitate each other: the spread of English accelerates global power discourses in the matters of democracy, capitalism and modernisation, and vice versa (Pennycook 2001). In this regard, we need to acknowledge the connection between the spread of English and its international discourse and to realise their important relation.

However, English linguistic imperialism, that is, the symbolic power of Englsh, remains. The power of the English language is connected with those international discourses, because the discourses of Anglophone economic-politics prevail. In this regard, it is doubtful that we can exclude discourses from EIL. This can lead to structuralism determinism in terms of linguistic discourses, in which the Engl sh language is predominant. Because of this, we need to resist linguistic Imperialism, for instance, resisting Linguistic Imperialism in teaching EIL (Canagarajah 1999).

In spite of his criticising structuralist determinism, such as Phillipson's argument, in the spread of English, Pennycook (2001) acknowledges inequalities in the spread of EILa structural reproducer of global inequalities, which is situated in linguistıc discourses at the global level, and a producer of inequality in the learner's own linguistic values, which the learner himself produces. Along with Canagarajah, he is, however, concerned with how to resist those inequalities-counter-discourse: 
As long as English remains intimately linked to the discourses that ensure the continued domination of some parts of the globe by others, an oppositional programme other than one that seeks only to limit access to English will be necessary. .. counter-discourses can indeed be formed in English and that one of the principal roles of English teachers is to help this formulation (Pennycook 2001: 87).

There are several possibilities for resisting linguistic imperialism and for forming counter-discourses in teaching EIL to rectify those inequalities. This perspective draws attention to critical ethnographic methods in teaching English in that these methods focus on culture and discourse and consider the behaviour and attitudes of English learners evident in their social interactions (Canagarajah 1999).

Along with ethnographic methods, 'talking back' can make those possibilities into practicalities (Hooks 1989: ii). Distancing, decentring, and dialogic work are also beneficial in being an intermediator or an intercultural actor in intercultural settings. 1 shall discuss this further with reference to the framework of ICM in 2.2.

With regard to what I have discussed so far, language teachers should consider different foci of EFL and EIL, global inequalities and the language of English classrooms: how they can conceptualise the inequalities in teaching EIL, and how they can teach the dominant language but resist linguistic imperialism (Canagarajah 1999; Pennycook: 2001).

To put it briefly, the aims and objectives of language education in the context of EFL are to attain the same level of language competence and cultural competence as Anglophone native speakers, with respect to grammar, pronunciation, gesture, facial expression and so on. Therefore, ideal teachers are regarded as Anglophone native speakers. On the other hand, the aims and objectives of EIL education are to improve the communicative level in international or intercultural situations and focus on interacting with people of various cultures rather than being focused on the English language itself. Therefore, learning EIL aims to acquire the model of international English by having a better intercultural understanding of EIL and cultures in global discourses. In the end, ideal teachers of EIL are regarded as English teachers, who have the same linguistic/cultural identity as students have and are able to interact appropriately, rather than English teachers of Anglophone native speakers.

After all, the spread of EIL along with rapidly globalising processes has increased the speed of multiculturalism, mentioned as 'The Fourth Wave,' and vice versa. 
This global phenomenon has led to teaching EIL for intercultural communication. Its basic paradigm should be the ways in which learners use EIL appropriately in intercultural interactions with all the others in this global world. Furthermore, in interacting with those who have different linguistic and cultural identities, we need to stand back from international discourses as well as our own discourses to be more objective in order to have a better understanding of the world.

\subsubsection{Situational Teaching and Learning EIL}

As I discussed, we need to connect schools with their broader society, i.e., the globalising world for intercultural education.

Because of the linguistic intercultural discourse relevant to EIL, language teachers of EIL need to consider intercultural education in practical terms of educating students to participate effectively in the globalising processes. For instance, teachers should consider

- What kind of criteria can educationists use to set aims and objectives of ICM in teaching EIL?

- What can teachers select as their teaching materials in order to achieve those aims and objectives in their English classroom?

- How can they teach their students how to communicate appropriately in intercultural situations with the focus on learning English through cultural studies or vice versa?

However, as discussed in terms of the relation between EIL and international discourses, the first thing to be considered is that there is little choice but to deal with the connection between EIL and intercultural situations in this global world. What is more, in the sociological perspective, an individual constructs his society and the society constructs the individual; language teachers should take socio-cultural contexts and socio-political contexts surrounding their language learners, into consideration.

With regard to socio-cultural discourses, I want to draw attention to three basic constituents of ICM-language, communication and culture. I do this because we can search for answers to the aforementioned three questions by reconsidering what the three constituents indicate.

First of all, language is regarded as a dynamic social phenomenon. Language is defined not as a formal system but as a medium for social life in a social-semiotic 
perspective (Halliday and Hasan 1989). The Bakhtin circle focuses on dynamic situations in language, that is to say, 'language is not static and that speakers-hearers exist within social-cultural effervescence' [author's italics] (Moraes 1996: 90). With reference to culture, language is not only a carrier of culture but also a signifier of cultural belonging with potent cultural and symbolic power (Rassool 1998).

These authors thus analyse the relationship of language and culture from a 'pure' linguistic perspective, and when we turn to an 'applied' perspective and to language teaching theory, we find that a similar view is taken when defining the context or 'object' of language teaching and leaming. There is an argument that it is desirable to teach language and culture in language education (Byram 1989, 1991; Kramsch 1993, 1997; Lorenz et al. 1994; Kress 1995; Gumperz 1996).

For instance, Byram suggests two possible approaches to a model for language and culture teaching.

...first, the use of leamers' first language as the medium of study of a foreign culture, taught according to the principles of appropriate disciplines,... Second, the integration of language and culture learning by using the language as a medium for the continuing socialisation of pupils is a process which is not intended to imitate and replicate the socialisation of native-speaker peers but rather to develop pupil's cultural competence from its existing stage, by changing it into an intercultural competence (Byram 1989: 137).

He illustrates that there are the four sectors:

- Language learning-skill-oriented: Foreign language (FL) focus mainly FL medium,

- Language awareness-sociolinguistic knowledge oriented: L1 (first language) medium: comparative focus,

- Cultural experience — knowledge oriented: FL medium; foreign culture focus, and

- Cultural awareness - knowledge oriented; L1 medium comparative focus.

Furthermore, he emphasises equalities of four sectors in terms of the size and time devoted to each. In this regard, we should consider ways to deal with the relation of language and culture in teaching and learning EIL.

Secondly, we should not forget communication, at which language ultimately aims. Communication is described as social and contextual interactions in symbolic processes. Communication is a social interaction in a cultural context beyond a 
linguistic exchange and a linguistic ability-using context as a tool to decode messages (Halliday and Hasan 1989; Gumperz 1992; Seelye 1997a).

Third and finally, culture is constructed through situational interactions in negotiation processes. Culture is an additional component to linguistic skills but it is a construct, in a broad sense, which encompasses almost anything humans have learned (Seelye 1997a). A cultural object provides a clue or a witness to the culture in the network of significant connections, and therefore is understood 'as signs signifying a spatiotemporal system' (Deetz 1984: 217).

Thus the above three constituents, language, communication, and culture, share their common and salient concept-symbols constructed through interactions between individuals and the society in dynamic social contexts. It implies that the symbolic construction is dependent on the dynamic interrelationship between the three: situational contexts link individual activity in social processes with social interactive activity at the social structural level (Gudykunst and Kim 1984; Byram 1997).

From this perspective on individual and social discourses, it is inevitable that English language teachers should teach their students EIL with situational ICM in intercultural contexts. Therefore, there is no doubt that the aim of teaching EIL is to foster intercultural competence (ICP) to participate effectively in intercultural contexts or situations of the global world, along with linguistic competence, in a word, intercultural discourses.

Therefore, English language teachers assume that objectives in teaching EIL share objectives with the humanities and the social sciences with respect to developing the intercultural awareness of an intercultural actor with individual identity.

Thus, the most important objective of learning EIL is to acquire a competence in ICM with people of different linguistic and cultural identities with intercultural awareness (Smith 1981: Lo Bianco et al. 1999; Hahn 1997). Moreover, its final goal is to move from ethnocentric stages to ethnorelative stages of a developmental model of Intercultural Sensitivity (Bennett 1993). Setting this goal and following it implies intercultural understanding, which is the sensibility 'to perceive the other as a cultural subject rather than as a cultural object' (Brière 1997: 563).

To sum up, in order to communicate with others, who have different linguistic and cultural identities, we need a medium. Therefore, English is used as an international language (EIL) with international or intercultural discourses. In other words, English 
language teachers should be concerned with international or intercultural discourses. Moreover, they should include socio-linguistic, socio-political, and socio-cultural contexts in the global world in teaching their students EIL-situational teaching of English as an international language.

\subsubsection{Multimodal Communication in Situational Teaching and Learning ElL}

Because of the attention given to situational teaching and learning of EIL, there is the need to construct or re-construct effective educational programmes including new curricula and teaching methods in order to meet expectations of teaching and learning EIL in relation to intercultural communication and competence.

At this stage, the first thing I should like to consider is what young people in the global world need to know in order to participate effectively in this contemporary culture in terms of ICM.

Let me take an example-websites. Websites are made of texts, images, and audiovisual material and are connected with people all over the world. We acquire an abundance of information, textual and audio-visual and have multisensory experiences through the websites. Websites are only one of numerous examples in which Information technology compresses the spatio-temporal dimension enough to communicate across time and space, as mentioned before, and to participate in a variety of new communication genres.

As indicated in the above examples, written language is still the medium of representation and communication but it is no longer as effective as in the past. According to Kress,

\footnotetext{
Several factors contribute to that dynamic. First, young people are formed and form themselves in the totality of their semiotic environment.... Second, through a multiplicity of factors,.... many texts are becoming multimodal in a pronounced way, using visual and verbal elements in quite new ways..... Textbooks are increasingly now visual rather than verbal objects (Kress 1996: 193).
}

Indeed, this is a fundamental change for the young generation-less dependent on logocentrism. Although written languages are still dorninant as the medium of information and communication, there are growing changes into a new communication genre, which provides both audio and visual elements. 
Also, Kenneth Burke criticises the limitations of literacy and the concentration in language teaching exclusively on the verbal, ignoring other dimensions of linguistic elements:

The [written] record is usually but a fragment of the expression (as the written word omits all telltale record of gesture and tonality; and not only may our "literacy" keep us from missing the omissions, it may blunt us to the appreciation of tone and gesture, so that even when we witness the full expression, we note only those aspects of it that can be written down) [brackets mine] (Burke 1962, 1969: 185).

As we observed in our Rhetoric of Motives, though one may scientifically distinguish simply between words and things, philosophically there must be not only the verbal and the nonverbal, but also the more-than-verbal, since "Reality" as a whole comprises not only the verbal and the nonverbal, but also the morethan-verbal [author's italics] (Burke 1966: 455).

Furthermore, in his book, Culture and Society, Raymond Williams also challenged scriptocentrism: '... performance and practical activity... which is always latent in the highly literate, is a mark of the observer's limits, not those of the activities themselves' (Williams 1983: 309).

Given the above criticism of literacy and verbal text, it can be inferred that there is a perspective to be taken into serious consideration in the sense that students can be given more than logocentric experiences in teaching and learning EIL. Furthermore, we must ask what we can do for our young generation in the totality of their semiotic environment, when there is no doubt that 'visual communication may prove more effective in that context than language can ever be' (Kress 1996: 194).

Given the above viewpoint, language teachers, particularly those teaching EIL, should be concerned with educating their students in order to engage in multimodal communication appropriately in the contemporary information-oriented society. This also implies that there is the need for students to have multisensory experiences in their learning activities. In other words, when language teachers are engaged in intercultural communication and the intercultural competence of their students, it appears that teachers ought to consider the involvement of new communication genres as well as multi-sensory experiences as communication genre.

This argument, and its application to teaching EIL, should lead to students having multi-sensory experiences. For instance, they can experience and learn which communication genres are appropriate and which forms and modes of the communication genres can be applied in certain contexts or situations. 


\subsubsection{ElL learners as Critical Ethnographers}

In relation to two kinds of global impacts, that is, information technology and multiculturalism, I have reviewed global changes in terms of teaching and learning English- [1] the predominant paradigm of EIL, [2] its appropriate situational teaching, and [3] the need for multimodal communication for intercultural communication and its competence in the global world. These changes generate a new tendency in terms of policies, objectives, teaching methods in English language education, which are appropriate to new socio-cultural contexts and situational learning environmentinterculturalism in multicultural societies. It has been shown that teaching and learning EIL is dependent on contextual and situational frameworks. In practical terms, English linguistic imperialism is more complicated than the extent to which English language teachers understand in their classrooms.

As reviewed in the previous subsections, it is the main point in English language education to approach teaching and learning EIL in socio-cultural approaches. This implies that changes in contemporary socio-cultural phenomena change teaching English as a second or foreign language, into English as an international language (EIL). Along with this transposition of the English language, its teaching objectives and methods, we should also reconsider English learners' needs in this new situation.

First, we have to consider what we should do in relation to the above impacts. It seems to me that there is the need to rectify the predominant paradigm of EIL, which is described as linguistic genocide (Day 1985) and English linguistic imperialism (Phillipson 1992). Furthermore, relevant teaching methods should be connected with international situations or intercultural discourses with multimodal communication.

With regard to this need, some language education scholars adopt ethnography to teaching the English language in that ethnography can be a method to enable learners to develop their understanding of other situational discourses and other languages in context (Byram et al. 1991; Holliday 1994; Hymes 1994; Barro et al. 1998; EganRoberton and Willett 1998; Canagarajah 1999; Roberts and et al. 2001).

Above all, as implied in the term itself, ethno (people) and graphy (describing), 'ethnography usually involves a holistic description of cultural membership...all relevant aspects of a culture's material existence, social system, and collective beliefs and experiences' [author's italics](Lindlof 1995: 20). Geertz (1983) points out the advantage of thick description: the more detail that goes into the description, the more 
multidimensional cultural learners' understanding, the more meaning each element of the culture holds for those who respond to them.

With regard to its holistic or thick description of all social discourses, we can adopt an ethnographic fieldwork model of teaching and learning EIL, which Byram et al. propose and provide EIL learners with opportunities to do fieldwork on intercultural situations:

The ethnographic fieldwork model is oriented towards developing a particular mode of thinking by teaching methods of cultural analysis rather than readymade accounts of the culture. The processes of ethnographic analysis are to be taught, not the products. It is not a question of replacing teachers' informal information by a more scientific ethnographic monograph on the other culture, but of teaching pupils how to produce an ethnographic monograph for themselves (Byram et al. 1991: 383-384).

As implied in the above, this model focuses on analysing activities, that is, critical thinking, as well as being process-centred, to develop cultural awareness through language learning.

Canagarajah, in even stronger terms, articulates his orientation to an ethnographic approach in order to rectify the inequalities of English and asserts that there is the need to adapt a critical ethnography to situational teaching of ElL. Furthermore, he illustrates the advantages of critical ethnography in situational teaching of EIL:

While traditional ethnography has claimed to treat the words of the informants from the community as sacrosanct, critical ethnography analyzes the words in relation to the larger historical processes and social contradictions, searching for the hidden forces that structure life. While descriptive ethnography would attempt to smooth over the contradictions among informants, critical ethnography considers the explanation of these contradictions to be its very quest (Canagarajah 1999: 48).

Thus, Canagarajah also emphasises critical ethnographic methods, which are accompanied by critical thinking about socio-linguistic, socio-political, and sociocultural contradictions surrounding English learners, in teaching English as an international language (EIL).

Along with this critical ethnographic way, I shall review further, how language teachers of EIL can help their students participate effectively in ICM in relation to basic paradigms and the framework of ICM in order to be intercultural actors in the next subsection. 


\subsection{ElL Learners to be Intercultural Actors}

EIL teachers have begun to consider the relationship between language learning and social identity formation, particularly with respect to national identity (Byram 1999). It appears to attract attention to the issue of cultural identities along with ICM and its competence in EIL classrooms.

At this stage, it is necessary to make distinctions between national identity and cultural identity. According to Smith (1991), we can list the fundamental features of national identity-[1] an historic territory, [2] common myths and historical memories, [3] a common, mass public culture, [4] common legal rights and duties for all members, and [5] a common economy with territorial mobility for members. Its fundamental features inhere in the nature of national identity and make national identity complex and abstract.

Also he explains national identity as follows:

The nation, in fact, draws on elements of other kinds of collective identity, which accounts not only for the way in which national identity can be combined with these other types of identity - class, religious or ethnio-but also for the chameleon-like permutations of nationalism, the ideology, with other ideologies like liberalism, fascism and communism. A national identity is fundamentally multi-dimensional [author's italics] (Smith 1991: 14).

As indicated, national identity is multi-dimensional. Additionally, national identity can be combined with cultural and political identity in that 'the nation...signifies a cultural and political bond, uniting in a single political community all who share an historic culture and homehand' (Smith 1991: 14-15).

However, we can say that national identity overlaps cultural identity in the case that one nation has one common culture. On the other hand, if one nation has two different cultures there are two cultural identities in the nation. Therefore, natıonal ident ty 'provides a powerful means of defining and locating individual selves in the world through the prism of the collective personality and its distinctive culture' (Smith 1991 17). On the other hand, cultural identity wherein we have a shared, unique culture enables us to know 'who we are' in the world. By dint of our cultural identity, we rediscover ourselves.

With regard to this relation between national identity and other ident ty each person lives with multiple identities at the same time, for instance, national identity cu tura identity, ethnic identity, religious identity, and so on. In other words, se f-dent ty s 
combined with these collective identities and these collective identities are roleidentities relevant to socio-cultural roles. Therefore, I can say that it occurs to people that when one of their collective identities brings about changes their self-identity can be paralysed by those changes. For instance, as Smith illustrates, Oedipus, in Sophocles's play, Oedipus the King, has many collective identities in terms of roleidentities, such as father, husband, king, brother, and hero. However, some of his socio-cultural identities are confused with others and this confusion eventually collapses his self-identity.

It occurs to me how EIL teachers can be involved in this sort of identity formation in their learners in terms of cultural identity. People have experiences of culture shock in daily life as well as in certain intercultural situations. They have unpleasant surprises or astonishment in their experiences of new cultures whereupon the experiences can have a great impact on their own cultural identity.

\subsubsection{Basic Paradigms of Intercultural Communication (ICM)}

One of the main arguments in favour of ICM in forming cultural identity is that the relation between individual and society contributes to the identity formation in the process of ICM. With regard to this formation, I should like to review the individualsocial relation. I intend to start with the connection between ICM and cultures, particularly the relation between individual single persons and all other social discourses surrounding them.

\subsubsection{Relations between Culture, ICM, Individuals, and Society}

The first thing to be considered is that the concept of culture is varied and can be defined depending on scholars' own historical, socio-cultural backgrounds.

I, however, would like to start with its general and basic definition, which is widely accepted among sociologists, that the concept of culture is explained as the symbolic, socially patterned nature and form of individual thought and action. Jorgensen et al. explained two meanings from their definition:

Cultures are what is called socially determined-for example, when you are born, you are born into an existing symbolic order; you don't choose the culture into which you are born.... Cultures are patterned in that they form relatively coherent packages of signs and symbols. It is this patteming which allows people to learn their culture and to transmit it over time (Jorgensen et al 1997: 32-33). 
Jorgensen et al are certainly correct that culture is socially determined as well as transmissible. The former is related to a situational structure patterned in a collective group process in the society, which is, in other words, a deterministic view; the latter is connected with individual cultural identity in an individual process. In the patterning process, there are deterministic factors in the social structure. Nevertheless, there are also probable factors to transmit cultures. It is natural to communicate individually as well as in a social dimension and to change cultural identities through the communication between individual and social dimensions. In other words, there is a crucial relationship between individuals and their society in changing their cultural identities.

Furthermore, it appears that culture and communication cannot be separable, as Hall (1976) claimed: culture is communication; communication is culture.

On the other hand, Kim pointed out the first steps of ICM as abstracting certain human-social phenomena and naming them as the central conceptual domain of the field and defined ICM as follows:

\begin{abstract}
... the crux of intercultural communication that distinguishes it from the rest of the field is the relatively high degree of difference in the experiential backgrounds of the communicators due to cultural differences.... Intercultural communication, then, refers to the communication phenomena in which participants, different in cultural backgrounds, come into direct or indirect contact with one other.... The focal point of intercultural communication, instead, is the communication process between individuals and groups [author's italics] (Kim 1984: 15-16).
\end{abstract}

Furthermore, Rey regards the intercultural dimension as a means, not as an end in itself and points out a twofold problem in intercultural teaching: 'how to put the guidelines into practice ('what can we do?') and how to assess the results ('what purpose does it serve?') (Rey 1991: 144). Moreover, he emphasises that teachers should consider designing intercultural activities 'in terms of strategies and dynamics,' along with the efficacy of these strategies. This, in the end, indicates that dynamics of ICM between different cultural qualities are based on determination but the dynamics can be transmissible through intercultural education.

Carey's perspective of ICM as a "symbolic process whereby reality is produced, maintained, repaired and transformed" is also relevant (Carey 1989: 23).

I agree with them in saying that ICM is a dynamic communicative process. I should like to consider how individuals and collective groups understand differences and how 
they interact with each other in their cultural backgrounds in the process of ICM within their situational social structures.

From this viewpoint, I shall discuss paradigms about dynamic processes of ICM in intercultural situations, and I intend to focus on symbolic interactionism (hereafter interchangeable with $S$ ), which came in multiple varieties including Goffman's dramaturgy (1959), and Bakhtin's dialogism (1981, 1984, 1986).

\subsubsection{The Self-Others Relation to ICM: Otherness in Symbolic Interactionism}

First of all, how do we interact, exchange, share different cultural signs and symbols with each other in daily life in ICM? We have intercultural experiences more often than before in daily life.

George H. Mead, in his book, Mind, Self and Society (1934), identified interior selfconversations and the place of the other in the act. The act is one of his key concepts in that the social self, which is described as the combination of the subjective ' $I$ ' and the objective 'me,' is constituted through acts. The self as a social object emerges where expression plays a crucial part and is structured by the principle of sociality, or the taking of the attitude of the other in a social situation.

In his explanation, he argued a reciprocal process of interaction between the self and the society, to which the self belongs. Also, he asserted that individuals develop the social self by coming to see themselves as others see them, that is, by reflecting themselves. Selves and the society are interrelated in that individuals develop the social self, or their self-awareness through others' eyes or otherness. However, I am more concerned with how individuals interact to develop their self-awareness. Mead (1934) illustrated levels of conduct and phases of action in relation to that development, as I present in Table 2.1:

Thus, the social self develops by using symbols or imagery through imagination, synthesising divergent perspectives into inclusive perspectives, and comprising better cognition and behaviour, that is, increased self-awareness. The social self is an internalisation of the social process.

Mead postulated that the 'I', who is, like the unsocialised self, a bundle of spontaneous wants and desires, is involved in a continual interaction with the 'Me,' that is, the social self, wherein the individual develops self-consciousness. The ' $\mathrm{Me}^{\prime}$ represents the 
Table 2.1 Stages in the Development of Self-Awareness in Meadian Perspective

\begin{tabular}{|c|c|c|c|c|}
\hline Stages & & $\begin{array}{l}\text { Main } \\
\text { Elements }\end{array}$ & Role-taking & Processes in the stage \\
\hline \multirow{3}{*}{$\begin{array}{l}\text { Preliminary } \\
\text { Distinction }\end{array}$} & 1 & $\begin{array}{l}\text { Conduct 1: } \\
\text { Some low level } \\
\text { of routine self- } \\
\text { awareness }\end{array}$ & $\begin{array}{l}\text { No role- } \\
\text { taking: } \\
\text { Habitual \& } \\
\text { Reflective } \\
\text { conduct }\end{array}$ & $\begin{array}{l}\text { [1] To direct toward immediate satisfaction } \\
\text { of impulses, with no regard for how the } \\
\text { consequences of this conduct will } \\
\text { affect others. } \\
\text { [2] Much habitual conduct in the egocentric } \\
\text { type. }\end{array}$ \\
\hline & 2 & $\begin{array}{l}\text { Conduct 2: } \\
\text { Some } \\
\text { awareness of } \\
\text { conflicting } \\
\text { impulses } \\
\text { to act }\end{array}$ & $\begin{array}{l}\text { Simple kind } \\
\text { of role-taking } \\
\text { in simple } \\
\text { Moral } \\
\text { cognition }\end{array}$ & $\begin{array}{l}\text { [1] To meet conflict and to inhibit conduct } \\
\text { because the individual does not know } \\
\text { which rule to follow. } \\
\text { [2] To take only the perspective of the } \\
\text { group to try to determine which specific } \\
\text { behaviour will be approved or } \\
\text { disapproved. } \\
\text { [3] Once this is determined, action } \\
\text { resumes. Although such rule-following } \\
\text { behaviour is often described as } \\
\text { implicating morality. it is largely a } \\
\text { matter of ritual conformity entailing no } \\
\text { complex moral cognition. }\end{array}$ \\
\hline & 3 & $\begin{array}{l}\text { Conduct } 3: \\
\text { Multiple } \\
\text { interests and } \\
\text { values- } \\
\text { embodied in } \\
\text { divergent } \\
\text { perspectives at } \\
\text { the stage of } \\
\text { compulsory } \\
\text { reconciliation }\end{array}$ & $\begin{array}{l}\text { Complex } \\
\text { role-taking in } \\
\text { more } \\
\text { complex and } \\
\text { interesting } \\
\text { moral } \\
\text { cognition } \\
\text { [moral } \\
\text { dilemmas] }\end{array}$ & $\begin{array}{l}\text { [1] For the inhibiting conflict to involve in } \\
\text { three perspectives: } \\
\text { 1> Conflict with whose values/ } \\
\text { moral standards among } \\
\text { the community members } \\
\text { 2> Conflict with whose interests/ } \\
\text { benefits among them } \\
\text { 3> Conflict with the whole community } \\
\text { such as the generalised other } \\
\text { [2] To the perspectives of all those persons } \\
\text { or groups whose interests and values } \\
\text { are at stake in the situation rather than } \\
\text { the perspective of the group over } \\
\text { against themselves }\end{array}$ \\
\hline \multirow{2}{*}{$\begin{array}{l}\text { Action } \\
\text { Distinction: } \\
\text { continuously } \\
\text { alternative } \\
\text { process } \\
\text { between covert } \\
\text { action and } \\
\text { overt action in } \\
\text { order to solve } \\
\text { moral problems } \\
\text { in the real } \\
\text { world }\end{array}$} & \multirow[b]{2}{*}{4} & $\begin{array}{l}\text { Covert Action } \\
\text { phase: } \\
\text { Thought }\end{array}$ & $\begin{array}{l}\text { Role taking } \\
\text { thought; } \\
\text { Parts of a } \\
\text { process of } \\
\text { adaptive } \\
\text { action in } \\
\text { moral } \\
\text { cognition }\end{array}$ & $\begin{array}{l}\text { [1] Thinking guides attempts to overcome } \\
\text { problems in order to reconcile } \\
\text { conflicting values and interests and find } \\
\text { a way for action to proceed } \\
\text { [2] It serves behaviour and the need to } \\
\text { satisfy impulses }\end{array}$ \\
\hline & & $\begin{array}{l}\text { Overt Action } \\
\text { phase: } \\
\text { Behaviour }\end{array}$ & $\begin{array}{l}\text { Role taking; } \\
\text { Observable } \\
\text { behaviour }\end{array}$ & $\begin{array}{l}\text { [1] Behaviour driven by impulses gives nse } \\
\text { both to satisfaction of impulses and, } \\
\text { because inhibiting problems often } \\
\text { arise, to thinking. } \\
\text { [2] To attempt tentative solutions into } \\
\text { practice } \\
\text { [3] To lead to new problems demanding } \\
\text { new thinking from the previous } \\
\text { behaviour }\end{array}$ \\
\hline
\end{tabular}


attitudes of the social group, the generalised other, and through role-taking in play and imaginative rehearsal of interaction, we internalise the group's values as our own.

According to Jary and Jary (2000), Mead saw human nature as part of evolution and nature, but put emphasis on language and symbolic communication as an important aspect of this evolution, enough to free human action from natural determinism.

As reviewed so far in the present subsection, Mead assumed and worked on three basic principles on the ground that there is a dialectical dynamic relationship between self and society:

[1] The individual and society are not separate. They are mutually supportive and dependent. [2] Action, emotion, and cognition are limited, changed, and some times determined by forces greater than the individual. [3] Society is sustained in the dynamic processes of interaction [brackets mine] (Callero 1991: 324).

The individual, or the self, is structured by the principle of sociality, or the taking of the attitude of the other in a social situation. That is to say, the Meadian SI is a view of the self-society interaction in a reciprocal process.

Other perspectives I should like to consider in SI are dramaturgy and grounded theory. Erving Goffman, in his book, The Presentation of Self in Everyday Life (1959), constructed a dramaturgical framework. According to Brissett and Edgley,

\begin{abstract}
Dramaturgy is a social behavioural (Mead) rather than cognitive or phenomenological image of human activity. Its focus is on the communicative dimensions of human interaction rather than on what the interactants might be thinking, feeling, or experiencing.... It is with communication in this broad sense that dramaturgy is interested-both discourse (speech and language) and nondiscourse (clothing, hair style, gestures-indeed, a myriad of objects that people use in their communication with others) (Brissett and Edgley 1990a: 4-5).
\end{abstract}

The dramaturgical perspective focuses on the communicative dimensions of human interaction with others in the society, and explains how people interact or communicate. Goffman focused on a dialectical process between the essential self and the presented self-a performer and a character.

More things to be considered in Goffman's concepts are distancing and emancipatory dimensions. The former, distancing or alienation, finds and measures the size of the gap between the objective, represented as the society, on the one hand, and the subjective, described as individual human understanding of those objective socral situations, on the other hand, in the dialectical process. 
The latter is acquired through the 'Magic If,' to enable us to reflect upon selfawareness in that the 'Magic If,' as it is called by a Russian director, Stanislavski, helps an individual to create intuitively, subconsciously by turning on his subconscious and then carrying out his truthful, logical, concrete action relevant to certain situations. Young regarded the power of the 'Magic if' as emancipating us. We mobilise, transform, evaluate, and progress in the human condition through the 'Magic If' to make ourselves emancipatory. Young defined an emancipatory dramatic event as the event that 'reflects upon the quality of social life, evaluates it against some standard of collective and personal good, and offers, as well as, an alternative vision of social life' (Young 1990: 273).

On the grounds of what I have reviewed so far, I consider, above all, the self-society dynamics in terms of how to interpret symbolic interaction in intercultural settings. This means how the social self communicates with others in intercultural situations. Now I can elucidate, the main concept is otherness, wherein there is a dialectical relationship between the subjective ' $\mathrm{I}$ ' and the objective 'Me,' and between the objective 'Me,' in other words, the social self or the generalised other, and the society, which the self belongs to. Thus, it is said that $\mathrm{SI}$ is a paradigm to search for intercultural identity through symbolic intercultural interactions or ICM.

\subsubsection{The Self-Identity Relation to ICM: Otherness and Outsidedness in Dialogism}

SI has a different name, 'structuralist interactionism' [author's italics] (Perinbanayagam 2000: xi). Nonetheless, SI has been still criticised from structuralistic perspectives: Mills (The Sociological Imagination 1970), Atkinson (Ethnomethodology: A Critical Review 1988), and Carey (Communication and Culture 1989). According to Denzin (1992) and Jary and Jary (2000), the main criticism converges on the point that it attaches more importance to micro-social processes and sub-institutional phenomena rather than to macroscopic structures and historical factors, such as economic forces and institutionalised political power. In addition, it is criticised for not being sufficient to investigate creative competence at its deep level and to be 'more internal to interpretive and symbolic interactionist tradition' by focusing on micro-social processes rather than dealing with external socio-cultural discourses (Jary and Jary 2000: 623).

There are alternatives to Meadian SI, whose principles are based on structural approaches, which deal with intercultural discourses in deep dimensions of ICMMills' sociological imagination and Bakhtinian dialogism. 
Sociological imagination means thinking sociologically about personal circumstances wherein we can think ourselves away from our own customs and habits and then develop a more profound understanding of them. The salient principle of the sociological imagination is that we think ourselves away from the familiar routines of our daily lives in order to look at them anew-distancing or alienation from the self.

Mills explicated functions of the sociological imagination as follows:

The sociological imagination enables its possessor to understand the larger historical scene in term of its meaning for the inner life and the external career of a variety of individuals. It enables him to take into account how individuals, in the welter of their daily experience, often become falsely conscious of their social positions.... The Sociological imagination enables us to grasp history and biography and the relations between the two within society. That is its tasks and its promise (Mills 1970: 5-6).

Described as an intellectual pivot for social analysis, the sociological imagination provides us with the capacity to shift from one perspective to another, for instance, from the political to the psychological, and from the most impersonal to the most intimate features of the human self. And then by dint of the imagination, we examine and assess comparatively the relations between the two perspectives. In the end, we can be conscious of ourselves as an outsider by distancing or alienating from ourselves temporarily and have a better understanding of social relativity and of the transformative power of history.

Mills emphasised the productivity of the sociological imagination in terms of developing self-consciousness or self-awareness and claimed that 'by their reflection and by their sensibility, they [those who use the imagination] realize the cultural meaning of the social sciences [brackets mine] (Mills 1970: 8).

In a stronger structural perspective than symbolic interationists' including Mills, the paradigms of Mikhail M. Bakhtin on language and culture are along the line of structuralistic approaches. I shall review Bakhtin's concepts in relation to this study, particularly focus on

- Language as social communicative process,

- The Bakhtinian self (the dialogic or interlocutive self and all the others)

- Open otherness, dialectical and dialogical

- Outsidedness for creative understanding of the self and,

- Thirdness between the centre and the not-centre 


\title{
Language as Social Communicative Process
}

First of all, for Bakhtin, language is a social communicative process in that, first, it has its ideological-semiotic value, second, it is a site for the embodiment of ideologies, and third, it exists in order to communicate with others. Therefore, he argues that language is a dynamic social entity and a socio-cultural phenomenon.

\begin{abstract}
Bakhtinian theory emphasizes that language is not passively assimilated by an individual; rather, language is a dynamic process that exists dynamically in the socio-historical arena.... In this sense, language is never a consummated outcome but a dynamic social entity and, therefore, a socio-cultural phenomenon (Moraes 1996: 92).
\end{abstract}

Thus, Bakhtin described language as a socio-cultural phenomenon in dynamic processes. In this regard, EIL should be taught in cultural contexts, that is, situational teaching and learning, as discussed in 2.1.

\section{The Dialogic or Interlocutive Self and All the Others}

The second thing to be considered is the concept of the self-other relation. Bakhtin's is similar to Mead's approach to the concept of dialogue principles, although Mead is based in the field of social psychology and Bakhtin, in literary criticism and theory of culture (Koczanowicz 2000; Gurevitch 2000; Holquist 2002). Similar to the functions of the Meadian self, producing and responding, Bakhtin also asserted that a social actor has two different roles - a participant, and a spectator to perceive and to be perceived at the same time.

He pointed out, however, that dialogue is not a dyadic or a binary phenomenon. Holquist illustrated the nature of Bakhtin's dialogue:

Dialogue is a manifold phenomenon, but for schematic purposes it can be reduced to a minimum of three elements having a structure very much like the triadic construction of the linguistic sign: a dialogue is composed of an utterance, a reply, and a relation between the two. It is the relation that is most important of the three, for without it the other two would have no meaning (Holquist 2002: 38).

As indicated above, dialogue exists only within the interrelation between the three, not within one single itself. Therefore, the existence is forged and is understood under the triadic construction between the three-an utterance, a reply, and their relation. 
There is a dialectical process in this tripartite structure. However, the dialectical process is based on open-ended conceptions of negation and negativity in neoKantian perspectives, rather than of the process of contradiction or opposition between two things like the structure of thesis-antithesis-synthesis in Marxian perspectives.

The binary oppositional relation is dialectical in Marxian concepts, whereas the differential relation is dialectical in neo-Kantian thinking. Britannica distinguishes between 'dialectic' in its Marxian concept and its Neo-Kantian concept:

\begin{abstract}
Among the classical Greek thinkers, the meanings of dialectic ranged from a technique of refutation in debate, through a method for systematic evaluation of definition, to the investigation and classification of the relationships between specific and general concepts. . . More recently, Immanuel Kant denoted by "transcendental dialectic" the endeavour of exposing the illusion involved in attempting to use the categories and the principles of the understanding beyond the bounds of phenomena and possible experience. G.W.F. Hegel identified dialectic as the tendency of a notion to pass over into its own negation as the result of conflict between its inherent contradictory aspects. Karl Marx and Freidrich Engels adopted Hegel's definition and applied to social and economic processes [bolds mine] (Britannica 2000a).
\end{abstract}

Thus, dialecticality in neo-Kantian perspectives takes place between ' $A$ ' and 'not- $A$ 'for instance, green-and-non-green relations such as green-and-blue, green-and-grey, and green-dark green, in a word, one and all the others. On the other hand, an example of Marxian dialecticality is a relationship between ' $A$ ' and 'anti-A'-for instance, green-and-red or black-and-white.

Bakhtin took this neo-Kantian concept of 'dialectic' and argued that it is based on the concept of his otherness. Let me describe the Bakhtinian concept of 'dialectical' with reference to the triadic construction of dialogue, which was reviewed earlier as the tripartite structure of an utterance, a reply, and a relation between the two-the utterance and the reply.

For instance, each interlocutor of the two people in conversation is, at the same time, a hearer as well as a speaker and recognises the 'l' existence through what the other speaks in the wholeness of the other's utterance. The relationship of the two interlocutors is the relationship between one interlocutor and the other, between the speaker and the responder, between the self and the other, and the individual and the society where he belongs. 


\section{Open Otherness: Dialectical and Dialogical}

This relationship is neither oppositional nor resistant relation but the differential relation between a center and all that is not that center' (Holquist 2002: 18). This is the core of Bakhtinian otherness, which is similar to Meadian otherness. The two interlocutors are conscious of their utterance on the basis of otherness. This otherness remains not merely a dialectical alienation, but also leads to otherness in dialogic consciousness to be synthesised at a higher dimension than a dialectical dimension.

With regard to this multiple relation between the centre and the non-centre in social discourses, the Bakhtinian concept of 'dialectical' is included in 'dialogic or dialogical.' The dialogic is re-cycled, as Bakhtin himself described 'Dialectics was born of dialogue so as to retum again to dialogue on a higher level (a dialogue of personalities)' [author's italics](Bakhtin 1986: 162). Thus, the Bakhtinian concept of 'dialogical' is pregnant with two key dynamic factors: dialecticality and continuity. Holquist and Liapunov described the wholeness and the continuity of the interlocutive self:

The interlocutive self is one that can change places with another. . . the two try to see what is there together.... Aesthetics is the struggle to achieve a whole, but a whole that must first of all be understood as a purely positional or relative construct: Second, such a whole is never a seamless oneness, insofar as it is always a negotiated relation between "two powers.... (Holquist and Liapunov 1990: xxvi-xxvii).

As implied in the above, however, Bakhtin emphasised three points: first, how relations between living subjects get ordered into categories of 'I' and 'another'; second, how 'I' forges the kind of tentative wholeness surrounding the 'I'; third, how entities relate to each other at various levels to achieve another whole (1990). This is one of his main issues about 'How to shape heterogeneous parts into a (positionally) consummated whole,' in his words, the objective of architectonics (Holquist and Liapunov 1990: xi).

Therefore, for Bakhtin, it is necessary for the self/other always to negotiate relations among unique individuals, that is, the interlocutive relation of 'l' to 'another' in order to achieve the objective of architectonics.

One more thing to be considered is the need for emancipatory discourses to achieve social change in the process of negotiating between two powers. At the first stage of this discourse, it is necessary to combat the illusions and fears, which are in the 
enslaved (Lukàcs 1990). At the later stages, the illusions and fears are released by changing the delicate balance between the two into equilibrium.

The interest in this sort of social mechanism in structuralistic perspectives led into Bakhtin's concept of speech genres, in other words, communication discourses surrounding a speaker and a listener. This concept is based on his claim that 'genre is not considered an individual achievement but an element of social reality...genre is the aggregate of the means of collective orientation in reality [author's italic] (Bakhtin and Medvedev 1991: 135). Also, Bakhtin asserted that 'Genres are of special significance. Genres (of literature and speech) throughout the centuries of their life accumulate forms of seeing and interpreting particular aspects of the world' (Bakhtin 1986: 5). Thus, genre can function as a repository of ideas and worldviews. Bakhtin's otherness seems to be open. That is to say, the concept of otherness is not limited within the context made between the speaker and the listener, but includes their social discourses, which is accumulated at their contemporary as well as at their historical collective dimensions. For instance, I can put the former into empathising a person in his perspective, but the latter inside and outside his perspective, i.e. through all the others including himself.

\title{
Outsidedness for Creative Understanding of the Self
}

As hinted in his concept of open otherness, Bakhtin criticised a one-sided approach to understanding an unfamiliar or alien culture:

\begin{abstract}
There exists a very strong, but one-sided and thus untrustworthy, idea that in order better to understand a foreign culture, one must enter into it, forgetting one's own, and view the world through the eyes of this foreign culture. This idea, as I said, is one-sided. Of course, a certain entry as a living being into a foreign culture, the possibility of seeing the world through its eyes, is a necessary part of the process of understanding it; but if this were the only aspect of this understanding, it would merely be duplication and would not entail anything new or enriching (Bakhtin 1986: 6-7).
\end{abstract}

He illustrated the disadvantages of one-sidedness in that it required one to forget one's own culture. Because of this, it could merely duplicate the foreign culture and would not entail anything new or enriching. I agree with him in the sense that it is scarcely possible to have critical thınking in one-sidedness wherein there is nothıng to compare with the unfamiliar or alien culture and nothing to assess in relation to the unfamiliar or alien culture That is to say, there are no dialectical dynamics.

In this regard, he suggested outsidedness as an alternative to one-sidedness: 
Creative understanding does not renounce itself, its own place in time, its own culture; and it forgets nothing. In order to understand, it is immensely important for the person who understands to be located outside the object of his or her creative understanding - in time, in space, in culture.... In the realm of culture, outsidedness is a most powerful factor in understanding' (Bakhtin 1986: 7).

As implied in the above, there are at least dynamical conditions in that the relation between one's own culture and the unfamiliar or alien culture provides one with dialectical dynamics in neo-Kantian terms.

The final aim of outsidedness is to understand one's own culture, although it penetrates other cultures, in other words, be inside and out of other cultures and return to one's own culture, and understands them, as well. With regard to this selfawareness in terms of cultural identity, Holquist explains Bakhtin's outsidedness as follows:

\begin{abstract}
the need first to use one's understanding to penetrate the other person or the other culture as deeply as possible; but then, having done this, he stresses in both cases the no less urgent need to retum to the perspective provided by our native self or our native culture (Holquist 1986: xiii).
\end{abstract}

Here we explained dialectical dynamics by stressing this returning to one's own perspective. In this regard, to be precise, I can define outsidedness as outside one'sown-ness, wherein one can have a creative understanding of oneself from the standpoint with oneself.

Let me return to his statement in relation to the starting point of the creative understanding. Bakhtin explained the reason why one should return to one's own perspective:

... a pure projection of myself into the other, a move involving the loss of my own unique place outside the other, is, on the whole, hardly possible, in any event it is quite fruitless.... Aesthetic activity proper actually begins at the point when we return into ourselves and to our own place outside the [other] person.... [author's brackets](Bakhtin 1979: 25-26 cited by Holquist 1986: xiii).

Thus, there is no doubt that it is necessary to have dialectical or critical thinking between being outside and being inside in order to have cultural awareness of understanding one's own culture as well as others; to put this in detail, there are four perspectives, that is, outside ownness, outside otherness, inside ownness, and inside otherness. 
Bakhtin, however, pointed out how to encounter the two cultures engaged in intercultural communication:

\begin{abstract}
A meaning only reveals its depths once it has encountered and come into contact with another, foreign meaning: they engage in a kind of dialogue..... Without one's own questions one cannot creatively understand anything other or foreign (but, of course, the questions must be serious and sincere). Such a dialogic encounter of two cultures does not result in merging or mixing. Each retains its own unity and open totality, but they are mutually enriched [author's italics](Bakhtin 1986: 7).
\end{abstract}

On the other hand, there arises a question of how one can encounter this dialogical condition wherein one is involved in outsidedness as well as otherness. My answer to the question is the Sociological Imagination (Mills 1970).

The sociological imagination enables people to grasp what is going on in the world, and to understand what is happening in themselves as minute points of the intersections of biography and history within society. Generally speaking, 'contemporary man's self-conscious view of himself as at least an outsider, if not a permanent stranger, rests upon an absorbed realization of social relativity and of the transformative power of history,' for instance, moving a trouble as a private matter into a public issue of social structure (Mills 1970: 7).

With regard to this, the concept of the sociological imagination can develop into another concept-the intercultural imagination, which I shall cover in 2.2.2.

\title{
Thirdness between the Centre and the Not-Centre
}

Finally, let me take the concept of thirdness, in which we are engaged from the dialectical relation between the two. As reviewed in the above, the Bakhtinian concept of 'dialectical' is not related to the opposition between the two, but the negation of the centre and the not-centre, or the self and all the others.

Holquist comments on this openness, and the continuity and the degree of Bakhtinian thirdness:

. . . in so far as my " $\mathrm{l}$ " is dialogic, it insures that my existence is not a lonely event but part of a larger whole. The thirdness of dialogue frees my existence from the very circumscribed meaning it has in the limited configuration of self/other relations available in the immediate time and particular place of my life. For in later times, and in other places, there will always be other configurations of such relations, and in conjunction with that other, my self will be differently understood. This degree of thirdness outside the present even 
insures the possibility of whatever transgredience I can achieve toward myself [author's bolds](Holquist 2002: 38).

From the above, I can say that a third place between the two is not restricted to the relationship between the Self and other in a certain context, but the third place can exist beyond spatio-temporal dimensions. That is, a third place is not closed within the relationship in a specific context, but is open to, for example, all those, which are not 'I.' Also, a third place emerges in other places and/or other times. That is, thirdness between the two does not always exist at the same place as each of them but it, along with all the others surrounding them, is always dynamic in different spatio-temporal dimensions. Therefore, thirdness creates changes at the individual level, which can produce, in the end, changeability of social discourses.

Therefore, there is plurality in thirdness, too. There is a multitude of third places between the self and the other. In this regard, I should like to consider diverse outcomes, for instance, capable of understanding various cultural values or different languages at interpersonal interaction level, and self-pluralistic thinking in intrapersonal communication. The last perspective can be regarded in that the intrapersonal communication is not restricted to the Self within only its individual dimension but is related to the social values intrinsic in the Self, that is, the Social Self.

Regarding what I have reviewed about Bakhtinian dialogic concepts, I can summarise with this formula: neo-Kantian Dialectics + Open Dialogism = Dialogics. Also, I present the characteristics of Bakhtinian otherness and outsidedness, in comparison with Meadian otherness, with Table 2.2 .

However, there are differences between the reflexivity of the Meadian concept and that of Bakhtinian concept in the above table. According to the Dictionary of Sociology (Jary and Jary), reflexivity is defined in ethnomethodology and symbolic interactionism (SI) as follows:

1. The capacity possessed by an account or theory when it refers to itself.... 2 . (particularly in ETHNOMETHODOLOGY and SYMBOLIC INTERACTIONISM) The idea that our everyday practical accounts are not only reflexive and selfreferring but also socially constitutive of the situations to which they refer. On this view, reflexivity is a capacity possessed by social actors which is decisive in distinguishing human actors from animals. It is a feature of reflexive social accounts and theories of all types that these accounts may also act to reproduce or to transform those social situations to which they refer (Jary and Jary 2000: 512). 
Table 2.2 Comparison of the Concept, Sociological Dialogue, between Mead's and Bakhtin's Perspectives

\begin{tabular}{|c|c|c|}
\hline Self-Identity & Meadian Dialogue & Bakhtinian Dialogue \\
\hline Standpoints & Social psychological & Cultural \\
\hline Interlocutors & The "l" and The "Me" & $\begin{array}{l}\text { The Self and Another or } \\
\text { The Self and All the others }\end{array}$ \\
\hline $\begin{array}{l}\text { Main attribute } \\
\text { of } \\
\text { Interlocutors }\end{array}$ & $\begin{array}{l}\text { The Dialectic Self } \\
\text { Through Otherness } \\
\text { in structuralistic perspectives }\end{array}$ & $\begin{array}{l}\text { The Dialogic or } \\
\text { Interlocutive Self } \\
\text { through Otherness and } \\
\text { Outsidedness } \\
\text { in structuralistic perspectives }\end{array}$ \\
\hline $\begin{array}{l}\text { Self- } \\
\text { Awareness } \\
\text { [ldentity] }\end{array}$ & $\begin{array}{l}\text { The Social Self: } \\
\text { Constructed by Others }\end{array}$ & $\begin{array}{l}\text { The Social Self: } \\
\text { Constructed through others by } \\
\text { the Social Self per se }\end{array}$ \\
\hline Objectives & $\begin{array}{l}\text { Self-awareness of the social } \\
\text { self through } \\
\text { Inside otherness: }\end{array}$ & $\begin{array}{l}\text { Self-awareness of the social self } \\
\text { through Wholeness including } \\
\text { One's-own-ness, Inside } \\
\text { otherness, and outside } \\
\text { otherness (outsidedness) }\end{array}$ \\
\hline Boundaries & Inside the Social Self & $\begin{array}{l}\text { Outside and } \\
\text { Inside the Social Self }\end{array}$ \\
\hline $\begin{array}{l}\text { Discourse } \\
\text { routes }\end{array}$ & $\begin{array}{l}\text { One-sided Otherness to } \\
\text { one's-own-ness } \\
\text { [1] otherness } \\
\text { [2] one's-own-ness }\end{array}$ & $\begin{array}{l}\text { Openness of thirdness } \\
\text { From inside through outside to } \\
\text { inside the Self } \\
\text { [1] one's-own-ness, } \\
\text { [2] otherness, } \\
\text { [3] outsidedness, and } \\
\text { [4] one's-own-ness }\end{array}$ \\
\hline $\begin{array}{l}\text { Range and } \\
\text { Structures }\end{array}$ & $\begin{array}{l}\text { Inside one's-own-ness and } \\
\text { inside othemess }\end{array}$ & $\begin{array}{l}\text { Inside one's-own-ness, inside } \\
\text { otherness, } \\
\text { outside otherness and } \\
\text { outside one's-own-ness }\end{array}$ \\
\hline Dynamics & $\begin{array}{l}\text { Horizontal } \\
\text { Binary Dialectical between } \\
\text { the self and the other } \\
\text { [The 'l' and the 'me'] }\end{array}$ & $\begin{array}{l}\text { Horizontal and Vertical } \\
\text { Dialectical and } \\
\text { Open Dialogical to all. } \\
\text { ['Vertical' dynamics emerge from } \\
\text { relationships between the Self } \\
\text { and the Not-Self in multi- } \\
\text { dimensions] }\end{array}$ \\
\hline Outcomes & $\begin{array}{l}\text { Reflection, } \\
\text { Self-reflection, and } \\
\text { Reflexivity* }\end{array}$ & $\begin{array}{l}\text { Reflection, } \\
\text { Self-Reflection, and Reflexivity }\end{array}$ \\
\hline & \multicolumn{2}{|c|}{$\begin{array}{l}\text { There are differences of refiexivity in the conceptual matter } \\
\text { between Mead's and Bakhtin's; Meadian reflexivity is Self- } \\
\text { reflective rather than reflexive. }\end{array}$} \\
\hline
\end{tabular}


As implied in the above, it is generally accepted that reflexivity is related to the concept that one reflects on oneself. With regard to SI's perspectives, reflexivity means that one reflects the self on others inside the self itself, which is similar to the self-reflection by Narcissus in Greek mythology.

In a critical statement, which implies differences between Meadian and Bakhtinian reflexivity, MacAloon defines reflexivity as the 'capacity of human beings to distance themselves from their own subjective experiences, to stand apart from and to comment on them' (1984a: 11). First of all, I agree with his definition, particularly in the matter of distancing.

He takes another definition of reflexivity in relation to Narcissus of Greek mythology and applies the term, reflexivity, to the concept of Meadian reflectivity in a different perspective:

\begin{abstract}
As G. H. Mead argued, it is the ability of individuals to take the attitude of the other that allows them to become objects to themselves. Kapferer, following Mead, rephrases this in performative terms: "reflexivity" is the act of becoming an audience to oneself.... This is the general sense... But on more particular grounds there is divergence. Some insist upon making a distinction between reflexivity and reflection. Of Narcissus, Babcock has written: "He is reflective, but he is not reflexive-that is, he is conscious of himself as an other, but he is not conscious of being self-conscious of himself as an other, and hence not able to detach himself from, survive, even laugh at this initial experience of alienation"(MacAloon 1984a: 11-12).
\end{abstract}

Also, I entirely agree with this comment. First, I can say that taking the attitude of the other to oneself is not reflexive but reflective, or rather self-reflective. I should like to define self-reflection as the capacity to be engaged in thinking ones' own or other's action to consider what one can do as one's own next action. In this process, one is not another or an outsider, nor alienated from oneself, but still keeps oneself inside it. Second, reflection can be defined as the capacity to be involved in regarding one's own or other's action as ground factors of producing other's action. In other words, Narcissus in the above case is engaged in self-reflection. We can be involved in selfreflection like the case of reflecting the Narcissus in a well in a part of the forest on his next action.

On the other hand, what is it like if we reflect the face in the well away from ourselves, for instance, in order to judge our facial impression from the standpoint of the well but with still keeping ourselves, not losing ourselves? That is, one reflects on oneself from the well, not from oneself. This is in reflexivity, not self-reflection, or reflection. Therefore, I can say this example can be applied to reflexivity in a Bakhtinian 
perspective wherein there is at least outsidedness in critical thinking and creative understanding.

In the end, regarding what I have reviewed selectively about Bakhtin's concepts with relation to this study, I can take a quotation from Tihanov, which summaries Bakhtin's concepts in cultural understanding:

\begin{abstract}
...Bakhtin's understanding of culture was shaped not only by neo-Kantian thinking or philosophy-of-life, but also by Hegelian ideas, and especially by the relativization of the boundaries separating the domains of objective and absolute Spirit in favour of a totalising idea of human culture [italics mine](Tihanov 2000: 22).
\end{abstract}

On the basis of these basic paradigms, I shall review intercultural communication (ICM) and explore an appropriate framework to improve ICM and competence (ICP) of EIL learners in the next subsection.

\title{
2.2.2 The Framework of ICM for EIL Learners
}

Through concepts given in the studies reviewed so far, I have demonstrated that EIL teachers should be concerned with the interrelation between the English language, ICM, and EIL learners. Also, I placed emphasis on otherness and outsidedness as inter-mediators between the self and the society, or between one's own culture and other cultures in ICM.

From such a view, I shall review the framework of ICM, especially in relation to drama. The first part of this review will develop from the framework of ICM in ethnographic perspective, but the latter part will move to its dramatic perspective.

\subsubsection{Factors of ICM (Intercultural Communication)}

As reviewed in 2.1, EIL is the salient medium in intercultural communicative processes. Equally, EIL teachers should consider the relation between language and its sociocultural contexts with Byram (1989), Kramsch (1993), Moraes (1996), Rassool (1998), and so on.

Furthermore, I have reviewed basic paradigms of communication through language, that is, the dynamic relation between the self and not- the-self, or all the others, in social communicative processes. Those processes also are connected with affective, cognitive, and behavioural dimensions at individual as well as at group levels, wherein 
we, as the dialogic or interlocutive self, are engaged in achieving otherness and outsidedness through 'third places.'

Through the review, it has emerged what language teachers should be concerned with in situational teaching of EIL in terms of ICM. The first thing to be considered is how we perceive the relation between EIL and our perception. For instance, we can suppose as follows:

- EIL can shape our perception differently to the way our own language does.

- there can be something in EIL that influences how we perceive the world.

- English speakers of EIL can see the world differently from Korean speakers of EIL.

- Chinese speakers of EIL cannot perceive identically what a certain African speaker of EIL says.

- even an English native speaker can perceive the world differently depending on his use of English as his mother tongue or EIL.

The thing to be considered here is what factors are related to intercultural communication through EIL.

As reviewed in 2.1, ICM scholars are concerned with cultural differences between communicators and search for how to overcome culture shock and to understand one's own culture as well as other cultures.

The concern about cultural differences should start with affective factors. If we see a certain cultural event that is very different from ours, what do we do normally? At first, we get a feeling of excitement or fear of the cultural event. And then we try to find out what's going on. After that, we might decide what we are going to do. Although these processes are not exactly linear, feelings about the situation affect how we are going to interact or behave appropriately in the intercultural situation, and perhaps much more.

What is more, experiences of a new or different culture cause communicators to lose all their familiar signs and symbols of social intercourse, whereupon they are in unpleasant surprise or shock, that is, anxiety in intercultural situations. Because of this feeling of loss, we can become alienated or strange in the new or different cultural situations (Furnham and Bochner 1986). Therefore, affective factors should be regarded as a basic ground to understand and to interact in intercultural situations. 
With reference to this affective influence, Gudykunst (1995) argues the importance of anxiety/uncertainty management in ICM. That is to say, effectiveness of communication is dependent on how an individual can manage his uncertainty and anxiety in intercultural situations.

On the other hand, Byram illustrates two criteria to assess successful ICM:

\begin{abstract}
I shall introduce the concept of the 'intercultural speaker' to describe interlocutors involved in intercultural communication and interaction. The success of such interaction can be judged in terms of the effective exchange of information, as has been the tendency in much communicative language teaching, but also in terms of the establishing and maintenance of human relationships' (Byram 1997: 32-33).
\end{abstract}

However, he also argues attitudinal factors relevant to human relationships between interlocutors affect the success of ICM.

\begin{abstract}
.. . for example the willingness of the interlocutors to expect problems of communication caused by lack of overlap in their respective knowledge of the world and of each other's country. It may depend on the ability of the interlocutors to accept criticism of the values they share with people in their usual social groups, and of which they may not have been consciously aware. It may also depend on their willingness to accept at least initially that they will be perceived by their interlocutor as a representative of a particular country, its values and its political actions, whatever their own views of these (Byram 1997: 33).
\end{abstract}

As indicated, he describes the salience of a non-judgemental attitude for ICM, that is, the willingness of the interlocutors who accept criticism of those values rather than evaluate those values in certain cultural perspectives.

Along with this non-judgemental attitude, he proposes a schema of the factors involved in ICM:

[1] Attitudes (savoir être) relativising self and valuing other,

[2] Knowledge (savoirs) of self and other and of interaction at individual as well as societal levels,

[3] Skills (savoir comprendre) to interpret and relate,

[4] Skills (savoir apprendre/faire) to discover and/ or interact, and

[5] Education (savoir s'engager) connected with political education into critical cultural awareness (Byram 1997).

On the other hand, Martin and Nakayama (1999) illustrate [1] motivation, [2] knowledge, [3] attitudes, [4] behaviours, and [5] skills, as basic factors relevant to the 
effectiveness of ICM and emphasise the interrelation among the five factors. They regard motivation as the most important dimension of communication competence. They emphasise the importance of self-knowledge and linguistic knowledge in the knowledge factor, and empathy and nonjudgementalness in the attitude factor, respectively. This is same as Byram but adds 'motivation.'

With regard to those factors, EIL teachers should be concerned with above all, attitudinal factors of EIL learners in teaching ICM through EIL. Equally, teachers should consider how to make the learners know about ICM and how to behave in ICM. In the end, to have better ICM means to interact with 'the right person, to the right degree, at the right time, for the right purpose, and in the right way, as Aristotle pointed out, in certain intercultural situations (Aristotle cited in Goleman 1996: xiii).

\subsubsection{Structure and Process of Intercultural Communication: Triadic, Third and Spiral}

In the previous subsection, I have reviewed factors in ICM with two schemata of Byram (1997) and Martin and Nakayama (1999). Here I shall compare between the two, particularly in terms of their attitudinal factors, which are basic points in ICM. Byram's schema, above all, is based on otherness, whereas Martin and Nakayama's is limited to self point of view. Let me take an example of their attitudinal factors. Byram emphasises 'relativisation of one's own and valuing of others' meanings, beliefs and behaviours' as desirable attitudinal factors (Byram 1997: 35). Here is an action where we compare the self with others, concern between the self and others, and value others. This means this sort of attitude is not limited to the self but is more than the self, that is, on boundaries of the self and others.

Meanwhile, Martin and Nakayama (1999) emphasise empathy and nonjudegementalness, along with tolerance of ambiguity as significant qualities in attitude components for ICM. I should like to consider empathy in terms of attitudes for ICM. According to Colman, empathy is defined as 'The capacity to understand and enter into another person's feelings and emotions or to experience something from the other person's point of view' [italics mine](Colman 2001: 241). Therefore, I can say their perspective on attitudinal factors is limited to the boundaries of the other person's point of view rather than on the boundaries of the self and the other.

With regard to this comparison, I can say dynamic foci in ICM are different depending on which factors are more emphasised. The different foci produce general dynamics in different ways. For instance, Byram's perspective can be more contextual than Martin 
and Nakayama's perspective in the attitudinal dimension in ICM. The former can be related to semi-open context on boundaries by conceiving 'a "threshold" at which learners become interculturally competent. . . as a point of orientation' (Byram 1997: 76). At this stage, I intend to take the concept of 'threshold' as a dynamic concept, emerging from the image of Janus. The dynamic concept provides EIL learners with spatio-temporal relation to take stock of intercultural situations as the boundary between being outside and being inside, whereas Martin and Nakayama's perspective can be related to close context within boundaries by focusing on how to "walk in another person's shoes" (Martin and Nakayama 1999: 320).

From this standpoint, I intend to explore how factors of ICM relate to each other in intercultural situations. First, I have argued that ICM has its main interest in cultural differences. In this regard, ICM aims at understanding the differences and its effectiveness means to interact appropriately in different cultural situations.

Now I should like to consider how factors relate to each within the triadic structure of ICM, that is, the dynamics of ICM. Camilleri describes the reason why we should understand the dynamic between cultures:

\begin{abstract}
Thus that which is contentious appears among the transmitters of culture as far surpassing problems of dissonance among systems considered in the abstract.... It is by taking as their point of departure explanations of such contentious situations that subjects may achieve the better intellectual orientation obtained through a correct understanding of the dynamic of culture, because their intelligence will be disengaged from the pernicious effects of subjective factors. It is at the end of this process, combining both intellectual and affective elements, that the cultural difference of others may be not only perceived but signified as it is [italics mine](Camilleri 1992: 146).
\end{abstract}

As indicated, understanding of the dynamic between different cultures provides interlocutors with distancing from the pernicious effects and combining affective and cognitive elements. Also, it is necessary to be transferable to both insidedness and outsidedness, not to restrict to one side.

\footnotetext{
... one should be concerned about the application of this method: besides a process of externalisation of feelings. . . . This objection commands clear acceptance of, on the one hand, what is expected of educators, and, on the other hand, the level of intercultural education which it is proposed that each should reach, by all internal and external means at one's disposal (Camilleri 1992: 146).
}

Thus Camilleri does not only emphasise the combination of cognitive and affective factors in the process of perceiving and signifying other cultural differences, but also 
accentuates internal and external means at one's disposal as an effective method to understand the dynamic.

In short, there is a question of how one combines intellectual with affective factors and at the same time applies internal and external means in the process of ICM.

Camilleri is more concerned about educating general attitudes of principle for ICM, that is, reciprocal interaction between individuals who have different cultural backgrounds:

That demands not satisfaction with a surface harmony, or politeness, easily obtained by educators, but that one facilitates between members of different groups reciprocal expressions of that which shocks them. One should not forget, above all, that those who are concerned are an integral part of the relational system.... (Camilleri 1992: 146).

Also, Byram and Zarate (1997) consider cultural learning through language involves more than the cognitive learning of culture. They argue that both affective and reflexive learning should be involved in the process of cultural learning, particularly with respect to decentring in the process of thinking about one's own cultural values and relating them to other cultural values.

With regard to what I have reviewed so far, there are important points involved in the process of ICM-

- the relation between affective and cognitive factors,

- distancing or decentring, and

- relative interaction between an individual and others.

However, we should consider how EIL teachers educate more than general attitudes or cognitive learning of ICM, in other words, how EIL learners connect what they learn cognitively about learning of different cultures, as well as their own cultures, with ICM in practical terms. Byram and Zarate suggest the principle of the spiral curriculum as a means of connecting cultural learning and cultural competence:

The principle of the spiral curriculum is a means of combining the concept of spheres of knowledge or areas of experience.... The same area of experience can be 're-cycled' and re-analysed at a larger stage in learners' development (Byram and Zarate 1994: 19). 
As implied in the concept of 're-cycled,' this principle is related to progression and levels of cultural competence in terms of the dynamic of ICM.

As reviewed, there are the factors in Byram's schema of ICM: [1] Attitudes (savoir etre) relativising self and valuing other, [2] Knowledge (savoirs) of self and other and of interaction at individual as well as societal levels, [3] Skills (savoir comprendre) to interpret and relate, [4] Skills (savoir apprendre/ faire) to discover and/ or interact, and [5] Education (savoir s'engager) connected with political education into critical cultural awareness (Byram 1997). These factors are considered 'in respect of empathy, but with a triadic definition which includes not only cognitive but also affective and behavioural aspects' (Byram and Zarate 1994: 20).

These five factors are not only inter-related with others but also involve the effectiveness of ICM through EIL in going through the following six stages of general processes. The factors and the stages comprise processes, which are non-linear and cumulative and even sometimes overlap. Byram and Zarate illustrate that the progression shows how the above five factors of ICM develop in the matters of its direction and its dynamics the progression.

Stage 1: The geo-political context-EIL learners feel and observe their situations at individual and societal levels. At this stage, individual learners are more dependent above all on attitudinal factors, although other factors are involved here. Those attitudinal factors involve their perception and observation of different cultural situations and then lead those factors to be relative to the self and others.

Stage 2: The learning context-Learners are more dependent on the Knowledge (savoirs) factor but still keep attitudinal factors and are imbued with their knowledge of other cultures with intercultural meanings and values as EIL learners.

Stage 3: The developmental factor-Learners move their focus onto the factor of skill to interpret cultural phenomena, beliefs and practices and to relate those parameters to themselves, along with factors involved in the previous stages.

Stage 4: Identification of objectives-Learners need to rely on all the three factors of attitude, knowledge, and skills, to interpret and relate as well as to discover and interact. Here learners are concerned with their cultural identities and operate their cultural learning in specific intercultural situations. 
Stage 5: The ICP threshold-Learners are taught political education in order to prepare to deal with international or intercultural discourses in terms of cognitive, evaluative and action orientations. Because of this, EIL learners have critical intercultural awareness.

Stage 6: Sequence in the curriculum-Learners consider their involvement in ICM as cultural learning in relation to all of the five factors of ICM and improve their ICP. ICP, which is different from the previous ICP, is based on another ICP threshold. This implies that intercultural sensitivity of EIL learners changes or improves in this progressive way.

This progression of ICP in relation to factors in ICM can be summarised as Figure 2.1.

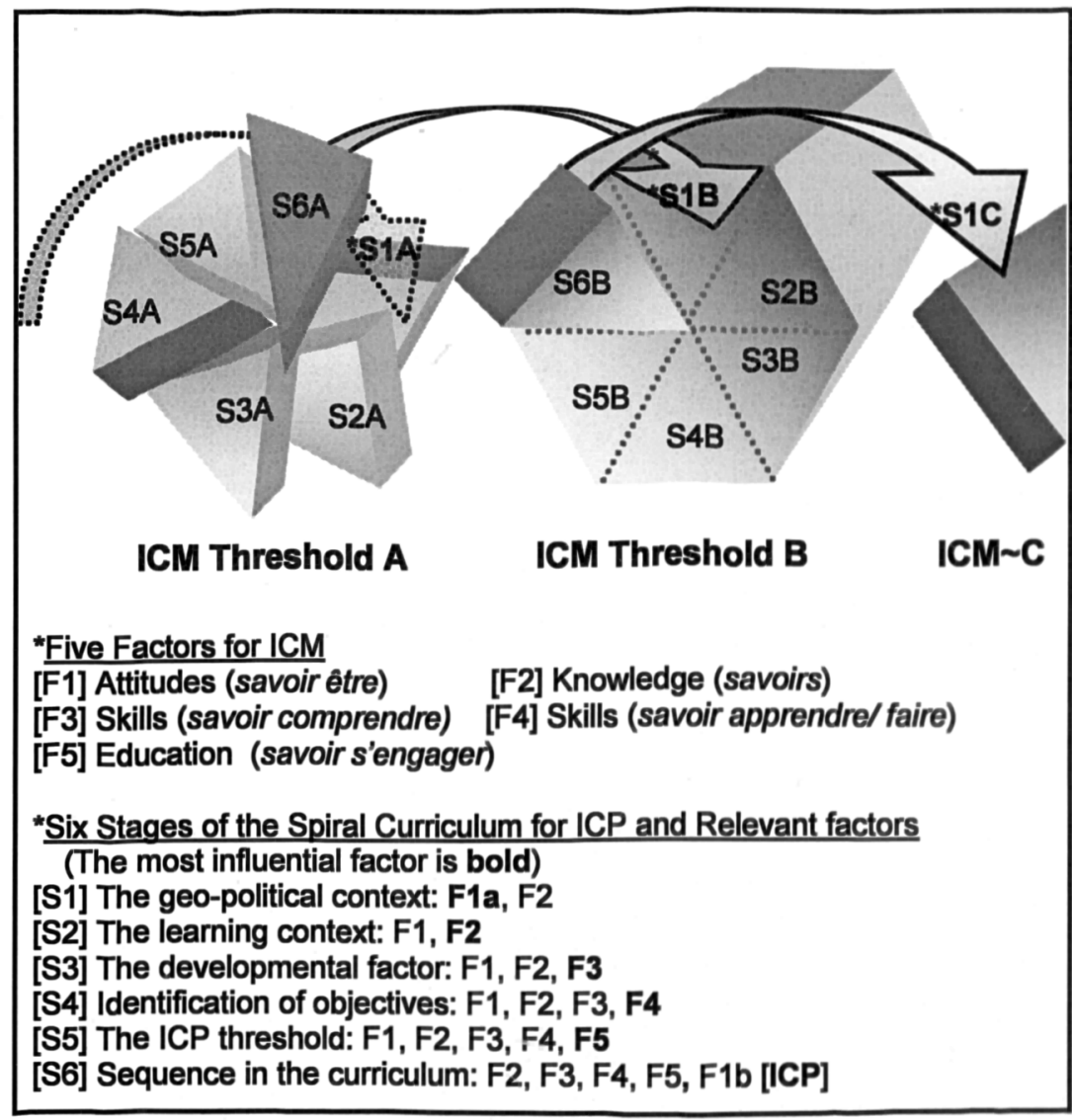

Figure 2.1 Factors and Progression of ICM in the Spiral Curriculum for ICP 
Summarised in the figure, five factors of ICM are not only inter-related to each other but also are cumulative as stages are taken. Also, those factors are connected with the six stages in the spiral progression of intercultural competence (ICP). That is to say, this progression comes about through the interaction of an EIL learner as an interlocutor with information, contexts, settings, situations, and other interlocutors. These interlocutors use the spiral process that is rooted in their previous experiences, affective, cognitive, and behavioural, for instance, fears, disappointments, prejudices, hopes, belief systems, values, and achievements.

With reference to what I have reviewed as the framework of ICM, EIL learners are taught to interact with different socio-cultural backgrounds through taking third places by dint of distancing themselves from those backgrounds or externalising them. In the process, they should be concerned with ICM at individual as well as group levels in their situational social structures.

On the other hand, their ICM and ICP are also on the ground that culture has the transmissibility, for instance, through thirdness or spiral progression in their triadic structure, affective, cognitive and behavioural. As discussed in 2.2.1, culture is socially determined but transmissible. This means cultural identity, although it is socially determined, in other words, is constructed at the social level, always is involved in the process of starting to change and develop, and of being. That is, cultural identity is transmissible-not static but changeable. From this standpoint of the changeableness of cultural identity, EIL teachers or Intercultural educators should be engaged in ICM, ICP, and intercultural education for young people.

\subsubsection{Pathway to Intercultural Actors with Dialogic Identity}

Here is another issue for EIL teachers-how they can lead EIL learners to change their cultural identity within ICM and ICP.

As I have suggested, intercultural learning through EIL does not merely mean learning general principles for ICM but also awareness of international or intercultural discourses through effective paradigms such as the dialogic dynamic self-society relationship. This relationship between individuals and socio-cultural or intercultural discourses through EIL involves their cultural identities as well as their national identities. This means EIL teaching can be regarded in terms of socialisation and identity formation of the learners, and the implications for teaching policy of EIL (Byram 1999). 
In this regard, EIL teachers should add moral-ethical dimensions to ICM and ICP. Willems emphasises the importance of moral-ethical capacity:

Interculturality has moral-ethical dimensions for it incorporates respect for what is different. It requires knowledge (of cultural factors), insight (into what constitutes cultural identity), readiness (towards opening up to cultural differences) and skills (in negotiating 'common territory' and identifying bridging gaps) (Willems 2002: 7).

Therefore, EIL learners need to be taught to become intercultural mediators or intercultural actors, who have effectiveness as well as ethics in ICM. Here I use the term of intercultural actors in the sense of persons who undertake intercultural action. This term is borrowed from social actors, which is used as the concept such as roles in sociology indicates. The term is applied in that this study involves intercultural action as well as drama activity.

Above all, we should be concerned with what the term of 'intercultural', implies. As reviewed before, intercultural communication (ICM) refers to the communication process between individuals and groups, who have differences in cultural backgrounds. In the process of contacting a new or different culture, an intercultural mediator or an intercultural actor seems to experience an unpleasant surprise or shock because of different cultural qualities. As said earlier, Furnham and Bochner state those experiences make people incur culture shock, 'partly because it is unexpected and partly because it may lead to a negative evaluation of one's own culture' (1986: 47).

However, although culture shock emerges with psychological problems for individuals at the individual level, EIL teachers should be also concerned with the sociological relationship between individuals and international/ intercultural discourses, particularly in terms of the self-society dynamic at the global level.

At this stage, the thing to be considered is how to teach EIL learners ICM, to develop their ICP, and to become intercultural actors.

According to Gudykunst and Nishida(1989) and Hall (1992), there are three main approaches to researching ICM and ICP: functionalist, interpretive, and critical approaches.

All of the approaches to ICM and ICP research have their strengths and limitations. As indicated in my review of basic paradigms of ICM in the present section, I should like 
to use the critical approach in intercultural education for this research study. This is, above all, because the approach emphasises the importance of studying the context wherein ICM occurs, that is, international/ intercultural discourse.

The critical research approach is unlike the functionist approach, which has identified psychological and sociological variable in the process of ICM, but is limited in that it fails to identify all of the variables that affect ICM, which is creative and dynamic. It is different from the interpretive approach, which provides an in-depth understanding of ICM patterns through field studies, observation, and participant observation in particular situations because of its emphasis on ICM in context. Nevertheless, it usually fails to compare ICM between different groups and it fails to develop learners as insiders who are good enough to communicate accurately.

This is a weakness because, in order to have an in-depth understanding, learners need the position as insiders as well as outsiders. And then, they can involve critical thinking about communication and explore the essential roles of EIL learners in ICM. As reviewed in 2.1.4, EIL teachers should be concerned with the role of EIL learners as critical ethnographers in the dual perspective of outsider as well as insider.

Moreover, teachers should be concerned with their learners as critical ethnographers. This is because the critical approach emphasises the power relations in relation to international/intercultural discourse but has its limitation of not focusing on face-to-face ICM. Martin and Nakayama explain the reason of the limitation:

... one limitation is that it does not focus on face-to-face intercultural interaction. Rather, most (though not all) critical studies focus on popular media forms of communication-TV shows, music videos, magazine advertisements, and so on. Such studies, with their lack of focus on face-to-face interactions, may yield less practical results' (Martin and Nakayama 1999: 42).

In order to overcome this limitation and to yield practical results more in intercultural communication, we can turn to drama.

As Fleming argues, 'exploration of interculturalism is likely to require more than intellectual enquiry, reasoned argument and acquisition of knowledge,' and we should try to supplement something else, such as emotion, to logocentric interculturalism (Fleming 2003: 97). In this regard, he argues 'drama as a form of intercultural education' to provide concrete contexts and affective engagement for the participants (Ibid). Before pursuing this, however, I shall consider another approach. 
Byram et al. suggest a structured and integrated way to intercultura educat on for instance, an ethnographic fieldwork model in language teaching. A so, they argue the way forward is rather to consider how a structured approach can be deve oped and introduced into language teaching' in order for EIL learners to be actively invo ved $\mathbf{n}$ ICM process in certain intercultural contexts or situations [bolds mine] (Byram et al 1991: 380-381).

As reviewed in 2.1.4, EIL learners as critical ethnographers are involved in critical thinking about socio-linguistic, socio-political, and socio-cultural contradictions at a global level in situational learning of EIL.

Nevertheless, how do they start critical thinking about certain international or intercultural contexts? The short answer is to start with an act of imagination, which is the hearer's ability to reconstruct the idea expressed by the words the hearer hears in the relation between speaker and hearer (Collingwood 1963). However, another way to be considered is to think critically about international or intercultural contexts. That is to say, EIL learners need to think critically at international or intercultural level, with intercultural imagination.

I should like to borrow the concept of The Sociological Imagination (Mills 1970), which I have reviewed in 2.2.1. Moreover, I intend to apply this concept to critical thinking in relation to understanding one's own culture as well as other cultures in ICM, and to call this sort of thinking the intercultural imagination.

In the process of the intercultural imagination, EIL learners need to think themselves away from their own familiar socio-cultural situations to look at them anew. Because of this, they are engaged in a multitude of third places between themselves and international/intercultural discourse surrounding them through distancing or outsidedness and are then involved in dialogic identities.

Mills (1970) articulates the capacity to shift from one perspective to another and the relation between distancing and identity in the Sociological Imagination. In this regard, the ultimate aim of the Sociological imagination is to lead EIL learners to realise the relativity between them and intercultural discourse and to transform themselves as well as the discourse.

As an alternative to ethnographic approaches, we can return to the argument for drama. Erlenwein regards dramatic activities as 'experiences of and reflections upon the holistic learning process' and points out that these dramatic activities 'should be 
recognised as primary tools of intercultural communication, and promoted and sponsored accordingly! [author's italics](Erlenwein 1993: 193, 200).

On the other hand, Schechner, from the viewpoint of performance studies, suggests a workshop as an approach to understand that the global world consists of thousands of distinct cultures.

\begin{abstract}
The workshop is a way of playing around with reality, a means of examining behavior by reordering, exaggerating, fragmenting, recombining, and adumbrating it.... The aim of the workshop is to construct an environment where rational, arational, and irrational behavior exist in balance. Or, to put it biologically, where cortical, brain-stem, motor, and instinctive operations exist in balance, leading to expressive, symbolic: playful, ritualised, "scripted" behavior" (Schechner 1988: 103-104).
\end{abstract}

More directly, Shaked regards drama/theatre activity as gateway to ICM and ICP:

Only a play can bring a past or distant culture to its audience in its authentic fullness...the confrontation of the theatrical process is always between two social paradigms.... In the eternal gap between any two social, cultural, and normative worlds, the audience confronted with a strange world is expected to make a constant effort at confrontation, translation, and cognisance both of what is similar and of what is different. That process is one of cultural awareness and understanding (Shaked 1989: 23).

Given these arguments for drama/theatre, I shall review in the next chapter how drama/theatre is inextricably interconnected with ICM and ICP in situational teaching of EIL.

\title{
2.3 Summary: EIL Learners to Intercultural Actors
}

In this chapter, I reviewed the literature that has affected this study. Beginning with the position of the English language as an International language in a global context, $I$ reviewed teaching EIL for intercultural communication (ICM) that comprises teaching methods of situational teaching and learning EIL and multimodal communication in that context.

Consequently, it emerged that EIL learners should be taught as critical ethnographers for ICM and intercultural competence (ICP) in international and intercultural discourses.

I situated my review in basic paradigms of ICM and demonstrated how individual EIL learners connect with their intercultural contexts and vice versa. Through a detalled account of those paradigms, I clarified the self-others relationship and its relation to 
intercultural identity in ICM through EIL. Also, I illuminated the need to develop ICM and ICP by otherness, outsidedness, thirdness, that is, the dialogic self.

Finally, I presented those basic paradigms of ICM and ICP that have influenced this study and explained the framework of ICM in situational teaching of EIL learners, that is, in terms of intercultural education. Also, I explained the approaches EIL teachers can take in their classrooms. I ended with an explanation of the ways in which drama is one of the most effective ways to have an in-depth understanding of ICM in situational teaching of EIL learners.

In the next chapter, I give details about how drama is involved in ICM and ICP in intercultural discourse. 


\section{Chapter 3.}

\section{Drama and its Approach to ICM (Intercultural Communication)}

One of the distinctive features in this global society, as argued in Chapter 2, is an increasing interconnection between the dialogic self of an individual person and the international and intercultural discourses surrounding him. The dialogic self is 'not a passive entity, determined by external influences; in forging their self-identities... individuals contribute to and directly promote social influences that are global in their consequences and implications' (Giddens 1991b: 17).

In my view, critical ethnographic fieldwork, workshop, or drama is effective in understanding and promoting the interconnection between the self and intercultural discourses. I propose that drama is one effective method of gaining an in-depth understanding of ICM in the situational teaching of EIL learners.

I have confidence in the argument of Hornbrook that

...art is not a genetic or psychological attribute but a cultural construct....
Drama's unique capacity to incorporate the other arts means that it probably
offers more scope for potential student achievement than any other art form'
(Hombrook 1998b: 61-62).

From this standpoint, I shall review drama and its approach to ICM (intercultural communication). The argument that 'Only a play can bring a past or distant culture to its audience in its authentic fullness' is also relevant [italics mine] (Shaked 1989: 23). There is another supportive argument-a dramatic approach is 'not merely to be regarded as so many additional aids to language teaching, but should be recognised as primary tools of intercultural communication, and promoted and sponsored accordingly!' [author's italics] (Erlenwein 1993: 200).

The purpose of this chapter is, first, to review drama as related to every day sociocultural life. EIL learners who participate in drama need to negotiate the relationship between their selves and international and intercultural discourses during their drama activities. Second, EIL teachers should consider drama as an effective method for developing ICM and ICP of EIL learners. 
The discussion will be presented in three sections. In the first section, I shall review situatedness as intrinsic to drama, in that the latter, as cultural morphology, figures in life and vice versa. Second, I shall show how individual participants in drama are engaged in ICM. My intention here is to point out that the nature and the dynamics intrinsic in drama, for instance, creativity, dialecticality, and dialogicality, lead EIL learners to interact in ICM in an effective way. Third, I shall present an integrated approach to ICM through drama.

The ensuing presentation emphasises psychological and socio-cultural dimensions of the understanding of persons in ICM through drama. Both Beckerman's figure-ground principle and Bakhtin's concepts relevant to art underpin the review in the present chapter.

\subsection{Drama and its Double with ICM}

According to Vendler, the understanding of a person happens not as scientific datum, theory, or projection, but as something private, subjective, and unobservable. In other words, we cannot say we understand him with only our knowledge of his superficial factors until we understand important things at his deep and private level. That is, it is necessary to understand something in his mind. As discussed in the previous chapter, the person, as an individual member within his socio-cultural structure, influences the structure and vice versa. Furthermore, a person is not a momentary being. Therefore, 'to understand a person is to imagine being in his situation...including not only his "external" circumstances but also his state of mind' (Vendler 1984: 211).

In this regard, I believe that 'the feat of understanding people is intimately tied to the practice of social sciences' along with the connection with their inner lives [author's italics] (Vendler 1984: 212). This is supported by the arguments of Shaked (1989) and Schewe (1998) for drama, namely that drama can provide EIL learners with the exploration of self-identity and the practice of the social sciences in terms of understanding people in intercultural situations.

\subsubsection{Art and Life: Figure and Ground of Drama}

According to Bakhtin, there are three domains of human culture, i.e., science, art, and life, but only the individual person who integrates them into his own unity can achieve the unity of the three. For instance, when a person is in art, he is not in life, and vice versa. Art and life can be integrated through connecting with the intrinsic elements in the person. This indicates that art and life must assume not only mutual answerability 
but also mutual liability. Therefore, it may be correct to say that 'Art and life are not one, but they must become united in myself-in the unity of my answerability' (Bakhtin 1919: 2).

This relationship can be explained as the figure with the double profiles, which a Gestalt psychologist, E. Rubin, first used as in Figure 3.1.

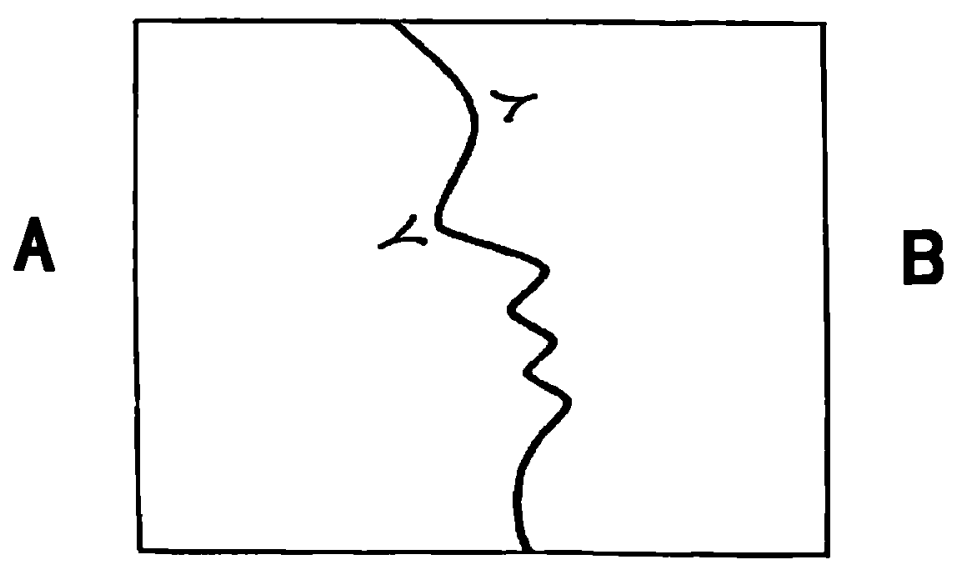

Figure 3.1 Rubin's Double Profiles (Ehrenzweig 1971: 23)

(' $A$ ' and ' $B$ ' are added)

As shown in the figure, we choose to see Face $A$ as the figure and then Face $B$ disappears from view to become the invisible ground and conversely. In other words, if we choose one, the other become invisible and consequently we cannot catch what the figure indicates with the double profiles. The double profiles cannot become one until they become united in ourselves. That is to say, in order to hold the entire picture we must pay two kinds of attention at the same time.

Therefore, I can say at this stage that the relationship between drama and life is the same as the relationship between the figure and the ground, as implied in Rubin's double profiles. Furthermore, drama and life sometimes overlap. Equally, I can say that EIL teachers, who use drama in their English classrooms, should consider this figure-ground relationship of drama and its socio-cultural discourses. I agree with the following argument of McLaren:

What is needed is not simply more time for drama and the related arts in schools, but the orchestration of the entire school day as a series of ritualized events.... Teachers could draw upon the works of Stanislavski, Bolton, Brecht and Grotowski in order to promote an awareness in students that they are active body-subjects that engage the world not just in their heads, but in their hands, their heart, their guts and their loins. Educational literature could include the writings of Bellah, Grimes, Turner, Goffman and Schechner in order to lay 
the groundwork for an emergent epistemology of learning theory by way of ritual embodiment [ltalics mine] (McLaren 1993: 243-244).

Thus, teachers should be concerned with awareness in students and the relationship between the students and the world wherein they are engaged. In other words, the EIL teacher should consider EIL learners and international and intercultural discourses surrounding the learners in terms of the figure-ground principle. EIL students, who participate in intercultural drama, cannot be separated from the intercultural discourses relevant to their involvement.

\title{
3.1.2 Drama as Cultural Configuration
}

At this stage, I would like to compare to understand with to know in relation to understanding people in relation to promoting awareness in students. First of all, we can consider their differences, that 'whereas know takes both simple and complex objects, understand is restricted to more involved objects' (Vendler 1984: 203-204). For example, there are two sentences: [1] The teacher knows all the pupils by name; [2] The teacher understands all the pupils by name. Sentence One makes sense but Sentence Two does not sound correct. The difference is explained by Vendler as follows:

\footnotetext{
Understanding requires a constructive effort of the mind to supplement features that are not observable to the senses. The whole conceptual environment of understand betokens the fact that understanding, unlike knowledge, is no mere passive state but a result of the mind's constructive labor [author's italics] (Vendler 1984: 205-206).
}

As implied in the above, the second sentence is not able to deliver its meaning but needs more description. Or, to use another image, we have to read between the lines in order to understand a novel.

In addition, Vendler emphasises time or cultural backgrounds surrounding the person:

\begin{abstract}
...the understanding of humans remote in time or cultural background would be impossible without the data and interpretations offered by such sciences as history and anthropology. On the other hand, the attempt to understand his subjects, that is, to reproduce their minds in his own, is likely to guide the scientist in his search for those features in their environment and cultural background that might have been instrumental in shaping those minds. (Vendler 1984: 212).
\end{abstract}


Thus, in order to understand the person, although the understanding may be only partial or incomplete, we should be concerned with his situations in temporal or cultural backgrounds.

With regard to this, I should like to replace awareness with understanding in the argument of McLaren (1993), which I have discussed in the previous subsection. Moreover, I can say that drama can be effective in the practice of intercultural discourses in order to understand people in intercultural situations. In other words, drama is a gateway for culture in the sense that it allows one to receive a foreign culture within a single, complete framework with clear contexts.

Deetz agrees to Geertz's argument that culture is an ensemble of texts read over the shoulders of those who live them out and give a further explanation that 'such an approach allows a holistic-contextual consideration of meaning grounded in the empirical, objective products of that culture' (quoted by Deetz 1984: 215-216). Moreover, he defines culture as 'morphological configurations composed of basic spatial and temporal orientations' [italics mine] (lbid: 216).

Wuthnow et al. (1984) regard culture as the symbolic-expressive dimension of behaviour in terms of the verbal discourse as well as the dramatisations of everyday life and the ritualised aspects of social arrangements.

From the viewpoint of drama, Britannica on line regards 'dramatic literature/drama as an expression of a culture' (Britannica 2000b). And Courtney states that 'drama is one aspect of a society, but a vital one. By its very nature, drama presupposes communication-and this is the primary social process' (Courtney 1968: 148).

In even stronger terms, Peter Brook argues that drama is not just interconnected with socio-cultural discourses. Drama is the force to counterbalance the fragmentation of our world and to discover the relationship 'between man and society, between one race and another, between the microcosm and the macrocosm, between humanity and machinery, between the visible and the invisible, between categories, languages, genres' through cultural links (Brook 1996: 66).

In relation to these cultural links, he provides an example of his experiences at the International Centre of Theatre Research. He presents from the example the cultural links achieved through the third culture between those who worked for the Centre: 
The core of our work at the International Centre of Theatre Research was to bring together actors from many different backgrounds and cultures.... It involved challenging all the elements that in all countries put the theatre form into a very closed bracket-imprisoning it within a language, within a style, within a social class, within a building, within a certain type of public. It was by making the act of the theatre inseparable from the need to establish new relations with different people that the possibility of finding new cultural links appeared. For the "third culture" is the culture of links.... Only cultural acts can explore and reveal these vital truths (Brook 1996: 65-66).

From Brook's experience with ICM, I am concerned with four main elements:

- All the socio-cultural elements, which emerge from different cultures, can be challenged;

- Nevertheless, we need to communicate with each other in certain situations;

- The way to start with ICM is to establish that a human relationship exists in the third culture, that is, thirdness; and

- It is through cultural acts for us to have intercultural understanding, that is, the interconnection between cultures and acts.

As indicated above, drama, culture, and ICM are integrated into intercultural understanding in human relationships in multicultural societies. Inside this successful ICM of Brook's, there seem to be practical factors of interrelationship between art and culture:

This realization, that to study an art form is to explore a sensibility, that such a sensibility is essentially a collective formation, and that the foundations of such a formation are as wide as social existence and as deep... A theory of art is thus at the same time a theory of culture (Geertz 1983: 99, 109).

Hornbrook's view quoted earlier is also relevant to this relationship.

Art is not a genetic or psychological attribute but a cultural construct.... Drama's unique capacity to incorporate the other arts means that it probably offers more scope for potential student achievement than any other art form (Hornbrook 1998b: 61-62).

Furthermore, Schechner (1988) insists on the importance of drama as the rehearsal process in terms of the cultural performance approach to ICM.

With reference to the above arguments, it seems that drama, both as art form and as cultural construct, can offer an arena to students, particularly, cultural learners, in order to make appropriate ICM and to have intercultural understanding. 
Hornbrook (1998b) argues that it is inevitable that young peope who produce and perceive socio-cultural communication in and through drama escape the nfluence of the society and the culture to which they belong. With respect to th $\mathbf{s}$ he proposes that drama education is, in some significant part, about cultural induction

With regard to this interrelationship between art, drama, and cu ture, it appears that drama is defined as cultural configurations and cultural performance Drama is a so applied to understanding people through these cultural links.

\title{
3.1.3 Situatedness: Situated Learning by Doing through Drama
}

Drama is thus concerned with culture. However, we need to ask more precisely about the interrelationship between drama and culture. In what sense does drama interrelate to culture? Firstly, we have to keep considering drama as cultural configuration. Secondly, the relationship between drama and culture is based on dramatic pattem. Courtney claims that 'Dramatic patterns...reflect in a detailed and emotionally important way the thought and life of the community' (Courtney 1968: 148).

On the other hand, Wuthnow et al., from the viewpoint of culture, assert that culture is the symbolic-expressive dimension of behaviour, which does not merely subsume verbal discourse, 'but also the dramatizations of everyday life and the ritualized aspects of social arrangements' (Wuthnow et al. 1984: 255).

Furthermore, Bolton, from the standpoint of drama education, illustrates the common ground between drama and real life, that is, their situatedness:

\begin{abstract}
Just as in 'life situations' we 'work at' our social contexts to make them believable to ourselves, so it is in 'dramatic playing'; just as in 'life situations' we initially describe to each other the kind of situation it is, so it is in 'dramatic playing';....just as in 'real life' participants may be relatively active or passive within the situation, so it is in 'dramatic playing'; just as in 'real life' participants operate spontaneously in the 'here and now', so it is in dramatic playing (Bolton 1992: 11).
\end{abstract}

As implied in the above, it is not possible to do drama without any context or any situation. When we are doing drama, we usually take the place of another, in consideration of who they are, where they are, and when they are.

This is not only because our values and behaviours are positioned in space and tıme but also our activities are dependent upon the contextual settings. Also even in our 
everyday lives, what we are is not exactly what we were, although we have been in the same place that we had been. On these grounds, we can start to reconsider what drama activity is like and what situated activity is like, and if they are related to each other and then how they are related. Lave and Wenger explain the term 'situated' in the following way:

On some occasions "situated" seemed to mean merely that some of people's thoughts and actions were located in space and time. On other occasions, it seemed to mean that thought and action were social only in the narrow sense that they involved other people, or that they were immediately dependent for meaning on the social setting that occasioned them (Lave and Wenger 1991: 32-33).

In the light of their definition that thought and action are situated in social or intercultural contexts, which are set by time and space, I believe that drama activity can be described as situated activity.

Furthermore, on the basis of this concept of situated activity, Lave and Wenger developed the situatedness of activity into situated learning:

\begin{abstract}
...it [the situatedness of activity] took on the proportions of a general theoretical perspective, the basis of claims about the relational character of knowledge and leaming, about the negotiated character of meaning, and about the concerned (engaged, dilemma-driven) nature of learning activity for the people involved [brackets mine] (Lave and Wenger 1991: 33).
\end{abstract}

From this explanation they give me the idea that learning is situated in a mutual construction between an individual person and society and therefore 'any "power abstraction" is thoroughly situated in the lives of persons and in the culture that makes it possible' (Ibid: 34$)$.

With respect to the ways in which drama activity implicates its situatedness in the society and the culture, drama activity can be seen as a form of action learning, in which participants learn by doing in their intercultural discourses in which power is situated.

Above all, drama action itself has not only its potential in its capacity to allow participants to draw from their socio-cultural backgrounds, but also the essential keys to learn how to learn and to cope in a teaching and learning environment for which they have not been prepared. 
Drama experiences, which the participants bring into their intercourses, therefore appear to be dependent upon two factors: [1] the participants themselves explore their current situations and approach to problems in the situations, in the sense that drama experiences can reflect their situated actions in every day life and vice versa; and [2] participants' experiences, their behaviours through drama actions, are explored in dramaturgical perspective.

Drama reflects socio-cultural life in the here-and-now situation. At this stage, a key factor is that we do not engage in drama activity only by putting ourselves into another's shoes through drama, but also by exploring the activities in order to have a better understanding of the other as well as ourselves.

So far, I can say that drama is thus supportive in situational teaching and learning of EIL with reference to its situatedness. In addition, drama, as a gateway to ICM and ICP, can be summarised as follows (Shaked 1989):

- Drama is a gateway for culture in the sense that it allows one to receive a foreign culture within a single, complete framework with clear contexts.Drama provides objective research by presenting facts and numbers outside their living contexts.Drama brings culture to its audience in its authentic fullness beyond spatial-temporal dimensions.Drama is always grasped as paradigmatic, no matter how much it also represents a world beyond the stage, although culture is illuminated from the limited point of view of a single observer.

\subsection{Drama Activity and ICM (Intercultural Communication)}

As reviewed in 3.1.2, we can apply drama to understanding people in cultural contexts. Then the thing to be considered is how we shift EIL learners towards performance in order to improve their ICM, as Byram suggested that 'Language Learning may well be largely rehearsal-oriented, with some communicative teaching techniques shifting the learner towards performance' with regard to the above advantages of drama (Byram 1991: 20).

\subsubsection{The Total Imaginative Activity through Drama in Intercultural Discourses}

Given the concept of situatedness in a global society and intercultural situations, I have argued that drama can be effective in understanding others. This, above all, is based on the perspective that by reflecting on socio-cultural or multicultural life we 
have a better understanding of other people in different cultures as well as ourselves by putting ourselves into another's shoes through drama.

From the viewpoint of cultural theory, Vendler states 'Persons...are not merely objects but also subjects of experience -of sensation, feeling, thought and action...the need for such a double representation' (Vendler 1984: 200). Furthermore, he emphasises the function of imagination, in other words, the 'Magic If in the Stanislavski acting system, in the process of understanding people:

... one can represent him, in imagination, as a subject of experience. After all, it is this way of thinking of him that provokes our sympathy, pity, and moral outrage.... Subjective states-sensations, feelings, and emotions-cannot be found, recognized, or discovered in bodies but are attributed to them on the basis of certain observable manifestations that warrant such attribution.... Thus the connection between subjective states and overt manifestation is to be found in one's own experience alone: And this is not a scientific datum. Yet, it enables one to represent, in imagination, the states of another mind. Without the power of the imagination, we all would be solipsists [italics mine] (/bid: 201).

He suggests how we can put ourselves into another's shoes, that is, by dint of empathy, through imagination in order to understand a certain person:

\begin{abstract}
There is a way...by imagining what it must be like for him to be in that situation. What would I feel, how would I react, if I were he? We have to watch out, though, concerning the sense of the "I" in this context. For it is one thing to imagine what $I$, that is, Z. V., would feel and do, given my temperament, background, beliefs and values, and so forth, and quite another thing to perform total transference and try to assume in addition our subject's beliefs, values, prejudices, hang-ups, and the rest. Obviously, to understand people, the latter move is called for (lbid: 208-209).
\end{abstract}

As implied in the above, Vendler argues that understanding people requires 'empathy, that is, the reproduction, by means of imaginary transference, of the agent's consciousness into one's own mind so that his conduct may appear as a result of free, but rational, choice' [italics mine](Ibid: 209).

However, there is something important to be considered here, that is, processes of total transference in relation to a person as a double representation, an object as well as a subject, of sensation, feeling, thought and action. In this regard, as Vendler mentions, we should consider in the imaginative process, firstly, what I as a certain person would feel, secondly, react in socio-cultural discourses surrounding me, and thirdly, perform total transference. That is to say, there is the need for three main processes, which involve empathy through imagination to total transference-feeling, reacting, and performing. 
Similarly, Collingwood argues that there are three pre-stages to total imaginative activity:

\begin{abstract}
The activity of consciousness, we have seen, converts impression into idea, that is, crude sensation into imagination. Regarded as names for a certain kind or level of experience, the words consciousness and imagination are synonyms: they stand for the same thing, namely, the level of experience at which this conversion occurs. But within a single experience of this kind there is a distinction between that which effects the conversion and that which has undergone it. Consciousness is the first of these, imagination is the second. Imagination is thus the new form which feeling takes when transformed by the activity of consciousness [italics mine](Collingwood 1963: 215).
\end{abstract}

Thus, Collingwood argues that we take three processes in having experiencesfeeling, consciousness, and imagination.

Abbs also emphasises the processes of the three and relates these processes to language learning:

\begin{abstract}
It is essential also that we perceive the contiguity between sensation and feeling, of sensory experience and sensibility. Again and again the practices of our language, the inherited conjunctions and the daily alliances of our speech, suggest the intimacy of this relationship.... The very word 'feel' embodies the conjunction; one can feel both feelings and objects and indeed, one can do both simultaneously. Our belief analysis discloses that the aesthetic involves both the perceptual and the affective. The education of aesthetic intelligence must therefore be concerned with the development of sensation and feeling into what is commonly called sensibility [author's italics] (Abbs 1989d: 4).
\end{abstract}

Thus, there tends to be three stages-feeling, consciousness, and imagination in understanding people and in explaining human experiences from the viewpoints of both cultural study and arts education. This indicates that three domains in relation to the aforementioned three-feeling, consciousness, and imagination, have influenced our experiences. In other words, the three factors, affective, cognitive, and behavioural, have influence on intercultural experiences of EIL learners through drama in terms of forming their pathos, logos, and ethos connected with the three, which will be shown in 5.3.2.

With respect to the relationship between the above three, there remains another question, particularly in terms of socio-cultural or intercultural experiences, of how EIL learners deal with imagination through drama in relation to ICM and intercultural discourses.

The first thing to be considered here is where we should focus imagination through drama in ICM. Kear and Callaway (2000) define imagination as a function and at the 
same time the product of arts education in that it is based on personal experience and the ability to imbue known phenomena with wondrous and unknown qualities. They pinpoint the productivity of imagination from personal experience and ability.

Furthermore, Best defines the relation of feeling to rationality and argues that the criteria of creativity should be found in the creative aspects of activities and of what is created:

Creativity is not to be found by an unintelligible delving into occult 'inner' processes, whether one's own or someone else's. It is to be found in the character of the work of art, the use of language, the way someone behaves. (Best 1992: 95).

Thus, Best emphasises the productivity of creativity by illustrating that the depth of the problem lays on its surface rather than an unintelligible delving into occult 'inner' processes.

Secondly, we should consider how we could facilitate the productivity of imagination in terms of ICM through drama. Reid describes the processes of the understanding of works of art including drama:

\begin{abstract}
The understanding of works of art in any depth is partly conditioned by knowledge of different kinds...knowledge-how, knowledge of facts, and cognitive feeling for values.... Then there is knowledge of techniques, in one way particularly important for working artists themselves as 'knowing-that' and 'knowing-how', but also important for discriminating appreciation. These form the background conditions or presuppositions for true artistic understanding [author's italics] (Leid 1989: 17-18).
\end{abstract}

He thus states knowledge-how, knowledge of facts, cognitive feeling for values, and knowledge of techniques as pre-stages of artistic understanding. But, he argues that a mere accumulation of those different kinds of knowledge could fail to understand works of art.

With regard to the above argument, let me return to imagination, particularly sociological imagination and intercultural imagination in ICM through drama. From the above argument, it emerges that imagination, along with creativity, is involved in understanding of works of arts, as well as understanding artists.

First of all, we should consider that imagination involves the constructive process of thought. Peacock illustrates two kinds of images in the process: 
Acknowledging the overlap between scientific copy-images and art-images helps towards understanding their divergence.... The former belong to a context of analytic seeing and scientific, or conceptual, truth. The latter belong to a context of aesthetic, or imaginal, seeing. They are both "constructions" in the sense that they are actively incorporated into processes of thought, the one with a conceptual aim, the other with an imaginative one (Peacock 1960: 9).

As Peacock suggests, the copy-images and the art-images overlap in the constructive process of thought.

Secondly, the thing to be considered is that they function in a different way: art-images provide aesthetic or imaginal contexts, whereas copy-images belong to conceptual contexts. At this stage, we should consider the advantages of 'Contexts of imaginative awareness, as distinct from perceptual or conceptual awareness, which enable us to make visual discoveries about the world' (Ibid: 12).

Thirdly, in this constructive process, the changes or substitutions all contribute to composition, and to a unity of meaning. Also, the changes are related to the meaning of the new construction by dint of the function of the imaginal contexts: 'All the images are influenced by this process; they are all "modified" or " reconstructed", as a colour varies in tone when juxtaposed with others' (Ibid: 30-31). That is to say, all the images in the constructive process are interconnected with others to be modified or reconstructed.

Fourthly, this constructive process in relation to imagination is not only engaged in producing and reproducing one's own works and lives, but also in the production and the re-production of other lives. However, at this stage, I intend to focus on only the former, and the latter will be reviewed in 3.2.4.

Finally, how, in this constructive process, could we apply imagination, particularly sociological imagination and intercultural imagination to ICM through drama as the reconstructive ability of imagination? This question follows four specific questions:

- How can we relate intercultural imagination to ICM through drama?

- How can we think our cultures away from the familiar routines of our daily lives in order to look at them anew?

- How can we think about other cultures in order to understand them?

- How can we reconstruct our own cultures as well as other cultures in relation to intercultural discourses surrounding us? 
Now, I intend to focus on the first question and the others will be reviewed in the next subsections. As the basic perspective of the application of imagination to ICM through drama, we need to return to what I discussed at the beginning of the present subsection-three interconnected factors in understanding people and their experiences, that is, affective, cognitive and behavioural.

Warnock (1977) argues that imagination is basic to arts including drama. She also explains that educating the imagination not only enables children to improve their reflective and perceptive capacity, but also leads them to a world, more interesting, better loved and understood. In this regard, the imaginative powers should be taken into consideration in education concerned with developing the sensory experience of each pupil as well as to perceiving the world surrounding them.

What is more, Witkin (1974) emphasises feeling among the component parts of imagination, although all of feeling, mood, and emotion influence the affective life.

This argument is interrelated to Amold's distinction between feeling and emotion in qualitative differences. According to Arnold (1960), emotions are various in their kind but are static; steadier as well as directed toward some object than feeling is. On the other hand, feeling gives an indication of a momentary state of mind because it focuses on only one of the aspects of an object. Therefore, it appears that feelings can vary, whereas emotion remains the same.

Collingwood uses the two words of feeling and emotion differently:

\begin{abstract}
...the word 'feeling' only with reference to the psychical level of experience, and not as a synonym for emotion generally. This level contains indeed a vast variety of emotions.... When thought comes into existence (and it is no part of my plan to ask how or why that happens) it brings with it new orders of emotions: emotions that can arise only in a thinker, and only because he thinks in certain ways. These emotions we sometimes call feelings;... I shall avoid so calling them, in order not to confuse them with the peculiar experiences we enjoy at the psychical level (Collingwood 1963: 164).
\end{abstract}

He explains that we can analyse that experience into two elements-sensuous and emotional, but cannot simply divide it into two experiences simply. Also we can not say that it is right to have an emotional element of the experience, because of its sensuous element (Collingwood 1963).

Despite the above distinction between feeling and emotion, I shall apply both terms of feeling and emotion to ICM interchangeably. This is because I intend to approach this 
research based on a holistic approach rather than approaching the psychological process of an individual subject.

From what I have reviewed so far in relation to imagination, I can say that feeling and emotion are basic components of imagination, and imagination can be educated for developing the reflective and receptive capacity of students. Furthermore, although scientific copy-images, which belong to conceptual contexts, and art-images, which belong to imaginal contexts, overlap, 'the education of aesthetic intelligence must therefore be concerned with the development of sensation and feeling into what is commonly called sensibility' (Abbs 1989d: 4).

Once more, the thing to be considered here is which one takes precedence among sensation, consciousness, and imagination in our experience at a certain situation. Collingwood regards experience as the continuum of impression-consciousness idea, or sensation-consciousness -imagination. He emphasises this process in the artistic experience by the following:

The artistic experience is not generated out of nothing. It presupposes a physical, or sensuous-emotional, experience. By an illegitimate comparison with craft, this psychical experience is often called its 'matter'; and it is actually transformed, somewhat (but not exactly) as a raw material is transformed, by the act which generates the artistic experience. It is transformed from sense into imagination, or from impression into idea (Collingwood 1963: 273-274).

As implied in the above, at the level of imaginative experience, a sensuous-emotional experience on the physical level is transformed into idealised emotion. The emotion loads on the experience of expression a given emotion and then is converted into a controlled activity of the organism, dominated by the consciousness which controls it, and this activity is language or art (Collingwood 1963). He coins the total imaginative activity in order to distinguish this aesthetic experience or artistic activity to express one's emotions from language or art.

Now we can answer how we can relate intercultural imagination to ICM through drama through the reconstructive ability of imagination. That is to say, it is through the total imaginative activity that we speak of communicating emotions with others in intercultural discourses.

In this regard, we can say that drama, as the total imaginative activity, supports the situational teaching and learning of EIL with reference to its situatedness. What is more, drama, as a gateway to ICM and ICP, provides a single, complete framework with clear contexts, as I have shown in the previous section. 


\subsubsection{Juxtaposition in the Duality of Drama}

As I mentioned before, I am going to consider how we might think about our cultures away from the familiar routines of our daily lives in order to look at them anew and how we can think about other cultures in order to understand them with intercultural imagination through drama. This, of course, is based on the total imaginative activity in intercultural discourses.

First of all, I should like to draw on what I have suggested about the figure-ground relationship between art and life in 3.1. As Bakhtin argues, 'Art and life are not one, but they must become united in myself-in the unity of my answerability' (Bakhtin 1919: 2).

There is the commonsense view of the relationship between drama and real life. Millward and Woolland describe this commonsense view:

This commonsense view of the relationship between drama and real life seems to hold true whether we see drama as a reflection of life or as a temporary release from harsh reality.... We can feel the force of this 'duality' between everyday and make-believe experience.... Drama may then be seen as 'concerned with [the] examination of human issues in specific social contexts' (Millward 1988: 9-11).

By focusing on an imagined, fictional world of make-believe, the drama (whether it's in a large theatre or in the classroom) draws our attention to aspects of the 'real' world; it helps us to recognise a sense of our own reality, and understand it better. Drama is essentially a social art form; it is concerned with how individuals relate to the world they live in; how individuals interact with each other and with society in a wider sense (Woolland 1993: 9).

Thus, we take backgrounds from our experience in real life to give meaning to the fictitious context.

Conversely, our real life compares to drama in dramaturgical perspective, that is, sociological perspectives-society as drama, as I have argued in 2.2. According to symbolic interactionism and dramaturgical sociologists, by dramaturgical society we learn how to be a social actor anywhere through 'Role Taking; process versus conformity' of self-roles and other-roles (Turner 1962: 85).

Therefore, we should consider the interrelationship between drama and real life. This is based on the commonsense of the juxtapositional relationship between the two. 
I intend to consider this interrelationship in terms of the notion of levels of making meanings in drama. Bolton articulates the juxtaposition of spatio-temporal dimensions in drama:

Drama is the most concrete of the art forms. The meaning is created from the juxtaposition of two concrete events: the actual use of time and space by the participants; and the simulated use of time and space in a fictitious context (Bolton 1981: 16).

Let me take an example of this juxtaposition. Whereas Student $A$, who participates in drama, is answering some questions which Student B asks Student A in their classroom, in the fictitious context an immigrant (Student $A$ in role) is being interviewed by an immigration officer (Student B in role) in Heathrow Airport in UK. In this way, the actual spatio-temporal context and the fictitious spatio-temporal context are juxtaposed in drama.

This juxtaposition is also related to another duality of production and perception in drama wherein performers are engaged. Arts Council of Great Britain describes the juxtaposition as the three activities-making, performing, and responding in drama in schools:

\begin{abstract}
The three activities which constitute the subject of drama in schools are making, performing, and responding.... It is possible to be engaged in more than one of these activities at the same time. Pupils improvising, for example, are simultaneously making, performing and responding. Similarly, the emphasis placed on each can change across the key stages. However, the principle of balance is as important within a subject field as it is across the curriculum as a whole [author's italics] (Arts Council of Great Britain 1992: 10).
\end{abstract}

As hinted, two drama activities, making and responding are overlapped by the other activity, performing drama. For instance, while a performer is performing his action, he can make drama activity and respond to performing activity, which other performers are doing, or to an audience's response.

The audience or spectator in drama makes drama different from other genres such as novels and poems:

Whereas novels and poems are addressed directly to their readers...plays are addressed to spectators through performers. Or, to put it differently, the reception of a play involves a "relay"-like process: the play is "received" first by performers and then by spectators. Nor does the plurality of addressees stop here [author's italics] (Chaudhuri 1984: 283). 
Thus, it appears to be the salience of drama, that is, plural interactions between the authorship of performers and the spectatorship of spectators.

What is more, the relationship between performer and audience is also involved in another juxtaposition in terms of drama world and real world, that is, theatricalism and realism. Gassner states that theatre experience is always a dual one:

Duality of experience exists to some degree in every theatre and for both the performer and his audience: action in the theatre is both make-believe and actual for them.... The experience of the theatre is always a dual one...to achieve a satisfactory synthesis of realism and theatricalism (Gassner 1956: 210, 212).

As implied in Gassner's statement, the event in drama and theatre itself does not have only a duality in the performers' perspective, but also in the audience's perspective, there is another duality of realism and theatricalism. For instance, an audience is 'both "in" and "out" of a stage production at the same time, and that the play is an extratheatrical and a theatrical experience for him at the same time' [author's italics] (Ibid: 212).

Furthermore, Bennett illustrates the dual role of audience in terms of cultural perspectives:

\begin{abstract}
Where audiences are consulted and involved in the structuring of the theatrical event, and are encouraged (at least in the immediate post-production period) to translate their reading of that event into action, then their role no longer maintains the fixity that dominant cultural practice assumes. In this way, the production/reception process acts bi-directionally in broader cultural perspectives. Cultural systems, individual horizons of expectations, and accepted theatrical conventions all activate the decoding process for a specific production, but, in turn, the direct experience of that production feeds back to revise a spectator's expectations, to establish or challenge conventions, and occasionally, to reform the boundaries of culture (Bennett 1990: 180).
\end{abstract}

Bennett shows that the theatre experience of audiences is not limited within the theatrical event, but is engaged in their cultural systems by applying their experiences to decoding production-reception process bi-directionally.

This description is similar to that of Beckerman (1970) who regards experience of the theatrical event as two different kinds of experience-the theatrical experience during the event itself and the memorial experience remembering the theatrical event. He regards the former as artistic experience and the latter as artistic effect. 
However, he argues that 'it is the artistic experience that we must use as the foundation for our analysis of drama,' although both artistic experience and artistic effect are equally important in appreciating a performance (Beckerman 1970: 157).

Thus, it appears that there are different kinds of duality in the theatrical event in perspectives of the performer as well as the audience. Therefore, as the Rubin's double profile indicates (Figure 3.1), it is necessary to decentre or alienate from the profile in order to have full understanding of the event, not to pay two different kinds of attention to it separately.

\title{
3.2.3 The Dialectic in the Duality of Drama
}

The juxtaposition of the real world and the drama world generates dramatic dynamics. First, the relationship between art and life as figure-ground, which I have reviewed in 3.1.1, is the axis of the dynamics in drama. Turner explicates the interrelationship between art and life:

\begin{abstract}
Life itself now becomes a mirror held up to art, and the livings now perform their lives.... Neither mutual mirroring, life by art, art by life, is exact, for each is not a planar mirror but a matricial mirror, at each exchange something new is added and something old is lost or discarded. Human beings leam through experience...in the circulatory or oscillatory process of their mutual and incessant modification (Tumer 1985: 300-301).
\end{abstract}

Turner shows that the relationship between art and life is reciprocal, rather than mutual mirroring, and regards its dynamic relationship between art and life as circulatory or oscillatory. Schechner elucidates this circulatory or oscillatory process between drama and life, in his terms, aesthetic drama and social drama:

\begin{abstract}
The "infinity loop" depicts dynamic positive feedback. Social dramas affect aesthetic dramas, aesthetic dramas affect social dramas. The visible actions of a given social drama are informed-shaped, conditioned, guided-by underlying aesthetic principles and specific theatrical/ rhetorical techniques. Reciprocally, a culture's visible aesthetic theatre is informed-shaped, conditioned, guided-by underlying processes of social interaction (Schechner 1988: 190).
\end{abstract}

It seems that there are everlasting oscillatory processes between social dramas and aesthetic dramas, that is, visible aesthetic theatre with inherent cultural values and social interaction.

As Figure 3.2 shows, workers in the world, for instance, politicians and activists use theatrical techniques on the stage in order to support social and political action, which is designed to change the social order or to maintain it. Meanwhile, theatre artists use 
the consequential actions of social life, as the ground of their art works. Although their main aim is to entertain, sometimes their works effect changes in perception, viewpoint, and attitude of audiences in terms of theatrical experience as well as memorial experience. In other words, aesthetic drama can take a role of a transformative agent to the audience.

These processes between social dramas and aesthetic dramas can be compared to 'the infinity loop' shown in Figure 3.2.

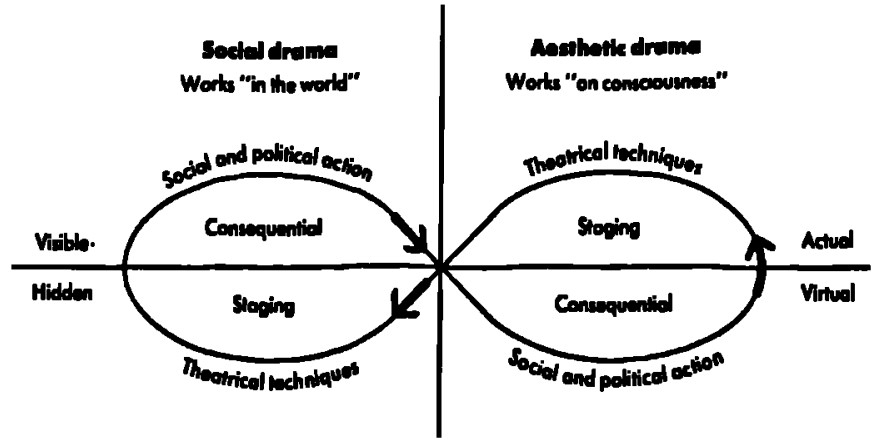

Figure 3.2 The Infinity Loop between Social Drama and Aesthetic Drama (Schechner 1988: 190)

What is more, the circulatory or oscillatory process, like the infinity loop between drama and life, can be applied as the basic dynamical principle to other kinds of duality of drama, such as the production-perception relationship in the performer, the relationship between the performer and audience, and so on.

On the other hand, along with the oscillatory process in the duality of drama, I should like to consider its dialecticality. The first thing to be considered here is that the essence of drama is in conflict and conflict resolution...in transformation' [underlines mine and author's italics] (Schechner 1988: 171). Beckerman articulates the relationship in the juxtaposition as follows:

In a parallel manner, Nietzsche polarized these contradictory views as the Dionysian and the Apollonian, the subrational, physical exaltation and the super-rational, intellectual vision. And, of course, the theatre encompasses both. It is flesh. It is spirit. The art of the drama is, to a large extent, the art of relating one to the other. I have called the flesh "activity," the spirit "inner life"...the dialectical interplay between the two (Beckerman 1970: 36).

Thus, it tends to be the dialectical dynamics between the flesh, activity, the spirit, inner life, and drama, in a broad sense. 
Just as part of the nature of human communication is dialectical, so the dialectic is the pattern of the change of any concept or meaning that results from the source of reference being placed in a new context of relevance, a new intentional perspective' Wright 1989: 14). That is to say, there are tripartite elements in the dialectic-one, the other, and a new one resolved between the two.

What is more, Beckerman argues that we can have an emancipatory experience by overcoming differences or resistances in the dialectic:

\begin{abstract}
Resistance is a key element in a dialectic act as well. It is the factor that the performer needs to overcome in order to realize a performance. It is the focus of the risk that resides in the act, and overcoming it produces an elemental pleasure for the spectators. In dialectic presentation, if there is a real question of control, the resistance has the distinct potentiality of defeating the performer. The tension between the will to do the act and the obstacle to its fulfilment is thus neatly calibrated so that when the performer prevails, there is not so much awe but release - that is, catharsis (Beckerman 1990: 57).
\end{abstract}

The dialectic thus is generated in the relations between other juxtapositional pairs and drama. Furthermore, the performer overcomes resistance or differences in the dialectic to provide a cathartic experience.

This dialectical dynamics can be simplified as the dialectic between the self and the fictitious context, but there is a multitude of the juxtapositional dialectic between two different elements in the drama event:

- 'Art is one of the enduring means for representing (for oneself and for other) those truths latent within our experience.... It has been my aim to describe in the arts a common rhythm, a cumulative sequence moving from self to community back to self, in an endless dialectical motion.' (Abbs 1989c: 209-210);

- 'The scheme is dialectical in character for the stages are conceived as a set of polarities or oppositional tendencies.... So individual and society are interwined.'(Stevens 1983: 56);

- Dialectical presentation between real resistance and illusory resistance (Beckerman 1990);

- Duel between the character and the performer (Beckerman 1990);

- The dialectical activity and the imagined act. . . a text, the lines of the characters, and a subtext, the inner life of the work (Beckerman 1970);

- Dialectical performance between performer and audience (Brecht's theory; Beckerman 1970; 1990);

- $\quad$...the dialectic of the dramatic process ensures that its audience must undergo the total treatment of transmission, reception and perception before it is felt.' (Styan 1975: 240); 
- Brecht's dialectical theatre: contradiction between experience and portrayal, empathy and demonstration, justification and criticism. (Mitter 1992).

- ...the nature of the experience the learner...in drama...to a shifting dialectic between experiencing and demonstrating. In other words the dialectical unity of the experience is made up of a submission to the experience and a reflection upon it' (Davis and Lawrence 1986: vii).

- 'Although the words "living through" imply that important sense of "being there in the present and presence," Heathcote's methodology also builds in its opposite of "being outside it." There is a mercurial inside-outside dialectic that heightens awareness.' (Bolton 1997: 374-375).

As mentioned earlier, the dialectical pairs in the drama event are too various to illustrate all of them in detail at this stage. The aforementioned quotations are some of those pairs.

As indicated in Figure 3.2, the infinity loop between Social drama and Aesthetic drama seems to be an endless dialectic between intrinsic form and emergent process in drama.

\subsubsection{Distancing and Defamiliarisation to Outsidedness and Reflexivity in Drama}

Given the juxtapositional dialectic and its process, I intend now to consider how we can understand the drama event fully. First, I should like to consider both the figureground relationship of Beckerman's notion and the unified attention of Rubin's double profiles as fundamental principles in understanding drama.

The second thing to be considered is a change in 'the aestheticisation of every day life as the cultural spreads throughout the social formation' in postmodern society (Usher and Edwards 1994: 13). In this regard, Usher and Edwards also regard us cultural consumers, who are actively engaged in the cultural event in every day life, as well as cultural producers.

This tendency to dissolve drama convention has started in drama culture. Brecht (1936) is one of the most outstanding in breaking the traditional drama convention. Brecht argues for dialectical theatre and emphasises the alienation effect in the dialectical theatre, particularly in terms of the positions of audience and performers, which does not fit with Aristotelian epic theatre and Stanislavski's empathy' 
The audience is to be nudged into a critical and inquiring attitude by a continual emphasis on the fictional status of the theatrical enterprise. The audience is not allowed to forget that it is sitting in the theatre, yet, at the same time, it must be prevented from bringing along the same set of expectations that has in life: an active productive state is to replace its passive consumer mood Wright 1989: 27).

This critical and inquiring attitude is associated with how we should pay attention to Rubin's double profiles. Brecht refutes Stanislavskian theatre, along with Aristotelian theatre, wherein the spectator is forced to be engaged in empathy and is entangled in the action. And then he suggests a way for better audience or performers-alienation or distancing from the drama event without the dissociation of audience from the performance or of the performer from his role.

That is to say, whereas Stanislavski emphasises emotion, Brecht's focus is on critical awareness. Let me take an example of their differences in the performer's status:

\begin{abstract}
Whereas in Stanislavsky, emotion occurs at the point of greatest resonance between action and character, in Brecht it marks the extent of the divergence between these worlds. In Stanislavsky the result is identification; in Brecht it is perspective.... Just as the identification of actor and character generates a corresponding influx of empathy in the audience, so also the dislocation of actors from their roles offers the audience an opportunity to deconstruct as malleable an orthodox formulation. Through their experience of defamiliarization in the theatre, they learn not to identify as well with the social conditions to which they are subject (Mitter 1992: 48-49).
\end{abstract}

Thus defamiliarisation, in other words, distancing or alienation, leads performers as well as audience to take critical and inquiring attitudes as active cultural producers in the postmodern society.

Furthermore, distancing and defamiliarisation are crucial notions in ICM, which EIL learners are taught in order to become intercultural actors in the global and postmodern world:

This distancing is essential to the notion of defamiliarisation and was realised in the structure of the second drama by the use of a play within a play.... Such devices serve to provide the type of allernative perspectives essential to the objective of developing cultural awareness (Fleming 1998: 156-157).

At this stage, with reference to the relationship of empathy, distancing, and defamiliarisation in ICM through drama, we should reconsider how to have nonjudgemental attitude in ICM (intercultural communication), which I reviewed in 2.2.2. That is to say, it is necessary to take a non-judgemental attitude with empathy and defamiliarisation. 
Above all, the aim is to have a better understanding of the drama event, as well as other events on its periphery, in other words, both the event per se and its situatedness. Moreover, we should try not to be entangled in a certain action owing to simply taking someone's position.

Therefore, I can compare the way to take this non-judgemental attitude to Brecht's three-stage rehearsal programme in building the character of a performer.

This is reflected in the three-stage rehearsal programme (significantly entitled 'Building a Character') outlined by Brecht for his company: (i) Before you assimilate or lose yourself in your character you must first become acquainted with it.... (ii) The second phase is that of empathy, the search for the character's truth in the subjective sense...becoming one with it.... (iii) And then there is a third phase in which you try to see the character from the outside, from the standpoint of society (Mitter 1992: 50).

Thus, Brecht emphasises three stages of characterisation-knowledge of the character, empathising with the character, and distancing to be outside the character and to build the character.

However, Brecht's theory is not only related to the performer to be characterising, but also the performer to be characterised. In other words, the performer is involved in audiences who watch other actors' performance. The performer takes his status as an audience who watch his own performance, creating a distancing and defamiliarisation from the performance. Whereas the former is involved in spectatorship, the latter is in self-spectatorship.

If, in the theatre, spectators can be transformed into actors without losing detachment, so in the drama lesson the participants can be transformed into observers without abandoning involvement. Both functions can coexist [author's bolds] (O'Neill 1989: 21).

In its creation and in its reception, drama is a communal experience, shaped and shared by individuals working or watching in groups.... Classroom drama is a similarly social and communal experience but more fluid and participatory in its form than most forms of theatre.... Just as the roles of actor and director may become blurred and co-exist simultaneously in one child, the roles of actor and audience can interchange several times within the one lesson Winston and Tandy 1998: vili-ix).

As implied in the above two, it seems that these two notions can be applied to drama more flexibly in schools than in professional theatre companies.

Regarding outsidedness in Brecht's three-stage characterisation, I intend to explore it in terms of its relation to self-spectatorship of performers or participants. Brecht 
suggests the performer's position from the outside, or from the society, as the final of three stages of characterisation, as I mentioned before. This means there is a need to be inside the character through empathy, and then to be outside the character to be distanced or alienated from it in order to have critical thinking through the dialectic between the character and the performer. The performer eventually retums to himself with an empathetic attitude toward the character. To sum up this procedural characterisation in the perspective of the performer, he takes several processes in different contexts for characterisation and action:

- The first phase to be inside the performer: to get knowledge about the character and socio-cultural or intercultural discourses surrounding the character.

- The second phase to be outside the performer to be inside the character: to empathise with the character without losing the performer himself.

- The third phase to return to be inside the performer, that is, to be outside the character, with his empathetic attitude.

- The fourth phase to build the character in the performer's creative understanding, which results from his critical thinking, and to do the total imaginative activity as the performer builds the character.

Whereas the first three phases in the above are related to reflection through otherness, the last phase is relevant to outsidedness, i.e., being outside to return to inside himself. That is to say, he is reflexive in order to build the character and perform his total imaginative acts, and eventually to have a better understanding of his performance as well as the character.

With regard to the characterisation and understanding of the performer, these processes can give us clearer answers to the four questions, which I have raised since 3.2.1:

- How could we relate intercultural imagination to ICM through drama?

- How could we think about our cultures away from the familiar routines of our daily lives in order to look at them anew?

- How could we think about other cultures in order to understand them?

- How could we reconstruct our own cultures as well as other cultures in relation to intercultural discourses surrounding us?

As Mead (1934) argues, reflexivity is the ability of human beings to take the attitude of the other that allows them to become objects to themselves. Brecht's three-stage 
programme also shows that reflexivity is the capacity of individuals to distance themselves from their own subjective experiences, to stand apart from and to comment on them.

MacAloon also emphasises the salience of reflexivity: "reflexivity" is the act of becoming an audience to oneself (1984a: 11). Furthermore, he introduces a comment on reflexivity, with the example of Narcissus in the Greek myth:

Of Narcissus, Babcock has written: "He is reflective, but he is not reflexivethat is, he is conscious of himself as an other, but he is not conscious of being self-conscious of himself as an other, and hence not able to detach himself from, survive, even laugh at this initial experience of alienation" (Ibid: 12).

As implied in the distinction, reflexivity involves distancing participants from the drama event, wherein they are engaged, without losing their involvement in it. In other words, participants are reflexive through being outside themselves and then returning to be inside themselves.

Boal applies this theory of distancing, outsidedness, and reflexivity to his theatre activity, particularly in terms of the notion of a triad of the spect-actor.

\begin{abstract}
A triad comes into being. The observing-I, the l-in-situ, and the not-I, that is, the other. The human being alone possesses this faculty for self-observation in an imaginary mirror.... Theatre-or theatricality -is this capacity, this human property which allows man to observe himself in action, in activity... An actor, acting, taking action, he has learnt to be his own spectator. This spectator (spect-actor) is not only an object; he is a subject because he can also act on the actor-the spect-actor is the actor, he can guide him, change him. A spectactor acting on the actor who acts [author's italics] (Boal 1995: 13).
\end{abstract}

Boal thus illustrates the dialectic in the triad of the observing-I, the I-in-situ, and the not-l, that is, the other, which the actor or performer should consider. Then, he emphasises the actor's capacity of self-observation through imagination as the spectactor, who can act as well as observe himself in action, in other words, as an actor as well as a spectator.

In this regard, we should consider the salience of self-spectatorship as actors as well as human beings in professional theatre works or in classroom drama. Let me take an example in drama education. Bolton defines acting behaviour as follows:

Acting behaviour is an act of fiction-making involving identification through action, a prioritising of determining responsibilities, the conscious manipulation of time and space and a capacity for generalisation. It relies on some sense of audience, including self-spectatorship (Bolton 1998: 270). 
Bolton thus articulates the involvement of self-spectatorsh $\mathbf{p} \mathbf{n}$ actorg behaviour Moreover, he returns to Holmes (1911; 1914) in order to explore the development of the notion:

\begin{abstract}
Holmes seems to be feeling towards the recognit on that drama promotes some kind of relationship between the 'self' and the world'. Of growng importance $\mathbf{n}$ the vocabulary of writers in recent years in the notion of 'self-spectator' a conception that enactment leads to seeing oneself reflected in the fict on one is making (Bolton 1998: 278).
\end{abstract}

Later, Bolton comments on other advocates of self-spectatorship in drama education, for instance, Heathcote (1976), Robinson (1980) and Fleming (1982; 1994).

\begin{abstract}
Thus 'self-spectatorship,' at its best, can be said to promote a double valence of being an audience to one's own creation and being an audience to oneself. Mike Fleming uses the term 'percipient' to combine the participant/spectator function in drama. Such a concept takes us beyond individual spectating to the collective feelings shared by all the players as 'audience' to what they are creating or presenting. It further extends the theory of self-spectatorship to take on board the notion of the 'percipient's' emotional engagement with what is going on. The dimensions of 'playwright' and 'director' could also be added. The four functions, 'dramatist', 'spectator', 'participant' and 'director' are occurring simultaneously [author's italics] (Bolton 1998: 266).
\end{abstract}

Thus Bolton shows that students who participate in drama activity have four functions simultaneously, and he regards students in drama as multi-reactive actors including self-spectatorship. The multi-reactive functions of students in drama as 'dramatıst', 'spectator', 'participant' and 'director' can be achieved through their acts of imagination, in a word, their total imaginative activity in the drama event and their socio-cultural intercultural discourses.

\title{
3.3 Drama Approaches to ICM (Intercultural Communication)
}

At this stage, I would like to re-consider what the most pressing issues are in the twenty-first century. According to Kidder (1995), leading thinkers around the world listed ethics as the most pressing issue in that ethics can rectify nuclear catastrophe and environmental accidents, which result from breakdown of moral values.

Then the question arises: what moral values should compose a global code of ethics? He answers the question with a 'set of precepts so fundamental that they dissolve borders, transcend races, and outlast cultural traditions' (Kidder 1995: 5). He also highlights the need for guidelines that enhance human interaction rather than detract from it, like generosity instead of greed or kindness instead of cruelty. Furthermore, he 
emphasises that there is no democratic form of government without an intelligent citizenry imbued with positive character traits.

\title{
3.3.1 Approaches to Interculturalism in the Contemporary Theatre and Drama Education
}

Thus, I would like to put ethics as the most pressing issue into relation to approaches in the contemporary theatre. How does the contemporary theatre approach the intelligent citizenry in the global society? As Collingwood argues, art is not contemplation, but action whereby an artist should utter, as a spokesperson of his audiences and his community, their secrets, not his own problems:

If art were contemplation, it could be pursued by an artist who constitutes himself a mere spectator of the world around him, and depicts or describes what he sees. But, as the expression of emotion and addressed to a public, it requires of the artist that they should participate in his public's emotions, and therefore in the activities with which these emotions are bound up (Collingwood 1963: 332).

\begin{abstract}
His business as an artist is to speak out, to make a clean breast. But what he has to utter is not, as the individualistic theory of art would have us think, his own secrets. As spokesman of his community, the secrets he must utter are theirs.... Art is the community's medicine for the worst disease of mind, the corruption of consciousness (lbid: 336).
\end{abstract}

Now, I intend to review briefly how artists in the contemporary theatre as spokespersons approach interculturalism with reference to the intelligent citizenry in the global society. In this regard, I shall single out some key points in theatre works of Pavis (1989; 1992; 1996), Brook (1968; 1989; 1993), Wilson (1984), Barba (1986), Schechner (1988; 1990; 1993; 2002), Brecht (1935; 1936; 1938), and Boal (1979; 1992; 1995), and find relations of their works to drama education in schools. All of them, with the exception of Brecht, are still actively engaged as scholar, artist, or director.

Here, I should like to divide the above theatre scholars or directors into two categories-whilst Pavis, Brook, Wilson, and Schechner place emphasis on intercultural performance, Brecht and Boal are more related to drama education than the others.

First, intercultural performance has enormous potential for open dialogue between cultures, according to Pavis, Brook, and Schechner. According to Pavis, there are three main forms of theatrical interculturalism at the crossroads of cultures; 
- Intercultural theatre: 'this creates hybrid forms drawing upon a more or less conscious and voluntary mixing of performance traditions traceable to distinct cultural areas' such as Brook's or Barba's theatre works, wherein they make use of Indian or Japanese traditions and create their plays [italics mine] (Pavis 1996: 8).

- Multicultural theatre: this form chooses its source from 'the crossinfluences between various ethnic or linguistic groups in multicultural societies (ө.g. Australia, Canada).... Cross-cultural influence is also and especially involved when a staging uses signs borrowed from a cultural universe without there being a direct relationship' [italics mine] (Ibid: 8-9).

- Cultural collage: unlike intercultural theatre, artists in this category 'cite, adapt, reduce, enlarge, combine and mix various elements without concern for a scale of importance or value. The intercultural becomes the unexpected and quasi-surrealist encounter of cultural debris or-more positively-of cultural material that has been repressed or discredited ' [italics mine] (Ibid: 9).

Particularly, Brook's and Wilson's theatrical directing are regarded as a new avantgarde in theatre in Europe and North America. Their concerns about 'foreign theatre cultures must lead-in Brook's theatre programmatically, in Wilson's rather implicitlytowards the development of a "universal language of theatre"'(Pavis 1996: 33). Meanwhile, Barba (1986) introduces the concept of The Stranger, of never being at home in any place, of always distancing from the world to look at it in the perspective of intercultural theatre.

Furthermore, Schechner approaches interculturalism in a socio-cultural perspective, particularly in ritual theory, and pinpoints two things to recognise in approaching Intercultural Performances:

\begin{abstract}
First, in today's world, cultures are always interacting-there is no totally isolated group. Second, the differences among culture are so profound that no theory of performance is universal: one size does not fit all. Nor are the playing fields where cultures interact level. The current means of cultural interactionglobalization-enacts extreme imbalances of power, money, access to media, and control over resources (Schechner 2002: 2).
\end{abstract}

As implied, he is concerned with intercultural dynamics and its unbalanced structure, and argues that theatre can provide new ethics for respecting differences by dint of the way to explore, understand, promote, and enjoy cultural diversity (Schechner 2002).

At this stage, I can say it appears that we could apply the above approaches to interculturalism in the contemporary theatre, that is, to intercultural education through 
drama. However, alongside them, we should consider pedagogical perspectives, which are interrelated to Brecht's as well as Boal's views.

As I have shown in 3.1 and 3.2, the contemporary theatre seems to be dependent upon non-Aristotelian theatre influenced by Brecht and Boal, rather than Aristotelian theatre, wherein traditional conventions are the concern, like Stanislavski $(1910 ; 1960)$. Furthermore, drama education seems to share with the former rather than the latter. Let me take the case of Dorothy Heathcote as an example for that:

\begin{abstract}
Boal espoused Freire's pedagogy....began to develop a theatrical form which addressed directly the problems identified by Freire, a form which would activate and change people from being passive 'spectators' of action into active participants in it - active transformers of the dramatic action. He describes these processes as the 'Theatre of the Oppressed'. Although there are undoubtedly a great many other influences on drama teachers today (notably Dorothy Heathcote, who herself acknowledges a considerable debt to Freire), and there may well not be wide grassroots knowledge and understanding of the work of Boal and Freire, much of the best practice in educational drama certainly exemplifies Freire's assertion that education is of necessity a form of 'cultural action' behaviour (Lacey and Woolland 1992: 85).
\end{abstract}

In this regard, Carroll may be correct when he says that:

\begin{abstract}
The methodology of critical theory and the transformational perspectives of drama research is a way of theory building and is grounded in the interpretation of actual data of the drama but it differs from other ethnographic forms in that it is a more narrowly focused inquiry. Participants in drama research work together in a dialectical way (Carroll 1996: 76-77).
\end{abstract}

What I have done so far is to identify a foundation of approaches to ICM through drama concemed with critical approaches. From this basic standpoint, I intend to discuss approaches to ICM through drama, i.e., approaches which are artistic, instructive, socio-cultural, therapeutic and integrated.

\title{
3.3.2 Integrated Approach to ICM through Drama in Schools
}

Let me return to the basic but ultimate question in intercultural education, whose answer intercultural educators have been searching for: how do we form or guide the development of intellect and character among the young in order to prepare for the global society? For me, drama is an ideal approach to developing ICM and ICP of young people.

Fleming (2003) regards drama as a form of intercultural education and illustrates that it can be applied to ICM and ICP in schools by 
- Exploring other cultures through their drama and theatre traditions,

- Participating in theatre in other languages/using drama in the modern language classroom,

- Exploring cultural differences through drama activities,

- Exploring aspects of one's own culture through drama, and

- Exploring the concept of 'other' in drama.

Drama teachers could specify or emphasise certain perspectives, artistic, instructive, socio-cultural, or therapeutic, depending upon their specific aims and objectives for ICM through drama.

I intend to illustrate that the aforementioned approaches should be seen from an inclusive standpoint in relation to intercultural education through drama, particularly in primary and secondary schools. That is to say, drama teachers should consider applying an integrated approach rather than a specific approach, which is applied in the contemporary theatre, in order to develop ICM and ICP of their students.

This is first because according to Kumpulainen and Wray (2002), cognitive perspectives on learning have begun to be replaced by the socio-cultural perspectives on individual's learning processes.

Secondly, I should like to consider the argument of Genesee that effective education of second language learners calls for a more integrative approach-academic, cognitive and social as well as linguistic, that is, 'The Whole Child, The Whole Curriculum, The Whole Community' [author's italics](Genesee 1994a: 2).

Thirdly, as suggested earlier, while it appears that there is no democratic form of government without an intelligent citizenry imbued with positive character traits, intercultural educators ought to take responsibility for educating their young citizens sufficiently to live in the global society.

The thing to be considered here is that the intelligent citizenry is not merely relevant to cognitive intelligence but also total imaginative activity wherein affective, cognitive, and behavioural factors are included. Let me take the concept of global identity as an example to illustrate the integrated approach to ICM and ICP. Young and Bagley describe global identity in relation to self-concept and self-esteem:

Identity has both cognitive (knowledge) and affective (evaluation) aspects, and these two aspects are related to self-concept and self-esteem respectively. We prefer to use the concept global identity as the highest-order concept, involving 
both self-concept and self-esteem in an integrated whole. Within global identity are the parts of the self, related to one another in particular configurations representing varying degrees of ego integration [author's italics](Young and Bagley 1982: 55).

As implied in the concept of identity and global identity, we should be concerned with an integrated approach to educating our young citizens to become intercultural actors with positive characters traits.

Fourthly, Fleming also argues for an integrated approach to the teaching of drama in primary and secondary schools, which will help practitioners to develop a theoretical rationale for their work. He suggests an integrated framework of the following pairs of polarised concepts:

$\begin{array}{ll}\text { Learning in drama } & - \text { content and form } \\ \text { Planning lessons } & \text { experience and structure } \\ \text { Planning schemes of work } & \text { means and ends } \\ \text { Assessing drama } & \text { internal and external experiences } \\ \text { Progression in drama } & \text { making and responding } \\ \text { Working with script } & \text { feeling and form } \\ \text { Performing drama } & \text { process and product } \\ \text { Drama and language } & \text { meaning and logic } \\ \text { Drama and aesthetics } & - \text { expression and representation }\end{array}$

(Fleming 2001: 7).

These pairs of juxtapositional concepts, which can be defined as dualistic oppositions rather than polarised couples, lead drama activity into 'dualistic ways of thinking...from Hegel (his notion of synthesis through dialectical thinking) as well as from more recent postmodern writers' (Fleming 2001: 6).

There is a supportive argument for his integrated approach. Eliasmith and Thagard (2001) argue the integrated approach of drama is effective because drama's successes are due largely to integrating structural and semantic constraints through the analogical mapping process of integrating structure and meaning. They claim that drama is evidence of using distributed representations to model high-level cognitive phenomena.

As I have shown, the juxtapositional structure in drama lays the foundation to generate dynamics as well as produces the catalytic action to make its contexts holistic, when participants are practically engaged in drama activity. 
In this regard, it is certainly correct when he says that:

The insight that human life begins in doing not thinking has implications for education. It suggests that exploration of interculturalism is likely to require more than intellectual enquiry, reasoned argument and acquisition of knowledge. Drama can provide concrete contexts and affective engagement for the participants and by its very nature can be seen as a form of intercultural education (Fleming 2003: 97).

As implied, we should consider approaches to ICM through drama in three perspectives-intellectual engagement, affective engagement, and drama action.

Drama is not only given historical roots in ritual, but also denotes active involvement in the world. Moreover, drama in education should be not only considered as drama per se and the related arts in schools, but also the orchestration of the entire school day as a series of ritualized events-events that truly nurture and celebrate the potential for human freedom instead of fulfilling an Apollonian passion for order and control' (McLaren 1993: 243-244).

That is to say, drama teachers should consider approaches to ICM and ICP through drama in perspectives of an Apollonian passion for order and control as well as 'a strong element of Dionysian animation, surging energy, creativity and significance onto the drama teaching scene' (Fleming 2001: 148).

In this regard, I can say that drama teachers should approach ICM through drama in an integrated approach to participants' experiences, affective, cognitive, and behavioural, through multiple communication media-visual, audio, and graphic.

Therefore, their drama experiences should be analysed in a vertical method, rather than a horizontal method, which is essentially literary, for instance, distinguishing elements of plot and character from a script. This is because the vertical method is essentially concerned with the progression of a play in its entirety such as the contextual frames within which the drama evolves, i.e., the dynamics of the play/drama (Beckerman 1970). With respect to the dynamics, we need to see the whole and the parts simultaneously in their spatio-temporal dimensions and to analyse drama activity in the vertical method.

\subsection{Summary: Dual, Dialectical, and Dialogical to Holistic}

In this chapter, I singled out the literature that has related to drama and its approaches to intercultural education. Starting with the duality of drama, which is an intrinsic 
quality in drama per se, I reviewed drama as cultural configuration and showed that consequently drama provides EIL learners with situated learning by doing.

Moreover, I reviewed drama activity in relation to ICM that demonstrates how participants are engaged in drama activity in intercultural situations, that is, from intercultural imagination to the total imaginative activity. I also reviewed the procedural involvement that shows what participants meet, that is, juxtapositional contexts in the duality of drama and their dialectical situation, and how they cope with those situations, through distancing and defamiliarisation.

I then reviewed ways in which the contemporary theatre approaches interculturalism and commented that those approaches can be applied to intercultural education through drama. I ended with an argument that drama teachers should apply drama in an integrated way rather than a specific way, in their approaches to ICM and ICP in schools. In the next chapter, I give details about the methodology that I followed in this study. 


\section{PART III. PRACTICUM: Methodology AND Findings}




\section{Chapter 4.}

\section{Research Methods}

In this chapter, having taken into consideration theoretical backgrounds underpinning the research issues, I intend to show how I investigated the key issues in this study including the empirical way of gathering data and analysing them. The purpose of this chapter is: firstly, to present a discussion of some varieties of Action Research (AR) and their strengths and weaknesses. Secondly, I will explain and justify the methodological choices made and the specific perspectives on AR which guided those choices.

This discussion will be presented in seven sections.

- First, I shall overview the research method.

- Second, I shall show how I developed my research design in this study.

- Third, I shall demonstrate what the context was for the research.

- Fourth, I intend to report how I did my pilot study.

- Fifth, I am going to demonstrate how I collected data from my main fieldwork.

- Sixth, I shall discuss how I analysed data.

- Seventh, I will discuss my research method in terms of its dependability and confirmability in relation to trustworthiness in the study.

\subsection{Overview of the Research}

\subsubsection{Parallelism in the Research Method}

"You've come to ask me what I know about the death of Betsy Clendenin." "Yes, Sir. I would like to talk about that, if ya don't mind." "What connects me to her death?" "She was workin' on a book about the FBI. She told somebody she was gonna interview you...." (Harrington 1998: 127).

'Yes, Sir. I sure will." Columbo stepped outside, where abruptly he stopped, just as Fritz was closing the door. "Oh, say, Sir, there is just one other little thing I meant to ask you. Prob'ly isn't important, but-Did you ever hear of a man named Harry Lehman?" Fritz Kloss tumed down the corners of his mouth and shook his head. "No, Lieutenant. I don't think I've ever heard that name in my life." "Well, thank ya again. It was just a long shot, that you might have known the man sometime." Fritz shook his head, and Columbo walked toward his car (Ibid: 130). 
The above extracts are from one of my favourite detective stories, the American TV series, Columbo, which I would like to illustrate the way in which drama is applied to this research study. Columbo was aired on NBC in the 1970s, with Peter Falk, as the shabby, bumbling, cigar-smoking Lieutenant. Each episode of the series would begin without the title character in the first scenes.

When watching one of its episodes, I, as a TV viewer, became involved in something private or secret, in an elaborately staged murder. In the beginning, I was told who "did it." However, in the middle of it, I realised that the enigma of the series did not focus on "who did it?" but "how will Lieutenant Columbo solve the crime?" And, in the end the Lieutenant proved how the victim was killed by the murderer by explaining what the murderer him/herself was doing. I enjoyed watching Columbo methodically figuring out how the murderer was involved in the crime scene.

By introducing this TV series at the start of the chapter, I intend to show how drama is used in this study and how drama is involved in students' actions throughout the research project, both in the approach to the research issue and the structure of its approach.

First of all, with the respect to the way in which students and I approached the issue, we explored the research issue on the basis of how drama is effective for enhancing social interaction and intercultural communication rather than whether or not drama is effective.

At this stage, I should like to distinguish the term of 'we' from the general 'we.' Hereafter the term, we, which indicates both students who participated in this study and me as the researcher of the study, is written in bold italic fonts in order to distinguish from 'we' which is used to refer to people in general. Possessives, reflexive, and personal pronouns are based on the same rule. On the other hand, participants and the project group were a little different in that a few participants could not be involved in the EDIE project to the end because of their irregular attendance or their personal reasons. Nevertheless, when mentioning only students, who participate in this study, I shall use interchangeably participants with the project group.

Let me return to the main point. The intrigue is not whether drama is effective in this sphere, since that is already known, but how it is effective. We asked questions, got our answers, analysed them, and collected evidence which could explain how drama was effective for intercultural communication just as Lieutenant Colombo did in order 
to solve the crime with his colleagues in the TV series. The students were deeply involved in their actions in the stance of students as researchers in their research project, whilst I not only did research as one of the participants in our research project but also as the researcher for my own project, which encompassed their project.

Another perspective is that the research structure in this study had a dual structure just as any drama has a dual structure, from two practical angles. From its functional angle, there is the dual structure of both the research for the students as researchers and the research for me as the researcher in this study. Similarly, Columbo has its dual structure: that which is interpreted by the characters, for instance, Lieutenant Columbo, who was played by the actor, Peter Falk, and that which is directed by the producer or the director.

From the perspective of its own duality of drama, this research has the structure of the drama world and the real world. Students in their roles were in the drama world, and out of their roles were in the real world, in the same way that the actor, Peter Falk, stood outside his role as Lieutenant Columbo in his everyday life.

However, I am going to add one more perspective to this dual structure, that of a 'drama within drama,' or metadrama, as Shakespeare sometimes devised a 'play within a play, for instance, in Hamlet. In order to clarify this point, I should draw attention to three points concerning a dramatic technique in drama education, 'the Mantle of the Expert,' invented by Dorothy Heathcote (Heathcote and Bolton 1995), which involves having the students take the role of 'experts' who are engaged in a professional area.

Firstly, throughout the study each student took the role of researcher, that is, each student wore the mantle of the expert as one of the researchers, to solve the research issue. In short, they played or were doing drama as researchers, in role, throughout the whole of their research project.

Secondly, they were doing drama activities as multiple reactive actors in drama sessions whilst doing drama as researchers in the research project. For example, students as researchers, who had been wearing the mantle of the expert in their basic roles, were doing drama as one of the characters, such as, the president of an American company or the president of a Japanese company in a drama situation where an international business meeting is being held. 
Thirdly and finally, this situation, when they as actors, were doing drama in drama sessions at the micro level and at the same time, as researchers throughout the drama at the macro level, can be defined as the situation of metadrama similar to the technique of 'play within a play' of Shakespeare.

On this basis, I present a comparison of the structure of the TV series, Columbo with the structure in this research study as Table 4.1 shows:

Table 4.1 Analogy of the TV series Columbo to the Research Study

\begin{tabular}{|c|c|c|}
\hline Categories & TV series, Columbo & The Research study \\
\hline Leader & TV producer/director & The researcher (me) \\
\hline $\begin{array}{l}\text { Stance of } \\
\text { Participants } \\
\text { [The project } \\
\text { group] }\end{array}$ & $\begin{array}{l}\text { "Actors (Peter Falk in } \\
\text { everyday life) } \\
\text { "Character (Columbo in } \\
\text { the TV series) }\end{array}$ & $\begin{array}{l}\text { "Students as Researchers } \\
\text { (cf 'mantle of the expert) } \\
\text { "Students as Multiple } \\
\text { reactive actors in drama }\end{array}$ \\
\hline The Structure & $\begin{array}{l}\text { Producer's (macro level) } \\
\text { Actors' (micro level) }\end{array}$ & $\begin{array}{l}\text { Researcher's (macro level): } \\
\text { the BGCH project } \\
\text { Students' (micro level): } \\
\text { the EDIE project }\end{array}$ \\
\hline The Target & The murder & Drama \\
\hline The Focus & How to solve the crime & How drama is effective \\
\hline The Context & The context around the murder & $\begin{array}{l}\text { Socio-cultural } \\
\text { Intercultural context }\end{array}$ \\
\hline $\begin{array}{l}\text { Procedures to } \\
\text { solve the } \\
\text { problem }\end{array}$ & $\begin{array}{l}\text { The general direction which the } \\
\text { author and the lieutenant first } \\
\text { decided on. }\end{array}$ & $\begin{array}{l}\text { Participatory action Research } \\
\text { (hereafter as PAR) and } \\
\text { Students in Participatory Action } \\
\text { Research } \\
\text { (hereafter as SPAR) }\end{array}$ \\
\hline $\begin{array}{l}\text { The way to } \\
\text { solve the } \\
\text { problem }\end{array}$ & $\begin{array}{l}\text { The specific route which the } \\
\text { author and the lieutenant took to } \\
\text { discover the decisive murder } \\
\text { scenes }\end{array}$ & $\begin{array}{l}\text { Grounded theory analysis } \\
\text { (hereafter as GTA) } \\
\text { Computer Aided Qualitative Data } \\
\text { Analysis Software (hereafter as } \\
\text { CAQDAS): } \\
\text { ATLAS.ti version } 4.2\end{array}$ \\
\hline $\begin{array}{l}\text { Institution and } \\
\text { Places }\end{array}$ & $\begin{array}{l}\text { TV Broadcasting Company } \\
\text { Police station and other places }\end{array}$ & $\begin{array}{l}\text { The School and EIL settings } \\
\text { in drama }\end{array}$ \\
\hline
\end{tabular}

By using the analogy of the TV series Columbo with my research framework, I have tried to show a brief map of how we, the project group and I, have approached this research through AR (Action Research) and GTA (grounded theory analysis).

My research project thus had its dual structure for participants and for me, that is, the research level of participants and my research level. In this dual research structure, both participants and I collected and analysed our actions and responses as the primary source of our own data, and drew conclusions through GTA, while attending 
to and exploring drama actions for intercultural communication in the context of teaching and learning English as an international language (EIL).

\title{
4.1.2 Drama: Action Learning by Situatedness
}

Before moving on to describe the research method aims, the main characteristics of the research, and how data were collected and analysed, it is important to consider the nature of drama actions. This aspect was a key factor in choosing the method for the research in the practical sense that participants and I were going to do drama activity by putting ourselves into others' shoes in drama sessions and to explore the activities in order to have a better understanding of the other as well as ourselves.

However, it is not possible to do drama without any context or situation. Generally speaking, when we are doing drama, we usually take the place of the other, in consideration of who they are, where they are, and when they are. This is because our values and behaviours are positioned in space and time. Their activities also are dependent upon the contextual settings.

Also, even in our everyday lives, what we are, is not exactly what we were, although we may still be in the same place. On these grounds, we can start to reconsider what drama activity is like and what situated activity is like, and if they are related to each other and then, how they are related. Lave and Wenger explained the term of 'situated' as follows:

\begin{abstract}
On some occasions "situated" seemed to mean merely that some of people's thoughts and actions were located in space and time. On other occasions, it seemed to mean that thought and action were social only in the narrow sense that they involved other people, or that they were immediately dependent for meaning on the social setting that occasioned them (Lave and Wenger 1991: 32-33).
\end{abstract}

In the light of their definition that thought and action are situated in social contexts which are set by time and space, I believe that drama activity can be described as situated activity.

Also, on the basis of this concept of situated activity, Lave and Wenger developed the situatedness of activity into situated learning:

...it [the situatedness of activity] took on the proportions of a general theoretical perspective, the basis of claims about the relational character of knowledge and leaming, about the negotiated character of meaning, and about the concerned (engaged, dilemma-driven) nature of learning activity for the people 
involved [brackets mine] (Lave and Wenger 1991: 33).

From this explanation, they gave me an idea that learning is situated in a mutual construction between an individual person and society and therefore situated learning is thoroughly situated in the lives of persons and in the culture that makes it possible (Lave and Wenger 1991).

With respect to the ways in which drama activity implicates its situatedness in the society and the culture, drama activity could be seen as a form of action learning in which participants learn by doing, in their socio-cultural environments where power is situated.

Above all, drama action itself has not only its potential in its capacity to allow participants to draw from their socio-cultural backgrounds, but also the essential keys to learn how to learn and to cope in a teaching and learning environment for which they have not been prepared.

Drama experiences, which the participants bring into their environment, therefore appear to be dependent on two factors:

- the participants themselves explore their current situations and approach problems in the situations, in the sense that drama experiences can reflect their situated actions in every day life and vice versa;

- participants' experiences, their behaviours through drama actions are explored in dramaturgical perspective.

In relation to this, the participants and I were going to analyse and compare their intercultural factors through drama actions, using a form of ethnographic approach to their intercultural interactions through drama.

\subsubsection{Intercultural interactions through Drama}

The research style and the written product of our research activity, which the drama research team were approaching with regard to ICM in drama, are not unlike ethnography. Our exploration has aimed to place our ICM in its social context in that we have focussed upon fundamental assumptions about nature, attitude, behaviour in intercultural interaction and the nature of intercultural knowledge of participants. This perspective was beneficial to our understanding of intercultural interaction in drama in several ways, above all, in terms of its holistic approach to ICM, as I discussed in 
Chapter 3.

The approach to ICM through drama, however, needs to be close to critical ethnography in the sense that the educational aim itself resides not only in the changes of human behaviour for the betterment of human life (Geertz 1983; Carspecken 1996). Critical ethnography, firstly, analyses human behaviour in relation to the larger historical processes and social contradictions, secondly, searches for the hidden forces that structure life, and thirdly, considers the explanation of these contradictions to be its very quest. In this research process, a critical ethnographer tries to balance his insider's experience near view of culture with his outsider's experience far perspective (Geertz 1983). In this regard, I can argue that the aim of critical ethnography inheres in the socio-cultural discourses in which power is situated, mentioned in the previous section. In addition, I can assume that that reality around participants, as critical ethnographers, is changeable, and I aim not only at understanding participants' behaviour but also at changing the lives of intercultural actors' ICM within the drama.

In order to have a better understanding of the human condition in this intercultural context, critical ethnographic research could be applied in the sense that participants and I were going to discuss features of group life. They would study, for instance, intersubjectivity, multi-perspectives, reflection, self-reflection, reflexivity and negotiability, through observation, participant-observation, interviews, and so on.

However, how do drama researchers approach group life and explore those features of the group life through critical ethnographic research? How do drama researchers find their topics engaging and challenging? The answer can be drawn from 'ethnographic imagination' (Atkinson 1990), borrowed from the concept of 'sociological imagination,' which Mills defines as 'the major common denominator of our cultural life and its signal feature' (Mills 1970: 14).

But what is more considered here is to research ICM through drama wherein the imaginative process results in participants and myself being made unfamiliar or strange by thinking ourselves away from the familiar routines of our daily lives in order to look at them anew. Therefore, I intend to emphasise the intercultural imagination, as I discussed in 2.2 .

Equally, drama itself was a useful tool to look at the unfamiliar (Fleming 1998). Wright (2000) gives support to Fleming arguing that in the context of drama education, its 
system helps teachers, students and researchers to step back from the topic, to imagine new constructions and new re-connections more clearly. The essential point from those perspectives can be drawn as this, that the unfamiliarity, which drama produces, enables us to analyse the intercultural interaction in that situation, to solve the problematic situation, and to achieve a better understanding through drama.

In short, building on this relationship between critical theory, sociological imagination and drama, I can say that the research paradigm in drama is dependent upon critical ethnography with sociological imagination, in approaching ICM through drama. This leads researchers to be engaged in a critical approach to ICM. This is more effective than approaches of Functionalism and Interpretivism, because Functionalists see research as instrumental, as a tool for discovering knowledge that existed out there, and Interpretivism develops as a paradigm in which researchers come to understand that knowledge is socially created. The essential ingredient in drama's approach to ICM is that it provokes critical thinking from defamiliarisation or alienation.

\subsubsection{Interest in the Effectiveness of Drama}

Many forms of drama have co-existed with a wide variety of techniques for achıeving a viable 'artificiality' (Beckerman 1970: 126) and there has undoubtedly been a tendency for the pure type (drama as art itself) to disappear and for the applied type (drama as a research method or drama therapy) to increase. This phenomenon introduces questions of how we research those various and applied types of drama in our educational context and how we can cope with the possibilities of change in drama education.

A few researchers through their chosen research processes, qualitative or quantitative, have explored the contradictions and problems of drama education through hypotheses or propositions by modifying existing researcher methodologies or creating new ways of researching in drama education. Drama researchers have defined the effectiveness of drama, an art form, as well as a learning medium, in Language Arts and Literature (Backstrom 1988; Buege 1991; Robbins 1998; PIl ay 2000).

Also, dramatic techniques, although they include some aspects of drama, for Instance simulation and role-play, began to be applied to intercultural education by Kaough (1987)' Elkins (1997)' Arnaut and Arnaut (1998), Ackroyd and Pilkington (1999), and Greenwood (2001). Furthermore, drama is applied to psychoanalysis as a therapeutic 
tool and similarly special education and counselling (Peter 1994, 1995; Slade 1998).

In relation to these studies, I wondered why drama has become extensively utilised in various approaches. In my case of ICM through drama in teaching EIL (English as an international language), I would like to search for the effective factor in drama. So, my key research question is: why is drama effective for ICM in teaching EIL? I planned to investigate effective factors in drama, in which the integrated structure of action and reflection exists, and through which participants interact with each other in their own social or educational situations.

Judging from this point, I was concerned to determine which research mode was the most appropriate in collecting and analysing ICM through drama for the investigation and finally, chose AR as my basic research model.

\subsection{Research Design}

The research paradigm described in this study has four main elements: [1] research motivations, [2] research questions, [3] the characteristics of data to collect and analyse for studying those questions, and [4] participants and me, as researchers. These elements normally make the researcher re-consider the original motivation and then influence us to carry out this whole study. In this respect, we can ask why and for what I intended to rely on PAR (Participatory Action Research). Now I shall explain why I chose AR as my research model.

\subsubsection{Features of Data to be Collected}

\subsubsection{Ethnographic Data: Intercultural Interaction through Drama}

First of all, I took the view that ICM in drama sessions could be ethnographic data. Swartz introduced dramaturgical perspective into educational research in her discussion and also pointed out that:

Each dramatic performance differed from non-ethnographic drama in that the 'script' and the thoughts of its author were continuously informed and reinformed by the 'actors' as they lived the actual events of their real lives. These 'actors' appear in each lesson through a remaking of events that intends to let their voices and mine come together to do the directing. All ethnographic accounts have implicit dramaturgical qualities; these 'performances' make such qualities explicit (Swartz 1998: 117). 
Here Swartz supports the possibility that an ethnographic approach through drama enables participants to explore their socio-cultural actions in everyday life and to have a better understanding of them by re-writing and re-reading these interactions in drama events.

Judging from the aforementioned, what participants and I were going to do in drama, was ethnographic in several points:

- first, we would describe the most relevant aspects of intercultural phenomena, and collective beliefs and experiences in our drama in sessions;

- second, we would make decisions about the appropriate mix of perspectives through those intercultural phenomena, not only within our situated context. Also, we would admit ourselves to scrutinise the other group with our mixed and open activities. This writing and reading of ICM would be realised through negotiation with participants, outsiders and me:

- third, in the end, the negotiation would likely be a dialectical phenomenon in fieldwork between ourselves as researchers and ourselves as cultural people.

Thus, it would seem that activities in classroom drama can be described as ethnographic drama and those activities have the characteristics of ethnographic data, to be collected and to be analysed by us in that context. Participants go to where people live and do field work through drama by asking questions, eating strange foods, learning a new language, watching ceremonies, taking field notes, washing clothes, writing letters interviewing informants, and many other things. All of these come true in the context of the 'Magic If,' the participants are doing ethnography, which 'is more than a tool for anthropologists to study exotic cultures... is a pathway into understanding the culture differences...' (Spradley 1979: v).

Judging from the above, there were no doubt participants in these drama lessons, as ethnographers, would explore their social interaction by living and working in society or a social setting, through drama. At the same time, they would reflect on their investigations in their everyday life, immersing themselves as fully as possible in their activities both by researching and by being researched, under observation. This is possible because their drama experience is always a dual one, which is a synthesis of theatricalism and realism-theatrical experience and extratheatrical experience (Gassner 1956); theatrical experience and memorial experience (Beckerman 1970); a matricial mirror between art and life (Turner 1985); aesthetic drama and social drama 
(Schechner 1988), as I discussed in Chapter 3.

Having recognised the ethnographic data of socio-cultural interaction through drama, I hereafter gave particular weight to PAR as the research model. There are two key points to evaluate with respect to AR. One is that the research is 'a form of research carried out with the aim of inducing changes in social activities (e.g. increased participation in cultural events), and of also studying these changes' (Jary and Jary 2000: 4). This point seemed to refine the question of why $I$, as a researcher, should do this research because a researcher can encourage participants for themselves to investigate their intercultural discourses and to improve their ICM and ICP in AR, where participants themselves are engaged.

The other is that PAR, which is modified from AR, could let us do research as insider researchers by participating inside the fieldwork, through moving toward 'trying to know, or understand aspects of society... to change that which is studied. For this reason it is inherently political and involves researchers and researched working together to identify and resolve issues in a particular area' (Jary and Jary 2000: 443444). This point seemed to give me more strength in that participants would examine their own socio-cultural interaction in and through drama.

\subsubsection{ICM through Drama in Dramaturgical Perspective}

ICM (intercultural communication) in drama sessions widened my view to include a dramaturgical perspective as well as an ethnographic perspective. In the attempt to understand the character and processes of social life, social psychologists, especially associated with Erving Goffman, necessarily utilise 'dramaturgy as one of the conceptual frameworks with which they are socialised and whose analytic perspective is that theatre, more accurately, drama is the basis of an analogy with everyday life' (Young 1990: 9). This perspective can provide a clearer framework connecting drama with life from a socio-cultural standpoint.

Equally, in approaching ICM through drama in the classroom teachers should be concerned about the issue which is investigated in this dramaturgical perspective. For instance, we are concerned with intercultural dramatic studies and how they relate to the development of intercultural communication. That is to say, it is reasonable to approach ICM through drama in a socio-cultural perspective along with an aesthetic one. 
Swartz (1998) showed in her article that a dramaturgical approach is effective in examining emancipatory concepts in the fields of multicultural education and cultural studies. Also, it has been suggested that Heathcote's drama teaching seems to be influenced as much by a sociological awareness as a humanistic awareness of educational problems, precisely because her method of teaching bears a close resemblance to some of the technique employed in socio-drama (Ball 1983). In brief, it is generally agreed that drama in the classroom is not inseparable from sociological awareness.

\title{
4.2.2 Research Paradigm for Social Interaction/ICM
}

My participants and I would collect and analyse our data in the drama session, ethnographically as well as dramaturgically, and we took these research procedures throughout the fieldwork in accordance with the principles and procedures of AR. Now how could we manage to do this through AR?

\subsubsection{Socio-Cultural Literacy through AR (Action Research)}

I decided to give my participants enough information to do research; to read and write their ICM. By having an introductory session relevant to the research, I could do the research together with the participants and incorporate intercultural literacy inside the research. Some of the following was presented to them in the introductory session, in drama sessions and our Saturday research meetings.

\subsubsection{The Aims of the Literacy in AR}

Currently, there are a wide variety of approaches to conceptualising, organising and supporting AR. AR is introduced in many different ways in educational settings. It is pointed out as the main reason for the variety of AR in Zeicher's 'Educational Action Research,' that:

\begin{abstract}
The dimensions along which action research in education have varied include the purposes and motivations of those who engage in the research, the conceptions of the action research process and the form and content of action research studies, the ways in which the findings of the research are represented by researchers to others, the relation of action research to externally produced research, the sponsorship and organizational location of the research, the structures in place to support the research, and the assumptions about knowledge and teacher leaming that are reflected in particular research programmes (Zeicher 2001: 276).
\end{abstract}

It is, however, doubtful that the variety of AR in educational settings, even though it 
could be spread into so many different sub-branches, could be disloyal to its basic feature, that AR is 'the systematic study of attempts to change and improve educational practice by groups of participants by means of their own practical actions and by the means of their own reflection upon the effects of those actions' [author's italics] (Ebbutt 1985: 156). Those many different types of AR adhere to these basic processes and procedures and are modified for the unique situation of each research case.

Kember lists the major characteristics of AR whose aims are the change and improvement of the educational milieu:

Action research is:

- concerned with social practice;

- aimed towards improvement;

- a cyclical process;

- pursued by systematic enquiry;

- a reflective process;

- participative;

- determined by the practitioners (Kember 2000: 24).

Kember gives particular weight to the dynamic aspects of AR, in conjunction with the basic aims of professional improvement and social change.

What I am more concerned about, however, is what makes AR more applicable as the research method in educational settings than other research paradigms. McNiff et al. (1996) pinpoint the characteristic differences of AR from other research frameworks as well as their commonalities.

Before pursuing this, however, I wish to highlight the term, 'educational action research,' because this can sometimes be confused with AR in educational settings. McNiff et al. suggest that Educational Action Research is defined in the light of the organisational level of the research. They stress that the adjective 'educational' before 'action research' is added so that it emphasises 'the point that action research aims to bring about an improved situation through a careful evaluation of action' (McNiff et al. 1996: 14). And it should be applied to the educational environment 'as an educational means of bringing about a good social order for all concerned, ' not as a manipulative device (lbid).

There is no doubt that this view is focused on what the research aims are rather than where the research is carried out. This comment suggests that educational AR is not 
confined to location, that is, educational settings, but is focussed on the aim of education itself-social change. Taking this into consideration, I intend to take educational AR and focus on its aim: AR for educational change, rather than on its research location.

What makes AR itself educational in consideration of the statement, educational AR? It is simply answered; the researcher should give weight to the basic characteristics of all good research and equally its own unique characteristics. In order to make this clearer, we can consider another statement which McNiff et al. make on the similarities and differences of educational AR to other research models. They present the points that AR share with other methods, as follows:

- it leads to knowledge

- it provides evidence to support this knowledge

- it makes explicit the process of enquiry through which knowledge emerges

- it links new knowledge with existing knowledge (McNiff et al. 1996: 14).

On the other hand, it is suggested that AR is different from all other research with its own special characteristics in the following points:

- it requires action as an integral part of the research process itself

- it is focused by the researcher's professional values rather than methodological considerations

- it is necessarily insider research, in the sense of practitioners researching their own professional actions. (lbid).

The first thing to be considered from the above characteristics is that $A R$ is selfreflective or reflexive, in that the researcher is involved in his/her own action as well as research in order to explore his/her professional values and change them. And the change has influence on change in the researcher.

This change, however, is not only within the confines of the professional development of the single individual researcher, but also eventually leads to social change.

- Firstly, because the research is applied to an educational environment which the researcher belongs to, as a form of collective self-reflective enquiry undertaken by participants, it can improve the rationality and justice of their own educational practices, as well as the understanding of these practices and the situations in which these practices are carried out.

- Secondly this AR is a social process between the researcher and all 
the situatedness surrounding him including the researched. Action research therefore is participatory, in that it is a process of learning by doing; through action-and learning with others, by changing the way they interact in a situated social world.

- Thirdly, AR is practical and collaborative in the sense that the researcher participates in the research as an insider and works together with others or the researched.

The advantages are still not sufficient enough to make AR more educational. With respect to this, new directions in AR recently have shown teachers have not actively implemented $A R$ as a more appropriate research paradigm in their educational environments. Although educational AR has improved and become more accessible to research studies, action research still has difficulties in being accessible even at the local level.

For instance, Zuber-Skerritt highlights emancipatory AR in which 'action researchers who have critically reflected on their theory and practice with a focus on emancipatory or critical action research, based on the Frankfurt School of Critical Theory' [author's italics](Zuber-Skerritt 1996a: 3). And she regards it as one of the new directions in AR. Also, Kemmis (1994) gives particular weight to emancipatory AR by differentiating it from both a technical one and practical one in their aims and motivations, and supports the critical (or emancipatory) perspective on educational change as an offer in the post-modern condition in education.

So, what did I regard as the best research model for this study? At this stage, I can say that my research framework is participatory, self-reflective, emancipatory, and critical as a social process.

\subsubsection{The Cycle of AR for the Literacy}

Practices are the crux of $A R$, and the process of such practices is 'a process in which the researcher is not solving a problem for the other but with the other. The learning is in the joint action, a process of joint learning' engaged by the researcher or the participant, and the researched or the other [author's italics] (Greenwood 1999: 10).

Action research thus is an operating system in action and the system requires a great deal of action-oriented practices. Furthermore, these practices are never static, but in a continual process, "a cycle of cycles" or a "spiral of spirals"' (McNiff 1996: 23). Models of practice research are suggested by Lewin (1946), McTaggart et al. (1982), Elliott (1985), Ebbutt (1985), McKernan (1991). Zuber-Skerritt (1996b), and Kember (2000). Among them, I used Zubers-Skerritt's as my basic spiral of AR cycles, which is 
presented in Figure 4.1 because its pattern is similar to the drama process, which I mentioned in the previous chapter.

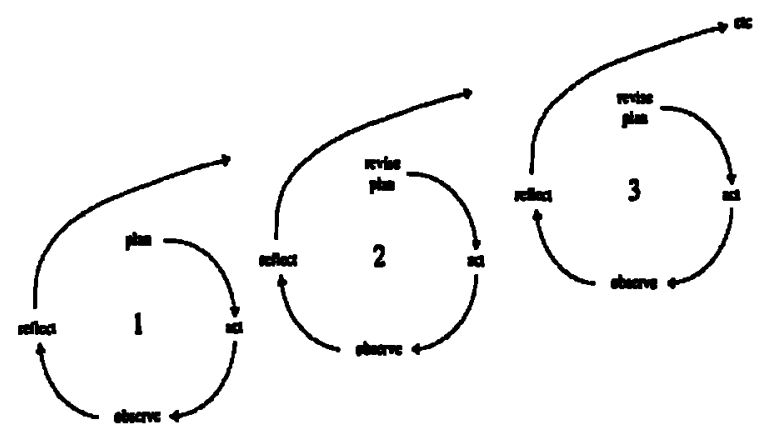

Figure 4.1 The Spiral of Action Research Cycles

Like the spiral-cum-cyclic process of Figure 4.1, participants in or through drama, especially socio-drama or ethno-drama, firstly, meet to plan what they study through drama and/or action together, establish a problematic situation, and then recognise its constraints and possibilities.

Secondly, they put the original plans into practice by casting characters, warming up actors and spectators (or audiences), and by acting out the situation. They perceive constraints to the plans by putting themselves into other's shoes, and are flexible in their actions.

Thirdly, participants, although they are both actors and audiences in the light of selfspectatorship, observe the results of acting-out and planning which are examined and negotiated.

Fourthly, they reflect on their situated actions which are acted out in or through drama by cutting the action, discussing and analysing those actions with a view to making sense of them. Through discussion, all participants as members of the AR team replan, based on previous observations and actions, and continue this cycle of critically informed, planning-action-observation-reflection.

In applying this cyclic model to drama sessions, we see that the spiral cycles of AR are comparable to the spiral cycles of drama, [A, B, C, D, and so on], as I mentioned in the previous chapter. 


\title{
4.2.3 The Less Illiterate, the More Literate through Drama
}

\subsubsection{Our Illiteracy through AR in Socio-Cultural Context}

I mentioned earlier that the characteristics of AR could make my research framework stronger, that is, participatory, self-reflective, critical and emancipatory, and also illustrated that the self-reflective spiral of cycles of the research could enable participants and me to carry out our research systematically and effectively. AR thus seemed to be supportive in exploring the socio-cultural action of this study, in which I intend to investigate how individual participants are engaged in intercultural situations, particularly in the matter of interactive sequences of individual social activity in the situated world.

However, I had doubts about applying this research method to my study because of two points. First of all, I took the comment on AR which Kemmis makes:

\begin{abstract}
Action research is not distinguished by the use of a particular set of research techniques. While it is common for educational action researchers to keep focused diaries about specific aspects of their practices, to make audiotape records of verbal interactions in classrooms or meetings, to carry out group interviews with students after particular lessons and so forth....(Kemmis 1993: 184).
\end{abstract}

Therefore, I used research techniques which provided instant data, such as taking photographs with a Polaroid camera and a drama technique, thought-tracking. However, I kept common techniques similar techniques to others such as diaries, audiotape records, interviews and the like. In this sense, I would apply AR to my study, as a method whereby students participate and explore ICM in certain intercultural situations overall, which needs to be supplemented by others. Thus, it is not a question of techniques specific to AR. The techniques which I used, are similar to other research methods. Therefore, it is the method of AR which is important, rather than the techniques.

Secondly, what this research led me to be concerned about was relevant to analysing ethnographic data through drama. There still remain the questions of how sociocultural action relates fundamentally to drama action in the classroom, how it is dealt with as their key factor in the method, and if a social actor in a socio-cultural phenomenon, connects with an actor in the drama world. Although regarded as being in the realm of Arts, drama is also seen as related to the social sciences in this study. It is consequently stated that some forms of drama action lend themselves to a sociological as much as an aesthetic interpretation and also, some social actions rely 
on drama in order to be investigated by sociologists, especially, who have 'the idea of dramaturgy as another way to represent or 're-present' ethnographic findings' (Swartz 1998: 116).

And then, we can raise a question of what my research context is precisely. First of all, I can indicate its dual structure of both the research for the researcher and the research in which students participated, mentioned as the analogy to the TV series, Columbo. Students were involved as co-researchers in this research by exploring their actions in drama sessions as well as out of drama sessions in the same way as myself. Secondly, we were going to investigate that which students communicate in sociocultural and intercultural settings in drama sessions. As I pointed out in the previous section, my participants would also deal with interaction/communication, intercultural, as well as social, as I would.

Hence, despite having acknowledged the advantages of AR, I still wondered if it could influence my research context enough to change the reality of my participants and to investigate issues in this study. I was a little doubtful that it was through AR that I could do my research which was concerned with diagnosing the problem in my specific research context and attempting to solve it in that context.

As I discussed earlier, AR is considered as the general method rather than the specific techniques. This means AR, needed to be supplemented by another specific method, particularly in terms of collecting and analysing data, which were the types of intercultural research and evaluation.

\subsubsection{Grounded AR is more Literate}

And then, what approach can support us to implement our research through AR? According to Zuber-Skerritt, grounded theory appears to be the answer to the above question. She pointed out grounded theory as one of the crucial directions which AR moves toward in order to be reinforced as emancipatory AR in a practical sense:

\footnotetext{
Action Research is emancipatory when it aims not only at technical and practical improvement and the participants' better understanding.... It also aims at the participants' empowerment and self-confidence about their ability to create 'grounded theory' (Glaser and Strauss 1967), i.e. theory grounded in experience and practice, by solving complex problems in totally new situations, collaboratively as a team or 'community of scholars,' everyone being a 'personal scientist' (Kelly 1963), contributing in different ways, but on an equal footing with everyone else (Zuber-Skerritt 1996a: 4-5).
} 
Thus, she put particular weight on grounded theory and emphasised the self-critical attitude of every single participant as a team member and the equal involvement of each participant in the research.

Hall and Callery (2001) also claim that grounded theory obtains its value from incorporating reflexivity and relationality, which are seen as attending to the effects of researcher-participant interactions on the construction of data in terms of power and trust relationships between researchers and participants.

Also, in a practical way, Rolfe (1996) utilises grounded theory as a supplementary tool to AR to close the gap between practice and theory, by trying to penetrate into practical situations through grounded theory. Baskerville and Pries-Heje (1999) seal another gap in AR by borrowing grounded theory in its data analysis stage. And they demonstrate how units of analysis and techniques from grounded theory can be integrated into the AR cycle in order to add rigor and dependability to the theory formulation process.

As the above two cases hint, although AR has been applied increasingly to classroom research, the number of action researchers who tend toward more grounded studies has become bigger (Cohen and Manion (1989); Kemmis (1993); Nias (1993); Spjelkavik (1999); Mienczakowski and Morgan (2001). This perspective is called grounded action research (Baskerville and Pries-Heje 1999).

Let me try to reply to my previous question: what approach can support us to implement our research through AR? My answer is also based upon grounded theory. Grounded theory can be complementary to the weakness of AR in terms of exploring socio-cultural interaction/communication, especially the practical socio-cultural situation and data analysis of interaction.

This is because firstly, grounded theory is 'any form of sociology theory that is built up gradually from the careful naturalist observation of a selected social phenomenon' (Jary and Jary 2000: 254); secondly because its function of sociological theorising can increase our self-critical attitude as practitioners, as well as developing problemsolving skills; thirdly, in accordance with May (1993) there is a constant relation between social science and social theory.

Therefore, in my case there is a clear accountability to grounded theory, and my research was designed as grounded AR. In the end, I have expected this sort of 
research design to emphasise participants' views and categories in shaping those practices and situations, and also to put AR at the centre of the research process. For example, students as social actors have a better understanding in their social/intercultural action through drama activity and become self-critical researchers.

In summary, I used grounded theory as a supplementary tool to AR. Firstly, this is because my data would be ethnographic. Secondly, it results from two facts: one is that the grounded theory would overcome the limitations of AR, in that the former is generally accepted not only as a discursive approach (Smith 1982) to qualitative research, but is based on theory building from the careful naturalist observation of a selected social phenomenon. The other is that grounded theory is regarded as a systematic strategy for data analysis. I planned to follow the principles of GTA (grounded theory analysis) and at the same time I was going to use computer aided qualitative data analysis software (CAQDAS), in order to reduce the limitations of my manual work.

\subsubsection{Drama as a Research Tool for ICM}

I mentioned earlier that AR has been modified or grounded in order to enhance its rigor, depending on the research context. Among the research techniques included, there are creative arts and photography, simulation, role-playing, and drama. However, all of these have their own limitations in terms of dimensions in which my participants and I were going to collect and analyse data. These techniques only deal with restricted actions and responses rather than holistic actions and responses.

Drama has advantages in investigating social/intercultural interaction, because researchers can collect and analyse ICM effectively through drama better than less holistic activity in Creative Arts, such as photography, simulation, and role-playing. Furthermore, role-playing, which is a dramatic technique, does not establish sociocultural contexts as effectively as drama in terms of characterisation. From this point of view, researchers have recently begun to pay attention to the effectiveness of drama for developing social skills and in training courses as well as in language arts and literature. I have therefore made an assumption that drama could be a more useful research strategy.

Nevertheless, my question remained why drama is effective, especially in intercultural settings. From this basic question, I was going to study the effectiveness of drama for developing ICM in teaching EIL (English as an international language). This research 
issue would be investigated from an intercultural perspective as if participants and I had taken the role of Lieutenant Columbo in TV Series Columbo, rather than from an artistic perspective. This grounded AR was supported not only by grounded theory in which the researcher collects and analyses data through inductive reasoning to evolving theory but also by drama which is used as a research tool.

In brief, AR has provided us with a research method about how to manage this study at a macro level. On the other hand, at its micro level, grounded theory and drama have supplemented the limitations of AR: grounded theory has focussed on how to collect and analyse ethnographic data; drama has been applied as a research tool for investigating holistically the ethnographic data in ICM.

\subsubsection{Students in PAR with Drama}

In the previous section, I mentioned that this research has a dual structure for the researcher and for participants. However, the research has another dual structure. The fact that drama was used as a research tool, has not only prompted me to ground drama in AR, but it has also ensured that I now have a clearer map of the process and the dynamics of the research.

There are two key dimensions in this research: one originates in the nature of AR itself-students were seen as actors as well as researchers; the other arises from the duality of drama. The former consists of two areas, action and research, firstly because researchers do actions and at the same time do research, and secondly because researchers are doing research and are being researched.

In the latter each participant took this dual role in drama sessions, as I discussed in the previous chapter.

- both in reality (out of role) and in theatrically (in-role),

- both as actor and audience/spectator,

- both as actor and self-spectator,

- both teacher and students.

Meanwhile we did our fieldwork; we carried out our own projects. As a matter of fact, the participants I worked with, before starting any drama sessions, were already regarding themselves as co-researchers required to work on a strange but special project which they had never done before. They were already framed in the functional 
role as co-researchers required by drama, as researchers.

To avoid confusing my fieldwork with their project, we called the students' project the Educational Drama in English project [hereafter the EDIE Project] and my fieldwork the research project in Busanjin Girls Commercial High School in 2001[hereafter the BGCHS project].

With a view to differentiating their research from my research in relation to this dual practical structure, I intend to label AR where students as co-researchers participated as students in participatory action research [SPAR]; and action research which I did, as participatory action research [hereafter PAR].

In relation to this dual structure, I should consider three factors: [1] the tıme process of the spiral cycles, which led us to follow the research project concretely and systematically, [2] their socio-cultural perception and production of ICM in drama sessions, and [3] both doing action and research in the research. I planned to explore actions chronologically, by the cycle, and to present them in this study.

This presentation will give an idea of the way that each spiral cycle in our research, supported us to perceive and make intercultural interaction, which we do, and to develop what we learned from the investigation inside or outside drama sessions. With regard to the aforementioned, I can say my research method in the end has logical connections with symbolic interactionism, which approaches cultural studies through social symbols.

\subsection{Contexts of the Research}

The project was executed on the grounds that $\mathrm{I}$, as a PhD candidate, had aimed at researching the effectiveness of drama for intercultural education as part of a research programme and have involved students who were in the second grade of Busanjin Girls Commercial High School in the city of Busan, Korea. Here I intend to show what the students were, both as the project group and as co-researchers, and what context they were in.

First of all, the dual relationship between researcher and researched has its characteristics of being dialectical and is never unrelated to situations, which they are in-the situated society. This situatedness has considerable influence on our research. In reality, within the situatedness we have limited procedures and processes for our 
research framework.

Participants and I, however, have tried to overcome the situatedness by shifting and balancing our insider's experience with our outsider's experience in intercultural interactions. With respect to this triadic relationship among the three-researcher, the researched, and the situated context, I shall describe the context of this research with respect to the project group and the situational context of the research.

\subsubsection{Construction of the Project Group}

\subsubsection{The Project Group: Participants as Co-Researchers}

\section{Representativity of the Project Group}

Sixteen participants gathered for this research project through volunteering and being chosen in each class at the second grade in Busanjin Girls Commercial High School in Busan, Korea. All of them, who were sixteen-year-old girls, were in the second grade in that school. Nine participants had attended the pilot study which I did in that same school in November $\mathbf{2 0 0 0}$ and the rest had never participated in learning activities through drama before except the activity of role-playing in their junior high schools.

There were no special criteria for participation. In a sense, this research project was open to every student who might have been interested in learning English as a foreign language (EFL) through drama in this school. However, having taken into consideration the optimum size of the project group for this qualitative method, I had asked each classroom teacher who took charge of the second graders to send me approximately two volunteers as participants in this research. Three factors, the cooperation of the teachers, the interests of students including the information from participants in the pilot study, and the limited number of volunteers were main variables in this process of volunteers' applying to participate in the research in each class.

The sample of participants was selected on a theoretical basis, i.e., I selected the school, in order to relate my study to vocational education and intercultural education. Also I chose the school because of its accessibility. I had worked in this school for a long time and had known teachers who were going to cooperate in this study.

In constructing my project group, I needed to give them roles of co-researchers. Therefore I had to obtain the permission from the school principal. The number of 
participants became smaller as grounded AR evolved and at the end of the research project, eleven participants were involved I shall demonstrate why five participants gave up in the middle of the research in the next chapter.

\section{Backgrounds of the Project Group}

Participants in this project were at the centre of the research, and their participation, also at the crux of this research, was expected to give me many ideas through drama which has ICM, and has been influenced by their situated lives. According to the Annual Scheme 2001 of the school, the participants got academic marks in their middle schools that were good enough to enter Academic High Schools in which they would prepare for higher education.

They, however, had to apply for this present school, on account of their home financial backgrounds rather than their own preference. They would not have chosen this school, because it is more focused on vocational education. So they were still considering higher education. As my first questionnaire showed, half of the participants planned to apply for higher education after having been employed.

Here we note the paradoxical point that participants planned to go into higher education but they were taking programmes for vocational education in this school. This means that they were highly motivated by a need to get good school records as well as to obtain professional qualifications to help them to gain employment.

Secondly, most of the participants were still afraid of learning Language arts and Literature in English, even English Conversation. Nevertheless, they had already accepted that English is considered as an international language as well as one of the most crucial subjects. This point considerably influenced the research whose basic context was in teaching/learning EFL.

Thirdly, the fact that their home financial difficulty was such that they could not apply for schools to prepare for higher education in a sense caused them to be dissatisfied with their parents or their working class, even though it was not always at a serious level. They tended to turn to their friends to talk about their problems. It appeared that they preferred their generation rather than the older generation, such as parents and teachers, who discouraged them from doing that. A survey by the School showed the

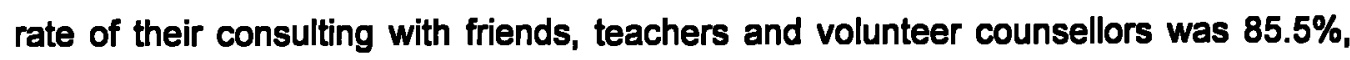
$11 \%$, and $3.5 \%$ respectively. 
What I considered here is that there is an intercultural clash between traditional cultural values and modem ones or local cultural values and global ones. For instance, Pratt et al. reported on young people in Korea under the title of 'Youth culture tunes into the West' (Pratt et al. 1999). Students have begun to think that Christianity is suitable for contemporary Korea and Buddhism is being revived, as nationalism has increased. On the other hand, older generations are still tied to Confucianism, the moral and ethical code that teaches respect for authority and the veneration of ancestors. Above all, young people in Korea are much more outspoken, more inquisitive and opinionated, more outgoing and expressive, unlike their parents, who were never allowed to exhibit all those traits within the confines of Confucian culture. In general, young Korean people are less interested in power and money than the previous generation and more concerned about their lives and loves, their friends, the environment and their future as modern, more progressive members of the world community. Therefore, they want very much to embrace other people and cultures and want not just political freedom in the form of democracy but also spiritual, intellectual and cultural freedom.

In this regard, the above statistics, which showed that the majority of students in the School consulted their problems with their friends, are reliable.

In short, there is an intercultural clash between traditional and modern, and between local and global. The project group has been under the influence of two distinct streams of culture in Korea: 'the atmosphere of official, corporate and intellectual discourse is very open to Confucianism... popular culture in Korea, like elsewhere, is increasingly guided toward teenagers and youth' (Callahan 1999: 353).

\section{The Perspective of the Project Group}

There were two key points which participants anticipated getting from this research activity: communicative competence in English and better understanding of other cultures. The former is a result of their reluctance to speak English which they must have got from their learning methods since they began to learn English in their junior high schools. The latter is caused by two factors: one is that the participants, the mono-ethnic group of Korean girls, had never travelled abroad; the other is that they had not heard enough about other cultures in classes of English, as well as other subjects.

Nonetheless, the general tendency of internationalisation and globalisation has urged them to get a feel for doing something more, for instance, having intercultural 
experiences, either directly or indirectly, even though they could get access to these intercultural situations through other channels such as mass media and cultural events.

In a word, along with internationalisation and globalisation, educational policy-making in Korea encourages participants to learn EIL, for the sake of their futures. Also, they wanted to get a better understanding of people from other countries, what they are like, and how they live, in order to communicate with them for the betterment of a future multicultural society, even in the present.

\subsubsection{The Teacher as Researcher}

In this study, I acted as the researcher as well as the teacher for two reasons. Firstly, I planned to make myself, as the insider-researcher, involved in the research by engaging directly with my participants, not just observing. Through this involvement I anticipated being able to monitor my own practices in drama sessions, to analyse classroom speech exchanges between students and me, and to get images of my work in contexts that would eventually provide me with more professional competence.

In reality, the role of the teacher enabled me to be closer to my participants than the role of the researcher, by exchanging teaching and learning activities between us during drama lessons, and by taking more opportunities for evaluating their activities, inside the sessions. Above all, the stance of the teacher-as-researcher could help me to develop my own professional competence through reflection on my own practices.

Another reason was that along with this image of a self-monitoring teacher I wanted to introduce drama into the English classroom. As a matter of fact, participants had seldom experienced learning EIL through drama because Korean teachers of English rarely seem to have introduced culture and drama into their classroom.

Therefore, I intended to apply this perspective to the educational research process, even though I was just a temporary teacher during the study. By putting these educational procedures into action strategically in a practical situation I intended both to understand my professional competence and to undertake strategic action in response to a present action context. In a word, I tried to test the adequacy of my understandings and of the situation into which practice would be introduced.

Thus, my status from the role of the self-reflective teacher to the stance of the practitioner researcher in this study was equivalent to 'action research itself, which is 
perceived as providing an understandable and workable approach to the improvement of practice through critical self-reflection' (Kemmis 1993: 181-182). With regard to my position, in which I functioned as the teacher as well as the researcher, I shall be called the instru-researcher in this study.

\subsubsection{Observers}

I had two types of observers: those regularly observing and those observing once or twice. Mrs. Choi, as a permanent observer, who had a five-year teaching career, not only monitored my study but also made audio and video records during the fieldwork. Mrs. Lee was another regular observer, a Korean teacher of English who was working for this school and was interested in introducing drama into her English classroom. On the other hand, both non-participant students in the school and others outside the schoolteachers, as irregular observers, came to monitor our drama sessions and public performances. The two regular observers provided our research team with their observation records regularly and the rest of the observers were asked to make contributions by being interviewed by the research team.

Although the interpretations of participants in this study would be directly relevant to the ongoing stages of this research, those observers who examined our situation, including the drama sessions, would provide significant factors in amending plans for further drama sessions. Therefore our research team treated them as perspectives of relevant otherness in our practices and regarded them as perspectives of objective critics of the research action.

\subsubsection{Situational Contexts of the Researchers}

\subsubsection{The Environment Inside the School: the School, the Teachers and the Curriculum}

Participants as well as observers were at the centre of this study. However, I considered the situational context around the researchers as another important factor, due to the triadic relation of the researchers, the researched and the socio-educational environment of the study, situated around the researchers. In this sense, it is appropriate to take stock of the environment both inside the school, the teachers working for it, the curriculum for participants, and outside the school, including the community to which participants belong and the local educational council.

First of all, Busanjin Girls Commercial High School, provides students with vocational 
education by focusing on the education of human resources for the information society, enabling the participants as second graders to apply for one of the three departments-international trade, management information, and computer application. The rate of employment of the school students was $98.05 \%$ in 2000 , compared with the nation-wide average for this type of school of $91.8 \%$ in 1996 . In addition, this school has set up a sisterly relationship with Shincho High School in Hiroshima, Japan, as one of its projects for Intercultural education. Judging from its achievements, since the establishment of the school, parents, the local educational council, and the community evaluate this school as being the best amongst Vocational High Schools in the city.

Secondly, seven teachers were teaching $E F L$, as English Conversation and Language arts and Literature in English. The Annual Scheme indicated that most of the teachers had participated in various programmes for teacher education and were especially interested in information technology. The Scheme, however, pointed out that teachers ought to pay more attention to the personality education of students as well as the development of teaching methods for enhancing the creativity of students.

Third and finally, the curriculum taught in the school had been constructed on the basis of the National Curriculum and had been divided into two parts of 34 academic units: subjects for general education and subjects for professional education.

- The first grader gets 21 units of general education including 3 units of English, 10 units of professional education, and three units of special activities;

- In the second grade, they get 16 units of general education including two units of English, 15 units of professional education including two units of Business English and three units of English Conversation, and three units of special activities;

- In the third and final grade 14 units of general education including three units of English, 18 units of professional education without any type of English sessions, and three units of special activities which consists of three activities: a classroom activity, for instance, the class meeting, club activity, through which students could develop their talented qualities, and group activity as part of its curriculum.

\subsubsection{The Environment outside the School: the Local Educational Council and the Community}

Up to the present, Korean education, whose arrangement is a linear school system of the 6-3-3-4 type, has developed continuously through various stages of foundation, extension, quantitative expansion, educational reform, and qualitative enhancement. 
The Seventh National Curriculum, which has been applied since 1997, highlights accomplishment of sound personality, pursuit of excellence, realisation of equality, and enhancement of hope for a better future. In addition, the globalisation and information society are emphasised as well.

Educational policies of the Ministry of Education are normally conveyed through the Local Educational Council to every school and consequently the council functions as the intermediary, to collect and control the management of schools rather than as the assistant to support school education for school effectiveness and school improvement. The local council could be one of the main factors in the hidden curriculum as well as the school curriculum (Meighan and Siraj-Blatchford 1997).

On the other hand, the community to which participants belong, and their sociocultural backgrounds, are also key elements in influencing the hidden curriculum. The industrial city of Busan, which is the second largest one in South Korea, is located in the south-eastern area of the Korean Peninsular nearer to Japan than China.

Busan people, like other Koreans, have strived to preserve the traditional, but at the same time tried not to return to the ancient but to get a new culture with identity and integrity based on tradition. They like to use the term of 'implant,' for instance; educational policy-makers have advised teachers to implant Western values in Korean values.

Influential as Buddhism and Taoism have been on their everyday lives, Confucianism is a body of ethical principles which have historically shaped the social and political structure in Korea (Slote 1996) and consequently is regarded as an analytic tool to understand the atmosphere of official, corporate and intellectual discourse (Callahan 1999).

However, more recently, Confucianism has been mobilised as part of the clash of civilisations between Korea and the West; for instance, it is not represented in popular culture as a positive force, especially with regard to teenagers and youth (Pratt et al.1999). Many citizens of Busan have its characteristic cultural homogeneity, in which 'public discourse on cultural identity, has been dominated by a mono-culturalist perspective under the rubric of "Korean national culture"'(Ryu 1998: 83) like other areas in Korea. They are now subject to the colliding binaries of East and West, and tradition versus modernity, all played out within the dynamic of tradition and globalisation. 


\subsubsection{The Natural History of My Research Study}

My teaching career for 18 years was based upon my own education. I finished my teaching career in order to study drama education in 1997. In the 1960s, during the period of quantitative expansion of Korean education, I was taught in overcrowded classrooms, in oversized schools, with a shortage of qualified teachers and educational facilities, and excessive competition in the college entrance examination. In the 1970s, during another decade of quantitative expansion in education, I was trained to be a teacher at primary level and then as a Korean teacher of English at secondary level. In the 1980 s and the 1990s, during a period of qualitative expansion, I taught EFL.

Throughout these drastic educational changes, I have had to utilize a vast amount of resources. My whole experience in the world of education has eventually led me to where I am now, studying drama education as a researcher.

\subsubsection{My Achilles' Heel in Teaching English as a Foreign Language: the Personal Context of the Research Topic}

Ever since I began teaching EFL in Korea, educators and teachers have been introducing many different teaching methods into English classrooms, at each level. Throughout this time, seven revisions of the National Curriculum relevant to teaching English have encouraged Korean teachers of English to apply them to their classrooms.

However, it has not been easy to shift teaching methods from the traditional to the progressive because the traditional grammar translation method has been firmly stuck to. Despite the various efforts to improve teaching methods, quite a few Korean teachers of English have failed to move on to teaching methods which could provide them with the model for English education and for actual international communication tasks. They have remained dependent upon the grammar-translation method.

I was one of those who had learned English in the grammar-translation method and had taught English in a similar method, with less focus on communicative competence such as listening and speaking skills. This limitation has led me to search for other methods to provide my students and me with ways to teach and learn the language, which is not static but dynamic, particularly in the context of becoming more global and multi-cultural. 


\subsubsection{Long Journey into Drama Education: Trials and Errors}

My search for new teaching methods began with the following two questions: How can I approach teaching English as a living language which is useful in our everyday lives? And then what is the nearest approach to everyday life?

The answer, to these questions came in the form of drama. The title of Goffman's book, The Presentation of Self in Everyday Life, itself was sufficiently exciting for me to cry out "Eureka! I have found it!" He dealt with people as wearing a persona, from the Latin 'mask,' presenting oneself in everyday life as an actor performs on the stage and off the stage (Goffman 1969).

I had taken a MA course in drama, which focussed upon American contemporary drama, such as Albee's plays. Such contemporary plays seemed to be much more similar to our everyday life and most teachers taught American English rather than British English. After having finished that course, I was not sure if I could yet introduce drama into teaching English as a living language because the MA programme seemed to be limited to literary study rather than a holistic approach to everyday life. Then I decided to get involved in drama as a performing art, and took another MA programme in drama, which would provide me with material much closer to everyday life than just studying play scripts. Based on what I got from the programme I ran workshops with other MA students for in-service teachers and also discussed it with would-be teachers, and applied the ideas to English classrooms, as well as 'club' activity sessions.

Incidentally, while I was studying drama I found out that drama education had been already developed considerably in the USA and the UK and established as academic departments in higher education. This was definitely another "Eureka" for me when comparing the complete lack of recognition given to the term of 'drama teacher' in Korea.

In the end, I gave up my teaching career to study drama education in the UK, whose drama education seemed to be process-centred rather than the product-centring focus in the USA. I hope my study of drama education might make a difference to Korean teachers, even one as small as a ripple in a pond, in their efforts to diversify their teaching methods within the educational context in Korea.

Since 1997, I have been studying drama education through the courses of diploma in 
the University of Central England from 1998 to 2000, MA and PhD in the University of Durham from 1997 to the present. This seems to be really a long journey to being a drama researcher in terms both of time and geographic distance.

\subsubsection{My Springboard for Education through drama in Korea}

The Seventh revision of the National Curriculum in Korea pursues an image of men and women as healthy, self-independent, creative and moral. Some people working in the field of education agree to the need for educational change more than others. However, it seems to me that educational change still lingers on in its peripheral rather than in its centre.

What can I do for that as a drama researcher as well as a former Korean teacher of English in such a situation? I intend to focus on improving teaching methods, moving from traditional teaching methods towards progressive ones, through drama.

- First of all, I wonder if I can improve student learning with a more discursive style through drama, especially in ElL classrooms but in other language classrooms as well, to enhance effective communication.

- Secondly, now that drama is regarded as cultural configuration, discussed in Chapter 2, it enables students to have a better understanding of cultural understanding of their own and other cultures, that is, intercultural understanding.

- Thirdly, drama activities are considered to be one of the key strategies in integrated projects inside schools.

- Fourthly, drama is applied to students with serious problems by counsellors in schools in the sense that drama itself has its own characteristics of being therapeutic as well as being cathartic.

- Fifthly and finally, drama could be introduced into activities outside schools such as art camp or group activity.

These illustrations explain the reasons, my motivation, and match my ultimate goals in this study.

\subsection{The Pilot Study}

My aim in the piloting was to get into contact with a preliminary sample of participants in the school, and to establish their views and preferences on a number of important aspects of my main fieldwork. These aspects included:

- performing drama in the classroom, 
- improving ICM in learning EIL through drama,

- understanding other cultures through drama in the intercultural context, and

- methods of how to explore their socio-cultural/ intercultural communication through drama, and of how to develop critical thinking about their communication or interaction.

Also, I tried to investigate the extent of their competence in presenting themselves in English and through drama, and to survey the general pattern of their learning style in the school.

Next to these aims, I intended to try out the sampling and administrative procedures for the main fieldwork, and to evaluate the effectiveness of several methods to be employed in the fieldwork.

\subsubsection{Students, Observers and Me in Participatory Activity}

Having taken into consideration how to relate my research questions to the field of investigation and to manage my pilot study, I chose Busanjin Girls Commercial High School in Busan, Korea, where I had taught students EFL for many years. Because Mrs. Lee, the Korean teacher of English, who was interested in drama education, wanted to co-operate with both pilot study and main fieldwork, I consulted with her on how to construct the group to participate in the pilot study. We decided to ask her students to be volunteers for the activity. In the end, I had 22 volunteers to participate in the activity among the first graders in the school.

Also, in addition to myself as researcher, I involved two observers: a former teacher who had a five-year teaching career and made video-records of the drama sessions both in this pilot study and the main fieldwork, and the Korean teacher of English. I could thus get three perspectives for this pilot study-students', observers', and researcher's.

\subsubsection{Procedures and Arrangements}

First of all, I had to make contact with the school in order to gain permission for the study. I got verbal permission from the school principal through the vice school principal. In October, I submitted my plan of the pilot study to the principal with a formal letter from the School of Education, the University of Durham and the consent form, which the principal would complete. 
After having visited the school on a couple of occasions to consult with the school principal and senior teachers on 4 November 2000, it became clear that traditional, conservative, and formal approach to schools, had not changed since the 1980 s.

\subsubsection{Action and Research in the Pilot Study}

Along with the consent from the principal, I obtained consent from volunteers for the pilot study and asked them to fill in a questionnaire, which is presented as Q1 (ASQ) in Appendix $A$, before starting the first drama session. This aimed to survey to what extent they had developed an awareness of intercultural sensitivity, at what level they thought they were in terms of their ICP (intercultural competence) in English, and to what extent they were satisfied with learning EIL in their classrooms. The questionnaire was set in Korean because the Korean version allowed participants a greater understanding of each question and to answer questions more precisely.

I gave five 90-minute drama sessions: Monday, 13 November; Thursday, 16 November, Friday, 17 November, Monday, 20 November, Tuesday, 21 November 2000. The five 90-minute drama sessions were intended to make participants get a feel for how to do drama in their classroom rather than for getting a better understanding of ICM. They had rarely experienced drama except in role-play exercises in school.

During the pilot study, I kept a diary to record briefly what I had done for each drama session, how participants had felt about the session and had got through the session, and what observers had commented on each session, if at all. Also, Mrs. Choi, the observer, and I, made audio and video records of the whole five drama sessions, and took photographs

After finishing all of the planned drama sessions, I interviewed some of participants to monitor our drama sessions for approximately twenty minutes during lunch break or after school. Also, I had another meeting with the principal and the Korean teacher of English to prepare for the main fieldwork.

With respect to collected data I had the questionnaire, their personal journals, audio taped interviews, and photographs, from participants; observation checklist papers from observers, and field diary from me, along with audio and video-taped drama sessions. 


\subsubsection{Report on the Pilot Study}

In this section, I will present what I got from three different sources in this pilot study: participants, observers and myself as the teacher researcher. First of all, feedback from participants was collected through various means, which were textual, graphic and visual, an analytic surveying questionnaire, personal journals, diaries, SelfQuestioning form, interviews, photographs, audio and videotape records, and the drama activity itself.

Each participant completed the questionnaire effectively except for a couple of items: one was a way to describe their birthday presented in the British way, and another problem was that I missed out the Internet in an item relevant to sources of information about cultural events.

I asked them to write either personal journals or to keep diaries, and to keep their entries brief to reduce their burden especially in terms of writing something in English, not in Korean. Only a few of them handed in both journals and diaries, and entries were intermittent. Despite being written in Korean, the Self-Questioning form (see Appendix $B$ ) seemed to be difficult for them to understand because the questions were made on the basis of searching for psychological conversations in their minds.

However, as the pilot study moved on toward the final stage, all of the participants became interested in doing drama and became more involved, although they still hesitated about expressing their feelings and actions. They preferred to do drama relevant to their everyday lives and everyday expressions in English. At the end of the pilot study, all participants became more confident in doing drama in English and were very satisfied with what they had achieved through drama in the study.

In general, the activities of participants in this pilot study were focussed on how they did action through doing drama rather than how they researched their drama activities in terms of the socio-cultural/intercultural context.

Feedback was collected mainly from two regular observers, mentioned earlier, and non-observers, such as the vice-principal of the school. Mrs. Choi gave me her general comments on participants' activities. She said, for example, that although they were not competent in speaking English, they were very enthusiastic about drama itself and became accustomed to presenting themselves, with expressions of 
everyday life in English through drama.

Mrs. Lee, the Korean teacher of English, pointed out that some teaching materials were too difficult for participants to understand and suggested that I give participants hints or explanations about certain material. But she added that it was clear that participants wanted to be involved in doing drama deeply, and acknowledged their participation could make a contribution to their learning process in the English classroom as well as in other learning activities.

What did I attain from my research activities as the researcher? I greatly enjoyed the pilot study and there is no doubt that it was very useful. First of all, I became more confident integrating drama into learning styles in teaching and learning EIL. Moreover, I believe this integration made participants see English, which they had thought of as boring and difficult, as a potentially fun exercise. Consequently, the participants became interested in doing drama in English.

Secondly, I had to re-consider my drama lesson plans in terms of students' competence in the language and their inability to express their feelings and opinions in English. In spite of their strong motivation and interest in doing drama, they sometimes hesitated about doing it in English. Also, we did not have enough time to complete my drama lesson plan for 90-minute sessions. We had to skip several activities of the plan and consequently participants did not have enough time to think out their ideas and then to act out them. In addition to lesson plans, they preferred to do drama with teaching materials and to adapt them, rather than creating dialogue in English.

Thirdly, I had difficulty in observing their activities during drama sessions and keeping records about them during and after sessions, particularly in terms of the dynamics of their interaction.

Fourthly, as far as technical management was concerned, I should have considered where to locate microphones well enough to catch their voices in making records of audio and videotapes, and photographs.

Regarding the last two points, I realised that collecting data could impact on analysing data and vice versa. For instance, I could get different results from photographs.

Fifth and finally, I realised that I had over-estimated participants' confidence and competence in communicating with each other in speech and writing letters or making 
documents in English and for doing drama in English.

Given all of these, it can be said that this pilot study revealed the possible limitations of the study due to my being a novice researcher and drama teacher. This therefore gave me food for thought for the main fieldwork, the next stage of my study.

\subsubsection{Implications for the Main Fieldwork}

What did this pilot study provide as a sort of base to my fieldwork? I intend to address those implications for the fieldwork by the procedural stages of AR: plan, act, observe, and reflect.

The First Stage: Planning: The closing time for each drama session in the fieldwork should be changed to an earlier time, or the period of the fieldwork should move into spring, summer or early autumn when the daytime is longer than in the rest of the year. Insofar as I planned to give participants drama sessions after school in winter time, they had hardly been dismissed from each drama session before most of the participants had to rush to home because it had already got dark and it took some of them nearly two hours to get home.

The Second Stage: Act. Firstly, I needed to correct some questions in the SelfQuestioning form, to make them clearer as well as easier. Secondly, I should extend the duration of each drama session to longer than 90 minutes per session. Thirdly, participants seemed to need to do a preliminary drama activity to get a feel for what doing drama is like in drama education because they had never done drama in their schools. Some of them were too shy to express their feelings and emotions through drama. Fourthly and finally, what participants as co-researchers did as research in an activity-based method encouraged me to change the plan of the fieldwork to a more activity based method, in other words; an equal focus on action and research in the fieldwork.

The Third Stage: Observe: Regular observers need to be managed more effectively and to have more information about drama education and observation skills; on the other hand, participants could be more involved in observing and researching their activities through research meetings, interviews, and publishing a booklet.

The Fourth Stage: Reflect. taking into consideration that participants were becoming interested in drama, presumably I could encourage them to do a public performance 
even on a small scale, although such performances tend not be open to everybody in the school. And for me, it was fairly certain that $I$, as the researcher, had still used questioning strategies as well as managing techniques on the basis of teacher authority rather than participant autonomy.

Therefore, on the grounds of the above, I made changes in the fieldwork:

- The fieldwork was done in March and April 2001, not December 2000.

- The duration of each session was extended from 90 minutes to 120 minutes

- The questionnaire was revised and made clearer and easier

- The frequency of personal journals was decreased by half.

- The diaries of participants were completely excluded.

- One more video camera was set up, and cameras, Polaroid as well as an ordinary one, were used.

- Research meetings were held on Saturdays with lunch outside the school.

- Participants were involved in interviewing non-participants.

- The booklet in a Korean version was published

- The public performance was made to smaller limited audiences

This section has focused on how I conducted the pilot study, which led into the main fieldwork. The pilot study was used to test ideas before putting my research scheme into the fieldwork, for instance, in exploring my research questions through collecting and analysing data, described in the following sections.

\subsection{Data Collection}

As shown in the pilot study, researchers should not approach data collection while entirely excluding data analysis. What is more, data collection cannot be separated from data analysis in the matter of grounded $A R$, as mentioned in 4.2. However, I shall present the division of these research actions in two parts, and I shall examine how to collect data in this section in three perspectives: [1] how to design the instruments and how to carry out drama workshops for data collection, [2] the procedures to collect data, and [3] the management of data collection. In 4.6, data analysis will be described. 4.5 and 4.6, aim at exploring my research questions. Each research question was focused on specific points, as described in Table 4.2. 
- Question 1: What kinds of role can drama take in teaching and learning EIL?

- Question 2: Can drama be effective for Intercultural Education?

- Question 3: Why can drama be effective for developing ICM?

- Question 4: How do participants do drama activity in the intercultural context?

- Question 5: Do participants take roles as intercultural actors with an intercultural identity?

Table 4.2 Research Questions and their Foci

\begin{tabular}{|c|c|c|c|}
\hline \multicolumn{2}{|c|}{ Research questions } & \multicolumn{2}{|c|}{ Categories of Their Foci } \\
\hline 1 & $\begin{array}{l}\text { What kinds of role can } \\
\text { drama take in teaching } \\
\text { and learning EIL? }\end{array}$ & 1 & $\begin{array}{l}\text { Effectiveness of drama as a learning } \\
\text { medium in learning ElL; listening, } \\
\text { speaking, reading, and writing. }\end{array}$ \\
\hline 2 & $\begin{array}{l}\text { Can drama be } \\
\text { effective for } \\
\text { Intercultural } \\
\text { Education? }\end{array}$ & 2 & $\begin{array}{l}\text { Effectiveness of drama as an arena to } \\
\text { provide aesthetic distance from the self; } \\
\text { unfamiliarity, reflection, perception, and } \\
\text { critical thinking. }\end{array}$ \\
\hline \multirow[t]{3}{*}{3} & $\begin{array}{l}\text { Why can drama be } \\
\text { effective for developing } \\
\text { ICM? }\end{array}$ & 3 & $\begin{array}{l}\text { Effectiveness of drama as a reflective } \\
\text { tool of the self on otherness; listening to } \\
\text { others, concerning about others, } \\
\text { understanding others, and giving way } \\
\text { to others. }\end{array}$ \\
\hline & & 4 & $\begin{array}{l}\text { Effectiveness of drama a reflexive tool } \\
\text { of the distanced self on the self; critical } \\
\text { to the self and projection of the } \\
\text { reflected self. }\end{array}$ \\
\hline & & 5 & $\begin{array}{l}\text { Effectiveness of drama as a workshop } \\
\text { to experience intercultural experience; } \\
\text { interest in different cultures, positive } \\
\text { participation, discussion with others, } \\
\text { and critical mediator. }\end{array}$ \\
\hline 4 & $\begin{array}{l}\text { How do participants do } \\
\text { drama activity in the } \\
\text { intercultural context? }\end{array}$ & \multirow[t]{2}{*}{6} & \multirow{2}{*}{$\begin{array}{l}\text { Effectiveness of drama as a laboratory } \\
\text { to take a role as intercultural actors; } \\
\text { knowledge of the self and otherness, } \\
\text { skills to interpret and relate to } \\
\text { intercultural actors, skills to discover } \\
\text { and or interact, and attitudes } \\
\text { relativising self and valuing other. }\end{array}$} \\
\hline 5 & $\begin{array}{l}\text { Do participants take } \\
\text { roles as intercultural } \\
\text { actors with an } \\
\text { intercultural identity? }\end{array}$ & & \\
\hline 6 & Other possibilities & 7 & Other things observed by the observer. \\
\hline
\end{tabular}




\subsubsection{Instrumentation for Data Collection}

In this section, I am going to present the instruments designed to gather data and how triangulation for trustworthiness and dependability of the instrumentation was carried out. I will examine firstly, the field diary and observation list kept by the researcher, secondly, questionnaires and Self-Questioning form from the participants; thirdly and finally, observation check lists from observers. I intend to describe further other methods and the triangular structure of data collection in 4.5.4.

\subsubsection{Field Diary}

I made use of my field diary and kept a record of as much as I could. My field diary was written about what I saw as well as heard, and how I was treating participants and was being treated by them throughout the study.

Also, I added to this fundamental information, several other things related to some questions. For instance, was my study worthwhile? What did I do in the drama sessions on the day? What was I going to prepare for the next day as the teacher as well as the researcher? How did the participants respond to their project? How could non-participants learn from our experience? I tried to make comments on various important things related to these. Because of this, this field diary functioned as the basic repository of the study, enough to be used as raw data. Basic categories of my field diary can be summarised as follows:

A. The perspective of the researcher

[1] Management and emotions on my individual dimension

[2] Research methods for data collection and data analysis

[3] Collaboration with participants and the school

B. The perspective of the participant

[1] Collaboration with participants and the school

[2] Responses from student researchers and observers

[3] Perception of student-participants

C. The perspective of the drama teacher

[1] Teaching methods and teaching materials

[2] Refining the teaching problem and approach

[3] Relations of drama activities to research questions

I thus wrote down what actions I did about the area I was researching and what I learned myself as the teacher-researcher while I was doing the research project. In the end, these recordings led me to make use of the field diary as the repository for my research action files, which is a collection of notes, ranging from some very trivial memos to empirical comments, in relation to this research study. 


\subsubsection{Questionnaires}

Two questionnaires, Q1 (ASQ) and Q2 (Post-Questionnaire), which are presented in Appendix $A_{1}$ were applied to the main fieldwork: the former one had been designed before I did the pilot study and was pilot tested; the latter was produced during the fieldwork to be used instead of interviews at its end.

The purposes of the former were, firstly, to survey the basic context related to the project group and to collect data to answer the research questions, mentioned in the introductory chapter and above. The second aim of this questionnaire was to measure, which stage student participants were at in terms of intercultural sensitivity, for instance, 'a developmental model of intercultural sensitivity' (Bennett 1993: 29). The third was to check their self-awareness as Korean teenagers. With a view to surveying and analysing these three points, I extracted some items from existing questionnaires (Bhawuk and Brislin 1992; Ballou 1996; Seelye 1994; Singelis 1998), modified them, and designed the questionnaire for my study.

The questions of the questionnaire are categorised into five parts:

- The first part (item number 1-6) is about personal data;

- The second part (item number 7-20) is about the basic context of their learning English in the school

- The third part (item number $21-30$ ) asks about what they thought of Koreans

- The fourth part (item number $31-51$ ) is related to their selfawareness and their general cultural patterns:

- The fifth part (item number $52-80$ ) covers feelings and thoughts of participants in terms of intercultural sensitivity: (This questionnaire and references to each item are presented in Appendix A).

The second questionnaire is based on tracing any changes of the project group while the participants were doing their project. I planned to compare their intercultural sensitivity at the beginning and at the end of the EDIE project by re-using items of the first questionnaire in the post-questionnaire, to see how much the intercultural sensitivity of participants changed throughout the project. But I had reconsidered my main research topic was to investigate how drama was effective rather than how much drama was effective, and decided not to focus on the change in detail, although I measured it.

However, I will present the result of how he project group evaluated the drama project 
in which was collected from the post-questionnaire. These items were put in successive questioning about each topic; the introductory items were produced in fivepoint scales, and the items evaluating their EDIE project were made in open questions. This questionnaire, as I mentioned earlier, took the place of interviews, which I planned to have with participants after the project.

\subsubsection{Semi-Structured Interview}

At the beginning of the project along with the questionnaire, I had scheduled interviews with participants. During these interviews I therefore tried to focus on Research Questions 1 and 2, which enquired about the relation between drama and learning EIL; at the same time, I made inquiry about what participants were expecting in the intercultural project, in order to take account of my key research questions, Question 3 and 4, mentioned in the previous section.

I made a semi-structured interview list and the main questions to be put to each participant. These questions aimed at checking the extent participants expected themselves to achieve learning through drama before they had been deeply involved in this research project. The questions were related to two main categories: drama as a learning medium in language education, especially in the context of EIL, and effective drama in ICM. The results from this interview will be discussed further in Chapter 6.

Although the above list had, as its purpose, the gathering of information about how much interviewees expected from this research project in terms of the aforementioned categories, I allowed more time to certain categories, which the interviewee wanted to emphasise more than other categories.

\subsubsection{Self-Questioning Form}

Meanwhile questionnaires were designed to collect data of overall opinions of participants relevant to the project; the Self-Questioning form was produced with the purpose of exploring interactions between an individual participant herself and the intercultural context around her while she was doing drama activities.

I used the Self-Questioning form as an instrument, which helped each participant to communicate a deeper understanding to herself and get her intercultural experiences through thinking more deeply about some aspects of her own interpersonal style in the process of drama activity. This form, which is attached as Appendix $B$, followed a 
pattern of dialogue in which the participant had a conversation between herself and her situations within the drama context.

I tried to explore the dialogue at two levels: its basic understanding level and its deeper understanding level. At the basic level each participant self-questioned and answered about her interpersonal situations, for instance, in what kind of pattern did she do drama actions on the day? What sort of relationship did she have with other participants in role? How did she feel about herself in role related to others? And what did she do in the described context? At the deeper understanding level, selfquestioning was related to dialogue between the self and otherness in terms of dialogical dynamics.

There were questions at her deeper level:

- Firstly, in terms of dialogue with otherness, she was asked to enumerate several ideas, which she had thought out in her head before taking actions in her situation and to write down the action, which she actually acted. And then she was asked to explain the reasons why she picked and acted out a certain action, and why she did not move other ideas into practical actions;

- Secondly, she considered which was the most difficult option to resolve among her ideas, considering resistance to the self in the intercultural context;

- Thirdly, in the light of reflection on otherness, how she would act in that situation if she were an Englishwoman or an American; and

- Fourthly and finally, in the matter of self-reflexivity from otherness, she considered what she would do in the similar situation to that in the future.

Through this self-questioning of participants, I also tried to collect data related to how each participant coped with her situations, especially focused on her dialogical interactions between the self and otherness in her mind.

\subsubsection{Observation Checklist}

Throughout the study, observers were asked to use the observation checklist, which I constructed, in order to focus the research questions in this study. I encouraged those observers including me to tick one cell, which indicated one of five rates for each item, and to make their own comments on each item. Items in this observation checklist, which will be presented in Appendix $C$, are divided into seven categories and these categories are related to research questions as shown in Table 4.2. 
Observers focused on exploring the effectiveness of drama for better ICM. The term 'effective' has the basic meaning-something works well and produces the results that were intended, as defined in 1.3.3.1. In the above list the effectiveness of drama means that drama is able to influence feeling and thinking patterns, and furthermore to manipulate necessary pre-requisites for eventual behavioural change.

The above checklist is what I asked observers to pay attention to, but it was not easy for observers to keep observation records with this checklist. I shall make further description of this in 4.5.4.

\subsubsection{Drama Sessions}

In the first week, we tried to get to know participants by playing games introducing who we are and what we see as important things. And then I asked participants to look at pictures of figures and to write down their first impressions of them under each picture. By experiencing how participants get their first impressions from the pictures and what factors could influence the impressions at their first meeting, they had an understanding of the importance and the meaningfulness of non-verbal language

This understanding was later going to be reflected in expressing what kind of role each student took. I introduced basic dramatic techniques, still-image (tableau), questioning-in-role (similar to hot-seating), and five-level exercises, as presented in Appendix $D$. The first two techniques aimed at understanding basic concepts of doing drama, for instance, that everything is very significant in terms of making meanings; the last two techniques were for having a better comprehension of the context which they were handling.

After this activity, we had another session to read a poem, 'Impression of a New Boy,' and to have a better understanding of the importance of non-verbal language through doing basic drama activity.

In the second week, participants were asked to use the dramatic form, for instance, roles and relationship, use of language, including speaking simultaneously, eye contact, and so on, in making duologue with their partners. Also, while presenting their duologues they used many pieces of cloth as costumes, which added some meanings to their duologues. Through this activity of using cloth as their costumes, they had an understanding of establishing a commitment to belief. 
These simple presentations had the purpose of providing the participants with the familiarity with drama and team spirit, which is necessary for this research project through drama.

In the third week, I asked participants to make groups of 4 and to play the Passport Game. Each group took collective responsibility for inventing their country, culture, language, and their passports in their created language. After this invention, each group dealt with procedures to immigrate into another country, which one of the others invented, by group improvisation in role as immigrants or immigration officers.

Also, in this week, participants were asked to reveal an understanding of the significance of the intercultural interaction between American and English, and between American and Japanese by using the drama form, for instance, role-play. Especially, role-play is a very beneficial technique to explore the context around the participants, as discussed in 4.1.

In the fourth week, participants were also asked to investigate intercultural interaction through drama in the context of international trade between an American firm and a Japanese firm in Tokyo. After analysing a text about an international business meeting, participants prepared for dramatising this and brought tension in stages. And then they developed theatre skills by carefully working towards a climax and eventually did a simple presentation. At the end of the drama session, I encouraged participants to discuss what they had experienced and how they could solve the intercultural difficulties.

Among the drama techniques I applied to these drama activities, dramatisation was aimed at understanding the text and presenting how much they understood about that through dramatising it. Another aim was to understand how to write a play script. After having a better understanding of that text, while they were acting out, I tried to elicit their dialogues in their minds through drama techniques of thought-tracking and discussion, as discussed in 4.5 and 4.6.

In the fifth week, participants had a better understanding of intercultural interaction through drama by exploring ICM in the following contexts. The first is between two friends when an American friend asks a Korean to go to the movie; the second is the context in which an American asks a Japanese about a small private matter of that Japanese family; the third, a situation in which an American has dinner with a Korean family. 
When I asked participants to make a public performance, they chose the script, War Paint, by David Walker (1977b), for the public performance. This was written about a conflict, between an English teenager and her mother, over the use of heavy make-up and returning home late; these issues of the play were common to the participants. After choosing the play, the project group analysed the script through studying its structure, its characters, and so on and read it like a radio drama, not to act out yet.

In the sixth week, participants and I prepared for the public performance, that is, rehearsals and dress rehearsal. This week focused on drama activity as an art form, discussed in 3.2.

In the seventh and eighth weeks, participants had an understanding of drama education in the classroom as participants as well as researchers by observing videotape reviewing their drama activities. In the last week, participants, who took roles of researchers through the dramatic technique of the mantle of the expert, made a report of their project by publishing a booklet.

Participants as co-researchers discussed their current problems in learning EIL and developing intercultural competence and tried to solve those problems throughout this research project. This participant-in-role was related to their dual functions of participants as well as co-researchers, addressed in 4.1 and also in the matter of drama conventions, reality and theatricality, during their drama sessions, discussed in 3.1.

During these drama sessions, participants took roles of multi-reactive actor, which I mentioned in 4.1.2, and at the same time they still had positions as researchers by using the drama technique, the mantle of the expert, described in 4.1.1. In other words, doing drama in the drama sessions is regarded as another drama technique, drama within drama.

As discussed in 4.5.2, because drama activity per se has its immediacy as well as its dynamics, in order to catch momentary actions we took photographs with a Polaroid camera. This camera provided us with discussion chances shortly after relevant actions.

These drama sessions can be summarised as shown in Appendix D. Photographs of some drama activities are also presented in Appendix E. 


\subsubsection{Procedures of Data Collection}

As mentioned in 4.2.4, I conducted my fieldwork with students as researchers from the beginning of March to the beginning of May 2001. The fieldwork had its dual structure in two perspectives-the AR model of SPAR and PAR, and a series of drama workshops. Both students as researchers and I as the researcher were involved in collecting data and analysing data in our own ways inside and outside the drama workshops.

In addition to the drama sessions just described, I had nine research meetings with students as researchers on Saturdays, through which students as researchers and I as the researcher had opportunities to review our actions in that week and re-plan the next actions. The whole programme of data collection in this fieldwork was carried out at six stages. At these stages, we explored the effect of our action on our competence by questioning observers and non-participants as well as ourselves in direct and indirect ways.

\subsubsection{Stage 1: Preparation for the Main Fieldwork}

I visited the school and gave a brief talk on my fieldwork to the school principal and the vice-principal. This was followed by these following steps:

- the discussion of the possibility of a doctoral study with the students in the school with the vice-principal;

- the permission of the principal in informal and formal ways; and

- the cooperation from the principal and secured cooperation from the teacher, who had been involved in the pilot study and interested in this study.

It took me several months to get through these procedures from asking informal permission including the pilot study to the moment I made this visit. At this fieldwork briefing, I held a preliminary negotiation to re-confirm the situations related to the fieldwork and to consult with the teacher about some situations, which had changed since the pilot. Also, I formed preliminary impressions regarding the school principal, teachers, their attitude regarding drama education in English, their students, the school management, and so on.

\subsubsection{Stage 2: Process-Centred Drama I}

I took into account that I would be working with half of the student-participants who 
had never attended educational drama except role-playing. Therefore, above all I had to focus on what drama is like in education, that is, on the participants' understanding of and use of drama in general. Before asking volunteer students to fill up their consent forms, I gave them an introductory session on what educational drama was like and what we were going to do through drama, that is, doing research through drama.

After this introductory session, the volunteer students gave their consent to participating in the study and hereafter their stance was transformed into student researchers from student volunteers. The student researchers were asked to fill up Analytic Surveying Questionnaires and to write their names in the interview timetable.

Two drama sessions on Wednesday and Friday and one research meeting on Saturday in each week followed this introductory session. At this second stage for two weeks I introduced to student-participants how drama, process-centred rather than product-centred, was applied to learning and teaching, and doing research in order that they might get a feel for educational drama and for doing research through drama. I recorded and reflected upon what I saw and heard inside and outside drama sessions.

As soon as participant students took roles as researcher through being influenced by a technique of drama education, the Mantle of the Expert, they became involved as researchers in this study. As this programme proceeded students became deeply engaged as researchers, and from the next stage, they became very active researchers in planning and realising the study.

\subsubsection{Stage 3: Process-Centred Drama II}

Process-centred drama was still dealt with at this stage. However, we, moved on to another perspective, to focus on exploring the key research question of why drama can be effective for developing intercultural competence in this three-week period. Student researchers conducted actively their project, EDIE project 2001, which I mentioned in 4.1.1.1 by planning the next steps and doing actions for those steps. For instance, they managed to interview the school principal and non-participant students on the impression they had of their EDIE project.

On the other hand, $l$, as the participant observer as well as the researcher, collected data from the student researchers and observers, and documentary data. At the same 
time, I not only revised and refined my understanding of problems related to using drama in this study, but also my practice as drama leader conducting this study. In the last week of this stage, we made a decision to make our public performance at the end of this project.

\subsubsection{Stage 4: Product-Centred Drama}

We focussed on product-centred drama as an art form in the intercultural context by making a public performance of a short play, War Paint, which was written about an English teenage girl by David Walker. We had only rehearsed this play for approximately twelve hours. The names of all student participants were on the casting list and they took charge of many things relevant to acting including their costumes and make-up.

Also, they managed the public performance itself, for example, the director, the sound manager, and the property manager. I directed the performance as the assistant manager. After the public performance, we discussed and reflected about productcentred drama. This discussion was focused on drama as an art form along with ICM.

\subsubsection{Stage 5: Review and Publication}

We reviewed what we had done in terms of action and research as student researchers and the researcher, and published a booklet, which covered this study but focussed on the students' project, EDIE project 2001 rather than my project, BGCHS project 2001. The title of the booklet, '쪼부랑 몸부립.' means 'Struggle for Learning English' and hinted that my project group had felt like this in learning English.

\subsubsection{Stage 6: Completion}

I re-visited the school in order to say thanks to the school principal and the relevant teachers for their co-operation, to report to them what student participants and I did for our projects, and to collect more governmental documents related to the study.

Throughout this study nearly every day student researchers were asked to fill in the Self-Questioning form after drama workshop of the day. Also, in the middle and at the end of the research fieldwork they were asked to evaluate their research action by writing their personal journals.

As I mentioned earlier, we had research meetings every Saturday during the study. 
Most of the activities throughout this study were audiotape recorded and all drama sessions were audiotape recorded as well as videotape recorded.

Thus I took six stages in collecting data and there were different collection methods or forms in these stages: Questionnaires, Personal Journals, Self-Questioning form, Observation List, audio and video taping, field diary, photographs, and so on. These techniques introduced into collecting data and the progress of data collection can be summarised as shown in Table 4.3.

In the next section, I shall address how to manage those data. In these procedures, several different research methods were employed. The methods, which were expected us to get high trustworthiness of the study, were used to create triangulation in data collection.

\subsubsection{Management of Data Collection: Trianqulation}

First of all, as I mentioned in the previous section, methods of data collection common to qualitative research were used such as personal observations, interviews, SelfQuestioning and personal joumals, and audio and videotape recordings. Also, quantitative methods, for example, questionnaires, were employed. All these techniques were conducted on the basic principle of triangulation in data collection.

Before moving on how to triangulate my data, here I would like to present what triangulation is. Cohen et al. defined triangulation as 'the use of two or more methods of data collection in the study of some aspect of human behaviour' (Cohen et al 2000 112).

Douglass (1976) lists three types of triangulation- [1] multiple sources from one technique can be compared, [2] multiple methods which can be applied to the same problem, and [3] multiple investigators, which are also used to compensate for their individual styles, biases, or shortcoming, or to exploit their specific strengths:

On the other hand, Denzin (1970) and Cohen et al. (2000) gave six types of triangulation-[1] time triangulation, [2] space triangulation, [3] combined level of triangulation, [4] theoretical triangulation, [5] investigator triangulation, and [6] methodological triangulation. 
Table 4.3 Procedures of Data Collection

\begin{tabular}{|c|c|c|c|}
\hline Stages & Dates & Actions to collect data & Research Techniques \\
\hline $\begin{array}{l}\text { Stage 1: } \\
\text { Preparation }\end{array}$ & $\begin{array}{l}\text { Pre-session period } \\
\text { of Drama sessions: }\end{array}$ & $\begin{array}{l}\text { To get the consent from the } \\
\text { school principal in informal and } \\
\text { formal ways, To visit the } \\
\text { school, and to consult teachers } \\
\text { with the fieldwork. }\end{array}$ & $\begin{array}{l}\text { Interviewing teachers including } \\
\text { the principal, and field diary. }\end{array}$ \\
\hline \multirow[t]{2}{*}{$\begin{array}{l}\text { Stage 2: } \\
\text { Process- } \\
\text { centred } \\
\text { Drama I }\end{array}$} & $\begin{array}{l}\text { The First week: } \\
\text { 13[introductory], } \\
14 \text { Wed], } \\
16[\text { Fri], \& } \\
\text { 17[Sat] March } 2001\end{array}$ & \multirow[t]{2}{*}{$\begin{array}{l}\text { Drama sessions: } \\
\text { How to do drama A } \\
\text { [Process-centred in the socio- } \\
\text { Cultural context] } \\
\text { [Situated learning by doing] } \\
\text { cf. Consent from students. }\end{array}$} & $\begin{array}{l}\text { Pre- Questionnaire, Interview, } \\
\text { Self-Questioning Form (SQF), } \\
\text { Observations, } \\
\text { Audio and video records, } \\
\text { Photographs, } \\
\text { Research meetings } \\
\text { (Tuesday and Saturday), and } \\
\text { Field diary. }\end{array}$ \\
\hline & $\begin{array}{l}\text { The Second week: } \\
\text { 21[Wed], } \\
\text { 23[Fri], \& } \\
\text { 4[Sat] March }\end{array}$ & & $\begin{array}{l}\text { SQF, Observations, Audio and } \\
\text { video records, Photographs, } \\
\text { Saturday Research meeting, \& } \\
\text { Field diary. }\end{array}$ \\
\hline \multirow[t]{3}{*}{$\begin{array}{l}\text { Stage 3: } \\
\text { Process- } \\
\text { centred } \\
\text { Drama II }\end{array}$} & $\begin{array}{l}\text { The Third week: } \\
\text { 28[Wed], } \\
\text { 30[Fri], \& } \\
31 \text { [Sat] March }\end{array}$ & \multirow{3}{*}{$\begin{array}{l}\text { Drama sessions: } \\
\text { How to do drama B } \\
\text { [Process-centred in the } \\
\text { Intercultural context: } \\
\text { intercultural drama] } \\
\text { [Situated learning by doing] } \\
\text { ("Participants did some drama } \\
\text { activities in English as well as } \\
\text { in Japanese in the Fourth } \\
\text { Week). }\end{array}$} & $\begin{array}{l}\text { SQF, Observations, Audio and } \\
\text { video records, Pholographs, } \\
\text { Saturday Research meeting, } \\
\text { and Field diary. }\end{array}$ \\
\hline & $\begin{array}{l}\text { The Fourth week: } \\
\text { 4[Wed], } \\
\text { 6[Fri], \& } \\
7[\text { Sat] April }\end{array}$ & & $\begin{array}{l}\text { SQ F, Observations, Audio and } \\
\text { video records, Photographs, } \\
\text { Personal Joumals (interim report } \\
\text { l), Saturday Research meeting, } \\
\text { \& Field diary. }\end{array}$ \\
\hline & $\begin{array}{l}\text { The Fifth week: } \\
11 \text { Wed], 12[Thurs], } \\
\& 14[\text { Sat] April }\end{array}$ & & $\begin{array}{l}\text { SQF, Observations, Audio and } \\
\text { video records, Photographs, } \\
\text { Saturday Research meeting, \& } \\
\text { Field diary. }\end{array}$ \\
\hline $\begin{array}{l}\text { Stage 4: } \\
\text { Product- } \\
\text { centred } \\
\text { Drama }\end{array}$ & $\begin{array}{l}\text { The Sixth week: } \\
\text { 18[Wed], } \\
\text { 20[Fri] \& } \\
\text { 21[Sat] April }\end{array}$ & $\begin{array}{l}\text { Drama sessions: } \\
\text { How to do drama C } \\
\text { [Product-centred in the } \\
\text { Intercultural context: } \\
\text { intercultural drama]: } \\
\text { Public performance } \\
\text { War Paint]: [Situated learning } \\
\text { by doing] }\end{array}$ & $\begin{array}{l}\text { Self-Questioning Form, } \\
\text { Observations, Personal Joumals } \\
\text { (interim report II), Interviews, } \\
\text { Audio and video records, } \\
\text { Photographs, Saturday } \\
\text { Research meeting, and Field } \\
\text { diary. }\end{array}$ \\
\hline \multirow[t]{2}{*}{$\begin{array}{l}\text { Stage 5: } \\
\text { Review and } \\
\text { Publication }\end{array}$} & $\begin{array}{l}\text { The Seventh week: } \\
\text { 28[Sat] April } 2001\end{array}$ & $\begin{array}{l}\text { Reviewing and Discussion } \\
\text { [Reflection, self-reflection, \& } \\
\text { reflexivity] } \\
\text { [Observed video-recorded data } \\
\text { of drama] }\end{array}$ & $\begin{array}{l}\text { Saturday Research meeting, } \\
\text { Observations, Interviews } \\
\text { Post-Questionnaire, discussions, } \\
\text { Reflection Notes, and the final } \\
\text { report }\end{array}$ \\
\hline & $\begin{array}{l}\text { The Eighth week: } \\
\text { 4[Fri] May } 2001\end{array}$ & $\begin{array}{l}\text { Publication: the booklet, } \\
\text { whose title '꼬부랑 모부림,' } \\
\text { (meaning of 'Struggle for } \\
\text { Learning English'). }\end{array}$ & $\begin{array}{l}\text { The last Research meeting, } \\
\text { Report (publication) } \\
\text { (Final evaluation report). }\end{array}$ \\
\hline $\begin{array}{l}\text { Stage 6: } \\
\text { Completion }\end{array}$ & $\begin{array}{l}\text { After the main } \\
\text { fieldwork }\end{array}$ & $\begin{array}{l}\text { To Re-visit the school and to } \\
\text { keep contact with some } \\
\text { participants }\end{array}$ & $\begin{array}{l}\text { Interview the principal and } \\
\text { teachers }\end{array}$ \\
\hline
\end{tabular}


Some of these triangular approaches were part of a double dual structure at the one level of both PAR and SPAR and at the other level of both a series of drama workshops in the real world and 'drama within drama' in the drama world.

Let me take an example of interview method. Students as researchers used the method of interviews when they collected data from audiences who had watched their performance and on the other hand, I as the researcher employed this interview method in collecting data about what teachers observed during drama workshops. Thus the method of interviews was used to collect data from the same perspective in terms of observers. Nevertheless, it was conducted at two different levels-at the micro level of EDIE project 2001 by students who wore their mantle of the expert as researchers and at the macro level of BGCHS project 2001 by me as the researcher. The former was collected from the workshop in the context of 'drama within drama'; the latter, from the project in the BGCHS project.

This double dual structure is thus again taken into account by ensuring that data should be managed efficiently in collection, and then we may consider how to refine the nature of the data and to arrange them. The structure of data collection led us to determine by whom the data were collected, as well as from whom data collectors got their data. With regard to this, I shall present how I directed these techniques in data collection at two different levels, the micro and the macro.

\subsubsection{The Macro Level: Researcher in PAR (the BGCHS project)}

\section{Data Collected from the Researcher Myself}

Field diary. The field diary began as a private diary, but became combined with a research diary.

- First of all, what I collected as data was recorded in the field diary, which was followed with my principles mentioned in 4.5.1.1, and served as the basic repository of my operations throughout this study.

- Secondly, this diary was not just used to keep records of collecting data about the action, but also it was used to interpret the data I had collected, to evaluate what I had done, to re-plan what I would do, and re-confirm what I had to do for the next action.

- Thirdly, I used this diary in recording my thoughts and feelings about the daily events of this study.

- Fourthly, it was convenient for me to make a time-line of this study with this diary because of its own characteristics-daily recordings.

- Fifthly and finally, this diary was used as raw data for this study 
because this functioned as the basic repository of my operations throughout this study.

The foremost of those principles was that I kept the diary on the basis of describing as I saw and heard rather than discussing, in the sense that the former is the more objective way to collect data than the latter is, although writing has the advantage of stimulating thought.

Observation: As soon as possible, after or during the drama workshops, I made audiotape records of my observations in my voice recorder, which recorded what I said more effectively than the sporadic written notes. Discussions with the assistant teacher as an observer were also recorded in this way. Later, when I returned home that evening, I translated them into a more permanent and detailed record of what I observed and heard in my field diary.

\section{Data Collected from Student Participants by the Researcher}

Analytic Surveying Questionnaire and Post-Questionnaire: The former questionnaire was used in order to survey the context in which students had been; learning EIL and their intercultural sensitivity. This questionnaire was made up of mostly closed questions so that the respondents might not skip certain items. I expected them not to like to make long sentences in such a questionnaire.

The latter questionnaire was used instead of interviews, which had been planned to have with students after the study, in order not to bother their preparation for the midterm examination in the school. This questionnaire had items to explore any changes of intercultural sensitivity how drama was considered after the EDIE project. Some items in the questionnaire were closed questions; others, open questions.

Interviews: One of the key methods in data collection was the personal interview. This method was done with a face-to-face personal interview at the beginning of this research project and the interviews were semi-structured-partially structured depending on factors concerning learning environments. All semi-structured interviews with student researchers were audio taped, meanwhile non-schedule-structured interviews with them were written down in my field diary.

Self-Questioning forms: This form was designed to draw out information about what they detected between their thoughts and their actions while they were participating in drama sessions, to probe into situations and to evaluate the manner and emotions in 
relation to those interactions.

Therefore, this technique was inclined to explore psychological factors of student participants. This Self-Questioning form, as a function of obtaining information about their inner dialogues, encourages them to clarify ideas and explain reasons behind their interactions. Student researchers were asked to fill in these forms immediately after the drama sessions so as not to forget what they had thought during those sessions.

Personal Journals: I asked student participants to write their personal journals in the middle and at the end of the study in order to get information about their assessment of the project by making them evaluate their activities.

Saturday research meetings (audio-recordings): We had research meetings on Saturdays after school. At these meetings we discussed what we had done in the last week, how we were going to change our original plan if we should, and what we were going to do in the next week. All research meetings on Saturdays were audiotape recorded. Throughout the meetings, all participants were asked to speak into small microphone in order to reinforce them in their role as co-researcher.

Reflection notes: Student participants were asked to make their short notes at the last session. The notes provided each participant with chances to reflect on their activities, what they had done, and what they thought of the others during the study.

Drama workshops (Video-recordings, audio-recordings and photographs): All drama workshops were videotape recorded by two video cameras: one camera covered drama activities overall; meanwhile, the other was focussed on specific activities.

Also, some crucial moments were photographed with a normal camera as well as a Polaroid camera by participants as well as me. The activity of which participants took photographs provided them with advantages of developing their literacy in terms of making meanings through drama. Especially photographs taken with the Polaroid camera were useful to discuss their drama activities immediately because drama itself has its own characteristics of immediacy and dynamics.

\section{Data Collected from Observers and Others by the Researcher}

Observation: One assistant teacher was asked to make observation records throughout the drama workshops and her observation form was open, although its focus was on intercultural interactions of participants through drama, whereas another 
teacher sometimes was given her observation list, whose form was structured, as shown in Appendix C. This teacher returned this observation list to me shortly after drama sessions.

Discussion and Interview. After drama workshops, observers and I discussed the dally drama activity of participants and I took notes in my field diary. Also, I wrote down interviews in an informal way with observers, who temporarily visited to encourage our study.

\subsubsection{The Micro Level: Students in PAR (the EDIE Project)}

Most of these data were used in data analysis by student researchers as well as by the researcher. This means the researcher re-analysed these data, which student researchers collected and analysed, that is, made second data analyses at the macro level.

\section{Data Collected from Students as Researchers}

As I mentioned in the foregoing section, student researchers were involved in collecting the following data—Self-Questioning form, Personal Journals, Saturday research meetings (audio-recordings), Reflection notes, and drama workshops (Videorecordings, audio-recordings and photographs). Data collected from these methods were shared with us, student researchers and $I$ as the researcher in the analysis stage in our own ways. On the other hand, a booklet was published with the student researchers' perspective at the end of the project. In order to make this booklet, we collected first drafts for publishing a booklet at the end of this research project or interviewed various people, for instance, other, student researchers, observers, audiences and non-participants (a teacher and parents), and me as the researcher.

\section{Data Collected from Students as Participants}

Students as participants were also engaged in the following data-Self-Questioning form, Personal Journals, Reflection notes and Drama workshops Nideo-recordings, audio-recordings and photographs), because the function of student participants overlapped with that of students as researchers.

\section{Data Collected from Non-Participants by Student Researchers}

Interviews: Student researchers used the face-to-face interview. Some student researchers had interviews with non-participants such as non-participant teachers and 
non-participant students in the school to get information about what they thought of drama education in English. These interviews were made on and off depending on the situations of interviewers and interviewees in Stage Three and Stage Four, which I presented in Table 4.3.

On the other hand, in Stage Five, other student researchers had interviews with members of the audience about their public performance of War Paint. These were audiotape recorded by those student interviewers.

What we used as the methods of data collection is thus part of the dual structure of this study. The triangulation of data collection structure also has its duality. This dual triangulation of data collection in this study can be summarised as Table 4.4 shows:

In the next stage, all audiotape-recorded data, which we collected through the drama workshops, interviews with student participants, interviews with audiences, and Saturday research meetings, were transcribed, as I wanted to use some parts of original audio-taped recordings.

Table 4.4 Dual Triangulation of Data Collection

\begin{tabular}{|c|c|c|c|c|}
\hline Data Collection & Researcher & \multicolumn{2}{|c|}{ Participants (students) } & $\begin{array}{l}\text { Observers } 8 \\
\text { Others }\end{array}$ \\
\hline $\begin{array}{l}\text { Project } \\
\text { type at the } \\
\text { Macro level: } \\
\text { PAR } \\
\text { Teacher- } \\
\text { researcher }\end{array}$ & $\begin{array}{l}\text { Fieldwork Diary } \\
\text { Discussion } \\
\text { Saturday } \\
\text { Research } \\
\text { me日tings } \\
\text { (Audiotape } \\
\text { recorded) } \\
\text { Booklet }\end{array}$ & \multicolumn{2}{|c|}{$\begin{array}{l}\text { Questionnaires } \\
\text { Interviews (Audio-taped) } \\
\text { Personal Journals } \\
\text { Self-Questioning } \\
\text { Seturday research meetings } \\
\text { (Audiotape recorded) } \\
\text { Reflection notes } \\
\text { Drama sessions } \\
\text { Videotape recorded, } \\
\text { Audiotape recorded \& } \\
\text { Photographs) }\end{array}$} & $\begin{array}{l}\text { Observation } \\
\text { Discussion } \\
\text { Interviews } \\
\text { Booklet }\end{array}$ \\
\hline \multirow{2}{*}{$\begin{array}{l}\text { Workshop type } \\
\text { at the Micro } \\
\text { Level: } \\
\text { SPAR } \\
\text { Student- } \\
\text { researchers }\end{array}$} & \multirow{2}{*}{$\begin{array}{l}\text { made secondary } \\
\text { analysis of all } \\
\text { data which } \\
\text { students as } \\
\text { researchers } \\
\text { had collected }\end{array}$} & $\begin{array}{l}\text { Student } \\
\text { researchers }\end{array}$ & $\begin{array}{l}\text { Student } \\
\text { participants }\end{array}$ & \multirow{2}{*}{$\begin{array}{l}\text { Interviews: } \\
\text { Audiences } \\
\text { (Audiotape } \\
\text { recorded) \& } \\
\text { Non- } \\
\text { participants }\end{array}$} \\
\hline & & $\begin{array}{l}\text { Personal } \\
\text { Journals } \\
\text { Self- } \\
\text { Questioning } \\
\text { Discussion } \\
\text { Saturday } \\
\text { research } \\
\text { meetings } \\
\text { (Audiotape } \\
\text { recorded) } \\
\text { Booklet }\end{array}$ & $\begin{array}{l}\text { Interviews } \\
\text { Reflection- } \\
\text { notes }\end{array}$ & \\
\hline $\begin{array}{l}\text { Italicised data v } \\
\text { The above met } \\
\text { each other. All }\end{array}$ & $\begin{array}{l}\text { overlapped in } \\
\text { is of collecting o }\end{array}$ & $\begin{array}{l}5 \text { of data sour } \\
\text { vere used in }\end{array}$ & $\begin{array}{l}\text { at both levels } \\
\text { bination with }\end{array}$ & \\
\hline
\end{tabular}


The drama sessions, as I mentioned in 4.5.2, were videotape-recorded with two video cameras. At the beginning of the drama workshops, students felt rather uncomfortable, but as the workshops went on, they did not care about the situation in which they were framed.

These videotape-recordings aided me in distancing from my experience and provided me with a means to check what we did and to reflect on what I did especially in terms of recalling aspects of my experience as a drama teacher as well as a conductor of this study.

As I have mentioned, this section has focussed on how we managed to triangulate data in the dual structure based on PAR and SPAR. This triangulation was in terms of the types of data source.

\subsubsection{Triangular Data in the Study}

The foregoing sections have made clear how I designed this research study, how I made the instrumentation for that, and how I triangulated in terms of data collection from different sources, e.g. interviews and/or documents. This is related to the question of trustworthiness which will be discussed in 4.7 .

The purpose of what I did in terms of empirical work is to enhance confirmability and dependability, of the study. According to Lincoln and Guba (1985), the former is concerned with reference to believable researches, wherein data and findings from those data are more credible in terms of building theories with the empirical meaningfulness by using different sources, different methods, and multiple investigators. Dependability is more concerned with accuracy or predictability of findings from data collection and analysis by avoiding any careless act in assessment process, instrumental decay, ambiguities and so on. With a view to accomplishing this object, triangular techniques of data collection contributed to the trustworthiness in my study (Cohen et al. 2000). Types of triangulations, which I described in the previous sections, can be summarised as follows:

\section{Tripodic Data Collection: Methodological Trianqulation}

The first triangulation described here is related to the design of the study. As I mentioned in Chapter 3, drama does not just function at linear level and not only at horizontal level. Drama is dynamic at its vertical level, too (Beckerman 1970). Also, the data I collected from drama activity are not static, but ethnographic data. Therefore, 
I believe drama should be explored in consideration of its dynamics. On this ground, my methodology was chosen and modified from three factors:

- PAR: students, as co-researchers and informants, are involved in the EDIE project at the micro level, for instance, by planning and evaluating their research actions in Saturday research meetings as well as in drama sessions and by collecting and analysing data through interviewing participants as well as non-participants (teachers, friends or parents);

- grounded theory enables participants to obtain values from their experience and practice, to improve their self-critical attitude by observing interactions on intercultural phenomena, that is the construction of data, and to develop problem-solving skills, for instance, trying to establish the EDIE club and the English Zone in order to have more opportunities to practice English skills of speaking and listening in everyday life;

- drama provides participants with its fullness, that is, affective, cognitive, and behavioural experiences, as I mentioned in 4.2. Participants kept their individual journals on their own drama activity in relation to ICM.

With respect to different methods, I collected data by different data collection modesinterview, questionnaire, observation, personal journals, SQF (Self-Questioning Form), audio and video-tape records of drama sessions, Saturday research meetings, reflection notes, photographs taken with a Polaroid camera, the booklet, field diary and so on.

\section{Triplex Data Collection: Combined Levels of Triangulation}

The second triangulation is on the grounds that the dynamics of drama is relevant to both participants in drama and the context around and inside those participants, as I mentioned 4.3. This triangular connection is based on the dynamical drama itself that has triadic relationship of, [1] the self of a participant, [2] otherness relevant to her society, and [3] participant's activity. For instance, the technique of SQF through selfquestioning was useful in exploring dynamic ICM through drama.

\section{Trilateral Data Collection: Investigator Triangulation}

The third triangulation is related to three sources to collect data from researchers (field diary, observation notes, and the booklet), participants (questionnaires, interviews, personal journal, SQF, photographs, audio and video-tape records of drama sessions, Saturday research meetings, and the booklet), and the third parties (interviews and observation comments), for instance, observers, and non-participants of students, teachers, and parents, described earlier. This tripod foundation of data collection is 
built at two levels: the $B G C H$ project at the macro level and the EDIE project at the micro level, mentioned in 4.1.1.

\section{Triform Data Collection: Form Triangulation}

The fourth and last triangulation described here, is connected to patterns of data: textual, audio, and visual data such as photographs and videotape recordings. This triangular type aimed at avoiding textualism and scriptocentrism, which I am going to discuss later. That is to say, I considered drama by its nature and collecting data through drama, that is, drama data should be collected in terms of how human beings present themselves in a certain time and space. Time and space in drama was dynamic. Furthermore, the medium by which human beings present themselves was their bodies, not written records.

What is implied from the above basic definition of drama is that:

- firstly, drama is not static but dynamic;

- secondly, drama is not horizontal but vertical;

- thirdly, drama is not single dialectical, but multiple dialogical;

- fourthly, drama is made up of not only audio images, but also visual and textual images.

Therefore I believe the study to research drama should be designed, its data should be collected and the data should be analysed in terms of an integrated or holistic approach, as was suggested by Fleming (2001) in drama education and Conquergood (2001) in drama studies. For instance, when a participant employs different modes of dramatic expression and the expression in sound, movement, verbal, and writing is seen side by side, it provides a better in-depth portrait of an individual participant than any single mode on its own. I shall have further discussion about these suggestions in relation to triangular analysis and contemporary research trends toward drama later, in 4.6.2.2.

The above-mentioned triangulations started with the basic concept of drama, developed through the dynamics of drama, and converged onto the dynamics of drama itself with Bakhtinian dialogism, mentioned in Chapter 3. And then how did I analyse these multiple triadic data? I shall answer this question in 4.6 and the relationship between triangulation, and confirmability and dependability in 4.7, respectively. 


\subsection{Data Analysis}

There are multiple perspectives and practices in the analysis of qualitative data and therefore there are a variety of techniques. These different techniques are often interconnected, overlapping and complementary. This variety and diversity in data analysis should be narrowed down to the purpose of the research and I believe the method of analysis should be integrated into the whole procedure of the research.

For me, as defined in 1.3.3.1, my purpose in this study was to investigate the effectiveness of drama for developing intercultural competence. The effectiveness of drama appears to stem from the fact that drama is able to influence feeling and thinking patterns, and furthermore to manipulate both, which are necessary prerequisites for eventual behavioural change. To analyse this effectiveness for developing ICM we could start with the following questions:

- Did participants feel different in real-life situations as a consequence of what they had done previously as a result of participating in their drama project?

- Did participants think differently about intercultural experiences from their previous experiences as a result of the project?

- Did participants behave differently in intercultural settings from what they had done?

- By what were participants influenced in the project?

- Or to what extent did they make changes as a result of the project?

These questions are relevant to whether drama can be effective, and how much drama can be effective if it is.

However, I focussed on the question of why drama can be effective, particularly, its dynamics, which can make drama effective rather than the aforementioned questions. Although I discussed the dynamics of drama education in Chapter 3, I shall take this topic further in connection with the data analysis.

My argument will be presented as follows:

- in the first part of this section, I will present how data relevant to the effectiveness of drama have been analysed by other researchers in general and what current issues can be raised in terms of approaches to data analysis of drama;

- in the second part, on what grounds I started to analyse data which 
I got from the drama project in the study.

- And then, in the third part, how I took procedures for data analysis;

- in the fourth and the final, how I managed to do this in manual ways and by using computer aided qualitative data analysis software (henceforth CAQDAS).

Most of the statements made here, aim at the emphasis on data analysis through grounded theory, following Punch (1998) that grounded theory is normally seen as an approach to data analysis in qualitative research.

\subsubsection{Drama and Its Analysis}

\subsubsection{Contemporary Trends in Data Analysis of Drama}

It is appropriate to discuss in general first the approaches to analysis of the effectiveness of drama and then to move on to how I approached drama data analysis in this study.

As pointed out earlier, the effectiveness of drama can be studied in various perspectives and its effectiveness can therefore be analysed from different angles. Their different approaches reflect the backgrounds and interests of researchers. Whilst some researchers focus on the content of drama, others have concentrated more on its form. Sometimes it appears that researchers tend toward emphasising one more than the other, although both cannot be separated from each other, but are complementary to each other.

First of all, I would like to divide research studies on effectiveness of drama since 1990s, into two categories-drama and education, and drama for education depending on their research foci. Researches in the sub-categories are related to, on the one hand, the content of drama and its form.

On the other hand, applied drama and its subcategories, the second category, is divided briefly into the field of language education, the field of the social studies including intercultural education and business management, the field of health education, and research methods (see Appendix F: Previous Researches on the Effectiveness of Drama Education). 


\subsubsection{Limitations of Previous Data Analyses of Drama}

As argued earlier, research interest in the effectiveness of drama has been widening over the last decades and it has turned out that drama is not only effective, but also research into socio-drama or ethno-drama, is expected to exert considerable influence on socio-cultural theory, and eventually on ICM theory.

There is, however, still very little research about how drama is effective in terms of its dynamics. Research remains sceptical about the range and depth of the investigations of this issue. This is because of what I referred to as general limitations of drama studies in Chapter 3, which still remain in many researches in Appendix $F$, because this research is over-dependent on textual data.

I would like to give an example of this. Dobson (1996) explored the effectiveness of drama with respect to its dynamics by investigating the way in which drama in education enables students to subject established consensus beliefs to critical examination through a process of dialogue and debate. Although his understandings in the study were developed and tested within the social domain of open, reasoned, critical debate, he limited classroom drama to its knowledge-centred manifestation and stopped further development of it as a polyphony of unfinished dialogues which open students to new and enlightened understandings.

Dobson (1996) did not show us properly what the moral dynamic was like in the study in the sense that he made only a statement that drama-in-education regains the moral dynamics lost in the solipsistic processes of its pedagogical methodologies, but made no further suggestion.

On the contrary, as mentioned in 3.3.2 and 4.3.1, Fleming (2001) provided a perspective to reduce contemporary limitations by suggesting his integrated approach to drama education. He emphasises the importance of relationship between theory and practice as an integrated approach to drama education and the significance of its sub-relationships in pairs; internal and external, in assessing drama; making and responding in progression in drama; feeling and form in working with script; meaning and logic in drama and language; expression and representation in drama and aesthetics.

This incorporated relationship as the integrated approach, I believe, can lessen contemporary limitations of research in drama, because this approach can provide 
drama researchers with, at least, a starting point using the following pairs:

- content and form;

- experience and structure;

- means and ends;

- internal and external experiences;

- making and responding;

- feeling and form;

- process and product;

- meaning and logic; and

- expression and representation (Fleming 2001).

This integrated approach can also be seen as similar to Conquergood's. Conquergood criticised contemporary limitations with a hierarchical division of labour between scholars/ researchers and artists/ practitioners. He condemned that division:

\begin{abstract}
A performance studies agenda should collapse this divide the hierarchical division] and revitalize the connections between artistic accomplishment, analysis, and articulations with communities; between practical knowledge (knowing how), propositional knowledge (knowing that), and political savvy (knowing who, when, and where). This epistemological connection between creativity, critique, and civic engagement is mutually replenishing, and pedagogically powerful [brackets mine] (Conquergood 2002: 153).
\end{abstract}

Also, he recommended this connection as the basic attitude for drama students, especially to $\mathrm{PhD}$ research students:

Very bright, talented students are attracted to programs that combine
intellectual rigor with artistic excellence that is critically engaged, where they do
not have to banish their artistic spirit in order to become a critical thinker, or
repress their intellectual self or political passion to explore their artistic side.
Particularly at the PhD level, original scholarship in culture and the arts is
enhanced, complemented, and complicated in deeply meaningful ways by the
participatory understanding and community involvement of the researcher (Ibid).

From this statement, what I should do as a drama researcher as well as a PhD candidate is, above all, to connect artistic accomplishment, analysis, and articulations with communities and become a critical thinker by participatory understanding and community involvement in researching drama studies.

From the aforementioned, I can summarise the integrated approach as being between theory and practice and the connection between artistic accomplishment, analysis, and articulations with communities in order to overcome contemporary limitations of data analysis of drama. 


\subsubsection{Approach to Drama Data Analysis in the Study}

For my case, I would like to take three critical points in researching the effectiveness of drama. Firstly, researchers overlook the effectiveness of the very nature of drama, which originates from its dynamics. Some of them seem to be more interested in using drama as a medium or a method to investigate their issues. Secondly, drama should not be explored only with written records. Thirdly, researchers must not forget a valuable factor of the effectiveness of drama-drama is dynamic, never static.

Most of my research procedures therefore aim at dynamics in drama, that is, how participants interact, communicate, and make changes through drama. This aim is based on how drama influences feeling and thinking patterns of the participants and their eventual behavioural change.

Now, the steps of data analysis will be presented in connection with GTA (grounded theory analysis) and drama, and multiple triadic approaches to data analysis of drama. After this presentation, a detailed description of my use of the computer programme for data analysis will be given in 4.6.4.2. This is because I believe an understanding of my methodological standpoint should precede an introduction to the software per se.

\subsubsection{GTA (Grounded Theory Analysis) and Drama}

\section{Relationship between GTA and Drama}

Both in 3.3.2 and in the previous 4.6.1.2, I discussed limitations of drama studies and limitations of some previous researches. On the other hand, in 4.2.3.2, I stated my choice of grounded AR as one of the main research methods in the matter of being related to [1] the connection between theory and practice, [2] emancipatory self-critical research attitude, [3] ethnographic data, and [4] participatory observation.

Bearing in mind the statements in the aforementioned subsections and my research case, I was asking myself which method of data analysis could be the most appropriate in terms of my research focus on why drama is effective. Above all, it seemed that I was going to analyse data on the basic grounds of how participants interact, communicate, and make changes through drama in the intercultural context between the individual, the self, and the society.

My final answer was found in GTA, as mentioned briefly in the introductory part of this chapter. The major reasons for selecting this method come from a body of sociological 
backgrounds in theory and identifying and developing socio-cultural theories through drama in practice.

Before moving on to the next stage of objectives and principles of GTA with drama data, I would like to give an account of the sociological backgrounds of grounded theory analysis from the following:

This approach [Grounded theory] to qualitative analysis was developed by Glaser and Strauss in the early 1960 s.... Contributing to its development were two streams of work and thought: first, the general thrust of American Pragmatism (especially the writings of John Dewey, but also those of George $\mathrm{H}$. Mead and Charles Pierce)...; second, the tradition of Chicago Sociology at the University of Chicago....[brackets mine] (Strauss 1987: 5-6).

Since Glaser and Strauss's discovery in 1967, and its application within sociological study, grounded theory has been used in many other fields.... It [grounded theory] is rooted in symbolic interactionism....'[author's italics] [brackets mine] (Cutcliffe 2000: 1476-1477).

In these brief overviews, I was interested in the words, 'discovery' and 'symbolic interactionism.' First of all, I would like to offer a definition of 'discovery.' According to Bradbury et al., 'discovery' means that 'If someone makes a discovery, they become aware of something or learn of something that they did not know about before' (Bradbury et al. 1995: 469).

And then what theory did they discover, which already existed in our human society? Here I would like to borrow a simple explanation about this from Jary and Jary:

\begin{abstract}
For symbolic interactionists, meanings 'do not reside in the object' but emerge from social processes. Emphasis is placed on the 'active,' 'interpretive,' and 'constructive capacities or competence, possessed by human actors, ... . The term was coined in 1937 by H. Blumer, who summarizes the main principles of the approach in terms of three propositions (Blumer 1969): (a) 'human beings act towards things on the basis of the meanings that things have for them'; (b) these meanings 'arise out social interaction'; (c) social action results from a 'fitting together of individual lines of action' (Jary and Jary 2000: 622-623).
\end{abstract}

Thus, it seems to me that Glaser and Strauss discovered self-awareness through social interaction or social dialogue, that is, triadic structure between the self, the mind, and the society. This sort of socio-cultural interaction or social dialogue already was discussed in relation to drama in Chapter 3. In this perspective, this theory has common characteristics with the triadic structure in drama actions. Furthermore, this commonness is related to the integrated approach to drama education suggested by Fleming (2001), which emphasises action, reflection, and self-reflection. 


\title{
Objectives of GTA with Drama Data
}

As stated previously, grounded theory appears to be generally accepted as an approach to data analysis in qualitative research. Therefore, it is defined as a research strategy rather than as a research design (Denzin and Lincoln 1994; Punch 1998). Furthermore, Strauss himself, who is one of the discoverers of the theory, explained this:

\begin{abstract}
The methodological thrust of the grounded theory approach to qualitative data is toward the development of theory, without any particular commitment to specific kinds of data, lines of research, or theoretical interests. So, it is not really a specific method or technique. Rather, it is a style of doing qualitative analysis that includes a number of distinct features (Strauss 1987: 5).
\end{abstract}

In the above statement, Strauss defined grounded theory, as a data analysis style, neither a research method or technique nor a research design in the sense that 'Grounded theories, because they are drawn from data, are likely to offer insight, enhance understanding, and provide a meaningful guide to action' (Strauss and Corbin 1998: 12).

Therefore, I can summarise grounded theory as an action style of qualitative data analysis.

Before describing how I used GTA, it is proper to precede this by saying that grounded AR is modified in order to overcome the limitations of PAR, as I showed by choosing it as my research method in 4.2.3.2. Grounded theory, as theorists explained earlier, made each participant in our research study become a critical thinker as well as a researcher about our research study. This is because this theory provides us with a set of tools to approach analysis-insight, understanding, and a meaningful guide and therefore enable us to explore and solve complex problems in intercultural situations, and to contribute in different ways to the study.

Secondly, in 4.1.2, I pointed out that GTA provides us with a tool to analyse drama activity, which implicated its situatedness in the intercultural context, whereas in 4.1.3 and 4.2.1.1 I classified drama data as ethnographic data by quoting Swartz (1998). In this sense, analysing drama data should be comparable to analysing ethnographic data. I therefore prefer to use similar theoretical backgrounds to those of ethnography.

As I already showed at the beginnings of 4.6.2.1, the root of GTA is in symbolic interactionism (Strauss 1987; Cutcliffe 2000; Jary and Jary 2000). Prus described the 
ethnographic research tradition as related retroactively to the Chicago school of sociology:

\begin{abstract}
While George Herbert Mead (philosophy), Ellsworkth Faris, and Herbert Blumer maintained a fairly sharp and consistent focus on the social essence of human group life, the other significant figures in the development of Chicago-style ethnography were more diffuse (eclectic) in their modes of analysis (Prus 1996: 113-114).
\end{abstract}

The aforementioned similar backgrounds between GTA, ethnographic research and drama research, I believe, can make my research study more valid and reliable in the sense that three research methods, similar but different, functioned as one of triadic structure in this study.

\title{
Principles of GTA and Drama
}

GTA is expected to function as a research action style for building theories for our team. Nevertheless, we need guidance as well as the techniques and procedures in order to develop that style with critical thinking. The development of the competence for that action style, in the end, enables research studies to achieve their confirmability and dependability of data analysis. In other words, their trustworthiness is partly dependent on researchers and 'the interplay between researchers and data' is one of the variables to their researches in the sense that worth of their results is dependent on how they analyse their data (Strauss and Corbin 1998: 13).

In this sense, I intend to present the principles of ground theory analysis, which we followed in analysing drama data here. I shall overview GTA by starting with terms and concepts in GTA, which are listed on Figure 5.2 by Punch (1998: 219).

As shown in the Figure, the overall procedures of GTA include the constant comparison method. This key constant comparative method consists of two essential operations-asking questions and making comparisons (Strauss and Corbin 1998).

Our project team also made comparisons consistently through not only asking questions of us as well as other researchers as participants but also comparing research actions through drama. In these processes, sensitivity, which is defined as 'the ability to respond to the subtle nuances of, cues to, meanings in data,' was a crucial factor in data analysis (Strauss and Corbin 1998: 35).

As discussed earlier, the interplay between researchers and data had considerable 
influence on analysing data and in the end, I believe, on the trustworthiness of this study. For instance, my participants as co-researchers had never been involved in this sort of drama project in schools, nor in other educational institutions. They were really beginners to drama, to collecting data and to analysing those data through their drama actions. As a result of this situation, the participants as co-researchers could not manage to do these research strategies as systematically and confidently as I, the researcher, did. The participants however followed this key principle of constant comparison of GTA at their level.

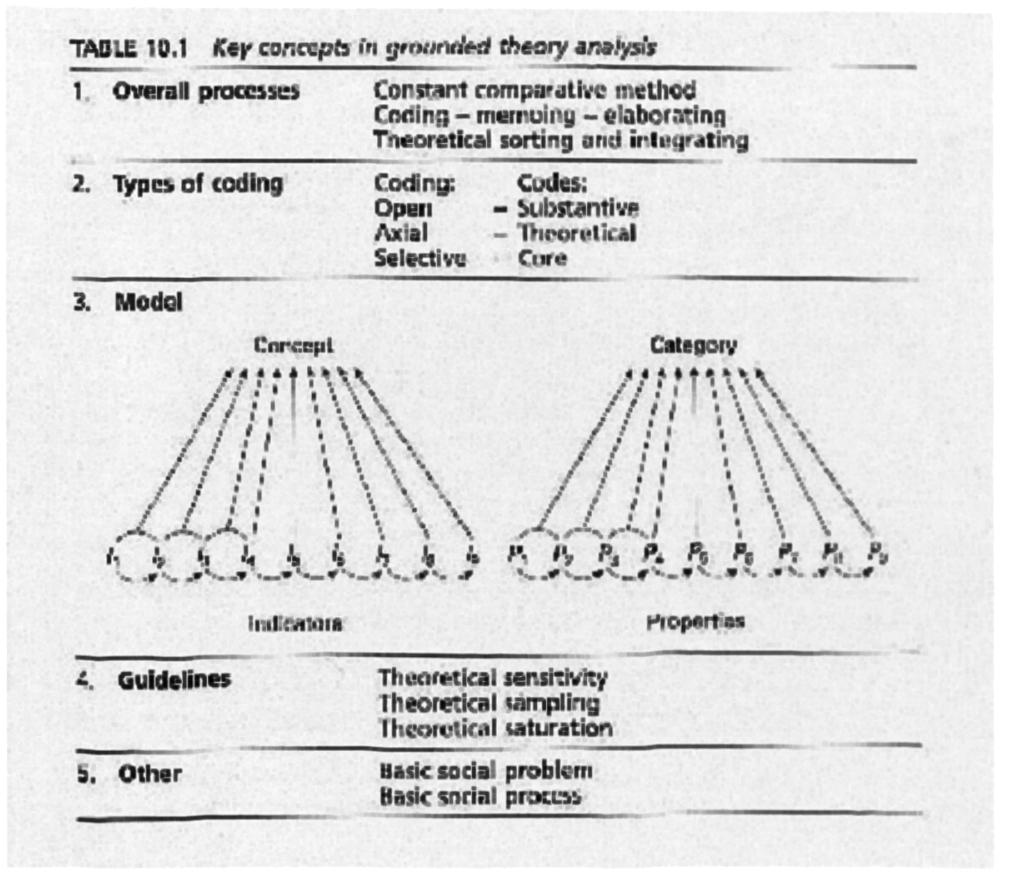

Figure 4.2 Key Concepts in GTA (grounded theory analysis)

With regard to the word we, which I am using in this section, it is being used more in relation to me, as the researcher, than the balanced meaning between student researchers and me in terms of GTA. Nevertheless, I want to keep this pronoun, we, as I defined in the introductory section of this chapter, in the sense that they still are using some concepts and skills of this analysis strategy.

Now, I shall return to the constant comparison method. This comparative method throughout our project helped us sort out and integrate theoretical meanings through the three important analytic processes in it. First of all, we carried out analytic processes through microscopic examination of drama data and in the processes we analysed the drama data line-by-line in detail. This aimed at generating initial categories at the beginning of analysis stages and suggesting relationships among 
those categories. This action, which is called coding, is the start activity and also a constant action throughout the analysis, and consequently is the central strategy.

On the other hand, I kept on making memos as a sort of written record of analysis in different types and forms. According to Glaser, memos are defined like this: 'A memo is the theorizing write-up of ideas about codes and their relationships as they strike the analyst while coding...it can be a sentence, a paragraph or a few pages...' (Glaser 1978: 83-84). Strauss and Corbin also defined memos as 'the researcher's record of analysis, thoughts, interpretations, questions, and directions for further data collection' (Strauss and Corbin 1998: 110).

Memoing thus does not follow coding actions but goes along with coding. Also, it is possible to make memos, which $I$ did, in different perspectives-substantive, theoretical, methodological and even personal.

We made three kinds of coding: open coding, axial coding and selective coding.

Each coding was treated at a different level, for instance,

- the purpose of open coding, labelling any significant indicators, was to find conceptual categories in the drama data, at a first level of abstraction;

- axial coding, theoretical relating, aimed at finding relationships between categories which were discovered through open coding; and

- the aim of selective coding, elaborating categories, was at the conceptualisation of these relationships at a higher level of abstraction.

As a result of these processes, we picked out both the central phenomenon around which other categories are integrated and the descriptive narrative about this central phenomenon. In a word, we tried to find out the core category and the story line of our drama data through GTA (Punch 1998).

However, in the matter of theoretical sensitivity, theoretical sampling, and theoretical saturation, the interplay between researchers and drama data, still remains a crucial variable. Furthermore, in terms of dealing with basic social problems and basic social process in the middle of data analysis, there were still challenges. 


\subsubsection{Multiple Triadic Approach to Data Analysis of Drama}

As said in 4.5.4, my drama data became multiple triadic because I tried to collect my data with several types of triangular structures

At this stage, I am going to emphasise Beckerman's definition of drama, introduced in 4.2.1.1 and 4.5.4.3 once more in a different perspective. The definition as 'human beings isolated in time and space' in his definition indicates a triadic dynamics of human being, time and space.

According to Beckerman, 'Units of time are, in fact, the contextual frames within which the drama evolves, and the first step in our dramaturgical analysis is to discern them in a dramatic work' (Beckerman 1970; 37). Furthermore, there are active dynamics inside those contextual frames, and therefore the drama data were contextual as well as dynamic. In a word, what I did throughout my fieldwork is contextualised collection and contextualised analysis, not isolated data.

As experienced in the pilot study, I kept on taking this contextual point of view in data collection and data analysis. I sometimes swayed between the two stages, as mentioned in 4.2. This swaying was related to sub-stages of the stages of data collection and data analysis and throughout the fieldwork, there were interaction between the sub-stages-data collection, data reduction, data display, and drawing and verifying for conclusions (Miles and Huberman 1994).

However, this 'interactive model' is integral to theory building in grounded AR because the cycle of collecting and analysing data repeats until theories are saturated with those data through the constant comparative method (Miles and Huberman 1994:12). Strauss and Corbin define theory as what 'was derived from data, systematically gathered and analysed through he research process' (Strauss and Corbin 1998: 12).

The above quotation shows that a theory is not just a set of findings. It should explain phenomena. Therefore, generating theories does not mean generating a set of findings but the development of a field of knowledge. Researchers determine which parts of a concept are valid and what new concepts can be added to the original conceptualisation. Researchers can also develop a set of concepts through stating their relationships, which constitute an integrated framework. On the basis of this meaning of theory, I shall explore my research questions. 


\title{
Formulation between Trianqulation, Limitations of Drama Research, and
} Integrated Approach to Drama

From here, I shall discuss how triangulation is relevant both to drama research which is over-dependent on text and to research which is holistic. Boring (1953), who is an early supporter of triangular strategy, argues that one operation dealing with a new construct, is a starting point; two operations provide corroboration, but multiple operations make that construct reified.

Lindlof also supported triangular techniques:

\begin{abstract}
... triangulation involves a comparative assessment of more than one form of evidence about an object of inquiry. Although it is typically a method of verification, triangulation can also be used to develop a concept, construct, or proposition (Lindlof 1995: 239).
\end{abstract}

Lindlof thus argues that the function of triangulation in analysis is comparative assessment, conceptualisation, and theoretical saturation. Triangular techniques are strategies for comparatively assessing multiple evidence about an object of inquiry, and developing that evidence into theories. Triangular analysis of my data made me reconsider the previous data collection for a particular issue and sometimes collect further evidence.

In the end, the purpose of triangulation in analysis as well as collection was to get evidence about every single issue from multiple angles and to find more trustworthy answers about each question related to the issue.

The following approaches to triangulation in analysis were taken:

\section{Tripodic Data Analysis in Methodological Triangulation}

This type is how to analyse drama. As I mentioned earlier, PAR, GTA including ATLAS.ti, and drama were applied in analysing data. For instance, we collected data of ICM, which were done as drama activity by participants in and through drama, and observed, discussed, and analysed those data in and through drama.

\section{Triplex Data Analysis at Combined Levels of Triangulation}

First of all, there is drama at the teacher research level and at the student research level. At the basic levels, data relevant to the dynamics of drama activities of participants, the context around and inside them, were analysed, as discussed in 4.3. 
This triangular type is based on dynamical drama itself that has a triadic relationship of participants, the self, and otherness in the society.

\section{Trilateral Data Analysis in Investigator Trianqulation}

This type is also related to drama between the teacher research level and the student researcher level. The type is called triangulation of sources. An item from actions of participants was analysed by three kinds of investigators-researchers, participants themselves, and the third parties (e.g. observers, non-participants of students, teachers, and parents).

As I explained in 4.5.4, the trilateral foundation of data was also analysed at two levels: one sub-structure of mine at the macro level and the other sub-structure of the students or participants at the micro level. Therefore, strictly speaking, data were collected and analysed in perspectives from six different investigators-the researcher, participants, observers at the macro level and students as researchers, students as participants, and the third party (including non-participant students, their teachers, or their parents), which was presented in Table 4.4 Dual Triangulation of Data Collection in 4.5.4.2.

\section{Triform Data Analysis in Form Triangulation}

This triangulation is related to different forms of data: textual, audio, and visual data such as photographs and videotape recordings, as discussed in 4.5.4.3.

Up to now in this section, I have explained why I committed to triangular techniques in data analysis, how I triangulated my multiple triadic data and what influences the triangulations exerted over my study. In the next, I shall present the process of analysis.

\subsubsection{General Procedures to Analyse Data}

As mentioned in 4.2 and 4.6.2.2, I sometimes swayed from data collection to data analysis in theory building. Moreover, the cycle of collecting and analysing data repeats until theories are saturated with those data through constant comparative method. Although the procedures were not sequential but interactive, in this subsection, I shall present procedures in a linear fashion in seven steps: 


\subsubsection{Step 1: Preparative Activities for Data Analysis}

As my starting point of data analysis, I recalled my research aims because my aim in this study is to answer the research questions, and to suggest some ways of solving the problems through this study. Both, especially my research questions, are the skeleton as well as the target of this study.

Therefore, it was necessary to analyse data with researcher problems and research questions in the study. Student researchers also took a similar position to mine and focused on their research questions about learning EIL and intercultural awareness through drama.

We also took into consideration how we interpret with data, as discussed in 4.6.2.1, including our objectivity and sensitivity to data analysis which are regarded as some crucial preparative activities. Objectivity enables us to be confident that our findings are a reasonable, impartial representation of a problem under investigation by achieving a certain degree of distance from our materials and representing them fairly. Sensitivity enables creativity and the discovery of new theory by responding to the nuances of, and cues to, meanings in drama data (Strauss and Corbin 1998). According to Strauss and Corbin, the researcher per se is an instrument of data analysis in qualitative studies and it is important for the researcher to maintain a balance between objectivity and sensitivity.

\subsubsection{Step 2: A Starting Point for Analysing Data}

I stated this stage of analysis by checking and analysing data which existed in the public sphere, for instance, newspapers, which had reported problems about teaching and learning EFL in schools, annual reports of the School, and documents from the Local Council of Education to the School.

Secondly, I analysed reports of research studies, which could be relevant to my findings in order to compare their findings with mine. In these two procedures, analysis included data reduction, for instance, identifying and rejecting data according to its relevance.

Thirdly, in the middle of the fieldwork, I analysed interviews, which I had audiotaperecorded at the beginning of the fieldwork. My field diary was also being analysed. Data collection and analysis overlapped at this point. Through analysing interviews and the field diary at this stage, I got some stimuli to think about properties and 
dimensions of concepts emerging from drama data to supplement following actions, and sessions.

And fourthly, I needed to transcribe the audiotape recording of the interviews, although I was going to use the original audiotape recording as well for the computer package of ATLAS.ti. Because the type of audio files occupied a huge space in my computer, parts of audiotape recordings of interviews, I had to transcribe audio-taped drama sessions and Saturday Research Meetings. Also, I had to format all the textual data for the package of ATLAS.ti.

\subsubsection{Step 3: Arrangement of My Data}

At this stage, my main activity for data analysis was to arrange data chronologically. Because time has great influence on patterning socially the nature and form of individual thought and action, sampling was going to become more specific with time. The relation of sampling to time comes from grounded theory.

In other words, the development of theory was influenced by the time factors because I had to look at action and interaction, and trace them over time in order to observe any changes.

\subsubsection{Step 4: Developing Coding Procedures}

My key activities at this step were to make codes and to categorise those codes through microscopic examination of data. This aim is to generate initial categories with their codes, properties and dimensions and to suggest relationships among categories through a combination of open coding and axial coding, as explained earlier.

These procedures led $u s$ to explore the conditions along which the properties of concepts vary by making comparisons and by making selective coding. I started with asking questions and was directed towards theoretical sampling through selective coding which contributed to the process of integrating and refining theories. Thus I gradually moved from describing events to conceptualising them. Those three forms of coding enhance the internal confirmability in the research.

\subsubsection{Step 5: Theoretical Sampling}

At this step I confirmed, extended and sharpened the theoretical framework that I got through coding and categorising. Strauss and Corbin define the purpose of theoretical 
sampling as follows:

Data gathering driven by concepts derived from the evolving theory and based on the concept of "making comparisons," whose purpose is to go to places, people, or events that will maximize opportunities to discover variations among concepts and to densify categories in terms of their properties and dimensions (Strauss and Corbin 1998: 201).

I took the following procedures for this sampling:

- Analytic questioning and comparing as a basic step for sampling, such as 'what would happened...? When? How? Or Where?

- Sampling in open coding through being open to sources that would provide the greatest opportunity for discovery;

- Relational and variational sampling in axial coding by looking for incidents that demonstrate dimensional range or variation of a concept and the relationships among concepts;

- Sampling in selective coding, that is, discriminate sampling through choosing sites purposefully to maximise or minimise differences.

\subsubsection{Step 6: Theoretical Saturation}

It is possible to know where to stop data analysis because of the concept of 'saturation.' When there was marginal improvement between raw data and theoretical sampling through conceiving concepts and formulating them into a logical, systematic, and explanatory scheme, it was possible to stop. This will be illustrated in the following chapters.

\subsubsection{Step 7: Evaluating Emergent Theories}

In this step, I compared my framework with other frameworks in the literature. Comparison with other similar frameworks provided me with the domain in which to generalise the findings of this study. I could therefore improve external confirmability; meanwhile the comparison with different frameworks enabled me to improve my definitions and consequently improve internal confirmability in my study.

\subsubsection{Management of Data Analysis}

There are multiple perspectives and practices in the analysis of qualitative data and therefore there is variety in their techniques. In the following two subsections, I shall address the limitations of manual data analysis, why I wanted to rely on CAQDAS and why I turned to the software package, ATALS.ti. 


\subsubsection{Manual Data Analysis and its Limitations}

At the very beginning of my research design, I had thought about using my own analysis cards, but $I$ expected my data to be too much to manage in the manual approach to data analysis, and take too much time.

Secondly I thought a manual method would be inadequate because, for example, it was not easy to keep track of which participants had done which activity with whom. Above all, non-textual data were difficult to manage with respect to interconnecting codes and categories and making selective codes.

\subsubsection{Using Computer Aided Qualitative Data Analysis Software (CAQDAS) and ATLAS.ti Programme}

I decided to use CAQDAS. The development of CAQDAS helped me get confident in using computer programmes, although some programmes had their limitations. There are two main purposes to use CAQDAS: One aims at facilitating the attachment of codes to the items of data; the other is to enable the researcher to make code-andretrieve approaches easier.

The main beneficial tasks can be summarised as:

- theory-based coding procedures;

- search and retrieval;

- data linking to others such as documents, codes, memos, categories, and networks (see Appendix G);

- adding analytic memos;

- graphic mapping, such as visible indicators and networks; and

- generating interim and final reports.

(Miles and Huberman 1994; Lewins 2001).

Especially by adopting ATLAS.ti (Muhr 1997) as the most appropriate programme for data analysis, I was about to analyse the data in ways appropriate to GTA and the capability for multi-modal analysis of ATLAS.ti was particularly suitable for dramaturgical data, which were textual, audio, video, and graphic. This allowed the triangulation of all types of data. However, I tried to code audio and video taped data as they were recorded through this software but I could not manage to do that except making a few codes, because the computers, which I used, were not upgraded enough. In the end, I had to rely on transcripts of audio-taped data and my observation of videotaped data. 
As discussed in 4.6.2.2, I believe I should analyse my multimedia data, which I collected through triangulation, in a triangular approach. After having considered the various forms of my qualitative data-textual, audio, video and image data, I made use of ATLAS.ti in analysing, managing, and theory building my qualitative data in this study.

\subsection{Trustworthiness: Confirmability and Dependability}

As mentioned in 3.1.3, there are three different perspectives on knowing in and through the artistic form-[1] being grounded on fact; [2] being contrary to the facts; and [3] creating a new reality from the facts rather than knowing the facts per se. This dialectical propensity made me reconsider how I could improve the trustworthiness in my study. The standpoint of these perspectives is that artistic meaning is abstracted and presented symbolically from everyday experiences, along with from literal interpretations and communicative medium through conventional language (Ball 1983).

Thus meaning-making in and through drama is involved in the three perspectivesliteral interpretations, communication through conventional language, and dramaturgical everyday life. If my findings in and through drama could not be empirically provable, then the study would be of doubtful trustworthiness.

My claims to trustworthiness in this research study come from my engagement with intercultural situations through drama, but eventually from dialogues relevant to selfreflexivity. This self-reflexivity, as mentioned in 3.2 and 4.2, focused on the interrelationship between the self, the mind, and the society of an individual participant.

What I had to consider was criteria for trustworthiness-internal validity, external validity, reliability, and objectivity (Lincoln \& Guba 1985), as discussed in 4.5.4. With a view to checking whether those criteria were met in this study, I set the following four aims, which were suggested by Lincoln and Guba: [1] Truth value for internal validity; [2] Applicability for external validity; [3] Consistency for reliability; and [4] Neutrality for objectivity. Also, I agreed to their argument that researchers can establish trustworthiness by 'reaching assessment of confirmability, dependability, and, as an optional feature, providing an external check on steps taken in relation to credibility' (Lincoln \& Guba 1985: 323).

The confirmability of my research, which I discussed in 4.5.4, in the end, is situated within the investigation of this single case study. Validation in investigating that sort of 
dialogic dynamics was challenging. Therefore, having recognised the impact that assumptions could have on the analysis and interpretation of ethnographic data through drama, I designed verification checks to try to minimise any inappropriate influence of context and assumptions. Above all, I tried to apply triangular approaches to exploring my research questions. As discussed in 4.2.2 and 4.2.3, the aims of $A R$ are to conceptualise, organise and support my study in terms of socio-cultural literacy, especially intercultural literacy. However, as my data were collected from drama activities and the data are ethnographic, I added grounded theory into AR. Therefore, my research method was modified into the combination between AR, grounded theory, and drama.

As mentioned in 4.6.2.1 and 4.6.3.1, the interplay between researchers and their data is significant for the trustworthiness of their research studies. Triangulation can produce divergent outcomes and improve confirmability and dependability, along with credibility, which is concerned with dealing with data appropriately, of the studies.

Triangulation could be applicable retrospectively to questions, which I discussed in 4.2, about drama for ethnographic research-drama as a research method, drama as a confirmability practice in intercultural situations, and drama as a medium of intercultural literacy.

The answers to those questions were found in dialogues between the self of participants and intercultural situations around them. I tried to apply drama techniques such as thought tracking, asking student researchers to fill up their Self-Questioning form after every drama session, and to write up their personal journals in the middle and in the end of their project. This triangular approach in terms of time-during drama sessions, shortly after drama sessions, and after drama sessions-provided me with different evidence on the same event.

However, it was not easy for student researchers to express what they were thinking within their selves because the process of their thinking was sometimes momentary as well as multidimensional.

Despite this difficulty, I searched for evidence valuable enough to build theories in this study through triangular approach, mentioned in 4.6.2.2 and 4.6.4.2, and dialogic process, mainly discussed in, 4.4.1, 4.5.1, and 4.6.3, respectively. It was here that ATLAS.ti was used to analyse my data. 
Furthermore, ATLAS.ti, enabled me to make codes of audiotape and videotaperecorded data as well as transcriptions. This function was essential for analysing drama data.

But the researcher should use the original data, audio and videotaped recordings rather than the transcribed data, because the original context of the data should be delivered with only minimum change. With regard to this, I tried to analyse the very crucial parts of photographs, audio and videotape-recorded data per se, rather than transcribed data.

This triangular analysis through ATLAS.ti allows an integrated approach or holistic research in drama research. Also it leads researchers to reduce the contemporary limitations in those areas, which I mentioned in 4.6.1.2.

\subsection{Summary: Metadrama, SPAR, and GTA}

In this chapter, I described in detail the underpinnings of my methodology. On the basis of this theoretical background, I have explained my research design in relation to triangular approach-participatory action research (PAR) and students in participatory action research (SPAR), grounded theory, and drama.

This triangular research design was adapted to the single case study, which I chose for my thesis. The procedures of data collection and analysis, in my study, have been described in terms of multiple triangular approaches to data. With a view to managing those procedures more systematically and more efficiently than I could do in my manual way, I have described the use of a computer package, ATLAS.ti.

Also, I mentioned that this package produced the possibility to analyse my data in a triangular approach and enhanced the speed and the rigour of data analysis. In the next two chapters, I shall analyse what I collected from the data and present what I found through these procedures.

In summary the important points of research methods in the study are the characteristics of the data-ICM through drama. As I discussed in Chapters 3 and 4, drama provides its fullness in exploring ICM and is a holistic approach to ICM and ICP. I managed to collect and analyse drama data in multiple triangulation in data collection as well as data analysis: 
- methodological triangulation (SPAR, GTA, and drama),

- combined levels of triangulation (macro and micro levels with metadrama, that is, 'play within a play'),

- investigator triangulation (researchers, participants themselves, and the third parties, for instance, observers, non-participants of students, teachers, and parents), and

- form triangulation (textual, audio, and visual data ATLAS.t).

In short, with respect to drama per se and the research limitations, the fullness and the holistic approach, which are based on drama by its nature, I investigated my research questions in multiple triangular ways in order to overcome the research limitations, of other researchers who have placed over-emphasis on textualism and scriptocentrism in their research. 


\section{Chapter 5.}

\section{Findings A: Drama Experiences in ICM (Intercultural Communication)}

The purpose of this chapter is first, to narrate what emerged from the data, in terms of what participants had experienced through participation in an event for intercultural communication in drama. Second, I shall narrate which experiences helped participants to do the particular activities for this communication.

There are many different analyses of experiences of drama, for instance, as discussed in Chapter 3-[1] a holistic/integrated approach; [2] artistic approach; [3] learning medium approach; [4] socio-cultural approach; [5] therapeutic approach; and [6] research methodological approach. $I_{1}$ however, shall unfold what experience participants had in doing drama in terms of three approaches: artistic, therapeutic, and socio-cultural.

Here are two main reasons why I intend to concentrate on these three rather than all of the six approaches. This is first because the three approaches are based on the nature of drama per se and second because I believe these approaches not only provide me with essential factors for investigating how drama is effective in developing intercultural competence, but also are the inevitable pathways to the other approaches.

In the first section, I shall start with the artistic experience of participants, which is based on the essence of drama as an art form, in which expressing feelings and emotions are included as its core.

In the second section, I shall describe therapeutic experience-how to release the feelings and emotions which participants felt and to handle them through drama in intercultural communication.

In the third section, I shall illustrate socio-cultural/intercultural experiences through drama. At this stage, I shall aim to discuss how to individualise their feelings and emotions in the drama world, to think about what they have done in the drama world, and to express what they have thought about their everyday life. 
The discourse about the aforementioned three experiences will show that drama's particular characteristics provided participants with practices for better intercultural communication. This will be my focus throughout this chapter and the next two.

\subsection{Artistic Experience in ICM: Drama as an Art Form}

First of all, my starting point of the narration is to posit drama as an art form, in which expressing feelings and emotions are included as its core. I shall therefore concentrate in this section on how participants experienced the process of expressing their feelings and emotions in ICM during their drama sessions.

My concern was with their artistic experiences, which are firmly grounded in their activities in doing drama and would have great influence on the next stages of this fieldwork. I analysed data recorded on audio and videotapes and my field diary related to the first drama session.

In the present section, I shall present what emerged in relation to how participants handled their feelings and emotions. As mentioned earlier this kind of artistic experience was, I believe, a springboard for better intercultural communication. My initial focus in this section is on how participants related their feelings and emotions to drama as an art form and then what they did with them while they were doing drama activities in intercultural situations.

\subsubsection{Expressing One's Own Emotions in Doing Drama: Knowing Oneself}

As mentioned in 3.2, Collingwood (1938) argued that 'The artistic experience, or artistic activity, is the experience of expressing one's emotions; and that which expresses them is the total imaginative activity....' (Collingwood 1938: 275).

How did my participants express their feelings and emotions in doing drama in the light of the aforementioned definition? The ideal way for the participants to express feelings and emotions is to do so appropriately. According to Aristotle, we can become angry easily. However, it is never easy to be angry with the right person, to the right degree, at the right time, for the right purpose, and in the right way (Aristotle, The Nicomachean Ethics cited in Goleman 1996).

While analysing my data, I realised that some participants had difficulties in expressing their own feelings and emotions. Either they restrained themselves too 
much in expressing feelings, or they were poor at expressing them. In the second drama session, we used a poem of the impression of a newcomer, written about the first day when the newcomer was introduced to his new classmates. Participants were asked to make still-images and to speak out in simple words as a group activity after reading the poem.

I shall take three examples of this-KimHJ, KimJA, and KimMS [Hereafter the names of participants will be italicised]. They filled up the Self-Questioning form about their doing drama in the first week for this as follows:

What have I thought but I couldn't do today during the above situation? I was planning to give the newcomer my feeling against her, for example, acting disliking her.

Why did I think this was the most difficult thing to act? That was because I should express everything in English [ltalics mine to indicate questions; SelfQuestioning form of KimHJ on 16 March 2001].

What have I thought but I couldn't do today during the above situation? I have thought about bullying the newcomer by shouting at and kicking her.

Why did I think this was the most difficult thing to act? That was because my poor English made me lose self-confidence in expressing something in English because of my poor English [Self-Questioning form of KimJA on 17 March 2001].

What have I thought but I couldn't do today during the above situation? I have wondered if I could do well enough to express what I was feeling and thinking.

Why did I think this was the most difficult thing to act? That was because I am an introvert and this is the first time in my life to do drama. In fact, I was so shocked to hear that we should do drama in English [Self-Questioning form of KimMS on 17 March 2001].

As the above examples show, KimHJ, KimJA, and KimMS had less self-confidence in doing drama in English, because of their poor English competence, personality and naive performance. At the beginning of this project, the difficulty in doing drama in English did not make just these three participants hesitate expressing their feelings and emotions. Most participants were embarrassed to do drama in English and avoided speaking out in drama as well as in English. That was not at all easy for all of my participants, for they had low self-confidence in speaking in English.

What made things worse, some Korean philosophies, which teach participants not to express their feelings and emotions facially, or force them to pay respect to people senior to themselves, sometimes impeded their drama activities. This relation between the expression and socio-cultural backgrounds will be illustrated in $\mathbf{5 . 3}$. 
As the drama project moved on to its next stages, every participant put herself into the position of feelings and emotions and acting them, and these activities eventually enabled her to express them. At the beginning of her expression, she was conscious of having a feeling or an emotion, but not conscious of what this feeling or emotion was and how to deal with that. Most of the participants filled in their Self-Questioning form even in the middle of the project like this: "We did feel something, but we didn't know what we felt. So, it is very difficult for us to complete this form. Would you explain us how to deal with it?" [At the second Saturday Meeting on 24 March 2001].

However, these expressive activities of her own speaking and acting through drama, got related to her consciousness, and, eventually, led her to become conscious of what the feeling and emotion was. Furthermore, this activity helped participants become aware of the way, in which they felt the emotion, and of relations with how they dealt with the consciousness of the feeling and emotion.

Thus, the more participants were engaged in their drama sessions, the better they handled their feelings and emotions. Let me take the comments of KimMH and LimEY, which were made in the Self-Questioning form at the early stage, and at the second last research meeting, on 21 April 2001, held immediately, in Korean, after the public performance of the very short play, War Paint (Walker 1977b). The following is translated from comments which participants gave at the meeting.

\footnotetext{
How did I feel about myself as a person who relates to others? I didn't get used to that situation yet.

What have I done today during drama sessions in practice in the above question? I felt very awkward.

What have I thought but I couldn't do in the above situation? Oh, no, that was not me at all, but I would just try to do that.

Why did you think that was the most difficult thing to resolve or to act? That was because I was not able to express that and didn't have proper emotion for that.

How could I act in that situation if I were one of the people who come from England or America? I seemed to act that situation more or less in a realistic way.

What do I want to do in a similar situation to that in the future? I thought, "Let's be in full activity."
}

[Self-Questioning form of KimMH on 16 March 2001]

KimMH: I think I have more self-confidence than before, although I have had an unsatisfied feeling for what I have been doing throughout the project.

The researcher. What do you mean by that?

KimMH: When speaking out about something in English, I was ill at ease and my action was handled clumsily. Now I feel more self-confident and I can do my action with less awkwardness. 
[Comments of KimMH at the Second Last Research Meeting on 21 April 2001]

What have I done today during drama sessions in practice in the above question? I put something over the newcomer and was unkind to her.

What have I thought but I couldn't do in the above situation? I wished I had more lines and acted in a realistic way, but I couldn't do that.

Why did I think that was the most difficult thing to make resolution? That was because I still felt too shy to take my role properly.

How could I act in that situation if I were one of the people who come from England or America? I seemed to act that situation without bashfulness.

[Self-Questioning form of LimEY on 16 March 2001]

How did I feel about myself as a person who relates to others? I would like to play with other characters.

What have I done today during drama sessions in practice in the above question? I danced with them and enjoyed that.

What have I thought but I couldn't do in the above situation? I wished I had danced better and made more lines in that situation. And I would like to memorise every line of mine.

Why did I think that was the most difficult thing to resolve or to act our? That was because I had not enough time to practice that.

How could I act in that situation if I were one of the people who come from England or America? I seemed to be very interested in dancing and acting in that situation.

What do I want to do in a similar situation to that in the future? I wish I did behave myself in my age, not pretend to be an adult.

[Self-Questioning form of LimEY on 21 April 2001]

As shown from the above, each participant experienced changes and more relaxation to express her feelings and emotions throughout the drama project. Participants were not stuck in the middle of a clumsy and reluctant way of describing their feelings and emotions. They became able to disclose these gradually through their bodies. Also, they could individualise feelings and emotions by referring to some situations around those feelings and emotions, as KimMH and LimEY self-evaluated what they had done through their drama project as illustrated in the above.

Through expressing feelings and emotions which participants felt, they were conscious of what their feeling and emotions were. This consciousness led them to handling feelings and emotions, which are individual or socio-cultural/intercultural, and to know themselves through handling this well enough to achieve effective communication, interpersonal or intercultural.

In the end, the main aim of drama is expressing feelings and emotions appropriately. This aim, however, is not only for drama, but also for interpersonal interaction in 
everyday life and better intercultural communication. As mentioned in 2.2, this is because feelings and emotions can disrupt interpersonal communication and intercultural interaction, or they can on the contrary enrich them. Therefore, it is crucial to be able to handle our feelings and emotions for communicative competence.

\subsubsection{The Total Imaginative Activity in Doing Drama: Making Oneself}

The main aim of drama mentioned in the previous subsection, however, led participants to involvement at another level in handling their feelings and emotions: this can be described as the "making" of the participants themselves as well as knowing themselves through expressing feelings and emotions.

I shall show how participants made themselves through expressing their feelings and emotions from their dialogues with themselves-in their Self-Questioning form on 28 March 2001. These dialogues were related to what participants, who are Koreans, acted about the intercultural situation, in which a young American couple were dating in the park on a very sunny day.

What have I thought but I couldn't do today during the above situation? I had several ideas about how I could act to express their dating, but I couldn't act them.

Why did I think this was the most difficult thing to resolve or to act? That was because I was so awkward and even ashamed to act as I have thought out.

What do I want to do in a similar situation to that in the future? I prefer to act in our own cultural way-a little bit be shy. I am proud of our culture, although I cannot express that sort of cultural factors very openly [Self-Questioning form of KimJA: bolds mine].

What have I thought but I couldn't do today during the above situation? I was planning to play as a tricky girl.

Why did I think this was the most difficult thing to resolve or to act? That was because I have no boyfriend and I don't know what the girl was going to play with her boyfriend. But I imagined and acted making a show of my putting on a perfume and telling a lie [Self-Questioning form of KimEL: bolds mine].

What have I thought but I couldn't do today during the above situation? I was going to act an over-caring guy for his girlfriend.

Why did I think this was the most difficult thing to resolve or to act? That was because I hated that kind of behaviour. However, I tried to do that a little bit because I supposed the guy to do that. That strange acting gave me gooseflesh in the arms, in fact... [Self-Questioning form of MunJM: bolds mine].

According to the above cases, each participant took three stages in order to act as the character or its partner: 
- Stage 1-participants got the feel of something awful in the situation;

- Stage 2-they made their maps of the situation clearer by talking to themselves or other participants;

- Stage 3-through thinking, imagining, or supposing what the situation would be like and how the couple acted in America.

This was helped by the use of all memories, which they had had until that moment, as their cultural backgrounds. Even though they had never been to America and were not accustomed to this situation, each participant got some idea of how she could act in this situation, that is, she made or wrote the scene in which her thoughts or intercultural imagination started with her feelings and emotions about the situation.

With regard to these activities throughout the drama project, LimEY made comments at the final stage of the project as follows:

\footnotetext{
LimEY: We have experienced what we had never done before this drama project, haven't we? For instance, acting, make-up for our public performance and so on. I have tried to do what I could do as much as possible and have really enjoyed all what I have done. This is because I have wondered when I can get this sort of chance again. If you had one more month to do this kind of drama project, we could, I think, do drama as professional theatre companies do it. [At the Second Lest Research Meeting, on 21 April 2001]
}

LimEY took participation in the project more deeply by getting engaged in drama activities and passing through various drama situations. These continuous drama activities provided her with more chances to release her shyness and to express her feelings and emotions in whatever situations she got involved during drama sessions.

If we compare this comment about her shyness at the beginning, which I mentioned earlier on her Self-Questioning form of LimEY on 16 March 2001-that was because I still felt too shy to take my role properly-with her self-confidence in drama now, great progress can be seen. Starting as a shy participant in classroom drama, she now felt confident enough to imagine herself taking part in a professional drama or theatre. Thus, I can say LimEY had a very powerful artistic experience to express her feelings and emotions appropriately and to get her competence for drama and theatre.

These artistic experiences starting in personal pathos with feelings and emotions and ending in personal ethos with the total imaginative activity through drama experiences can be summarised as shown in Network 5.1: Sensation through Sensibility into Sensitivity through drama experience. 


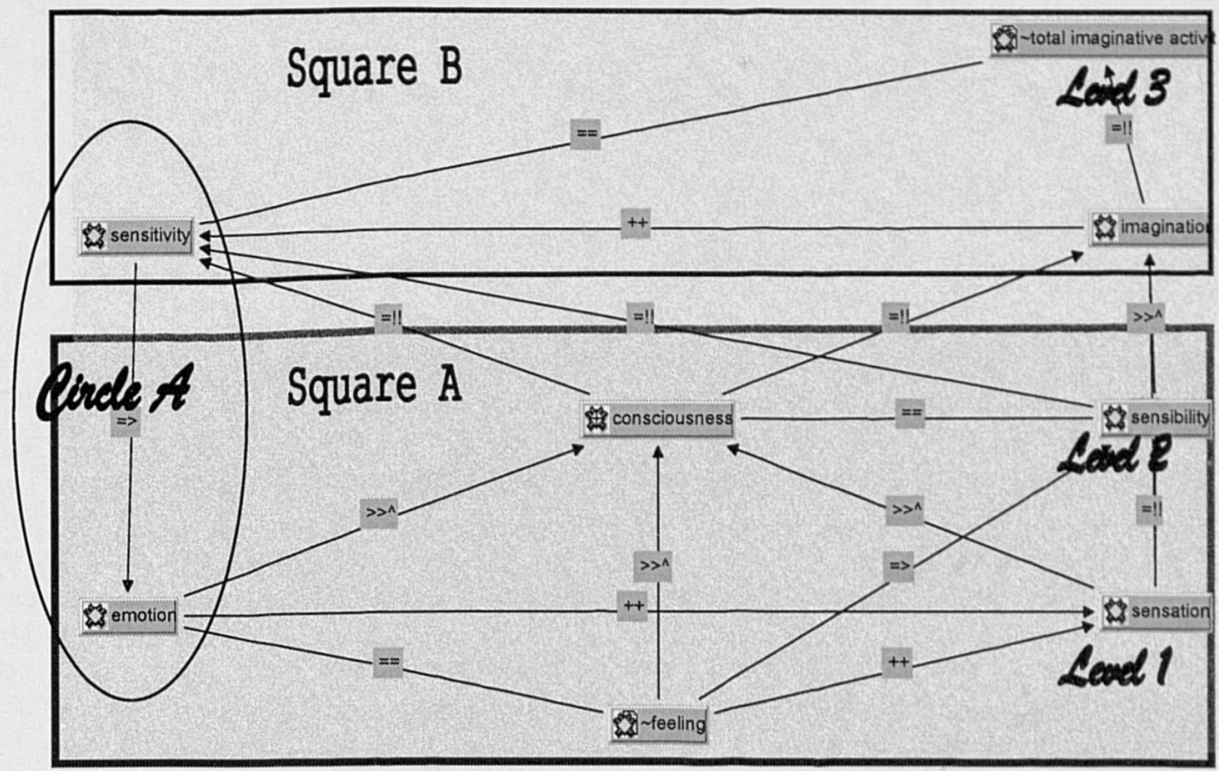

Network 5.1 Feeling through Consciousness into Total Imaginative Activity (Artistic Experience through Drama) by the Semantic Layout

In the process of making or writing the scene, there was the involvement of what factors each participant chose for the situation. Her choice was involved at three levels:

- the first is to sense her feeling and emotion in that situation using her own background, pathetic and emotional, from which she got its feel;

- the second to speak out or think out the feeling she got; and

- the third and final, to make or write her own scene through imagining how she wanted to act the situation.

In other words, each participant developed her feelings and emotions as a starting point at the pathos level via her own previous conscious act to her total imaginative activity at the ethos level through drama. As shown in Network 5.1, each arrow indicates the relation between the two connected concepts. Emotion is associated with feeling [==]. Emotion and feeling get benefit from sensation [++], in other words, sensation, or body feeling with a specific body condition, produces feeling and emotion as a mental condition.

All of emotion, feeling, and sensation react to consciousness [ $>\wedge$ ]. Sensation resolves sensibility [=!!]. Feeling generates sensibility [ $=>$ ], and so on (see Appendix G: How to Read Networks of ATLAS. (t). 
The first step was taken at the level of her sensation-the psychical sensuous level, at which the participant got feelings and emotion. At this level, the participant senses pathos in the relevant situation. Discussed in 3.2.1, pathos is a quality, as of an experience or a work of art that arouses feelings of pity, sympathy, tenderness, or sorrow and the quality of emotions, traits, or experiences, which are personal, restricted, and evanescent, in the participant's character.

Therefore what the participant senses at the pathos level is transitory and idiosyncratic dispositions or feelings as distinguished from those which are universal and deepseated in character-opposed to ethos. Because of transitory and idiosyncratic feelings, which have a physiological or temperamental peculiarity, sometimes participants said they realised they felt something but they did not know exactly what that was at the beginning stage.

The second step was taken at the level of her sensibility-the conscious level, at which she got to know to what she was feeling, indicated at the top of Square $A$ in Network 5.1. Through this conscious level, each participant was engaged in their ethos, opposed to pathos, and developed into the third level. Ethos, discussed in 3.2.1, is the disposition, character, or fundamental values peculiar to a specific person, people, culture, or movement, and also is defined as the spirit which not only actuates manners and customs but also, the characteristic tone or genius of an institution or social organization.

Therefore, the third step was taken at the level of her sensitivity-the total imaginative level, at which she had imaginative and artistic experience. This level, which is indicated as Square B in Network 5.1, does not just provide participants with the level of knowing themselves by expressing feelings and emotions but also leads them into the level of making themselves by engaging thinking and imagining from the feelings and emotions.

The sensitivity of each participant had influenced her sensation in the psychical sense and the process from sensation to sensitivity is repeated as shown in Circle $A$ of Network 5.1. This relation, however, will be made clearer with other factors, dialectical, dialogical and spiral, in Chapter 6.

Now, I can summarise that artistic experience through intercultural drama is related to exploring sensibility. Through this, participants have intercultural experience to gain from sensation into sensitivity during their drama activities, as discussed in 3.2. We perceived the continuous links of feeling and emotion, which is sensory experience, 
with sensibility and then with sensitivity in doing drama. Also, we practised doing drama activities with this contiguous relationship of sensation, sensibility and sensitivity. This is because the practice, in the end, can enhance an act of intercultural imagination, which is the participant's ability to reconstruct from pathos to ethos in her own consciousness the ideas, expressed by all signals including words she perceives.

\subsection{Therapeutic Experience in ICM: Drama as a Reflexive Tool}

In the previous section, I drew from participants' experiences that there is a continuum in the process of expressing their feelings and emotions from sensation into sensitivity, which is relevant to the interrelationship between individual pathos and individual ethos in artistic experience.

Moreover, at the level of sensitivity participants have engaged in the total imaginative activity based on what they perceived, which, in the end, is associated with individual ethos. From my analysing the Self-Questioning form and audio and videotaped drama sessions, I realised that another ingredient helped participants to have drama experience throughout their project-drama is therapeutic: individual pathos and ethos are related to therapeutic experience through drama.

\subsubsection{Drama is Emancipatory}

In the process of engaging in the total imaginative activity-knowing and making participants themselves-participants expressed their feelings and emotions which they had experienced or never had before but wanted to experience.

How was it possible for the participants to express feelings and emotions that they wanted to have, from individual pathos to ethos, through drama? The answer is so simple-starts with the broad Magic If $^{\prime}$ and then focuses on the specific situation of the here-and-now.

Let me take an example from the drama sessions-Passport Game, which was done on 28 March 2001 and on which feedback came in the next session. In this game, participants made groups of five and each group established a new country, its own language, their own passports in the new language, and its cultural factors such as costumes. At this stage, I asked all participants in each group to have the same nationality and same culture because I was afraid at this beginning stage they could 
be confused with concepts relevant to different identities-ethnic, national, cultural and so on.

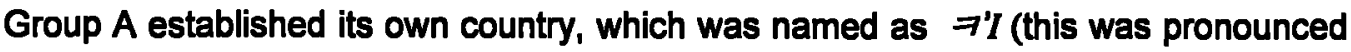
as [gi]); whereas Group B and Group C chose Spain and China, respectively. Although they were asked to make up their own new nation, they preferred these nations. However, other ingredients of their passports were made up. For example, they created a new mark, 00 , which was made up of two round shapes with different sizes and indicated a female person. KimMH invented this mark. When asked what she meant by that mark and why she wanted to use that mark instead of $F$, internationally used, she answered that the mark came from the image that a woman should have a baby and was always carrying that baby. Her explanation gave me a piece of strong evidence of a heavy emphasis on childbirth and fostering for a woman in Korean cultural values.

One more example, Group B filled up '000,' which consisted of three zeros and whose meaning was forever, in the cell of expiry date. This symbol will be referred to in relation to their socio-cultural experience in 5.3.

After they had established their nations, languages, cultural factors through making up their own passports, I gave them another situation-the moment of taking immigration procedures as travellers to the other countries, not returning to their own country. While passing through the procedures, each group did situational drama.

After this activity, they discussed what they went through, for instance, their feelings, their attitudes, and their ideas about the procedures, in which they took roles of immigrant, tourists and immigration officers. Further presentation about this discussion will be in my discussion of the sensibility of participants in the next chapter.

As shown in this Passport Game, without any limitations on time, space, objects and people, participants were opposed to the real time, space, objects, and people. As a consequence, they could be involved in any situation and were able to express what they had never experienced or they wanted to experience, or to release what they desired to speak out by taking 'as if and then getting the hero-and-now situations through drama.

Stanislavski named this limitless situation the 'Magic If (Moore 1965, The Stanislavski System: 27). And, this sort of aesthetic space, the here-and-now, provided whoever 
participates in drama with the context that fiction is pure reality, and reality is fiction' and they meet an emancipatory factor which is therapeutic in that space (Boal 1995: 20).

In this process of liberating their feelings and emotions through memory and imagination in the aesthetic space, they were concerned about how to appropriately express feelings and emotions, which they were conscious of, that is, they were involved in their total imaginative activities, mentioned in 5.1. Therefore, I can say this emancipatory experience is an element of the total imaginative activities which participants did in doing drama.

\subsubsection{Drama is Self-Reflexive}

The plasticity of the here-and-now through the Magic If' enabled participants not only to have emancipatory experiences in doing drama but also to have dreams and to make those dreams concrete and in the end to have self-reflexive experiences toward the total imaginative activities. This factor eventually contributed to searching for selfidentity, which is defined as 'the self-concept; the self as reflexively understood, as a continuing project' (Jary and Jary 2000: 544). This relation between self-reflexive drama and self-identity will be dealt with in Chapter 6 . I am going to focus the present subsection on the concept of self-reflexive drama which emerged from my data.

It appears that although emancipatory experience and self-reflexive experience through drama overlapped with each other, the former experience is more deeply involved in the stage of knowing oneself, whereas the latter experience is involved in the stage of making of oneself, mentioned in the previous section on artistic experience through drama. Emancipatory experience is related to being aware of oneself by liberating feelings and emotions from oneself, whereas self-reflexive experience is to be creative of oneself by reflecting on oneself, on what feelings and emotions one has liberated from oneself in the situation in and through drama, and making of oneself someone who is not the same as one has been.

While analysing the case of KimMS, which gave me many pieces of strong evidence for this sort of self-reflexive experience through drama, I reconfirmed that whoever participates in drama can have self-reflexive experiences through drama, whether those experiences come to them in the middle of or after the drama activity.

In her pre-questionnaire, which was filled up at the pre-stage of this drama project, on 13 March 2001, KimMS described herself as an introvert. Moreover, her Self- 
Questioning form, which asked about her second drama session on 16 March 2001, was filled up as follows:

Q A: First of all, I will write down one thing: what action do I want to talk about more to myself among today's drama activities? My classmates were surrounding me and assailing me with blows.

Q B: What do I think is the situation related to? What relationship did I have to others? That seems to be related to being bullied and I felt so frightened.

Q C: How did I feel about myself as a person who relates to others? I felt so terrified but I couldn't accept that situation very seriously.

Q D: What have I done today during drama sessions in practice in the above question? I couldn't express that feeling properly.

Q E: What have I thought but I couldn't do today in the above situation? I wished I would do well, I have wondered if I could do well enough to express what I was feeling and thinking and I asked myself whether I could express my feelings well.

Q F: Why did I think that was the most difficult thing to act or to resolve? That was because I am an introvert and this is the first time in my life to do drama.

Q G: How could I act in that situation if I were one of the people who come from England or America? I could do drama more naturally and seriously.

Q H: What do I want to do in a similar situation to that in the future? I will carry out what role I take and express my feelings and ideas in actions, not keep them inside my mind and head [From Self-Questioning form of KimMS on 16 March 2001].

By filling up the above Self-Questioning form, through which KimMS had a conversation between herself out of role and her character in role, she took three stages of being self-reflexive. In the first stage of distancing, relevant to Questions $A$, $B, C$, and D, she got distant from herself as well as her character, reviewed and thought critically what she had done during today's drama session, and then understood her drama activity from an objective perspective.

In the second stage of negation, she took a negative perspective on herself as well as her activities as shown in answers to Questions $E$ and $F$. In the answer of Question $E_{\text {, }}$ she thought her lack of self-confidence was negated doing drama activities; whereas in the answer to Question F, she evaluated her introvert personality and her inexperience in drama which caused her to lose her drama competence.

In the third stage of reflexivity, she described how disharmonic situations between what she wanted to do, what she has done and what she could not do were dealt with, as shown in answers to Questions $G$ and $H$. In the answer of Question $G$, she reflected what she has done on other people, who had Western cultural backgrounds; meanwhile Question $\mathrm{H}$ covered considering her activities and expressing how to act as the character with her own perspective. Through these two questions, she mirrored 
to others and herself what she has done. This action took on a self-reflexive aspect, which provided her with a continuing task to understand herself reflexively. Her continuing actions with this self-reflexivity, in the end, became self-awareness and self-identity. I shall present what I found in relation to self-reflexivity throughout this drama project in a deeper analytic perspective in Chapter 6.

Her continuing actions relevant to the self-reflexivity were kept going throughout the drama project as the following shows: How could I act in that situation if I were one of the people who come from England or
America? I could control my feelings and emotions properly to concentrate on
my acting.
What do I want to do in a similar situation to that in the future? I am going to do my
best for my roles and do better acting-out. And I will control my emotions and
act well [Self-Questioning form of KimMS on 21 March 2001].

How could I act in that situation if I were one of the people who come from England or America? I could give my voice clearly and strongly to relevant situations.

What do I want to do in a similar situation to that in the future? I am going to listen to Mum and keep my promises. I will be the best daughter for her, indeed [Self-Questioning form of KimMS on 23 March 2001].

How could I act in that situation if I were one of the people who come from England or America? I could express my feelings and emotions in a proper way.

What do I want to do in a similar situation to that in the future? I hope I can adopt more positive attitude if I have this sort of opportunities again [SelfQuestioning form of KimMS on 21 April 2001].

Her main concern was the change necessary to express effectively what she felt and thought, and sufficiently to be in a full activity. In a word, she hoped to become an extrovert person rather than to remain an introvert person.

Eventually, she had changed enough for all participants to agree unanimously about the radical changes. They commented on her reflection notes as well at the second last research meeting shortly after the public performance on 21 April. I would like to show what they did at the meeting.

LimEY: I have been very VERY surprised to see how much KimMS had changed. Throughout this drama project she seems to have changed enormously. She has changed a lot, indeed. And RyuKW was absent from many drama sessions because of her club activities, whereas, in fact, KimMS was never absent from any drama sessions and any Saturday research meetings. If RyuKW had attended every drama activities, she also could have changed more than she has, too.

All participants: Right, right! Yes, that's really true. (Everybody applauds to show hor approval for LimEY's comments.) 
The Researcher: The next, KimMS?

RyuKW: You have really a lot of things to say, don't you?

All participants: (encourage KimMS to say something)

LimEY: She is delighted to call herself the Nickname, the BIZARRE and IRON girl, or No. 28.

KimJA: She prepared for properties, for instance, a beer can, costume, and so on.

All participants: (Everybody applauds to show her approval for KimJA's comments.)

The assistant teacher. Why do you call her Big Senior?

KimJA: At the beginning of this project, she was really quiet ....

All participants: The atmosphere! What an apathetic and unfeeling girl she looked like!

LimEY: Now? Totally differentl

KimMH: At the beginning she didn't do as she does, did she? She looked very threatening to us, indeed.

KimJA: She looked controlling over all of us.

OhHJ: at that time, she said; only "I'm happy."

The researcher. How about now?

All participants: No, no, no... (all of them burst out laughing).

LimEY: She said, "Let's love, shall we?"

ParkJM: She has expressed something in English and....

She has become talkative and made some jokes.

Most of the participants: (Keep talking about episodes relevant to the changed behaviour of KimMS).

From the above comments, I found three main changes of KimMS's behaviour: being positive, being emancipatory in terms of emotions and ideas, and speaking in English.

The changes are what KimMS said she wanted to change. In self-questioning at the very beginning of this project, as mentioned earlier, she thought she had difficulties in her lack of self-confidence in not being able to express feelings and emotions, in her very introvert personality, and her inexperience in drama in Korean as well as in English. At the end of the project, she had changed a lot, as shown in the above comments, and also had been very proud of herself, about what she had done throughout the drama project.

In relation to her therapeutic experience through drama, I can illustrate its relevant factors as Network 5.2 shows. 


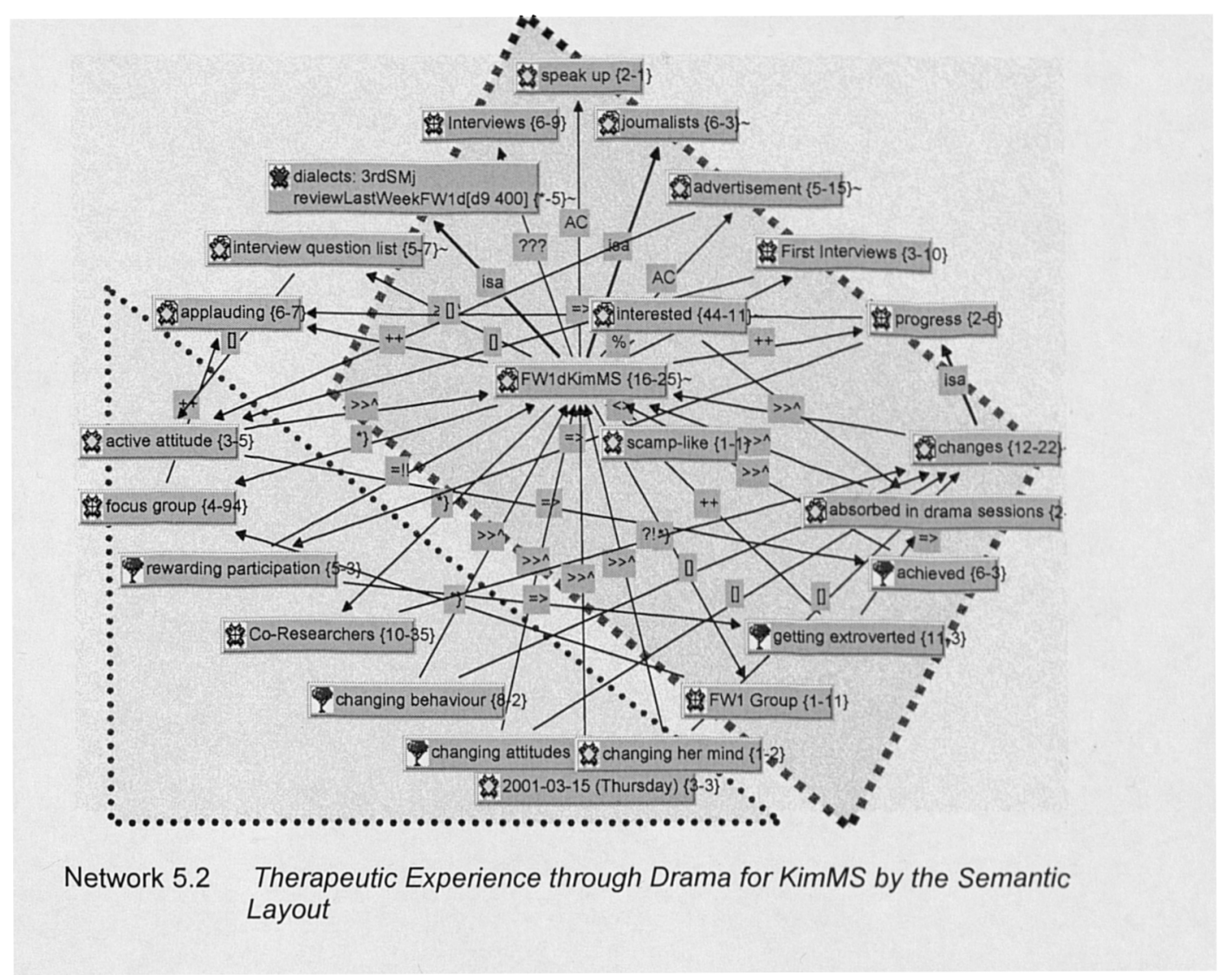

First of all, factors in the triangular area, her readiness or motivation for the EDIE project-15 March 2001, changing her mind, changing attitudes, changing behaviour, active attitude, co-researchers and so on. Meanwhile what is shown in the square, outside the triangular area indicates what she was becoming in the middle and did by the end of the project. The above network drew my attention to two concepts-'being interested,' which came from her own eagerness for EDIE, and 'scamp-like,' that is, first impression which participants were given from KimMS, closer to her than any other in the semantic dimension.

Therefore, I can say that KimMS was engaged in the EDIE project as co-researcher, and as a result of her involvement, she had therapeutic experience of releasing her pathos and ethos and of reflecting herself inside and outside herself through drama, that is, by thinking seriously to express considered thoughts about not only what she had wanted in her mind and how she presented what she had wanted, but also what she thought about her own presentation itself and the relation between her own presentation and her perception of the presentation.

I believe that drama activities throughout the project thus provided participants with therapeutic experience considerably as well as artistic experience, which I mentioned 
in the previous section, although not every participant had that sort of experience as much and powerfully as KimMS has.

Participants thus had artistic and therapeutic experiences through drama and these experiences helped the participants get to know themselves and to develop themselves from their pathos into engaging in the total imaginative activity, which establishes their ethos.

\subsection{Socio-Cultural Experience in ICM: Drama as Cultural Morphology}

There was, however, another experience through drama, which is as crucial as the aforementioned two experiences, artistic and therapeutic. That experience is with regard to the perspective of drama as cultural morphology, which is socio-cultural experience through drama.

The socio-cultural/ intercultural experience of an individual is not only concerned with the individual level of pathos and ethos, but also is imbued with the sociocultural/intercultural pathos and ethos, that is, at the individual and collective levels.

Mentioned in 5.1, the total imaginative activity is the participant's ability to reconstruct in her own consciousness the idea expressed by all signals including words she perceives from others as well as herself in doing drama. In the process of engaging in the total imaginative activity, that is, knowing herself and making herself, her own socio-cultural backgrounds were deeply involved, because drama is a cultural morphology, in the sense that drama per se has cultural ingredients in the matter of its form and structure, as well as being an art form.

In 5.3.1, I shall pick up the first point, how participants dealt with what they perceived from others as well as themselves. In 5.3.2, I will deal with relations between the experiences in doing drama and their everyday life.

\subsubsection{Drama to Individualise in a Whole Socio-Cultural Context}

As mentioned in the previous two sections, expressing feelings and emotions was not easy for all of my participants, for they had seldom had any previous opportunities to do drama in their classes. This kind of drama project was the first time for all of them. 
This difficulty, however, did not come only from their naivety in doıng drama but a so from their socio-cultural backgrounds.

I stated in 5.1.2 that in this process of making or writing the scene, the choice of each participant was made in three steps: recalling her memory about relevant factors to the situation from her whole background; thinking or imagining new ideas from that memory; and making her own scene proper for the situation.

Let me return to the example of the dating situations. All participants got the same intercultural situation, with which Korean participants were going to deal as an American couple. Participants focussed this situation on different behaviours. One focused her feeling on the shamefulness of dating itself. Another concentrated on the behaviour of the girl who attracted attention from her boyfriend, and the other, on the "disgusting attitude" of an over-caring person for his girlfriend.

Nevertheless, they had a common idea of the situation that sexuality seemed to be shameful and a taboo subject, although they made their scenes differently. This kind of evidence, where they were in the same situation and had similar feelings but they made the acting different and individual, was not shown only in this situation, but throughout the drama project.

Let me take another piece of evidence. In our public performance, we chose two participants, $\mathrm{OhHJ}$ and $\mathrm{KimHJ}$ to act a daughter role, Angie-double casting for that character, in order for all of the participants to have chances to act in the performance. Self-Questioning of both participants indicated what they have done with that character, as compared in Table 5.1.

In the table, there are cultural clashes between Korean culture, in which two Korean participants were doing drama, and English culture, in which the play was written about a generation conflict between Mum and her daughter in modern English society by an English playwright, David Walker. While having developed their character during rehearsals and the public performance, each participant, OhHJ and KimHJ, has individualised the same intercultural situation within their own socio-cultural boundary. OhHJ focused her activity on acting-out itself-how could she empathise with the character in order to act well as one of the main characters in the play? Meanwhile KimHJ, focused on the context itself-how could she solve this sort of difficult human relationship? The one was nearer to drama as an art form and the other, to drama as cultural configuration. 
Table 5.1 Comparison of Individualising the Situation between OhHJ and KimHJDouble Casting

\begin{tabular}{|c|c|c|}
\hline Self-Questioning & OhHJ as Angie & $\mathrm{KimHJ}$ as Angie \\
\hline $\begin{array}{l}\text { First of all, I will write down } \\
\text { one thing: what action I want } \\
\text { to talk about more to myself } \\
\text { among today's drama } \\
\text { activities? }\end{array}$ & $\begin{array}{l}\text { Empathising part of having } \\
\text { conversation with two } \\
\text { policemen }\end{array}$ & $\begin{array}{l}\text { The first scene at which I ran } \\
\text { out of my room. I, as Angie, } \\
\text { could have solved the } \\
\text { problem through having } \\
\text { conversation with Mum. }\end{array}$ \\
\hline $\begin{array}{l}\text { What relationship did I have } \\
\text { to others? }\end{array}$ & $\begin{array}{l}\text { Team members of this } \\
\text { project out of role and } \\
\text { policemen and citizens in } \\
\text { role in the play }\end{array}$ & $\begin{array}{l}\text { Mum, Dad, two policemen, } \\
\text { and manager in the } \\
\text { discothèque }\end{array}$ \\
\hline $\begin{array}{l}\text { How did I feel about myself } \\
\text { as a person who relates to } \\
\text { others? }\end{array}$ & $\begin{array}{l}\text { Not empathising well } \\
\text { enough and being poor at } \\
\text { expressing feelings }\end{array}$ & $\begin{array}{l}\text { Not expressing feelings well } \\
\text { enough. }\end{array}$ \\
\hline $\begin{array}{l}\text { What have I done today } \\
\text { during drama sessions in } \\
\text { practice in the above } \\
\text { question? }\end{array}$ & $\begin{array}{l}\text { Speaking my lines in a low } \\
\text { voice }\end{array}$ & $\begin{array}{l}\text { Making many mistakes } \\
\text { because I couldn't remember } \\
\text { my lines. }\end{array}$ \\
\hline $\begin{array}{l}\text { What have I thought but I } \\
\text { couldn't do today during the } \\
\text { above situation? }\end{array}$ & $\begin{array}{l}\text { Trying to cry out? To jump } \\
\text { about? To drink up? To rush } \\
\text { out into the street? }\end{array}$ & $\begin{array}{l}\text { I was very sad to have to } \\
\text { take the character, Angie, a } \\
\text { bad daughter for her Mum. I } \\
\text { really hope to be a good } \\
\text { daughter. }\end{array}$ \\
\hline $\begin{array}{l}\text { Why did I think this was the } \\
\text { most difficult thing to resolve } \\
\text { or to act? }\end{array}$ & $\begin{array}{l}\text { Emphasising without proper } \\
\text { consideration }\end{array}$ & $\begin{array}{l}\text { That was because the } \\
\text { character, a bad daughter, } \\
\text { didn't match me, who I think } \\
\text { is really a good daughter to } \\
\text { my Mum, in reality. }\end{array}$ \\
\hline $\begin{array}{l}\text { How could I act in that } \\
\text { situation if I were one of the } \\
\text { people who come from } \\
\text { England or America? }\end{array}$ & $\begin{array}{l}\text { Americans could express } \\
\text { their feelings variously and } \\
\text { positively because English is } \\
\text { their mother tongue. }\end{array}$ & $\begin{array}{l}\text { Probably, they could be } \\
\text { same. }\end{array}$ \\
\hline $\begin{array}{l}\text { What do I want to do in a } \\
\text { similar situation to that in the } \\
\text { future? }\end{array}$ & $\begin{array}{l}\text { Well, they could do similar } \\
\text { behaviours to everybody in } \\
\text { the situation in terms of } \\
\text { being conservative and } \\
\text { doing what they want to do, } \\
\text { although each has different } \\
\text { response to it. }\end{array}$ & $\begin{array}{l}\text { I would like to try to solve } \\
\text { that kind of problem through } \\
\text { dialogues with the relevant } \\
\text { person. }\end{array}$ \\
\hline
\end{tabular}

Main common ideas between $\mathrm{OhHJ}$ and $\mathrm{KimHJ}$ are [1] expressing their feelings, [2] empathising with the character, and [3] difficulties in speaking their lines in English from the data of Self-Questioning form of the two participants on 21 April 2001. KimHJ was very self-reflexive in terms of the daughter-mother relationship and focused her role on its problem-solving, meanwhile OhHJ was more interested in acting in English rather than in its socio-cultural/intercultural context. 
Both of them, however, have common ideas from the cultural clashes between cultures, Korean and English: [1] expressing their feelings, [2] empathising with the character, and [3] difficulties in speaking their lines in English, as shown in Table 5.1.

These ideas came from their own socio-cultural backgrounds. The first common idea, is that it is somewhat difficult for Koreans to express their feelings through their bodies because of their traditional philosophies. For the second, they had less self-confidence in putting themselves into the role of an English girl because they had never been abroad. And for the third, they were afraid of speaking English. Most of the participants said that they had some phobia about learning English, although they realised their language competence in English was important as an international language (from interviewing with participants at the first week of the drama project).

As emerged in the examples, each participant, who was a Korean girl, individualised differently the same intercultural situation which each of them perceived depending on her own sensation, sensibility, and sensitivity to the situation-how she felt it, was conscious of it, and imagined it within her socio-cultural context.

Now, I intend to discuss further the influences of Korean philosophies on drama activities of participants. I said in 5.1.1 that some Korean philosophies, which taught participants not to express their feelings and emotions facially, or forced them to pay respect to people senior to themselves, sometimes impeded their drama activities. Let me analyse more the dating situation, which I already discussed in 5.1.2, by focusing on individualising in the situation in intercultural perspective, in particular, from the cases of KimJA, as shown in Network 5.3:

As indicated, at Level 1, Sensation of the network, KimJA felt something strange and produced an embarrassed smile on the dating situation psychically at her sensuous level [Level 1: Sensation]. Moreover, she put the situation into the here-and-now through the 'Magic If.' Because of this, she liberated her feelings about dating in that context by gesturing and speaking [being emancipatory]. Then she realised that she had feelings of shame for dating as a Korean teenager [Level 2: Sensibility]. So far, she had taken the stage of knowing herself with respect to feelings and emotions. This emerges from her own intercultural pathos, which she got from the cultural clash between two different cultures.

However, after this she was taking the stage of making herself with respect to feelings and emotions. She considered that situation was not in Korea, but in America and the couple, who were dating, were young Americans. Then she recalled all her memory 


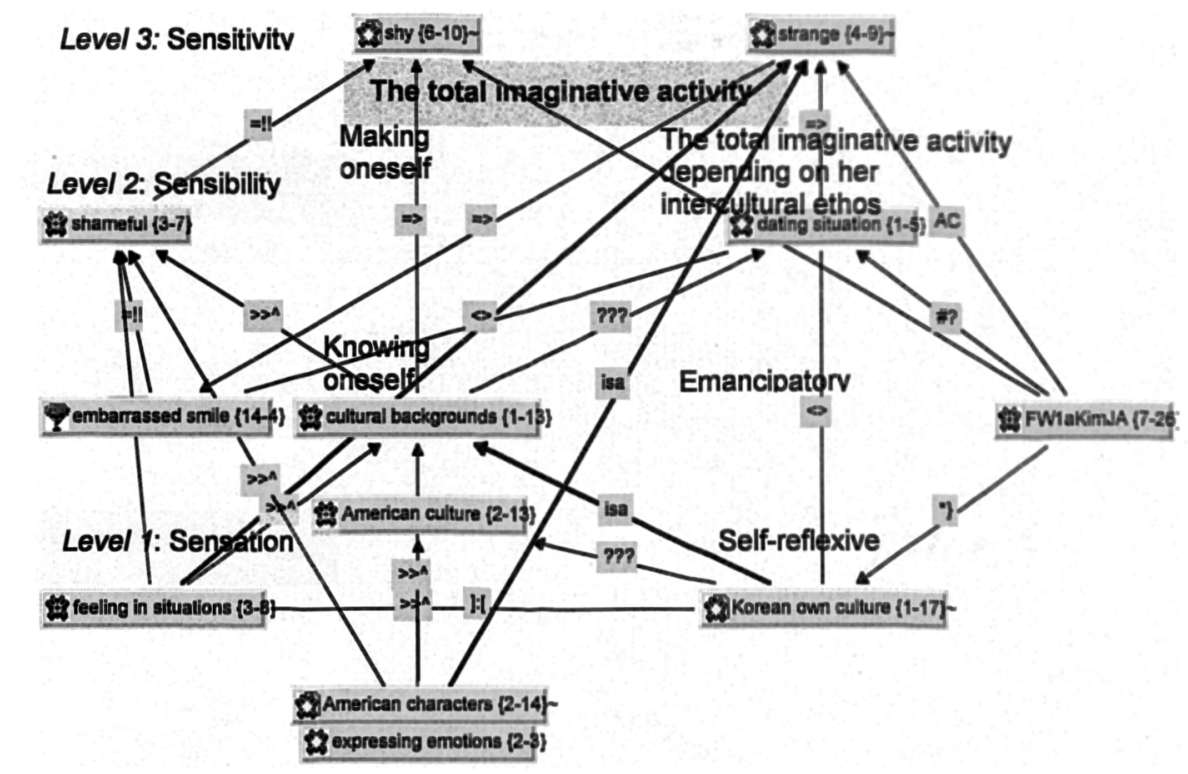

Network 5.3 KimJA's Individualising the Dating Situation in Intercultural Context by the Semantic Layout

about young Americans dating-this means she thought the situation in relation to other cultural factors to express considered thoughts. After taking ideas relevant to the situation into consideration, she made her acting, that is, characterised the American young girl.

Nevertheless, she could not carry on as she planned, because that action seemed to her to be still shameful, even though the character belonged to American culture. This means she thought about the situation in relation to her own Korean cultural standards and expressed considered thoughts about the situation which was produced through her consciousness, self-reflexive from her inside, wherein she perceived American cultural values as a Korean, to her outside, wherein she perceived American cultural values as Korean but in American perspective, that is to say, from the Korean actor to the American character, and vice versa, as well as from her outside in Korean perspective within American perspective to Korean perspective [from the Korean actor taking the role of the American character to the Korean actor].

Therefore, she decided to act as she felt comfortable and acted well enough to express her feelings about that situation-being shy [Level 3: Sensitivity; her total imaginative activity on the dating situation]. In other words, her own intercultural ethos, which could be accepted within her Korean socio-cultural/intercultural values, didn't cope with the dating situation of her American character because she couldn't accept that sort of public behaviour. 
I present her context that Korean cultural elements were more influenced by her action on the dating situation in which the Korean actor and the American character were engaged, in a linear list, as shown in Network 5.4.

However, what is more considered here is that American cultural elements, along with Korean culture, were also influenced by her action. This means she didn't reject American cultural elements but accepted them and related American cultural values to her action. In other words, she changed her attitude from an ethnocentric stage to an ethnorelative stage.

The relations positioned from the upper left to the lower right and a node with the least dependencies is made the first node positioned in the upper left corner, and the node with the most dependencies on other nodes is positioned in the lower right corner of the Network (ATLAS.ti manual 1997).

As emerged in the Network, the American character, which KimJA acted by expressing emotions and feelings at Level 1, Sensation, itself, was indicated as the least dependent on individualising the situation. Meanwhile her being shy at Level 3 , Sensitivity of the total imaginative activity emerged as the most dependent on her individualisation. This means her Korean socio-cultural values influenced the individualisation rather than the American socio-cultural values of the character did. In other words, the high sensitivity of participants in intercultural experience through drama depends on how the participant feels in a certain situation, is aware of what she feels, and how she reconstructs her ideas from her own socio-cultural backgroundsher intercultural ethos.

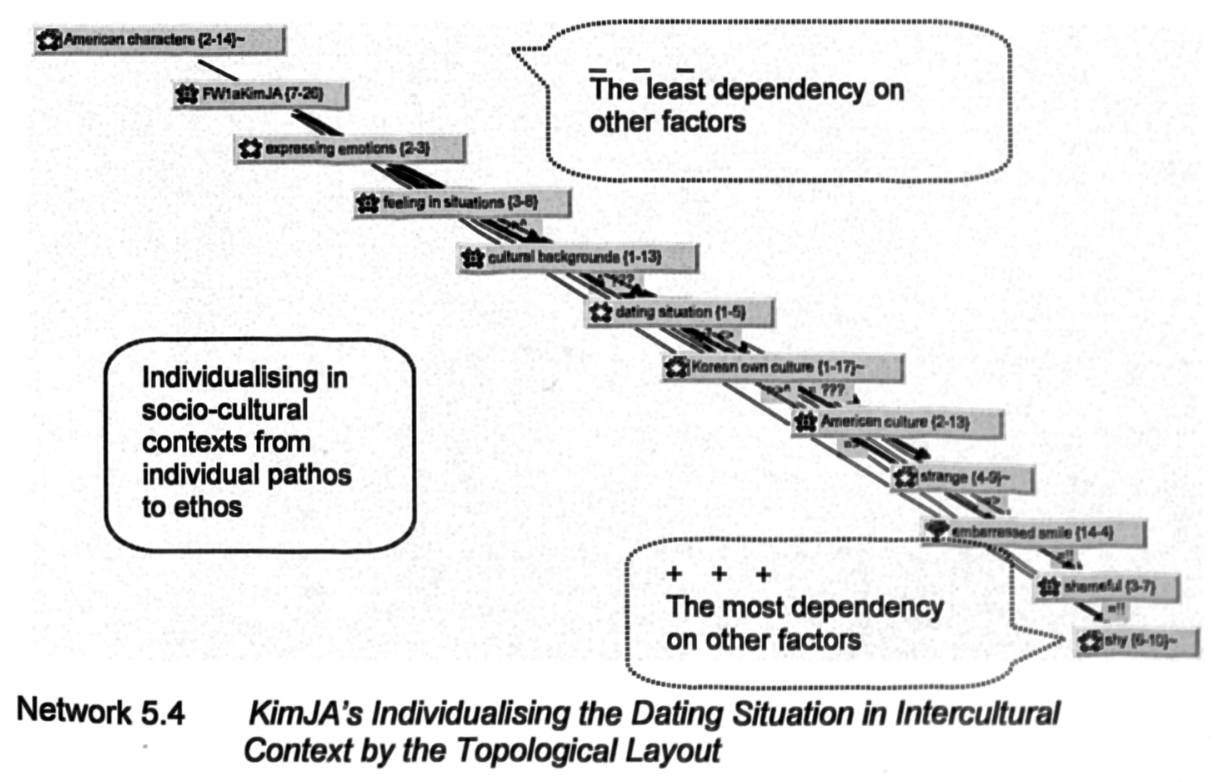


In short, it emerged that socio-cultural backgrounds have considerable influence on how participants feel in a certain situation and how they are aware of it. How can we use this powerful cultural background to improve intercultural communication?

Above all, I believe, drama provides participants with essential experiences for better intercultural communication-artistic, therapeutic and socio-cultural. I shall answer this question in Chapter 6.

\subsubsection{The Drama World and Everyday Life}

In the previous subsection, I presented factors to be applied to individualising activities in the reconstructive process-individual perspective as well as socio-cultural perspective in the sense that the former is imbued with the latter. However, contrary to this, has the former also been assimilated into the latter-from individual into sociocultural?

In this subsection, I shall present this dissemination from individual to intercultural in doing drama by connecting two points of view. One is the relation between life and art (Schechner 1988), and between social drama and aesthetic drama (Ibid), which I discussed in 3.2.3; the other is activities of participants in the drama project, which had its dual structure, like 'play within a play,' mentioned in 4.1.1, with drama events in their EDIE project.

I shall start my discussion with two still-images. I asked participants to express a situation in which a Japanese Firm and an American Firm sent their representatives to have a business meeting abroad. Both of the two companies were going to appoint the President of the company as one of their key delegates. How would each company see off their representatives before their departure?

My participants had never been to any foreign companies, nor to any foreign countries; they had to imagine what those sorts of companies were like. After recalling their memory about foreign companies, sharing some information, and discussing about that, eventually they made their still-images about that situation.

In this process, we made raw ingredients, like the aforementioned memory and information, into cooked food, that is, the still-images which they made. In other words, 'Art is cooked and life is raw. Making art [from something in life] is the process of transforming raw experience into palatable forms' [brackets mine] (Schechner 1988: 38). 
At this stage, I would like to re-state a point, which I argued in 3.1.1. This is that while we had cooking experiences in the process, it influenced, at least, choosing raw stuff for another recipe, despite the statement "There is no way for raw food to "come after" cooked food' (Ibid).

We took photographs of those still-images, which they made after their deep discussion and short rehearsal between them. MunJM, as a researcher-photographer, and $\mathrm{I}$, took photographs in order to analyse what we did for the relevant context. The following photographs are two pairs. Figures 5.1 and 5.2 are presented as the original pictures without any marking for analysis; Figures 5.3 and 5.4 are added with my marks.

The two still-images, which participants made as the images of the two firms were different from each other, in the first place, in their non-verbal language, as Table 5.2, presented immediately after the still-image photographs shows:

Each participant shared what she has known about those sorts of companies with other participants and each group constructed the image of the relevant company with information collected from each group member. Their performance of these stillimages is relevant to the concept of 'the whole binary continuum efficacy/ritualentertainment/theatre' (/bid: 141)

Participants put themselves into the characters, which they had never been, to make things happen, to entertain, to get results, to collect meanings, and so on. Of course, these activities were things relevant to participants' ethos and sociological factors, and everything collected from individual participants was perceived in their socio-cultural contexts. Those things, however, were individualised by the individual perceiver and were influenced under her own knowledge.

And then they came out from the perceiver and were used to reconstruct her activity as the actor, who was still also the perceiver. Thus, as the socio-cultural saturates the individual, the individual saturates the socio-cultural again, although the second sociocultural will be never the same as the first. In other words, participants moved continuously one actuality into another actuality by means of their performance. This process was repeated and this will be mentioned in relation to the spiral process of drama in 6.4. 


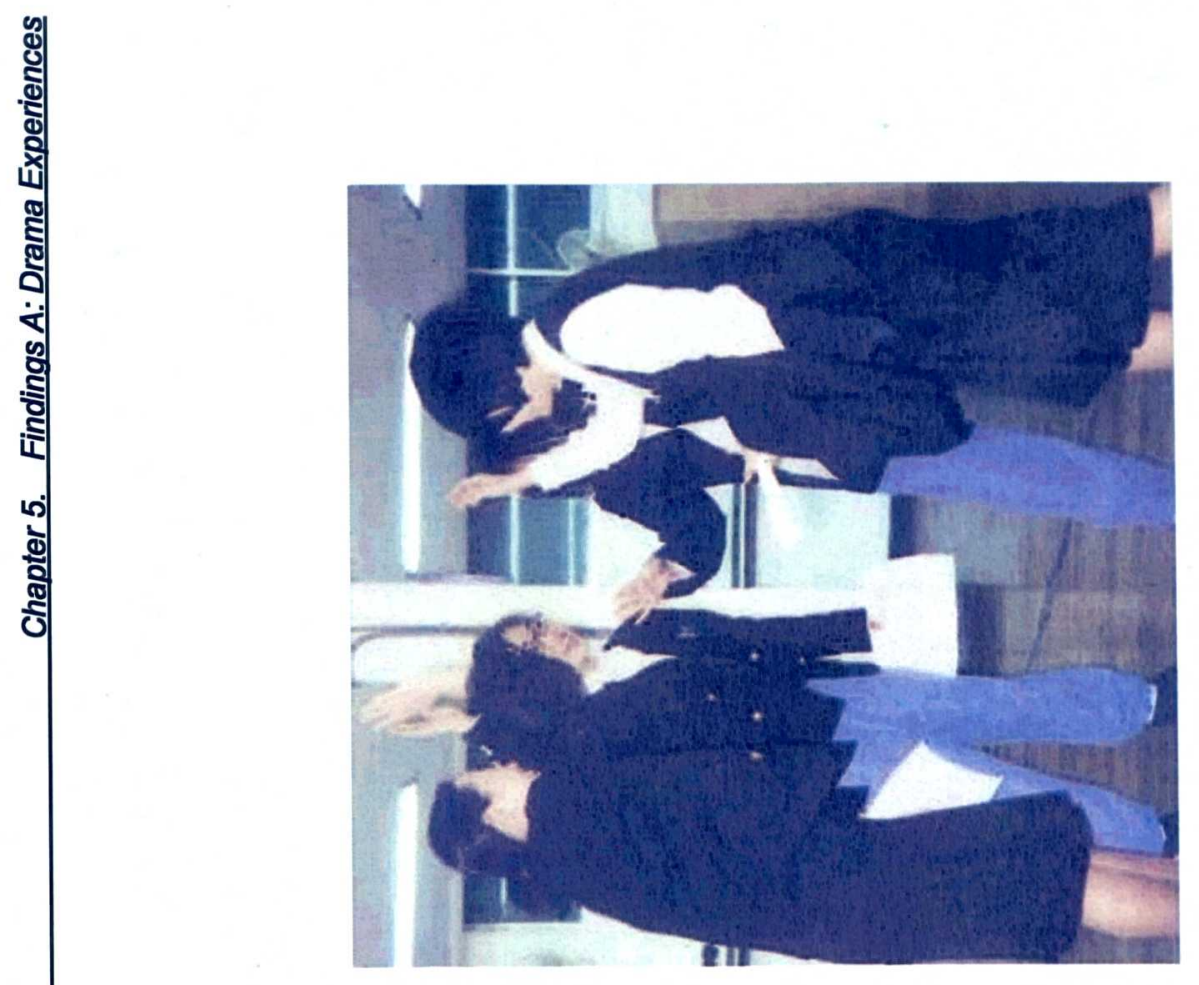

¿ू๊

ฐ

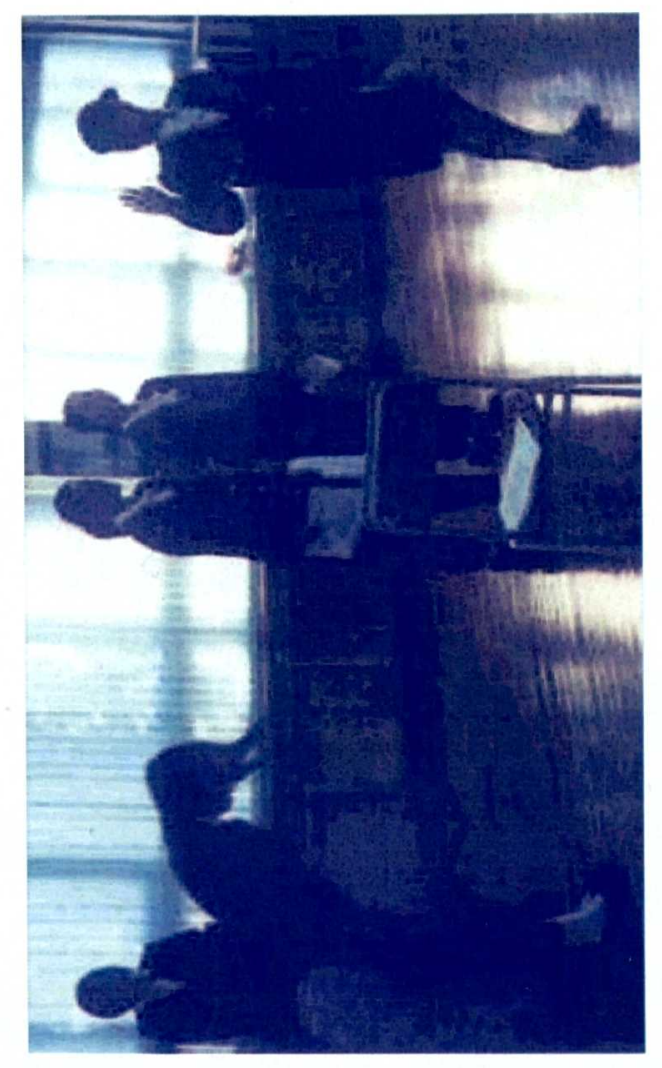

ठ 


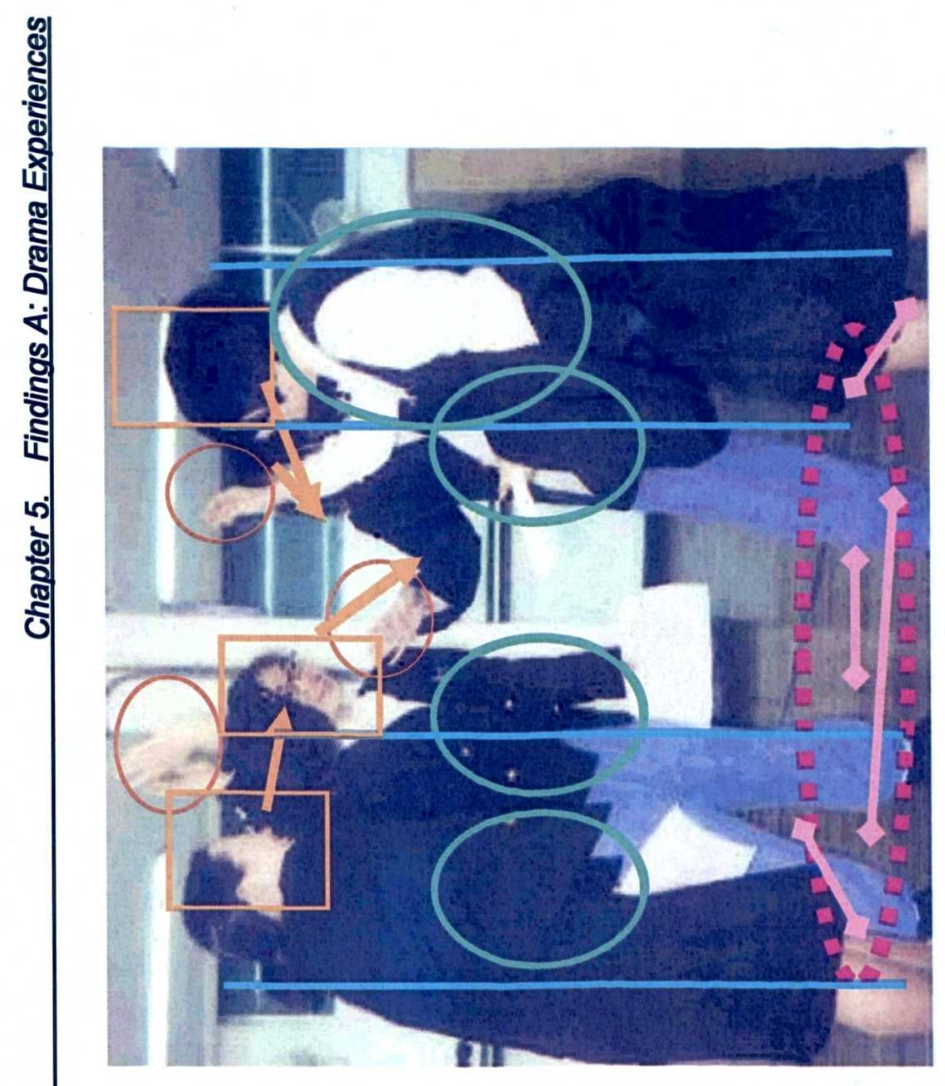

है
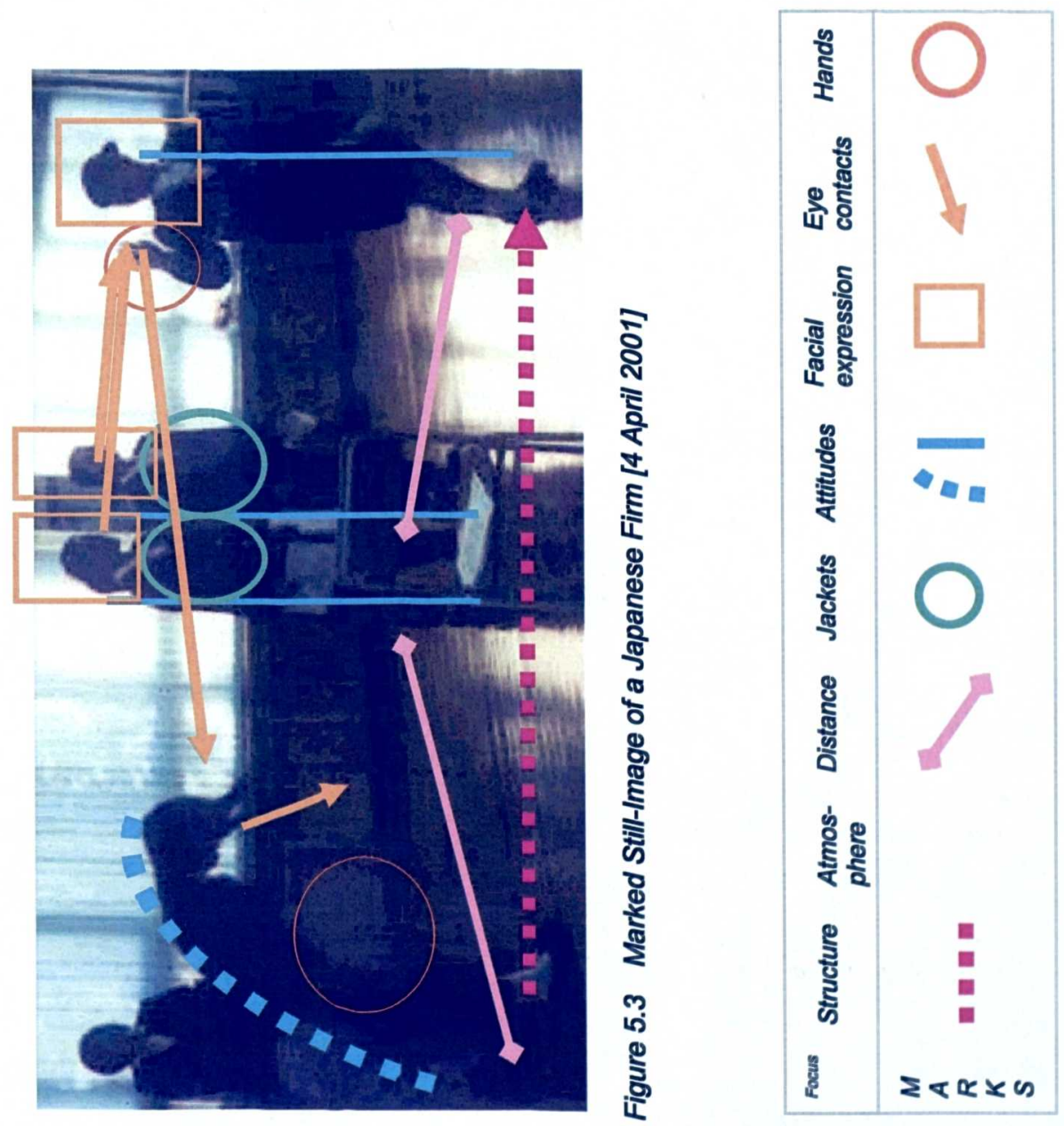

$\stackrel{\cong}{\Omega}$ 
Table 5.2 Comparison between Two Still-Images of Figures 5.3 and 5.4

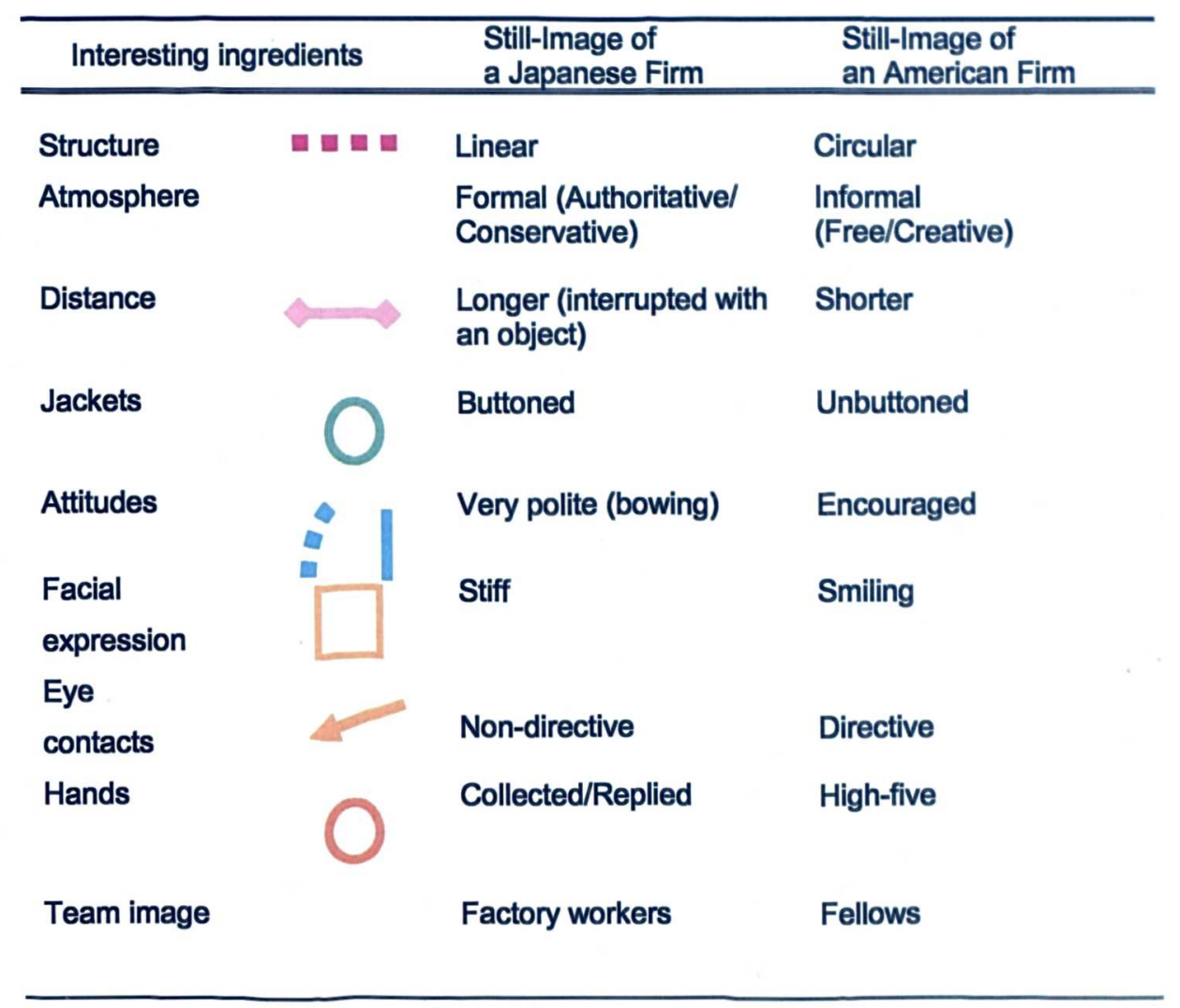

Some images which they made, for instance, that the Japanese firm seemed hierarchic and the American firm democratic, were filled with some of the thoughts which they have got about the two firms from their everyday life. The sequel of these drama activities led them to get a better understanding of business contexts in intercultural situations. In the end, the drama activities, which were in the Hidden Curriculum during drama sessions, provided them with opportunities to learn by doing drama things relevant to the subject of Trade Business in the National Curriculum. Therefore, I believe, the aforementioned drama activities became beneficial to their vocational education in the school. This was not only because of taking advantages relevant to the above subject but also because they were being educated as would-be career women immediately after leaving the school throughout their whole school life.

In the end, as discussed in 3.2.3, the aesthetic drama of these still-images, which participants made from their raw ingredients, acquired a hidden structure of what they could communicate with people from other cultures in intercultural situations and developed its visible structure of social drama, i.e. how they should behave as intercultural actors in those situations. 
What I found from the development of these still-images into more advanced contexts will be presented with other findings in Chapter 6, in which I will state why drama is effective in terms of its dynamics.

From this moment, 1 intend to move the question of the dissemination of drama activities from their individual level into their everyday life on to another perspective. At the beginning of the present subsection, I mentioned participants were involved in the drama project, which had its dual structure of 'play within a play.' This context, mentioned in 4.1.1, means each participant took two roles through the project- a performer, which I prefer rather than a 'spec-actor' at this stage, when she was doing drama activities, and a student researcher throughout the project.

This dual perspective is different from what I mentioned earlier in terms of overlapping relations between drama sessions and everyday life. The former, which means a 'spect-actor' level, is related to drama activities only in the situation of participants-inrole as each participant fulfilled their two functions of an actor and a spectator. While participants were taking their roles, they acted what they wanted to do as actors and at the same time they observed what they acted as self-spectators as well as what other participants acted as spectators.

The latter, a participant-and-researcher level, is relevant to their drama project. At this level, each student took two roles as a participant and a researcher and each role fulfilled two functions. That is to say, a student had two double positions-not only the participant with both an actor and a spectator, but also the researcher with both a researcher who was researching and a researcher who was researched throughout the project.

And then, how did students think about their drama activities in relation to their everyday life in the dual perspective? Most students who participated in the project had a lack of self-confidence, evident when they opened their hearts to me many times at personal interviews, drama sessions, Self-Questioning form and Saturday Meetings throughout the project.

I was very interested in how participants were going to handle their common difficulties through the drama project, because they said they were very afraid of learning English and even sometimes did not like to study English at all but they knew very well they had to overcome that. From this point of view, my analysis about their solving the problem included perspectives of socio-cultural drama, in which 
participants focus on exploring their social problems and finding out how to oppose some uncertainty and predictability and to solve those things through drama.

As mentioned earlier, participants already discovered their problem with speaking EIL to raise that as an issue from their social drama via their aesthetic drama in intercultural situations [here I could say intercultural drama]. The issue is how they could overcome their difficulty in learning English, especially in terms of English conversation, because of the great importance of their communicative competence in English as would-be professionals and ordinary people in their everyday life, in a word, in EIL.

They could not ignore the importance of English conversation any more in their everyday life-in their school as well as their future workplaces. While attending the drama project-expressing, performing, and acting in English and also observing and discussing about that, participants were experiencing and realising how effective this sort of drama activities is in improving their English.

They thought it would be helpful to keep this kind of drama activities even after the project was over. Students who participated as co-researchers in the EDIE (Educational Drama in English) project, evaluated the project as very effective in the matter of getting interest in the project, taking its advantages of developing English competence and expressing emotions and feelings, becoming interested in EDIE, having intercultural awareness, intercultural education, and so on, in their PostQuestionnaire at the end of the project, as Table 5.3 shows.

Table 5.3 General Evaluation of the EDIE Project by Participants

\begin{tabular}{|c|c|c|c|c|c|}
\hline $\begin{array}{l}\text { Participants' Evaluation } \\
\text { [the project: hereaster } \\
\text { EDIE in this table }\end{array}$ & $\begin{array}{l}\text { Strongly } \\
\text { Agree (\%) }\end{array}$ & Agree (\%) & $\begin{array}{l}\text { Neutral } \\
(\%)\end{array}$ & $\begin{array}{l}\text { Disagree } \\
\text { (\%) }\end{array}$ & $\begin{array}{l}\text { Strongly } \\
\text { Disagree } \\
\text { (\%) }\end{array}$ \\
\hline Interest in EDIE & 81.8 & 18.2 & 0 & 0 & 0 \\
\hline Benefits from EDIE & 72.7 & 27.3 & 0 & 0 & 0 \\
\hline $\begin{array}{l}\text { Interest in English } \\
\text { through EDIE }\end{array}$ & 45.5 & 54.5 & 0 & 0 & 0 \\
\hline $\begin{array}{l}\text { Intercultural awareness } \\
\text { through EDIE }\end{array}$ & 27.3 & 63.6 & 9.1 & 0 & 0 \\
\hline $\begin{array}{l}\text { Intercultural education In } \\
\text { Teaching ElL }\end{array}$ & 27.3 & 72.7 & 0 & 0 & 0 \\
\hline $\begin{array}{l}\text { Drama in the subject of } \\
\text { English }\end{array}$ & 36.4 & 63.6 & 0 & 0 & 0 \\
\hline Participation in EDIE & 18.2 & 45.4 & 27.3 & 9.1 & 0 \\
\hline Performance in EDIE & 0 & 72.7 & 27.3 & 0 & 0 \\
\hline
\end{tabular}


And they tried to change their educational conditions, for instance, obtaining two spaces in their school campus-the English Zone, where many students could have more opportunities to use English in their everyday life as much as they could, and the room for their activities of EDIE as co-researchers, who were deeply involved in the EDIE project.

Since the Saturday Meeting on 24 March 2001, they had been thinking about establishing their club of EDIE and an English Zone in their school campus. Here is an extract from the transcript of the Saturday Meeting:

\begin{abstract}
They discussed this possibility-who will have its membership, what its members can do, how they manage the club and the English Zone, when they can consult on their proposal with the senior teachers and the School Principal, who is the best person to talk with the Principal and how many participants are going to do that, when they can advertise the club and the English Zone in order to get more members except participants themselves, what they can do for the advertisement, and so on [From Transcript of the Second Research Meeting on 24 March 2001].
\end{abstract}

At another Saturday Meeting on 7 April 2001, I reported to my participants what I had said in relation to the club and the English Zone with the Vice principal. At this Saturday Meeting, they decided to go and interview the Principal about their proposal. As the first stage, an English teacher, who had been observing our drama sessions, was asked if she could arrange the interview for participants and did her best.

On the other hand, KimMS, who took a role of a journalist in the drama project, made a list of questions so as not to feel too nervous while interviewing the Principal. Nevertheless, they could not move on to the further stage because the principal suspended his being interviewed until participants would do well enough to satisfy him.

In fact, the Principal avoided being interviewed because he was afraid that he could not contribute to our project. This was because most school principals had been under the considerable influence of their management relations with the Local Council of Education in Korean educational milieu. Principals were reluctant to make any independent decision beyond regulations or rules ordered by the Council. For instance, if the priority in the Council were things relevant to Computer Education, principals would provide a computer room rather than a language lab. In worse cases, language labs could be replaced by computer rooms.

In the end, despite our attempts, which resulted from the research throughout the EDIE project, to make a change in the school in terms of learning English in their 
everyday life, we failed to obtain any space good enough to use as the English Zone or the EDIE Club until we finished the project as shown in the following comments.

KimJA: My school gave us the caretaker office, which nobody would use any longer.

The researcher: What?

KimJA: It is the caretaker office that we are given, the caretaker unfit for use!

The researcher: Are you sure?

KimJA and OhHJ: Look at that!

The researcher: I know what it is like. The Principal has allowed you to use the office as your clubroom, hasn't he?

KimJA: Yes, he has, indeed. The office is too small for four students to enter and to furnish two desks. So, we are really very disappointed with this.

[Comments of KimJA as the representative of the project in the Last Saturday Meeting on 28 April 2001]

We have learned from that situation that all participants should remember that there is nothing that can convey meaning in a vacuum or outside of history (Barthes et al. 1972). Even a tiny socio-cultural element exists within its macro level, that is, its sociocultural discourse surrounding the event

Participants in the drama project wanted to change everyday life because their experience in their drama world influenced their everyday life. They believed it would be very helpful for them to release their fear, to get interested in learning English and eventually to enhance their communicative competence in English.

At this stage, I can say that their imaginative activities through drama enabled students as co-researchers to transit from aesthetic drama to social drama/ intercultural drama, because they moved their focus onto socio-cultural factors in intercultural situations and explored their ICM.

The participants thus were not stuck in the imagined, fictional world of make-believe drama in our drama classroom, but turned their attention to aspects of their everyday life, the real world, where they should live and deal with some influential issue such as communicative competence in English and intercultural competence, as shown in Network 5.5.

Their action, establishing the English Zone and the room of EDIE club, is an example of transiting their aesthetic drama into social drama for solving their problems which were raised through their drama activities in the project. It also illustrates a changing of their current problems to match the ultimate aim of their SPAR as co-researchers 
engaged in the EDIE project as well as my PAR as the researcher involved in the BGCHS project.

Their action, which came from their common intercultural ethos, however, had to negotiate with other intercultural pathos and ethos, which non-participants, including the school principal, had at that moment and gave as relatively influential factors in making the decision of the English Zone and the room of EDIE club. The context of the campaign included considerable complicated factors, shown in Network 5.5.

Most concepts in Square $A$ were categorised into intercultural ethos from the perspective of the researcher, whereas concepts in Square $B$ were put into intercultural ethos from the perspective of Koreans who lived in Korea at that moment.

To my surprise, the semantic side of co-researchers exactly overlapped that of the school principal; despite this, they were deeply engaged in the project and raised the campaign for the English Zone and the room of EDIE club. Again, the BGCHS project which was led by the researcher, took its place in Square $B$.

This means the project itself was influenced by the educational conditions of the school in Korea, that is, it is still strongly dependent on the intercultural pathos and ethos around the project. From this semantic network, I would like to point out that the side of Square $A$ shared the importance of foreign language education and intercultural education with the side of Square $B$.

Also, as shown in Network 5.6, EDIE as an art form appeared to be a teaching and learning medium beneficial enough to EIL education as well as intercultural education and to be highly recommended for teaching and learning EIL.

Influential factors on the campaign, as indicated in Network 5.5 and 5.6, interacted with others in terms of the vectors of factors between pathos, logos, and ethos, at two levels, individual and other's:

- The individual level;

[1] his individual pathos,

[2] his individual logos,

[3] his individual ethos,

[4] his intercultural pathos,

[5] his intercultural logos,

[6] his intercultural ethos; 


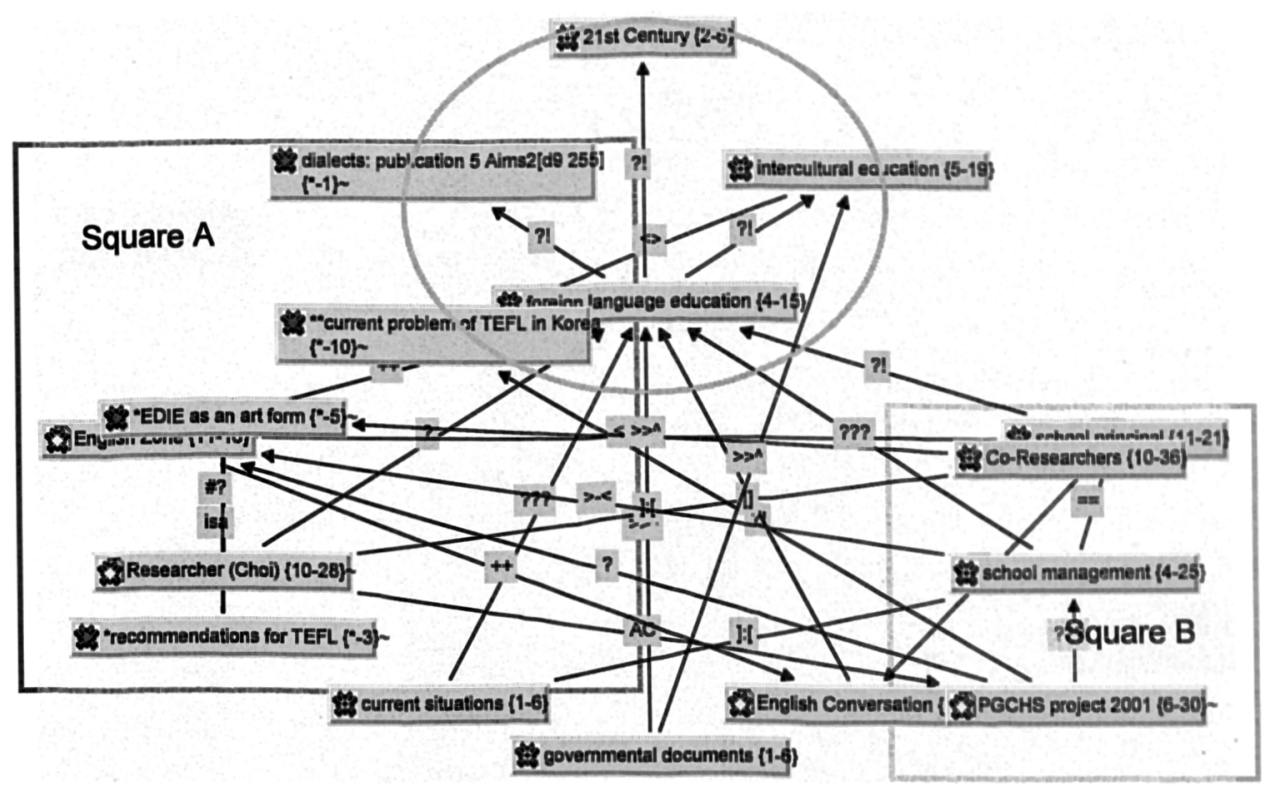

Network 5.5 Resolution for EIL Education and the English Zone in the Projects by the Semantic Layout

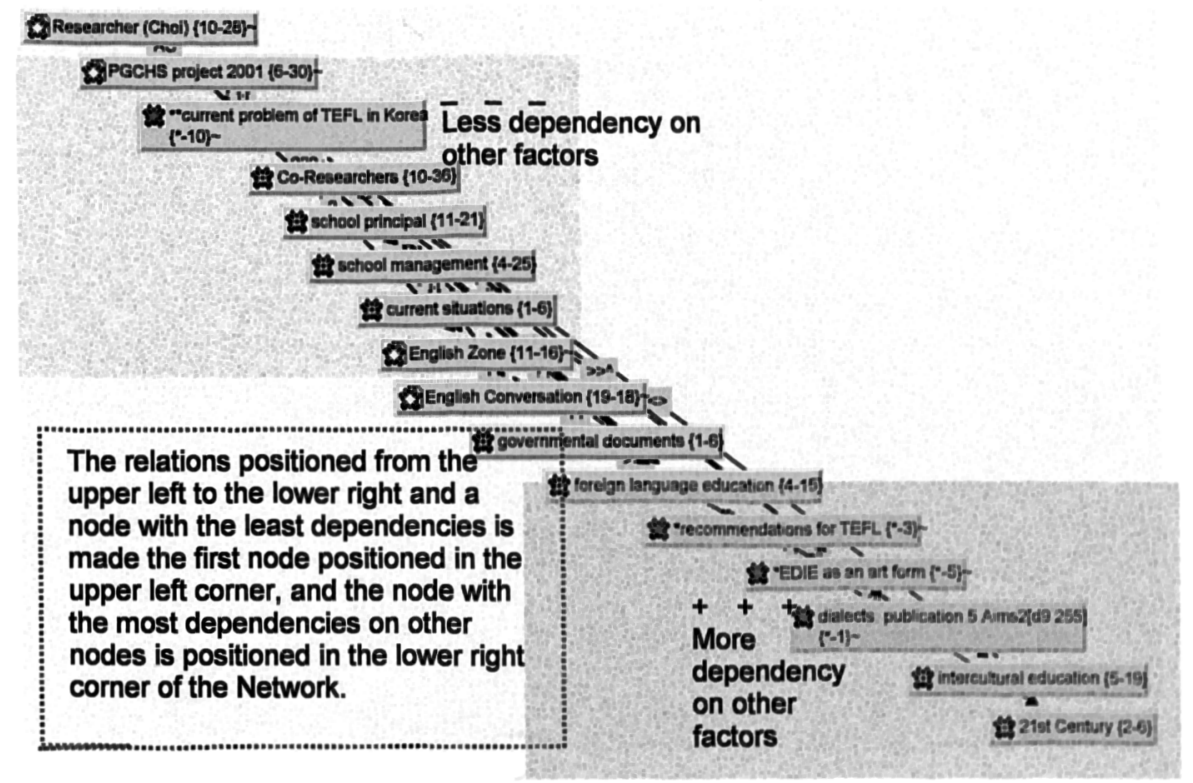

\section{Network 5.6 Resolution for EIL Education and the English Zone in the Projects by the Topological Layout}


- The other's level;

[1] other's individual pathos, [2] other's individual logos,

[3] other's individual ethos, [4] other's intercultural pathos,

[5] other's intercultural logos, [6] other's intercultural ethos.

The mechanism of interrelated factors makes their interrelated discourses different For instance, the other's intercultural pathos and intercultural ethos is shared with others in the society, although the participants applied the first two, the individual pathos and ethos, to the total imaginative activity in their own ways. Intercultural pathos and ethos in the society, to which participants belonged, were too powerful in making the decision about their campaign for them to end in a positive way. In the end, they failed to get any appropriate room to the English Zone and the EDIE club.

Up to the present, I have presented intercultural experiences which my participants had in intercultural communication in doing drama from different perspectives-artistic, therapeutic and socio-cultural. I have also presented the evidence that those intercultural experiences had started with individual pathos, in which participants had got feelings and emotions, and had produced their total imaginative activities for intercultural communication with their individual ethos and intercultural ethos. I have shown the interrelationship between individual pathos, individual ethos, intercultural pathos, and intercultural ethos in/around participants.

In a sense I can say I have done research about searching for the way to encourage the participants to practice getting the right vectors between those relations for better intercultural competence-from individual pathos through the self into intercultural ethos. In the end, what I want to say is how we can communicate to others with those twelve factors in an appropriate way.

My main interest of this study therefore is in investigating something relevant to the interrelationship between these factors to lead into a better intercultural competencedynamics of the interrelationship. How did participants handle those areas for the right intercultural communication when they were in the intercultural context in doing drama? This is going to be my next work in Chapter 6. 


\subsection{Summary: Total Imaginative Activity through Drama-Artistic, Therapeutic, and Socio-Cultural}

In the present chapter, I have presented the experiences participants had in doing drama from a psychological and sociological point of view-artistic, therapeutic, and socio-cultural/ intercultural experiences interrelated to each other.

These experiences emerged from their drama activities, whose essential ingredient was to express their feelings and emotions through the act of their consciousness about those feelings and emotions into their ideas, in the end, to their total imaginative activities. These procedures were taken in stages: participants' sensation in the psychically sensuous stage, their sensibility in the conscious stage, and their sensitivity in the aesthetic stage. Their experiences transferred from pathos to ethos, or vice versa, in individual as well as in socio-cultural/intercultural dimensions, and some experiences overlap at the same time.

In relation to the mutual relations between pairs of dimensions, for instance, individual pathos and other's pathos, I have found from the data that these drama experiences provided participants with opportunities to get a better understanding of themselves as well as others. What is more, these experiences led to changes both in their everyday life and in intercultural communication, in other words, to interact with each other in appropriate ways for getting at the total imaginative activity with intercultural sensitivity.

And then some questions can be raised. What, in particular, I was more interested in among what $I$ found from the data, is how the aforementioned experiences of participants functioned in and through drama and, secondly, what related to their activities for better intercultural communication through drama by understanding intercultural pathos and ethos from others-the dynamics in their drama experiences. What emerged in relation to these two questions will be my work in the next chapter. 


\section{Chapter 6.}

\section{Findings B: Dynamics of Drama in ICM (Intercultural Communication)}

The purpose of the present chapter is to investigate the effectiveness of drama in terms of its dynamics relevant to intercultural communication. In order to facilitate the investigation, I have presented the kind of drama experiences which took place in intercultural situations in the previous chapter.

Now I intend to move onto how participants got to those experiences and how they handled their experiences in intercultural communication through drama. I shall therefore focus on the effectiveness of drama with respect to its dynamics. It has already been argued that drama is effective in various perspectives, such as an art form, a learning medium, a therapeutic tool or a socialising activity. This investigation about how, not what, will be on the basis of four contexts, in which participants engaged through this research study:

- everyday life,

- their EDIE project, which we called Educational Drama in Education [EDIE], as mentioned in Chapter 4,

- drama sessions for the project, and

- drama activities during the sessions.

I shall focus on two dynamic points-first, how participants handled what occurred in and between these contexts and, second, how the ways in which they handled them were effective in improving intercultural communication.

The argument will be presented in four sections. In the first section, I shall show how participants had drama experiences in intercultural communication in or between those four contexts. What occurred in or between contexts will develop from both the duality of drama-the duality of its actuality and its theatricality, and the duality-its production cycle and its perception cycle.

These dualities are the key axes of dynamics in drama at the general drama level as well as in one single drama action, as discussed in 3.1. They are the main factors, which make drama dialectical and develop its dialecticality into dialogicality, which leads into otherness and outsidedness, as argued in Chapter 3. 
In the second section, therefore, I intend to describe and analyse how participants handled two different elements from the aforementioned duality in intercultural communication with respect to the dialecticality.

In the third section, I shall move further onto the dialogicality in which the participants were involved. The plurality of their drama activities influences this dialogicality, as I discussed in 3.3. With respect to this dialogicality, it will emerge that it is related to other concepts such as learning by situated doing, otherness and outsidedness, and also becomes relevant to reflection, self-reflection, and the reflexivity of participants in their contexts.

Fourth and last, I shall draw on the dialogical-cum-spiral dynamics of drama to analyse the process of chronological development of drama activities.

The following presentation emphasises the dynamical mechanism of drama activities for improving intercultural communication through drama in the context of teaching/learning EIL. There are two main bases for the illustrations-Beckerman's concept of the dynamics of drama and Bakhtin's concept of dialogism in drama, presented respectively in 3.1 and 3.2. The former is based on the psychological perspective of drama itself and argues for the duality of figure and ground in drama, whereas the latter is based in the socio-cultural perspective of drama and develops from dialecticality to dialogicality in drama.

The dynamics of drama illustrated in the present chapter, along with drama experiences presented in the previous chapter, will lead to the conclusion of this study, and to suggestions about how we can prepare for improving intercultural competence.

\subsection{Dualities of Drama Actions in ICM}

What I am trying to demonstrate from now on is from the process standpoint of drama experiences. This standpoint is based on Beckerman's figure-ground principles, that is, how participants began to get their intercultural experiences through drama, particularly from where to where and how they carried out their ICM.

This shifting interaction will emerge as a basic key factor in drama dynamics, which will be much further developed in the matters of dialecticality and dialogicality of drama in the next sections. This how viewpoint is different from what participants got through drama in the intercultural contexts. 
My focus therefore will be kept on how to handle ICM through drama rather than what to do in those situations, although the latter, in the end, will not be excluded from my discussion.

\subsubsection{The Duality of Actuality and Theatricality in Bi-Dual Contexts-Everyday Life, the EDIE Project. Drama Sessions, and Drama Activities}

In 4.1.1, I made the analogy between the TV series Columbo and my research structure in terms of emphasising the how standpoint in this study. I designed my research methods for this study in a bi-dual structure. The whole structure provided for participants not only a dual structure of the real world and the drama world, but also a bi-dual structure, which I presented as 'play within a play.' First, let me clarify each of the four contexts-everyday life, the EDIE project, drama sessions, and drama actions.

I have just mentioned a bi-dual structure and 'play within a play' in my research design. This means my participants could cope with two different 'plays' at the same time, although the plays sometimes overlapped: one 'play' was more related to everyday life and the EDIE project; the other 'play,' relevant to drama sessions and drama actions themselves.

I labelled the EDIE project as the fictional context of Play $A$ and everyday life, as the real context of Play A-its non-fictional context. In the former, participants made the relevant fictional or drama context and took roles of co-researchers with the help of a drama technique, 'mantle of the expert.' However, in the latter they returned to their school life as students in the real context.

On the other hand, I categorised drama actions or activities as the fictional context of Play $B$, whilst drama sessions were the real context of Play $B$-its non-fictional context. The real world and the drama world can be interchangeable as the real context and the fictional context, respectively, in this study. Furthermore, I shall use Play A's $R$ as the abridged name of the real context in Play A, Play A's $T$ for the fictional or drama context in Play A-the EDIE project, Play B's R for the real context in Play B, Play B's $T$ for the drama context in Play $B$, respectively in the present chapter and the next one (For a diagrammatic representation see Figure 6.1 in 6.1.1.2). 


\subsubsection{Interaction between Contexts of Drama, Drama Sessions, and Everyday Life}

Now I intend to show how participants made the shift between those four contexts to interact with the contexts in terms of the duality of drama-actuality and theatricality. One context inter-related to another or others, or sometimes overlapped with another. Their relations are on the ground of the duality in drama-actuality and theatricality, discussed in 3.1.1.

In other words, their interactions in the bi-dual contexts emerged between actuality and theatricality in their drama actions. Let me return to the case of KimMS, who made changes in terms of her attitudes and behaviours while participating in this study, as a representative example of the relation between everyday life and drama actions of participants.

As illustrated with regard to therapeutic experiences through drama in 5.2.2, KimMS was involved in the situations, reflective as well as self-reflective by taking herself in role and out of role during drama sessions. Her Self-Questioning papers and Personal Journals showed how KimMS handled her participation in her drama sessions with respect to being reflective and self-reflexive.

According to her Self-Questioning papers written on 16 March 2001, at the beginning, KimMS confessed that she was introverted and was a pure participant in drama activities. She had difficulties in doing drama because other participants made her laugh. Her subsequent Self-Questioning about the next drama sessions revealed that she laughed a lot and at the same time learned a lot (Self-Questioning form on 21 March 2001). She did not exclude this point from her attention during her participation in this study.

This means she kept releasing her emotions, not suppressing them, and discovering herself through observing and thinking about what she did during drama sessions, and then finally acquired a form of critical thinking about herself. For instance, she made the criticism that she did not control her emotions appropriately and did not speak out properly and wished to voice her opinions clearly and strongly (Self-Questioning form on 23 March 2001). Also, she had difficulties in her limited vocabulary and poor brainstorming (Self-Questioning form on 30 March 2001).

Eventually she stated her participative activities in the drama session were satisfactory (Self-Questioning form on 4 April 2001). In her mid-Personal Joumal her changes until 
that that time were described from three main perspectives: first, she became more interested in learning English; second, her drama activities provided her with chances to change her personality; and third, it was good for her to begin understanding other cultures (Personal Joumal on 6 April 2001).

This consecutive dialogue between herself and her drama activities in drama sessions enabled her to interact with different contexts or shift other contexts from herself, her everyday life, and her drama activities in drama sessions and to reflect about herself and the others. What I would like to emphasise about Self-Questioning again is the crucial function that self-questioning took to remind her of the inner conversation she had while doing drama, although it was written after each drama session.

Self-questioning between herself, everyday life and drama sessions, in other words, shifting into other contexts, such as Play A's R, Play B's R, and Play B's T, enabled her to step away from herself as well as her character, to review and think critically about what she had done during each day's drama session, and then understand her drama activity in an objective perspective.

From this example, I can say that interrelations in bi-dual contexts, led participants in drama to have drama experiences of actuality and theatricality as well as to get more understanding of themselves, in the end, of their identities.

\subsubsection{Interaction between Contexts of Drama, the EDIE Project, and Everyday Life}

Let me return to the drama action, which KimJA performed in the episode of the International Business Meeting-drinking a beer as the American president, as an example of the relation between everyday life and drama actions of participants. Her action, "the American president is drinking a beer in a pub" is in the drama context. Before taking this drama action, KimJA is in the real context in which she participated in her drama session in that real time in that real dancing room [the verve for the work]. However, she changed that real context into a fictional context on the basis of the play script with the help of the 'Magic If-the 'here-and-now' situation. In the end, this shift enabled KimJA to take her drama action into the drama context.

Because of this process, the real context was shifted into the fictional context and at the same time actuality in the former shared with theatricality in the latter. This is because KimJA still kept her own identity but took another identity as the American president even in the drama context in Play $B$. 
In other words, actuality and theatricality co-existed in relation to her drama actiondrinking a beer as the American president. In the real world of Play A (Play A's R) KimJA was mimicking drinking something with her bare hands in the room at that time, but in the drama world of Play A (Play A's T) the American president, whose role was taken by KimJA, was drinking a beer with a beer glass in a pub at a certain time. The former is a fact or condition that really exists in that real place, the dancing room, at that real time, that is, actuality of that action, meanwhile the latter is a condition that the American president was drinking a beer in the pub at a certain time, that is, theatricality of that action.

However, the actuality was the ground for the drama action, which emerged through its theatricality as its figure. Also, in the process of expressing her own emotions through doing the drama action, what she had in her everyday life was reflected in her acting.

From the above example, I can say that participants were engaged in multiple contexts-Play A's R, Play B's R, and Play B's T, that is, this multiplicity applies to each context and every drama. They experienced ICM in those multiple contexts, because those contexts given in the drama world of Play $A$ and Play $B$, socio-culturally different from the contexts provided in the real world relevant to Play $A$ and Play $B_{1}$ that is, to the real world of participants themselves.

It appears that drama experiences of participants, who shifted between contexts, can be produced like Figure 6.1 .

As Figure 6.1 shows,

- Context 1, in which participants have their own cultural factors, refers to every day life of participants in relevance to Play $A_{i}$

- Context 2, where participants handled intercultural factors, including own as well as different, encompasses the EDIE project, in which participants were engaged in the drama world by making a fictional context in which participants took co-researcher roles;

- Context 3, also where they managed intercultural factors because of their learning materials, provides for drama sessions relevant to Play $B$; and

- Context 4, in which the drama world provides participants with different cultural factors and therefore participants performed drama actions in ways different to their own culture, that is, drama activities within drama sessions. This context is related to the concept of 'play within a play' or metadrama. 


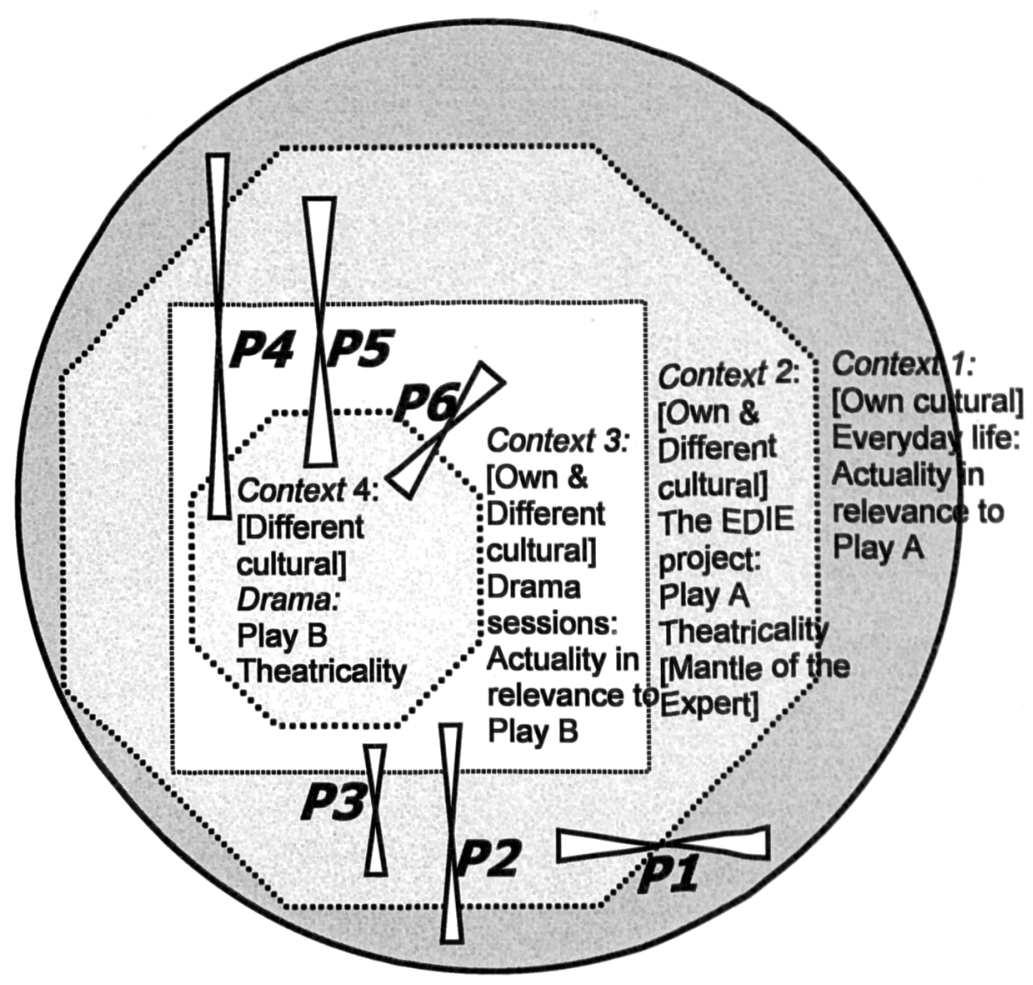

I produced this figure from a bird's eye view and arranged its four contexts in different layers but its general shape is like a pyramid. Participants made shifts between these contexts. [P: shifting pattem between contexts].

Figure 6.1 Duality of Participants' Drama Experiences: Actuality and Theatricality in Contexts, Bi-dual and Intercultural

Participants therefore took different functional roles in each context, respectively:

- Students in their school life in Context 1 of the real world (for instance, a student can say, 'I am a second-year student at BGCHS.').

- Co-researchers in the EDIE project in Context 2 of the drama world (Participants-in-role in the research part of Student Participatory Action Research [SPAR] ('I am an interviewer in the project going to interview my school principal).

- both Co-researchers in drama sessions (Participants-in-role in the action part of SPAR) and Performers in drama sessions and drama (performer-out-of-role) in Context 3 of the drama world and the real world relevant to Context 4, ('I am a researcher doing research to analyse photographs which are taken about our drama actions at the moment, and I am afraid I cannot member my lines and act naturally').

- Finally, Characters in drama (performer-in-role) in Context 4 of the drama world ('I am Gregory Jones, a president of the American company'). 
These multi-functional participants moved into one of six shifting patterns-from P1 to P6, depending on the situations, in which they found themselves. For instance, the case of KimMS, demonstrated in the previous subsection, made main shifts of P1, P2, P4, and P6-everyday life, drama sessions and drama; meanwhile, the case of KimJA, illustrated in the present subsection, mainly interacted between P1, P2, P4, and P5everyday life, the EDIE project, and drama.

With regard to the above cases, I can say that shifting or interacting between these contexts, first, gave participants opportunities to get involved in ICM, second, to have more critical thinking because of distancing from the relevant context, and third, to have a better understanding of other cultures as well as their own, that is, of ICM.

\subsubsection{The Duality of Production and Perception in Bi-Dual Contexts-Everyday Life, the EDIE Project, Drama Sessions, and Drama Activities}

In Chapter 5, I described the drama experiences of the participants in the light of their intrinsic qualities so far-artistic, therapeutic, socio-cultural, and dual between actuality and theatricality. It is necessary to add however the duality of Production and Perception to the above qualities.

As discussed in 3.3.2, participants were engaged in two different cycles-production and perception, or drama writing and drama reading. With regard to this, I shall present the production-and-perception cycle in drama from two perspectives, extroversive and introversive, of each participant.

\subsubsection{Production and Perception at the Extroversive Level of a Participant in Drama}

With a view to illustrating the production-and-perception cycle where participants were engaged among participants, first of all, I intend to re-consider the starting points of the Passport Game done during drama sessions on 28 March 2001, which was presented in the light of emancipatory drama experiences in 5.2.1.

In the first part of the Passport Game, participants of each group were asked to establish a new country, its own language, their own passports in the new language, and its cultural factors like costumes. They began to be involved in the production cycle for this drama activity. One group, for instance, invented its own country, which was named as $=1$. According to the explanation from KimEL, there were three 
principal reasons for that word: first, $\Rightarrow$ was originated from one of consonants in their own Korean language; second, the mark, ', for a voiced sound of the consonant, $\Rightarrow$, was borrowed from a mark used in the Japanese language: third and finally, $I$ came from the alphabet of the English language. Members of the group therefore pronounced that word as [gi].

Other group members including me realised how to create it, to pronounce it, and to interpret it as the meaning of spirits or strength for the country. Through realising the backgrounds and the meaning of the word, other group members got involved in the perception cycle of that drama activity, that is, they perceived the word, which other participants invented in their production cycle.

At this stage participants applied their own thoughts, ideas, and feelings to others, rather than themselves, that is, their predominant concerns were the outside world rather than within themselves. With respect to this, I can say that participants were involved in the production-and-perception cycle at the extroversive level.

One more thing, here, is about the order of the application of languages-Korean, Japanese, and English. They started from their own standpoint [Korean] and spread their viewpoint farther and farther [Japanese and English]. I can say that in their production cycle of inventing this word they must have been most comfortable with the closeness to their own socio-cultural values. This attitude matched their perception cycle relevant to the International Business Meeting between the Japanese president and the American president, where participants took the Japanese side rather than the American one. The same can be said in reference to their spectatorship but I shall not present this perspective at this stage. The spectatorship together with the selfspectatorship will be illustrated in terms of dialectical interactions of participants in 6.1.2.3.

\subsubsection{Production and Perception at the Introversive Level of a Participant in Drama}

Now I shall move on to production and perception of drama at the introversive level. Contrary to the extroversive level among participants illustrated in the previous section, production-and-perception cycle at the introversive level occurred within the participant herself.

Here is an example for production-and-perception cycle at the introversive level-the case of the scene in which the American couple were dating in the park, presented in 
5.3.1. I analysed this scene to show that participants individualised an event into drama even in a whole socio-cultural context where the participants were all together, because each participant had her own individual socio-cultural values.

Now judging from the standpoint of the introversive level, it seems that each participant's individual socialising process is also taken into consideration in terms of her own perception cycle. Participants, for instance, KimJA, KimEL, and MunJM were asked to act the same dating scene during the drama session on 28 March 2001. According to their Self-Questioning about the scene, which was presented in 5.1.2, the participants perceived that same drama context differently.

For instance, KimJA felt so awkward and even ashamed to act what she thought about the dating scene. KimEL realised that she could not know how the American girl was going to behave with her boyfriend because she did not have her own boyfriend. Moreover, MunJM, with far stronger words, hated that kind of behaviour. These three participants experienced their own emotions or thoughts in their perception cycle within themselves, not in relation to the perception of others.'

On the other hand, in their production cycle there was no interaction with others. KimJA reached her decision in her own cultural way to act a bit shy because she was proud of her culture, KimEL relied on her imagination, and finally acted 'putting on her perfume' along with telling a lie. Moreover, MunJM tried to do a little bit but still felt strange, even got gooseflesh on her arms.

Each of the above participants thus faced the same intercultural situation between her Korean culture and the American culture but accepted that situation differently and acted the situation in different ways. This is because each of them perceived the context of the text in the American pathos and ethos which the participant expected but took stock of her perception in her individual pathos and ethos with respect to that scene. Furthermore, this is because she acted according to how she made the scene with her individualised imaginative activity for the scene. That is, she was engaged in the perception cycle in the first two stages, whereas she was in the production cycle at the last stage. Consequently, they took perception-and-production cycle within themselves, or at its introversive level.

Let me take another example from participants' individualising contexts, which I mentioned in 5.3.1. The leading role of a daughter, Angie, was assigned to two participants, $\mathrm{OhHJ}$ and $\mathrm{KimHJ}$-double casting. Both participants took the same role but took different interests in that role while acting as the character. According to their 
Self-Questioning form on 21 April 2001, presented as Table 5.1, OhHJ paid more attention to her speaking lines, particularly how she could make the pitch or tone of her lines more appropriately in harmony with her drama context. Meanwhile KimHJ took into more consideration how she could characterise the role and based the character on her relationship with her own mother. To put it simply, OhHJ focused on her linguistic competence of ICM through drama, whereas KimHJ focused on the socio-cultural competence through drama.

It thus appears that participants were involved in the production-and-perception cycle at the extroversive level as well as the introversive level in drama, shifting between the four contexts, which I mentioned in the previous subsection-everyday life, the EDIE project, drama sessions, and drama activities. The relations to other contexts and the shift in reference to the production-and-perception cycle can be summarised as in Figure 6.2.

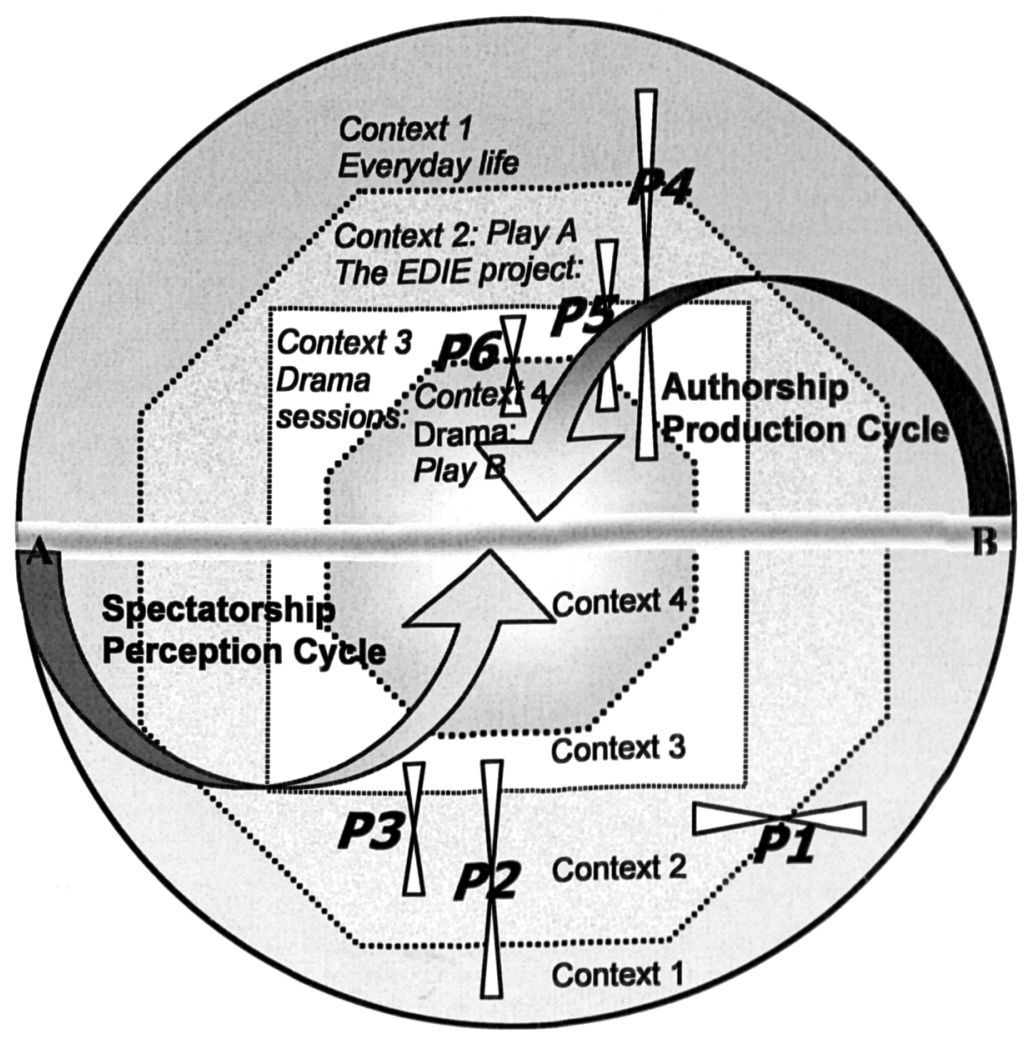

Figure 6.2 Duality of Participants' Drama Experiences:

Production-and-Perception Cycle in Bi-dual Contexts 
The figure has the same basic contexts, Context 1 to Context 4, and the same shifting patterns, $P 1$ to $P 6$, as Figure 6.1. Participants shifted from the production cycle into the perception cycle or vice versa within one context or between contexts. Depending on the relevant situation, participants made these shifts differently.

This is regarded as a very basic procedure to handle the intercultural situations in which participants were and the beginning stage for participants to be engaged in ICM. On the basis of this starting point, participants took cognisance of the duality of drama in the two dual matters of actuality and theatricality as well as production and perception.

Participants had experiences through their drama activities and made connections with more complicated procedures-dialecticality, dialogicality, and spiral movement through drama. Also, both the production and perception at the introversive level enabled participants to be engaged in their self-spectatorship as well as their spectatorship, and this perspective will be described in terms of dialectical interactions of participants in the next section.

\subsection{Dialecticality of Drama Actions in ICM}

It has been shown in the previous section that participants had drama experiences in ICM through shifting in or between four contexts and were also engaged at the extroversive level as well as the introversive level of the production-and-perception cycle in the shifting process. Their shifting and engagement in those dualities led participants to be confronted with two or more factors, different or contradictory to each other, in intercultural drama.

How then did the participants handle their difficult situations through drama? Providing the answer to this question is the major reason for this study and will provide the profession with a body of knowledge of drama for intercultural education and dynamics of drama, identifying and developing intercultural competence in ICM

\subsubsection{Dialecticality between Everyday Life, the EDIE Project, and Drama Sessions}

In order to search for answers to the aforementioned question, I am going to start with analysing participants' shifting contexts between everyday life, the EDIE project, and drama sessions. For example, the English language was used in everyday life as well as in their drama sessions, as a communicative medium in intercultural settings in 
their EDIE project. This is because the duality of drama led them into the relation between their school life as everyday life and their drama activities.

\subsubsection{Participants in Everyday Life - Co-Researchers in the EDIE Project [Play A]}

I intend to show how participants managed their actions in the contradictory context, which existed between their real world and their drama world and found a resolution. In fact, in the process of constructing the project group for this study, I wondered how many students wanted to participate in this research study because their school, which I chose intercultural education in order to connect vocational education, had students, who were basically afraid of learning English.

Despite regarding EIL as an essential part of professional competence, many students in the school seemed to be still reluctant to be involved in any sort of activities related to the English language. The situation in which participants did drama activities in English, had many demands throughout the project, and I had to explain English vocabulary more frequently than I had expected.

At their first interviews, most participants asked me if their poor English would be acceptable for drama activities in English, although at the end of their interviews they told me that they were becoming more interested in using English than before [from my interviewing participants in the first week of the project]. From the second week of their project they faced their dual attitudes to English: one was their fear of learning English in school; the other, their willingness to use English more in the project as they became more engaged in the EDIE project.

How did participants as co-researchers manage these contradictory factors throughout the project? As disclosed in my first interview with them, participants in Play A's real world, as a first step, faced their uneasiness with English at their pathos level. Later, in spite of this anxiety, they developed their easiness, or rather willingness, at their ethos level by a better understanding of the situations when they became interested and pleased in using English, and realised how important it was for them to use English properly and competently.

The issue of how they could handle the contradictory situation of English seemed to become an important task for us (both participants as co-researchers and me as the researcher/ teacher), after starting the discussion in the Second Saturday Meeting on 24 March 2001. In the end, we unified two contradictory factors into a third action- 
practising English in the English Zone without being afraid of English within the school campus.

We planned to suggest to the school principal that the school provide students with a certain space where some of them could go to speak English in an informal way, not like in the English classroom. We called this place the English Zone, whose aims were to get more opportunities for students to practise daily expression in English in their everyday life contexts. We took actions, such as the consult the Vice-principal for the English Zone in order to solve the aforementioned tension between the uneasiness and the willingness, as shown in Figure 6.3 and the tension was solved in a dialectical way.

I put their uneasy feelings (indicated as Factor 1) about English in everyday life (Play A's real world), at their pathos level, on the bottom left side of the triangle in Figure 6.3, and their willing attitude (indicated as Factor 2) in the EDIE project (Play A's Drama world), on its bottom right side. Factor 1 (their uneasy emotion) was controlled and resisted by Factor 2 (their willing attitude).

We, or rather students as co-researchers, considered their two very different factors and sought one possibility with a view to solving the tension. In this process of producing the English Zone as their total imaginative activity at the ethos level, their uneasy emotion controlled their willing attitude, and vice versa.

With regard to this resolution, I suggest a tripartite dialectic formula, as illustrated in Figure 6.3.

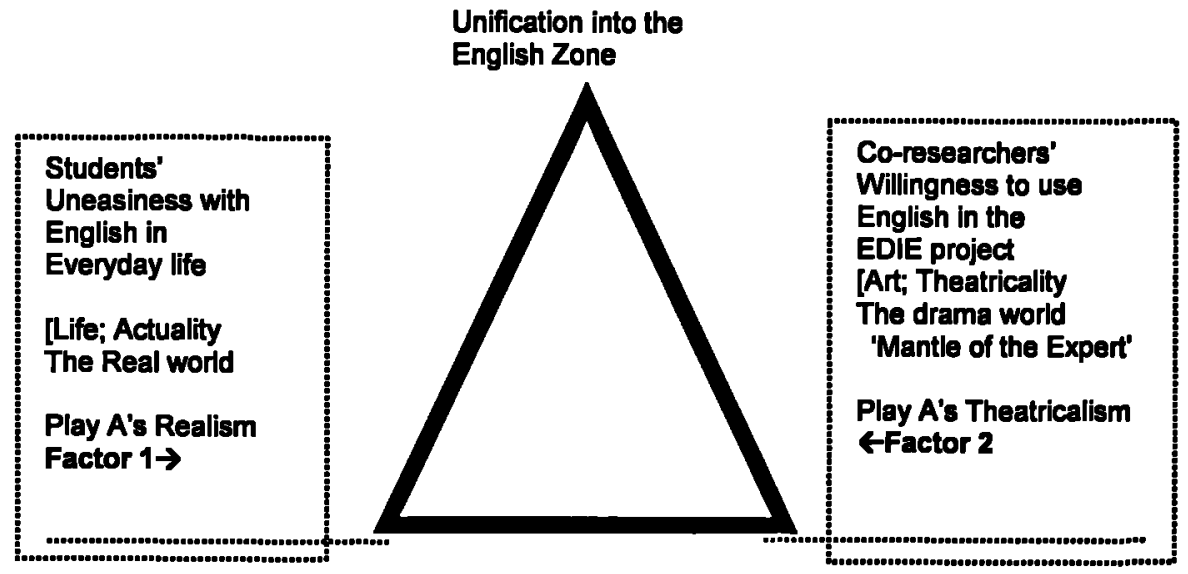

Figure 6.3 Being Dialectical in the Dual Attitudes to English (Students' Uneasiness vs. Co-Researchers' Willingness) 
I can therefore say that there was dialecticality in the dynamics in solving the resistance or tension with the duality of students' situation between participants in everyday life (Play A's Real world) and co-researchers in the EDIE project.

This dialectical interaction between the dual attitudes developed into dialogical interactions in the multiple contexts. In the process of dialogical interactions, external variables came to the fore such as the school management and educational milieu. I shall describe these external variables in reference to otherness through extroversive dialogicality in 6.3.2.

\subsubsection{Participants in Everyday Life - Participants-in-Role in Drama Sessions [Play B's T]}

I shall illustrate another dialectical example where the real world controls the drama world and vice versa, in relation to drama sessions in the present subsection. Let me take a case of how a participant handled her intercultural situation in role as an American character.

On 4 April 2001 we had drama sessions, in which participants, who were all Korean girls, participated in a drama about negotiating across cultural boundaries between an American firm with its small, family-type climate and a Japanese one, which was a well-established and highly respected contracting firm. KimJA took the role of Greg Jones, the 25-year-old American president, who was dressed casually in slacks and a polo shirt and was going to make a trade agreement with Goto Yasuhira, the 60-yearold president of the Japanese firm, who dressed formally. KimJA as Greg Jones was going to act in the following context, described in a text given to the students:

\footnotetext{
Jones was already sitting at a table in the restaurant [in Japan] at which everyone had agreed to meet when Goto and his two assistants arrived. The Japanese were directed to the American's table and were shocked to see a youthful American man, casually dressed, drinking a beer [brackets mine] (Singelis 1998: 119-120).
}

We, KimJA as the American President, MunJM as the Japanese President, RyuKW as a Japanese subordinate, and I (teacher-in-role) as another Japanese subordinate, were about to act this ICM but KimJA didn't feel comfortable in that situation at all. The following transcript captures the moments at which KimJA faced the tension between herself and her character, the American president.

\footnotetext{
Teacher-out-of-role: (To KimJA) wait a minute. Did you drink a beer before that action?

KimJA-out-of-role: I sat and .... .
} 
Teacher-out-of-role: All right! You sit there and drink a beer. (To MunJM and RyuKW And then what can we do while we are approaching to him, I mean KimJA?

MunJM and RyuKW: (show some gestures)

Teacher-out-of-role: (To KimJA) and how about you?

KimJA: I see the Japanese representatives entering and approaching to me, and .... The next action is to shake hands with them?

Teacher-out-of-role: Yeah.

......

KimJA-in-role, Jones: What did we all forget our lines? Where's the... (KimJAout-of-role) what I can say?

Teacher-out-of-role: script.

KimJA-in-role, Jones: Where is the script? (Laugh unnaturally) huh, huh, huh! (KimJA-out-of-role laughs very loudly because of her embarrassment in this uncomfortable situation)

[From transcript of the Drama Session on 4 April 2001]

KimJA did not seem to feel comfortable in performing the aforementioned ICM, as she signalled her embarrassment in her body language with three periods of silence and loud laughter. This embarrassing emotion was felt at her sensation level, which I mentioned with Network 5.1 in 5.1.2, and her embarrassment derived from her emotional disagreement with the American character's behaviour. I can therefore suggest another tripartite dialectic formula in relation to the process which produced her embarrassment, Figure 6.4, which is the same pattern as Figure 6.3.

\section{Embarrassment of KimJA as the American president at drinking a beer}

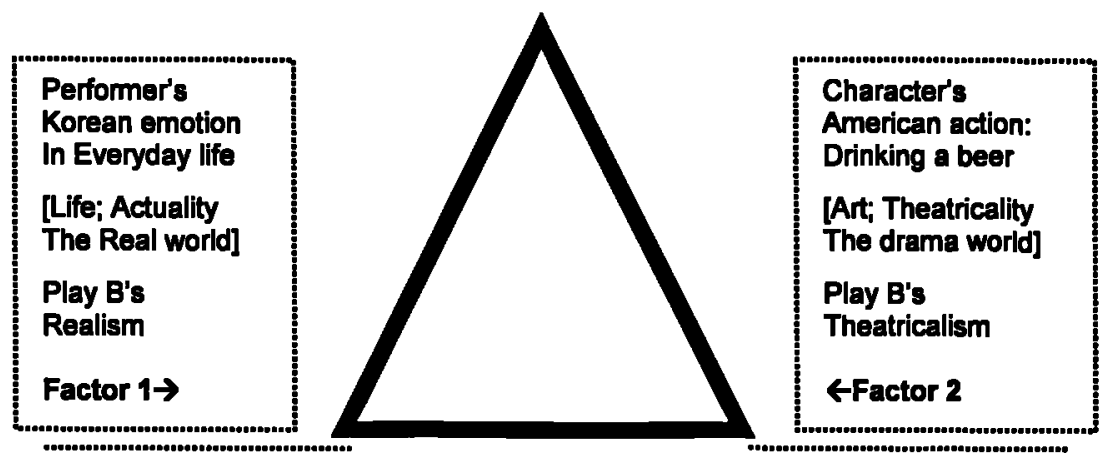

Figure 6.4 Being Dialectical in the Cultural Clash of KimJA (Penformer's Korean Emotion vs. Character's American Action on 4 April 2001)

KimJA as a student with a Korean attitude in the real world, imbued with Korean culture, is at the bottom left of the triangle, whereas KimJA as the American president drinking a beer in the drama world is at the bottom right. 
And then, I wondered whether KimJA only looked embarrassed or really was embarrassed and how she negotiated and acted the aforementioned tension in that intercultural context. To consider this, $I$ intend to move to the next levels-the sensibility level and the sensitivity level, which were mentioned along with the first level of sensation in 5.1.2. I shall take her Self-Questioning form, in which she conducted a dialogue with herself shortly after the drama session on that day. The following was her Self-Questioning about that moment.

\footnotetext{
What action do I want to talk about more to myself among today's drama activities? I would like to talk about Jones, whose behaviour is nonsensical or rather with big self-indulgence.

What relationship did I have to others? I was the American president, and my counter-partner was the Japanese president with his two Japanese subordinates. We had a trade business meeting in a restaurant in Japan.

How did I feel about myself as a person who relates to others? I thought I took the Japanese side and I was friendly to the Japanese characters because Japan has similar culture to us.

What have I done today during drama sessions in practice in the above question? I took the role of the American president. Nevertheless, my actions were brought in the Japanese perspective.

What have I thought but I couldn't do in the above situation? I don't like Jones, the American president. I think he is very impertinent.

Why did I think that was the most difficult thing to resolve? That was because I couldn't understand that kind of people. I haven't grown up to have such an impertinent manner.

How could I act in that situation if I were one of the people who come from England or America? They seem to cope with that situation far better than I because my character, the American president, has the same culture that they have.

What do I want to do in a similar situation to that in the future? I think it is necessary to promote cultural interchange and to educate that kind of intercultural communication in order to overcome cultural differences. So, Jones should have understood what Japan is like in terms of his cultural dimensions.
}

[From Self-Questioning form of KimJA on 4 April 2001]

According to the above Self-Questioning, the aforementioned embarrassment led KimJA to understand that she regarded the American character's behaviour as nonsensical or self-indulgent at the sensibility level. In the end, she expressed her embarrassment at his rudeness by laughing awkwardly, as her total imaginative response to that context.

My focus relevant to her cultural conflict is on her dialectical response to two sides, Korean [Factor 1 in Square A of Figure 6.4] and American [Factor 2 in Square B of Figure 6.4]. The American president, whom she acted as in the drama world, was more affected by a Korean performer rather than the American character. That is to say, KimJA relied on the Korean side related to her everyday life, the real world which 
KimJA as a Korean high school girl lived in and belonged to, rather than the American side connected with her drama activities in drama sessions, the drama world, which KimJA as the American president should put herself into and act in.

Her action of the American president's drinking a beer started with her Korean emotions such as feeling embarrassed, mentioned in relation to Figure 6.4 in 5.1.2. At the next level of sensibility, she understood the American action was nonsensical and that understanding was closer to Korean culture in the real world than American culture. Furthermore, what she acted in the end at the last level of sensitivity was like ridicule and that acting was far nearer to Korean culture, or rather I can say the American president was ridiculous in her perspective as Network 6.1 indicates.

This adjacency also indicates her acting was most dependent on her own Korean culture, among other factors in this intercultural context, as shown in Network 6.2. I therefore can say that KimJA was still at 'the ethnocentric stages' rather than 'the ethnorelative stages' despite the fact that she was doing the action in an American cultural background-drinking a beer (Bennett 1993: 29).

So far, I have explored two dialectical cases between realism and theatricalism in ICM through drama: one was examined to achieve a satisfactory synthesis of participants in everyday life and participants-in-role as co-researchers in their EDIE project; the other investigated how to negotiate a cultural conflict between participants in everyday life and participants-in-role in their drama sessions. These two cases were explored in the common context of being in and out of everyday life and moving into the drama world, Play A's theatricalism or Play B's theatricalism.

As a result of this investigation, I can reconfirm the belief that 'Every-day life became a source for improvisation, for the study of the stage-character in order to create an original role' (Lewicki 1996: 21). Also, everyday life has powerful factors in intercultural drama, and everyday life, which is imbued with cultural backgrounds, is more dependent on the dialectical process of performers' characterisation, especially in the ethno-centric cases, which I explored in this study. 


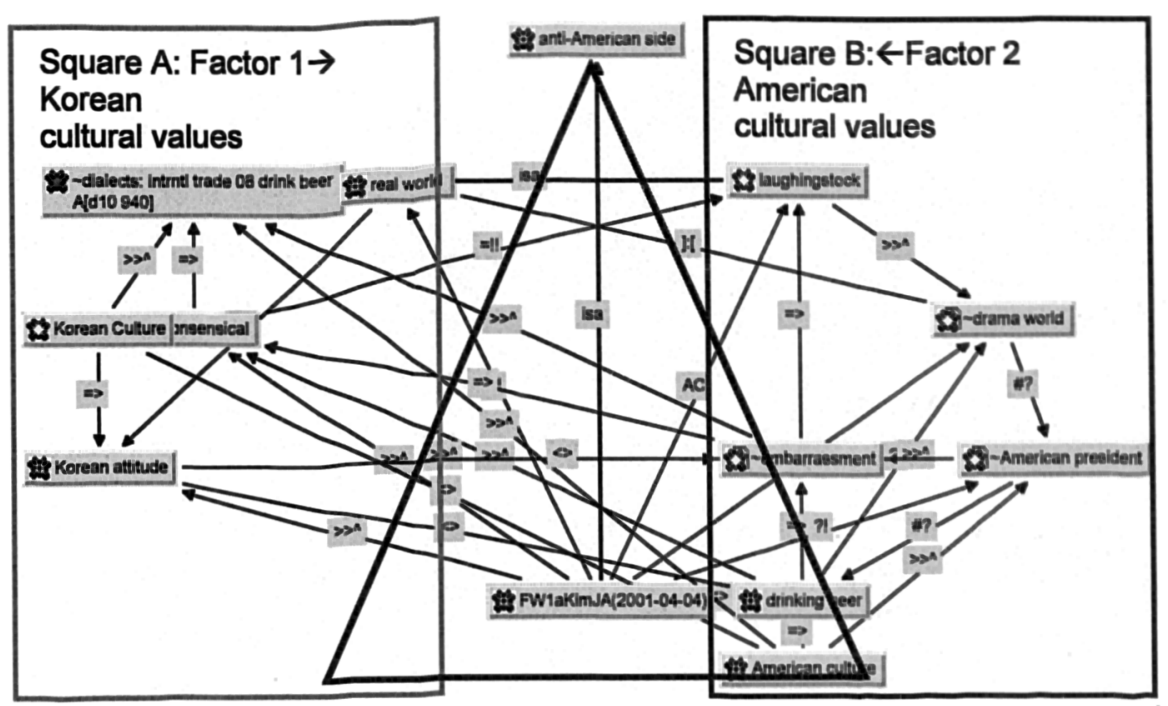

Network 6.1 Dialecticality between a Participant in her Real World and her Drama World in ICM by the Semantic Layout

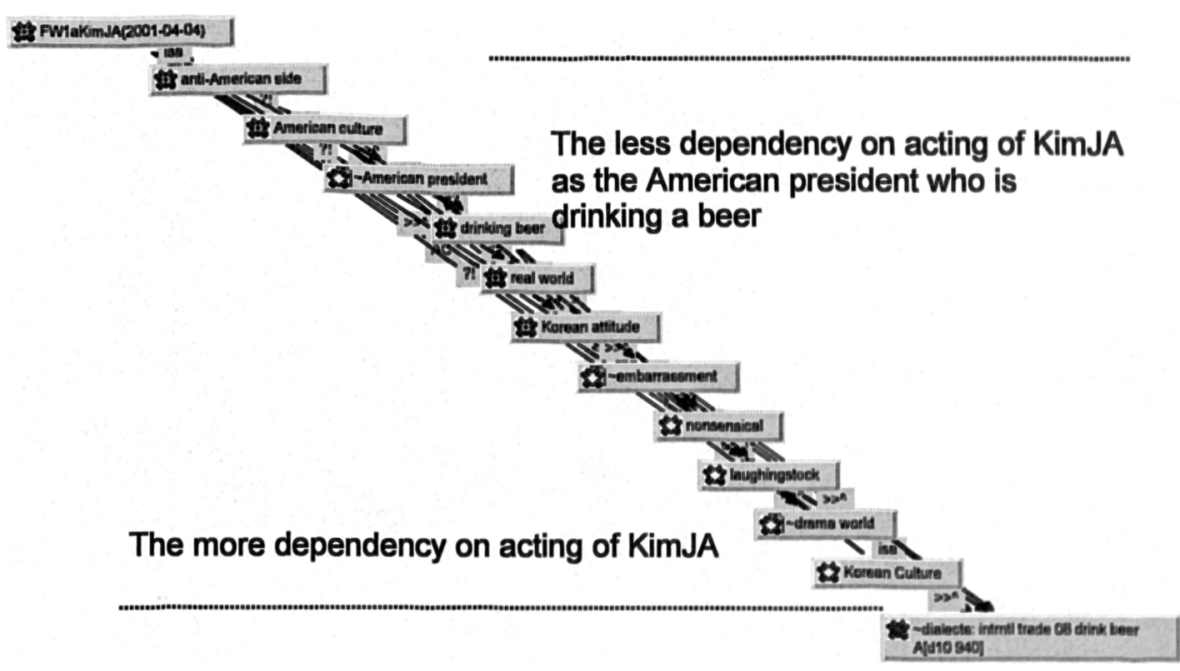

Network 6.2 Dialecticality between a Participant in her Real World and her Drama World in ICM by the Topological Layout 


\subsubsection{Dialecticality in the Dual Drama Structure [Play B in Play A] in ICM}

In the present subsection, I intend to explore the dialecticality between being in and out of a stage production in intercultural Play $B$ [drama in drama sessions] throughout the EDIE project, that is, Play B within Play A. There are three different kinds of time and space in Play $B$ within Play $A$, and I already described their differences in Figure 6.1:

[1] Play A's Theatricalism, with which participants-in-role were connected by doing action as co-researchers in the EDIE project with the help of a drama technique-mantle of the expert.

[2] Play B's Realism, to which participants-out-of-role were related through discussing and rehearsing in the process of their performing as performers in drama sessions.

[3] Play B's Theatricalism, in which participants-in-role lived by acting as their characters in drama sessions.

On the basis of the above patterns, I shall identify the dialecticality of intercultural drama first by examining how the inner lives of participants were related to their drama activities in analysing the intercultural contexts of co-researchers. Second, I shall explore how participants performed their characters that had different cultural backgrounds from performers', and third, I shall investigate how they did drama as performers and observed their actions as spectators-and-performers in intercultural drama.

\subsubsection{Sub-Text, Text and Meta-Text in Participants' Drama Writing}

The present subsection focuses on how participants handled a text extract which they had to perform. In the process of handling the extract, the participants were mainly engaged as co-researchers, performers, and characters in Contexts 2, 3, and 4, to make shifts between those contexts. The extract was written about the International Business Meeting, described earlier. At this stage, however, the relation between the intercultural extract and co-researchers will be examined in terms of another tripartite dialectic formula between subtext, text, and meta-text.

I shall begin with Beckerman's introductory comments on avoiding the imbalance between the aforementioned:

Present understanding of the dialectics of activity and the imagined act is influenced considerably by the teaching of Stanislavski. In play analysis one speaks of a text, the lines of the characters, and a subtext, the inner life of the 
work. Robert Hethmon quotes Lee Strasberg as saying that the actor must "concern himself with the subtext, the inner life of the character; he must avoid an overconcern with words and cues." This approach presupposes a subordination of activity to inner life.... To avoid this imbalance, we must go beyond Stanislavski's pioneering influence in close analysis toward a more comprehensive approach (Beckerman 1970: 27-28).

Thus, Beckerman criticised Lee Strasberg saying that if the inner life is given undue emphasis at the expense of the texture and shape of the activity, the artistic balance between the two is weakened in the end.

What I try to make clear from the quotation is to define sub-text as the inner life of the co-researchers, in which they live with their pathos and ethos at their individual and socio-cultural level, whereas text is a context or an extract, which they are given. On the basis of these definitions, I shall define meta-text as that which they produce or write with the text in terms of drama literacy.

On 4 April 2001 participants as co-researchers were given the intercultural extract to dramatise for their performance. At the first stage, they were asked to read the extract with my explanation about some vocabulary relevant to business, as well as general terms. At the next stage, they made two still-images about the two firms and took photographs of them with a Polaroid camera, which allowed us to compare and discuss those still-images immediately after their activities. The photographs were presented as Figures 5.1 to 5.4 in relation to socio-cultural experience in drama as cultural morphology in 5.3.2.

Their first response to the context after the above activities was their great surprise, embarrassment, and even anger. The following is what I wrote in my field diary in relation to their response to the context:

\begin{abstract}
What I was so surprised at is that most of participants complained about the attitude of the President of the American Company, Jones, and that even some participants were angry at his attitude and behaviour. They felt the American president, Jones, was so rude to the Japanese representative of the Japanese Company. I thought the thought of participants about this situation seem to be the similar one to the situation in which participants cope with this sort of young American president. My participants seem to be more conservative than I expected them to be [My field diary on 4 April 2001]
\end{abstract}

They were very embarrassed to discover the fact that the American president was described differently in the extract from what they had expected him to be like. They felt the 25-year-old American president of the small company, for instance, was too young to face the 60-year-old Japanese president of the well established and highly 
respected contracting firm and to manage their international business meeting in Japan and he appeared to be very rude to the senior Japanese president.

Co-researchers described those kinds of feelings in their Self-Questioning papers, especially, in the Self-Questioning paper of MunJM, who took the role of the Japanese president. My assistant teacher also confirmed that participants got uncomfortable with the behaviour of the American president while discussing with me after the drama session.

Furthermore, there was another source of development for them related to their use of language. Co-researchers, participants-in-role, thought they were to use only the English language because of their involvement in the EDIE (Educational Drama in English) project, but they realised that they should use the Japanese language in their performance along with English because in part of the scene Japanese characters had conversations with each other in their own language, Japanese. They were excited at using two different foreign languages, both English and Japanese, in one class, although they had been learning the Japanese language as a second foreign language in the National Curriculum.

This means they were concerned about three different languages-Korean, English, and Japanese. They felt as if they had been in a real intercultural situation. This was because they communicated with each other in the three different languages while they were acting that scene. [From the transcript of Drama Sessions, Self-Questioning, and Discussion with the assistant teacher's comments on 4 April 2001].

I can again explain their emotion with the dialectical tripartite formula between their Korean cultural values, American cultural values, and their emotion at the sensuous level. Co-researchers could not accept American behaviour which was depicted in the extract in the light of their cultural values. It can be seen from Figure 6.5 that they resisted the text against the sub-text and produced a third one-their meta-text, which led them to move onto Levels 2 and 3-sensibility and sensitivity.

Emotion at the sensation level, Level 1, was mixed up in two different intercultural contexts. One intercultural context was given between the real world and the drama world inside and outside the extract.

Participants were more dependent in the real world at this stage of analysing the text than the next stages, such as rehearsing and performing its play which was dramatised by them. This was because their Korean culture remained in the inner lives 


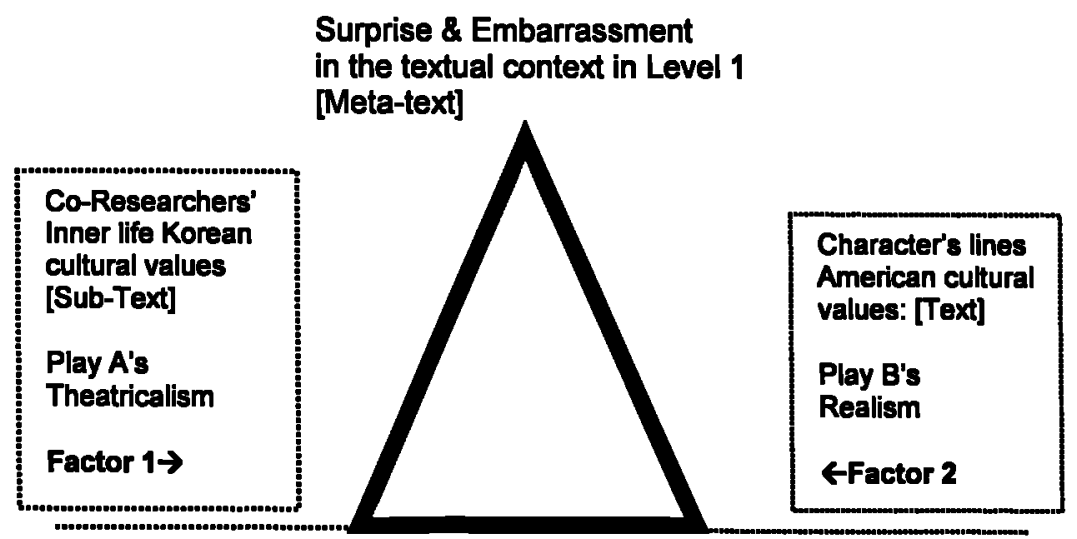

Figure 6.5 Dialecticality between Sub-Text and Text in an Extract of the Intemational Business Meoting

of co-researchers, whereas the American and Japanese cultures interacted with each other inside the extract. Another intercultural context was given within the extractbetween the American cultural and the Japanese culture. Both intercultural contexts, whichever participants put more weight on, were involved in participants' meta-textual process between sub-text, text, and meta-text.

Let me find an example of the former case of focusing on intercultural context given inside and outside the extract. In this case, analysing the extract, co-researchers stood by the Japanese side or against the American side, as shown below from SelfQuestioning papers of participants.

How did I feel about myself as a person who relates to others? I thought I took the Japanese side and I was friendly to the Japanese characters because Japan has similar culture to us.

What have I done today during drama sessions in practice in the above question? I took the role of the American president. Nevertheless, my actions were adopted from the Japanese perspective.

What have I thought but I couldn't do in the above situation? I don't like Jones, the American president. I think he is very impertinent.

- [From Self-Questioning form of KimJA on 4 April 2001, mentioned before in 6.1.1]

How did I feel about myself as a person who relates to others: It didn't look good to me that the American president behaved in another country as he does in his own country, especially in the different case between two countries.

What do I want to do in a similar situation to that in the future? I would like to teach people such as the American president how to behave themselves in communicating with foreigners.

[From Self-Questioning form of RyuKW on 4 April 2001] 
What have I done today during drama sessions in practice in the above question? I as the Japanese president was shocked and seemed to be reluctant to have the meeting at its beginning. In its middle, I left my subordinates after giving them my delegated order.

[From Self-Questioning form of MunJM on 4 April 2001]

Participants did not take sides with the American president but regarded his behaviour from the Japanese position alongside their Korean position.

In focusing on the intercultural context given within the extract, however, participants put themselves in a different position from the above, although they still were in favour of the Japanese representatives with their Korean attitudes.

What action do I want to talk about more to yourself among your today's drama activities? I would like to know how Americans behave themselves in meeting people who have different cultures from them.

How did I feel about myself as a person who relates to others: I [as the American president] was very embarrassed because I didn't know about their Japanese culture.

What have I done today during drama sessions in practice in the above question? I as the American president was drinking a beer before the Japanese representatives arrived there.

What have I thought but I couldn't do in the above situation? I encouraged myself to do my best in acting.

Why did I think that was the most difficult thing to resolve? Because I am not a good English speaker my English pronunciation sounds bad. Also, I haven't practised enough.

[From Self-Questioning form of LimEY on 4 April 2001]

What have I thought but I couldn't do in the above situation? It was difficult for me [as a Japanese subordinate] to express the situation with only gesture, because I didn't know about what the Japanese is like. Also it's difficult to handle my facial expression.

Why did I think that was the most difficult thing to resolve? This was because I didn't understand the Japanese culture enough to act that.

How could I act in that situation if I were one of the people who come from England or America? They may work differently because they have different cultures from the Japanese culture.

[From Self-Questioning form of KimEL on 4 April 2001]

The above two participants pointed out their insufficient understanding of the Japanese culture although they took different roles: LimEY as the American president and KimEL as a Japanese subordinate.

With respect to this intercultural meeting context, participants were classified into three groups depending on their foci. This is because each participant had her own cultural 
values, that is, her own sub-text, to speak in relation to the topic of this subsection. The first group of KimJA, RyuKW, and MunJM put weight on the American president's behaviour in the context, meanwhile the second group of LimEY and KimEL paid more attention to their misunderstanding of the Japanese culture relevant to the context. Also, there was the neutral group between these two groups, which I describe next.

Each participant resisted the intercultural context in the script depending on what subtext she had, and therefore responded to it in different perspectives. In other words, this neutral group was neither against the American side, nor for the Japanese side, so much as the first group was. Thus each participant analysed the same extract in her own way and individualised it for her performance, which is called individual metatextuality in 5.3.1 in the sense that each participant individualises the context.

I therefore can say that sub-text is regarded as the co-researchers' background, which is expressed by social, or individual, actions, treatment of the extract, and a code of attitude to which those actions adhere. Sub-text is a mediating element between text and meta-text comparable to the relation of ground-figure. In other words, the phenomenon of meta-textuality was created on the ground of their sub-text and was figured as meta-text. Both sub-text and text function as mediating elements in the dialectical process of meta-textuality.

Furthermore, this dialectical meta-textuality is conceived as another dialectical relationship between an individual person and the society, to which the person belongs. Also, the individual person experiences that sort of dialectical format if he or she faces two different societies. The latter meta-textuality will be examined in intercultural situations, where participants faced two cultures, such as co-researchers' society and the society of their characters, in the next subsection.

\subsubsection{Co-Researchers [Play A's T], Performers [Play B's R], and Characters [Play B's T]}

How did co-researchers manipulate the process of meta-textuality in the given intercultural situations between their society and the society of their characters? To examine this question, I shall take an example of MunJM, who took the role of the Japanese president in the context of the International Business Meeting. Moreover, I intend to focus on what emerged in the process of her handling the ICM from the standpoint of the co-researcher rather than in the other two perspectives. 
During the discussion, which was followed by the analysis of the aforementioned extract about the International Business Meeting, MunJM made her predictions as a co-researcher about the success of the meeting as shown in the following transcript of the drama session on 4 April 2001:

The teacher. I am wondering whether this meeting will be successful or not...

Some co-researchers: (at the same time) Unsuccessfull

The teacher. Why do you think so?

Some of them: Because of differences in their way of thought, opinions, and their embarrassment, it can be unsuccessful.

The teacher. Why are they embarrassed?

MunJM: The American president seems to be easy-going and to be in a hurry; on the other hand, the Japanese representatives try to handle the meeting in a formal way. So, one has very great differences from the other.

ParkJM: They are too different, aren't they? So, you have to accept that....

MunJM: No. We should understand that. We have different ways of thought, don't we?

[From transcript of the Drama Session on 4 April 2001]

MunJM as a co-researcher presupposed that the meeting would be unsuccessful by emphasising that because people had their own values it was acceptable to have their differences even in an intra-cultural situation, where they shared their cultural values with others within their society. Her prediction was made from attitudes of characters that did not think about their differences in that intercultural situation, rather than because of the differences per se.

At this stage, her Self-Questioning paper needs to be examined in terms of how she got to the prediction-the process of her meta-textuality.

What action do I want to talk about more to myself among today 's drama activities? I would like to talk about Goto, who is the Japanese president.

What relationship did I have to others? The Japanese president was conservative and old.

How did I feel about myself as a person who relates to others? I was very surprised at the young American president. What a surprise to meet such a young president . ...

What have I done today during drama sessions in practice in the above question? I as the Japanese president was shocked and seemed to be reluctant to have the meeting at its beginning. In its middle, I left my subordinates after giving them my delegated order.

What have I thought but I couldn't do in the above situation? Ah, it's Japanese! I had to speak the Japanese language, as well. Oh, dear....

Why did I think that was the most difficult thing to resolve? Because I am not a Japanese person it is very difficult for me to express what I want to do in Japanese. 


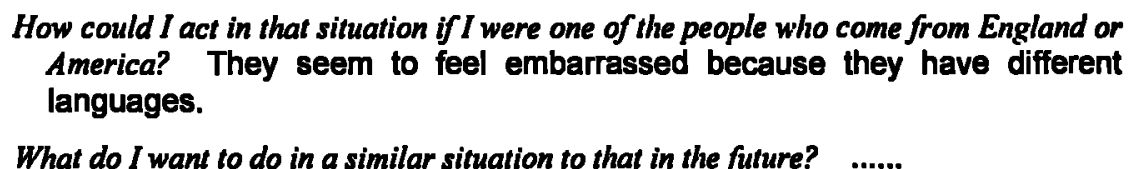

[From Self-Questioning form of Mun IM on 4 April 2001].

According to the above Self-Questioning, I can say her surprise was the key moment in the intercultural context. She was surprised to face two facts-the very young American president and the Japanese language. The former was given from the standpoint of a co-researcher in Play A's theatricalism as well as the character of the Japanese president in Play B's theatricalism. She considered the Japanese language from the viewpoint of a performer in Play B's realism. Her surprise at the Japanese language is on the simple ground that she could speak not only English but also Japanese in that drama session. This was because she did not expect to speak two different foreign languages in her foreign language classes as well as in the EDIE project.

Her surprise at the young American president, however, is not so simple. Her surprise at the young boy, the American president, was perceived from her three standpoints of co-researcher, performer and character. Therefore, her response to the young American president was the key moment in analysing the ICM. This is because her surprised emotion was connected with the real world in Play B's realism as well as the drama world in Play A's and Play B's theatricalism, as shown in Network 6.3.

More factors were interrelated to this process of dialecticality in relation to that response, as shown in the network. I shall focus on dialecticality in the tripartite formula of $C, D$, and $F$ from her standpoint as the co-researcher in Area $F$, although dialecticality of seven more tripartite formulas can be discussed.

Let me start with the presentation of the network: Lines a-a' and $b-b^{\prime}$ in the above network are indicated as basic boundaries: Line $a-a$ ' indicates the transitional area between Play $A$ and Play $B$, meanwhile Line $b-b^{\prime}$ covers the transitional area between the real world and the drama world. Because of this demarcation, four relevant areas mean respectively as follows:

- Area $C$ in the upper left of the network-the drama world in Play $B$ [as characters];

- Area $D$ in its right upper-the real world of Play $B$ [as performers];

- Area $E$ in its lower right-the real world of Play $A$ [as students];

- Area $F$ in its lower left-the drama world of Play $A$ [as co-researchers]. 


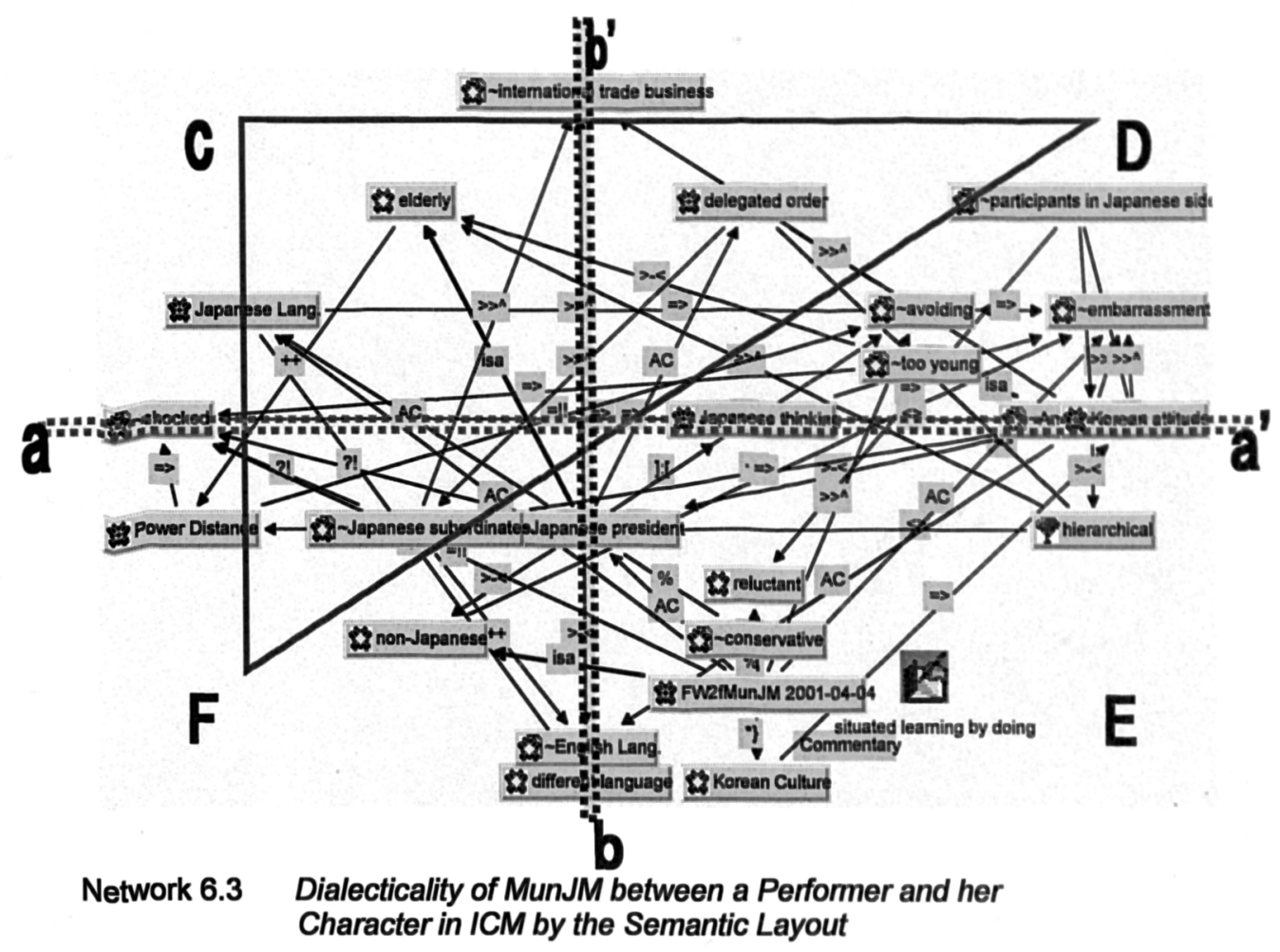

MunJM as a co-researcher realised with the help of a drama technique, 'mantle of the expert' in Play A's theatricalism (indicated in Area F) as follows: first of all, she felt shocked to meet the young American president and then this shock generated from her attitude in reference to power distance, which Hofstede (1980) points out as one of cultural features. Her resolution of power distance indicated in Area $F$ was taken into consideration on two different grounds- her character side (Area $C$ ) where the elderly Japanese president required respect because he was old, and meanwhile her performer side $(A r e a D$ ) where she felt embarrassed because the American president was too young.

The key point in this context was how old they were and how others should treat them and in the end, MunJM as a co-researcher regarded the main cause of unsuccessful communication in that intercultural context as power distance, which the Japanese and Korean societies have.

In the process of analysing the intercultural context, I can say, MunJM as a coresearcher thus paid two kinds of attention to this moment in relation to Beckerman's concepts of ground-figure. On one hand, Play B's realism was regarded as the ground on which the co-researcher was, that is, her uncomfortable feeling about the young American guy in her performer dimension with Korean attitude and Japanese thinking 
in Play B's realism (Area D). On the other hand, Play B's theatricalism, seen as the figure which she made, that is, her resisting the young American president as her counter partner in her character dimension as the elderly Japanese president in Play $B$ 's theatricalism (Area C).

This position as the co-researcher in Context 2 enabled her to get distance from her given context in her Play B. Therefore she had more objectivity and a more holistic view in investigating her context by considering both her performer dimension in Context 3 and her character dimension in Context 4.

This reminds me of Rubin's double profiles, mentioned in 3.1.1, on the ground that MunJM contemplated how she acted by paying simultaneous attention to both dimensions in Contexts 3 and 4. As a result of this, she found the existence of power distance in that ICM-dialecticality connected with power distance between the American and Eastern cultures. The perspective in which the co-researcher analysed Play $B$ (Areas $C$ and $D$ ) in Play $A$ 's theatricalism (Area $F$ ) provided her with the outsidedness where she analysed Play $B$ outside it and eventually with the reflexivity for herself as a student in everyday life.

\subsubsection{Reception and Production of Participants as Spectators in Play B' s R and T}

The meta-textual image of the performer standing between the spectator and the text examined in the present subsection overlaps with the meta-textual image given in the dissociation from the performer's role.

In the present section, however, I shall examine this from a different perspective from the meta-textual image described in the previous subsection. Its focus will be on dialecticality between authorship of a participant as the performer and her spectatorship as the spectator. I intend to pick cases where participants encourage other participant performers in Play B's theatricalism because their encouraging each other appeared to be one of the most effective factors in carrying out the EDIE project.

In order to do this, performances done in the drama sessions on 11, 12 April 2001 and their discussion after their public performance on 21 April 2001 will be examined. All these cases are chosen in connection with the participants' nervousness about learning English as a foreign language, which has to be overcome in order to become an intercultural actor, mentioned in 2.2.2. 
First of all, let me remind the reader that participants were wondering, or rather were nervous about whether they spoke their lines in English well enough or not. One of their difficulties was to say their lines in an appropriate way, for instance, a proper intonation for a certain context. Participants were asked to do practice exercises relevant to this activity with simple dialogues in classroom drama. The following episodes show participants were afraid of speaking English, as they revealed in my interview with them, and therefore they needed to encourage each other to try to speak the language without becoming more nervous.

KimMS: (out of role in her authorship) It's me who should speak the most important part. ...

LimEY: (in role in her spectatorship) Yes. Why not?

KimMS: (out of role in her authorship) All rightl All right, all right!

All of the participants in role in their spectatorship are laughing.

KimJA: (in role in her spectatorship) (with her big smile) We have no time for that! (Clears her throat).

KimMS: (in role in her authorship) Robert! You are a GENIUS!

KimHJ: (in role in her authorship) Yes, I AM.

All of the participants in role laugh again.

KimJA: (in role in her spectatorship) Well done, jolly good!

LimEY: (in role in her spectatorship) Oh, you did too well, indeed!

[Transcript of the Drama Session on 11 April 2001]

ParkJM: (in role in her spectatorship) (look at YunKE who is performing) Look at her face, turned red!

KimMH: (look at YunKE) Even so, I am sure she will do well!

RyuKW and YunKE keep acting in English and participants in role in their spectatorship sometimes laugh.

[Transcript of the Drama Session on 12 April 2001].

As indicated in the above two episodes, participants (LimEY, KimHJ, KimJA and KimMH) helped nervous performers not to become more nervous. Those episodes, however, are different in their positions and in a chronological sense. I shall explain the former episode with Beckerman's Figure-Ground relation. LimEY and KimJA as participants in spectator roles related KimMS's performance to two main factors-the performer's introverted personality and her performance with audiences, and expected the performer to be in a contradictory situation between herself and her performance.

With respect to this, the two spectators intended to encourage the performer, KimMS, to reduce her nervousness immediately before the performer did. At that encouraging moment, the two spectators were engaged in Context 3 of fellow participants as well 
as in Context 4 of spectators on the basis of being in Context 2 of co-researchers. Thus, the participants function as a dialectical resolution to solve a conflict between KimMS's personality and her anxiety about the performance in English by contemplating both of the factors as the Ground and the Figure in the situation where KimMS was.

Moreover, this is relevant to another Figure-Ground case of performer-spectator during their performance in the positions of spectators rather than fellow performers. Participant spectators helped another performer, YunKE. Shortly after hearing from a participant spectator, ParkJM, that YunKE, who was about to perform, turned red, YunKE became more nervous. After that, another participant spectator, KimMH, encouraged YunKE to perform well. KimMH's encouragement was against the innocent but discouraging response from ParkJM, functions as a dialectical resolution to solve a conflict between YunKE's anxiety about performing in English and the spectator's response of stimulating the anxiety of the performer, YUnKE.

Spectators thus have a dialectical relationship to performers in that situation of their common uneasiness about learning English as a foreign language. YunKE as a performer and KimMH as an audience or a spectator communicated with each other. Participants passed through this sort of dialectical experiences in the EDIE project along with dialecticality of drama per se between actuality and theatricality of drama.

However, what is taken more into consideration from the YunKE's case is the function of $K i m M H . K i m M H$ as a spectator was not just engaged in reading drama in the perception cycle, but also was participating in writing drama with her authorship by encouraging the performer not to become more nervous as a spectator at that moment.

This interaction between YunKE and KimMH, that is, between performers and spectators/audiences makes drama become more participatory and in the end can break down the boundary between performers and spectators, like Boal's works in our contemporary drama/theatre, mentioned in 3.3.1.

It thus emerged from the above two cases that participants did not just get their spectatorship in the position of spectators or audiences by getting involved in the performance, by observing or reading it, but also applied the spectatorship to participating in writing drama by responding to performers' acting. I have presented the above two cases in relation to dialecticality of spectatorship between performers 
and spectators/ audiences, not from the standpoint of performers but from that of spectators.

And then I wondered about another standpoint, what the spectatorship is like from the viewpoint of performers-how did the performers get through the common apprehension among themselves in terms of the spectatorship during the public performance? I speculated about what participants as performers observed and how they handled their common tension about speaking English among performers themselves within their big public performance, where they should speak their lines in English much more and longer than in the two simple classroom dramas.

For the above question, I shall take our second last research meeting we had shortly after the public performance, which was done on 21 April 2001. We exchanged what we observed and thought about other performers from the perspective of the spectatorship as co-researchers (Play A's Theatricalism) as well as performers (Play B's Realism) during the performance. The following parts are selected from the meeting.

KimHJ: I didn't remember my lines. And I stammered out all the time. To make it worse, I couldn't manage even remembered lines. KimEL, I am really terribly sorry about this. I made you very CONFUSED.

KimEL: Oh, don't be silly. I also did nearly make mistakes.

Participants: (all together) me, tool (All participants laugh at the same time).

YunKE: To be honest with you, I didn't have many lines. Nevertheless, I didn't remember all of my lines and therefore I feel really sorry for not remembering them. Furthermore, I am very sorry about that because even other participants who I think did very well talked about their leaving much to be desired. I didn't remember even my simple lines, meanwhile the other many participants almost managed to do their lines. I am very sorry, indeed! (All participants laugh again) Anyway, I feel really sorry for that.

LimEY: We seem to feel more apologetic than you.

KimHJ: Yes, indeed! She is a fairly representative group of great regret. (AII participants laugh again).

KimEL: Well, I took...um.... I took Mum's role as counter partner of Daughter, which KimHJ took the role of. We should speak our lines one after another, shouldn't we? By the way, she forgot her lines and I forgot mine as well. However, we tried to speak our lines as if we had had conversation with each other in our real world in order to match that present moment properly. I know we made so many mistakes because we tried to change and add our lines to different parts from their planned ones. Nevertheless, we could perform together in harmony. I think it was not bad at all. Our lines seemed to be natural and to go smoothly.

KimHJ: That is because you did well, isn't it?

[From transcript of the Saturday Research Meeting on 21 April 2001] 
According to the above, performers felt that there were many things yet to do but had a feeling of self-achievement. For instance, they managed to make the big performance in spite of their low self-confidence in speaking English. Each performer encouraged other performers, and vice versa. In the first part, KimHJ and YunKE mentioned that they felt sorry about forgetting their lines and therefore about making other performers uncomfortable during the performance. KimHJ felt a conflict between her bad performance of her lines and good performance of her partner, KimEL, and tried not to spoil the performance as a whole and her partner's performance. That is to say, apologetic feeling and desperate efforts of $\mathrm{KimHJ}$ is the dialectical resolution from her conflict between her speaking her lines clumsily and confusing her partner's.

On the other hand, KimEL, the partner of KimHJ, in the second part, recognised that her partner, KimHJ, forgot her lines too often to keep faithful to the script while they were performing. KimEL, herself forgot to speak her lines occasionally. Therefore, she needed to solve the problem of speaking their lines in order to keep their lines natural and smooth within the whole context of the play. Her resolution in reference to speaking English lines was to imagine her role as Mum as if she were speaking in her everyday life.

This imagination freed her from that uneasiness about her forgotten English lines as well as her partner's. This resolution in her spectatorship was made from the dialecticality of the tripartite formula between her lines, her partner's lines, and the performance, which they wanted to make successful. Also, she didn't like to discourage her partner, KimHJ, who forgot her lines quite often and during the meeting still encouraged KimHJ not to feel so sorry about her performance.

Participants thus had dialectical experiences to solve two different factors through their spectatorship, in the position of a performer as well as a spectator. It appears that participants did not just observe other performers from the standpoint of performers as well as spectators, but also observed themselves from the viewpoint of the performer. As discussed in 3.2.1, the former was relevant to the spectatorship, whereas the latter, to self-spectatorship, which will be investigated in the next section.

Another to be considered is that participants had more difficulty in their performance because they had to perform the play in English. This difficult situation led them into more difficult intercultural situations, which were related to both linguistic and sociocultural competence and drama competence. As indicated in the above parts, participants as intercultural actors had difficulty in speaking their lines in English in the middle of the big performance. 
Nevertheless, performers were encouraged by other performers, and vice versa, by the spectatorship in their position of performers as well as spectators/audiences, in Play B's Theatricalism. This encouragement in the end provided participants with opportunities to overcome their common apprehension and to become more interested in learning English as a foreign language or a lingua franca, as mentioned in their two Personal Joumals (on 6 and 21 April 2001). In a word, without their mutual encouragement participants could not have done the public performance and could not have become more interested in using English in everyday life. This statement will be discussed with respect to what to teach students who live in a global society in the last chapter of this study.

\subsection{Dialogicality of ICM in Drama Activities}

In the previous section, I described dialectical interactions based on Neo-Kantian dialectics whereby a resolution emerged between two different constituents, which are intrinsic to the multiple contexts and the production-perception duality. In the present section, however, I am going to widen the focus to describe the dialogical interactions with ICM through drama.

\subsubsection{Dialogicality of ICM in Drama Activities}

Bakhtin (1961), in Problems of Dostoevsky's Poetics (1984), caught the meaning of human existence in communication:

To be means to communicate.... To be means to be for another, and through the other, for oneself. A person has no internal sovereign territory, he is wholly and always on the boundary; looking inside himself, he looks into the eyes of another or with the eyes of another [author's italics] (Bakhtin 1961: 287).

As implied in the above statement, Bakhtin first relates the meaning of human existence to human communication with respect to being 'for another,' and then as its next stage advances the idea of communication 'through the other, for oneself.' The ultimate aim of the communication is not just at otherness to communicate with another, but also decentring from our internal views into the eyes of another or with the eyes of another. This means that to be outside ourselves is the better way to communicate with oneself rather than to be inside ourselves.

From this perspective on otherness and outsidedeness, I am going to start with dialogicality in simple cases. These cases will be taken from the Passport Game of 
drama activities to analyse how participants responded to and handled their ICM through drama and what outcomes they got from what they did.

\subsubsection{Dialogicality in the Extroversive Interaction of Participants with Intercultural Factors in Drama Activities}

As described in the first section of the present chapter, participants as human beings were inextricably in communication with another, in terms of the two dualities in drama shown in Figure 6.1 and 6.2-actuality and theatricality; production and perception. These dualities led participants to be involved in dialecticality in order to resolve two different factors in ICM through drama. The dualities did not restrict participants but allowed them to arrive at the stage of dialectical resolution to get involved in the stage of 'Self-pluralism,' through which participants obtained dialogical outcomes (Altrocchi 1999: 170).

It appears appropriate to define these aspects of the dialogical outcomes as dialogicality, as I argued in 2.2. The dialogicality however relates to two different dialogues-extroversive dialogues with others and introversive dialogues through others with self.

In this subsection I shall first give an idea about basic extroversive dialogues of participants, that is, how participants interacted dialogically with others, for instance, between participant actors and participant audiences, or between actors in ICM through drama. And then introversive dialogicality will be presented in the next subsection.

In order to pinpoint the potential implications for participants of the extroversive dialogicality and its effectiveness, I shall present a crucial mid-phase from the Passport Game. This will be used as a means of showing how those who have different cultural values, and therefore culturally different identities, handled ICM in and through drama.

The Passport Game was done in drama sessions on 28 and 30 March 2001. We began by cutting papers with left-handed scissors. This activity with the left-handed scissors enabled the participants, who all were right-handed, to get a feel of what an alien experience was like. And then participants, who were divided into three groups, were asked to create their own countries which each group imagined they belonged to: their own languages that they spoke there, their own costumes improvised with 
some clothes, and their own ways to greet. In the next stage participants made their own passports in order to travel to other countries, which the other groups created, and at the end of the Passport Game they created immigration procedures by turns as immigration officers as well as immigrants with their passports which they had produced in their own imaginative ways.

From the aforementioned activities, I will take a sample of participants' experience in creating immigration procedures, and explore first how they communicated and second what they got from that ICM.

First of all, the observer, who was my assistant teacher, monitored the whole drama session and observed:

It was revealed that participants had difficulties in communicating with each other in English while they were doing the Passport Game in today's drama session.... They should have had more experiences of understanding what they could do in the process of the immigration through their proper conversation between immigrants [participants-in-role] and immigration officers [participantsin-role]. Some of them tried to ask only questions, which were familiar and simple to them [Brackets mine] [From Observation Records of the assistant teacher on 28 March 2001].

The assistant teacher pointed out participants could not speak English well enough to have an appropriate interaction between the immigrants and immigration officers. I therefore wondered what dialogues they had with whom or what elements in that situation. The following Self-Questioning gave me some clues:

What feeling did I have as the character? To be honest, I couldn't understand the
questions totally but I was very interested in adding our new words, such as
'yeob' at the end of each sentence,
What have I thought but I couldn't do today during lhe above situation? I had several
ideas, but I followed others and was more dependent on others' ideas and
did the drama activity with them. And I was not accustomed to yet
brainstorming. (Bold font mine).

[From Self-Questioning form of YunKE on 28 March 2001]

What have I done today? I tried to do my best; my English was not good enough, though.

What have I thought but I couldn't do today during the above situation? I wished other performers had asked those questions, easy and simple. (Bold fonts mine).

[From Self-Questioning form of KimMH on 28 March 2001]

In the above, it appeared that participants were nervous about interacting with immigrants or immigration officers in English in that drama context. In particular, they 
were afraid of becoming the focus of criticism in their interaction. In other words, I can see that they thought about or actually or had dialogues with their poor English skills in terms of interacting with 'other' participants rather than performing their language. Participants thus focused on extroversive dialogues with external elements, such as other participants on their English competence in Context 1 and other participants-inrole in Context 4 in the perception cycle of other performers rather than the production cycle of performers themselves.

On the other hand, my field diary had comments on the drama activity itself, which is significant in terms of another extroversive dialogue in the intercultural situation:

\begin{abstract}
Drama Activity in today's sessions was not good enough to deliver their content in terms of visible perspective in comparison with the drama activity itself. This is because I was focusing their activity on the perspective of their cultural experience. While all participants were taking the process of immigration at the Immigration office in drama sessions, some participants felt frightened and others felt very unfamiliar as well as very strange. After stepping out of their roles, some participants seemed to recognise that their immigration officers don't intend dealing kindly with immigrants in the sense that they should protect their country from other external factors [From the Field Diary on 28 March 2001].
\end{abstract}

The above Field Diary revealed how I thought the participants felt about the immigration activity-while doing their immigration drama activity in the Passport Game, participants seemed to feel frightened, unfamiliar, and strange. After the immigration drama activity, we discussed how they felt and what concerned them in that process. I picked out their comments on that intercultural situation:

The teacher. When immigration officers asked you, what kind of feeling did you have? If you remembered the feeling, you can write down about that.

LimEY: Pit-a-pat, pit-a-patl

The teacher. Pit-a-pat! (To other participants) What kind of feeling do you have?

KimJA: They seem to be rude.

The teacher: Rude? What do you mean by rude?

KimJA: I just thought.

The teacher. (Finds a piece of paper on which 'dirty' was written) Dirty? Do you feel dirty? Somebody takes a bribe? Why do you think the immigration officer is dity?

KimJA: Answer, the person who made this note, please.

The teacher. Did you feel very comfortable when we were passing through the immigration office? Do you feel comfortable?

Some participants: (at the same time) No! Really uncomfortable!

The teacher. Not at all? Why not? 
KimHJ: Stately ... strict... and commanding....

The teacher. You said the atmosphere in the office was authoritative. Why do you think they needed to do that?

ParkJM: Strict precautions might be essential in the immigration countries where native people should live with immigrants who come from different countries.

KimEL: That's true. Immigration officers could not identify immigrants until they finished inquiring into their identities, could they?

ParkJM: Immigration officers could be suspicious about which aims immigrants would like to come into the country. (Bold fonts mine)

[From Transcripts in the Drama Session on 28 March 2001]

From the above discussion, it emerged that participants perceived differently those immigration drama activities depending on which roles they took, immigrants or immigration officers, in Context 4, Play B's Drama World. The immigrant roles gave them a rather inferior impression, such as a feeling of nervousness and discomfort. Converse to this, the immigration officer roles left a superior impression, such as a feeling of powerfulness and strictness. In Context 4, participants observed and perceived the immigration drama activity which other participants performed, that is, had dialogues with performances of other participants within Context 4. And then through their discussion in Context 3 , they realised that they had the aforementioned feelings and why other participants tried to perform the activity in that particular way.

Participants thus saw that performance in order to get better intercultural awareness in that sort of intercultural situation is necessary in Context 3 rather than solely their selfawareness, although their socio-cultural backgrounds in Context 1 influenced the discussion in the production-and-perception cycle. I therefore can say these extroversive dialogues are construed as reflections on otherness through drama activities.

In other words, through their dialogical thinking in multiple contexts with their production-and-perception cycle, first, they realised why immigration officers-in-role were characterised as stately and august characters and second, they were aware that the immigration officers acted uncomfortably and in a commanding fashion for the security of their countries. These are the extroversive dialogues.

According to the Passport Game, participants above all had dialogues with one common feeling in the context-anxiety about uncertainty, which arose particularly in the use of language as a communicative tool with strangers. Participants thus related their communication to concern for others and had a better understanding of others or others' situations. 
In the end, I can say that participants were engaged with extroversive dialogicality to get involved in otherness, in which participants focused what they felt, realised, and understood others or other things through this Passport Game, for instance, what and why immigration officers could do for their jobs and how and why immigrants could feel in that situation. This sort of otherness will be further described in 6.3.2.

\subsubsection{Dialogicality in the Introversive Interaction of Participants with Intercultural Factors in Drama Activities}

The previous subsection introduced the idea about basic extroversive dialogues of participants who interacted with other participant actors, or other things in ICM through drama. These sorts of dialogues are focused on others and otherness rather than themselves; in short, the dialogues are based on putting participants' self into the other's shoes to make them communicate for others. Equally, participants had their introversive dialogues with themselves. Their introversive dialogues are focused on how participants interacted dialogically through others with themselves, for instance, between participant actors and their own characters, or between their drama situations in which their characters were, and their real situations in which they themselves were.

In order to show these introversive dialogues of participants, I am going to take a case of one participant, KimEL, who learned introversive dialogicality by experience of using a pair of left-handed scissors. In the drama session, participants were requested to cut papers with two different kinds of scissors, right-handed and left-handed, without any previous information about the scissors. While cutting papers, they talked about that activity with other participants.

KimMH: This [a pair of left-handed scissors] is more difficult to take hold of. Ah, I get it! I should grasp this in this way.

The teacher. All right then, can you try to do that again, everyone?

(All participants took hold of two pairs of scissors by tums).

KimMH: This...seems to be a pair of left-handed scissors.

KimJA: That is something left-handed....

LimEY: Left-handed?

KimMH: Yes, it seems....

Some participants: Ah, it is, indeedl.... [Brackets mine].

.............

KimJA: Are you sure? You could be wrong, couldn't you?

LimEY: Wow, look, look! This is very interesting! 
$K i m H J:$ It is not easy for my fingers to come out from this part.

KimJA: This piece of papers should have come upward, shouldn't they?

ParkJM: Yes, I should have held this in this way.... That's why I felt uncomfortable.

KimEL: this paper went up strangely....

KimHJ: I see, I felt uncomfortable.... Um, I see.

LimEY: It seemed to me that something was strange

[From parts of the transcript in the Drama Session on 28 March 2001]

\begin{abstract}
Participants, who were right-handed, had a feeling of awkwardness in person in using a pair of left-handed scissors. This enabled participants to get experiences of how difficult and uncomfortable it is for people to meet different customs and cultures from theirs.
\end{abstract}

[From the Observation Records on 28 March 2001]

As shown in the above transcript and the observation record, participants quickly found in Context 3, the drama session, that they did not feel comfortable or why they felt something strange. This is because those who were right-handed cut papers with a pair of left-handed scissors, which were different from the right-handed scissors they were accustomed to using. Their understanding of others through the scissors activity led participants into the intercultural imagination, as I argued in 2.2 and 4.1, and built a bridge to enter Context 4 where they did the Passport Game. Of course, I can say that in the process of this intercultural imagination, participants made shifts from Context 3 through Context 1 to Context 4 or vice versa in relation to their socio-cultural experiences in and through drama, 5.3.

In relation to this scissors activity, the case of KimEL particularly provided an example of how participants had introversive dialogues with activities in drama sessions. KimEL described how she felt through that scissors activity and what dialogues she had with that activity in our Saturday Research Meeting on 31 March 2001:

KimEL: I couldn't help remembering the activity for the next few days after that and that came into my head even in bedtime.... To be honest, I didn't know even the fact that there is a pair of left-handed scissors. So, how could I notice that was a pair of scissors for a left-handed person?... However, when I tried to do that once more I realised that I felt something wrong with the handle part of the scissors.... Therefore, this activity reminded me of something very important: one thing, when I see a matter, I shouldn't observe it from only one angle, that is, do not catch only its routine aspects, but should turn my perspective to observe that matter in a rather different way; Secondly I had better look for what is important from the matter and its importance not only for me but also other people. One more thing to surprise me is that we talked about the possibility to extend what experiences we got through that scissors activity into the issue of what we, as South Koreans, can do for North Koreans in order to prepare for or after the Reunification of two Korean countries. At that moment, I was very surprised to reach that point. I was really deeply impressed, indeed [From the Saturday Research Meeting on 31 March 2001]. 
At the beginning of the above, KimEL mentioned that she did not know there was another kind of scissors from a pair of right-handed ones nor had she paid any attention to the awkwardness for left-handed persons having to use right-handed scissors. The first half of her statement shows that she had just used a pair of scissors without any serious thinking but now realised what she was used to were pairs of scissors for right-handed persons. Her dialogues at that moment made shifts from Context 3, where she began to consider left-handed people through the scissors activity, to Context 1 , where she became aware that she had not been aware of or understood the awkwardness. In Context 3, she reacted in the production cycle, whereas in Context 1 she reacted in the perception cycle.

The second half of the above revealed that her dialogues were much deeper in Context 1 rather than in Context 3, reflecting the effect from the scissors activity on her self-awareness. In Context 1 , she was conscious of the inconvenience to others, left-handed people, and developed her concern for otherness-how to observe, perceive, manage others who were different from her and otherness around her.

In a word, the experiences she had in Context 3 made shifts to Context 1 in her perception cycle, and were reflected in her own thoughts or values in reference to otherness. I can argue this awareness of otherness took place from introversive dialogicality, in which the participant focused what she produced and perceived from her drama on herself. This is the opposite of otherness from extroversive dialogicality, in which a participant focused her drama activities on others or other things, described in the previous subsection.

As described so far in the present section, the dualities, which took place because of multiple contexts from actuality and theatricality and the production-and-perception cycle in drama, didn't let participants limit their involvement in the stages of dialectical resolution but allowed them to get involved in the stage of 'self-pluralistic thinking,' through which participants obtained dialogical outcomes.

Finally, I can summarise the dialogicality, extroversive and introversive in drama activities as shown in Figure 6.6. 


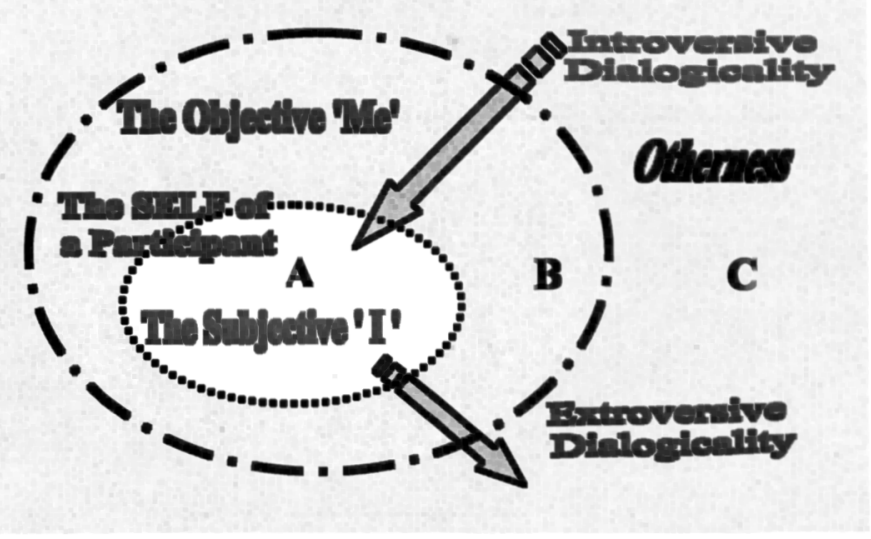

Figure 6. 6 Othemess from Dialogicality, Extroversive and Introversive, in Drama Activities

So far, I have focused on two couples of duality in drama, actuality and theatricality, and production and perception. As shown in the above figure, there are two areas within the self of a participant-the subjective 'I' (Circle $A$ in Figure 6.6), the objective 'Me' (Circle B), and the self per se belongs to the society (Square C). The Subjective 'I' is the unsocialised infant with a bundle of spontaneous wants and desires. The Objective 'Me' is the social self which enables the individual to develop selfconsciousness by coming to see themselves as others see them, as argued in $\mathbf{2 . 2}$.

However, it would appear that both the 'I' and the 'Me' in the self of a participant interacted with two different sorts of dialogicalities between the self and non-the-selfextroversive and introversive. These two dialogicalities provided participants with the experiences of otherness, which gave them a better understanding of their situations through drama.

Thus, I can state participants got involved in the dualities of drama in their drama experiences, which provided for participants both dialectical thinking and self-plural thinking through intercultural imagination. Through this involvement, participants were engaged in dialogicalities, extroversive and introversive, through drama and acquired better understanding of the ICM they handled throughout their project.

As illustrated above, the dialogicality in and through drama is an effective dynamic factor for participants to improve their ICM. Our drama activities, as I argued before, mainly in 3.2, 3.3, and 5.3, are to be socio-cultural/intercultural experiences as socialising activities relevant to factors, affective, cognitive, and behavioural. It appears that extroversive and introversive dialogicalities are essential for social 
awareness as well as self-awareness in terms of drama dynamics. Then my next question is: What did participants do with these two different dialogicalities?

\title{
6.3.2 Otherness and Outsidedness: Dialogicality of Participants in Drama Activities
}

In reality, as briefly mentioned in the introductory part of 6.3.1, I emphasised dialogicalities in participants' interactions in the matters of, first, communicating for another, and through the other, for oneself and second, decentring into the eyes of another or with the eyes of another for self-awareness. I wondered at this stage how a participant related the self to these two experiences.

I contend that the self pivots on exploring the participants' interactions. Bakhtin (1984), introduced in 6.3.1, emphasises the territory established from socio-cultural situations around a person rather than his/her internal sovereign territory and therefore regards the way to self-awareness as being through otherness and outsidedness. He argues that otherness and outsidedness eventually focus on the self and return to the self, as discussed in 2.2 and 3.2 .

Beckerman also argues about the self as an axis of activities:

\begin{abstract}
In dramatic activity, the truth lies in and arises from a broader context, because the spectator witnesses not merely a superficial activity but an activity with an inner life. A continuous signal reports to his imagination, informing him whether or not what he is seeing is "true." In parallel fashion, the dramatist or performer, in selecting and preparing his activity for presentation, is guided by inner radar (Beckerman 1970: 24).
\end{abstract}

It thus appeared that dialogical interactions of participants were led into either extroversive or introversive directions by their inner lives. Then my next question is raised: How did their inner radars guide these two different dialogicalities?

I shall further investigate their dialogical interactions in order to get answers for the above question. In practice, as mentioned in the introductory part of 6.3.1, the relationship of dialogicalities to self-plural thinking and dialogical interactions produced participants with better intercultural imagination, which enables participants to engage with critical thinking about intercultural situations, and led them into otherness. 


\subsubsection{Otherness: Extroversive Dialogicality- Reflection of Co-Researchers on the Event of 'English Zone'}

Let me begin with the simple example of YunKE, which I have already presented in 6.3.1.1. According to her Self-Questioning form on 28 March 2001,

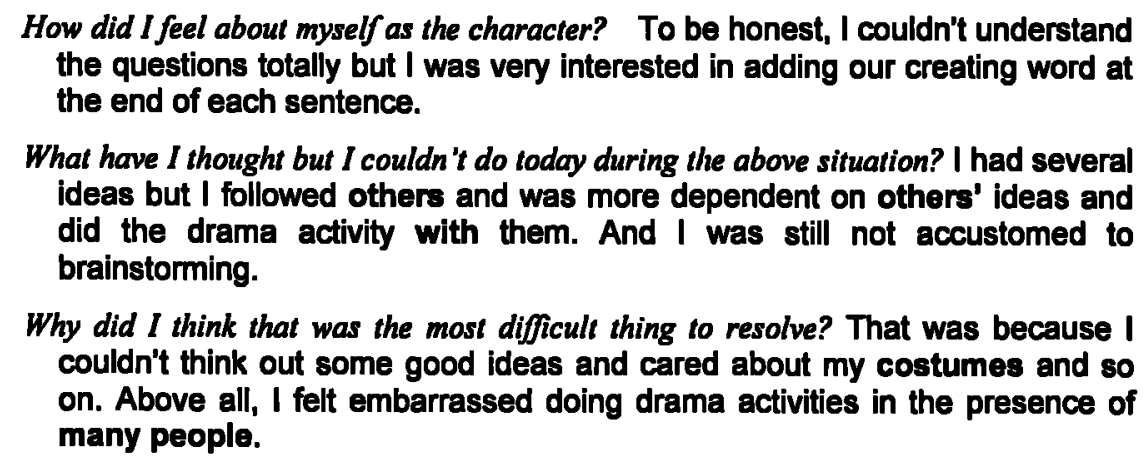

[Self-Questioning form of YunKE on 28 March 2001: Bolds mine].

As made explicit in the above Self-Questioning, YunKE gave way to others. YunKE had several ideas. Nevertheless, she would not urge other participants to select her ideas. The subjective 'I' of YunKE was given up for others and the objective 'Me' was taken and this choice got involved in dialogicality, not in introversive but extroversive.

There are several factors, which are interconnected with her extroversive dialogical interactions in that situation, evident in the above Self-Questioning.

- Responsibility for doing drama actions as co-researchers at the production cycle in Context 2

- Responsibility for brainstorming for drama activities at the production cycle in Context 3

- Cooperation for drama session at the production cycle in Context 3

- Nervousness about the performance at the production cycle in Context 4

- Handling costumes during her performance at the production cycle in Context 4

- Not fully understanding questions which she was given in the Passport Game at the perception cycle in Context 4

- Unfamiliarity with ICM in the Passport Game at the perception cycle in Context 4

- Interest in that drama activity at the perception cycle in Context 4

- Acceptance of others' ideas at the perception cycle in Context 3

- Self-awareness of the discomfort in brainstorming at the perception cycle in Context 3 
- Fear of the audience at the perception cycle in Context 4.

She acquired otherness through this extroversive dialogicality, which I can assume may have more than the factors illustrated in the above. She interacted dialogically with others' drama activities in an extroversive way, in which she communicated on behalf of others, thought about what she produced and perceived in the aforementioned contexts in that situation, and applied that to understanding others rather than herself. In the process of the above extroversive dialogical interactions, she responded to her authorship and spectatorship. For instance, factors at the production level interacted with the authorship of her drama activities in that situation, whereas factors at the perception level interacted with the spectatorship.

As a result of this, she had a better understanding of that situation including other relevant participants. This is the way in which she reflected-getting involved with otherness through the extroversive dialogicality and reflecting on others rather than the self. In the end, I can say she relied on her ethos rather than her pathos level and the group ethos rather than her own ethos in that situation.

At this stage, contrary to the above case, in which YunKEs extroversive dialogical interactions with others produced a way to shift from herself to others, $I$ intend to present another extroversive dialogical interaction to show another way to shift from others to others.

In order to relate our project to practicality in the field, we set goals of establishing the club with EDIE and the English Zone within the school campus. The EDIE club was going to provide its members with learning environments to improve their communicative competence in English; the English Zone was for any student at the School. Both the EDIE club and the English Zone would encourage participants to practice English Conversation to improve their intercultural competence, especially linguistic skills in English in their everyday life situations.

In the process of our project, there were differences between our ethos for the English Zone and the school ethos in terms of pedagogical priority, which both sides gave for the sake of students in the school. I shall describe how participants interacted with the difficulties using the concept of extroversive dialogicality.

First of all, participants transformed themselves into co-researchers and took the lead handling the task and we discussed what we could do for that task at the Second 
Saturday Research Meeting on 24 March 2001. Some important matters, which we took into consideration, included the following:

- Period and time of an ordinary club activity

- School regulation about the membership of a club activity

- Possibility to establish the Club of EDIE [EDIE was a tentative title for the Club]

- Activities by the Club of EDIE including public performances

- Tutor(s) for the Club of EDIE

- Possibility to obtain a room for the Club of EDIE

- Learning materials and facilities in the club room

- Consulting about the room with senior teachers

- Giving the EDIE club its appropriate name

- Public relations of the club to other students

- Naming the Room as 'the English Zone'

- Expecting the School principal to support us for the room

- Consulting about the task with senior teachers.

We carried out the above items by dividing actions for the task among us until the last week of the EDIE project. We planned to have a personal interview with the School principal for this task as well as a booklet, which we would publish, at the end of this project. KimMS, as a journalist for the publication, prepared for the interview; particularly what questions she could ask him. We sorted out the aforementioned items as best we could.

Nevertheless, we did not succeed in the task for the Club. We did not realise that several external factors militated against our task and that the English Zone was no more than just our suggestion for foreign language education at that moment. This is revealed in Networks 6.4 and 6.5 .

As shown in the Networks, extroversive dialogical matters in Quarter A and Quarter B were influential factors on succeeding in the task. For instance, the School principal and his school management were less dependent elements along with participants and their EDIE project than others. This means the former are less influential than the latter. For instance, if there were a spare room, a principal would permit a sports club to use the room rather than a language club because of regulations given from the Local Council of Education. He/she is reluctant to breach those regulations, as I discussed in Chapter 4. Above all, because there was no room in the school, the School principal could not help the participants to give any room for the English Zone or the EDIE club. That is to say, there are limitations on both the School principal and 
participants, on establishing the English Zone. Participants had not sought appropriate support for the Zone from their school by the end of the EDIE project and consequently the task was no more than a suggestion for foreign language education.

Thus, an unsuccessful outcome occurred in external factors rather than internal ones within the project and its researchers. First of all, we could not obtain sufficient members necessary for establishing the club because EDIE seemed to be too much for the first graders. Second, the school could not provide a room for the club for coresearchers. Third, the Local Educational Council and the Ministry of Education sometimes controlled the school management. Fourth and finally, general educational milieu around the co-researchers was not ready for applying EDIE into school activities yet.

The reasons why our attempts met with failure in terms of the extroversive dialogical interactions are shown in Networks 6.6 and 6.7, which follow Networks 6.4 and 6.5.

Above all, from the networks I can say the school management acted as an intermediary for the EDIE project and the School principal took charge of that management. Therefore, the School principal was a go-between between the project group and the Local Council of Education, as presented as Square A, Circle $B$ and Triangle $C$ in Network 6.6. This means he could not give his whole support to the task because he had to take educational policies and their relevant regulations from educational authorities into consideration and gave a priority to them in making his decisions.

That unsuccessful outcome thus resulted, I believe, from the external factors. We tried to interact dialogically with extroversive elements, rather than the internal elements within the EDIE project. Once more, there was a gap between co-researchers' pathos and ethos relevant to the EDIE project, and socio-cultural pathos and ethos in the educational milieu where the co-researchers were educated, such as pathos and ethos of the Ministry of Education.

In other words, this is a gap between Context 2 and Context 1, which I presented in Figures 6.1 and 6.2: co-researchers' pathos and ethos inside the EDIE project, and others' pathos and ethos outside the EDIE project, which co-researchers contacted in their everyday lives. With regard to this, I can say that some educators or the authorities relevant to EDIE were not ready for EDIE for drama education itself, as well as teaching EIL and intercultural education. 


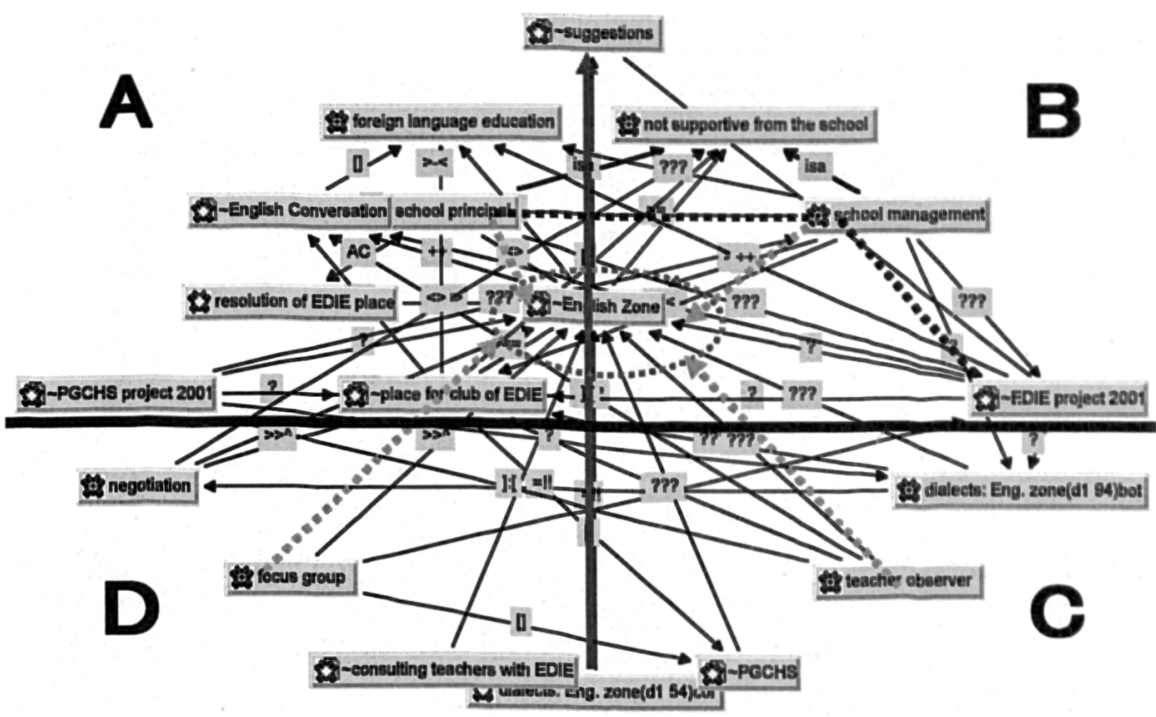

Quarter A and Quarter B cover extroversive dialogical matters outside the EDIE project, and Quarter $C$ and Quarter $D$, introversive ones within the Project. Moreover, Quarter $A$ and $Q u a r t e r D$ have relations to taking actions but Quarter $B$ and Quarter $C$ is relevant to managing the Task.

Network 6.4 Extroversive Dialogical Interactions at the Stages of the Task for the English Zone by the Semantic Layout

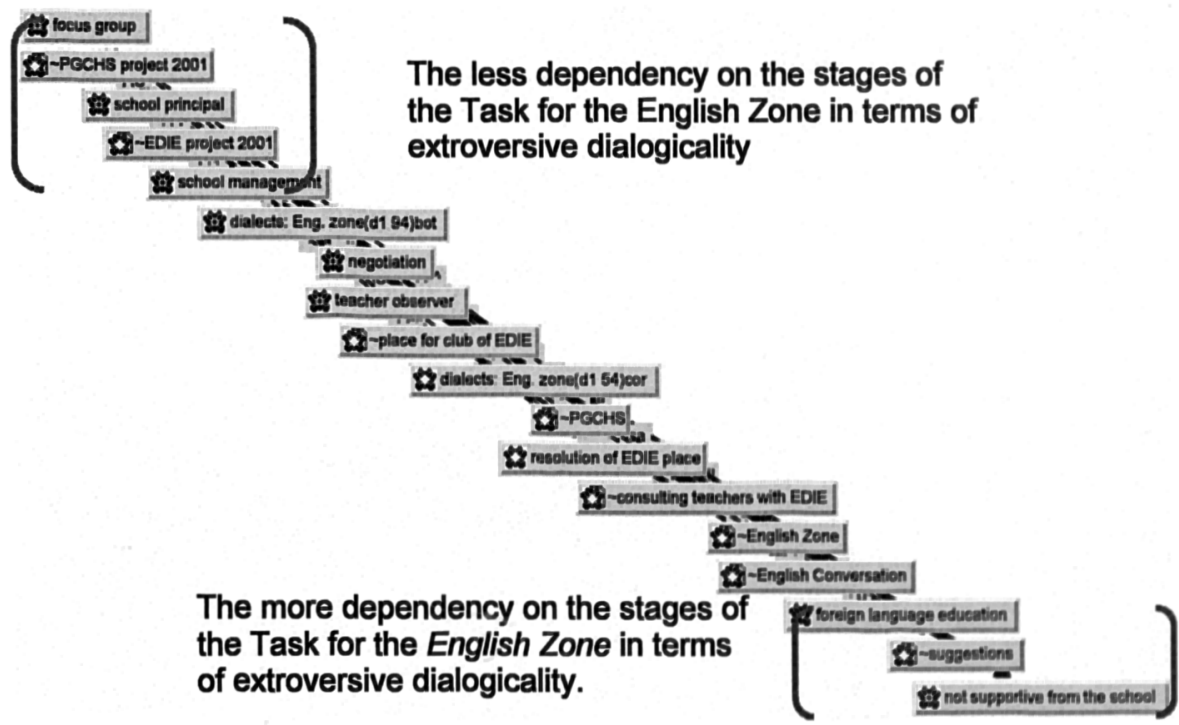

Network 6.5 Extroversive Dialogical Interactions at the Stages of the Task for the English Zone by the Topological Layout 


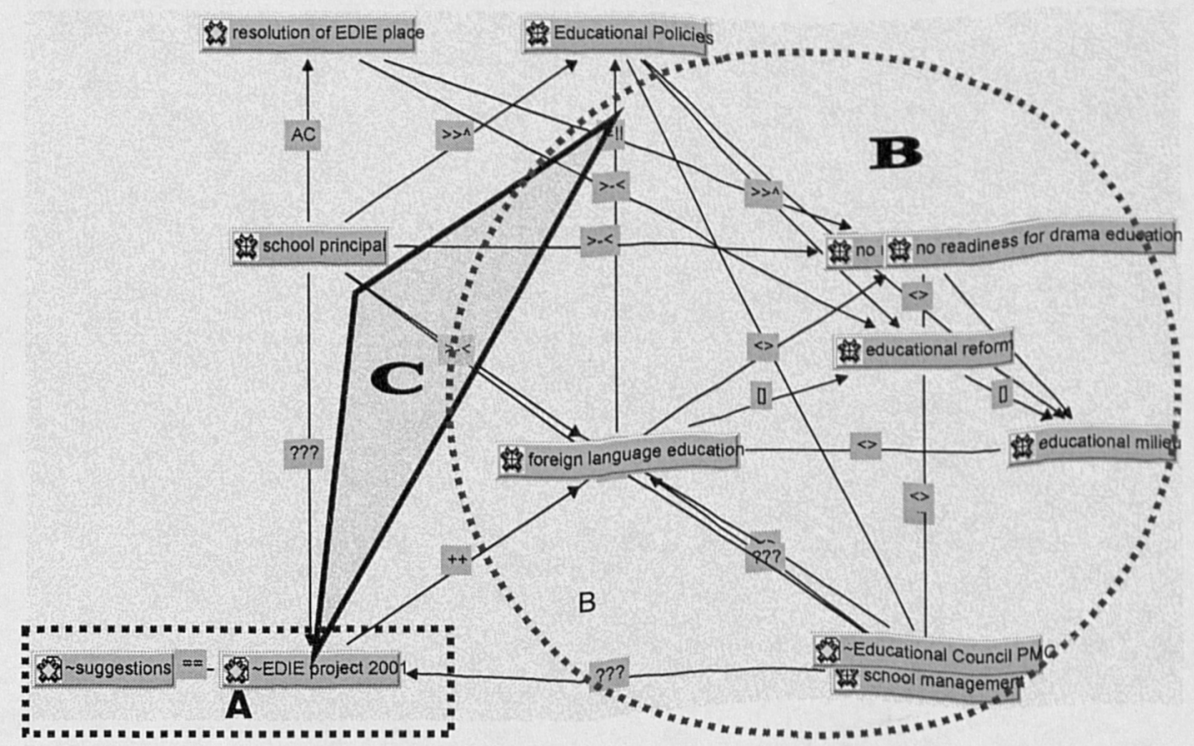

NETWORK 6.6 External Factors against the Room for the club EDIE in the School by the Semantic Layout

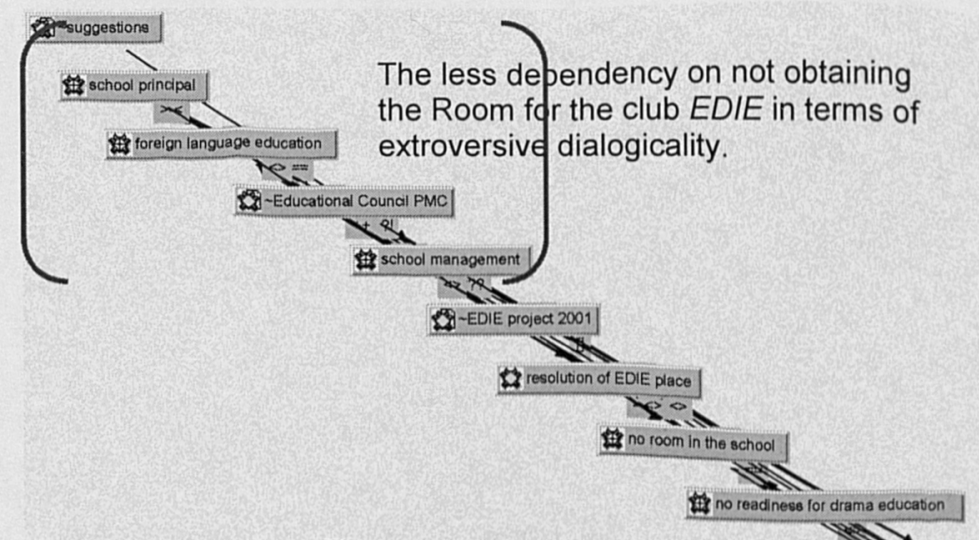

The more dependency on not obtaining Edu the Room for the club EDIE in terms of extroversive dialogicality.

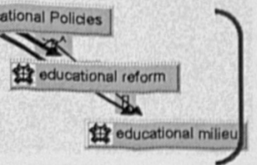

Network 6.7 External Factors against the Room for the Club EDIE in the School by the Topological Layout 
Let me take one single, but crucial, example of the problems in applying drama education to the National Curriculum. The Korean Education Newspaper, Hanguk Gyoyuk Shinmun, introduced the recent development of educational drama to its readers in a favourable light. On the other hand, the papers were critical of educational policies relevant to drama education, as follows:

The Ministry of Education in Korea included 'Drama and Film' as an optional subject at the secondary level in the National Curriculum, which will be enforced from 2003 and permitted the Departments of Theatre and Film of the Universities throughout the country to set up studies for the teaching profession in order to prepare for the enforcement.... In the end, it is only possible to establish the subject of 'Drama and Film' on a legal basis of the National Curriculum and in reality there are no drama teachers to teach the subject, no teaching materials and no teaching methods ["Drama, as a syllabus at the secondary level.... [my translation] (Seo 2002: 5).

According to the above, drama can be chosen as an optional subject by students at the secondary level from 2003. Nevertheless, there are still no qualified drama teachers, no teaching materials published, and no teaching methods to introduce in an appropriate way. In a word, it is impossible in actuality that students at the secondary level can choose 'Drama and Film' as their optional subject in 2003.

What I have presented in the present subsection are two different extroversive dialogical interactions with others, that is, reflection both from the self to others, and from others to others. The former is YunKE's case, whereas the latter, the case of the EDIE task. YunKE followed others' ideas rather than insisted on her ideas in developing drama activities, meanwhile we realised that the School principal was dependent on educational policies of the Ministry of Education in managing the school.

As the two cases showed, we became aware of what we got through the extroversive dialogical interactions with others around us: the awareness on re-mapping how a participant cooperated with other participants in the former case and what educational milieu were like at that moment, especially in terms of teaching English as a foreign language or teaching drama, in the latter case. Now, I can say both cases revealed the inconvertible inter-relationship between the self, which shapes others, and others, which shape the self. In a word, the self and others are not separable as individual entities but inclusive as co-existing within an entity. This perspective will be discussed further in the following subsections. 


\title{
6.3.2.2 Otherness: Introversive Dialogicality - Self-Reflection of Performers through Characters in the play, War Paint
}

Extroversive dialogicality of both cases in the previous subsection was described in terms of the authorship and the spectatorship through intercultural drama activities. In the present subsection, however, introversive dialogical interactions with others will be explored in terms of the self-authorship and the self-spectatorship. I shall start this exploration with Bakhtin's statement on architectonics, in which he emphasised the interrelationship between oneself and all others and its simultaneity in dialogical interactions. He argued:

\begin{abstract}
For cognition, "there is no absolutely inconvertible relationship of $I$ and all others....for a particular person, in a particular place at a particular time, and thus constrained by all the conditions peculiar to such a unique placement, "the interrelationship of 'I-the other' is not convertible for me in lived life in any concrete way." Since it is not convertible, it must be viewed prospectively (Holquist \& Liapunov, 1990: xxii-xxiii).
\end{abstract}

However, his argument for the interrelationship of 'I-the other' does not concern binary oppositions but the interrelations between the two. Holquist \& Liapunov regard the Bakhtin's concept as togetherness, not opposition.

\begin{abstract}
But what is essential for Bakhtin is not only the categories as such that paired in author/hero, space/time, self/other, and so forth, but in addition the architectonics governing relations between them. What counts is the simultaneity that makes it logical to treat these concepts together. The point is that Bakhtin honours both things and the relations between them-one cannot be understood without the other. The resulting simultaneity is not a private either/or, but an inclusive also/and. In other words, the logic of Bakhtin's simultaneity is—dialogic [authors' italics] (Holquist \& Liapunov 1990: xxil-xxiii).
\end{abstract}

According to the above, Bakhtin explained the differences in conditions which are shaped by who, what, when, where, why, and how-situational differences, and therefore emphasised the inconvertible interrelationship between an individual person and the conditions surrounding him and an inclusive simultaneity between the self and all others. That is, one is not exchangeable to the other because they co-exist inclusively within an entity.

I already described in 5.3.1, one of the factors which shapes different conditions. When an individual gets involved in drama they use their whole socio-cultural context or experience, which is different from individual to individual. Each has their unique placements. However, in the present subsection I intend to focus on how participants got introversive dialogical interactions when performing the play, War Paint, in a word, how they governed dialogic relations in their situations rather than what dialogues they had. 
I shall take two main perspectives here-performer's perspective on herself from her public performance and performer's perspective on herself from her character or characterisation. The former will be focused on how to interact as a performer with the public performance and how to reflect on herself; the latter will be concentrated on how to characterise her character and how to think about her characterisation to consider herself. I intend to take LimEYs case, and RyuKW's and KimMS's cases in the play, War Paint, as examples of the above standpoints, respectively.

The case of LimEY, first of all, shows that she took the role of Julia in the play, War Paint, who longed to go to places such as the discotheque, and LimEY didn't have many difficulties in carrying out the public performance. She had internal dialogues with herself in self-questioning on 21 April, immediately after filling up the SelfQuestioning form.

What action do I want to talk about more to myself among today's drama activities? I would like to explore how to act being embarrassed or being very surprised.

What relationship did I have to others? Angie, Rod, and Greg.

How did I feel about myself as a person who relates to others? I would like to play an older girl than I am.

What have I done today during drama sessions in practice in the above question? I did enjoy dancing in the discotheque, even in the play.

What have I thought but I couldn't do in the above situation? I thought I had to dance well enough and better than this. I was going to accentuate one part of my lines, 'Great' with a big gesture at the scene when I entered the discothèque with my friends.

Why did I think that was the most difficult thing to resolve? I couldn't practice speaking my lines enough.

How could I act in that situation if I were one of the people who come from England or America? They might dance and enjoy that.

What do I want to do in a similar situation to that in the future? Do behave yourself as old as you are, not like a grown-up.

[Self-Questioning form of LimEY on 21 April 2001]

As implied in the above Self-Questioning, LimEY was very motivated by acting as the English teenage girl, Julia, to characterise the personality as the girl who yearns to be a grown-up and feel free from the limitations of the teenager. In fact, the way she performed Julia was closer to LimEY herself than to the character in the play. For instance, LimEY confessed, 'I did enjoy dancing in the discotheque, even in the play.' Here she mixed the drama context of Play $B$, where Julia enjoyed dancing with the real context of Play $B$, where LimEY herself did.

This sort of mixture was described in her Final Personal Journal, which she wrote after the above Self-Questioning: 


\begin{abstract}
Above all, the most impressive one to me is making-up before this performance. I really enjoyed that! I am proud of myself because of what I have done for the performancel I took a role of 'Julia' and I was very pleased to make up in order to express the character. I am wondering if I can get this kind of chance again. After guessing "Maybe not!" I did make up for the character as much as I could. I am very happy with this. [Her Final Personal Joumal on 21 April 2001]
\end{abstract}

LimEY said, 'I took the role of 'Julia' and I was very pleased to make up in order to express the character,' but she wanted to feel free to make-up using this opportunity. Here again she mixed make-up for the character with getting her own feeling of a female grown-up. In the end, LimEY got self-confidence of acting through the performance and said, 'we rehearsed only for 10 days, but if we had enough time for a professional theatre company to rehearse, for instance, for a month, we could perform very well' [From LimEYs Comments at the Sixth Saturday Research Meeting on 21 April 2001].

LimEY thus tried to characterise the role of Julia in the play, War Paint but described 'Julia' as another herself rather than as the character by dialogically interacting with herself rather than with the character and mirroring Julia on herself. In a word, the relation between the character, Julia, and the performer, LimEY, appears to be a mirror image. Because of this mirror image, she did not have many difficulties in acting the character through dialogical introversive interactions with her character.

Alternatively, RyuKW and KimMS appear to be self-reflective from the standpoint of the self-actualised person, in whom 'polarities, or opposites or dichotomies... were resolved, the polarities disappeared, and many oppositions thought to be intrinsic merged and coalesced with each other to form unities' (Maslow 1970: 178-179).

RyuKW and KimMS had some difficulties in characterising their roles which seemed to have very different personalities from theirs, what I call, cross-characters to themselves. Actually, both of them were very embarrassed at being cast as Rod and Greg, respectively on 12 April 2001. Nonetheless, they took their roles and presented the characters in their own ways. KimMS looked more involved in the performance and also voluntarily took another role, Radio Voice, which was only an announcement, though. I really wondered how they would manage to act.

At first, RyuKW was introverted, well behaved, and very conservative but she took a male teenager role, Rod, who seemed to be part of a group of bums who flirted with young teenage girls, Angie and Julia, and danced with them at the discotheque in the play. RyuKW sometimes smiled awkwardly while she was acting the character, Rod, because he seemed to behave too differently from her own behaviour. This is on the 
surface of her performance. Therefore it is necessary to explore her self-questioning about characterising the character, Rod. She had dialogues with herself in the SelfQuestioning, which was done immediately after the performance:

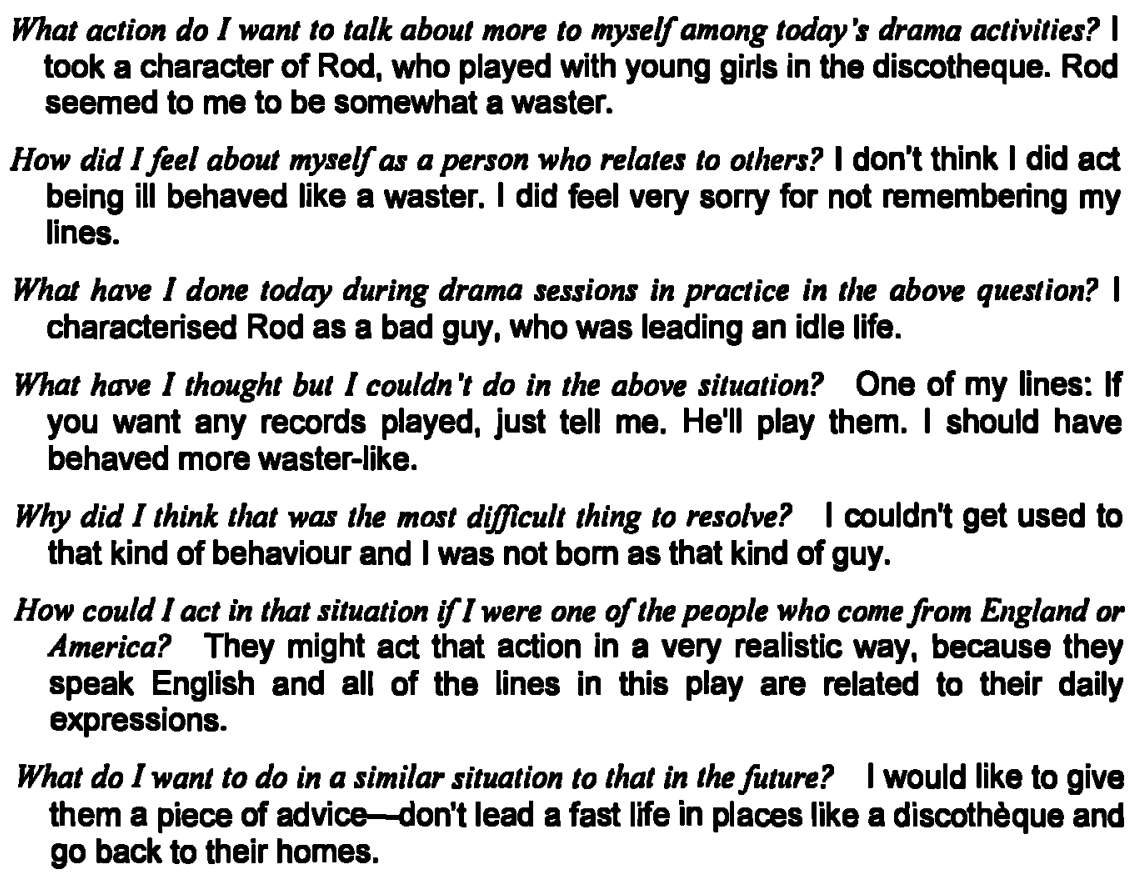

[From Self-Questioning form of RyuKW on 21 April 2001]

RyuKW, as the above implies, focused herself on the characterisation of Rod and speaking her lines. At the beginning she was afraid not to do well enough in the performance because of her personality-as she confessed: 'I couldn't get used to that kind of behaviour and I was not born as that kind of guy.' Nevertheless, she rather regretted not acting in a more ill behaved way.

There is a clash of two opposite or quite different personal attitudes between RyuKW relevant to Contexts 1, 2, and 3, and Rod in Context 4:

- in Context 1, RyuKW faced a sort of juxtaposing situation between herself in everyday life -female, conservative, well behaved, introverted, a Korean teenager, and a Korean native speaker,

- in Context 2, as a co-researcher,

- in Context 3, as a performer, and

- in Context 4, her character-male, liberal, ill behaved, extroverted, an English teenager, and an English native speaker.

However, she accepted all of Rod's traits that were contrary to her own and merged the two opposite sides to form unities of her acting. As the last two questions of the above Self-Questioning imply, she accepted situations around the character, Rod and 
at the same time kept her own values, for instance, conservative and well behaved. In the end, both acceptance and synergic dialogues in her mind, in Context 4, enabled her to overcome her embarrassment, which she felt at taking the role of Rod, and acting the character. At the same time, in Contexts 1, 2, and 3, the acceptance and the introversive dialogical interaction made her have a better understanding of English teenage culture. This understanding not only improves her cognitive competence but also her attitudinal competence and interactive competence and eventually leads her to become a better intercultural actor, which I argued in 2.2 .

The case of KimMS is similar to that of RyuKW-self-reflection from the standpoint of the self-actualised person. KimMS also had a clash of two opposite or quite different personal attitudes between herself and another waster character, Greg. However, KimMS, who took the role of Greg, appears to be different from RyuKW in terms of being self-reflective through others. Other performers recognised their differences in this point and LimEY, in particular, made comments on how much KimMS changed in terms of her attitude and behaviour since joining the EDIE project. Drama experiences of KimMS, in relation to the change, already were described in the discussion as being emancipatory and self-reflective in $\mathbf{5 . 2}$.

And then what are the differences between RyuKW and KimMS in terms of overcoming their cross-characters [Rod and Greg]? In the first place, I shall compare RyuKW-Rod interactions with KimMS-Greg interactions in the following two networks, which I produced through the ATLAS.ti software programme.

On the whole, as shown in the networks below, it appears that RyuKW and KimMS were very different from each other in terms of characterising their cross-characters, Rod and Greg, respectively. I would like to pick out two different points in relation to their introversive dialogical interactions with the characters.

One is that whereas RyuKW was inclined to characterise her character, KimMS had a tendency to focus on being self-reflective through dialectical thinking between herself and her cross-character. This difference is revealed by comparing Square $A$ in Network 6.8 with Square $B$ in Network 6.9. KimMS became more self-reflective through others than RyuKW did by trying more often to have empathy with her crosscharacter, Greg, in comparison to RyuKW. 


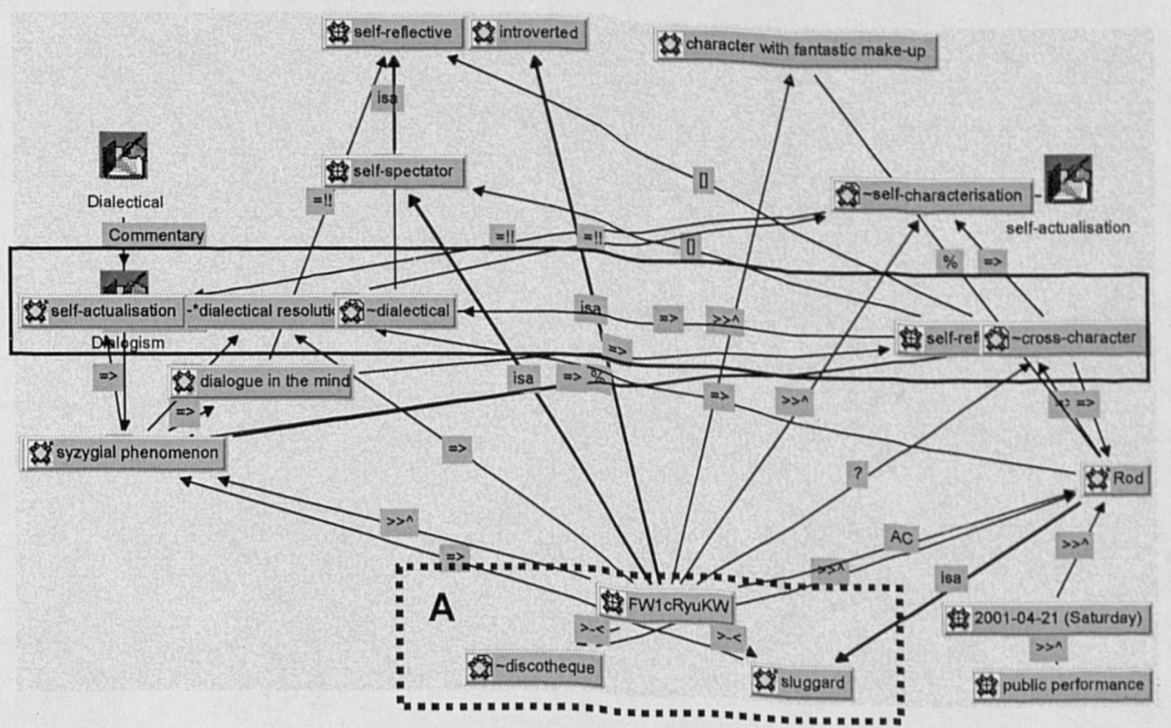

Network 6.8 Rod [Cross-Character to RyuKW] by the Semantic Layout

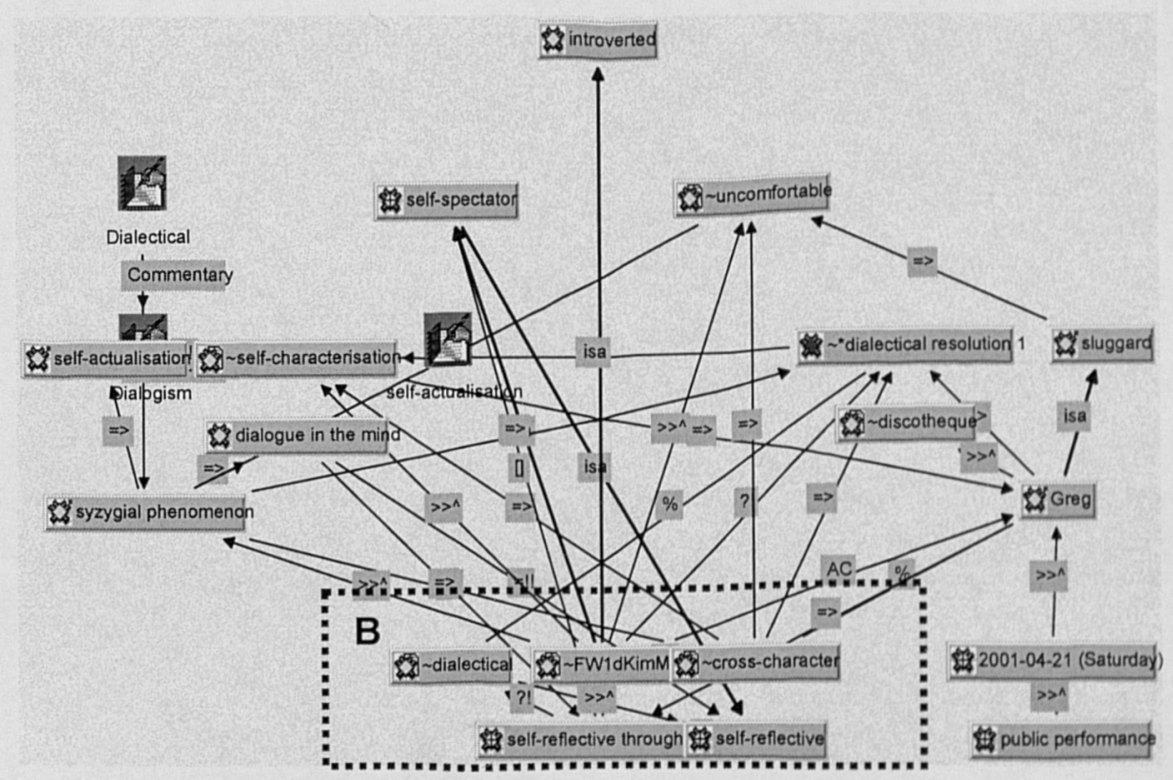

Network 6.9 Greg [Cross-Character to KimMS] by the Semantic Layout (See Appendix G) 
The aforementioned three performers in the play, War Paint, LimEY, RyuKW, and KimMS had introversive dialogical interactions with intercultural factors in two ways: self-reflection through identification with her character like a mirror-image with LimEY, and self-reflection through cross-character challenge to RyuKW and KimMS. Although it appears that the three were different in both their approaches to their ICM and the foci of their approaches, each of them was engaged in interactions with different intercultural factors, which were provided for them through their characters. They thought about what they experienced in relation to the performance and applied to themselves in and after the process of characterising and performing those characters.

As a result of this, they achieved a better understanding of "English teenager culture" as well as their own teenager culture. In short, their characters gave the three Korean teenagers the opportunity to improve intercultural competence-otherness for ICM through introversive dialogical interactions with the characters.

\subsubsection{Outsideness: Extroversive and Introversive Dialogicality-Reflexivity of Participants in Drama Activities of the International Business Meeting}

Participants, in addition to otherness, had a better understanding of ICM in another way-outsidedness through reflexivity in drama activities. They shifted from one cultural context into another cultural context to understand the two cultural values, their own as well as one different from theirs. This reflexive process is relevant to both extroversive and introversive dialogical interactions, which were earlier described in reference to reflection and self-reflection.

Participants had drama experiences with this sort of reflexive dialogical interaction, particularly in drama activities about the International Business Meeting. This was introduced earlier. In the event of this International meeting between a junior American president and a senior Japanese president accompanied by his two Japanese subordinates, participants faced intercultural situations. The crux in their situations was relevant to the cultural clash of the East and the West. I shall return to this event with reference to the reflexive dialogicality.

First of all, after reading the extract about this international meeting, participants felt surprised at the intercultural situation. They perceived ICM at this stage, Level 1, of sensation, which I described in 5.1. At the next stage, we compared the two cultural factors, American and Japanese, at first by making still-images of each firm, comparison of the still-images and producing our comparison table from the relevant 
extract on the two firms. I displayed these images as Figures 5.1, 5.2, 5.3, 5.4 and Table 5.2 in 5.3.2. I can classify this comparison stage into Level 2 of sensibility because participants understood what they felt and why they did that through comparing one firm with another.

ParkJM: The meeting could be successful.....

The teacher. All right, I am wondering whether or not the meeting will be successful. Who wrote that note which was described as 'red-faced'?

KimJA: The Japanese president becomes red-faced.

The teacher. Why?

KimJA: Because he looks upset when he sees the young American president rude....

The teacher. All right, then. How about the meeting itself? Successful or not?

Some participants: Not very successful, because they are thinking in different ways and have different opinions and they get embarrassed.....

MunJM: They get embarrassed...

The teacher: embarrassed? Why do they get embarrassed?

MunJM: Because they seem to be different from each other.... The American president tries to handle this meeting in an easy-going and swift way, meanwhile the Japanese representatives try to deal with it in a ponderous and formal way.

ParkJM: They are too different, aren't they?

MunJM: But we should accept their differences of thoughts.

The teacher. All right, according to your opinions, the meeting could be successful or not. They have different way of thoughts. How about a Korean president, then? What will happen if a Korean president had a similar international business meeting?

Participants: the Korean president will respond to that in the same way as the Japanese president....

The teacher: how about yourself? What would you do if you were the Japanese president or a Korean president?

KimEL: I would not buy the American products because the American president doesn't know about our culture very well and he doesn't care about the meeting partners. In a word, he is somewhat unrestrained.

The teacher. Do you mean the American president should become the target of criticism?

KimJA: He should enter the meeting with his understanding of his partner in order to trade with our country. Furthermore, he wants to sell his products here, not to buy something.

The teacher: And then, what should the Japanese president do after all?

MunJM: He shouldn't buy the American products.

ParkJM: But he is trying to understand the young American president....

KimEL: But the American president looks impolite, indeed. He doesn't know about his partner at all.

KimJA: However, the Japanese president tried to understand him. So, he ordered his subordinates to keep on this meeting instead of him. He could turn down the meeting if he didn't want to buy the products from the young American president, couldn't he? 
All participants: (seem to agree with KimJA and laugh all together).

[Transcript of the Drama Session on 4 April 2001].

While comparing them, participants thus realised that there were considerably different cultural values particularly in power distance between the two sides, which were displayed in the aforementioned figures and the table (Hofstede 1980). In this process of comparison, participants produced the dialectical resolution through discussing the ICM from two perspectives: [1] misunderstanding between the junior American president and the senior Japanese president, [2] their anticipated completion of the international meeting, successful or unsuccessful.

This discussion enabled each participant to achieve extroversive dialogical interactions with that intercultural situation together with other participants and to reflect what she had in her cognitive map of this sort of ICM. For instance, contrary to the beginning of the above transcript, KimJA eventually changed her attitude toward getting more understanding of the Japanese president at the end of the transcript.

The effect of this comparison through the still-images and the discussion is for the participants to reach the next level of sensitivity where they could handle that intercultural situation through dialogical interactions, both extroversive and introversive, that is, self-reflection and reflexivity stage, as displayed in Network 6.10.

Axis $A$, in the Network, is relevant to visible drama activities of participants in the drama session; meanwhile Axis $B$ indicates the attitudes which they were conscious of through extroversive dialogical interactions. Axis $C$ shows the dialectical and dialogical resolution of how they reflected and performed the intercultural situation in their performance.

Participants, however, not only applied their intercultural imagination to extroversive dialogical interactions but also to introversive dialogical interactions. In other words, participants tried to reflect on themselves through critical thinking in terms of sociological imagination or ethnographic imagination, mentioned in 2.2, for example, how she would handle that intercultural situation if she had been one of the characters. 


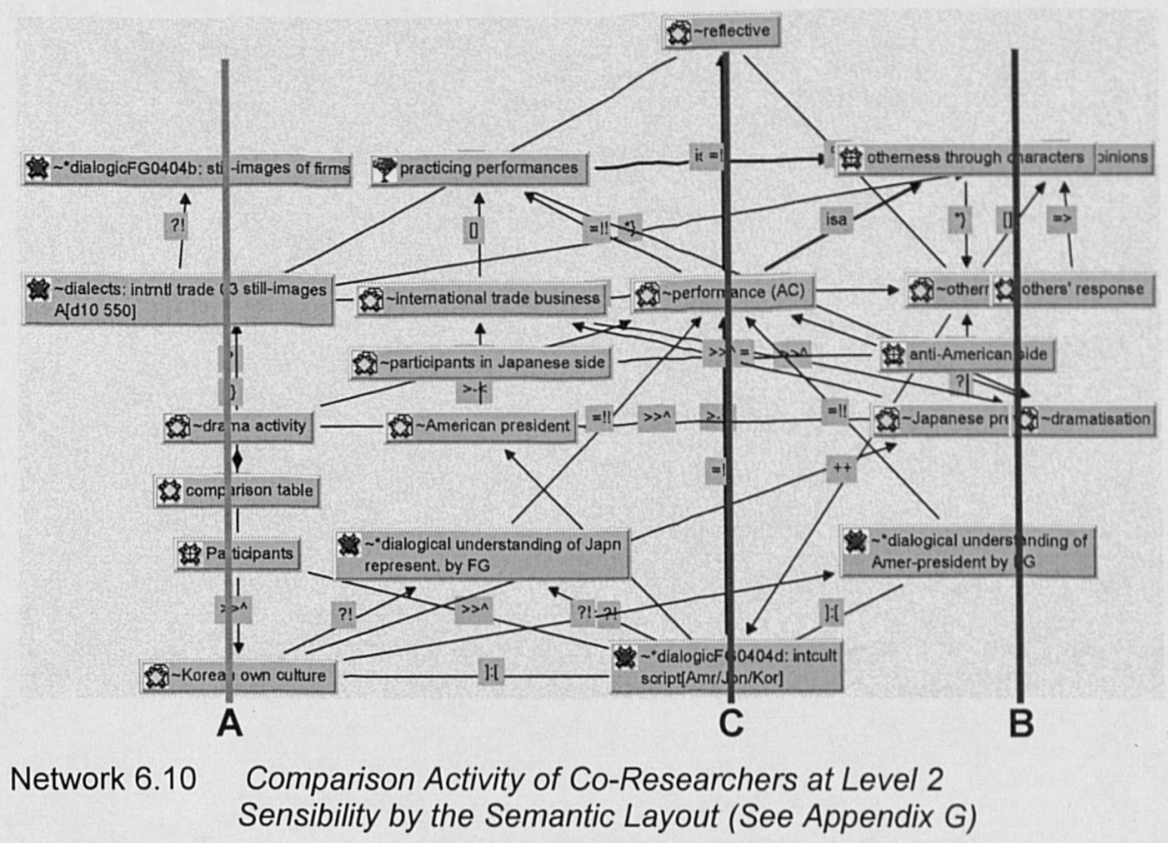

At this stage, which we took in the next drama session on 6 April 2001, I led participants to become involved in Level 3 of sensitivity in relation to that intercultural event. At first, I asked participants to write down what they imagined characters would think between their lines and note as many as they could, and then attach all of the notes to the aforementioned comparison table. In a word, I applied the drama technique of thought-tracking, which 'reveals publicly the private thoughts/reactions of participants-in-role at specific moments in the action so as to develop a reflective attitude towards the action and to contrast thinking-for-self with outward appearances or dialogue' (Neelands 1990: 54). Here are the thoughts in their minds as the American president, the Japanese president or the Japanese subordinate.

\section{[A] Thought-tracking notes of the American president}

$\Rightarrow$ Here they come! They are somewhat old. I will do my best!

$\Rightarrow I$ will succeed in this meeting. Smile as much as I can.

$\Rightarrow$ Let's go, men! I can do it, indeed!

$\Rightarrow$ Why don't they sit down but just stand there blankly?

$\Rightarrow$ What are they doing? What's up?

$\Rightarrow$ Are they trying to get down to business? What's wrong with me?

I am trying to do my best.... I wonder why....

$\Rightarrow$ I cannot get any start with them.

$\Rightarrow$ There must be something wrong. 
$\Rightarrow$ I wonder if it is good enough for them to look at me in the face in order to make the contract with me.

$\Rightarrow$ Should I leam Japanese?

$\Rightarrow$ They don't seem to like to talk enough.

$\Rightarrow$ Why do they speak only Japanese between themselves? Look, the person is leaving. Why is he leaving?

[B] Thought-tracking notes of the Japanese representatives

$\Rightarrow$ (Look at Mr. Jones. What a shock!) Where is Mr. Jones?

$\Rightarrow$ Impossible!

$\Rightarrow$ Is he the president to make a successful contract with us? It's the real president or a person instead of the president?

$\Rightarrow$ Are you really the business boss?

$\Rightarrow$ I am really wondering if they do know the business decorum. I am really embarrassed!! I want to tum him down. Oh, my godl

$\Rightarrow$ What an ill-bred boy he is! Shitl If this were not the business meeting, I would....

$\Rightarrow$ Is he insane? Why is he doing that? Can he laugh loudly in the presence of his grandfather?

$\Rightarrow$ What is he coming for?

$\Rightarrow$ I must be dreaming, not in reality. He is an extremely different president from the person whom we expected. How can he be the president?

$\Rightarrow$ I cannot make the contract with this person. He is not polite at all. I cannot see any politeness from him.

$\Rightarrow$ My president looks angry.

$\Rightarrow$ Is he the right person as the American president? Look at his clothes and behaviour at such an important meeting like this. He must be not polite.

$\Rightarrow$ I would rather take a rest in my office than do business with that person.

$\Rightarrow$ I will search for another firm, which can provide us with the products. I have wasted my time in coming here.

As indicated above, participants in character, revealed their miscommunication and misunderstanding between the two sides. Thought-tracking the American president is relevant to its main point-the American president's attitude, positive but wondering, toward meeting his partner. On the other hand, the Japanese representatives are focused on the first impression relevant to his behaviour, appearance and age from their meeting partner rather than the business meeting itself. Furthermore, participants had extroversive dialogical interactions with the Japanese side, with more sympathy than with the American side. Or rather, they made the American president the target of criticism in that intercultural miscommunication.

With regard to Level 3 of sensitivity, I would like to describe more the participants' attitude in terms of reflexivity through outsidedness by focusing on MunJM, who took the role of the Japanese president, along with KimJA, who acted as the American 
president. These two participants, in the positions of co-researchers, took the roles of the Japanese president and the American president respectively. Not just MunJM but also KimJA took the anti-American side with Japanese thought-tracking. This thinking was considerably influenced by two factors-their embarrassment at the unexpected situation and their outsidedness which stopped them having introversive dialogical interactions toward themselves outside the position of co-researchers.

MunJM, however, had different dialogical interactions with the intercultural situation, because of her role as the Japanese president, who might have similar cultural values to her own. In other words, it was easier for her to explore the character in terms of getting distance from and returning to herself than for KimJA to do that.

First of all, one of the differences between MunJM and KimJA, was the drama experience in which MunJM spoke some of her lines in the Japanese language and not just in English.

\footnotetext{
What action do I want to talk about more to myself among today's drama activities? I would like to talk about Goto, who is the Japanese president.

What relationship did I have to others? The Japanese president was conservative and old.

How did I feel about myself as a person who relates to others? I was very surprised at the young American president. What a surprise to meet such a young president....

What have I done today during drama sessions in practice in the above question? I as the Japanese president was shocked and seemed to be reluctant to have the meeting at its beginning. In its middle, I left my subordinates after giving them my delegated order.

What have I thought but I couldn't do in the above situation? Ah, it's Japanese! I had to speak the Japanese language, as well. Oh, dear....

Why did I think that was the most difficult thing to resolve? Because I am not a Japanese it is very difficult for me to express what I want to do in Japanese.

How could I act in that situation if I were one of the people who come from England or America? They seem to feel embarrassed because they have different languages.

What do I want to do in a similar situation to that in the future? ......

[From Self-Questioning form of MunJM on 4 April 2001, already displayed in the previous section]
}

As indicated in her Self-Questioning above, what she was mainly concerned about was the age of her character and the languages, particularly the Japanese language in the Drama session with the English language. 
Secondly, MunJM had different dialogical interactions with the intercultural situation depending on her position as the performer, the character and the co-researcher, as shown in Network 6.11.

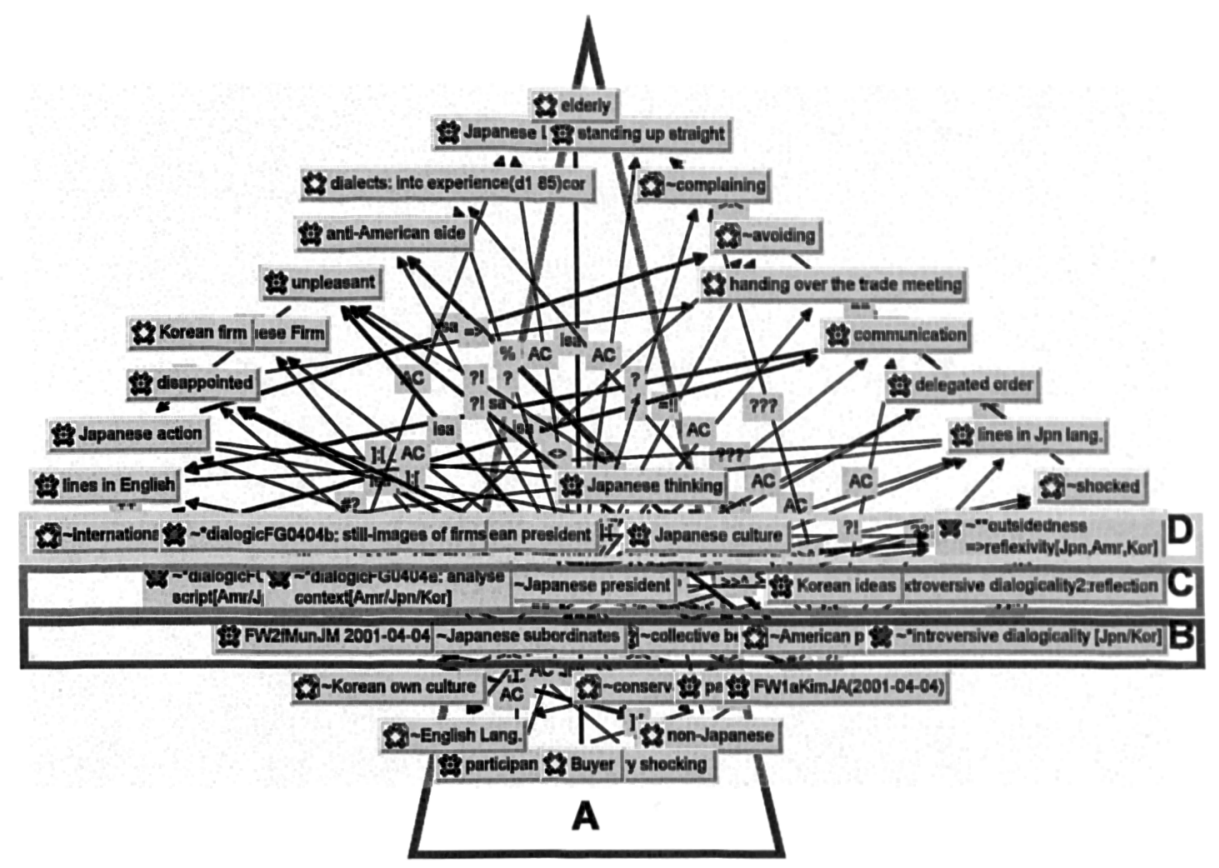

Network 6.11 Dialogicality of MunJM as the Japanese President in the Drama Event of the Intemational Business Meeting by the Semantic Layout

In the network, Triangle $A$ includes the basic context about what the Japanese president, as one of the characters, did in the drama event-Participant-in-role, an elderly buyer accompanied by his Japanese subordinates, the Japanese language and so on. It is the key point in Triangle $A$ that the elderly Japanese president is the very-shocked buyer. The left side of Triangle $A$ relates to her drama activities in Contexts 2 and 3, meanwhile the right side of Triangle $A$, relates mainly in Context 4, which I described in Figure 6.1.

MunJM, as the performer, shown as Square $B$, in this basic context interacted with the intercultural situation introversively through comparing the American president with the Japanese subordinates in terms of collective behaviour. MunJM, as the character, shown as Square $C$, was involved in extroversive dialogical interactions, meanwhile MunJM as the co-researcher, shown as Square $D$, was engaged in the reflexive outsidedness. 
Furthermore, MunJM, as the performer shown in Square B of the Network above, was more involved in the reflexivity of dialogical interactions with the intercultural situation. I can display this more clearly in Network 6.12.

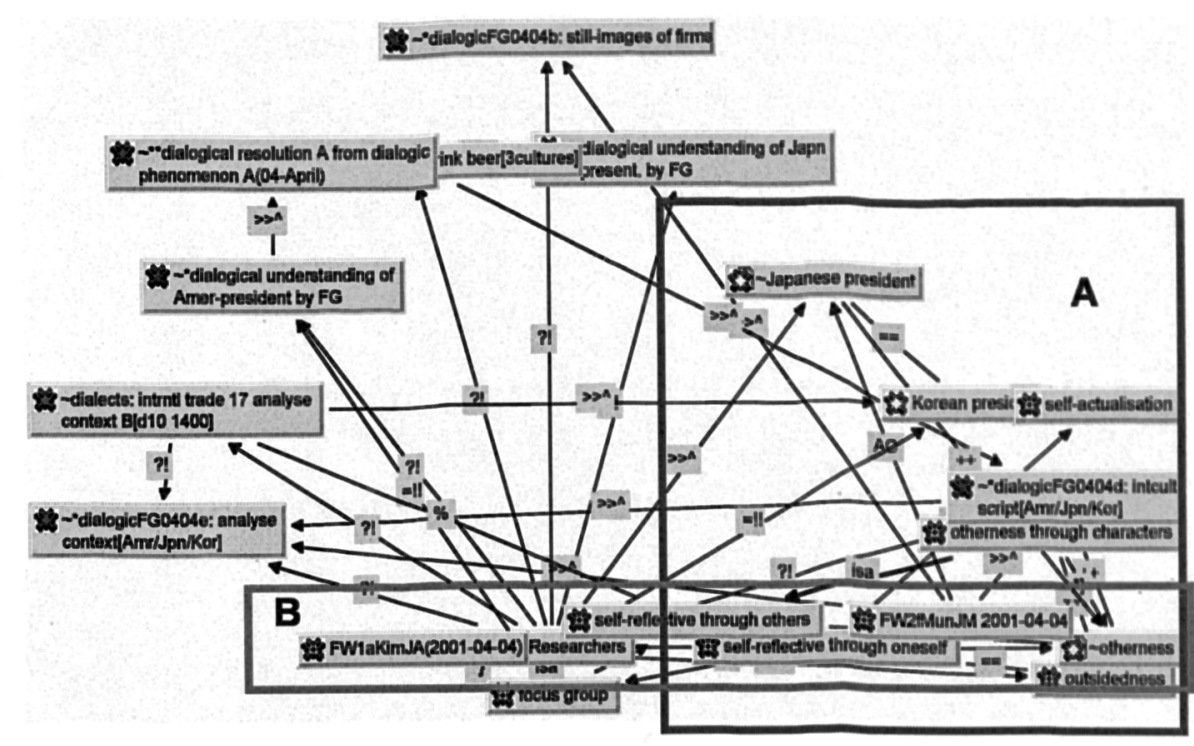

Network 6.12 Dialogicality of MunJM as the Japanese President on a Korean President in the Drama Event of the Intemational Business Meeting by the Semantic Layout

As indicated in Square $A$ of the network, MunJM was more positive than KimJA, when we reflected what they had performed, through drama, on a Korean president. This is because it is easier for MunJM to be engaged in outsidedness, in the sense that she could identify her character, the Japanese president, with the Korean president. This identification is possible in the general sense that the similarity of Japanese cultural values to Korean cultural values, especially in power distance or hierarchical structure in places of business.

At this stage, I asked participants first how they would have handled the situation if a Korean president had been the Japanese president, in a reflective way. Second, I led them to intercultural imagination on how participants would have solved the difficult situation if the Japanese president had been each of them, in a self-reflective way. And third, I moved toward a way of reflexivity by enquiring of participants what they would do in the situation if they had been a Korean president. On the basis of the similarity and these three steps, I can say that MunJM, who was a Korean girl taking the role of the Japanese president, was more easily involved in outsidedness than KimJA, another Korean girl taking the role of the American president. 
According to its semantic layout through the computer programme, ALTALS.ti, all of the factors in Square $A$ [Network 6.12] are in a queue, but I modified the original network into the above in order to show all of them. Particularly, the factor of otherness completely overlaps with that of outsidedness, and the two factors of selfreflective through others and FW2fMunMJ 2001-04-04 overlap that of self-reflective through oneself. This means MunJM, who was performing as the Japanese president, accessed dialogical interactions in the more introversive and extroversive way with her intercultural situation more than KimJA, and the other co-researchers. Easier access, I would argue, came from the general viewpoint that Japanese cultural values are similar to Korean cultural values in a sense, especially power distance, which is the key point in this intercultural situation.

I can summarise with Network 6.13 what I have described in relation to the International Business Meeting through drama in the present section.

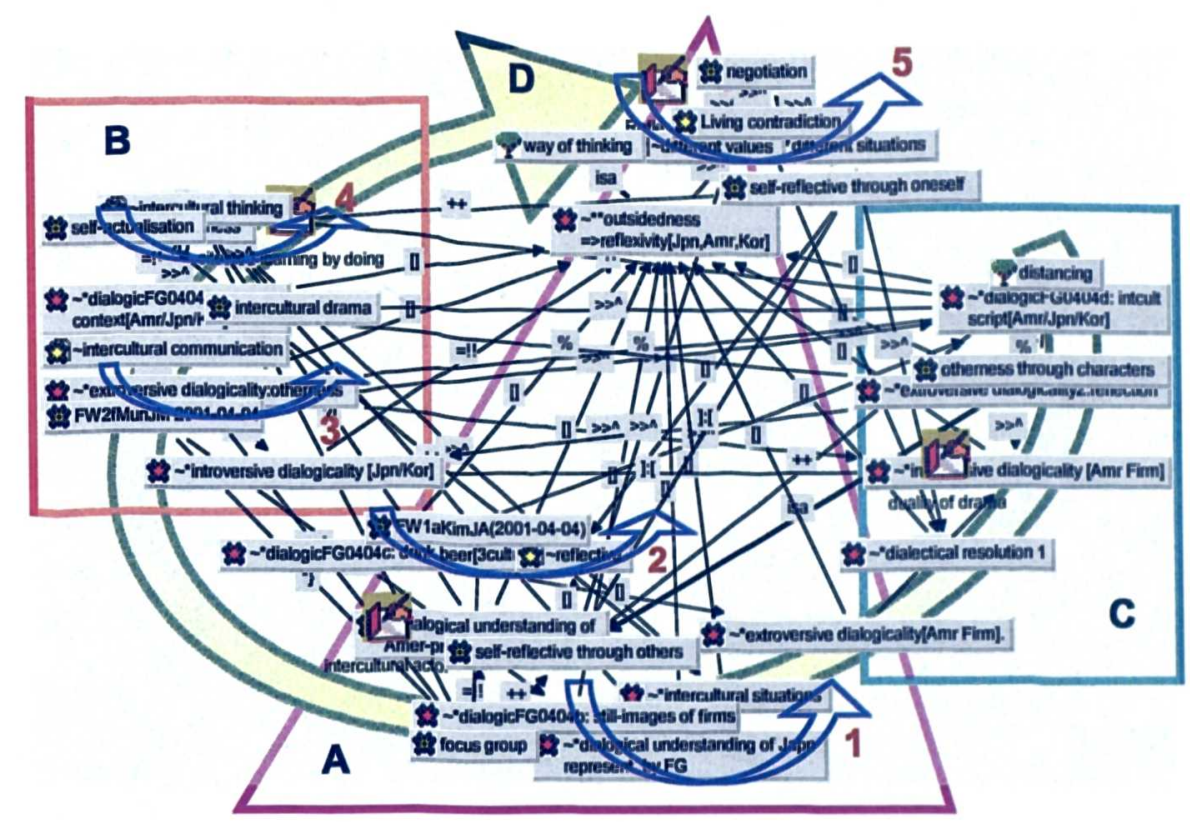

Network 6.13 Dialectical-cum-Dialogical Circle: Reflexive Dialogicality through Outsidedness of Participants in the Event of the International Business Meeting through Drama by the Semantic Layout

In the network, Triangle $A$ indicates the axis of participants' drama activities for managing the intercultural situation. Whereas Square B covers mainly participants' dialogical interactions with the Japanese representatives, Square $C$ is related to their dialogical interactions with the American president. I therefore can say in a broad 
sense, participants made their dialectical resolution between Square B and Square C in another tripartite structure, similar to structures described in 6.2. Within the tripartite structure of $A B C$, participants interacted in the dialectical-cum-dialogical way with the intercultural situation as Big Circular Arrow $D$ shows and got their equilibrium in that different situation.

Dialogical details are described in the Network above. Because each of five arrow parts, from Arrow 1 to Arrow 5, was overlapped, I modified them to disclose all the factors relevant to each blue arrow:

- Arrow 1-dialogical understanding of the Japan president by the project group (focus group), dialogical FG0404b: Still-images of firms, and intercultural situations;

- Arrow 2-dialogical FG0404c: drink beer [three different cultures], reflective, and FW1aKimJA (2001-04-04);

- Arrow 3-extroversive dialogicality: otherness and FW2fMunJM (2001-04-04);

- Arrow 4-self-actualisation and intercultural thinking;

- Arrow 5-different values, different situations, Living contradiction, and reflexivity, respectively.

Participants, thus, achieved distance from themselves by shifting contexts through drama, and had self-spectatorship with which participants performed, and, at the same time, observed what they were performing. In the middle of this self-spectatorship, it appeared that they were in the position of being distant from and outside the self and then of reflecting on themselves. I can put this with Marjorie L. Hourd's idea that 'to lose oneself is to gain oneself (cited in Bolton 1998: 266) and regard this stage of 'losing oneself temporarily as the threshold at which participants entered the reflexivity in the ICM, which Kapferer stated:

In a sense, reflexivity as a process can lead to its own negation.... Reflexivity, or the capacity to think about and to reflect upon one's own actions and the actions of others, is promoted, or mediated, by the structures that organize action and that establish contexts of meaning.... We need, therefore, to look at the degree to which the organization of ritual performance influences the participation of individuals and groups and distributes them along a continuum between total engagement and disengagement (Kapferer 1984: 188-189).

As stated above, every individual participates in everyday life in communicating with others and making meanings through the act of reflexivity, by engaging in dialogical interactions with the self and others introversively as well as extroversively. In other 
words, the negotiation between the individual person and the organisation which he/she belongs to converges at the choice between rejecting him/herself to accept others and vice versa, or between rejecting him/herself for him/herself and for others in socio-cultural/intercultural situations. In the end, an individual needs to lose him/herself to have a better understanding of him/herself, that is, understanding by distancing from him/herself.

Participants also were engaged in this sort of reflexivity in their drama activities with reference to the International Business Meeting. Participants had, at first, intercultural situations in the event of the International Business Meeting through drama and got a feel for that situation. Secondly, they researched that situation through drama activities such as still-images, thought-tracking and its performance, got dialogical dynamics, and were conscious of that situation. And thirdly, they decided how they would handle that sort of ICM. In other words, they realised that reflexivity through outsidedness led them to negotiate their living contradiction, different situations or different values, by allowing them to be self-reflective through themselves as well as others.

It thus appears, so far in the present section, those participants as intercultural actors at first faced their living contradictions in dialectical ways. These contradictions provided them with intercultural situations by making shifts in culturally different contexts. Secondly, they became involved in extroversive and introversive dialogical interactions with the intercultural situations through otherness and outsidedness. Thirdly, by dint of this sort of dialectical and dialogical interaction they thought about their drama experiences and applied to themselves as well as to others. Finally, I can produce the cone-shaped framework of drama dynamics in relation to ICM in this study. More details of the framework are in Figure 6.7.

As presented in the figure below, participants were engaged with three different sorts of dialogicality: extroversive, introversive, and both. First, the extroversive dialogicality led each participant to think about what she produced and perceived for this research study to apply it to someone else or something else except her, that is, she was involved in reflective ways.

Second, the introversive dialogicality enabled her to reflect her production-andperception activities on herself, that is, she was involved in self-reflective ways. 


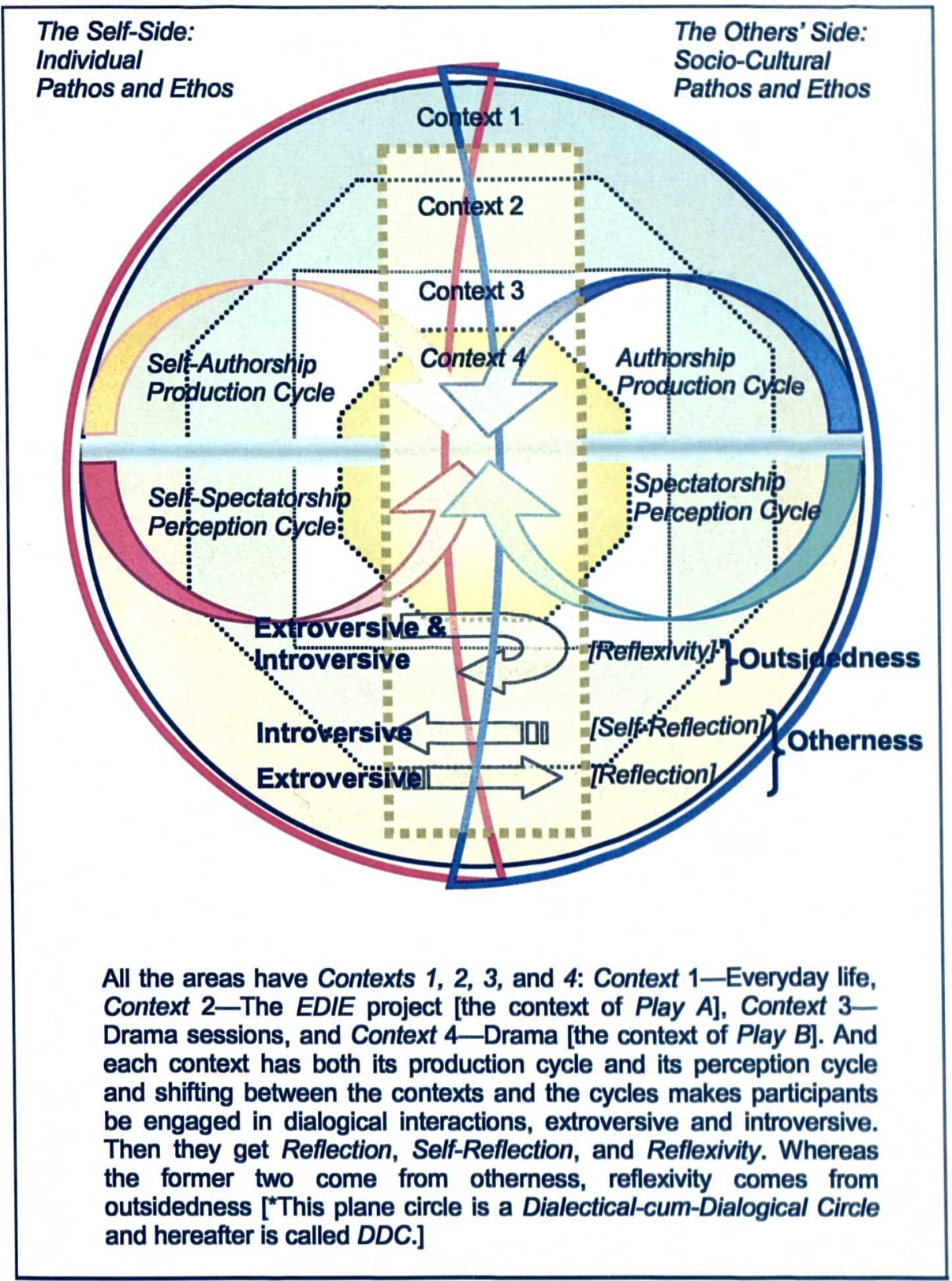

Figure 6.7 A Dialectical-cum-Dialogical Circle (DDC) and its Cone-Shaped Framework of Dialectical Dialogicality in Drama Dynamics throughout the EDIE project 
Third and finally, the dialogicality, taking two stages reflects her production-andperception activities, which got through decentring into otherness, on herself, that is, she was engaged in reflexive ways.

The dialogicalities presented, with respect to self-reflection and reflexivity in the above figure, are related to Bakhtin's otherness and outsidedness, which I introduced in terms of self-awareness through decentring into the eyes of another or with the eyes of another at the beginning of 6.3.1. By dint of this, the four cycles-authorship, selfauthorship, spectatorship, and self-spectatorship in the figure above develop in drama as well as in everyday life.

\subsection{Cyclical Process with Dialectical and Dialogical Interactions in ICM through Drama}

I believe that 'Drama occurs when one or more human beings isolated in time and space present themselves in imagined acts to another or others' and with the lapse of time, the imagined acts change through a multitude of dialectical dialogical interactions [author's italics] (Beckerman 1970: 20). Therefore, drama activity has sequential value in the sense that drama occurs through a series of dialectical and dialogical interactions.

Now, I intend to move my focus from dialecticality and dialogicality of drama dynamics to time, and to relate drama activities to the aspect of time in terms of their development. This perspective, I believe, leads me to examine another crucial mechanism in drama along with the two key mechanisms of dialecticality and dialogicality.

In the previous examination, I have treated drama activity as a single entity in some respects. There is however no doubt that drama activity was not static but constantly changing and progressing. My question therefore is-how did participants proceed to do drama activity, or dialectical and dialogical interactions one after another through drama?

\subsubsection{Dialectical Cycles in Drama Activities}

When each participant faced a situation, she first had a feeling for the situation. From her feeling through her conscious activities, relevant to the sensation, she was aware of what the feeling was that she was conscious of. In the end, she produced her total 
imaginative activity with her awareness of the situation. This process was described with three levels, sensation, sensibility, and sensitivity in 5.1. This successive progression to artistic experiences through drama is a series of temporal sensation, temporal awareness and temporal activity in a dialectical way. These temporal dialectical experiences are fitted within the next temporal one as well as the larger dialectical circle, like the circle shown in Figure 6.7 Cone-Shaped Framework of Dialectical Dialogicality. The experiences moved on to the next dynamical factor in a sequential way.

I shall consider how participants made certain resolutions in drama. The analysis will follow the characterising of an English character, Mum, in the play, War Paint, which participants rehearsed for their public performance, and it will focus particularly on their discussion of Mum's costume.

As I mentioned earlier, we chose the play, War Paint, for the public performance whose running time was approximately $\mathbf{3 0}$ minutes, assigned parts to every participant, and then had a brief discussion on how we could perform this play at the Fifth Saturday Research Meeting on 14 April 2001:

KimEL (cast for the role of Mum): What shall I prepare for Mum?

KimJA: Apronl

OhHJ: That's rightl Apron. And how about wearing a very curly permanent?

KimHJ: I agree. And then she can wear a pair of Japanese female trousers and put some very red lipstick on her lips.

(Participants are still thinking out their ideas)

The Teacher. You should think she is not a Korean Mum but an English Mum [1], shouldn't you?

LimEY: She is blonde and does her hair in a chignon.

(Participants keep talking on this between themselves).

KimHJ: And then, how about wearing her evening dress?

LimEY: Oh, no. That's too much [2].

KimJA: All right, then, what about a pair of trousers [3] in order for her to look active?

KimHJ: And she puts on her apron over that.

ParkJM: She is just an ordinary Mum who wears her apron but a somewhat elegant middle-aged Mum [4]. What about that?

KimJA: By the way, how would she wear her apron at night? [5] She should put on her nightgown, shouldn't she?

The teacher. That's a good point! We are wondering here whether or not the situation, where it's time for Angie to be about to go to a discothéque in the evening, is the same situation that Mum is worried about Angie, who hasn't returned home until nearly midnight.

Participants: Definitely different! 
The teacher: How can you show the differences? You can make the differences in your own way [6]. Think about that, will you?

KimEL (cast for the role of Mum): Shall I prepare for an evening dress as my evening costume?

KimJA: And one more, an erotic pink nightgown that Mums wear.

KimHJ: That's right! A sexy and sensual thing! [7]. And it should be very soft, as well. Men do like that, don't they?

KimEL: Well, I will think about that but....

(Eventually KimEL wore a long black skirt with her apron at the public performance)

(Participants are still interested in Mum's costume).

(Transcript of the Fitth Saturday Research Meeting on 14 April 2001: Underlined, numbered and bold, mine, and related in Table 6.1).

Participants met resistance seven times in the transcript above, the resistant factors I marked with the underlined and numbered parts. I picked out the moment when participants, as both co-researchers and performers, discussed Mum's costume because this sort of discussion reveals how those who were Korean teenager participants started characterising the English character of Mum in that play.

The first resistant factor is relevant to the intercultural dimension between English and Korean cultural factors. I was resistant to their discussion because participants still stayed in Context 1, their real world, and did not seem to take the English character into consideration, but talked about a Korean Mum. The next three resistant comments were related to the intracultural dimension and particularly to social status. They wanted to characterise the Mum as a middle class woman. Moreover, we met the fifth resistance, when KimJA refused the apron in terms of mismatching it with the time at the midnight scene. And the last two comments returned to the cultural clash between English cultural values and Korean cultural values, or between modern Western cultural values and traditional Korean cultural values.

In this consecutive process, each dialectical resolution made after a resistant comment is the source of the next resistant comment and the next resistant comment is influential on another dialectical resolution. At these successive stages, participants are 'affected by two operative factors that determine the suitability of any dramatic activity-selection and feedback' (Beckerman 1970: 23).

With regard to this, I can say there is a series of dialectical resolutions, that is, dialectical cycles. More details are shown in Table 6.1: 
Table 6.1 The Cyclical Process of Dialectical Resolutions in Characterising 'Mum' in the English Play, War Paint, at the Fifth Saturday Meeting

\begin{tabular}{|c|c|c|c|c|c|}
\hline \multirow{3}{*}{$\begin{array}{l}\text { Influential } \\
\text { Points } \\
\text { Sequential } \\
\text { Process: } \\
\text { [1] [7] inthe } \\
\text { transcript d } \\
\text { the front page }\end{array}$} & \multirow{3}{*}{ Key points } & \multicolumn{3}{|c|}{ The tripartite structure of each cycle } & \multirow{3}{*}{$\begin{array}{l}\text { Interactive } \\
\text { position } 8 \\
\text { contexts relevant } \\
\text { to Each } \\
\text { Dialectical } \\
\text { Resolution'DR }\end{array}$} \\
\hline & & $\begin{array}{l}\text { Two Differen } \\
\text { which were r } \\
\text { each other: } \\
\text { [Underlined } \\
\text { in the transc }\end{array}$ & $\begin{array}{l}\text { Factors, } \\
\text { esistant to } \\
\text { numbered } \\
\text { ipt above] }\end{array}$ & $\begin{array}{l}\text { Dialectical } \\
\text { Resolution [DR]: } \\
\text { Bold parts in the } \\
\text { transcript on the } \\
\text { front page }\end{array}$ & \\
\hline & & \multicolumn{3}{|c|}{$\begin{array}{l}\text { EX-DR: Extroversive dialectical Resolution } \\
\text { IN-DR: Introversive dialectical Resolution }\end{array}$} & \\
\hline $\begin{array}{l}\text { [1] Cycle 1: } \\
\text { Inter-cultural } \\
\text { [Level } 1 \text { of } \\
\text { Sensation] }\end{array}$ & $\begin{array}{l}\text { Confusing } \\
\text { the drama } \\
\text { context }\end{array}$ & $\begin{array}{l}\text { English } \\
\text { cultural } \\
\text { values }\end{array}$ & $\begin{array}{l}\text { Korean } \\
\text { cultural } \\
\text { vales }\end{array}$ & $\begin{array}{l}\text { English Side } \\
\text { through EX-DR } \\
\text { [blonde] }\end{array}$ & $\begin{array}{l}\text { Production } \\
\text { [Authorship] } \\
\text { Contexts } 1 \& 2\end{array}$ \\
\hline $\begin{array}{l}\text { [2] Cycle 2: } \\
\text { Inter-cultura] } \\
\text { [Leve/ } 2 \text { of } \\
\text { Sensibility] }\end{array}$ & $\begin{array}{l}\text { Imbalance } \\
\text { between } \\
\text { the cultural } \\
\text { vales }\end{array}$ & $\begin{array}{l}\text { English } \\
\text { cultural } \\
\text { values }\end{array}$ & $\begin{array}{l}\text { Korean } \\
\text { cultural } \\
\text { vales }\end{array}$ & $\begin{array}{l}\text { Korean side } \\
\text { through IN-DR } \\
\text { [A pair of } \\
\text { trousers] }\end{array}$ & $\begin{array}{l}\text { Perception } \\
\text { [Spectator-ship] } \\
\text { Contexts } 2 \& 3\end{array}$ \\
\hline $\begin{array}{l}\text { [3] Cycle 3: } \\
\text { Intra-cultural } \\
\text { [Leve/ } 2 \text { of } \\
\text { Sensibility] }\end{array}$ & $\begin{array}{l}\text { Social } \\
\text { status }\end{array}$ & $\begin{array}{l}\text { Middle } \\
\text { Class }\end{array}$ & $\begin{array}{l}\text { Working } \\
\text { Class }\end{array}$ & $\begin{array}{l}\text { Middle-class } \\
\text { through EX-DR } \\
\text { [apron] }\end{array}$ & $\begin{array}{l}\text { Production } \\
\text { [Authorship] } \\
\text { Contexts } 2 \& 3\end{array}$ \\
\hline $\begin{array}{l}\text { [4] Cycle 4: } \\
\text { Intra-cultural } \\
\text { [Level } 2 \text { of } \\
\text { Sensibility] }\end{array}$ & $\begin{array}{l}\text { Social } \\
\text { status }\end{array}$ & $\begin{array}{l}\text { Working } \\
\text { class }\end{array}$ & $\begin{array}{l}\text { Middle } \\
\text { Class }\end{array}$ & $\begin{array}{l}\text { Half-middle } \\
\text { Class through } \\
\text { IN-DR } \\
\text { [elegant] }\end{array}$ & $\begin{array}{l}\text { Perception } \\
\text { [Spectator-ship] } \\
\text { Contexts } 2 \text { \& } 3\end{array}$ \\
\hline $\begin{array}{l}\text { [5] Cycle 5: } \\
\text { Intra-cultural } \\
\text { [Level } 2 \text { of } \\
\text { Sensibility] }\end{array}$ & $\begin{array}{l}\text { Play } \\
\text { Directing }\end{array}$ & $\begin{array}{l}\text { Time in } \\
\text { the drama } \\
\text { context }\end{array}$ & Costumes & $\begin{array}{l}\text { Appropriate } \\
\text { Costume-designer } \\
\text { through } \\
\text { EX-DR } \\
\text { [night gown] }\end{array}$ & $\begin{array}{l}\text { Production } \\
\text { [Authorship] } \\
\text { Contex } 3\end{array}$ \\
\hline $\begin{array}{l}\text { [6] Cycle 6: } \\
\text { Inter-cultural } \\
\text { [Level } 2 \text { of } \\
\text { Sensibility] }\end{array}$ & Acting & $\begin{array}{l}\text { Acting the } \\
\text { Westem } \\
\text { Mum }\end{array}$ & $\begin{array}{l}\text { The } \\
\text { Korean } \\
\text { Mum } \\
\text { Image }\end{array}$ & $\begin{array}{l}\text { Discomfort in } \\
\text { acting in that very } \\
\text { Western costume } \\
\text { through } \\
\text { IN-DR } \\
\text { [evening dress] }\end{array}$ & $\begin{array}{l}\text { Perception } \\
\text { [Self-Spectator- } \\
\text { ship] } \\
\text { Contexts } 3 \& 4\end{array}$ \\
\hline $\begin{array}{l}\text { [7] Cycle 7: } \\
\text { Inter-cultural } \\
\text { [Into } \\
\text { Leve/ } 3 \text { of } \\
\text { Sensitivity] }\end{array}$ & $\begin{array}{l}\text { Non- } \\
\text { Feministic } \\
\text { Attitude }\end{array}$ & $\begin{array}{l}\text { Traditional } \\
\text { Korean } \\
\text { cultural } \\
\text { values }\end{array}$ & $\begin{array}{l}\text { Modem } \\
\text { Korean } \\
\text { cultural } \\
\text { values }\end{array}$ & $\begin{array}{l}\text { Rejecting Non- } \\
\text { Feministic Attitude } \\
\text { through } \\
\text { EX-DR \& IN-DR } \\
\text { [a long black skirt } \\
\text { with her apron] }\end{array}$ & $\begin{array}{l}\text { Perception } \\
\text { [Self-spectator- } \\
\text { shop] \& } \\
\text { Production } \\
\text { [Self-authorship] } \\
\text { Contexts } 3 \& 4\end{array}$ \\
\hline
\end{tabular}

Thus, whenever meeting resistance in the above, although participants did not treat the two different factors equally, they made dialectical resolutions between the different factors and one resolution followed another, that is, sequentially, as marked with bold fonts in the transcript on the previous pages. These dialectical resolutions can also be applied in the broad sense, for instance, between production and perception in the drama activities of participants. This is in the sense that different factors were in their same contexts or in different contexts from each other. Furthermore, all of these dialectical cycles form part of an accretion process which occurs through dialectical interactions. These then fit into another bigger process of dialogical interactions. 
In this respect, I can apply a dialectical process to two different perspectivesdialectical resolutions in a narrow sense and dialectical interactions in a broad sense. That is, while the former leads into dialogical interaction, the latter is concerned about dialectical relations in the duality of drama, such as production and perception, theatricality and reality, or performer and spectator. In the end, I can apply this dialectical cyclical process of their drama activities, in the broader sense, to the dialectical interplay between the two'-drama activity and inner life (Beckerman 1970: 36).

\subsubsection{Dialogical Spirals in Drama Activities}

Participants handled their drama activities with a series of dialectical cycles for single resolutions as well as for other bigger processes of the dialogical circle relevant to them. Then how did the dialogical circle develop with those drama activities?

Once more, I intend to return to the drama event of the International Business Meeting in order to pursue how to develop the dialogical circle in the process of drama activities. There is no doubt that 'Activity...is a way of grouping motion into coherent units of time' (Beckerman 1970: 28). With regard to this, let me first show chronologically the drama activities, which participants did in the event, and then follow the process of those activities.

I gave participants three drama sessions relevant to the above-mentioned event on 4 and 6 April 2001 and the main activities during those drama sessions are described in chronological order:

[1] Reviews of the previous session's work, particularly cultural icebergs about understanding cultural differences

[2] To skim the extract of the Intemational Business Meeting and to get their first impression from that meeting

[3] To make still-images of two firms, American and Japanese

[4] To talk about the beginning part of the extract where the American president is drinking a beer [the First cultural clash].

[5] To discuss what the American president is doing and why he does that in the beginning part

[6] To discuss how the Japanese representative respond to the American president at its beginning part.

[7] To read through the extract

[8] To dramatise the extract in order for participants to give their short performance.

[9] To perform it by groups

[10] To reflect what participants understood through their performance in understanding that sort of international business meeting

[11] To compare the Japanese president with an ordinary Korean president 
[12] To analyse the intercultural context of the meeting and to relate to three different cultures: American, Japanese, and Korean.

[13] To think about the intercultural situation to apply it to characterising the Korean president in the Korean side of participants

[14] To try to make reflexive dialogical interaction with the intercultural situation

[15] To get dialogical resolution through reflexive dialogicality from the dialogical phenomenon

Participants followed the above main drama activities one after another, at an individual level. For instance, KimJA added her previous drama activity, whose resolution she got from two different elements in her dialectical interaction, to another drama activity. Before completing this addition, she faced another resistance or difference between the previous activity and a new element in this situation, and she needed to make a dialectical resolution. In the end, this sort of dialectical resolution was added to the next and larger dimension over a period of time; that is, dialogical interactions with her intercultural situations. This process of her accretion continued until she achieved a balance between herself and otherness: her appropriate performance as the American president in the drama event. The balance that she tried to get through drama can be defined simply as the dialectical balance between herself and otherness. To put this in detail, it is the balance between Pathos and Ethos with dialogical interaction at her individual and socio-cultural levels, between the Drama world and the Real world, between Production and Perception and so on, through reflexivity in her drama activities.

On the other hand, I found that there was also a series of dialogical circles in the drama event at the level of the whole participant group. The aforementioned activities were presented in their semantic dimensions as shown in Network 6.14.

According to Network 6.14, which I produced in its semantic layout through one of CAQDAS (computer-aided qualitative data analysis software), ATLAS.ti, the main drama activities in the drama event take steps forward in the spiral pattern of circles through making shifts of contexts.

Participants did do each main drama activity, which was given its number in the network above, after making a series of dialectical resolutions and dialogical interactions in the process of handling the intercultural situation. Following the drama activities in chronological order, a series of dialectical-cum-dialogical circles are drawn, as marked in the network. 
There are three circles and each circle covers both the right and the left side of Line ab. This coverage reveals that participants made shifts between contexts. Activities on the right side of the line are mainly related to their drama experiences as students, coresearchers, and performers-out-of-role in Contexts 1, 2, and 3, respectively. On the other hand, activities on the left are mainly relevant to their drama experience as performers-in-role in context 4 , in the drama world.

The first $D D C$ (Dialectical-cum-Dialogical Circle), which is marked with red arrows with slant lines, consists of five dialogical interactions from [1] dialogicFG0404a: review cultural icebergs to [5] dialogical understanding of the American president. In this first circle, participants experienced feelings against the American president from their first reading of the extract of the International Business Meeting at their sensation level. Then they became conscious of how the American president behaved in that drama context through extroversive dialogical interactions at their level of sensibility.

The second $D D C$, marked with green arrows with dotted lines, begins with [5] understanding the American president through extroversive dialogical interactions and contains [6] dialogic understanding of the Japanese president, [7] dialogicFG0404d: intercultural script to dramatise for their performance, and [8] participants-in-role. Participants in this circle focused on understanding the drama situation from the position of performers as well as co-researchers and reflected on our Korean cultural situation. From here on participants connected the intercultural situation with their Korean one and were involved at their sensitivity level through producing how they did their total imaginative drama activities in order to perform in that situation.

The third and biggest $D D C$, marked with blue arrows, starting with [8] participants-inrole, includes activities, from [9] to [15], relevant to reflection, self-reflection and reflexivity at the final stage of that drama event. They thought about what they got from a drama event to apply it to that sort of intercultural situation, situations around themselves such as the subject of Trade Business, and themselves through otherness. A series of reflection and reflexivity continued during the performance as well as after the performance at their levels, of sensation, sensibility and sensitivity in the sense that there is 'the sequence of presentational reactions from the rearrangement of those reactions into a unified response' (Beckerman 1970: 131).

Two different kinds of drama experiences of participants were involved within this circle-their 'reactions during production of the theatrical experience and the remembrance of the production the memorial experience' (Ibid). 


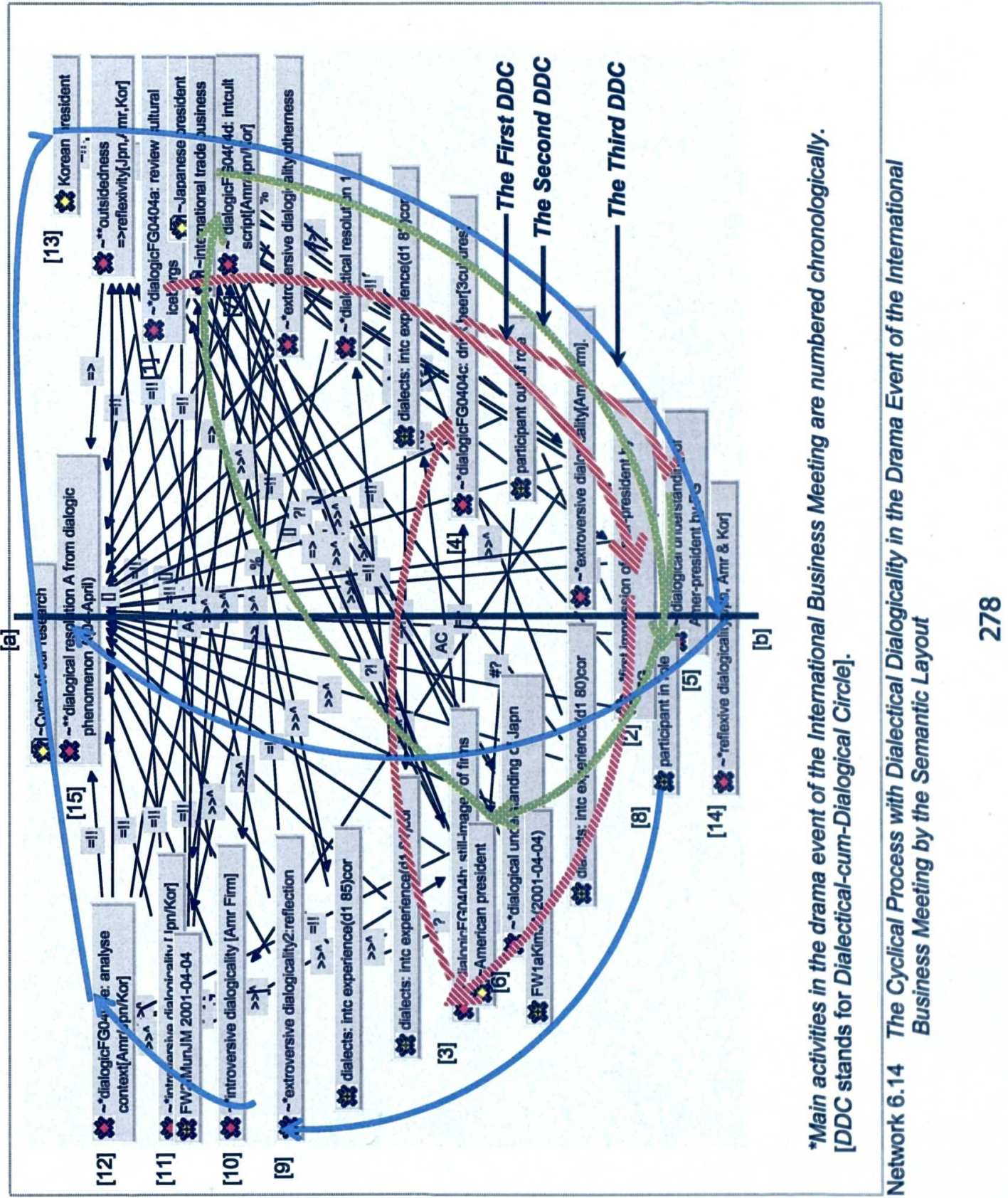


Furthermore, I can say that the final stage of this circle contains, in terms of dialogical interaction, extroversive and introversive elements with the ICM in that type of intercultural situation, rather than just a particular international business meeting. The circle therefore is drawn as the biggest one among the three circles at its semantic level and the circles make a pattern of dialectical and dialogical spirals, although each circle is different from the others in its size and its meaning.

What I would like to add to these circles is that participants seemed to be more dependent on dialogical interactions rather than dialectical resolutions. As can be seen in Part A of Network 6.15), which when laid out in its topological perspective through ATLAS.ti, dialectical experiences of participants are less dependent factors on the outcome of [15] dialogical resolutions produced through super-super coding rather than dialogical interactions in Part $B$ and $C$.

This, however, eventually reveals that dialogicality compromises dialecticality in dynamics of drama, but dialecticality is 'in a state of intense and essential axiological interaction' with extroversive and introversive dialogicality (Holquist and Liapunov 1990: xxvii). Also, the dialecticality leads participants into changing their cognitive map of the first impression from the American president because of their cultural clash in Part $D$ in the network.

The essential axiological interaction with both dialecticality and dialogicality is to struggle between the self and others with their culture. Abbs argues with respect to this:

...the arts are not ends in themselves but epistemological tools for the clarification of experience... in the arts a common ihythm, a cumulative sequence moving from self to community back to self, in an endless dialectical motion. Our new ground is that of creative mimesis in which the self struggles to become itself within its own culture, dynamically conceived (Abbs 1989c: 209-210).

There are a series of circles with the fifteen main drama activities, whose essential axis is dialecticality and there appears eventually to be no dialogicality without an endless dialectical motion between the self and the society within its own culture.

With regard to this, I can return to dialogical interactions of participants, extroversive and introversive, in drama. Participants in the EDIE project made consecutively dialectical and dialogical interactions with the intercultural situations through making 


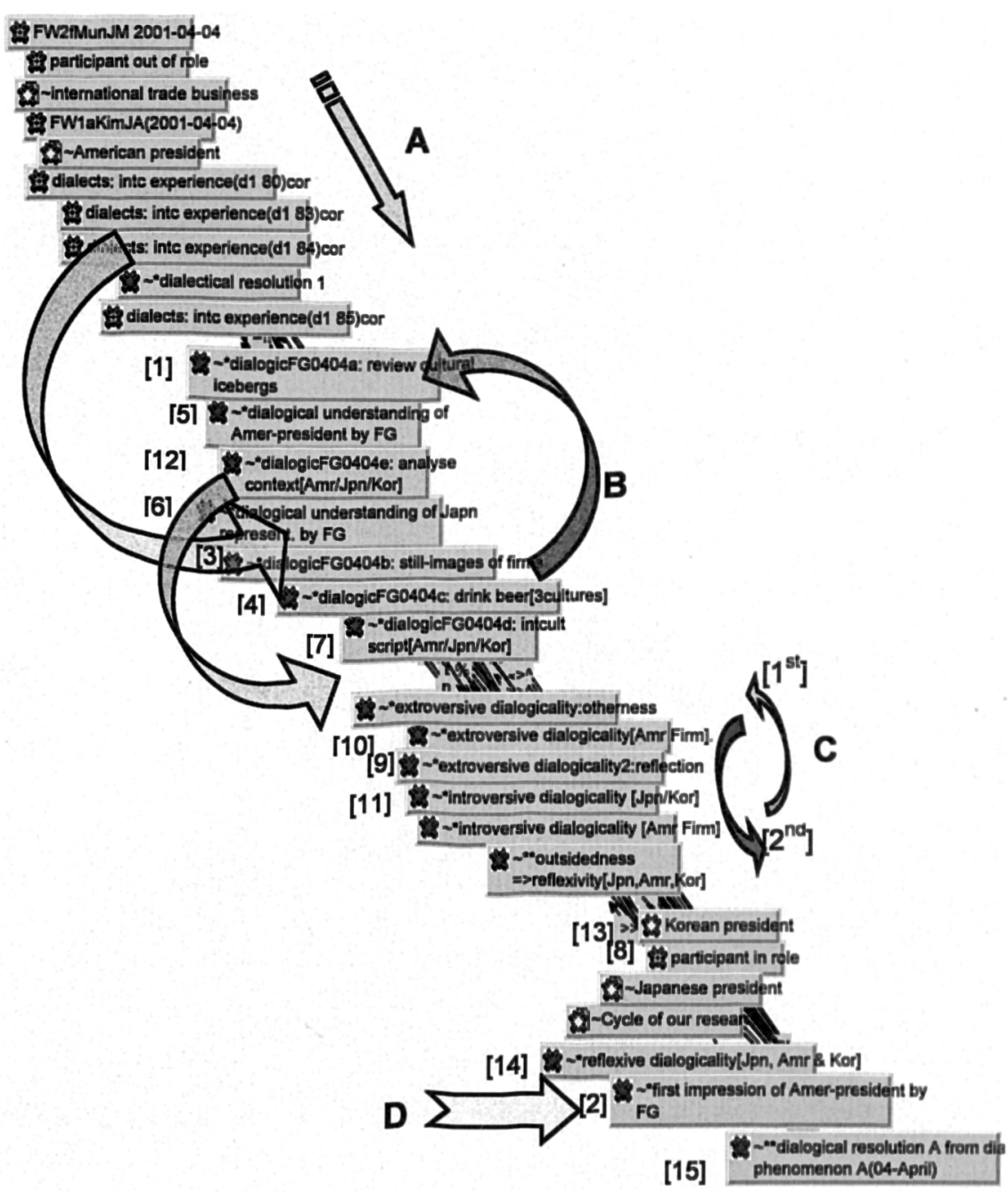

*Main activities in the drama event are given numbers in the chronological order.

Network 6.15 The Cyclical Process with Dialectical Dialogicality in the Drama Event of the International Business Meeting by the Topological Layout

shifts between contexts, which I mentioned in Figure 6.7 in 6.3.2. In this process, they revealed their struggling between themselves and their situated educational milieu.

It was possible for participants to make shifts between contexts, that is, being 'in' and 'out' of themselves and their situations because of the duality in drama, actuality and theatricality. Participants took drama as the analytical model for understanding foreign cultures through penetrating those cultures, via the American president and the Japanese president in the case of the drama event of the International Business Meeting, as stated above. Moreover, they returned to their own cultural values to think about their drama experiences to apply it to characterising a Korean president, 
who could be in a similar intercultural situation in that meeting. It is thus certain that outsidedness leads to better ICM, as Bakhtin stated:

\begin{abstract}
There exists a very strong, but one-sided and thus untrustworthy, idea that in order to better to understand a foreign culture, one must enter into it, forgetting one's own, and view the world through the eyes of this foreign culture.... Creative understanding does not renounce itself, its own place in time, its own culture; and it forgets nothing. In order to understand, it is immensely important for the person who understands to be located outside the object of his or her creative understanding -in time, in space, in culture.... In the realm of culture, outsidedness is a most powerful factor in understanding [author's italics] (Bakhtin 1970: 6-7).
\end{abstract}

In this 'being inside and outside' process, that is, in 'a dialogic encounter of two cultures' (Ibid: 7), participants took different roles of participants, co-researchers, performers, and characters with other cultural identities throughout the EDIE project. The participants, in other words, multi-reactive intercultural actors, retained their own cultural unity and open totality, such as outsidedness, to have a better creative understanding of their own and other cultures.

Another to be considered is how participants functioned as multi-reactive actors throughout the EDIE project with respect to the following argument of Collingwood:

\begin{abstract}
If art is not a kind of craft, but the expression of emotion, this distinction of kind between artist and audience disappears.... The poet is not singular either in his having that emotion or in his power of expressing it; he is singular in his ability to take the initiative in expressing what all feel, and all can express (Collingwood 1963: 118-119).
\end{abstract}

In common with the ways in which 'the poet' takes the initiative in expressing his feelings and emotions, I can argue that participants, as performers, take their initiative to express their emotions in acting in the production cycle and they, as audiences, concede the initiative to other participants in observing in the perception cycle. Furthermore, even in acting, participants take roles of performers as well as spectators, in a word, spect-actors or multi-reactive participants.

On this basis, all of these functions of participants in four contexts are eventually unified into the self of each participant, which is not exactly the same as she had been before, and is going to be fitted into another dialectical-cum-dialogical interaction on and on.

Now, I can model dynamics through drama on the grounds of what I found through analysing my drama data as displayed in Figures 6.8, 6.9 and 6.10: 


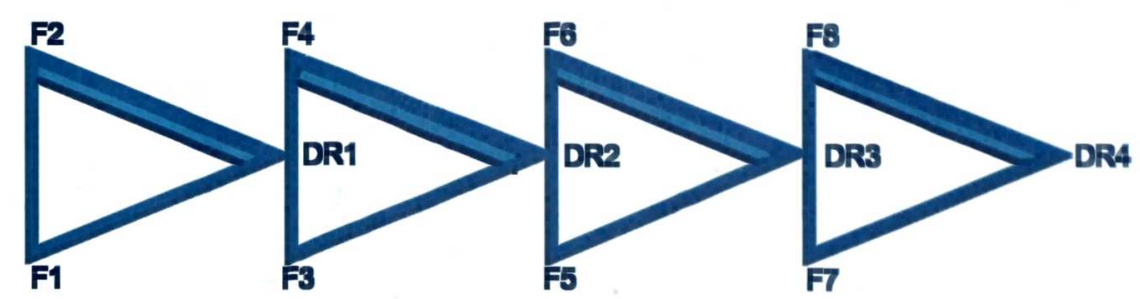

Participants get the balance between two different factors and then make Dialectical Resolution between them. [" F: Factor, DR: Dialectical Resolution]

Figure 6.8 The Cyclical Process for Dialectical Resolutions between Two Different Factors through Drama

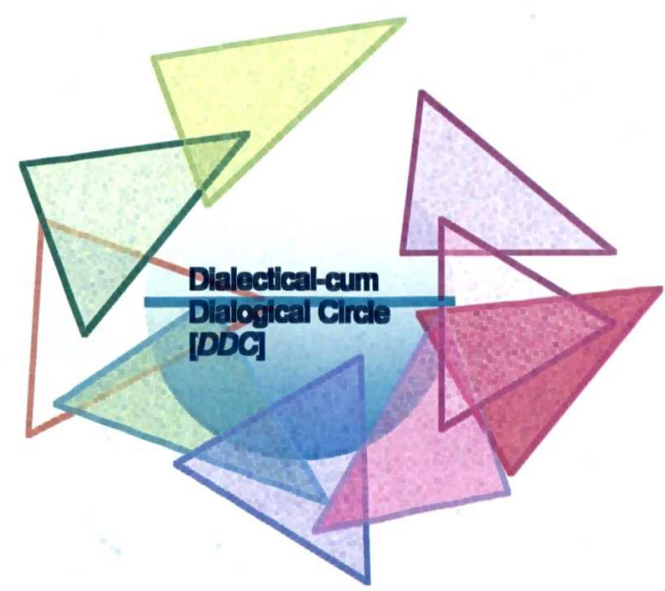

A series of dlalectical resolutions, which are marked with triangles, are fitted into their bigger interactions, that is, dialogical interactions in a Dialectical-cumDialogical circle (DDC). The two halves of the Circle are dialectical in the broad sense:
[1] The SelfXOthemess;
[3] PerceptionXProduction;

[2] The Real World X The Drama World; [4] PathosX Ethos, etc.

There are a multitude of dialectical and dialogical interactions in the circle.

\section{Figure 6.9 The Simplified Dialectical-cum-Dialogical Circle (DDC) of the Cone- Shaped Framework in Dynamics of Drama}

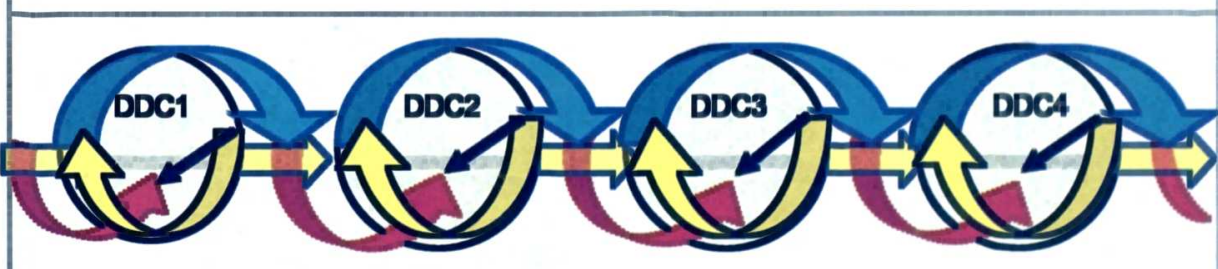

The bottom half of each circle is the ground of the top half and the latter is the figure of the former. One half is reflected on the other. Participants undergo this spiral process, dialectical and dialogical, until they get their own levels of equillibrium.

[' DDC: Dialectical-cum-Dialogical Circle].

Figure 6.10 The Spiral Cycles with Dialectical Dialogicality through Drama 


\title{
6.5 Summary: Dual, Dialectical, Dialogical and Cyclical
}

\begin{abstract}
Life by its very nature is dialogic. To live means to participate in dialogue: to ask questions, to heed, to respond, to agree, and so forth (Bakhtin 1984, Problems of Dostoevsky"s Poetics: 293).
\end{abstract}

In the present chapter, on the basis of the statement above I analysed my participants' drama activities in their EDIE project, which I led with Students in Participatory Action Research [SPAR] at a secondary school, Korea, in 2001

Taking a horizontal method of drama analysis and focused on their drama experiences in terms of the structure of drama in its linear patterns such as Levels 1, 2, and 3, sensation, sensibility, and sensitivity, in the previous chapter, I found that drama experiences of participants were artistic, therapeutic, and socio-cultural in ICM through drama.

On the other hand, in the present chapter, I took a vertical method of drama analysis to analyse the aforementioned data. Through this vertical method, I found that there is a duality, dialecticality, dialogicality and a cyclical process with dialectical dialogicality through drama.

In the first section, I found that the duality is the very basic factor, which makes drama a different type. Owing to its duality, 'Drama occurs when one or more human beings isolated in time and space present themselves in imagined acts to another or others' [author's italics](Beckerman 1970: 20). The duality is established between actuality and theatricality, and perception and production through drama. Through making shifts between these dual contexts, participants have drama experiences in managing ICM.

In the second section, I illustrated my finding that the above-mentioned duality makes participants become engaged in making dialectical resolutions and accrete those resolutions as they advance drama activities.

In the third section, those dialectical resolutions are fitted into dialogical interactions with intercultural situations in the drama event in three ways; extroversive, introversive, or both extroversive and introversive. Participants reflect on others, on themselves, and through others on themselves, that is, reflection, self-reflection, and reflexivity, respectively through these three ways. 
In the fourth and final section, I described the cyclical process of drama activities. Each dialectical resolution has its tripartite pattern and this pattern repeats and accretes to be fitted into dialogical interactions as participants advance their drama activities. Furthermore, the dialogical interactions also repeat in their cyclical process along with the development of their drama activities.

I therefore can say that there are dynamics in drama, dialectical, dialogical, and cyclical, in extroversive and introversive ways. The drama dynamics, along with drama experiences relevant to their sensation, sensibility, and sensitivity, can give participants better understanding of ICM to improve their intercultural competence. 


\section{Part IV.}

\section{CONCLUSION}




\section{Chapter 7. \\ Conclusion and Discussion}

The present chapter leads me into the conclusion as the final stage of this research. However, as I have learnt in this thesis, the conclusion gives me another task to have dialectical-cum-dialogical imagination for the next researches. This final chapter will be the basis for another beginning chapter; there is nothing final for us. It is said that 'nothing is absolutely dead: every meaning will have its homecoming festival' (Bakhtin 1986: 170). Regarding this, I intend to complete this chapter to take stock of the study at the threshold of entering another.

\subsection{Conclusion: Drama as an Arena of ICM}

\subsubsection{Drama and its Effectiveness}

This research study has been a long journey for me into drama education. My original intentions with the study, as a teacher and researcher, were to identify whether drama could be applied to language education, particularly in teaching EIL, and intercultural education. Both my career of teaching EFL in Korea and my intercultural experiences here, in Durham, UK, have given me opportunities to integrate theoretical and practical perspectives. As I discussed in Chapters 2 and 4, and judging from my own case, it became apparent to me that intercultural identities of EIL teacher or drama teacher could affect not only their teaching activities but also an interactive relationship between teachers and students to become better intercultural actors in the intercultural discourses surrounding themselves.

So far, the Ministry of Education and teachers in Korea have tried to participate in educational reform movements, whereby drama has recently been introduced as an optional subject at the secondary level in the National Curriculum.

However, non-traditional teaching methods have failed to be applied successfully in educational environments in Korea. For example, as I have discussed in Chapters 1, 2 and 3, there is the need to replace the position of English as a foreign language (EFL) with English as an international language (EIL), teaching intercultural discourses, and to apply drama as a subject per se as well as to other subjects such as EIL as integrated projects. 
In this educational milieu, this research study, whose main topic is the development of ICM and ICP through drama in teaching EIL, has been carried within my feeling that I would take a part of the responsibility for that milieu and respond to the responsibility with respect to pedagogical methods. As I discussed in Chapters 1 and 5, there were few researches in drama education and EDIE experiences in schools and the EDIE project seem to lay the groundwork in the field of ElL classrooms and intercultural education in Korea. That was why $I$, as a teacher and researcher, have taken the responsibility for suggesting drama as an effective pathway for intercultural education as well as teaching EIL in the Korean context.

From this standpoint, I have tried to take the responsibility for searching for the effectiveness of drama in my own way in this study.

\section{Drama in ElL Learners: Holistic Activity of Sensation, Sensibility, \& Sensitivity}

At the beginning of this study, I wondered what elements of drama were effective in teaching and learning EIL and how those elements could be applied to ElL education.

A main aim of teaching and learning EIL is to communicate with other people in intercultural discourses, as I have reviewed in Chapter 2. Furthermore, in order to have better intercultural communication (ICM), EIL learners do not merely need to acquire key factors at affective, cognitive, and behavioural levels to enhance intercultural competence (ICP). EIL learners, also, need to improve competence in other respects, such as [1] empathy in the symbolic interactionist's perspectives, [2] intercultural imagination through critical thinking, [3] decentring or alienation of feeling like a stranger, and [4] otherness, outsidedness, and thirdness in intercultural discourses.

I have explored what was able to satisfy these needs for EIL learners. As reviewed in Chapter 3, it became apparent to me that drama was beneficial to improving the ICM and ICP of learners. As I have found in Chapters 5 and 6, it appeared that drama provides cultural configurations as well as an art form to bring different cultures to the EIL learners in their authentic fullness (Scolnicov \& Holland 1989; Hornbrook 1998b; Fleming 2003).

This means that drama provided EIL learners with intercultural contexts of situational teaching and learning as an art form, a learning medium, a socialising activity, a therapeutic tool and a research method at affective, cognitive, and behavioural levels. 


\section{Effectiveness of Drama Experiences for ICM and ICP}

Drama was effective in this study. At this stage, we need to recall the figure-ground relation between art and life, which I introduced in Chapter 2. Drama was shown as cultural configurations and provides participants with the authentic fullness of relevant culture(s).

Let me take another analogy, which Schechner articulates-'Art is cooked and life is raw. Making art is the process of transforming raw experience into palatable forms. This transformation is a mimesis, a representation' (Schechner 1988: 38). In addition, he points out that some food can be raw but other needs to be cooked. This means that something in life can be used as material in art like some fruits and vegetables in food. Moreover, the process is irreversible, but previous experiences in cooking affect choosing raw materials for another cooking. Thus, "the boundaries between "life" and "art"—raw and cooked-are blurry and permeable' (Ibid).

Through this interconnection between drama art and life, as I have discussed in Chapter 5, participants used their experiences in everyday life as their material to learn ICM through drama by making contextual shifts between the drama world and the real world. Furthermore, their experiences in drama and everyday life were unified in the self of each participant and were expressed as total imaginative activities, which are 'in the unity of my answerability' (Bakhtin 1919: 2).

In short, drama led participants to intercultural experiences, and made them explore those experiences to have a better understanding of ICM.

In the process of their exploration through drama, participants were engaged in the characteristics of drama based by its nature on its duality, as described in Chapter 6 . The duality invited participants to be involved in its dynamics, such as dialecticality, spectatorship, self-spectatorship, distancing (otherness), and dialogicality, and these consecutive experiences enhanced the ICM and ICP of EIL learners in relation to intercultural discourses.

\section{Drama Dynamics for Enhancing ICM and ICP of Participants}

As I have shown in Chapters 5 and 6, first of all, participants were engaged in drama activities through their intercultural imagination of intercultural situations and made shifts of different contexts, between the real world and the drama world, between authorship and spectatorship/self-spectatorship, and so on. 
At the beginning of their drama activities in certain intercultural situations, participants mainly started with the intercultural imagination through the Magic If in the Stanislavski System. Then, participants were engaged in dynamics through drama, inroles and out-of-roles, such as juxtapositional duality, dialecticality, dialogicality, cyclic spirals, distancing, otherness, outsidedness, insidedness, thirdness, and so on.

And then they took stock of what they had understood, figured out what characters to create and how to characterise those characters in their own perspectives, and express those characters in third ways. Furthermore, these dynamics were not originated only from a specific domain of participants' experiences, but from all of the three domains in human experiences-affective, cognitive, and behavioural.

Above all, these dynamical factors in drama, along with intercultural imagination, were effective in developing dynamic ICM, and the ICP of EIL learners, who participated in intercultural drama activities. That is to say, the more they stretched intercultural imagination about relevant situations and thought about them more critically, dialectically, and dialogically, the further they distanced themselves from those situations or intercultural discourses. This distancing led them into otherness and outsidedness to have a better intercultural understanding of their own culture as well as others'.

\section{Development of Participants as Intercultural Actors in Intercultural Sensitivity}

It does not appear that my participants reached the level at which they became intercultural 'actors' in the fullest sense. However, their intercultural attitudes changed enough to overcome their ethnocentric attitudes. We should consider the fact that whereas a period of six months or more is desirable, the project group in this study participated in this sort of drama project for only a two-month project.

However, I can say that the more they had engagement in the intensive project, the more the participants became familiar with intercultural drama, were involved in drama activities with intercultural thinking, and had a better intercultural understanding. In short, the project gave opportunities to enable participants to overcome the limits of learning EIL in their English classrooms by releasing the learners from ethnocentric attitudes at a mono-cultural stage, and achieving ethnorelative attitudes at the intercultural stage.

Another thing to be considered here is that because there are no didactic programmes of intercultural learning at school, we should fill the vacuum of the programmes in a 
dialectic sense, particularly in 'the aspect of intercultural competence with development directed outwards and the aspect directed inwards' (Kordes 1991: 304). Participants overcame limits of intercultural learning in their ElL classrooms and found possibilities to be intercultural actors with cultural identities, through drama, dialectical and dialogical.

At the end of this research project, as I confirmed in data from the post-questionnaire, in 5.3.2 [see Table 5.3], participants highly recommended EDIE in that [1] they became interested in EIL through drama, that [2] they were concerned about their cultural identity with ethno-relative attitudes in and through drama, and [3] they had opportunities to handle ICM in and through drama in appropriate ways for intercultural actors, who stabilised their self-identity in the process of ICM, and mediated other cultural values to stabilise their self-identity in intercultural perspectives.

Thus what I have said so far suggests that drama is an effective way to prepare for better ICM and ICP of EIL learners in the fast-globalising world.

\section{Other Possibilities for Research at the Crossroads of ICM and Drama}

I have found that there remain things yet to research. We have limits of intercultural learning in EIL classrooms and therefore we need to search for possibilities to overcome those limits and appropriate teaching programmes. In this regard, we could recommend models of ethnographic fieldwork, wherein EIL learners or foreign language learners are taught to develop their ICM and ICP. However, it doesn't seem to be easy for all EIL learners, particularly at the primary and secondary levels, to be involved in that sort of programme. For instance, there are year-abroad projects, but they are too young to stay abroad without proper supervision of their teachers or parents for an extended length of time.

Also, there is an interest in intercultural education through using media, such as films or TV programmes. These sorts of teaching methods still remain disadvantageous in that there is no face-to-face interaction. The most important thing to research is the issue that we should have ICM with direct human contact, never only mechanical contact, through multiple communication media, not too much textualism or logocentralism, as I discussed in Chapters 2 and 3. 
Furthermore, I am still wondering what an ideal intercultural identity is like in relation to discourses, national, international, and global, and what transcultural identity or cosmopolitan citizenship should be developed for whom, to whom, and for what.

\subsubsection{Reflections on the Study: Active, Reactive, \& Proactive}

In my research, drama was applied as a research tool. I also used drama as perspectives of an art form, a learning/teaching medium, a socialising activity, and a therapeutic tool, and all of them were of equal significance in the project. In this subsection, I intend to discuss how drama was effective in inviting EIL learners to research their ICM and its achievement.

I designed my research methods with PAR (participatory action research) and grounded theory, and invited EIL learners to participate in SPAR (students in participatory action research) in drama activities as co-researchers.

That is to say, throughout this research project, they had to be engaged in a drama technique, 'play within a play,' as I presented in Chapters 4 and 6. They engaged in stages of PAR with the spiral cycles-a cycle of plan, act, observe, and reflect, and another cycle, and so on. In the process of all the stages, participants took two roles: in the macro-structure (throughout the research project), they functioned as coresearchers; meanwhile in the microstructure (during drama sessions), they became performers, who were in role and out of role.

By dint of this technique, 'play within a play,' or metadrama, ElL learners did not simply participate in the drama research project. Instead of the simple participation, they used drama as a research method for thinking about everyday life, a way of perceiving and producing it with dialectical-cum-dialogical critical thinking about ICM, that is, intercultural imagination. In short, they introduced to their project the concept that 'drama is not a mirror held up to nature, but rather a gauge' to explore intercultural situations and to develop ICM and ICP (Hornby 1986: 27).

It seemed to participants that this metadrama was both reflective and expressive in researching their drama activities about ICM, and also its figure-ground relation led them into dynamical thinking through making shifts of four contexts, as presented in Figures 6.1 and 6.2 .

Furthermore, 'play within a play' or metadrama provided participants with coherence, intuitive as well as rational. For instance, participants as co-researchers benefited 
more by being engaged in otherness and outsidedness, by observing their activities at more distant perspective rather than the performer's perspective.

In other words, they unified two kinds of attention to their drama sessions in relation to juxtapositional dualities, at a distance from the sessions, as indicated in Rubin's double profiles. Consequently, I can say that the research design of 'play within a play' or metadrama enhanced the effects of distancing, otherness, and outsidedness.

In brief, in this research study, I can conclude that ElL learners were invited to an arena to explore and develop ICM and ICP by participating in intercultural drama. In addition, I can summarise the difference between my research and previous researches as follows:

- I approached the teaching of the English language in a different perspective from EFL or ESL to EIL (English as an International Language), as said in Chapter 1, although some scholars already established the issue, I aimed at introducing the perspective of EIL with respect to bilingual/plurilingual education in non-Anglophone countries. That is to say, EIL perspective in this study was sociocultural, but for the purpose of taking an advantage of intercultural communication throughout the world. This perspective leads students into the position of intercultural actors.

- EDIE (Educational Drama in English) was laid as the groundwork in researching in the context of Korean schools, as I said in Chapters 1 and 5.

- Drama was applied in intercultural education in consideration of its own characteristics, that is, as cultural configuration with the fullness to explore our life, as I said in Chapters 1 and 2.

- In the process of the application of drama in developing ICM and ICP in EIL classrooms, I operated a concept of imagination in a different perspective. That is, students were involved in ICM through intercultural imagination and the total imaginative activity, which I discussed in Chapters 2 and 3.

- Both intercultural imagination and the total imaginative activity in and through drama led students, as co-researchers, to their EDIE project in the multiple triangular research, which I discussed in Chapter 4. That is to say, students experienced as co-researchers and multiple reactive actors at the levels of textualism as well as beyond it.

- Along with SPAR (students in participatory action research), my research methods were developed to collect and to analyse my data, which were textual, graphic, and visual. In this regard, I used ATLAS.ti. through which my findings were visualised and were integrated from those different types of data. 
However, it became apparent to me that the impact of drama experiences of students went beyond the drama sessions. As I discussed with respect to both theatrical experience and memorial experience of drama in Chapter 3, students had not only drama experiences within drama activity per se, but also transferred them to everyday life. In other words, we should consider the effectiveness of drama in its holistic perspective, and that it can lead to moral education or 'character education' for young people (Hall 2000).

\subsection{Implications: Drama and its Holistic Effectiveness in the National Curriculum}

\subsubsection{Cross-Curricular or Integrated Projects of Drama in Intercultural Education with Subjects}

Drama was based on a holistic perspective of the total imaginative activity and provided dialectical-cum-dialogical dynamics in relation to certain intercultural discourses. For instance, drama was therapeutically dialogical enough for students to be released from fear or mistakes and to encourage each other.

The first implication of this study is that I should like to say that ICM approaches to other subjects, such as EIL, moral education, Social Studies, or Politics, can be supplemented by drama approaches and they would therefore increase their effectiveness more than in their own methods.

In this respect, I would argue that cross-curricular or integrated projects of drama with other subjects are more effective in intercultural education than the approaches certain subjects take.

However, we should consider how to develop the intercultural curriculum in order to apply this cross-curricular or integrated approach to intercultural education in practice.

In this research case, I applied the cross-curricular or integrated approach of drama and EIL to intercultural education in a study, which was focused on how to communicate in intercultural situations through intercultural imagination. Alternatively, we could apply this approach to a sort of 'drama camp' with the purpose of developing ICM and ICP. For instance, students camp out to participate in certain drama activity for a short time, and drama as its main activity is combined with other activities to be integrated into the camping event. That is, drama is applied in a holistic perspective of the whole participant and the whole group throughout the whole camping event. 


\subsubsection{Drama in Intercultural Education with Ethics}

It is also possible to integrate drama into moral education regarding citizenship. This is more related to general education, particularly in terms of ethics. It implies that drama education and moral education overlap, although there remains the question of what we should consider as the optimal interrelationship between the two. It seems to be possible to educate EIL learners in order to develop their appropriate identities or citizenship at national, international, and global levels. In this regard, I can say that ethics could be taught in and through drama.

On the other hand, as Byram (2002) argues, foreign language education should engage with political education in relation to situatedness surrounding young people, and with moral education in the preparation of the young to live in the multilingual and intercultural discourses. In this regard, drama can overlap with parts of political education and moral education with reference to intercultural situatedness surrounding young EIL learners as would-be intercultural actors.

There still remains how we should develop ICM and ICP of young people in practice, together with the arts including drama in interculturalism in schools. This means we have to educate students within the constraints of our limited educational situations, wherein we cannot teach beyond our curricula in schools, particularly the National Curriculum.

The Korean National Curriculum says that the salient aim of intercultural education is to produce better members of multicultural societies, in other words, better intercultural actors in such societies, people who behave appropriately in intercultural discourses. Furthermore, the National Curriculum emphases issues about democracy, community, society and citizenship, addressed at local, national, international, and global levels. With reference to intercultural education, it is implied that young people should be taught how they can become the catalysts for change and improvement at a local, national and international level for their nation and how they can participate effectively in public life and prepare to be full citizens.

Thus it appears that the aforementioned approaches to ICM, both integrated and drama projects, in the end, come under the umbrella of the aims and objectives of the National Curriculum. This means that those approaches should consider drama education as a programme for social construction through the Curriculum, where national identity and ideology is taught, where the issue of building the power of the nation-state itself remains (Goodson 1998). Furthermore, it is reasonable to pay 
attention to how to educate young people for citizenship in terms of social and moral responsibility, community involvement and political literacy. Here we could face difficulties if there is a clash between educational responsibility and sociocultural/political literacy. That is to say, we as educators should be more concerned about education rather than non-educational discourses. Our concerns with education, in the end, converge on both objects of moral inquiry at the individual level and political philosophy but ethics applied to society at the collective level, such as groups, nations, and humankind (Berlin 1990).

\subsection{Recommendations: Intercultural Education through Drama for National and World Citizenship}

\subsubsection{Ethical Issues-ICM in Intercultural Discourses and the Hidden Curriculum surrounding Young People}

Regarding the possibility of the above clash between educational responsibility and socio-cultural/political literacy, let me return to the fullness of drama as an art form. It appeared that drama provided participants with an authentic fullness of discourses, socio-cultural, political, economic, and so on, in multiple communication media. In consideration of the function of drama in the fullness of human experience, drama as the total imaginative activity was relevant to holistic experiences of each student, which have an influence on her moral values or ethics.

And, as was emphasised in Chapter 3, ethical issues should be re-considered as the philosophical axis for those who live in this world. Furthermore, intercultural education aims ultimately at living with and communicating with others with cultural identities, either same or different. My main point here is the function of drama to connect ethical issues with intercultural education.

Therefore, I can say the ultimate object in this research study is to consider how we live with and communicate with each other in respectful perspectives in certain intercultural discourses rather than just to investigate how drama is effective in developing ICM and ICP.

However, I cannot eliminate from my head that that we, as educators, have still problematical matters concerning the appropriateness of certain identities, national, intercultural and transcultural, and optimal conditions of cosmopolitan citizenship. 
This is because educators are engaged in education, which by its nature takes responsibility for forming both national identity and world citizenship in specific contexts and cannot be entirely separable from its surrounding discourses, sociocultural, economic, and even political.

Therefore, approaches to ICM and ICP through drama, either integrated or nonintegrated, should be considered in terms of its holistic involvement, that is, with the partnership between the whole learner, the whole curriculum, the whole community, and the whole intercultural discourses. For instance, when we explore ICM between a British American, a Korean American, and a Korean, we should consider cultural differences in ICM between the three people in terms of Western and non-Western value orientations from the holistic viewpoint and have a better understanding of other cultural values.

Here I can point out again that we have difficulties in, what we call, the Hidden Curriculum at national as well as international levels in reference to interculturalism. As I discussed, the National Curriculum, as a social construction, underpins democracy, community, society and citizenship at local, national, international, and global levels. Furthermore, the Curriculum underpinning political projects leads into another dimension, the Hidden Curriculum in the form of discourses about the nation, socio-cultural and economic, 'for the hidden curriculum of the National Curriculum is a reassertion of the power of the state in nation-building' (Goodson 1998: 162). Also, it seems that 'this project is diametrically opposed to the alternative project of educating pupils, from a plurality of cultures, for active citizenship in a democracy' (Ibid).

It appears, however, that there are limits of a National Curriculum in relation to its influential power on young people, as Giddens points out:

The formal school curriculum is only one part of a more general process of cultural reproduction influenced by many informal aspects of learning, education and school settings. The 'hidden curriculum' plays a significant role in cultural reproduction (Giddens 1997: 430).

Similarly, Best (1992) argues that human response and behaviour have an instinctive propensity to emulate social environments. In addition, he points out that those responses and actions are learned from being assimilated by growing up in and emulating the practices of a social environment, rather than from explicit teaching. That is to say, it is ordinary people in socio-cultural contexts, not their teachers in schools, who educate young people —in the socialisation rather than from the National Curriculum. 
With reference to the powerful Hidden Curriculum and socialisation in social environments, I asked myself how young people could understand certain unequal intercultural situations and how I could introduce those situations to young people and lead them to overcome the situations in the Hidden Curriculum.

And then, how could we explore the ideal of compulsory equality from the sociocultural or intercultural point of view?

My answer is simple, but not easy at all to implement that in the Hidden Curriculum and in the whole community as well as the formal school curriculum-not to simplify standards but to diversify them, that is, cultural diversity such as various norms, multicultural, intercultural, or transcultural.

However, there still remains how we could deliver those aims at a realistic level in current intercultural discourses. It seems to me that there is no possibility to achieve our undertaking enough to make a breakthrough. However, I believe that we could, at least, reduce or relieve anxiety about those kinds of intercultural situations.

What I should like to say is that in order to avoid monocultural discourses we should consider inclusion/integration rather than assimilation/accommodation from the standpoint of ICM and ICP either in the formal school curriculum or in the Hidden Curriculum. Inclusion/integration is based in the perspective of cultural diversity, whereas assimilation/accommodation aims at the simplification or standardisation of cultural capital and eventually at the unification of socio-cultural norms into one monocultural norm. It is said that the concept of the former compares to salad bowl, wherein each material still keeps its own characteristics, meanwhile the concept of the latter, to vegetable soup, wherein qualities are changed entirely.

\subsubsection{Dialogic Identity through Drama-ICM into Integration/lnclusion in Balanced Intercultural Discourses}

Referring to integration/inclusion, I can say that intercultural imagination in and through drama assists students to be concerned about intercultural inclusion. This is because, above all, students use the imagination rather than real experience in and through drama in exploring certain intercultural situations in which they are. As I discussed in Chapters 3,5, and 6, the 'Magic If' in relation to intercultural situations, is beneficial to the development of drama experiences, along with their cathartic experiences: 
- to make shifts between different contexts or situations,

- to re-construct an actual situation by thinking about that imaginatively through the 'Magic If,'

- to have imaginative insight into their own viewpoints as well as other people's,

- to plan their drama activities, and

- to interrelate between emotions, consciousness, and behaviour as total imaginative activities.

Then, it seems that we should consider intercultural inclusion at school level as well as societal level, that is, in the National Curriculum as well as in the Hidden Curriculum, and how we can achieve intercultural inclusion effectively. Here, we should reconsider why we have experiences of cultural shock in certain intercultural situations, why some of us are more shocked than others are, or rather why certain people suffer from those same intercultural situations. Let us re-structure or re-interpret that situation in intercultural imagination.

In this total imaginative way, along with reconstructing or reinterpreting the situation, we can realise other aspects of the situation in which we empathise with characters. That is to say, imagination in and through drama makes it easier to fit the situation into individual existence but at a distance, that is, while being inside and outside. This emotional involvement with an unfamiliar point of view enables us to become more aware of our own reactions and prejudices, and those of others. This insight can transfer to intercultural inclusion in our real situation.

In this regard, I can say that we can educate the intercultural imagination of young people in and through drama. Furthermore, drama has its own dynamic characteristics-duality, dialecticality, and dialogicality, whereby we have dialogic identity to change unequal factors into balanced intercultural discourse as a consequence of intercultural inclusion or integration.

The ultimate aim of intercultural education is to develop the cultural identity of young people into transcultural identity, global identity or cosmopolitan citizenship (Kordes 1991; Parmenter 1999; Osler \& Vincent 2002).

However, we can reach this aim better through intercultural inclusion/ integration rather than intercultural assimilation/ accommodation, although it is said that 'a fully unified, completed, secure and coherent identity is a fantasy, and it will be neither easy nor simple to develop those cosmopolitan cultural identities (Hall 1992: 277). 
In consideration of this difficulty, I should like to again suggest drama as an effective medium for educating intercultural inclusion/integration. Drama, as I discussed in Chapters 3,5, and 6, provides the fullness of intercultural discourses, particularly at the three-dimension level rather than at the bi-dimensional level. That is to say, participants are engaged in an endless mountain-climbing with spectatorship and selfspectatorship through drama: first, living and observing in towns, second, exploring at the foot of a mountain, second, at its hillside, third, at the summit of the mountain, fourth, coming down to live in towns, and fifth, climbing another mountain.

In this way, the process in and through drama requires us to have bird's eye perspectives, not just perspectives of 'for' and 'against.' This perspective is beneficial to reciprocal communication with different people in balanced intercultural discourses.

\subsection{From Epilogue to Prologue}

I have suggested the effectiveness of drama as an arena for ICM and ICP with dialecticality and dialogicality. Here are two further reasons: students had an opportunity to experience multiple communication media, and were able to overcome or solve problems of the real, culturally shaped world without any risks through drama.

No matter how difficult and hopeless it may appear that we shall have intercultural or transcultural utopia, 'the capacity of developing perspectives and identity is, finally, the most important condition to ensure that big or small Auschwitzes are not repeated, ever again' (Kordes 1991: 306).

In the end, I will complete this epilogue, which is another prologue, by raising an intercultural question: what could I do and how should I be more concerned in the contemporary intercultural discourses? Above all, why should I take responsibility, not others? Instead of defining that you, not I should take the responsibility for an outcome in certain situations or saying that he, not you should act, I really wonder which person, as a better intercultural actor, should be more concerned with his dialogic identity in intercultural communication. In other words, all of us should make more efforts in order to live with all the others in multicultural societies in the single global world, that is, for inclusion in ICM.

Therefore, drama is for all. 


\section{Bibliography}

\section{References}

Abbs, P. (1989a). A is for Aesthetic: essays on creative and aesthetic education. London: Falmer Press.

Abbs, P. (1989c). 'The Pattern of Art-Making', in P. Abbs (ed) (1989b). The Symbolic Order. A Contemporary Reader on the Arts Debate. London: Falmer Press. 198-210.

Abbs, P. (1989d). 'Aesthetic Education: An Opening Manifesto,' in P. Abbs (ed)(1989b). The Symbolic Order. A Contemporary Reader on the Arts Debate. London: Falmer Press. 3-11.

Abbs, P. (1994b). 'Educational Drama as Cultural Dispossession', in P. Abbs (1994a). The Educational Imperative: A Defence of Socratic and Aesthetic Learning. London: Falmer Press: 117-137.

Ackroyd, J. and Pilkington, A. (1999). 'Childhood and the construction of ethnic identities in a global age: a dramatic encounter,' Childhood, 6(4): 443-454.

Altrocchi, J. (1999). 'Individual Differences in Pluralism in Self-Structure,' in J. Rowan and M. Cooper (eds)(1999). The Plural Self: Multiplicity in Everyday Life. London•Thousand Oaks: Sage Publications. 168-182.

Aristotle. The Nicomachean Ethics. Ctd in D. Goleman (1996). Emotional Intelligence: Why it can matter more than IQ. London: Bloomsbury. IX.

Arnaut, S. and Arnaut, L. (1998). 'There are no Shortcuts to Intercultural Learning: it can be fun but it won't be easy,' European Journal of Intercultural Studies, 9 (Supplement): S49-S54.

Atkinson, P. (1988). 'Ethnomethodology: A Critical Review,' Annual Review of Sociology, 14: 441-65.

Atkinson, P. (1990). The Ethnographic Imagination: Textual Constructions of Reality. London and New York: Routledge.

Atweh, B., et al. (eds.).(1998). Action Research in Practice: Partnerships for Social Justice in Education. London and New York: Routledge.

Auerback, E. R. et al. (1996). Adult ESL Literacy from the Community to the Community: A Guidebook for Participatory Literacy Training. Mahwah, NJ: Lawrence Erlbaum.

Backstrom, E. L.. (1988). The effects of creative dramatics on student behaviours and attitudes in literature and language arts, Ed.D. Dissertation, University of California. 
Bakhtin, M. M. (1919). 'Art and Answerability,' in M. M. Bakhtin (1990). Art and Answerability: Early Philosophical Essays by $M$. M. Bakhtin. eds and trans, by M. Holquist and V. Liapunov. Austin: University of Texas Press. 1-4.

Bakhtin, M, M. (1970). 'Response to a Question from the Novy Mir Editorial Staff,' in M. M. Bakhtin: Speech Genres \& Other Late Essays. Trans by V. W. McGee and ed. by C. Emerson and M. Holquist. Austin: University of Texas Press. 1-9.

Bakhtin, M. M. and Medvedev, P. N.(1991). The Formal Method in Literary Scholarship. trans. by A. Wehrle. Baltimore - London: The Johns Hopskins University Press.

Bakhtin, M. M. (1961). 'Toward a Reworking of the Dostoevsky Book,' in M. M. Bakhtin, (1984). Problems of Dostoevsky's Poetics. Ed..and Trans. C. Emersion. Minneapolis, MN: University of Minnesota Press. 283-302.

Bakhtin, M. M. (1979). 'Avtor I geroj v estetixeskoj dejatel'nosti,' Estetika slovesnogo tvorchestva. Moscow: Iskusstvo. [25-26] Cited in M. M. Bakhtin: Speech Genres and Other Late Essays. Trans. V. W. McGee. Austin: Unviersity of Texas Press. xiii.

Bakhtin, M. M. (1981). The Dialogical Imagination. Ed. M. Holquist; Trans. C. Emersion and M. Holquist. Austin, TX: University of Texas Press.

Bakhtin, M. M. (1984). Problems of Dostoevsky's Poetics. Ed. and Trans. C. Emersion. Minneapolis, MN: University of Minnesota Press.

Bakhtin, M. M. (1990). Art and Answerability: Early Philosophical Essays by M.M. Bakhtin. Austin: University of Texas Press.

Ball, H. L. (1983). A Critical Evaluation of the Nature and Function of Drama in Education and in the Training of Teachers. PhD dissertation, the University of Wales.

Ball, S. D. (1993). Drama and theatre in education with specific reference to personal, social and health education. Ph.D. thesis, University of Manchester.

Ballou, C. D. (1996). A Comparative Assessment of the Effectiveness of International/ Intercultural Education in the General Education Program at American University, Ph.D. dissertation, The American University.

Barba, E. (1986). Beyond the Floating Islands. New York: Performing Arts Journal Publications.

Barnet, R. J. and Cavanagh, J. (1994). Global Dreams: Imperial Corporations and the New World Order, New York: Simon and Schuster.

Barro, A. et al. (1998). 'Cultural practice in everyday life: the language learner as ethnographer,' in M. Byram and M. Fleming (eds)(1998). Language Learning in Intercultural Perspective: Approaches through Drama and Ethnography. Cambridge: Cambridge UP. 76-97

Barthes, R. et al. (1974). Structural analysis and Biblical exegesis: interpretational essays. trans. by A. M. Johnson, Jr. Pittsburgh : Pickwick Press, 
Baskerville, R. and Pries-Heje, J. (1999). 'Grounded action research: a method for understanding IT in practice,' Accounting Management and Information Technilogies, 9(1): 1-23.

Basourakos, J. (1999). 'Moral voices and Moral Choices: Canadian drama and moral pedagogy,' Journal of Moral Education, 28 (4): 473-489.

Beckerman, B. (1970). Dynamics of Drama: Theory and Method of Analysis. New York: Alfred A. Knopf.

Beckerman, B. (1990). Theatrical Presentation: Performer, Audience and Act. Posthumous ed. By G. B. Beckerman and W. Coco. London: Routledge.

Bennett, M. J. (1993). 'Towards Ethnorelativism: A Developmental Model of Intercultural Sensitivity', in R. M. Paige (2 ${ }^{\text {nd }}$ ed).(1993). Education for the Intercultural Experience. Yarmouth, Maine: Intercultural Press. 21-71.

Bennett, S. (1990). Theatre Audiences. London: Routledge.

Berger, P. (1963). 'Sociological Perspectives-Society as Drama,' in D. Brissett and C. Edgley (eds)(1990). Life as Theater. A Dramaturgical Source Book. New York: Aldine De Gruyter. 51-61

Berlin, I. (1990). The Crooked Timber of Humanity: Chapters in the History of Ideas. ed. by H. Hardy. London: John Murray Ltd.

Best, D. (1992). The Rationality of Feeling: Understanding the Arts in Education. London: Falmer Press.

Bhawuk, D. P. S.and Brislin, R.(1992). 'The measurement of intercultural sensitivity using the concepts of individualism and collectivism,' International Journal of Intercultural Relations, 16 (4): 413-436.

Block, D. and Cameron, D. (eds)(2002). Globalization and Language Teaching. London and New York: Routledge.

Boal, A. (1992). Games for Actors and Non-actors. London: Routledge.

Boal, A. (1979). Theatre of the Oppressed. London: Pluto Press.

Boal, A. (1995). The Rainbow of Desire: The Boal Method of Theatre and Therapy. London: Routledge.

Bolton, G. (1981). 'A Statement outlining the contemporary view held of drama in education in Great Britain,' in D. Davis and C. Lawrence (eds)(1986). Gavin Bolton: Selected Writings. London: Longman. 12-17.

Bolton, G. (1992). New Perspectives on Classroom Drama. Herts: Simon \& Schuster Education.

Bolton, G. (1996). 'Afterward: Drama as Research,' in P. Taylor (ed). Researching Drama and Arts Education: Paradigms and Possibilities. London: Falmer Press. 187-194. 
Bolton, G. (1997). A Conceptual Framework for Classroom Acting Behaviour. Ph.D thesis, University of Durham.

Bolton, G. (1998). Acting in Classroom Drama: A Critical Analysis. Birmingham: Trentham Books.

Boring, E. G. (1953). 'The role of theory in experimental psychology,' American Journal of Psychology, 66: 169-184.

Bourne, J. and Reid, E. (eds)(2003). World yearbook of education 2003: Language Education. London: Kogan Page.

Bradbury, J. et al. (eds)(1995). Collins CoBuild English Dictionary. London: HarperCollins Publishers Ltd.

Brecht, B. (1935). 'From Theatre for Pleasure or Theatre for Instruction,' in R. Drain (ed) (1995). Twentieth Century Theatre: A Sourcebook. London: Routledge: 112-113.

Brecht, B. (1936a). 'Alienation Effects in Chinese Acting', in J. Willett (2 ${ }^{\text {nd }}$ ed)(1990). Brecht on Theatre: the Development of an Aesthetic. London: Methuen.

Brecht, B. (1938). 'From the Popular and the Realistic,' in R. Drain (ed) (1995). Twentieth Century Theatre: A Sourcebook. London: Routledge: 188-192.

Brière, J. (1997). 'Cultural Understanding through Cross-Cultural Analysis,' in P. R. Heusinkveld (ed.) (1997). Pathways to Culture: Readings on Teaching Culture in the Foreign Language Class. Yarmouth, ME: Intercultural Press. 561-569.

Briggs, J. L. (1992). 'Mazes of Meaning: How a child and a Culture,' New directions for child development, no. 58 (winter 1992): 25-39.

Brissett, D. and Edgley, C. (1990). 'The Dramaturgical Perspective,' in D. Brissett and C. Edgley $\left(2^{\text {nd }}\right.$ eds)(1990). Life as Theater: A Dramaturgical Source Book. New York: Aldine De Gruyter. 1-46.

Britannica (2000a). 'dialectic,' http://search.eb.com/bol/topic?eu=30745\&sctn=1 [accessed on 17 April 2000: www.britannica.com/bcom/eb/article/printable/6/ $0,5722,30745,00 . h t m l]$.

Britannica (2000b). 'dramatic literature/drama as an expression of a culture,' http://search.eb.com/bol/topic?eu=119377\&sctn=6 [accessed on 22 February 2000: www.britannica.com/bcom/eb/article/printable/6/0,5722,109456,00.html]

British Council (1995). English in the World: The English 2000 Global Consultation Report. Manchester: The British Council.

Brook, P. (1968). The Empty Space. Harmondsworth: Penguin.

Brook, P. (1993). There are no secrets: Thoughts on acting and theatre. London: Methuen.

Brook, P. (1996). "The Culture of Links," in P. Pavis (ed) (1996). The Intercultural 
Performance Reader. London and New York: Routledge. 63-66.

Bruce R. (1998). 'Creative Dramatics in the Language Arts Classroom', ERIC Clearinghouse on Reading and Communication Skills Bloomington IN. ERIC Digests (ERIC Identifier: ED297402) http://www.ed.gov/ databases /ERICDigests/ed297402. html [accessed on 02 December 2000].

Brutt-Griffler, J. (2002). World English: A Study of its Development. London- BuffaloToronto- Sydney: Multilingual Matters Ltd.

Buege, C. J. (1991). The effects of creative dramatics and social skills training on attitude and self-concept. Ph.D thesis, The University of Wisconsin - Milwaukee.

Burke, K. (1966). Language as Symbolic Action: Essays on Life, Literature, and Method. Berkeley= Los Angeles: University of California Press.

Burke, K. (2 ${ }^{\text {nd }}$ ed)(1969). A Rhetoric of Motives. Berkeley: University of California Press.

Busanjin Girls Commercial High School (2001). The Annual Scheme 2001 of Busanjin Girls Commercial High School. Busan: Busan Girls Commercial High School Press. (Korean Version).

Byram, M. and Zarate, G. (1997). The Sociocultural and Intercultural dimensions of Language learning and teaching. Clevedon: Multilingual Mattes.

Byram, M. (1989). Cultural Studies in Foreign Language Education. Clevedon: Multicultural Matters.

Byram, M. (1991). 'Teaching Culture and Language: Towards an Integrated Model,' in D. Buttjes and M. Byram (eds)(1991). Mediating Languages and Cultures: towards an intercultural theory of foreign language education. Clevedon: Multilingual Mattes. 17-32.

Byram, M. (1997). Teaching and assessing Intercultural Communicative Competence. Clevedon: Multicultural Matters.

Byram, M. (1999). 'Questions of Identity in Foreign language learning,' in J. Lo Bianco et al. (eds)(1999). Striving for the Third Place: Intercultural Competence through Language Education. Melbourne: Language Australia. 91-100.

Byram, M. (2002). 'Keeping Europe Multilingual': A Guide for Language Education Policies for Linguistic Diversity and Plurilingualism [Executive Summary]. Strasbourg: Council of Europe.

Byram, M. and Zarate, G. (1994). A Common European Framework for Language Teaching and Learning: Definitions, Objectives and Assessment of Sociocultural Competence. Strasbourg: Council of Europe.

Byram, M., Esarte-Sarries, V. and Taylor, S. (1991). Cultural Studies and Language Learning: A Research Report. Clevedon: Multilingual Matters, LTD. [Booklet: 1 myself]. 
Callahan, W. A. (1999). 'Negotiating Cultural Boundaries: Confucianism and Trans/national Identity in Korea,' Cultural Values, 3 (3): 329-364.

Callero, P. L. (1991). 'Conclusion,' in M. Byram et al. (1991). Cultural Studies and Language Learning: A Research Report. Clevedon: Multilingual Matters, LTD. 323-331.

Camilleri, C. (1992). 'From Multicultural to Intercultural: How to Move from One to the Other', in J. Lynch et al. (eds)(1992). Cultural Diversity and the Schools One: Education for Cultural Diversity Convergence and Divergence. London: Falmer Press. 141-151.

Canagarajah, A. S. (1999). Resisting Linguistic Imperialism in English Teaching. Oxford: Oxford University Press.

Carey, J. W. (1989). Communication as culture: Essays on media and society. Boston: Unwin Hyman.

Carroll, J. (1996). 'Escaping the Information Abattoir: Critical and Transformative Research in Drama Classrooms,' in P. Taylor (ed)(1996). Researching Drama and Arts Education: Paradigms and Possibilities. London: Falmer Press. 72-84.

Carspecken, P. F. (1996). Critical Ethnography in Educational Research: A Theoretical and Practical Guide. New York and London: Routledge.

Chaudhuri, U. (1984). 'The Spectator in Drama/ Drama in the Spectator,' Modem Drama, 27 (3): 281-298.

Cohen, L. et al. (5 $5^{\text {th }}$ ed) (2000). Research Methods in Education. London: Routledge.

Cohen, L.and Manion, L. ( $3^{\text {rd }}$ ed.)(1989). Research Methods in Education. London: Routledge.

Collihole, M. (1984). 'Impression of a New Boy,' in B. R. Marney et al. (1984). Poetry Plus: People are Strange? [Book 5]. Huddersfield: Scholfield \& Sims Ltd. 53.

Collingwood, R. G. (1938, 1963 printed). The Principles of Art. Oxford: Clarendon Press.

Colman, A. M. (2001). A Dictionary of Psychology. New York: Oxford University Press.

Conquergood, D. (2002). 'Performance Studies: Interventions and Radical Research,' The Drama Review, 46 (2): 145-156.

Council of Europe (Language Division) (2002). Guide for the Development of Language Education Policy in Europe: From Linguistic Diversity to Plurilingual Education [short version, Provisional text: no. 7]. Strasbourg: Council of Europe.

Courtney, R. (1968). Play, Drama \& Thought: The Intellectual Background to Dramatic Education. London: Cassell.

Crystal, D. (1997). English as a Global Language. Cambridge: Cambridge University Press. 
Cushner, K. (1994). 'Preparing teachers for an intercultural context,' in R. W. Brislin and T. Yoshida (eds.)(1994). Improving Intercultural Interactions: Modules for Cross-Cultural Training Programs (Volume 2)(Multicultural Aspects of Counselling Series 8). Thousand Oaks-London: SAGE.

Cutcliffe, J. R. (2000). 'Methodological issues in grounded theory,' Journal of Advanced Nursing, 31(6):1476-1484.

Dascal, M. (1996). Endangered Languages. Tel Aviv: Lester \& Sally Entin Faculty of Humanities, Tel Aviv University, manuscript.

Davis, D. and Lawrence, C. (eds)(1986). Gavin Bolton: Selected Writings. London: Longman.

Day, R. (1985). 'The Ultimate inequality: linguistic genocide,' in N. Wolfson and J. Manes (eds)(1985). Language of Inequality. Berlin: Mouton. 163-181.

De l'Etoile, S. K. (2002). 'The effectiveness of music therapy in group psychotherapy for adults with mental illness,' The Arts in Psychotherapy, 29 (1): 69-78.

Deetz, S. (1984). 'Metaphor Analysis,' in W. B. Gudykunst and Y. Y. Kim (eds). Methods for Intercultural Communication Research. Beverly Hills. London: Sage [in cooperation with Speech Communication Association's Commission]. 215-228.

Delamater, J. H. and Prigozy, R. (1998). The Detective in American Fiction, Film, and Television. Westport, Connect. London: Greenwood press.

Denzin, N. K. and Lincoln, Y. S. (eds)(1994). Handbook of Qualitative Research, Thousand Oaks, CA: SAGE.

Denzin, N. K. (1970). The Research Action in Sociology: A Theoretical Introduction to Sociological Method. London: The Butterworth Group.

Denzin, N. K. (1992). Symbolic Interactionism and Cultural Studies: the Politics of Interpretation. Oxford-Cambridge: Blackwell.

Dobson, W. (1996). Truth in dialogue: a knowledge-centred approach to drama in education. PhD. Thesis, University of Sussex.

Douglas, J. D. (1976). Investigate social research. Berverly Hills, CA: Sage.

Ebbutt, D. (1985). 'Educational Action Research: some General Concerns and Specific Quibbles,' in R. G. Burgess (ed)(1985). Issues in Educational Research:

Qualitative Methods. East Suses, UK: Falmer Press.

Egan-Roberton, A. and Willett, J. (1998). 'Students as Ethnographers, Thinking and Doing Ethnography: A Bibliographic Essay,' in A. Egan-Robertson and D. Bloome (eds)(1998). Students as Researchers of Culture and Language in their own communities. Cresskill, NJ: Hampton Press, Inc.

Ehrenzweig, A. (1971). The Hidden Order of Art: A Study in the Psychology of Artistic Imagination. Berkeley: University of California Press. 
Eliasmith, C. and Thagard, P. (2001). 'Integrating structure and meaning: a distributed model of analogical mapping,' Cognitive Science, 25(1): 245-286.

Elkins, M. R. (1997). An Investigation of Educational Gaming/ Stimulation as an Instructional Strategy for Teaching Intercultural Sensitivity. Ph.D. dissertation, Southern Illinois University.

Elkins, M. R. (1997). An Investigation of Educational Gaming/ Stimulation as an Instructional Strategy for Teaching Intercultural Sensitivity. Ph.D. dissertation, Southern Illinois University.

Erlenwin, P. (1993). 'From Movement to the Word: Experiences of and Reflections upon the Holistic Learning Process,' trans by P. Shaw. In M. Schewe and P. Shaw (eds)(1993). Towards Drama as a Method in the Foreign Language Classroom. Frankfurt am Main: Peter Lang Pub. 193-200.

Errington, E. (1993). 'Teachers as Researchers:Pursuing Qualitative Enquiry in Drama Classrooms,' Youth Theatre Joumal, 7 (4): 31-36.

Fantini, A. E.(ed.).(1997). New Ways in Teaching Culture (New Ways in TESOL Series II). Bloomington, II.: Teachers of English to Speakers of Other Languages, Inc.

Fleming, M. (1982). A Philosophical Investigating Into Drama in Education. Ph.D. Thesis. University of Durham.

Fleming, M. (1994). Starting Drama Teaching. London: David Fulton.

Fleming, M. (1998). 'Cultural awareness and dramatic art forms,' in M. Byram and M. Fleming. (eds)(1998). Language Leaming in Intercultural Perspective: Approaches through drama and ethnography. Cambridge: Cambridge UP. 147157.

Fleming, M. (2001). Teaching Drama in Primary and Secondary Schools: An Integrated Approach. London: David Fulton Publishers.

Fleming, M. (2003). 'Intercultural Experience and Drama,' in G. Alred, M. Byram and M. Fleming (eds)(2003). Intercultural Experience and Education [Languages for Intercultural Communication and Education 2; Series Editors: M. Byram and A. Phipps]. Clevedon·Buffalo. Toronto.Sydney: Multilingual Matters Ltd. 87-100.

Furhman, A. and Bochner, S. (1986). Culture shock: psychological reactions to unfamiliar environments. London: Routledge.

Gassner, J. (1956). Form and Idea in Modern Theatre. New York: Holt, Rinehart and Winston.

Geert, C. (1983). Local Knowledge: Further Essays in Interpretive Anthropology. New York: Basic Books.

Genesee, F. (1994). 'Introduction,' in F. Genesee (ed)(1994). Educating Second Language Children: The whole child, the whole curriculum, the whole community [Cambridge language education; series editor Jack C. Richards]. 
Cambridge: Cambridge University Press. 1-12.

Giddens, A. (1991b). 'Modernity and Self-Identity: Self and Society in the Late Modern Age,' in F. Franscina and J. Harris (eds) (1992). Art in Modern Culture: An Anthropology of Critical Texts. London and New York: Phaidon Press.

Giddens, A. (1994). 'Living in a post-traditional society,' in U. Beck et al. (1994). Reflexive Modernization: Politics, Tradition and Aesthetics in the Modern Social Order. Cambridge: Polity Press. 56-109.

Giddens, A. (2 ${ }^{\text {nd }}$ ed)(1993). Sociology. Cambridge: Polity Press.

Giddens, A. (3 $3^{\text {rd }}$ ed)(1997). Sociology. Cambridge: Polity Press.

Glaser, B. (1978). Theoretical Sensitivity. Mill Valley, CA: Sociology Press.

Glaser, B. G. and Strauss, A. L. (1967). The Discovery of Grounded Theory: Strategies for Qualitative Research. New York: Aldine de Gruyter.

Goddard, A. (1999). 'Culture and drama: The role of financial control systems in the organisational process in three local government organisations,' The International Journal of Public Sector Management, 12(6):516-532.

Goffman, E. (1959; 1969). The Presentation of Self in Everyday Life. New York: Anchor Books. London: Penguin Press.

Goldie, P. (1999). 'How We Think of Others' Emotions,' Mind and Language, 14 (4): 394-423.

Goleman, D. I (1996). Emotional Intelligence: why it can matter more than IQ. London: Bloomsbury.

Goodman, N. R. (1994). 'Intercultural education at the university level: teacher-student interaction,' in R. W. Brislin and T. Yoshida (eds.)(1994). Improving Intercultural Interacitons: Modules for Cross-Cultural Training Programs (Volume 2).(Multicultural Aspects of Counseling Series 8). Thousand Oaks-London: SAGE.

Goodson, I. (1998). "Nations at Risk' and "National Curriculum': Ideology and Identity,' in I. Goodson (1998). Subject Knowledge: Readings for the Study of School Subjects. London: Falmer Press. 150-164.

Graddol, D. (2001). 'English in the Future,' in A. Burns and C. Coffin (eds)(2001). Analysing English in a Global Context: A Reader [Series of Teaching English Language Worldwide]. London and New York: Routledge. 26-32.

Greenwood, D. J. (1999). Action Research: From practice to writing in an international action research development program. Amsterdam: John Benjamins Publishing Co.

Greenwood, J. (2001). 'Within a Third Space,' Research in Drama Education, 6(2): 193-205. 
Grossman, F. K. (1999). 'Evaluating a Collective-Drama Approach to Wilfred Laurier University Presenting Violence Against Women-Commentary: Power, Social Change, and the Deborah Mahlstedt Process of Feminist Research Reflections on a Feminist Research Project,' Intemational journal of educational development, 19 (3):117-138.

Gudykunst, W. B. and Kim, Y.Y. (eds)(1984). Methods for intercultural communication research. Beverly Hills London: Sage.

Gudykunst, W. B. and Nichida, T. (1989). 'Theoretical perspectives for studying intercultural communication,' in M. F. Asante and W. B. Gudykunst (eds) (1989). Handbook of international and intercultural communication. Newbury Park, CA: Sage. 17-46.

Gudykunst, W. B. (1995). 'Anxiety/uncertainty management (AUM) theory: Current status;' in R. Wiseman (ed) (1995). Intercultural Communication theory. Thousand Oaks, CA: Sage. 8-58.

Gumperz, J. (1992). 'Contextualisation and understanding,' in A. Duranti and C. Goodwin (eds)(1992). Rethinking Context: Language as an Interactive Phenomenon. Cambridge: Cambridge University of Press. 229-252.

Gumperz, J. (1996). 'The linguistic and cultural relativity of conversational inference,' in J. Gumperz and S. Levinson (eds)(1996). Rethinking Linguistic Relativity. Cambridge: Cambridge University Press. 374-406.

Gurevitch, Z. (2000). 'Plurality in Dialogue: A Comment on Bakhtin,' Sociology, 34 (2): 243-263.

Hahn, S. L. (1997). 'Strategies for Increasing Cross-cultural Awareness,' in P. R. Heusinkveld (ed.)(1997). Pathways to Culture: Readings on Teaching Culture in the Foreign Language Class. Yarmouth, ME: Intercultural Press. 499-522.

Hall, B. J. (1992). 'Theories of culture and communication,' Communication Theory, 1: 50-70.

Hall, E. T. (1976). Beyond Culture. New York: Anchor Press/Doubleday.

Hall, S. (1992). 'The question of cultural identity,' in S. Hall et al.(eds)(1992). Modernity and Its Futures. Cambridge: Polity. 274-316.

Hall, S. K. (2000). Using Picture Storybooks to teach Character Education. Phoenix, Arizona: The Oryx Press.

Hall, W. A. and Callery, P. (2001). 'Enhancing the Rigor of Grounded Theory: Incorporating Reflexivity and Relationality,' Qualitative Health Research, 11(2): 257-272.

Halliday, M. A. K. and Hasan, R. ( $2^{\text {nd }}$ ed)(1989). Language, Context, and Text: Aspects of Language in a Social-semiotic perspective. Oxford: Oxford University Press.

Harland, J. et al. (1998). The effects and effectiveness of ants education in schools 
Commissioned by the Royal Society for the encouragement of Arts, Manufactures \& Commerce (INTERIM REPORT 1). Slough, Berkshire: the National Foundation for Education Research.

Harrington, W. (1998). Columbo: the Hoover Files [Based on the Universal Television series Columbo]. New York: A Forge Book.

Harvey, B. and Tony, J. S. (2000). 'Evaluation of a drama-in-education programme to increase AIDS awareness South African high schools: a randomised community intervention trial,' International Journal of STD \& AIDS, 11(2): 105-111.

Heathcote, D. (1976). "From Drama as s Process for Change", in R. Drain. (ed) (1995). Twentieth Century Theatre: A Sourcebook. London: Routledge. 199-203.

Heathcote, D. and Gavin B. (1995). Drama for Learning: Dorothy Heathcote's Mantle of the Expert Approach to Education. Portsmouth: Heinemann.

Hermans, H. J. M. (1999). 'The Polyphony of the mind: a multi-voiced and dialogical self,' in J. Rowan and M. Cooper (eds)(1999). The Plural Self: Multiplicity in Everyday Life. London•Thousand Oaks: Sage Publications. 107-131.

Hofstede, G. (1980). Culture's Consequences: International Differences in WorkRelated Values [Volume 5, Cross-cultural research and methodology series]. Beverly Hills : Sage.

Holliday, A. (1994). Appropriate Methodology and Social Context. Cambridge: Cambridge University of Press.

Holmes, E. (1911). What is and What Might Be. London: Constable.

Holmes, E. (1914). In Defence of What Might Be. London: Constable.

Holquist, M. (1986). 'Introduction,' in M. M. Bakhtin (1986). Speech Genres and Other Late Essays. Eds by C. Emerson and M. Holquist; Trans. V. W. McGee. Austin: University of Texas Press. Ix-xxiii.

Holquist, M. (2 ${ }^{\text {nd }}$ ed)(2002). Dialogism: Bakhtin and his world. London: Routledge.

Holsbrink-Engels, G. A. (1997). 'The Effects of the Use of a Conversational Model and Opportunities for Reflection in Computer-Based Role-Playing,' Computers in Human Behaviour, 13 (3): 409-436.

Hooks, B. (1989). Talking Back: Thinking Feminist, Thinking Black. Boston: South End Press.

Hornbrook, D. (1991). Education in Drama. London: Palmer Press.

Hornbrook, D. (1998a). Education and Dramatic Art. London: Routledge.

Hornbrook, D. (1998b). 'Crafting Dramas,' in D. Hornbrook (1998c). On the Subject of Drama. London: Routledge. 51-67.

Hornby, R. (1986). Drama, Metadrama, and Perception. Cranbury, NJ: Associated 
University Press.

Hourd, M. L. (1951). Some Emotional Aspects of Learning. Melbourne and London: William Heinemann Ltd.

Hymes, D. (1994). 'Toward Ethnographies of Communication,' in J. Maybin (ed)(1994). Language and Literacy in Social Practice [Series: Language and Literacy in Social Context]. Clevedon- Philadelphia. Adelaide: Multilingual Matters Ltd in association with the Open University.

Jarvis, S. (2000). 'Semantic and conceptual transfer,' Bilingualism: Language and Cognition, 3 (1): 19-21.

Jary, D. and Jary J. ( $3^{\text {rd }}$ ed)(2000). Dictionary of Sociology. Glasgow: HarperCollins Publishers.

Jorgensen, N., et al (1997). Sociology: An Interactive Approach. London: Collins Educational.

Kachru, B. B. (1992b). 'Teaching World Englishes,' in B. B. Kachru (ed)(1992). The Other Tongue: English Across Cultures. Urbana: University of Illinois Press. 356-365.

Kaough, J. B. (1987). Drama as Cross-cultural literature for foreign students in American colleges: an Institutional model. Ph.D. thesis, the University of Texas at Austin.

Kapferer, B. (1984). 'The Ritual Process and the Problem of Reflexivity in Sinhalese Demon Exorcisms,' in J. J. MacAloon (ed.) (1984b). Rite, Drama, Festival, Spectacle: Rehearsals Toward a Theory of Cultural Performance. Philadelphia, Pen: ISHI. 179-207.

Kember, D. (2000). Action Leaming and Action Research: Improving the quality of teaching and learning. London: Korgan Page Limited.

Kemmis, S. (1993). 'Action Research,' in M. Hammersley (ed.).(1993). Educational Research: Current issues. London: P.C.P. 177-190.

Kemmis, S. (1994). 'Emancipatory Aspirations in a Postmodern Era,' in O. ZuberSkerritt (ed)(1996). New Directions in Action Research. London.Washington: Falmer Press. 199-242.

Kidder, R. M. (1995). 'Universal Human Values: Finding an Ethical Common Ground,' Public Management, 77 (6): 4-9.

Kim, Y. Y. (1984). 'Searching for creative Integration,' in W. B. Gudykunst and Y. Y. Kim (eds)(1984). Methods for intercultural communication research. Beverly Hills London : Sage [in cooperation with Speech Communication Association's Commission]. 13-30.

Koczanowicz, L. (2000). 'Freedom and Communication: The Concept of Human Self in Mead and Bakhtin', DIALOGISM: An International Journal of Bakhtin Studies, Issue 4: 54-66. 
Kordes, H. (1991). IntcFgLangLit/3choi\$ 'Intercultural Learning at School: Limits and Possibilites', in D. Buttjes and M. Byram (1991). Mediating Languages and Cultures: Towards an Intercultural Theory of Foreign Language Education. Clevedon and Philadelphia: Multicultural Matters, Ltd. 287-305.

Kramsch, C. (1993). Context and Culture in Language Teaching. Oxford: Oxford University Press.

Kramsch, C. (1997). 'Culture and Constructs: Communicating Attitudes and Values in the Foreign Language Classroom,' in P. R. Heusinkveld (ed.).(1997). Pathways to Culture: Readings on Teaching Culture in the Foreign Language Class. Yarmouth, ME: Intercultural Press. 461-485.

Krauss, M. (1992). The World's languages in crisis,' Language, 68 (1): 4-10.

Kress, G. (1995). Writing the Future: English and the making of a culture of innovation. Sheffield: NATE.

Kress, G. (1996). 'Internationalisation and globalisation: rethinking a curriculum of communication,' Comparative Education, 32 (2): 185-196.

Kress, G. (ed)(1988). Communication and Culture: An Introduction. Kensington NSW Australia: New South Wales University Press.

Kumpulainen, K. and Wray, D. (2002). Classroom Interaction and Social Learning: From theory to practice. London and New York: Routledge-Falmer Press.

Lacey, S. and Woolland, B. (1992). 'Educational Drama and Radical Theatre Practice,' New Theatre Quarterly, 8 (2): 81-91.

Lave, J. and Wenger, E. (1991). Situated learning: Legitimate peripheral participation. Cambridge: Cambridge University Press.

Lewicki, T. (1996). From 'Play Way' to 'Dramatic Art': A historical survey about 'drama in education' in Great Britain. Roma: LAS.

Lewin, K. (1946). 'Action Research and Minority Problems,' Journal of Social Issue, 2: 34-36.

Lewins, A. (2001). 'Computer Assisted Qualitative Data Analysis,' in N. Gilbert (2 ed)(2001). Researching Social Life. London. Thousand Oaks· New Delhi: Sage. 302-323.

Lincoln, Y. S. and Guba, E. G. (1985). Naturalisitic Inquiry. Newbury Park·Lodnon·New Delhi: SAGE.

Lindlof, T. R. (1995). Qualitative Communication Research Methods [Current Communication: An Advanced Text Series Vol. 3]. Thousand Oaks: SAGE Publications.

Lukàcs, G. (1990). History and Class Consciousness. (trans. R. Livingstone London: Merlin Press. 
Lykes, M. B. (2001). 'Creative Arts and Photography in Participatory Action Research in Guatemala,' in P. Reason and H. Bradbury (eds)(2001). Handbook of Action Research: Participative Inquiry and Practice. London and Thousand Oaks: Sage Publications. 363-371.

MacAloon, J. J. (1984a). 'Introduction: Cultural Performances, Culture Theory,' in J. J. MacAloon (1984b). Rite, Drama, Festival, Spectacle: Rehearsals Toward a Theory of Cultural Performance. Philadelphia, Pen: ISHI. 1-15.

Martin, J. N. and Nakayama, T.K. (1999). Intercultural Communication in Contexts. Mountain View, California: Mayfield Publishing Co.

Martin-Smith, A. (1993). A Conceptual Change in the Teaching of Drama in Education. Ph.D. thesis, University of Toronto.

Maslow, A. H. $\left(2^{\text {nd }}\right)(1970)$. Motivation and Personality. New York•London: Harper and Row.

May, T. (1993). Social Research. Buckingham: Open University Press.

McKernan, J. (1991). Curriculum Action Research: A Handbook of Methods and Resource for the Reflective Practitioner. London: Kogan Page.

McLaren, P. ( $2^{\text {nd }}$ ed). (1993a). Schooling as a ritual performance: Towards a political economy of educational symbols and gestures. London: Routledge.

McNiff, J. (1988). Action research: principles and practice. London: MacMillan.

McNiff, J. et al. (1996). You and your action research project. London: Routledge.

Meighan, R. and Siraj-Blatchford, I. $\left(3^{\text {rd }}\right.$ ed)(1997). Sociology of Educating. London: Cassell.

Merryfield, M. (1995). 'Teacher Education in Global and International Education', ERIC Clearinghouse on Reading and Communication Skills Bloomington IN. ERIC Digests [ERIC Identifier. ED384601: http://www.ed.gov/databases/ERIC_ Digests/ed384601.html, accessed on 02 December 2000].

Mienczakowski, J. and Morgan, S. (2001). 'Ethnodrama: Constructing Participatory, Experiential and Compelling Action Research through Performance,' in P. Atkinson, et al. (eds.)(2001). Handbook of Ethnography. London.Thousand Oaks: SAGE. 218-227.

Miles, M. B. and Huberman, A. M.(2 ${ }^{\text {nd }}$ ed $)(1994)$. Qualitative Data Analysis. Thousand Oaks, London and New Delhi: Sage.

Mills, C. W. (1970/1959 $3^{\text {rd }}$ ed). The Sociological Imagination. New York: Oxford University Press.

Millward, P. (1988). The Language of Drama: A study of the way in which people accomplish the dramatic presentation of experience. Ph.D. Thesis, University of Durham. 
Mitter, S. (1992). Systems of Rehearsal: Stanislavsky, Brecht, Grotowski and Brook. London: Routledge.

Moje, E. B. and Wade, S. E. (1997). What case discussions reveal about teacher thinking,' Teaching and Teacher Education, 13 (7): 691-712.

Moore, S. (1965)(New Rev.ed). The Stanislavski System: The Professional Training of an actor. Harmondsworth, England: Penguin Books Ltd:

Moraes, M. (1996). Bilingual Education: A Dialogue with the Bakhtin Circle. New York: State University of New York Press.

Moss, B. (2000). 'The use of large-group role-play techniques in social work education', Social Work Education, 19 (5): 471-483.

Muhr, T. (1997). ATLAS.ti: Visual Qualitative Data Analysis Management Model Building in Education, Research \& Business [User's Manual and Reference]. Berlin: Scientific Software Development.[cf. http://www.atlasti.de/ATLMAN.PDF].

Neelands, J. (1992). Learning through Imagined Experience. Kent: Hodder \& Stoughton.

Nias, J. (1993). 'Rrimary Teachers Talking: Reflexive Account of Longitudinal Research,' in M. Hammersley (ed.).(1993). Educational Research: Current issues. London: The Open University. 177-190 .

O'Neill, C. (1989). 'WAYS OF SEEING: audience function in drama and theatre,' 2D, 8 (2): 16-29.

O'Sullivan, C. and Gray, A. (1998). 'The Behavioural Stakes: a study of the effects of drama on undisciplined children at primary school level', Broadsheet, 14 (1): 2836.

Oberg, K. (1960). 'Cultural Shock: Adjustment to new cultural environments,' Practical Anthropology, 7: 177-182.

Osler, A. and Vincent, K. (2002). Citizenship and the Chanllenge of Global Education. Stroke on Trent: Trentham Books.

Parmenter, L. (1999). 'Constructing National Identity in a Changing World: Perspective in Japanese education,' British Journal of Sociology of Education, 20 (4): 453463.

Pavis, P. (1989). 'Problems of translation for the stage: interculturalism and postmodern theatre,' in H. Scolnicov and P. Holland (eds)(1989). The Play Out of Context: Transferring Plays From Culture to Culture. Cambridge: Cambridge UP. 25-44.

Pavis, P. (1992 ). Theatre at the Crossroads of Culture. London: Routledge.

Pavis, P. (ed) (1996). The Intercultural Performance Reader. London and New York: Routledge. 
Peacock, R. (1960). The Art of Drama. London: Routledge and Kegan Paul.

Pennycook, A. (1994). The Cultural Politics of English as an International Language. London: Longman.

Pennycook, A. (2001). 'English in the world/ the world in English,' in A. Burns and C. Coffin (eds)(2001). Analysing English in a Global Context: A Reader [Series of Teaching English Language Worldwide]. London and New York: Routledge. 7889.

Perinbanayagam, R. S. (2000). The Presence of Self. Lanham, Maryland: Rowman \& Littlefield Publishers, Inc.

Peter, M. (1994). Drama of All: Developing drama in the curriculum with pupils with special educational needs. London: David Fulton Pub.

Peter, M. (1995). Making Drama Special: Developing drama practice to meet special education needs. London: David Fulton Pub.

Phillion, J. (1999). 'Narrative and Formalistic Approaches to the study of Multiculturalism,' Curriculum Inquiry, 29 (1): 129-140.

Phillipson, R. (1992). Linguistic Imperialism. Oxford: Oxford University Press.

Pillay, K. (2000). 'Nondualism: a theoretical and experiential perspective for the practice of educational drama and theatre,' Research in Drama Education, 5(1): 115-116.

Pratt. G. et al. (1999). 'Youth Culture Tunes into the West: Korea Study Guide,' The Times Educational Supplement (TES) (London), 1 October 1999, 1-11.

Prus, R. (1996). Symbolic Interaction and Ethnographic Research: Intersubjectivity and the Study of Human Lived Experience. Albany: State university of New York Press.

Punch, K. F. (1998). Introduction to Social Research: Quantitative and Qualitative Approaches. London-Thousand Oaks-New Delhi: SAGE Publications.

Rassool, N. (1998). 'Postmodernity, cultural plurialism and the nation-state: Problems of language rights, human rights identity and power,' Linguistic Sciences, 20 (1): 89-99.

Rassool, N. et al. (1999). 'Postmodern culture and the Plural Self,' in J. Rowan and M. Cooper (eds)(1999). The Plural Self: Multiplicity in Everyday Life. London. Thousand Oaks: Sage Publications. 93-106.

Reason, P. and Bradbury, H. (eds)(2001). Handbook of Action Research: Participative Inquiry and Practice. London.Thousand Oaks: Sage Publications.

Reid, L. A. (1989). 'The Arts within a Plural concept of knowledge', in P. Abbs (ed)(1989b). The Symbolic Order. A Contemporary Reader on the Arts Debate. London: Falmer Press: 12-20. 
Rey, M. (1991). 'Human Rights and Intercultural Education,' in H. Starkey (ed.).(1991). The Challenge of Human Rights Education. London: Cassell. 135-151.

Robbins, B. (1998). 'Creative Dramatics in the Language Arts Classroom', ERIC Clearinghouse on Reading and Communication Skills Bloomington IN. ERIC Digests (ERIC Identifier. ED297402) http://www.ed.gov/databases/ERICDigests/ed297402. html Accessed on 02 December 2000].

Robertson, R. (1997). 'Social Theory, Cultural Relativity and the Problem of Globality,' in A. D. King (ed)(1997). Culture, Globalization and the World-System: Contemporary Conditions for the Representation of Identity. Minneapolis: University of Minnesopa Press. 69-90.

Robinson, K. (ed.) (1980). Exploring Theatre and Education. London: Heinemann.

Rolfe, G. (1996). 'Going to extremes: action research, grounded practice and the theory-practice gap in nursing,' Journal of Advanced Nursing, 24: 1315-1320.

Rubin, J. E. and Ruffin, R. (1993). 'Drama/Theatre and Multicultural Education', Proteus: A Joumal of IDEAS, 10 (1): 39-42.

Ryu, H. (1998). 'Multiculturalism in the Korean Context,' Korea Focus, (May-June 1998): $79-88$.

Said, E. (1983). The World, the Text and the Critic. Cambridge, MA: Harvard University Press.

Said, E. (1993). Culture and Imperialism. New York: Random House.

Schechner, R. and Appel, W. (1990). By means of performance: Intercultural Studies of theatre and ritual. Cambridge: Cambridge UP.

Schechner, R. (1990). 'Magnitudes of performance', in R. Schechner and W. Appel (1990). By means of performance: Intercultural Studies of theatre and ritual. Cambridge: Cambridge UP. 19-49.

Schechner, R. (1993). The Future of Ritual: Writings on Culture and Performance. London: Routledge.

Schechner, R. (2002). Performance Studies: An Introduction. London and New York: Routledge.

Schechner, R.(revised)(1988). Performance Theory. London: Routledge.

Schewe, M. (1998). 'Culture through literature through drama,' in M. Byram and M. Fleming (eds)(1998). Language Learning in Intercultural Perspective: Approaches through drama and ethnography. Cambridge: Cambridge UP. 204221.

Seelye, H. N. (1994). Teaching Culture: Strategies for Intercultural Communication. Lincolnwood, III.: National Textbook Com. 207-234.

Seelye, H. N. (1997a). 'Introduction,' in P. R. Heusinkveld (ed)(1997). Pathways to 
Culture: Readings on Teaching Culture in the Foreign Language Class. Yarmouth, ME: Intercultural Press, Inc. 97-105.

Seelye, H. N. (1997b). 'The Cultural Mazeway: Six Organizing Goals,' in P. R. Heusinkveld (ed)(1997). Pathways to Culture: Readings on Teaching Culture in the Foreign Language Class. Yarmouth, ME: Intercultural Press, Inc. 97-105.

Seo, H. (2002). "Drama, as a syllabus at the secondary level," Hanguk Gyoyuk Shinmun (Seoul, Korea), 29 August, 2002, 5.

Shaked, G. (1989). 'The play: gateway to cultural dialogue (trans. J. Green),' in H. Scolnicov and P. Holland (ed)(1989). The Play Out of Context: Transferning Plays From Culture to Culture. Cambridge: Cambridge UP. 7-24.

Silverman, D. (2000). Doing Qualitative Research: A Practical Handbook. London: SAGE Publications.

Singelis, T. M. (1998). Teaching About Culture, Ethnicity, \& Diversity: Exercises and Planned Activities. Thousand Oaks-London: SAGE.

Slade, P. (1998). 'The Importance of Dramatic Play in Education and Therapy,' Child and Adolescence Mental Health, 3(3): 110-112.

Slote, Walter H.(1996). 'Koreans Abroad in Therapy,' in A. Roland (1996). Cultural Pluralism and Psychoanalysis: The Asian and North American Experience. New York and London: Routledge. 187-198.

Smith, A. D.(1991). National Identity. Harmonsworth: Penguin.

Smith, H. L. (1999). 'Bilingualism and Bilingual Education: The Child's Perspective,' Intemational Joumal of Bilingual Education and Bilingualism, 2 (4): 268-281.

Smith, L. E (ed)(1981). English for Cross-cultural Communication. London: MacMillan Press.

Spjelkavik, Ø. (1999). 'Applied Research or Action Research?: Different or Complementary Methods,' in D. J. Greenwood (1999). Action Research: From practice to writing in an international action research development program. Amsterdam: John Benjamins Publishing Co. 117-129.

Spradley, J. P. (1979). The Ethnographic Interview. Orlando: Holt, Rinehart and Winston, Inc.

Stanislavski, C. (1916-1920). 'From Inner Impulses and Inner Action; Creative Objectives', in R. Drain (ed) (1995). Twentieth Century Theatre: A Sourcebook. London: Routledge:

Stanislavski, C. (Digested and $2^{\text {nd }}$ Edited by S. Moore)(1960). The Stanislavski System: the professional training of an actor. Harmondsworth, Middleses: Penguin Books.

Stevens, R. (1983). Erik Erikson: An Introduction. Milton Keynes: The Open University Press. 
Strauss, A. L. and Corbin, J. (1998). Basics of Qualitative Research: techniques and Procedures for Developing Grounded Theory. Thousand Oaks: SAGE

Strauss, A. L. (1987). Qualitative Analysis For Social Scientists. Cambridge: Cambridge University Press.

Styan, J. L. (1975). Drama, Stage, and Audience. London: Cambridge University Press.

Swart, E. (1998). 'Using Dramaturgy in Educational Research,' in S. R. Steinberg and J. L. Kincheloe (eds) (1998). Students as Researchers: Creating Classrooms that Matter. London: Falmer Press. 113-135.

Taylor, P. (1992). Our Adventure of Experiencing: Drama Structure and Action Research in the Grade Seven Social Studies Classroom, Ph.D. Thesis, New York University.

Tihanov, G. (2000). The Master and the Slave: Lukács, Bakhtin, and the Ideas of Their Time. Oxford: Oxford University Press.

Timothy, A. (1991). An Analysis of Creative Drama Structures in Relation to Intrinsic Learning in a Cultural and Social Contest. Ed.D thesis, University of Toronto.

Toffler, A. (1980). The Third Wave. London: Pan Books in association with Collins.

Turner, R. H. (1962). 'Role Taking: Process versus Conformity,' in D. Brissett and C. Edgley (eds)(1990). Life as Theater. A Dramaturgical Source Book. New York: Aldine De Gruyter.

Turner, V. (1985). On the Edge of the Bush. Tucson: University of Arizona Press.

Usher, R. and Edwards, R. (1994b). 'Postmodernism, Postmodernity and the Postmodern movement', in R. Usher and R. Edwards (1994a). Postmodemism and Education. London: Routledge. 1-32.

Usher, R. (2002). 'Putting Space Back on the Map: globalisation, place and identity,' Educational Philosophy and Theory, 34 (1): 41-55.

Vendler, Z. (1984). 'Understanding People,' in R. A. Shweder, and R. A. LeVine (1984). Culture Theory: Essays on Mind, Self, and Emotion. Cambridge: Cambridge University Press. 200-213.

Wagner, B. J. (1999a). 'Attitudes, Behaviour, and Moral Reasoning,' in B. J. Wagner (ed)(1999b). Building Moral Communities through Educational Drama. Stamford, Connecticut: Ablex Publishing Corporation. 137-158.

Waldschmidt, E. D. (1998). 'Bilingual Teachers Learning About Creative Drama: A Transformative Process,' Youth Theatre Joumal, 12 (1): 96-108.

Walker, D. (1977b). Play Read 2: Three Teenage Plays for Classroom Reading. London: Edward Amold (Publishers). Ltd.

Warnock, M. (1977). Emotion and Personality [series: education and philosophy] 
London: Faber.

Willems, G. M. (2002). Language teacher education policy promoting linguistic diversity and intercultural communication: $A$ Guide for Language Education Policies for Linguistic Diversity and Plurilingualism. Strasbourg: Council of Europe.

Williams, R. (1958,1983). Culture and Society. Oxford: Oxford Universtiy Press.

Wilson, R. (1984). The CIVIL warS, a tree is best measured when it is down. Cologne. [Play directed by Wilson].

Winston, J. (1998). Drama, Narrative and Moral Education: Exploring Traditional Tales in the Primary Years. London: Falmer Press.

Winston, J. (1999). 'Theorising Drama as Moral Education,' Journal of Moral Education, 28 (4): 459-471.

Witkin, R. W. (1974). The Intelligence of Feeling. London: Heinemann.

Woolland, B. (1993). The Teaching of Drama in the Primary School. London: Longman.

Wright, D. (2000). 'Drama Education: a 'Self-organising system' in pursuit of learning,' Research in Drama Education, 5 (1), March 2000: 23-32.

Wright, E. (1989). Postmodem Brecht: A Re-Presentation. London: Routledge.

Wuthnow R. et al., (1984). Cultural analysis: the work of Peter L. Berger, Mary Douglas, Michel Foucault, and Jurgen Habermas. Boston-London: Routledge and Kegan Paul.

Young, L. and Bagley, C. (1982). 'Self-Esteem, Self-Concept and the Development of Black Identity: A Theoretical Overview,' in G. K. Verma and C. Bagley (1982), Self-Concept, Achievement and Multicultural Education. London: MacMillan.

Young, T. R. (1990). The Drama of Social Life: Essays in Post-Modern Social Psychology. New Brunswick (USA)-London (UK): Transaction Publishers.

Zeicher, K. (2001). 'Educational Action Research,' in P. Reason and H. Bradbury (eds)(2001). Handbook of Action Research: Participative Inquiry and Practice. London and Thousand Oaks: Sage Publications. 273-283.

Zuber-Skerritt, O. (1996a). 'Introduction: New Directions in Action Research,' in O. Zuber-Skerritt (ed)(1996c). New Directions in Action Research. London. Washington: Falmer Press. 1-9.

Zuber-Skerritt, O. (1996b). 'Emancipatory Action Research for Organisational Change and Management Development,' in O. Zuber-Skerritt (ed)(1996c). New Directions in Action Research. London. Washington: Falmer Press. 83-105. 


\section{Other Sources Consulted}

Abbs, P. (1994a). The Educational Imperative: A Defence of Socratic and Aesthetic Learning. London: Falmer press.

Abbs, P. (ed) (1989b). The Symbolic Order. A Contemporary Reader on the Arts Debate. London: Falmer Press.

Alred, G. et al. (eds) (2003). Intercultural Experience and Education [Languages for Intercultural Communication and Education 2; Series Editors: Michael M. Byram and Alison Phipps]. Clevedon: Multilingual Matters Ltd.

Arts Council of Great Britain. (1992). Drama in schools. London: Roy Wood, Arts of Council of Great Britain.

AAsante, M. F. and Gudykunst, W. B. (eds) (1989). Handbook of international and intercultural communication. Newbury Park, CA: Sage.

Aston, E. and Savona, G. (1991). Theatre as Sign-System: A Semiotics of Text and Performance. London: Routledge.

Atkinson, P. et al. (eds)(2001). Handbook of Ethnography. London: Sage.

Atweh, B. et al. (eds). Action Research in Practice: Partnerships for Social Justice in Education. London: Routledge.

Bamgbose, A. (2001). 'World Englishes and globalization,' World Englishes, 20 (3): 357-363.

Barlow, S. and Skidmore, S. (1994). DRAMAFORM: a practical guide to drama techniques. London: Hodder and Stoughton.

Barry, C. A. (1998). 'Choosing Qualitative Data Analysis Software: Atlas/ti and Nudist Compared' Sociological Researcher Online, 3 (3) [20 Feb 2003].

Beck, U. et al. (1994). Reflexive Modernization: Politics, Tradition and Aesthetics in the Modern Social Order. Cambridge: Polity Press.

Bessis, S. (1995). From Social Exclusion to Social Cohesion: A Policy Agenda [Management of Social Transformations (MOST), UNESCO, Policy Paper 2. Paris: UNESCO.

Bhabha, H. K. (1994). The Location of Culture. London and New York: Routledge.

Booth, T. and Ainscow, M. (des)(1998). From them to us: an international study of inclusion in education. London: Routledge.

Bourne, J. and Reid, E.n (eds)(2003). World yearbook of education 2003: Language Education. London: Kogan Page

Bowman, R. L. (1997). 'Joking' with the Classics: Using Boal's Joker System in the Performance Classroom,' Theatre Topics, 7 (2): 139-151. 
Brandist, C. (1997). 'The Bakhtin Circle,' The Internet Encyclopedia of Philosophy. http://www.utm.edu/research/iep/b/bakhtin.htm. The Internet Encyclopedia of Philosophy] (Bakhtin Centre, the University of Sheffield).[accessed on December 2001]

Bray, M. and Lee, W. O. (1997). 'Education and Political Transitions in East Asia: Diversity and Commonality,' in M. Bray and W. O. Lee (eds) $\left(2^{\text {nd }}\right.$ Ed.) Education and Political Transition: Themes and Experiences in East Asia (CERC Studies in Comparative Education 1). HongKong: Comparative Education Research Centre.1-18.

Brink, D. O. (1999). 'Objectivity and Dialectical Methods in Ethics', Inquiry, 42: 195212.

Brinker-Gabler, G. (ed)(1995). Encountering the Other(s): Studies in Literature, History, and Culture. Albany: State University of New York Press.

Brissett, D. and Edgley, C. (eds)(1990). Life as Theater: A Dramaturgical Source Book. New York: Aldine De Gruyter.

Brooker, P. (1988). Bertolt Brecht: Dialectics, Poetry, Politics. London: Croom Helm.

Brown, J. S., Collins, A. and Duguid, P. (1989). 'Situated Cognition and the Culture of Learning,' Educational Researcher, Vol. 18: 32-42.

Bruner, J. S. et al. (eds) (1976). Play-lts Role in Development and Evolution. Middlessex: Penguin.

Burgess, R. G. (ed)(1985). Issues in Educational Research: Qualitative Methods. East Sussex, UK: Falmer Press.

Burns, A. and Coffin, C. (eds)(2001). Analysing English in a Global Context: A Reader [Series of Teaching English Language Worldwide]. London: Routledge.

Buttjes, D. and Byram, M. (eds)(1991). Mediating Languages and Cultures: towards an intercultural theory of foreign language education. Clevedon: Multilingual Mattes.

Byram, M. and Fleming, M. (eds)(1998). Language Learning in Intercultural Perspective: Approaches through drama and ethnography. Cambridge: Cambridge UP.

Byram, M. et al. (1991). Cultural Studies and Language Leaming: A Research Report. Clevedon: Multilingual Matters, LTD. (Co-authors: Byram, M., Esarte-Sarries, V. and Taylor, S.)

Byram, M. et al. (1994). Teaching-and-Learning Language-and-Culture. Clevedon: Multilingual Matters Ltd.

Cabral, B. (1994). 'Signs of a Post-modern, Yet dialectical practice', CORE 18(2). June 1A 04-13.

Caligiuri, P. M. et al. (2000). 'The Attitudinal and Behavioral Openness Scale: scale 
development and construct validation,' International Journal of Intercultural Relations, 24(1): 27-46.

Cameron, A. (1968). The Identity of Oedipus the King: Five Essays on the Oedipus Tyrannus. New York: New York University Press.

Carlson, M. (1990). Theatre Semiotics: Signs of Life. Bloomington· Indianapolis: Indian University Press.

Carlson, M. (1996). Performance: a critical introduction. London: Routledge.

Choi, Y. (1995). A Study of the effectiveness in Creative Drama in Education. MA. Dissertation. Kyungsung University, Korea [Korean version].

Choi, Y. (1998). Dialectical Dynamics of 'Living-through' Drama in Primary Schools. MA special study. University of Durham.

Choi, Y. et al. (1996a). A Study of Performing Arts [special issue on educational drama], spring 1996. Busan: Institute of Performing Arts, Kyungsung University. [Korean version]

Choi, Y. et al. (1996b). Workshop Report: 'theory and practice in educational dramafocused on its application to classroom drama,' Institute of Performing Arts, Kyungsung University, 30 November 1 December 1996. [Korean version].

Collier, Jr. J. and Collier, M. (1986). Visual Anthropology: Photography as a Research Method. Albuguerque: University of New Mexico Press.

Counsell, C. and Wolf, L. (eds)(2001). Performance Analysis: an introductory coursebook. London and New York: Routledge.

Couroucli-Robertson, K. (2001). 'Brief drama therapy of an immigrant adolescent with a speech impediment,' The Arts in Psychotherapy, 28: 289-297.

Cushner, K. and Brislin, R. W. (1997). Improving Intercultural Interactions: Modules for Cross-Cultural Training Programs (Volume 2) [Multicultural Aspects of Counseling Series 8]. Thousand Oaks: Sage.

Davis, D. and Lawrence, C. (eds)(1986). Gavin Bolton: Selected Writings. London: Longman.

Delanty, G. (1999). 'Self, Other and World: Discourses of Nationalism and Cosmopolitanism', Cultural Values, 3 (3): 365-375.

Denzin, N. K. (2 ${ }^{\text {nd }}$ ed)(2001). Interpretive Interactionism (Applied Social Research Methods Series Volume 16). Thousand Oaks: Sage.

DES (Department of Education and Science)(1989). Drama from 5 to 16 (Curriculum Matters 17 an HMI Series). London: HMSO.

DES (Department of Education and Science)(1990). The Teaching and Learning of Drama: Aspects of Primary Education. London: HMSO. 
Dillon, J. T. (1990). The Practice of Questioning (International series on communication skills). London: Routledge.

Drain, R. (ed) (1995). Twentieth Century Theatre: A Sourcebook. London: Routledge.

Duranti, A. and Goodwin, C. (eds)(1992). Rethinking Context: Language as an Interactive Phenomenon. Cambridge: Cambridge University of Press.

Easthope, A. and McGowan, K. (eds) (1992). A Critical and Cultural Theory Reader. Buckingham: Open University Press.

Edmiston, B. (1994). 'More than talk: a Bakhtininian perspective on drama in education and change in understanding,' NADIE Journal, 18: 25-36.

Edmiston, B. (2000). 'Drama as Ethical Education,' Research in Drama Education, 5 (1), March 2000: 63-84.

Egan, G. (1977). You and Me: The Skills of Communicating and Relating to Others. Belmont, Cal: Wadsworth Publishing Company, Inc.

Eikeland, O. (2001). 'Action Research as the Hidden Curriculum of the Western Tradition' in P. Reason and H. Bradbury (eds)(2001). Handbook of Action Research: Participative Inquiry and Practice. London: Sage.145-155.

Elam, K. (1980). The Semiotics of Theatre and Drama. London: Methuen.

Elliott, J. (1991). Action Research for Educational Change. Buckingham: Open University Press.

Emmison, M. and Smith, P. (2000). Researching the Visual: Images, Objects, Contexts and Interactions in Social and Cultural Inquiry. London: Sage.

Esslin, M. (1976). The Anatomy of Drama. London: Temple Smith.

Esslin, M. (1987). The Field of Drama: How the Signs of Drama create meaning on stage and screen. London: Methuen.

Friedrich, R. (1999). 'Brecht and Postmodernism' Philosophy and Literature, 23 (1): 44-64.

Gagnon, P. D. (1998). 'Acting Integrative: Interdisciplinarity and Theatre Pedagogy,' Theatre Topics, 8 (2): 189-204.

Garrick, D. A. (2001). 'Performances of Self-Disclosure: A Personal History,' The Drama Review, 45 (4): 94-105.

Gasparro, M. and Falletta, B. (1994). 'Creating Drama with Poetry: Teaching English as a Second Language through Dramatization and Improvisation', ERIC Clearinghouse on Reading and Communication Skills Bloomington IN. ERIC Digests (ERIC Identifier. ED368214). http://www.ed.gov/databases/ ERICDigests/ed368214.html [accessed on 3 August 2000].

Genesee, F. (ed)(1994). Educating Second Language Children: The whole child, the 
whold curriculum, the whole community [Cambridge language education; series editor J. C. Richards]. Cambridge: Cambridge University Press.

Giddens, A. (1991a). Modernity and Self-Identity: Self and Society in the Late Modem Age. Stanford, California: Standard University Press.

Giddens, A. (4 ${ }^{\text {th }}$ ed)(2001). Sociology. Cambridge: Polity Press.

Goalden, P. (1996). 'Educational drama and children's historical writing: process and product,' The Curriculum Journal, 7 (1): 75-92.

Gómez-Peňa, G. (2001). 'The New Global Culture: Somewhere between Corporate Multiculturalism and the Mainstream Bizarre (a border perspective), 'The Drama Review, 45 (1): 7-30.

González, K. P. (2001). 'Inquiry as a process of learning about the other and the self,' Qualitative Studies in Education, 14 (4): 534-562.

Goodman, N. R. (1994). 'Intercultural education at the university level: teacher-student interaction,' in R. W. Brislin and T. Yoshida (eds.)(1994). Improving Intercultural Interacitons: Modules for Cross-Cultural Training Programs (Volume 2)[Multicultural Aspects of Counseling Series 8]. Thousand Oaks: Sage. 129147.

Gudykunst, W. B. and Kim, Y. Y. (eds)(1984). Methods for intercultural communication research. Beverly Hills: Sage.

Gudykunst, W. B. and Nishida, T. (2001). 'Anxiety, uncertainty, and perceived effectiveness of communication across relationships and cultures,' International Journal of Intercultural Relations, 25 (1): 55-71.

Gudykunst, W. B. et al. (1985). 'A Cross-cultural test of Uncertainty Reduction Theory: Comparisons of Acquaintances, Friends, and Dating Relationships in Japan, Korea, and the United States,' Human Communication Research, 11 (3). Spring 1985: 407-454.

Gundara, J. S. (2000a). Interculturalism, Education, and Inclusion. London: Paul Chapman Publishing Ltd.

Hall, S. K. (2000). 'What Character is', in S. K. Hall (2000). Using Picture Storybooks to teach Character Education. Phoenix, Arizona: The Oryx Press. (pp.xvii--xxxi).

Hall, T. et al. (2000). 'Young People, Citizenship and the Third Way: A Role for the Youth Service?, Joumal of Youth Studies, 3 (4): 461-472.

Hammersley M. (ed.).(1993). Educational Research: Current issues. London: The Open University.

Harland, J. et al. (2000). Arts Education in Secondary Schools: Effects and Effectiveness. Slough, Berkshire: National Foundation for Education Research.

Harvey, D. (1990). The Condition of Postmodernity. Malden, Massachusetts: Blackwell Publishers. 
Hesse, B. (1997). 'It's Your World: Discrepant M/Multiculturalisms,' Social Identities, 3 (3): 375-394.

Heusinkveld, P. R. (ed)(1997). Pathways to Culture: Readings on Teaching Culture in the Foreign Language Class. Yarmouth, ME: Intercultural Press, Inc.

Hirschkop, K. and Shepherd, D. (eds)(1989). Bakhtin and Cultural Theory. Manchester: Manchester University Press.

Holland, D. et al. (1998). Identity and Agency in Cultural Worlds. Cambridge, Massachusetts: Harvard University Press.

Hornbrook, D. (ed)(1998c). On The Subject of Drama. London: Routledge.

Howard, J. A. and Callero, P. L. (eds)(1991). The self-society dynamic: cognition, emotion, and action. Cambridge: Cambridge University Press.

Hudson, L. (1968). Frames of Mind: Ability, Perception and Self-perception in the Arts and Sciences. London: Methuen and Co. Ltd.

Huxley, M. and Witts, N. (eds)(1996). The Twentieth-Century Performance Reader. London: Routledge.

Jaschok, M. and Jingjun, S. (2000).'Outsider within': Speaking to excursions across cultures,' Feminist Theory, 1 (1): 33-58.

Jewitt, Carey, et al. (2001). 'Exploring Learning Through Visual, Actional and Linguistic Communication: the multimodal environment of a science classroom,' Educational Review, 53 (1): 5-18.

Joachim, H. H. (1951). Aristotle: the Nicomachean ethics [a commentary]. ed. by D. A. Rees. Oxford: Clarendon Press.

John-Steiner, V. et al. (eds)(1994). 'Mediational processes, ' in V. John-Steiner, et al. (1994). Sociocultural approaches to language and literacy: An interactionist perspective. New York: the Press Syndicate of the University of Cambridge. 139-146.

Kachru, B. B. (ed)(1992). The Other Tongue: English Across Cultures. Urbana: University of Illinois Press.

Kamppinen, M. (1998). 'Evolution and culture-the Danwinian view on infosphere,' Futures, 30 (5): 481-484.

Kempe, A. (reprinted 1995). The GCSE Drama Coursebook. Cheltenham: Stanley Thornes.

Kim, T. C. and Harrison, R. (eds)(1999). Self and Future Generations: an intercultural conversation. Cambridge: The White Horse Press.

Kress, G. and et al. (1997). 'Discourse semiotics,' in T. Van Dijk (ed)(1997). Discourse as Structure and Process. London: Sage. 257-291. 
Landy, R. J. (1993). Persona and Penformance: The Meaning of Role in Drama, Therapy, and Everyday Life. New York: The Guilford Press.

Lawley, J. (2000). Social Inclusion and School Improvement: Can we have both?' (Head teacher's bulletin, Issue 46, May 2000). Surrey: Croner CCH Group.

Levine, R. and Adelman, B. $\left(2^{\text {nd }}\right.$ ed.)(1993). Beyond Language: Cross-cultural Communication. Englewood, NJ: Regents/Prentice Hall.

Lewis, R. B. (1998). 'ATLAS/ti and NUD.IST: A Comparative Review of Two Leading Qualitative Data Analysis Packages,' Cultural Anthropology Methods, 10 (3): 41-47.

Link, A. (1992). Mirrors from the heart: Emotional identity and expression through drama. Ontario, Canada: Elora. Lippe, W.A. (1992). 'Stanislavski's affective memory as a therapeutic tool,' Joumal of Group Psychotherapy, Psychodrama \& Sociometry, 45(3): 102-111.

Lomax, P. (2000). 'Coming to a Better Understanding of Educative Relations through Learning Individuals' Representations of their Action Research,' Reflective Practice, 1(1): 43-53.

Lorenz, E. et al. (eds.). (1994). Teaching Culture in Grades K-8: A Resource Manual for Teachers of French. Washington, D.C: Department of Academic Programs, Division of Curriculum etc.

Luyckx, M. (1999). 'The transmodern hypothesis: towards a dialogue of cultures,' Futures, 31: 971-982.

Lyle, S. (1996). 'Making Meaning: the voices of children talking about a dramatised story,' Educational Studies, 22 (1): 83-97.<D: 09>

Lynch, J. (1983). The Multicultural Curriculum (Series of Education in a Multicultural Society: Maurice Craft (editors). London: Batsfor Academic and Education Ltd.

Lynch, J. et al. (eds)(1992). Cultural Diversity and the Schools Vol. One: Education for Cultural Diversity Convergence and Divergence. London: Falmer Press.

MacAloon, J. J. (ed.). (1984b). Rite, Drama, Festival, Spectacle: Rehearsals Toward a Theory of Cultural Performance. Philadelphia, Pen: ISHI.

Macintyre, P. D. (1995). 'How Does Anxiety affect Second Language Learning? A Reply to Sparks and Granschow,' The Modern Language Journal, 79 (1): 90-99.

Marranca, B. and Dasgupta G. (eds)(1991). Interculturalism and Performance: Writings from PAJ. New York: PAJ publications.

Maths, J. A. et al. (1980). 'Psychodrama and sociodrama in primary and secondary education,' Psychology in the Schools, 17 (1): 96-101.

May, L. (2002). "Putting Yourself in Other People's Shoes": the use of Forum theatre to explore refugee and homeless issues in schools,' Joumal of Moral Education, 31 (1): 21-34. 
May, S. (ed)(1999). Critical Multiculturalism: Rethinking Multicultural and Antiracist Education. London· Philadelphia, PA: Falmer Press.

May, T. (1996). Situating SocialTtheory. Buckingham.Philadelphia: Open University Press.

McClelland, C. A. (1972). 'On the Fourth Wave: Past and Future in the Study of International Systems, in J. N. Rosenau et al. (1972). The Analysis of International Politics. New York: the Free Press. 15-40.

McGuire, Brian (1998). The Student Handbook for Drama: ideal for Key Stages 3 and 4. Cambridge: Pearson Publishing.

Min, E. (2001). 'Bakhtinian Perspectives for the Study of Intercultural Communication,' Journal of Intercultural Communication, 22 (1): 5-18.

Mitter, S. (1992). Systems of Rehearsal: Stanislavsky, Brecht, Grotowski and Brook. London: Routledge.

Morgan, N. and Saxton, J. (1987). Teaching Drama: A mind of many wonders. Cheltenham: Stanley Thornes.

Munro, T. (1949). The Arts and Their Interrelations. New York: The Liberal Arts Press.

Neelands, J. (1990). Structuring Drama Work. Cambridge: Cambridge University Press.

Neelands, J. (1992). Leaming through Imagined Experience. Kent: Hodder \& Stoughton.

Neelands, J. (1998). Beginning Drama 11-14. London: David Fulton Pub.

Nicolopoulou, A. (1993). 'Play, Cognitive Development, and the Social World: Piaget, Vygotsky, and Beyond,' Human Development, 36 (1): 1-23.

Nieto, Sonia (2002). Language, Culture, and Teaching Critical Perspectives for a New Century. Mahwah, NJ: Lawrence Erlbaum Associates, Inc.

Nuckolls, C. W. (1996). The Cultural Dialectics of Knowledge and Desire. Wisconsin: The University of Wisconsin Press.

O'Hare, D. (1981). Psychology and the Arts. Sussex: The Harvester Press.

O'Neill, C. (1995). Drama Worlds: A Framework for Process Drama. Portsmouth: Heinemann

O'Toole, J. (1992). The Process of Drama: Negotiating Arts and meaning. London: Routledge.

Olneck, M. R. (1993). 'Terms of Inclusion: Has Multiculturalism Redefined Equality in American Education?' American Joumal of Education, 101, part 3: 234-260. 
Oppenheim, A. N. (1992). Questionnaire design, interviewing and attitude measurement. London: Pinter.

Paige, R. M. (2 ${ }^{\text {nd }}$ ed).(1993). Education for the Intercultural Experience. Yarmouth, Maine: Intercultural Press.

Pandit, N. R. (1996). 'The Creation of Theory: A Recent Application of the Grounded Theory Method,' The Qualitative Report, 2 (4): (http://www.nova.edu/ssss/ QRI QR2-4/pandit.html).[accessed on 1 June 2001].

Pascual-Leone, J. (1987). 'Organismic Processes for a dialectical causual account of cognitive development,' International Journal of Psychology, 22: 531-570.

Pascual-Leone, J. (1995). 'Learning and development as dialectical factors in cognitive growth,' Human Development, 38 (6): 338-348.

Pfister, M. (1988). The Theory and Analysis of Drama. Trans. by J. Halliday. Cambridge: Cambridge University Press.

Phillipson, R. (2003). English-Only Europe?: Challenging Language Policy. London and New York: Routledge.

Planalp, S. (1999). Communicating Emotion: Social, Moral, and Cultural Processes [Series in emotion and social interaction, Series Editors: Keith Oatley and Antony Manstead]. Cambridge: Cambridge University Press and Editions de la Maison des Sciences de l'Homme in Paris

Raffo, C. and Reeves, M. (2000). 'Youth Transitions and Social Exclusion: Developments in Social Capital Theory', Joumal of Youth Studies, 3 (2): 147166.

Reid, L. A. et al. (1983). Art education: heritage and prospect. Ed. by A. Dyson. London: Institute of Education, University of London.

Richardson, R. (1999). Inclusive Schools, Inclusive Society: race and identity on the agenda. London: Trentham Books.

Riegel, K. F. (1979). Foundations of Dialectical Psychology. New York: Academic press.

Roberts, C. et al. (eds)(2001). Language Learners as Ethnographers (Modern Languages in Practice 16, Series Editor: Michael Grenfell). Clevedon·Buffalo: Multigual Matters Ltd.

Roland, A. (1996). Cultural Pluralism and Psychoanalysis: The Asian and North American Experience. New York and London: Routledge.

Ross, M. H. (2001). 'Psychocultural Interpretations and Dramas: Identity Dynamics in Ethnic Conflict,' Political Psychology, 22 (1): 157-178.

Rowan, J. and Cooper, M. (eds)(1999). The Plural Self: Multiplicity in Everyday Life. London-Thousand Oaks: Sage Publications. 
Sampson, E. E. (1993). Celebrating the Other. A Dialogic Account of Human Nature. New York and London: Harvester Wheatsheaf.

Savignon, S. J. and Sysoyev, P. V. (2002). 'Sociocultural Strategies for a Dialogue of Cultures,' The Modem Language Journal, 86 (4): 508-524.

Savingnon, S. J. (2003). 'Teaching English as Communication: a global perspective,' World Englishes, 22 (1): 55-66.

Schechner, R. and Appel, W. (1990). By means of performance: Intercultural Studies of theatre and ritual. Cambridge: Cambridge UP.

Schewe, M. and Shaw, P. (eds.) (1993). Towards Drama as a Method in the Foreign Language Classroom. Frankfurt am Main: Peter Lang Pub.

Schneider, J. J. and Jackson, S. A. W. (2000). 'Articles-Process drama: A special space and place for writing-Drama is a tool for instruction and learning that supports literacy development while also fostering children's inclination to imagine,' The Reading teacher, 54 (1): 38-53.

Scolnicov, H. and Holland, P. (ed)(1989). The Play Out of Context: Transferring Plays From Culture to Culture. Cambridge: Cambridge UP.

Seidlhofer, B. (2001). 'Closing a conceptual gap: the case for a description of English as a lingua franca,' Intemational Joumal of Applied Linguistics, 11 (2): 133-158.

Shweder, R. A. and LeVine, R. A. (eds)(1984). Culture Theory: Essays on Mind, Self, and Emotion. Cambridge: Cambridge University Press.

Skutnabb-Kangas, T. (2000). Linguistic Genocide in Education-or Worldwide Diversity and Human Rights? Mahwah, NJ: Lawrence Erlbaum Associates, Inc.

Smith, P. B. and Bond, M. H. $\left(2^{\text {nd }}\right.$ ed)(1998). Social Psychology Across cultures. London: Prentice Hall Europe, 1998

Smith, R. A. (1989). The Sense of Art: A Study in Aesthetic Education. New York: Routledge.

Somers, J. (1994). Drama in the Curriculum. London: Cassell.

Spradley, J. and McCurdy, D. W. (2000)(10 $10^{\text {th }}$ ed). Conformity and Conflict: Readings in Cultural Anthropology. Needham Heights, MA: Allyn and Bacon.

Steinberg, S. R. and Kincheloe, J. L. (eds) (1998). Students as Researchers: Creating Classrooms that Matter. London: the Falmer Press.

Sutherland, M. B. (1971). Everyday imagining and education. London: Routledge and Kegan Paul.

Szondi, P. (1987). Theory of the Modern Drama. Trans. by Hays, M. Minneapolis: University of Minnesota Press. 
Taylor, P. (ed)(1996). Researching Drama and Arts Education: Paradigms and Possibilities. London: Falmer Press.

Tehranian, Katherine Kia (1998). 'Global communication and pluralisation of identities,' Futures, 30 (2/3): 211-217.

Tomlinson, A. (1999). 'The Sociological Imagination, the New Journalism and Sports Writing and Research,' in A. Tomlinson (1999). The Game's up: Essays un the cultural analysis of sport, leisure and popular culture [Popular Cultural Studies: 15]. Aldershot: Ashgate Publishing Ltd. 41-60.

Torres-Reyes, Maria Luisa (1997). 'Theatre as an encounter of cultures.' TRI, 22 (1 sup) (1997): 61-68.

Turner, V. (1983). 'Body, Brain and Culture,' Zygon, 18 (3): 221-45.

Usher, R. and Edwards, R. (1994a). Postmodernism and Education. London: Routledge.

Verma, G. K. and Bagley, C. (1982). Self-Concept, Achievement and Multicultural Education. London: MacMillan.

Vygotsky, L. S. (1971). The Psychology of Art. Cambridge, Mass; London: MIT press.

Wagner, B. J. (ed)(1999b). Building Moral Communities through Educational Drama. Stamford, Connecticut: Ablex Publishing Corporation

Walker, D. (1977a). Next Stage: Thirteen plays for written or improvised extension. London: Edward Arnold (Publishers). Ltd.

Ward, C., Bochner, S. and Furnham, A. (2 ${ }^{\text {nd }}$ ed)(2001). The Psychology of Culture Shock. Hove: Routledge.

Wells, G. (1999). Dialogic Inquiry: Towards a Socio-cultural Practice and Theory of Education. Cambridge: Cambridge University Press.

Wertsch, J. V. (1991). Voices of the mind: a sociocultural approach to mediated action. Cambridge, Mass: Harvard Univ. Press.

Wildman, P. (1998). 'From the monophonic university to polyphonic multiversities,' Futures, 30 (7): 625-633.

Wilhelm, J. D. and Edmiston, B. (1998). Imagining to Learn: inquiry, ethics, and integration through drama. Portsmouth, NH: Heinemann.

Willett, J. ( $2^{\text {nd }}$ ed)(1990). Brecht on Theatre: the Development of an Aesthetic. London: Methuen.

Williams, R. (1975). Drama in a Dramatised Society. Cambridge: Cambridge University Press.

Winston, J. and Tandy, M. (1998). Beginning Drama 4-11. London: David Fulton Pub. 
Wiseman, R. (ed)(1995). Intercultural Communication theory. Thousand Oaks, CA: Sage.

Woods, P. (1993). 'Towards a Theory of Aesthetic Learning,' Educational Studies, 19 (3): 323-338.

Woods, P. (1996). Researching the Art of Teaching: Ethnography for Educational Use. London and New York: Routledge.

Yea, S. (1999). 'The culture and politics of resistance in South Korea,' Futures, 31: 221-234.

Zuber-Skerritt, O. (ed)(1996c). New Directions in Action Research. LondonWashington: Falmer Press.

\section{Other Websites Consulted}

http://uww.moe.go.kr/english/edukorea/edukorea1.html [the Ministry of Education in Korea]

http://uww.askasia.org/ [a K-12 resource of the Asia Society; accessed on 24 October 1999].

http://uwww.internationaled.org/ [National Campaign for International Education in the Schools, U. S. Department of Education]

http://humanities.byu.edu/linguistics/p/lpc1.html [more culture lesson plans at Department of Linguistics, Brigham Young University; accessed on 30 June 2002]

http://www.channel4.com/learning [Channel 4 learning in UK; accessed on 20 March 2002]

http://www2.soc.hawail.edu/css/de...esources/lntercultural/Study.html [accessed on 5 March 2002].

http://www.siu.edu/ ekachai/terms.html [Communication Across Cultures; More Lecture Handouts, Southern Illinois University, Carbondale Campus; SPCM 301accessed on 4 November 2001].

http://www.cabinet-office.gov.uk/...ations/reports/htm//bmezip/02/htm [National Strategy for Neighbourhood Renewal, Minority Ethics Issues in Social Exclusion and Neighbourhood Renewal; accessed on 1 April 2002].

http://uww.oup.com/elt/global/ [English language teaching worldwide: Oxford University Press; accessed through http://unw.oup.com/contents/elt/0-19437214-6/ on 25 January 2002]

http://uww.forefrontpublishers.com/esimusic/lesson plans/index.htm [Lesson plans of English teaching language through music; California State University; accessed on 30 June 2002] 
http://www.lle.mdx.ac.uk/die/pages/docs/lessons/yrg/jo.html [more lesson plans in the Drama in Education Site at the University of Middleses University] [accessed on 30 August 1999]

lhtto://www.pearsonpublishing.co.uk/education/html/secDr1.html [accessed through http://www.pearson.co.uk/cgi-win/M.exe?...\&2=Drama\&3=Citizenship\%2Fsocial +Studies on 19 September 2000].

http://members.iinet.net.au/ kimbo2/pd/index.htm [Drama Education in a Global Perspective: Learning in, with and through Drama; accessed on 8 January 2003].

http://unww.chalkface.com/html/drama.shtml [The Chalk Project provided by http://www. curriculumonline.gov.uk Department for Education and Skills]

http://www.ccsr.ac.uk/methods/ [Research Methods: an ESRC research programme]

http://www.nova.edu/ssss/QR/web.html [The Qualitative Report and Qualitative Research Web Sites at Nova Southeastern University]. 


\section{Appendices}

\section{Appendix A: Q1 (ASQ) and Q2 (Post-Questionnaire)}

\section{May I ask Questions about your Experiences?}

- This was the Pre-Questionnaire, in which I asked participants to fill in order to survey their cultural sensitivity of their own culture and others, including the English language.

This survey aims at exploring your feeling and experience of people who come from different countries and their different cultures in contacting other cultures and in leaming English in your school.

Would you read carefully each of the following items? You can tick a possible answer just simply. There are no right or wrong answers. You should work fast and record your first response.

Please fill up the following:

1. Your birthday:

(day)

(month) 19

(year)

2. Are you MALE [

] or FEMALE [

]

3. I consider my self to tend toward to be:

Extroverted [ ] or Introverted [

4. Are you planning to get a job [ ] ]

or to apply for a college or an university [ ] after your graduation from this school?

5. What languages can you speak fluently?

KOREAN ONLY [ ] KOREAN AND OTHERS [ ]

6. Which language can you speak, even if not very fluently?

(You cannot tick more than two)

$\begin{array}{llllll}<1>\text { English } & {[} & 1 & <4 \text { Japanese } & \text { [ } & \text { ] } \\ <2>\text { French } & {[} & 1 & <5>\text { Chinese } & {[} & \text { ] } \\ <3>\text { German } & {[} & 1 & <6>\text { Other } & {[}\end{array}$

Following is a list of statements about English. Place a check before each statement with which level, you think, you are at.

7. In the English language, I could say the days of the week.

\begin{tabular}{|l|l|l|l|l|}
\hline Very Good & Good & Middle & Bad & Very Bad \\
\hline
\end{tabular}

8. In the English language, I could give the current date (day-month-year).

\begin{tabular}{|l|l|l|l|l|}
\hline Very Good & Good & Middle & Bad & Very Bad \\
\hline
\end{tabular}


9. In the English language, I could order a simple meal in a restaurant.

\begin{tabular}{|l|l|l|l|l|}
\hline Very Good & Good & Middle & Bad & Very Bad \\
\hline
\end{tabular}

10. In the English language, I could talk about my favourite hobby using appropriate vocabulary.

\begin{tabular}{|l|l|l|l|l|}
\hline Very Good & Good & Middle & Bad & Very Bad \\
\hline
\end{tabular}

11. In the English language, in face-to-face conversation, I could understand a native speaker who is speaking slowly and carefully (deliberately adapting his or her speech to suit me).

\begin{tabular}{|l|l|l|l|l|}
\hline Very Good & Good & Middle & Bad & Very Bad \\
\hline
\end{tabular}

12. In the Enqlish language, I could tell what I plan to be doing five years from now, using appropriate future tenses.

\begin{tabular}{|l|l|l|l|l|}
\hline Very Good & Good & Middle & Bad & Very Bad \\
\hline
\end{tabular}

13. In the English language, in face-to-face conversation I could understand a native speaker who is speaking to me as quickly and as colloquially as she or he would to another native speaker.

\begin{tabular}{|l|l|l|l|l|}
\hline Very Good & Good & Middle & Bad & Very Bad \\
\hline
\end{tabular}

14. In the English language, I could state and support with examples and reasons a position on a controversial topic such as nuclear arms or abortion.

\begin{tabular}{|l|l|l|l|l|}
\hline Very Good & Good & Middle & Bad & Very Bad \\
\hline
\end{tabular}

Following is a list of statements about learning methods which you are experiencing or want. Place a check before each statement with which you agree.

15. I am satisfied with my English textbook which I am using in your English class.

\begin{tabular}{|l|l|l|l|l|}
\hline $\begin{array}{l}\text { Strongly } \\
\text { Agree }\end{array}$ & Agree & Neutral & Disagree & $\begin{array}{l}\text { Strongly } \\
\text { Disagree }\end{array}$ \\
\hline
\end{tabular}

16. It is more effective to learn English from native English teachers rather Korean English teachers?

\begin{tabular}{|l|l|l|l|l|}
\hline $\begin{array}{l}\text { Strongly } \\
\text { Agree }\end{array}$ & Agree & Neutral & Disagree & $\begin{array}{l}\text { Strongly } \\
\text { Disagree }\end{array}$ \\
\hline
\end{tabular}

17. I want to learn their cultural backgrounds of those who speak English in the English class?

\begin{tabular}{|l|l|l|l|l|}
\hline $\begin{array}{l}\text { Strongly } \\
\text { Agree }\end{array}$ & Agree & Neutral & Disagree & $\begin{array}{l}\text { Strongly } \\
\text { Disagree }\end{array}$ \\
\hline
\end{tabular}

18. I want to leam English through Games.

\begin{tabular}{|l|l|l|l|l|}
\hline $\begin{array}{l}\text { Strongly } \\
\text { Agree }\end{array}$ & Agree & Neutral & Disagree & $\begin{array}{l}\text { Strongly } \\
\text { Disagree }\end{array}$ \\
\hline
\end{tabular}

19. I want to learn English through Drama activity.

\begin{tabular}{|l|l|l|l|l|}
\hline $\begin{array}{l}\text { Strongly } \\
\text { Agree }\end{array}$ & Agree & Neutral & Disagree & $\begin{array}{l}\text { Strongly } \\
\text { Disagree }\end{array}$ \\
\hline
\end{tabular}

20. I think English is an international language to communicate with other people in this world.

\begin{tabular}{|l|l|l|l|l|}
\hline $\begin{array}{l}\text { Strongly } \\
\text { Agree }\end{array}$ & Agree & Neutral & Disagree & $\begin{array}{l}\text { Strongly } \\
\text { Disegree }\end{array}$ \\
\hline
\end{tabular}


Following is a list of statements about the people of Korea. Place a check before each statement with which you agree. Put a check in the position that best indicated the direction and intensity of your feeling with regard to each pair of descriptors.

21. Koreans in general tend to be for the benefit of their group:

\begin{tabular}{l|l|l|l|l|l|}
$\begin{array}{l}\text { Very } \\
\text { Self-sacrificing }\end{array}$ & +2 & +1 & 0 & -1 & -2
\end{tabular} \begin{tabular}{l}
$\begin{array}{l}\text { Not self- } \\
\text { sacrificing }\end{array}$ \\
\cline { 2 - 6 }
\end{tabular}

22. Koreans in general tend to be envious of others.

Envious

\begin{tabular}{|l|l|l|l|l|}
\hline+2 & +1 & 0 & -1 & -2 \\
\hline
\end{tabular}

Not Envious

23. Koreans in general tend to be:

God-fearing

\begin{tabular}{|l|l|l|l|l|}
\hline+2 & +1 & 0 & -1 & -2 \\
\hline
\end{tabular}

God-ignoring

24. Koreans in general tend to be:

Self-indulgent

\begin{tabular}{|l|l|l|l|l|}
\hline+2 & +1 & 0 & -1 & -2 \\
\hline
\end{tabular}

Self-indulgent

25. Koreans have an ideal home life

\begin{tabular}{|l|l|l|l|l|}
\hline $\begin{array}{l}\text { Strongly } \\
\text { Agree }\end{array}$ & Agree & Neutral & Disagree & $\begin{array}{l}\text { Strongly } \\
\text { Disagree }\end{array}$ \\
\hline
\end{tabular}

26. Koreans prefer a son to a daughter.

\begin{tabular}{|l|l|l|l|l|}
\hline $\begin{array}{l}\text { Strongly } \\
\text { Agree }\end{array}$ & Agree & Neutral & Disagree & $\begin{array}{l}\text { Strongly } \\
\text { Disagree }\end{array}$ \\
\hline
\end{tabular}

27. Koreans think a woman's place, truly, is at home.

\begin{tabular}{|l|l|l|l|l|}
\hline $\begin{array}{l}\text { Strongly } \\
\text { Agree }\end{array}$ & Agree & Neutral & Disagree & $\begin{array}{l}\text { Strongly } \\
\text { Disagree }\end{array}$ \\
\hline
\end{tabular}

28. Koreans in general do not like to receive unannounced visitors at home.

\begin{tabular}{|l|l|l|l|l|}
\hline $\begin{array}{l}\text { Strongly } \\
\text { Agree }\end{array}$ & Agree & Neutral & Disagree & $\begin{array}{l}\text { Strongly } \\
\text { Disagree }\end{array}$ \\
\hline
\end{tabular}

29. Koreans in general enjoy feeling that they are looked upon as equal in worth to their supervisors.

\begin{tabular}{|l|l|l|l|l|}
\hline $\begin{array}{l}\text { Strongly } \\
\text { Agree }\end{array}$ & Agree & Neutral & Disagree & $\begin{array}{l}\text { Strongly } \\
\text { Disagree }\end{array}$ \\
\hline
\end{tabular}

30. Koreans in general can eat dogs as one of their own traditional dishes.

\begin{tabular}{|l|l|l|l|l|}
\hline $\begin{array}{l}\text { Strongly } \\
\text { Agree }\end{array}$ & Agree & Neutral & Disagree & $\begin{array}{l}\text { Strongly } \\
\text { Disagree }\end{array}$ \\
\hline
\end{tabular}

Following is a list of statements about YOURSELF. Place a check before each statement with which you agree. Put a check in the position that best indicated the direction and intensity of your feeling with regard to each pair of descriptors.

31. I think of myself as a person who in general is:

Happy

\begin{tabular}{|l|l|l|l|l|}
\hline+2 & +1 & 0 & -1 & -2 \\
\hline
\end{tabular}

32. I think of myself as a person who in general tends to be:

Good

\begin{tabular}{|l|l|l|l|l|}
\hline+2 & +1 & 0 & -1 & -2 \\
\hline
\end{tabular}


33. I think of myself as a person who in general tend to be:

Fair

\begin{tabular}{|l|l|l|l|l|}
\hline+2 & +1 & 0 & -1 & -2 \\
\hline
\end{tabular}

34. I think of myself as a person who in general tends to be:

Selfish

\begin{tabular}{|l|l|l|l|l|}
\hline+2 & +1 & 0 & -1 & -2 \\
\hline
\end{tabular}

35. I think of myself as a person who in general tends to be:

Valuable

\begin{tabular}{|l|l|l|l|l|}
\hline+2 & +1 & 0 & -1 & -2 \\
\hline
\end{tabular}

36. I think of myself as a person who in general tends to be:

Beautiful

\begin{tabular}{|l|l|l|l|l|}
\hline+2 & +1 & 0 & -1 & -2 \\
\hline
\end{tabular}

37. I think of myself as a person who in general tends to be:

Sharp

\begin{tabular}{|l|l|l|l|l|}
\hline+2 & +1 & 0 & -1 & -2 \\
\hline
\end{tabular}

38. I think of myself as a person who in general tends to be:

Strong

\begin{tabular}{|l|l|l|l|l|}
\hline+2 & +1 & 0 & -1 & -2 \\
Weak
\end{tabular}

39. I think of myself as a person who in general tends to be:

Hot

\begin{tabular}{|l|l|l|l|l|}
\hline+2 & +1 & 0 & -1 & -2 \\
\hline
\end{tabular}

40. When talking about my own accomplishments I think of myself as a person who in general tends to be:

Modest

\begin{tabular}{|l|l|l|l|l|}
\hline+2 & +1 & 0 & -1 & -2 \\
\hline
\end{tabular}

Following is a list of statements about your every day life. Place a check before each statement with which you agree.

41. It is important to develop a network of people in my community who can help me out when I have tasks to accomplish.

\begin{tabular}{|l|l|l|l|l|}
\hline $\begin{array}{l}\text { Strongly } \\
\text { Agree }\end{array}$ & Agree & Neutral & Disagree & $\begin{array}{l}\text { Strongly } \\
\text { Disagree }\end{array}$ \\
\hline
\end{tabular}

42. I have respect for the authority figures with whom I interact.

\begin{tabular}{|l|l|l|l|l|}
\hline $\begin{array}{l}\text { Strongly } \\
\text { Agree }\end{array}$ & Agree & Neutral & Disagree & $\begin{array}{l}\text { Strongly } \\
\text { Disagree }\end{array}$ \\
\hline
\end{tabular}

43. I say "no" directly when I have to.

\begin{tabular}{|l|l|l|l|l|}
\hline $\begin{array}{l}\text { Strongly } \\
\text { Agree }\end{array}$ & Agree & Neutral & Disagree & $\begin{array}{l}\text { Strongly } \\
\text { Disagree }\end{array}$ \\
\hline
\end{tabular}

44. I like to speak English

\begin{tabular}{|l|l|l|l|l|}
\hline $\begin{array}{l}\text { Strongly } \\
\text { Agree }\end{array}$ & Agree & Neutral & Disagree & $\begin{array}{l}\text { Strongly } \\
\text { Disagree }\end{array}$ \\
\hline
\end{tabular}

45. I often think about the kind of world we are creating for future generations.

\begin{tabular}{|l|l|l|l|l|}
\hline $\begin{array}{l}\text { Strongly } \\
\text { Agree }\end{array}$ & Agree & Neutral & Disagree & $\begin{array}{l}\text { Strongly } \\
\text { Disagree }\end{array}$ \\
\hline
\end{tabular}

46. It is very important to me to choose a career in which I can have a positive effect on the quality of life in the global village. 


\begin{tabular}{|l|l|l|l|l|}
\hline $\begin{array}{l}\text { Strongly } \\
\text { Agree }\end{array}$ & Agree & Neutral & Disagree & $\begin{array}{l}\text { Strongly } \\
\text { Disagree }\end{array}$ \\
\hline
\end{tabular}

47. I define the other person's status by paying attention to name, gender, age, and other demographic attributes.

\begin{tabular}{|l|l|l|l|l|}
\hline $\begin{array}{l}\text { Strongly } \\
\text { Agree }\end{array}$ & Agree & Neutral & Disagree & $\begin{array}{l}\text { Strongly } \\
\text { Disagree }\end{array}$ \\
\hline
\end{tabular}

48. I enjoy being emotionally close to the people with whom I work.

\begin{tabular}{|l|l|l|l|l|}
\hline $\begin{array}{l}\text { Strongly } \\
\text { Agree }\end{array}$ & Agree & Neutral & Disagree & $\begin{array}{l}\text { Strongly } \\
\text { Disagree }\end{array}$ \\
\hline
\end{tabular}

49. I would not allow my subordinate to promote his nephew if there is someone marginally better than him. The person who is better must be promoted at all costs.

\begin{tabular}{|l|l|l|l|l|}
\hline $\begin{array}{l}\text { Strongly } \\
\text { Agree }\end{array}$ & Agree & Neutral & Disagree & $\begin{array}{l}\text { Strongly } \\
\text { Disagree }\end{array}$ \\
\hline
\end{tabular}

The following is a set of scenarios. Each scenario is followed by four options. Please imagine yourself in those situations, and place an $X$ next to the option you consider the best. Just tick your opinion of the most right or appropriate for you.

50. You and your friends decided spontaneously to go out to dinner at a restaurant. What do you think is the best way to handle the bill?

[ ] a. Split it equally, without regard to who ordered what.

[ ] b. Split it accordingly to how much each person makes.

[ ] c. The group leader pays the bill or decides how to split it.

[ ] d. Compute each person's charge, according to what the person ordered.

51. You are buying a piece of art for your office. Which one factor is most important in deciding whether to buy it?

[ ] a. It is a good investment.

[ ] b. Your co-workers will like it.

[ ] c. You just like it.

[ ] d. Your supervisor will approve of it.

52. You are deciding whom to vote for, for an important political job. Which is the most important consideration in deciding how to vote?

[ ] a. A high-status member of your community.

[ ] b. Powerful (influences national policy).

[ ] c. A relative.

[ ] d. Someone who appeals to you personally.

53. You are buying some new clothing. Which is the most important factor that you will consider in choosing the style? The style that is

[ ] a. Most suitable for your unique personality.

[ ] b. Most impressive in social situations.

[ ] c. Wom by your friends

[ ] d. Recommended by your parents

54. You are starting a new business, and you are looking for a partner. Which is the most 
Important factor in choosing a partner?

[ ] a. Someone which the same business interests.

[ ] b. Someone who has been successful in previous business ventures.

[ ] c. A close friend

[ ] d. A senior, successful, experienced member of the community.

55. Which one of these four books appears to you to be the most interesting?

[ ] a. How to make friends

[ ] b. How to succeed in business.

[ ] c. How to enjoy yourself inexpensively.

[ ] d. How to make sure you are meeting your obligations.

56. In my opinion, the best society is one where

[ ] a. People get more income if they contribute more to the society.

[ ] b. People have somewhat equal incomes.

[ ] c. People can do their own thing without being noticed.

[ ] d. People do their duty and enjoy it.

57. When people ask me about myself, I talk about

[ ] a. My ancestors and their traditions.

[ ] b. My friends and what we like to do.

[ ] c. My accomplishments.

[ ] d. What makes me unique.

58. How do you prefer to handle difficult class assignments?

[ ] a. Work alone.

[ ] b. Work with a group

[ ] c. Discuss the assignment with the teacher to get ahead of the others who did not to that.

[ ] d. Take charge of a group; parcel out tasks to make each person's job easier and the completion of the assignment successful.

59. You are away at office and have time to write a letter. Whom will you write to?

[ ] a. My boss at work - it will help me get ahead.

[ ] b. My parents.

[ ] c. The person I feel happy writing to

[ ] d. My brother or sister

The following is a list of statements about your feeling and thought related to those people live in other countries. Place a check before each statement with which you agree. Put a check in the position that best indicated the direction and intensity of your feeling with regard to each pair of descriptors.

60. Please estimate the number of programs or activities (excluding class activities) you went to in the last four months in which the focus was on another country (e.g. political speaker, presentation on the dance or art of another culture, foreign film, etc.).

$<1>$ None

$<2>$ One
$<4>$ Three or four

$<5>$ Five or more 
$<3>$ Two

61. Which do you consider the main source of the information you acquire conceming different cultural events? (Mark one).

$<1>$ Newspapers

<4> Radio

$<2>$ Magazine.

$<5>$ Friends/Family

$<3>$ Television

$<6>$ etc

62. Mark one.

<1> I have only travelled within my own country.

<2> I have made only 1 or 2 brief trips outside my own country.

$<3>$ I have travelled outside my own country for 3-9 weeks.

$<4>1$ have travelled outside my own country for more than 9 weeks.

$<5>$ I have actually lived in a community and/or attended school outside my own country for more than 9 weeks.

63. Indicate the total length of time you have spent travelling, living, or studying outside my own country. (Mark one).

$<1>$ Not at all.

<4>. 4-6 months

$<2>$ Less than one month

$<5>7-12$ months

$<3>1-3$ months

$<6>$ More than 1 year

Check all the statements that you agree with the following. Do not think about where foreigners come from. Please work rapidly

64. I decorate my home or office with artefacts from other countries.

\begin{tabular}{|l|l|l|l|l|}
\hline $\begin{array}{l}\text { Strongly } \\
\text { Agree }\end{array}$ & Agree & Neutral & Disagree & $\begin{array}{l}\text { Strongly } \\
\text { Disagree }\end{array}$ \\
\hline
\end{tabular}

65. I do not like to meet foreigners.

\begin{tabular}{|l|l|l|l|l|}
\hline $\begin{array}{l}\text { Strongly } \\
\text { Agree }\end{array}$ & Agree & Neutral & Disagree & $\begin{array}{l}\text { Strongly } \\
\text { Disagree }\end{array}$ \\
\hline
\end{tabular}

66. I wouldn't mind having a foreigner as a close friend.

\begin{tabular}{|l|l|l|l|l|}
\hline $\begin{array}{l}\text { Strongly } \\
\text { Agree }\end{array}$ & Agree & Neutral & Disagree & $\begin{array}{l}\text { Strongly } \\
\text { Disagree }\end{array}$ \\
\hline
\end{tabular}

67. I enjoy trying to understand people's behaviour in the context of their culture.

\begin{tabular}{|l|l|l|l|l|}
\hline $\begin{array}{l}\text { Strongly } \\
\text { Agree }\end{array}$ & Agree & Neutral & Disagree & $\begin{array}{l}\text { Strongly } \\
\text { Disagree }\end{array}$ \\
\hline
\end{tabular}

68. Are any of your friends from other cultures or countries? (Mark one)
$<1>$ No
<3> 1-3 Friends
$<2>2$ Acquaintances
<4> More than 3 Friends

69. I would not mind working on the job with foreigners.

\begin{tabular}{|l|l|l|l|l|}
\hline $\begin{array}{l}\text { Strongly } \\
\text { Agree }\end{array}$ & Agree & Neutral & Disagree & $\begin{array}{l}\text { Strongly } \\
\text { Disagree }\end{array}$ \\
\hline
\end{tabular}

70. I would not object to having foreigners move in next door as neighbours.

\begin{tabular}{|l|l|l|l|l|}
\hline $\begin{array}{l}\text { Strongly } \\
\text { Agree }\end{array}$ & Agree & Neutral & Disagree & $\begin{array}{l}\text { Strongly } \\
\text { Disagree }\end{array}$ \\
\hline
\end{tabular}


71. I would not mind if my brother or sister married a foreigner.

\begin{tabular}{|l|l|l|l|l|}
\hline $\begin{array}{l}\text { Strongly } \\
\text { Agree }\end{array}$ & Agree & Neutral & Disagree & $\begin{array}{l}\text { Strongly } \\
\text { Disagree }\end{array}$ \\
\hline
\end{tabular}

72. I think of myself not only as a citizen of my country but also as a citizen of the world.

\begin{tabular}{|l|l|l|l|l|}
\hline $\begin{array}{l}\text { Strongly } \\
\text { Agree }\end{array}$ & Agree & Neutral & Disagree & $\begin{array}{l}\text { Strongly } \\
\text { Disagree }\end{array}$ \\
\hline
\end{tabular}

73. I feel an obligation to speak out when I see my government doing something I consider wrong.

\begin{tabular}{|l|l|l|l|l|}
\hline $\begin{array}{l}\text { Strongly } \\
\text { Agree }\end{array}$ & Agree & Neutral & Disagree & $\begin{array}{l}\text { Strongly } \\
\text { Disagree }\end{array}$ \\
\hline
\end{tabular}

74. The needs of my own country must continue to be the highest priority in dealing with other countries.

\begin{tabular}{|l|l|l|l|l|}
\hline $\begin{array}{l}\text { Strongly } \\
\text { Agree }\end{array}$ & Agree & Neutral & Disagree & $\begin{array}{l}\text { Strongly } \\
\text { Disagree }\end{array}$ \\
\hline
\end{tabular}

75. Generally, an individual's actions are too small to have a significant effect on the ecosystem.

\begin{tabular}{|l|l|l|l|l|}
\hline $\begin{array}{l}\text { Strongly } \\
\text { Agree }\end{array}$ & Agree & Neutral & Disagree & $\begin{array}{l}\text { Strongly } \\
\text { Disagree }\end{array}$ \\
\hline
\end{tabular}

76. We all have a right to hold different beliefs about God and religion.

\begin{tabular}{|l|l|l|l|l|}
\hline $\begin{array}{l}\text { Strongly } \\
\text { Agree }\end{array}$ & Agree & Neutral & Disagree & $\begin{array}{l}\text { Strongly } \\
\text { Disagree }\end{array}$ \\
\hline
\end{tabular}

77. My country's values are probably the best.

\begin{tabular}{|l|l|l|l|l|}
\hline $\begin{array}{l}\text { Strongly } \\
\text { Agree }\end{array}$ & Agree & Neutral & Disagree & $\begin{array}{l}\text { Strongly } \\
\text { Disagree }\end{array}$ \\
\hline
\end{tabular}

78. The United States is enriched by the fact that it is comprised of many people from different cultures and countries.

\begin{tabular}{|l|l|l|l|l|}
\hline $\begin{array}{l}\text { Strongly } \\
\text { Agree }\end{array}$ & Agree & Neutral & Disagree & $\begin{array}{l}\text { Strongly } \\
\text { Disagree }\end{array}$ \\
\hline
\end{tabular}

79. Chinese culture, especially Confucianism, is still influenced on your everyday life.

\begin{tabular}{|l|l|l|l|l|}
\hline $\begin{array}{l}\text { Strongly } \\
\text { Agree }\end{array}$ & Agree & Neutral & Disagree & $\begin{array}{l}\text { Strongly } \\
\text { Disagree }\end{array}$ \\
\hline
\end{tabular}

80. In the long run, my country will probably benefit from the fact that the world is becoming more interconnected.

\begin{tabular}{|l|l|l|l|l|}
\hline $\begin{array}{l}\text { Strongly } \\
\text { Agree }\end{array}$ & Agree & Neutral & Disagree & $\begin{array}{l}\text { Strongly } \\
\text { Disagree }\end{array}$ \\
\hline
\end{tabular}

81. When I hear that thousands of people are starving in an African country, I feel very frustrated.

\begin{tabular}{|l|l|l|l|l|}
\hline $\begin{array}{l}\text { Strongly } \\
\text { Agree }\end{array}$ & Agree & Neutral & Disagree & $\begin{array}{l}\text { Strongly } \\
\text { Disagree }\end{array}$ \\
\hline
\end{tabular}

82. I feel very concerned about the lives of people who live in politically repressive regimes.

\begin{tabular}{|l|l|l|l|l|}
\hline $\begin{array}{l}\text { Strongly } \\
\text { Agree }\end{array}$ & Agree & Neutral & Disagree & $\begin{array}{l}\text { Strongly } \\
\text { Disagree }\end{array}$ \\
\hline
\end{tabular}

83. I sometimes feel irritated with people from other countries because they don't understand 
how we do things in my country.

\begin{tabular}{|l|l|l|l|l|}
\hline $\begin{array}{l}\text { Strongly } \\
\text { Agree }\end{array}$ & Agree & Neutral & Disagree & $\begin{array}{l}\text { Strongly } \\
\text { Disagree }\end{array}$ \\
\hline
\end{tabular}

84. My opinions about national policies are based on how those policies might affect the rest of the world as well as my own country.

\begin{tabular}{|l|l|l|l|l|}
\hline $\begin{array}{l}\text { Strongly } \\
\text { Agree }\end{array}$ & Agree & Neutral & Disagree & $\begin{array}{l}\text { Strongly } \\
\text { Disagree }\end{array}$ \\
\hline
\end{tabular}

85. I am able to affect what happens on a global level by what I do in my own community.

\begin{tabular}{|l|l|l|l|l|}
\hline $\begin{array}{l}\text { Strongly } \\
\text { Agree }\end{array}$ & Agree & Neutral & Disagree & $\begin{array}{l}\text { Strongly } \\
\text { Disagree }\end{array}$ \\
\hline
\end{tabular}

86. In fact, there is nothing I can do about the problems of the world.

\begin{tabular}{|l|l|l|l|l|}
\hline $\begin{array}{l}\text { Strongly } \\
\text { Agree }\end{array}$ & Agree & Neutral & Disagree & $\begin{array}{l}\text { Strongly } \\
\text { Disagree }\end{array}$ \\
\hline
\end{tabular}

87. In fact, it is not important to me to consider myself as a member of the global community.

\begin{tabular}{|l|l|l|l|l|}
\hline $\begin{array}{l}\text { Strongly } \\
\text { Agree }\end{array}$ & Agree & Neutral & Disagree & $\begin{array}{l}\text { Strongly } \\
\text { Disagree }\end{array}$ \\
\hline
\end{tabular}

88. It is important that universities and colleges provide program designed to promote understanding among students of different ethnic and cultural backgrounds.

\begin{tabular}{|l|l|l|l|l|}
\hline $\begin{array}{l}\text { Strongly } \\
\text { Agree }\end{array}$ & Agree & Neutral & Disagree & $\begin{array}{l}\text { Strongly } \\
\text { Disagree }\end{array}$ \\
\hline
\end{tabular}

\section{Thank you so much for your cooperation!!!}

Your answers will make important contributions to my doctoral research. I will do my best to deliver your messages cleanly and appropriately in my thesis. I hope you have a wonderful time in your school life. 


\section{What and how have YOU experienced throughout the EDIE project? What would you like to say to us?}

-This was the Post-Questionnaire, which participants answered on the last day of the project.

I would like to know what and how you have experienced throughout the EDIE project.

Would you share that with me?

What do you think of the project?

Would you read very each item to tick on the part to which you agree? There is neither right nor wrong answers. Therefore, put the tick simply.

The following is a list of statements about the EDIE project, in which you participated. Place a check before each statement with which you agree.

1. After attending the EDIE, I realised that it was beneficial to learn languages with their cultural factors

\begin{tabular}{|l|l|l|l|l|}
\hline $\begin{array}{l}\text { Strongly } \\
\text { Agree }\end{array}$ & Agree & Neutral & Disagree & $\begin{array}{l}\text { Strongly } \\
\text { Disagree }\end{array}$ \\
\hline
\end{tabular}

2. I was interested in the EDIE project.

\begin{tabular}{|l|l|l|l|l|}
\hline $\begin{array}{l}\text { Strongly } \\
\text { Agree }\end{array}$ & Agree & Neutral & Disagree & $\begin{array}{l}\text { Strongly } \\
\text { Disagree }\end{array}$ \\
\hline
\end{tabular}

3. The EDIE project was useful to me.

\begin{tabular}{|l|l|l|l|l|}
\hline $\begin{array}{l}\text { Strongly } \\
\text { Agree }\end{array}$ & Agree & Neutral & Disagree & $\begin{array}{l}\text { Strongly } \\
\text { Disagree }\end{array}$ \\
\hline
\end{tabular}

4. Give reasons in relation to, the previous item, [3].

(1)

(2)

(3)

5. While I was participating in the EDIE project, I became interested in learning English.

\begin{tabular}{|l|l|l|l|l|}
\hline $\begin{array}{l}\text { Strongly } \\
\text { Agree }\end{array}$ & Agree & Neutral & Disagree & $\begin{array}{l}\text { Strongly } \\
\text { Disagree }\end{array}$ \\
\hline
\end{tabular}

6. Give reasons in relation to the previous item, [5].

(1)

(2)

(3)

7. While attending the EDIE project, I had a better understanding of other cultures.

\begin{tabular}{|l|l|l|l|l|}
\hline $\begin{array}{l}\text { Strongly } \\
\text { Agree }\end{array}$ & Agree & Neutral & Disagree & $\begin{array}{l}\text { Strongly } \\
\text { Disagree }\end{array}$ \\
\hline
\end{tabular}

8. State how you tried to understand them in relation to the previous item, [7], particularly in 
that you interacted with other cultural factors.

(1)

(2)

(3)

9. I could not adjust to the project with ease at its beginning.

\begin{tabular}{|l|l|l|l|l|}
\hline $\begin{array}{l}\text { Strongly } \\
\text { Agree }\end{array}$ & Agree & Neutral & Disagree & $\begin{array}{l}\text { Strongly } \\
\text { Disagree }\end{array}$ \\
\hline
\end{tabular}

10. Give reasons in relation to the previous item, [9].

(1)

(2)

(3)

11. I can carry out my task as a member of the society, along with understanding our own culture as well as others.

\begin{tabular}{|l|l|l|l|l|}
\hline $\begin{array}{l}\text { Strongly } \\
\text { Agree }\end{array}$ & Agree & Neutral & Disagree & $\begin{array}{l}\text { Strongly } \\
\text { Disagree }\end{array}$ \\
\hline
\end{tabular}

12. We should be taught Anglophone cultural studies in English classes.

\begin{tabular}{|l|l|l|l|l|}
\hline $\begin{array}{l}\text { Strongly } \\
\text { Agree }\end{array}$ & Agree & Neutral & Disagree & $\begin{array}{l}\text { Strongly } \\
\text { Disagree }\end{array}$ \\
\hline
\end{tabular}

13 State what the Minister of Education, schools, teachers, and students should do in relation to the previous item, [12].

(1)

(3)

14. Drama activity should be included in English Classrooms.

\begin{tabular}{|l|l|l|l|l|}
\hline $\begin{array}{l}\text { Strongly } \\
\text { Agree }\end{array}$ & Agree & Neutral & Disagree & $\begin{array}{l}\text { Strongly } \\
\text { Disagree }\end{array}$ \\
\hline
\end{tabular}

15. State what the Minister of Education, schools, teachers, and students should do in relation to the previous item, [14].

(1)

(2)

(3)

16. I was actively engaged in the project.

\begin{tabular}{|l|l|l|l|l|}
\hline $\begin{array}{l}\text { Strongly } \\
\text { Agree }\end{array}$ & Agree & Neutral & Disagree & $\begin{array}{l}\text { Strongly } \\
\text { Disegree }\end{array}$ \\
\hline
\end{tabular}

17 Give reasons in relation to the previous item, [16].

(1)

(2)

(3)

18. I participated in the project to exercise my creative power. 


\begin{tabular}{|l|l|l|l|l|}
\hline $\begin{array}{l}\text { Strongly } \\
\text { Agree }\end{array}$ & Agree & Neutral & Disagree & $\begin{array}{l}\text { Strongly } \\
\text { Disagree }\end{array}$ \\
\hline
\end{tabular}

19 Give reasons in relation to the previous item, [18].

(1)

20. I enjoyed myself to the full in expressing what I felt and thought throughout the project.

\begin{tabular}{|l|l|l|l|l|}
\hline $\begin{array}{l}\text { Strongly } \\
\text { Agree }\end{array}$ & Agree & Neutral & Disagree & $\begin{array}{l}\text { Strongly } \\
\text { Disagree }\end{array}$ \\
\hline
\end{tabular}

21. Give reasons in relation to the previous item, [20].

(1)

22. State what else you would like to say.

Following is a list of statements about your every day life. Place a check before each statement with which you agree.

23. It is important to develop a network of people in my community who can help me out when I have tasks to accomplish. [Item 41 in the first questionnaire, ASQ]

\begin{tabular}{|l|l|l|l|l|}
\hline $\begin{array}{l}\text { Strongly } \\
\text { Agree }\end{array}$ & Agree & Neutral & Disagree & $\begin{array}{l}\text { Strongly } \\
\text { Disagree }\end{array}$ \\
\hline
\end{tabular}

24. I have respect for the authority figures with whom I interact. [Item 42 in the first questionnaire, ASQ].

\begin{tabular}{|l|l|l|l|l|}
\hline $\begin{array}{l}\text { Strongly } \\
\text { Agree }\end{array}$ & Agree & Neutral & Disagree & $\begin{array}{l}\text { Strongly } \\
\text { Disagree }\end{array}$ \\
\hline
\end{tabular}

25. I say "no" directly when I have to. [item 43 in the first questionnaire, ASQ].

\begin{tabular}{|l|l|l|l|l|}
\hline $\begin{array}{l}\text { Strongly } \\
\text { Agree }\end{array}$ & Agree & Neutral & Disagree & $\begin{array}{l}\text { Strongly } \\
\text { Disagree }\end{array}$ \\
\hline
\end{tabular}

26. I like to speak English with others. [Item 44 in ASQ].

\begin{tabular}{|l|l|l|l|l|}
\hline $\begin{array}{l}\text { Strongly } \\
\text { Agree }\end{array}$ & Agree & Neutral & Disagree & $\begin{array}{l}\text { Strongly } \\
\text { Disagree }\end{array}$ \\
\hline
\end{tabular}

27. I often think about the kind of world we are creating for future generations. [Item 45 in ASQ].

\begin{tabular}{|l|l|l|l|l|}
\hline $\begin{array}{l}\text { Strongly } \\
\text { Agree }\end{array}$ & Agree & Neutral & Disagree & $\begin{array}{l}\text { Strongly } \\
\text { Disagree }\end{array}$ \\
\hline
\end{tabular}

28. It is very important to me to choose a career in which I can have a positive effect on the quality of life in the global village. [Item 46 in ASQJ. 


\begin{tabular}{|l|l|l|l|l|}
\hline $\begin{array}{l}\text { Strongly } \\
\text { Agree }\end{array}$ & Agree & Neutral & Disagree & $\begin{array}{l}\text { Strongly } \\
\text { Disagree }\end{array}$ \\
\hline
\end{tabular}

29. I define the other person's status by paying attention to name, gender, age, and other demographic attributes. [ttem 47 in ASQJ.

\begin{tabular}{|l|l|l|l|l|}
\hline $\begin{array}{l}\text { Strongly } \\
\text { Agree }\end{array}$ & Agree & Neutral & Disagree & $\begin{array}{l}\text { Strongly } \\
\text { Disagree }\end{array}$ \\
\hline
\end{tabular}

30. I enjoy being emotionally close to the people with whom I work. [tem 48 in ASQ].

\begin{tabular}{|l|l|l|l|l|}
\hline $\begin{array}{l}\text { Strongly } \\
\text { Agree }\end{array}$ & Agree & Neutral & Disagree & $\begin{array}{l}\text { Strongly } \\
\text { Disagree }\end{array}$ \\
\hline
\end{tabular}

31. I would not allow my subordinate to promote his nephew if there is someone marginally better than him. The person, who is better, must be promoted at all costs. [Item 49 in ASQ].

\begin{tabular}{|l|l|l|l|l|}
\hline $\begin{array}{l}\text { Strongly } \\
\text { Agree }\end{array}$ & Agree & Neutral & Disagree & $\begin{array}{l}\text { Strongly } \\
\text { Disagree }\end{array}$ \\
\hline
\end{tabular}

Check all the statements that you agree with the following. Do not think about where foreigners come from. Please work rapidly

32. I regard English as an International Language to communicate with others.

\begin{tabular}{|l|l|l|l|l|}
\hline $\begin{array}{l}\text { Strongly } \\
\text { Agree }\end{array}$ & Agree & Neutral & Disagree & $\begin{array}{l}\text { Strongly } \\
\text { Disagree }\end{array}$ \\
\hline
\end{tabular}

33. I decorate my home or office with artefacts from other countries. [ttem 64 in ASQ].

\begin{tabular}{|l|l|l|l|l|}
\hline $\begin{array}{l}\text { Strongly } \\
\text { Agree }\end{array}$ & Agree & Neutral & Disagree & $\begin{array}{l}\text { Strongly } \\
\text { Disagree }\end{array}$ \\
\hline
\end{tabular}

34. I do not like to meet foreigners. [ltem 65 in ASQ].

\begin{tabular}{|l|l|l|l|l|}
\hline $\begin{array}{l}\text { Strongly } \\
\text { Agree }\end{array}$ & Agree & Neutral & Disagree & $\begin{array}{l}\text { Strongly } \\
\text { Disagree }\end{array}$ \\
\hline
\end{tabular}

35. I would not mind having a foreigner as a close friend. [tem 66 in ASQ].

\begin{tabular}{|l|l|l|l|l|}
\hline $\begin{array}{l}\text { Strongly } \\
\text { Agree }\end{array}$ & Agree & Neutral & Disagree & $\begin{array}{l}\text { Strongly } \\
\text { Disagree }\end{array}$ \\
\hline
\end{tabular}

36. I enjoy trying to understand people's behaviour in the context of their culture. Item 67 in ASQ].

\begin{tabular}{|l|l|l|l|l|}
\hline $\begin{array}{l}\text { Strongly } \\
\text { Agree }\end{array}$ & Agree & Neutral & Disagree & $\begin{array}{l}\text { Strongly } \\
\text { Disagree }\end{array}$ \\
\hline
\end{tabular}

37. I would not mind working on the job with foreigners. [ltem 69 in ASQ].

\begin{tabular}{|l|l|l|l|l|}
\hline $\begin{array}{l}\text { Strongly } \\
\text { Agree }\end{array}$ & Agree & Neutral & Disagree & $\begin{array}{l}\text { Strongly } \\
\text { Disagree }\end{array}$ \\
\hline
\end{tabular}

38. I would not object to having foreigners move in next door as neighbours. [Item 70 in ASQ].

\begin{tabular}{|l|l|l|l|l|}
\hline $\begin{array}{l}\text { Strongly } \\
\text { Agree }\end{array}$ & Agree & Neutral & Disagree & $\begin{array}{l}\text { Strongly } \\
\text { Disagree }\end{array}$ \\
\hline
\end{tabular}

39. I would not mind if my brother or sister married a foreigner. [ltem 71 in ASQ].

\begin{tabular}{|l|l|l|l|l|}
\hline $\begin{array}{l}\text { Strongly } \\
\text { Agree }\end{array}$ & Agree & Neutral & Disagree & $\begin{array}{l}\text { Strongly } \\
\text { Disagree }\end{array}$ \\
\hline
\end{tabular}

40. [B61] I regard myself not only as a citizen of my country but also as a citizen of the world. [Item 72 in ASQ]. 


\begin{tabular}{|l|l|l|l|l|}
\hline $\begin{array}{l}\text { Strongly } \\
\text { Agree }\end{array}$ & Agree & Neutral & Disagree & $\begin{array}{l}\text { Strongly } \\
\text { Disagree }\end{array}$ \\
\hline
\end{tabular}

41. [B52] I feel an obligation to speak out when I see my government doing something I consider wrong. [ltem 73 in ASQ].

\begin{tabular}{|l|l|l|l|l|}
\hline $\begin{array}{l}\text { Strongly } \\
\text { Agree }\end{array}$ & Agree & Neutral & Disagree & $\begin{array}{l}\text { Strongly } \\
\text { Disagree }\end{array}$ \\
\hline
\end{tabular}

42. The needs of my own country must continue to be the highest priority in dealing with other countries. [Item 74 in ASQ].

\begin{tabular}{|l|l|l|l|l|}
\hline $\begin{array}{l}\text { Strongly } \\
\text { Agree }\end{array}$ & Agree & Neutral & Disagree & $\begin{array}{l}\text { Strongly } \\
\text { Disagree }\end{array}$ \\
\hline
\end{tabular}

43. Generally, an individual's actions are too small to have a significant effect on the ecosystem. [Item 75 in ASQ].

\begin{tabular}{|l|l|l|l|l|}
\hline $\begin{array}{l}\text { Strongly } \\
\text { Agree }\end{array}$ & Agree & Neutral & Disagree & $\begin{array}{l}\text { Strongly } \\
\text { Disagree }\end{array}$ \\
\hline
\end{tabular}

44. My country's values are probably the best. [Item 77 in ASQ].

\begin{tabular}{|l|l|l|l|l|}
\hline $\begin{array}{l}\text { Strongly } \\
\text { Agree }\end{array}$ & Agree & Neutral & Disagree & $\begin{array}{l}\text { Strongly } \\
\text { Disagree }\end{array}$ \\
\hline
\end{tabular}

45. The United States is enriched by the fact that it is comprised of many people from different cultures and countries. [Item 78 in ASQ].

\begin{tabular}{|l|l|l|l|l|}
\hline $\begin{array}{l}\text { Strongly } \\
\text { Agree }\end{array}$ & Agree & Neutral & Disagree & $\begin{array}{l}\text { Strongly } \\
\text { Disagree }\end{array}$ \\
\hline
\end{tabular}

46. In the long run, my country will probably benefit from the fact that the world is becoming more interconnected. [ltem 80 in ASQ].

\begin{tabular}{|l|l|l|l|l|}
\hline $\begin{array}{l}\text { Strongly } \\
\text { Agree }\end{array}$ & Agree & Neutral & Disagree & $\begin{array}{l}\text { Strongly } \\
\text { Disagree }\end{array}$ \\
\hline
\end{tabular}

47. When I hear that thousands of people are starving in an African country, I feel very frustrated. [Item 81 in ASQ].

\begin{tabular}{|l|l|l|l|l|}
\hline $\begin{array}{l}\text { Strongly } \\
\text { Agree }\end{array}$ & Agree & Neutral & Disagree & $\begin{array}{l}\text { Strongly } \\
\text { Disagree }\end{array}$ \\
\hline
\end{tabular}

48. I feel very concerned about the lives of people who live in politically repressive regimes. [ltem 82 in ASQ].

\begin{tabular}{|l|l|l|l|l|}
\hline $\begin{array}{l}\text { Strongly } \\
\text { Agree }\end{array}$ & Agree & Neutral & Disagree & $\begin{array}{l}\text { Strongly } \\
\text { Disagree }\end{array}$ \\
\hline
\end{tabular}

49. I sometimes feel irritated with people from other countries because they don't understand how we do things in my country. [Item 83 in ASQ].

\begin{tabular}{|l|l|l|l|l|}
\hline $\begin{array}{l}\text { Strongly } \\
\text { Agree }\end{array}$ & Agree & Neutral & Disagree & $\begin{array}{l}\text { Strongly } \\
\text { Disagree }\end{array}$ \\
\hline
\end{tabular}

50. My opinions about national policies are based on how those policies might affect the rest of the world as well as my own country. [Item 84 in ASQ].

\begin{tabular}{|l|l|l|l|l|}
\hline $\begin{array}{l}\text { Strongly } \\
\text { Agree }\end{array}$ & Agree & Neutral & Disagree & $\begin{array}{l}\text { Strongly } \\
\text { Disagree }\end{array}$ \\
\hline
\end{tabular}

51. I am able to affect what happens on a global level by what I do in my own community. [Item 85 in ASQ]. 


\begin{tabular}{|l|l|l|l|l|}
\hline $\begin{array}{l}\text { Strongly } \\
\text { Agree }\end{array}$ & Agree & Neutral & Disagree & $\begin{array}{l}\text { Strongly } \\
\text { Disagree }\end{array}$ \\
\hline
\end{tabular}

52. In fact, there is nothing I can do about the problems of the world. [tem 86 in ASQ].

\begin{tabular}{|l|l|l|l|l|}
\hline $\begin{array}{l}\text { Strongly } \\
\text { Agree }\end{array}$ & Agree & Neutral & Disagree & $\begin{array}{l}\text { Strongly } \\
\text { Disagree }\end{array}$ \\
\hline
\end{tabular}

53. In fact, it is not important to me to consider myself as a member of the global community. [Item 87 in ASQ].

\begin{tabular}{|l|l|l|l|l|}
\hline $\begin{array}{l}\text { Strongly } \\
\text { Agree }\end{array}$ & Agree & Neutral & Disagree & $\begin{array}{l}\text { Strongly } \\
\text { Disagree }\end{array}$ \\
\hline
\end{tabular}

54. It is important that universities and colleges provide program designed to promote understanding among students of different ethnic and cultural backgrounds. Item 88 in ASQJ.

\begin{tabular}{|l|l|l|l|l|}
\hline $\begin{array}{l}\text { Strongly } \\
\text { Agree }\end{array}$ & Agree & Neutral & Disagree & $\begin{array}{l}\text { Strongly } \\
\text { Disagree }\end{array}$ \\
\hline
\end{tabular}

Below there are 46 statements that are clustered in pairs. Tick the statement in each matched pair that you are most comfortable with. There are no wrong answers. Muke your choice as spontaneously as possible.

1a. ( ) A positive association in society is with whatever is rooted in tradition.

1b. ( ) A positive association in society is with whatever is 'new.'

2a. ( ) Impersonal "truth" is stressed and can, in principle, be obtained from any competent person.

2b. ( ) Personal "wisdom" is stressed and is transferred in the relationship with a particular teacher (guru).

3a. ( ) A teacher should respect the independence of his or her students.

3b. ( ) A teacher merits the respect of his or her students.

4a. ( ) One is never too old to learn; continual education.

4b. ( ) The young should learn; adults cannot accept a student role.

5a. ( ) Students expect to learn how to do.

5b. ( ) Students expect to learn how to leam.

6a. ( ) Student-centred education (value is placed on student initiative).

6b. ( ) Teacher-centred education (Nalue is placed on teacher-ordered learning).

7a. ( ) Students expect teacher to initiate communication.

7b. ( ) Teacher expects students to initiate communication.

8a. ( ) Teacher expects students to find their own paths.

8b. ( ) Students expect teacher to outline paths to follow. 
9a. ( ) Individual students will speak up in class in response to a general invitation by the teacher.

9b. ( ) Individual students will only speak up in class when called upon personally by the teacher.

10a. ( ) Individuals will speak up in large groups.

10b. ( ) Individuals will only speak up in small groups.

11a. ( ) Large classes are split socially into smaller cohesive subgroups based on particularist criteria (e.g., ethnic affiliation).

11b. ( ) Subgroupings in class vary from one situation to the next based on universalist criteria (e.g., the task at hand).

12a. ( ) Students may speak up spontaneously in class.

12b. ( ) Students speak up in class only when invited by the teacher.

13a. ( ) The teacher is seldom contradicted and rarely criticised.

13b. ( ) Students are allowed to contradict or criticise teacher.

14a. ( ) Confrontation in learning situations can be beneficial; conflicts can be brought into the open.

14b. ( ) Formal harmony in learning situations should be maintained.

15a. ( ) Effectiveness of learning is related to the excellence of the teacher.

15b. ( ) Effectiveness of leaming is related to the amount of two-way communication in class

16a. ( ) Neither the teacher nor any student should ever be made to lose face.

16b. ( ) "Face-saving" is of little importance.

17a. ( ) Education is a way of improving one's economic worth and self-respect based on ability and competence.

17b. ( ) Education is a way of gaining prestige in one's social environment and of joining a higher status group.

18a. ( ) Outside class, teachers are treated as equals to students.

18b. ( ) Respect for teachers is also shown outside of class.

19a. ( ) Diploma certificates are important and displayed on walls.

19b. ( ) Diploma certificates have little importance.

20a. ( ) In teacher-student conflicts, parents are expected to side with the student.

20b. ( ) In teacher-student conflicts, parents are expected to side with the teacher. 
21a. ( ) Older teachers are more respected than younger teachers.

21b. ( ) Younger teachers are more liked than older teachers.

22a. ( ) Acquiring competence is more important than acquiring certificates.

22b. ( ) Acquiring certificates is more important than acquiring competence.

23a. ( ) Teachers are expected to give preferential treatment to some students (e.g., based on ethnic affiliation or on recommendation by an influential person).

23b. ( ) Teachers are expected to be strictly impartial.

\section{Thank you very much!!}

Your answering will make great contribution to my

PhD study. I will do my best for delivering your

experiences clearly and using them appropriately

in my study. I hope you enjoy your school life

increasingly. Thank you again

I borrowed parts of questionnaires in the following references: some items were used in the

study as they had been; meanwhile others were modified to describe questions clearer in

relation to the Korean context. Items of post-questionnaire, which were relevant to the

evaluation of the EDIE project, were produced for the study.

[B] Ballou, C. D. (1996). A Comparative Assessment of the Effectiveness of International/ Intercultural Education in the General Education Program at American University, Ph.D. dissertation, The American University.

[IC] Bhawuk, D. P. S.\& Brislin, R.(1992). 'The measurement of intercultural sensitivity using the concepts of individualism and collectivism,' Intemational Joumal of Intercultural Relations, $16(4)$ : 413-436.

[BY] Byram, M. et al. (1991). Cultural Studies and Language Leaming: A Research Report. Clevedon: Multilingual Matters, LTD. [Booklet :1 myself].

[IC] Elkins, M. R. (1997). An Investigation of Educational Gaming/ Stimulation as an Instructional Strategy for Teaching Intercultural Sensitivity. Ph.D. dissertation, Southern Illinois University.

[1a-23b] Goodman, N. R. (1994). 'Intercultural education at the university level: teacher-student interaction,' in R. W. Brislin \& T. Yoshida (eds.)(1994). Improving Intercultural Interacitons: Modules for Cross-Cultural Training Programs (Volume 2)(Multicultural Aspects of Counseling Series 8). Thousand Oaks \& London: SAGE.

[SE] Seelye, H. N. (1994). Teaching Culture: Strategies for Intercultural Communication. Lincolnwood, III.: National Textbook Com. 207-234.

[SI] Singelis, T. M. (1998). Teaching About Culture, Ethnicity, \& Diversity: Exercises and Planned Activities. Thousand Oaks \& London: SAGE. 


\section{Appendix B: Self-Questioning Form (an example)}

* I gave out this form in Korean Version to participants at the end of each drama lesson.

\section{My Name: KimJA, who took a role of Gregory Jones as the American president Drama Session Title: the International Business Meeting Today: 4 April 2001}

I am going to have dialogues with myself. I really want to know about what I have done in specific situations. So, I will try to ask myself what I did in that situation and to recall why I did that. This might be helpful to having a better understanding of what I have experienced through taking my role.

\begin{tabular}{|c|c|c|}
\hline \multicolumn{2}{|c|}{ What I would like to talk with myself } & $\begin{array}{l}\text { What I wanted to say and to act, I should have } \\
\text { done. Now it's time to speak out why I couldn't. } \\
\text { Actually, I had... }\end{array}$ \\
\hline \multirow{4}{*}{$\begin{array}{l}\text { How did I } \\
\text { do in } \\
\text { participat- } \\
\text { ing in } \\
\text { drama } \\
\text { activity } \\
\text { with } \\
\text { others? }\end{array}$} & $\begin{array}{l}\text { [A] Drama Action Pattern: } \\
\text { What action do I want to talk } \\
\text { about more to myself among } \\
\text { today's drama activities? }\end{array}$ & $\begin{array}{l}\text { I would like to talk about Jones, whose } \\
\text { behaviour is nonsensical or rather with big self- } \\
\text { indulgence. }\end{array}$ \\
\hline & $\begin{array}{l}\text { [B] Relationship: } \\
\text { What relationship did I have to } \\
\text { others? }\end{array}$ & $\begin{array}{l}\text { I was the American president, and my counter- } \\
\text { partner was the Japanese president with his two } \\
\text { Japanese subordinates. We had a trade } \\
\text { business meeting in a restaurant in Japan. }\end{array}$ \\
\hline & $\begin{array}{l}\text { [C] Feelings \& Emotions: } \\
\text { How did I feel about myself as a } \\
\text { person who relates to others? }\end{array}$ & $\begin{array}{l}\text { I thought I took the Japanese side and I was } \\
\text { friendly to the Japanese characters because } \\
\text { Japan has similar culture to us. }\end{array}$ \\
\hline & $\begin{array}{l}\text { [D] Drama Activity: } \\
\text { What have I done today during } \\
\text { drama sessions in practice in the } \\
\text { above question? }\end{array}$ & $\begin{array}{l}\text { I took the role of the American president. } \\
\text { Nevertheless, my actions were brought in the } \\
\text { Japanese perspective. }\end{array}$ \\
\hline $\begin{array}{l}\text { What } \\
\text { dialogues } \\
\text { about my } \\
\text { role and } \\
\text { my } \\
\text { situation } \\
\text { did I have } \\
\text { in my } \\
\text { mind? }\end{array}$ & $\begin{array}{l}\text { [E] Difficulty: } \\
\text { What have I thought but I } \\
\text { couldn't do in the above } \\
\text { situation? } \\
<1>\text { Let's write down what I } \\
\text { thought as many as I can. } \\
<2>\text { Underline the most dimicult } \\
\text { thing to do action of }<1>\text { list. }\end{array}$ & $\begin{array}{l}\text { I don't like Jones, the American president. I think } \\
\text { he is very impertinent. }\end{array}$ \\
\hline $\begin{array}{l}\text { Why was I } \\
\text { not able to } \\
\text { express } \\
\text { what I } \\
\text { thought } \\
\text { easily? }\end{array}$ & $\begin{array}{l}\text { [F] Reasons of the above Difficulty } \\
\text { in [E]. } \\
\text { Why did I think that was the } \\
\text { most difficult thing to resolve or } \\
\text { to act? (Write down the reasons } \\
\text { as many as I considered). }\end{array}$ & $\begin{array}{l}\text { That was because I couldn't understand that } \\
\text { kind of people. I haven't grown up to have such } \\
\text { an impertinent manner. }\end{array}$ \\
\hline $\begin{array}{l}\text { What could } \\
\text { I do in } \\
\text { another's } \\
\text { position? }\end{array}$ & $\begin{array}{l}\text { [G] How could I act in that } \\
\text { situation if I were one of the } \\
\text { people who come from England } \\
\text { or America? }\end{array}$ & $\begin{array}{l}\text { They seem to cope with that situation far better } \\
\text { than I, because my character, the Amencan } \\
\text { president, has the same culture that they have. }\end{array}$ \\
\hline $\begin{array}{l}\text { How would } \\
\text { I see } \\
\text { myself and } \\
\text { what would } \\
\text { I say to } \\
\text { myself in } \\
\text { other's } \\
\text { position? }\end{array}$ & $\begin{array}{l}\text { [H] What do I want to do in a } \\
\text { similar situation to that in the } \\
\text { future? }\end{array}$ & $\begin{array}{l}\text { I think it is necessary to promote cultural } \\
\text { interchange and to educate that kind of } \\
\text { intercultural communication in order to } \\
\text { overcome cultural differences. So, Jones should } \\
\text { have understood what Japan is like in terms of } \\
\text { her cultural dimensions. }\end{array}$ \\
\hline
\end{tabular}




\section{Appendix C: Observation Checklist}

Observer.

Date:

Observed group/participant

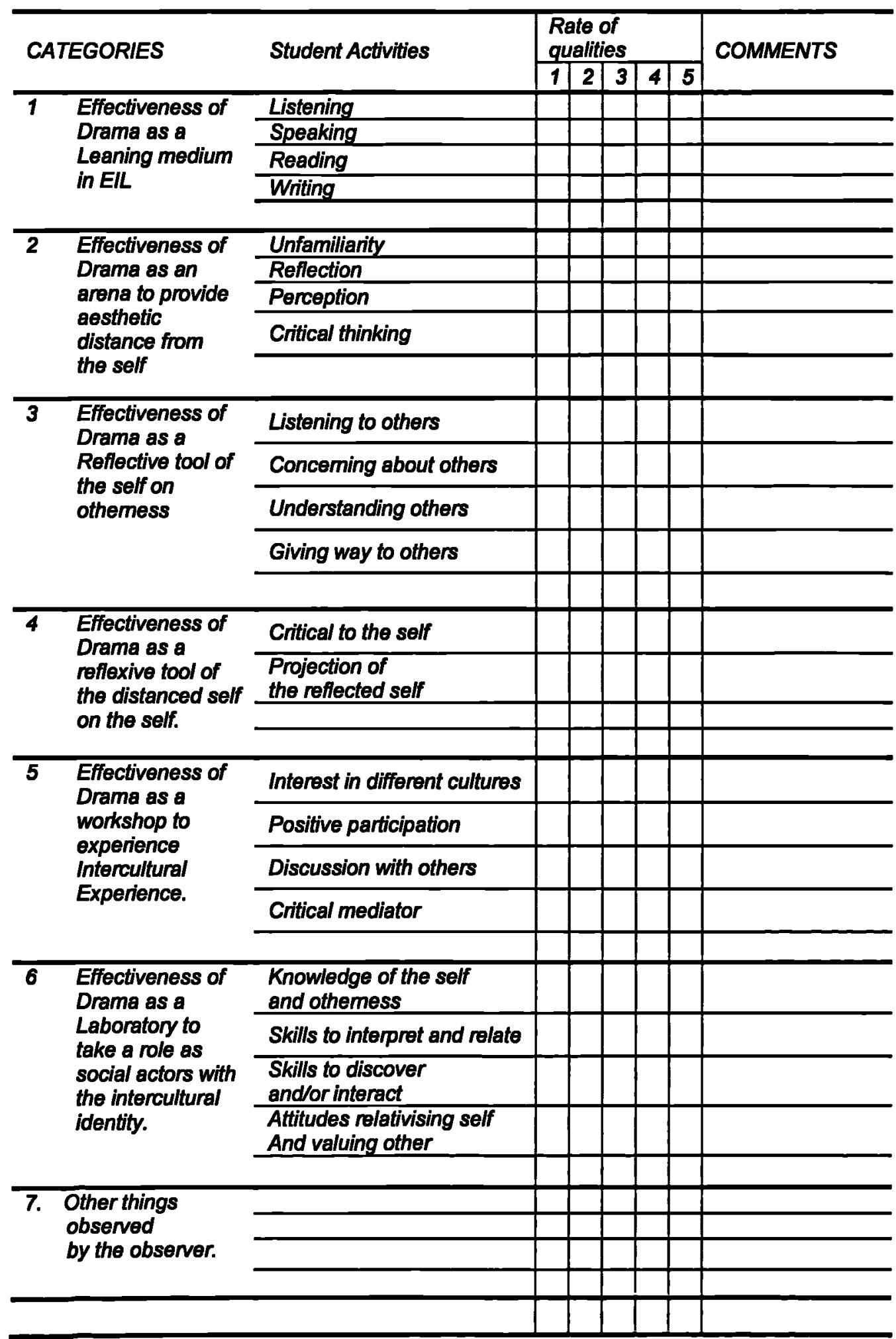




\section{Appendix D: Drama Sessions in the Study}

DRAMA SESSIONS IN THE STUDY

\begin{tabular}{|c|c|c|c|c|}
\hline $\begin{array}{l}\text { Pe- } \\
\text { riod }\end{array}$ & Titles & Objectives & Content & $\begin{array}{l}\text { Methods } \\
\text { (Including Polaroid } \\
\text { Camera) }\end{array}$ \\
\hline \multirow[t]{2}{*}{$\begin{array}{c}\text { Week } \\
1\end{array}$} & $\begin{array}{l}\text { Get known to } \\
\text { each other }\end{array}$ & $\begin{array}{l}\text { To communicate } \\
\text { with each other } \\
\text { To understand } \\
\text { non-verbal } \\
\text { language }\end{array}$ & $\begin{array}{l}\text { To get intimate with other } \\
\text { participants } \\
\text { To understand the figures which I } \\
\text { brought }\end{array}$ & $\begin{array}{l}\text { Still-Image } \\
\text { Five-level exercise }\end{array}$ \\
\hline & $\begin{array}{l}\text { Impression of } \\
\text { a New Boy }\end{array}$ & $\begin{array}{l}\text { Understanding } \\
\text { otherness }\end{array}$ & $\begin{array}{l}\text { Social inclusion through a new boy } \\
\text { in the school }\end{array}$ & $\begin{array}{l}\text { Questioning in role } \\
\text { Still-image }\end{array}$ \\
\hline \multirow[t]{2}{*}{$\begin{array}{l}\text { Week } \\
2\end{array}$} & Face to face & $\begin{array}{l}\text { Verbal language } \\
\text { through drama }\end{array}$ & $\begin{array}{l}\text { Duologues in the British context: } \\
\text { developing roles }\end{array}$ & $\begin{array}{l}\text { Simple } \\
\text { presentation }\end{array}$ \\
\hline & $\begin{array}{l}\text { What is } \\
\text { culture like? }\end{array}$ & $\begin{array}{l}\text { To understand } \\
\text { culture and cultural } \\
\text { things }\end{array}$ & $\begin{array}{l}\text { To introduce image of an iceberg } \\
\text { as the image of culture }\end{array}$ & Group work \\
\hline \multirow[t]{2}{*}{$\begin{array}{l}\text { Week } \\
3\end{array}$} & $\begin{array}{l}\text { Passport } \\
\text { game }\end{array}$ & $\begin{array}{l}\text { To understand } \\
\text { different cultures }\end{array}$ & $\begin{array}{l}\text { To create new cultures and to act } \\
\text { out in that cultural context: cartoon }\end{array}$ & $\begin{array}{l}\text { Questioning- in } \\
\text { role } \\
\text { Role-play }\end{array}$ \\
\hline & $\begin{array}{l}\text { American in } \\
\text { England }\end{array}$ & $\begin{array}{l}\text { To understand } \\
\text { intercultural } \\
\text { interaction }\end{array}$ & $\begin{array}{l}\text { Setting up intercultural situations: } \\
\text { American-English, American- } \\
\text { Japanese }\end{array}$ & Role-play \\
\hline \multirow[t]{2}{*}{$\begin{array}{l}\text { Week } \\
4\end{array}$} & $\begin{array}{l}\text { Meeting that } \\
\text { Wasn't }\end{array}$ & $\begin{array}{l}\text { To understand } \\
\text { intercultural } \\
\text { interaction }\end{array}$ & $\begin{array}{l}\text { International business meeting in } \\
\text { Japan (American-Japanese) }\end{array}$ & $\begin{array}{l}\text { Dramatisation } \\
\text { (Interpretation of a } \\
\text { story) }\end{array}$ \\
\hline & $\begin{array}{l}\text { Meeting that } \\
\text { Wasn't }\end{array}$ & $\begin{array}{l}\text { To understand } \\
\text { intercultural } \\
\text { interaction through } \\
\text { drama }\end{array}$ & $\begin{array}{l}\text { Intemational business meoting in } \\
\text { Japan (American-Japanese) } \\
\text { "participants spoke lines in English } \\
\text { as well as in Japanese in the } \\
\text { performance. }\end{array}$ & $\begin{array}{l}\text { Mantle of the } \\
\text { expert } \\
\text { Thought-tracking } \\
\text { Performance } \\
\text { Discussion }\end{array}$ \\
\hline \multirow[t]{2}{*}{$\begin{array}{l}\text { Week } \\
5\end{array}$} & Two friends & $\begin{array}{l}\text { To understand } \\
\text { intercultural } \\
\text { communication } \\
\text { through drama }\end{array}$ & $\begin{array}{l}\text { An American asks a Korean to go } \\
\text { to the movies }\end{array}$ & $\begin{array}{l}\text { Improvisation } \\
\text { Discussion }\end{array}$ \\
\hline & $\begin{array}{l}\text { Chit-chat } \\
\text { Dinner with a } \\
\text { Korean } \\
\text { Family }\end{array}$ & $\begin{array}{l}\text { To understand } \\
\text { intercultural } \\
\text { interaction through } \\
\text { drama }\end{array}$ & $\begin{array}{l}\text { Conversation in the workplace: } \\
\text { (American-Japanese) } \\
\text { Dinner dialogues: } \\
\text { (American-Korean) }\end{array}$ & $\begin{array}{l}\text { Improvisation } \\
\text { Still-image } \\
\text { Discussion }\end{array}$ \\
\hline \multirow[t]{2}{*}{$\begin{array}{l}\text { Week } \\
6\end{array}$} & War Paint & $\begin{array}{l}\text { To understand } \\
\text { intercultural } \\
\text { interaction }\end{array}$ & $\begin{array}{l}\text { Structuring the play } \\
\text { (Conflict between parents and their } \\
\text { teenage daughter) } \\
\text { Rehearsals }\end{array}$ & $\begin{array}{l}\text { Script analysis } \\
\text { Reading play }\end{array}$ \\
\hline & WarPaint & $\begin{array}{l}\text { To understand } \\
\text { intercultural } \\
\text { interaction }\end{array}$ & Rehearsal and dress-rehearsal & $\begin{array}{l}\text { Public } \\
\text { performance }\end{array}$ \\
\hline $\begin{array}{l}\text { Week } \\
7\end{array}$ & Review & $\begin{array}{l}\text { To understand } \\
\text { drama sessions as } \\
\text { both participants } \\
\text { and researchers }\end{array}$ & $\begin{array}{l}\text { After the public performance } \\
\text { Observing all drama sessions }\end{array}$ & $\begin{array}{l}\text { Observation } \\
\text { Discussion }\end{array}$ \\
\hline $\begin{array}{l}\text { Week } \\
8\end{array}$ & Publication & $\begin{array}{l}\text { To evaluate their } \\
\text { project }\end{array}$ & $\begin{array}{l}\text { Publishing a booklet } \\
\text { As researchers }\end{array}$ & $\begin{array}{l}\text { Mantle of the } \\
\text { expert }\end{array}$ \\
\hline
\end{tabular}




\section{Appendix E: Photographs of Drama Activities}

"The following photographs were taken during drama sessions by the researcher and a student participant.

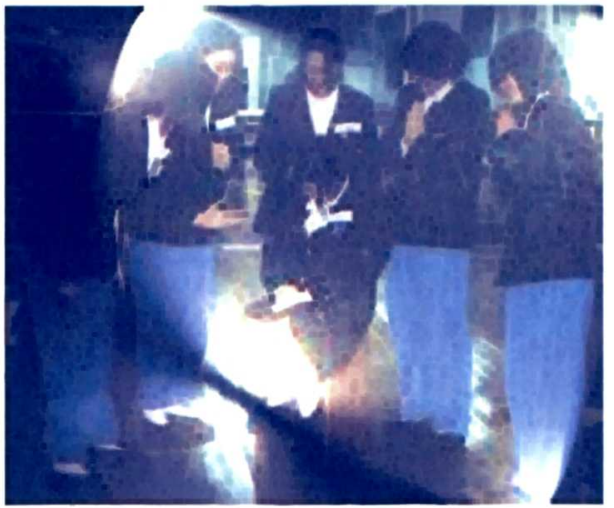

This drama activity aimed at understanding what a Newcomer's feeling was like through a poem, 'Impression of a New Boy.' A group made a positive still-image about how they act in that situation. A student, who was sitting on a chair, was a new boy in this context. [Drama activity in the first week, related to 5.1.1].

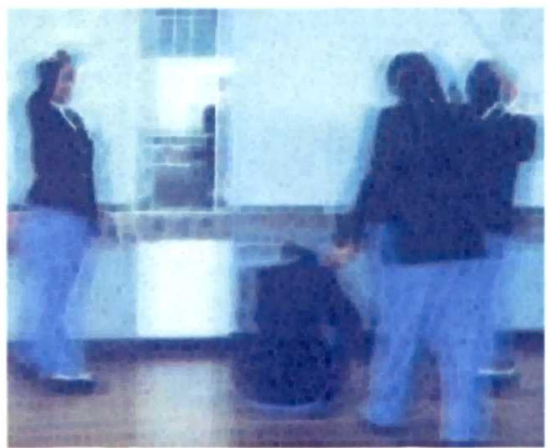

The left photograph, which is very different from the above still-image, shows a negative image. The new boy was bullied by other students. This activity is related to sociocultural inclusion.

[Drama activity in the first week, related to 5.1.1].

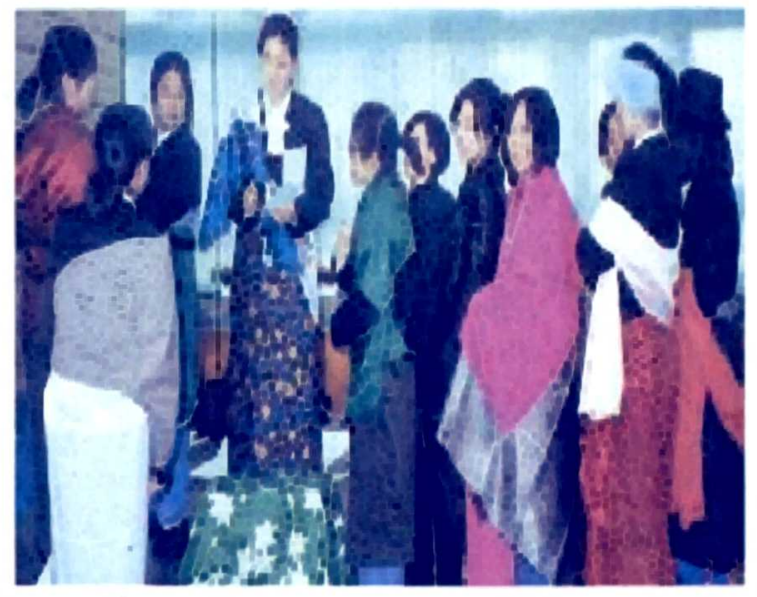

This scene was a part of a drama event, Passport Game, in which participants took immigration procedures in order to travel other countries. A participant in the centre was in a key role as an immigration officer.

Before this activity, each group created their new cultures and new countries. And they were engaged in an intercultural context through the "Magic If" and tried to understand what could happen there.

[Drama activity in the third week, related to 5.2.1, 6.3.1, and 6.3.2]. 


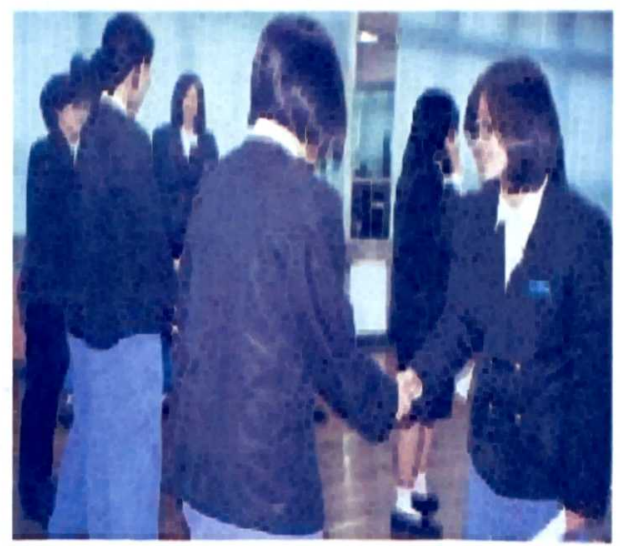

This still-image was made about how the English people greet in general contexts. Participants had an Impression that the English people like to behave themselves in a formal way and seem to be conservative. [Drama activity in the third week, related to 5.3.1].

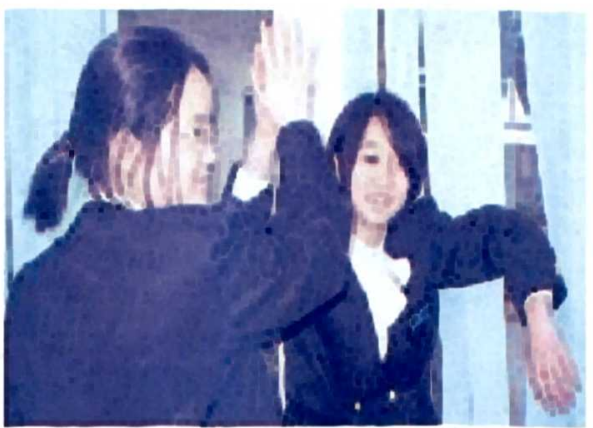

The left image is contrary to the above. This still-image was presented about how Americans greet in general contexts. Participants had an impression that Americans seem to behave themselves in an informal way and seem to feel freely. [Drama activity in the third week, related to 5.3.1].

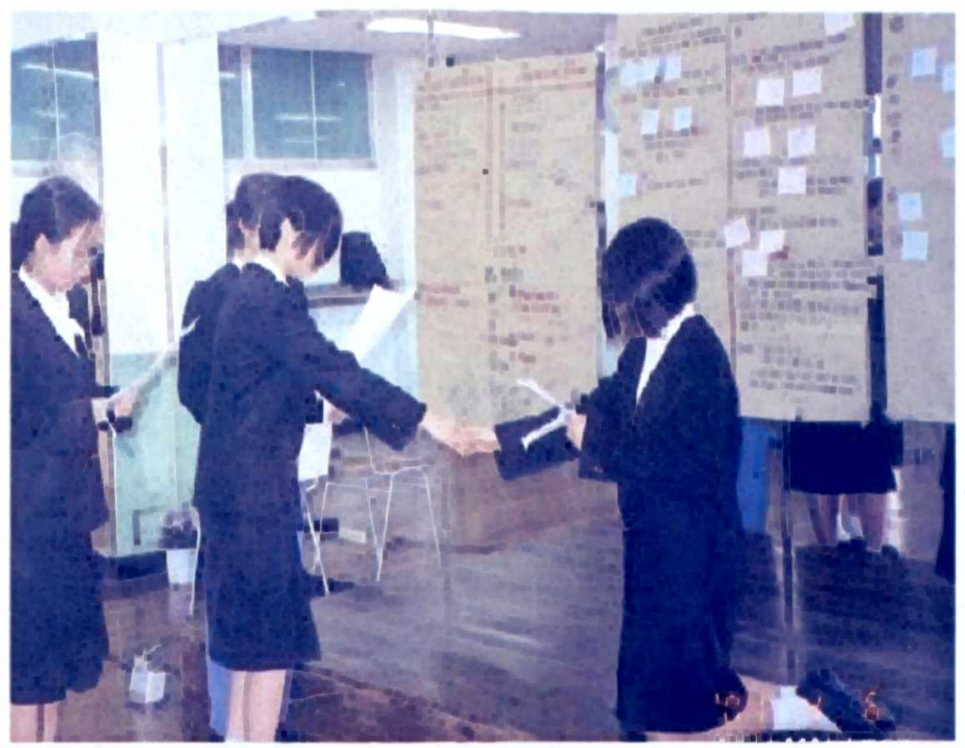

The above is a scene wherein participants performed Intemational business meeting. Before this drama activity, they analysed an extract of an international business meeting between a Japanese company and an American company, dramatised that extract, and did drama activity of thought-tracking, a drama technique.

A participant in the right was in role as a president of the American company.

And participants wrote their thoughts on the pink and blue notepads behind them.

[cf. Japanese's thoughts were on the pink notepads and American's thoughts were on the blue notepads].

[Drame activity in the fourth week, related to 6.3.2 and 6.4.2, Figure 6.5, and Networks 6.11-6.15. 


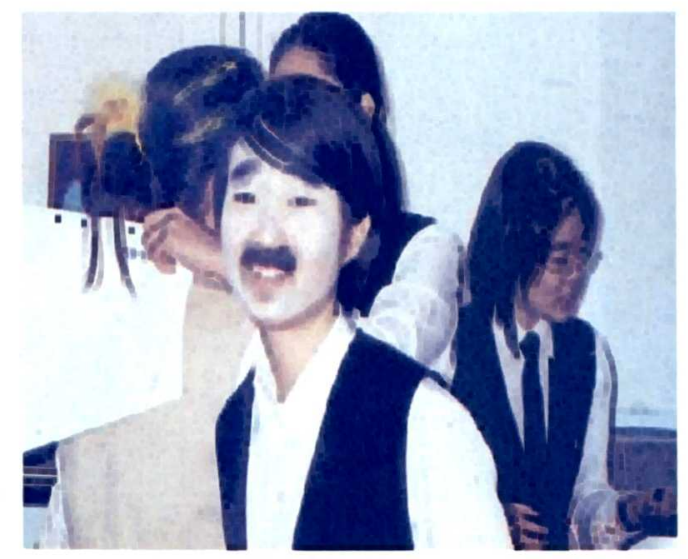

Participants were preparing for their dress rehearsal. They were going to perform a short play, War Paint, which is a teenager story to deliver a relationship between a mother and her daughter in an English context. In the whole process of this drama event, participants encouraged each other to overcome their difficulties in speaking English lines and acting.

[Drama activity in the sixth week, related to 6.2.2 and 6.3.2.

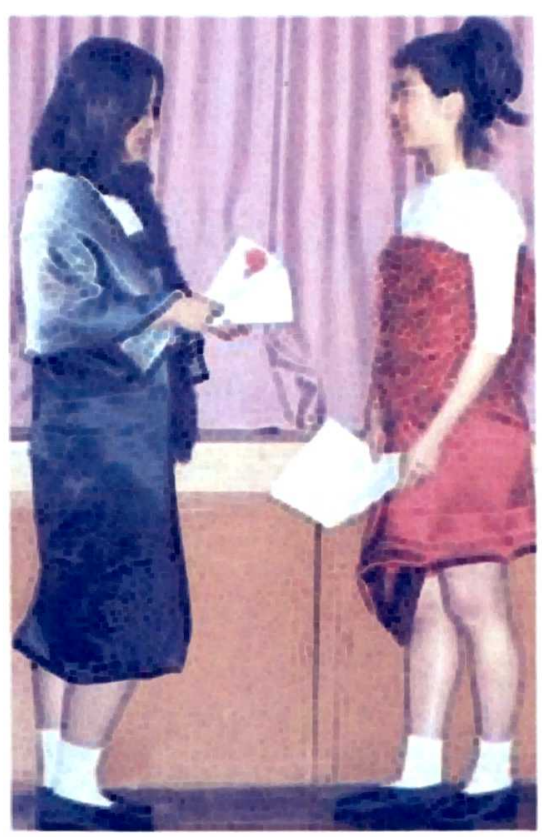

In this scene two teenagers were talking about their situation in a discotheque in the late evening. A participant in the left was double cast with another participant in the left in the below.

The participant in black was concentrated on speaking her English lines well; meanwhile the participant in red was focused on her acting.

[Drama activity in the sixth week, related to 5.1.1, 5.3.1, 6.1.1, 6.1.2, 6.2.2, 6.3.2, and Table 5.1]

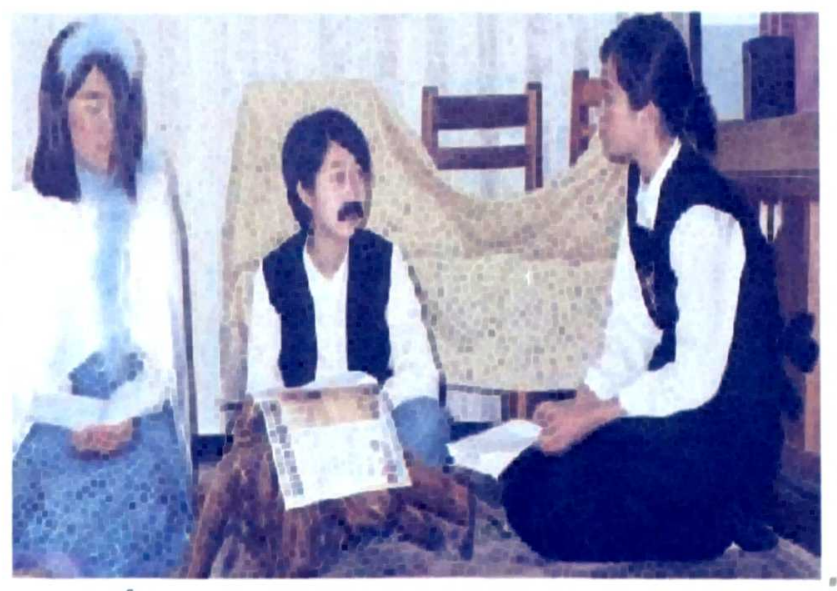

Throughout this performance, participants compared the English context with their Korean context and understood teenager's culture and motherhood in their ICM between English and Korean cultural values. Participants were in roles of the teenager, father, and mother [from the left]. [Drama activily in the sixth week, related to 5.3.1, 6.1.1, 6.1.2, Tables 5.1 and 6.1]. 


\section{Appendix F: Previous Researches on the Effectiveness of Drama Education}

\section{Previous Researches on the effEctiveness of Drama Education}

\begin{tabular}{|c|c|c|}
\hline $\begin{array}{l}\text { Research } \\
\text { Categories }\end{array}$ & $\begin{array}{l}\text { Research } \\
\text { Sub-categories }\end{array}$ & Research studies \\
\hline \multirow[t]{3}{*}{$\begin{array}{l}\text { Drama and } \\
\text { education }\end{array}$} & Content of drama & $\begin{array}{l}\text { Hornbrook (1991; 1998a); } \\
\text { Lacey and Woolland (1992); } \\
\text { Harland et al. (1998) }\end{array}$ \\
\hline & Form of drama & $\begin{array}{l}\text { Neelands (1992); } \\
\text { O'Neill (1995); } \\
\text { Lyle (1996); } \\
\text { Jewitt et al. (2001) }\end{array}$ \\
\hline & $\begin{array}{l}\text { Integrated drama } \\
\text { [content and form] }\end{array}$ & $\begin{array}{l}\text { Fleming (2001a); } \\
\text { Conquergood (2002) }\end{array}$ \\
\hline \multirow[t]{6}{*}{$\begin{array}{l}\text { Drama for } \\
\text { education }\end{array}$} & $\begin{array}{l}\text { Language and } \\
\text { Literature education }\end{array}$ & $\begin{array}{l}\text { Goalen (1996); } \\
\text { Robbins (1998); } \\
\text { Waldschmidt (1998); } \\
\text { Schneider and Jackson (2000) }\end{array}$ \\
\hline & $\begin{array}{l}\text { Personality } \\
\text { development and } \\
\text { Moral education }\end{array}$ & $\begin{array}{l}\text { O'Sullivan and Gray (1998); } \\
\text { Basourakos (1999); } \\
\text { Winston (1998; 1999); } \\
\text { Wagner (1999a): } \\
\text { Wright (2000) }\end{array}$ \\
\hline & Socio-cultural skills & $\begin{array}{l}\text { Buege (1991); } \\
\text { Timothy (1991); } \\
\text { Briggs (1992); } \\
\text { Abbs (1994b); } \\
\text { Holsbrink-Engels (1997); } \\
\text { Goldie (1999); } \\
\text { Grossman (1999); } \\
\text { Moss (2000) }\end{array}$ \\
\hline & Intercultural education & $\begin{array}{l}\text { Rubin and Ruffin (1993); } \\
\text { Elkins (1997); } \\
\text { Ackroyd (1999); } \\
\text { Byram and Fleming (1998); } \\
\text { Schewe (1993; 1998), } \\
\text { Greenwood (2001); } \\
\text { Day (2002) }\end{array}$ \\
\hline & Management training & Goddard (1999); \\
\hline & Health education & $\begin{array}{l}\text { Ball (1993) [including personal and social } \\
\text { education]; } \\
\text { Peter (1994; 1995); } \\
\text { Slade (1998); } \\
\text { Harvey and Tony (2000) }\end{array}$ \\
\hline $\begin{array}{l}\text { Drama for } \\
\text { research }\end{array}$ & Research method & $\begin{array}{l}\text { Swartz (1998); } \\
\text { Mienczakowski and Morgan (2001) }\end{array}$ \\
\hline
\end{tabular}




\section{Appendix G: How to Read Networks of ATLAS.ti}

ATLAS.ti was produced on the basis of grounded theory analysis. Thomas Muhr (1997), the developer of this computer programme, mentioned that he had consulted on the methods of this programme with Anselm Strauss at the beginning of producing the programme.

According to GTA (the grounded theory analysis), we have three different codes to make, open, axial, and selective, in analysing the data. Open codes are made in doing microanalysis through careful scrutiny of data, line by line. After this coding, we make another two sorts of codesaxial and selective. Axial coding relates categories to their subcategories depending on the level of properties and dimensions; meanwhile selective coding integrates and refines the theory through organising categories around a central explanatory concept.

In the programme, ATLAS.ti we produce codes by open coding, similar to a sort of open codes at the level of the microanalysis in GTA, and super-codes, super-super-codes, and networks, which are similar to axial and selective codes in GTA.

A code is usually a short piece of data, textual, graphic, audio, or video, and may reference many pieces. Also, codes may carry a comment, describing their meanings in further detail or describe how they should be used in coding.

We take another process to group codes, that is, to relate codes to make networks, which are used for conceptual theory building. The nodes in these networks are usually codes, which are linked with specifiable relations to form semantic networks. (See the below table of Code-Code Relations, presented in the study)

There are networks illustrated with detailed explanation in the next pages.

Code-Code Relations

\begin{tabular}{|c|c|c|c|c|}
\hline Intemal ID & $\begin{array}{l}\text { Symbol } \\
1\end{array}$ & $\begin{array}{l}\text { Symbol } \\
2\end{array}$ & Menu text & $\begin{array}{l}\text { Formal } \\
\text { property }\end{array}$ \\
\hline ACTION & $>n$ & AC & does & Transitive \\
\hline AT & ++ & $\overline{\mathrm{AT}}$ & gets advantage of & Transitive \\
\hline ASSO & $=0$ & $\overline{\mathbf{R}}$ & Is associated with & Symmetric \\
\hline BTP & $\overline{0}$ & $\bar{G}$ & Is a part of & Transitive \\
\hline CAUSE & $\Rightarrow$ & $\mathbf{N}$ & causes & Transitive \\
\hline CHARCT & $\%$ & $\mathrm{CH}$ & Is characteristic of & Symmetric \\
\hline CONSID & $?$ & $\mathbf{T}$ & considers & Transitive \\
\hline CONTRA & $<$ & $\bar{A}$ & contradicts & Asymmetric \\
\hline CTRAST & $><$ & CTS & contrasts & Asymmetric \\
\hline DIALOG & ?! & D & has a dialogue with & Transitive \\
\hline ISA & $=$ & 0 & Is a & Transitive \\
\hline PLAN & $\# ?$ & $\overline{\mathbf{P L}}$ & plans & Transitive \\
\hline PROP & 3 & $\overline{\mathbf{P}}$ & Is a property of & Asymmetric \\
\hline RE-ACT & $\gg x$ & $\mathbf{R A}$ & reacts & Transitive \\
\hline REFLEX & ]$:[$ & $\bar{M}$ & reflects & Symmetric \\
\hline RESOLV & $=! !$ & $\overline{\mathbf{R S}}$ & resolves & Transitive \\
\hline WONDER & ??? & $\overline{\mathbf{W}}$ & is wondering & Transitive \\
\hline
\end{tabular}




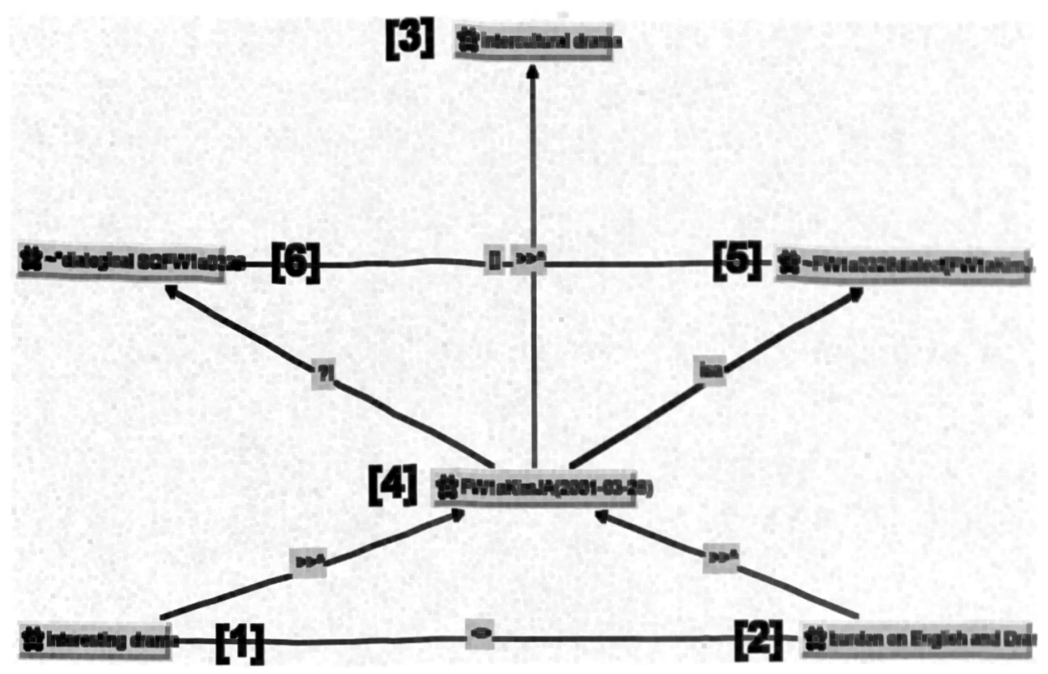

Notwork on Dialectical SQ06FWMa328 by the Semantic Layout

A network places its nodes within the window using the semantic layout algorithm. This algorithm places the nodes into optimal positions using an invisible matrix of default positions, for instance, the nodes with the highest connectivity into centre positions, and the rest of the nodes using neighbouring positions of the first node placed (Muhr 1997).

The network above has four codes or nodes (11] [4]), codes, shown as yellow nodes, and [ [] and [6] super codes, shown red nodes: [1] interesting drama, [2] burden on English and Drama, [3] intercultural drama, [4] FW1aKimJA (2001-03-28), [5] FW1a0328dialect [FW1aKimJA], and [6] "dialogical SQFWIaKimJA.

They are related to each other as follows [cf. the table of Code-Code relations on the previous page]:

- Burden on English reacts (>>A) FW1aKimJA(2001-03-28).

- Interesting drama reacts ( $>0$ ) FW1aKimJA(2001-03-28).

- Interesting drama contradicts $(\diamond)$ burden on English and Drama.

- FWIaKimJA(2001-03-28) reacts (>>n) intercultural drama.

- FWIaKimJA(2001-03-28) is (isa) FW1a0328dialect [FW1aKimJA].

- FW1aKimJA(2001-03-28) has dialogue with (?1) "dialogical SQFW1aKimJA.

- -FW1a0328dialect [FW1aKimJA] is a part of (II) -"dialogical SQFW1aKimJA.

Regarding code relations shown in the network on -FWIa0328dialect [FW1aKimJA], I interpreted this conceptual network as follows:

KimJA had difficulties in doing drama and expressing herself in English because of participating in the EDIE project at the first stage. Nevertheless, she was interested in drama. In this contradictory situation, she made dialectical resolution to continue attending the project covering intercultural drama. Her dialectical resolution, at the end, led her to dialogical thinking. 


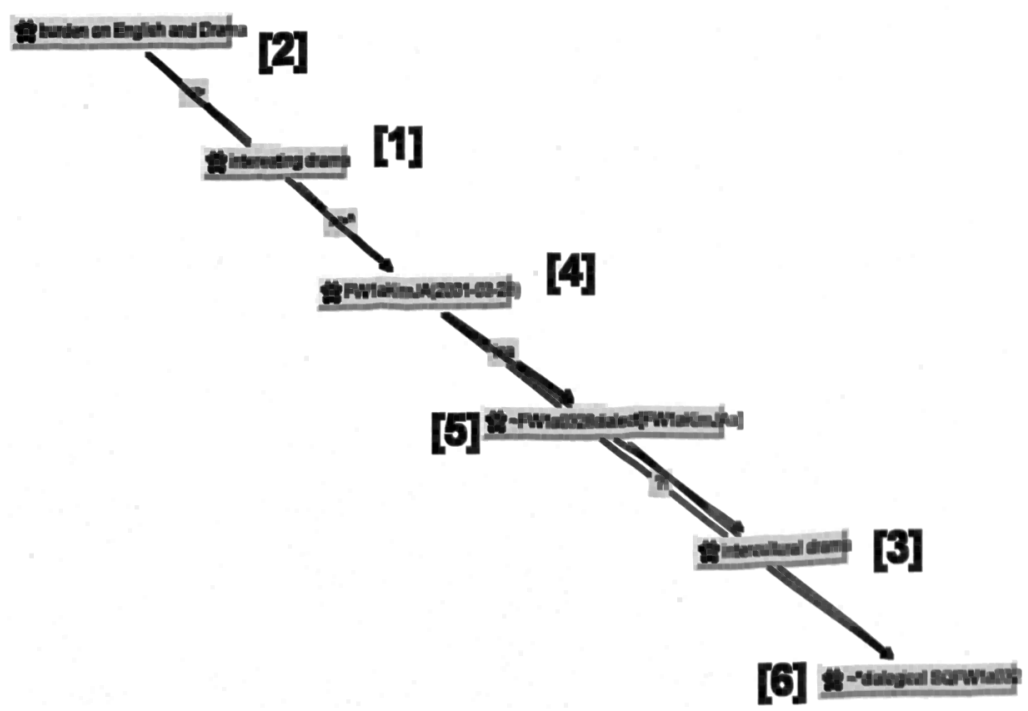

Network on Dialectical SQ06FWIa0328 by the Topological Layout

The network above was produced from a topological perspective on the previous network on Dialectical SQ06FW1a0328, which had a semantic layout.

This sort of topological layout creates a linear list of nodes positioned from the upper left to the lower right. This linear sequence is 'the result of a depth first traversal of the graph....a node with the least dependencies is made the first node positioned in the upper left corner, and the node with the most dependencies on other nodes is positioned in the lower right comer' in the window (Muhr 1997: 153).

With regard to this, I Interpreted the network above with the topological layout as follows:

Although KImJA had difficulties in doing drama and expressing herself in English, she would like to participate in intercultural drama. The dialogicality of drama, above all, led her to the participation.

Thus, networks are more sophisticated than code families: the former allows a strong structure, which provides meaningful semantic relationships between elements through relationships of code-code, code-memo, memo-quotation, and so on, whereas the latter treats sets of elements as similar. Therefore, networks show the relations of concepts, that is, those relations are viewed as role indicators for concepts.

With respect to the above, I shall take specific networks, Networks 6.9 and 6.10, which were presented in 6.3.2.2, and describe how we can read them. 


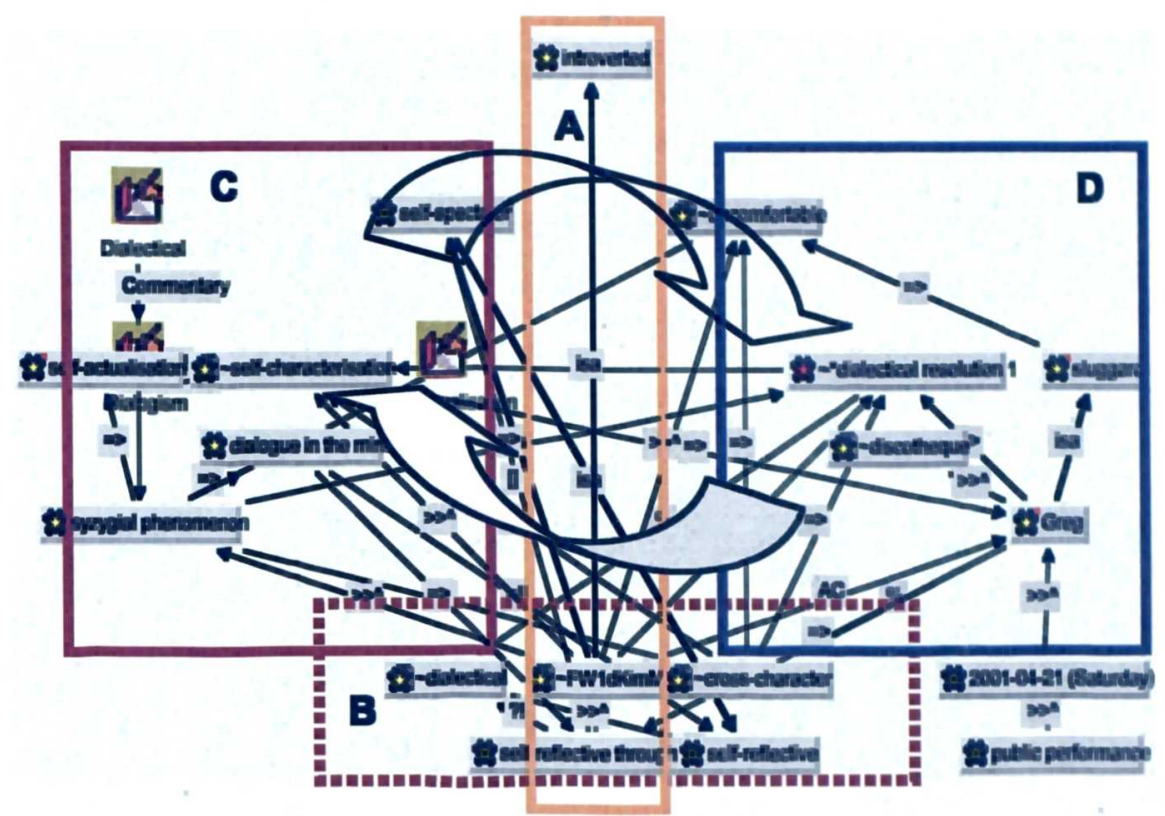

Network 6.9 Greg [Cross-Character to KimMS] by the Semantic Layout

According to my analysing relations of the data, which were put into ATLAS.ti, the software analysed them and laid out the above network on the concept of 'Greg [Cross-Character to KImMS].' This network is focused on a code, 'cross-character to KimMS' and is concerned with the context where KimMS took the role of Greg, whose character was different from herself. All codes and memos in the network are related to the code of 'cross-character to KimMS,' that is, its concept of how KimMS characterised her cross-character, Greg.

The location of each code or memo indicates how the code or memo are relevant to other codes or memos, that is, other concepts between eighteen codes (yellow nodes), one supercode (a red node), and three memos (shown as memo icons). That is to say, depending on how close they are or If they share with one another or other concepts I can divide this network into four conceptual areas, Square A, Square B, Square C, and Square D, and interpret them as follows:

[1] Square A covers the factual situation of who she was and what she wasKImMS was an introverted participant in the EDIE project.

[2] Square $B$ is related to her given situation of what she should do in the project-KImMS was cast as Greg, whose character was different from hers and had to give the public performance on 21 April 2003.

[3] Square C is relevant to inside situations of herself where she was concerned with how she was handling the dialectical condition, performing the cross-character for herself-KimMS met a syzygial phenomenon, which is like the combination of two feet into a single metrical unit, between her own character and Greg's character and had dialogical thoughts of selfactualisation and self-characterisation with the character, Greg.

[4] Square D shows situations outside herself before and during the performance-KimMS felt uncomfortable in rehearsing her cross-character, Greg but she resolved to act the character, Greg, who was a sluggard. 
Another thing is to be considered in the network is implied-the dynamics between Areas A, B, C, and D, particularly between Areas C and D. Let me take the concept of Self-Concept. KimMS observed herself in her mind while she was acting out her cross-character and kept on her dialogues between Areas $C$ and $D$.

According to this network, I found how KImMS carried out ICM in her situations and I discussed 'Otherness: Introversive Dialogicality—Self-Reflection of Performers through Characters in the play, War Paint in 6.3.2.2

Network 6.10 can be also analysed in a similar way, although it shows a different conceptual structure-'outsidedness: extroversive and introversive dialogicality—reflexivity of participants in drama.'

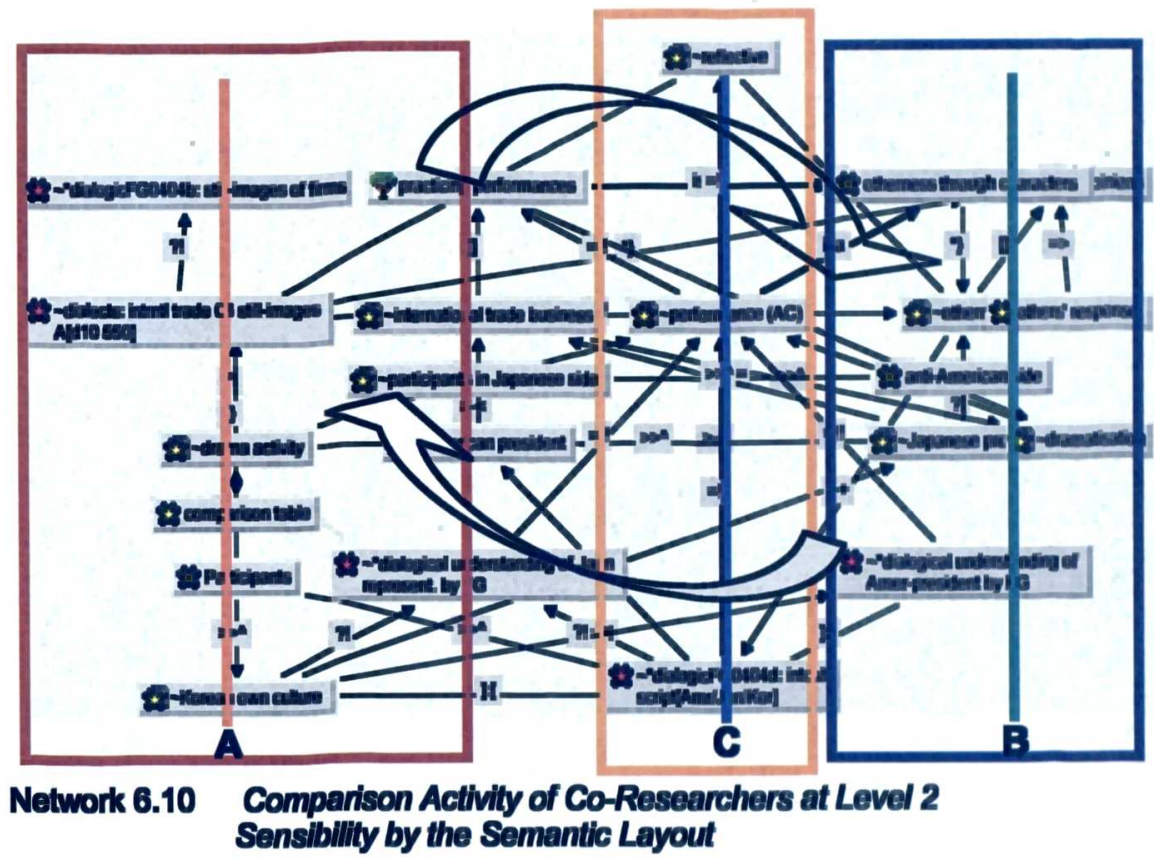

As I discussed earlier, locations of codes indicate what they mean in the above network. I focused on three conceptual areas, Square A, Square B, and Square C, and interpret them as follows:

[1] Square $A$ covers visible drama activities in the drama session which participants did as performers - participants were involved in the drama activities by analysing and practising intercultural siftuations of an Intemational Business Meeting.

[2] Square $B$ is related to those drama activities but participants were concerned with them as researchers-participants were distant from the performer's position, that is, outside the performer. That is to say, they had critical thinking about those intercultural situations, in other words, intercultural imagination.

[3] Square $C$ is about how participants performed in the intercultural situations through dialogical reflection between Squares $A$ and $B$, that is, between inside themselves as performers and outside themselves as researchers. 
In this regard, participants, as performers as well as co-researchers, were engaged in their drama activities through outsidedness: extroversive and introversive dialogicality, as I discussed in 6.3.2.3.

As indicated above, we need to have some knowledge of ATLAS.ti in order to interpret this sort of network and to find theoretical points just the same as we need to know statistics relevant to SPSS in interpreting tables produced through that software. Moreover, in the end, the better a researcher has theoretical sensitivity, the better he/ she achieves trustworthiness in his/her research. 